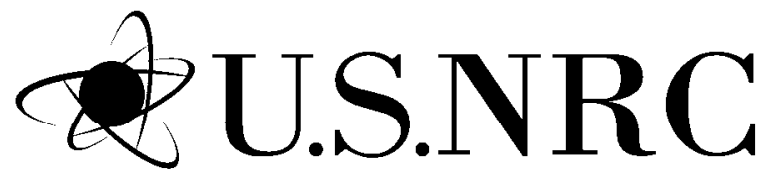

United States Nuclear Regulatory Commission

$\overline{\text { Protecting People and the Environment }}$

NUREG/CR-7127

ANL/EVS/TM/11-5

\title{
New Source Term Model for the RESRAD-OFFSITE Code Version 3
}





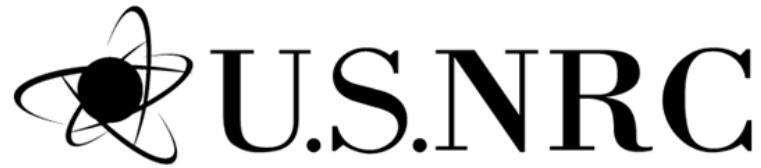

NUREG/CR-7127

ANL/EVS/TM/11-5

United States Nuclear Regulatory Commission

Protecting People and the Environment

\section{New Source Term Model for the RESRAD-OFFSITE Code Version 3}

Manuscript First Draft: September 2011

Manuscript Revision Completed: May 2013

Date Published: June 2013

Prepared by

C. Yu, E.K. Gnanapragasam, J.-J. Cheng, S. Kamboj, and S.Y. Chen

Argonne National Laboratory

9700 South Cass Avenue

Argonne, IL 60439

John Randall, NRC Project Manager for the First Draft

Wendy Reed, NRC Project Manager for the Revision

NRC Job Code N6731 (First Draft) \& V6360 (Revision) 



\section{Abstract}

This report documents the new source term model developed and implemented in Version 3 of the RESRAD-OFFSITE code. This new source term model includes: (1) "first order release with transport" option, in which the release of the radionuclide is proportional to the inventory in the primary contamination and the user-specified leach rate is the proportionality constant, (2) "equilibrium desorption release" option, in which the user specifies the distribution coefficient which quantifies the partitioning of the radionuclide between the solid and aqueous phases, and (3) "uniform release" option, in which the radionuclides are released from a constant fraction of the initially contaminated material during each time interval and the user specifies the duration over which the radionuclides are released. The source term model implemented in RESRADOFFSITE Version 2 computes the release of radionuclides from the contaminated zone, as well as the concentration and distribution of the radionuclides within the contaminated zone. The model calculates the atmospheric release of particulates due to resuspension and that of volatiles due to diffusion and evapotranspiration, the surface water release due to erosion by runoff water, and the groundwater release due to leaching by infiltrating water. The release rates are used by the code to compute the transport of contaminants and exposure at offsite locations, whereas the concentration and distribution of radionuclides within the contaminated zone are used to compute the direct external exposure from the primary contamination, as well as any exposure from onsite activities. The newly developed source term models for RESRADOFFSITE code Version 3 also consider these releases and distribution in the primary contaminated zone. The new release mechanisms are not limited to treating soil materials as the source (primary contamination). Furthermore, the new release mechanisms are also not limited to uncontainerized sources because the new source release models assume that the releases are from a distinct phase of source material; once released into surrounding soils, they are transported in the soil in the region of primary contamination. The new source release models implemented in RESRAD-OFFSITE code Version 3 are compared with DUST-MS code and the GoldSim software. The results of comparison are presented and discussed in the report. A detailed description of how to conduct sensitivity and probabilistic analyses using RESRAD-OFFSITE code is presented, and the results of using sensitivity and probabilistic analyses on the new source release input parameters are discussed. The revisions and additions to Version 3.1 included a time-delay feature for the source release mechanism. This time-delay feature provides the ability to model scenarios where the release of radionuclides is delayed and/or distributed over time. The "uniform release" option of Version 3.0 beta is now part of the time-distributed "equilibrium desorption release" option. The time-distributed, firstorder, rate-controlled release is an additional option available in Version 3.1. Another new feature in Version 3.1 facilitates the calculation of area factors for small areas of elevated 
activity. This area factor calculation was performed using the probabilistic analysis feature of the RESRAD-OFFSITE code.

Paperwork Reduction Act Statement

This NUREG does not contain information collection requirements and, therefore, is not subject to the requirements of the Paperwork Reduction Act of 1995 (44 USC 3501, et seq.).

Public Protection Notification

The NRC may not conduct or sponsor, and a person is not required to respond to, a request for information or an information collection requirement unless the requesting document displays a current valid Office of Management and Budget control number. 


\section{Contents}

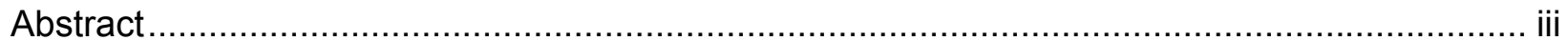

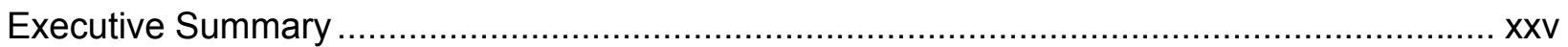

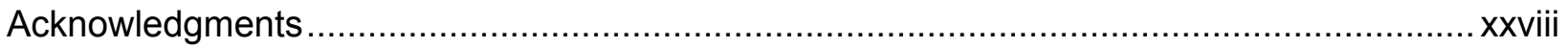

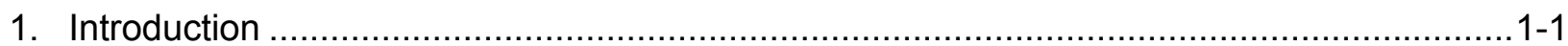

1.1 Overview of the Source Release Model in RESRAD-OFFSITE Version 2...............1-1

1.2 Considerations for Expanding the Source Term Model.......................................... 1-2

1.3 The Proposed Approaches and the Implemented Source Term Models ...................1-3

1.4 Area Factors for Offsite Exposure Scenarios ...................................................... 1-4

2. Primary Contamination and the Source Term ...................................................... $2-1$

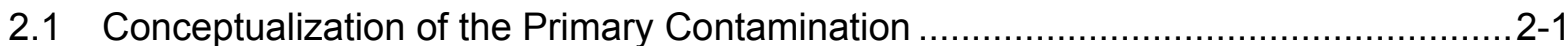

2.2 Derivation of Mathematical Expressions for the Conceptual Primary

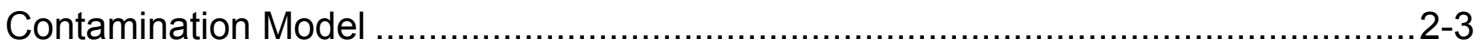

2.2.1 Thickness of the Primary Contamination ............................................. $2-3$

2.2.2 Concentration of Radionuclides in the Primary Contamination ......................2-5

2.2.2.1 Concentration of Radionuclides in the Primary Contamination under the First Order Release Model ........................................ 2-5

2.2.2.2 Concentration of Radionuclides in the Primary Contamination under the Uniform Release Model ............................................2-6

2.2.2.3 Concentration of Radionuclides in the Primary Contamination under the Equilibrium Desorption Model .......................................2-8

2.2.3 Surface Soil Mixing Model .............................................................. $2-8$

2.2.3.1 Density of Soil in the Mixing Zone .............................................. 2-9

2.2.3.2 Volume Fraction of Soil from the Primary Contamination in the Mixing Zone ........................................................................ 2-10

2.2.3.3 Concentration of Radionuclides in the Surface Soil above the Primary Contamination ......................................................... 2-12

2.2.3.4 Three-Layer Model ................................................................. 2-12

2.2.4 Release by Surface Runoff................................................................. $2-13$

2.2 .5 Release to Groundwater...................................................................... $2-14$

2.2.5.1 Transfer of Radionuclides to Soil Moisture under the First Order Release Model ....................................................... 2-14

2.2.5.2 Transfer of Radionuclides to Soil Moisture under the Uniform Release Model..................................................................... 2-15 


\section{Contents (Continued)}

2.2.5.3 Transport of Radionuclides from the Point of Transfer to the Bottom of the Primary Contamination for the Rate-Controlled Release Options ................................................................. 2-16

2.2.5.4 Release of Radionuclides to Groundwater under the Equilibrium Desorption Model.................................................2-16

2.2.6 Release to the Atmosphere in the Form of Dust .................................. 2-17

2.3 Implementation of the Models for the Primary Contamination ............................... 2-17

2.3.1 Shape and Dimensions of the Primary Contamination ...............................2-17

2.3.2 Thicknesses of the Primary Contamination, Cover, Clean Cover, Contaminated Mixing Zone, and Undisturbed Primary Contamination...........2-18

2.3.3 Concentration of Radionuclides in the Primary Contamination .....................2-19

2.3.3.1 Concentration of Radionuclides in the Primary Contamination under the First-Order Release Model .......................................2-19

2.3.3.2 Concentration of Radionuclides in the Primary Contamination under the Uniform Release Model ........................................2-20

2.3.4 Depth of Penetration of the Mixing Zone ..............................................2-20

2.3.5 Density of Soil in the Mixing Zone ...................................................... 2-21

2.3.6 Volume Fraction of Soil from the Primary Contamination in the Mixing

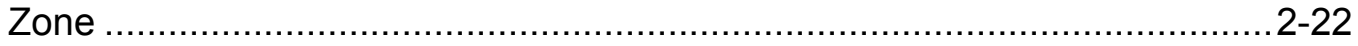

2.3.7 Concentration of Radionuclides in the Surface Soil above the Primary Contamination ..................................................................................... 23

2.3.8 Release by Surface Runoff........................................................... $2-23$

2.3.9 Transfer to Groundwater under the First-Order Release Model ...................2-24

2.3.10 Transfer to Groundwater under the Uniform Release Model.......................2-24

2.3.11 Release to Groundwater under the Two Rate-Controlled Release Options

2.3.12 Release to Groundwater under the Instantaneous Desorption Equilibrium Release..

2.3.13 Release to the Atmosphere in the Form of Dust .....................................2-25

2.4 Users Guide to the Release Options in the RESRAD-OFFSITE Source

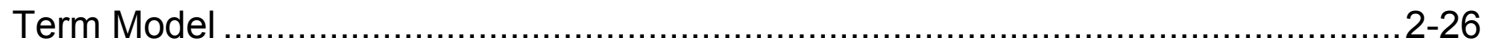

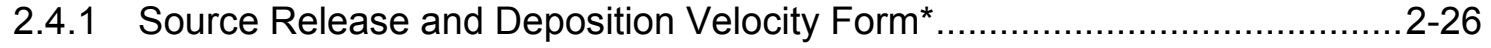

2.4.1.1 Version 2 Release Methodology .............................................2-26

2.4.1.2 First-Order Release with Transport .........................................2-28

2.4.1.3 Equilibrium Desorption Release .......................................... 2-29

2.4.1.4 Uniform Release ............................................................ 2-30 


\title{
Contents (Continued)
}

\author{
2.4.1.5 Release of Progeny Produced by Radiological \\ Transformations of an Initially Present Parent.............................2-30 \\ 2.4.1.6 Transport of the Radionuclides within the Primary

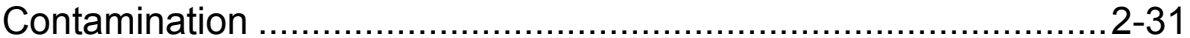 \\ 2.4.1.7 Temporal Plots of the Different Release Options .........................2-32 \\ 2.5 Overriding the RESRAD-OFFSITE Source Term Model .....................................2-37
}

3. Benchmarking of Radionuclide Release Rates Calculated by RESRAD-OFFSITE Version 3 and DUST-MS

3.1 Conceptualization of Radioactive Sources within the Contaminated Zone for

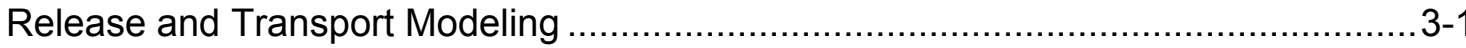

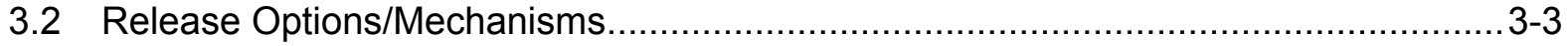

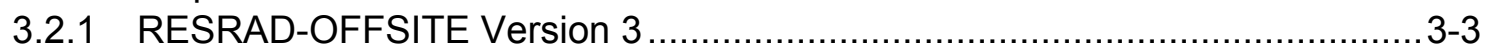

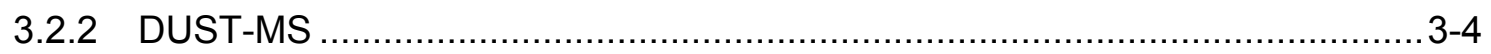

3.2.3 Options/Mechanisms Selected for Comparison .................................... 3-5

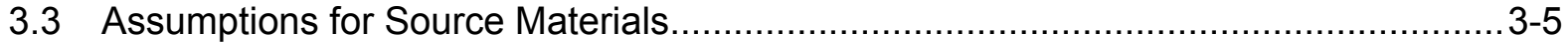

3.3.1 Release Sources in Different Comparison Cases ....................................... $3-5$

3.3.2 Input Parameters Used for RESRAD-OFFSITE ...................................... $3-6$

3.3.3 Input Parameters Used for DUST-MS .............................................. $3-7$

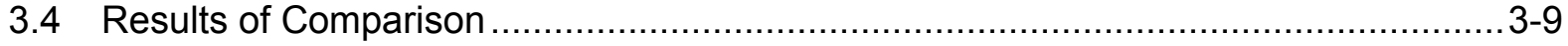

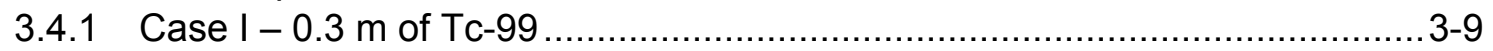

3.4.1.1 No Dispersion ................................................................... $3-9$

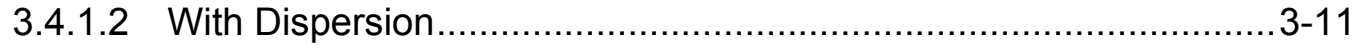

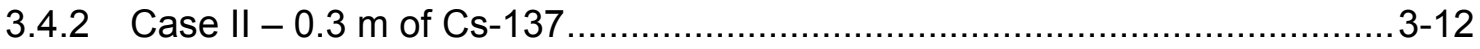

3.4.2.1 No Dispersion ................................................................. $3-14$

3.4.2.2 With Dispersion........................................................... 3-14

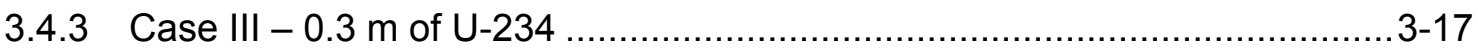

3.4.3.1 No Dispersion ................................................................ $3-17$

3.4.3.2 With Dispersion.................................................................. 3-21

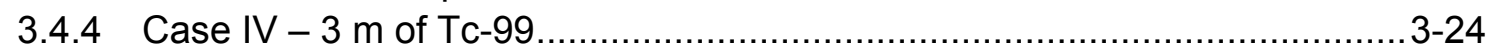

3.4.4.1 No Dispersion .............................................................. 3-24

3.4.4.2 With Dispersion................................................................ $3-26$

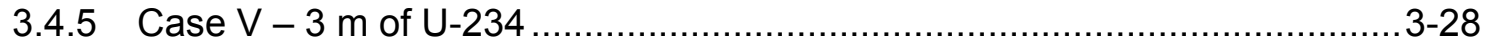

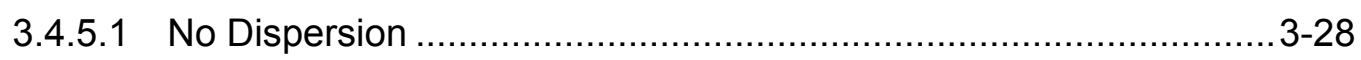

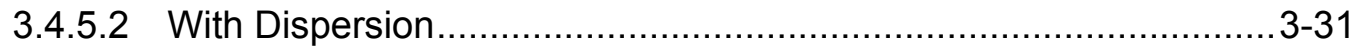

3.5 Summary of Comparison........................................................................ 31 


\section{Contents (Continued)}

4. Sensitivity Analysis to Identify Influential Inputs ......................................................

4.1 Hypothetical Scenario to Illustrate Identification of Influential Inputs .......................4-1

4.1.1 Site Layout and Dimensions ............................................................ $4-1$

4.1.1.1 Radionuclides in the Primary Contamination .............................. 4-2

4.1.1.2 Preliminary Data for the Hypothetical Site ................................... 4-2

4.1.2 Initial Ranges or Distributions of Inputs ............................................. 4-4

4.1.3 Relationships or Correlations between Inputs ........................................ 4-5

4.2 Three-Point Single Input Sensitivity Analysis ................................................... 4-6

4.2.1 Advantages and Disadvantages of Three-point Single-input Sensitivity

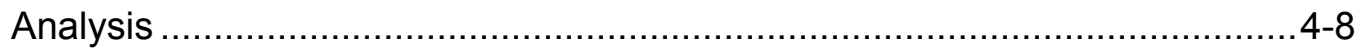

4.2.2 Conclusion about the Use of 3-point Single-input Sensitivity Analysis ..........4-10

4.3 Multiple Input Sensitivity Analysis ................................................................ 4-41

4.3.1 Advantages and Disadvantages of Multiple Input Sensitivity Analysis ..........4-44

4.3.2 Conclusion about the Use of Sensitivity Analysis..................................4-45

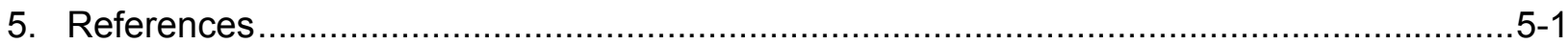

Appendix A Letter Report - Proposed Approach for the Task of Expanding

the Source Term Model for RESRAD-OFFSITE ......................................... A-1

Appendix B Additional Benchmarking of Radionuclide Release Rates ............................ B-1

Appendix C Sensitivity Analysis Using Probabilistic RESRAD-OFFSITE ......................... C-1

Appendix C.I Hypothetical Scenario for Multi-Input Sensitivity Analysis ........................... C-121

Appendix D Source Release with Time-delay Options in RESRAD-OFFSITE

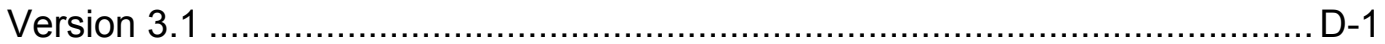

Appendix E Users' Guide to RESRAD-OFFSITE Code Area Factors ............................... E-1

\section{Figures}

2.1 Source Release and Deposition Velocity Form - Version 2 Option .............................2-27

2.2 Source Release and Deposition Velocity Form - New Release Options .....................2-29

2.3 Distribution Coefficients Form ..................................................................................

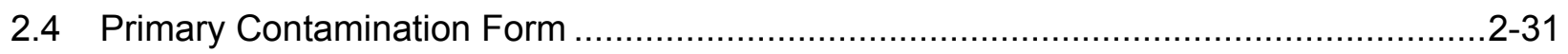

2.5 Temporal Profiles of Flux Out of the Primary Contamination for Different Release Options 


\section{Figures (Continued)}

2.6 Temporal Profiles of Flux Out of the Primary Contamination for the

First-Order Release Option with Different Rates of Transport ...

2.7 Temporal Profiles of Flux Out of the Primary Contamination for the Uniform Release Option with Different Rates of Transport

2.8 Temporal Profiles of Flux Out of the Primary Contamination for the

Equilibrium Desorption Release Option with Different Rates of Transport

3.1 Conceptualization of Radioactive Sources Within the Contaminated Zone

3.2 Various Designs for Subdividing the Domain of Analysis for DUST-MS Calculation .........3-8

3.3 Comparison of RESRAD-OFFSITE and DUST-MS Results for Case I Concerning Release from a 0.3-m Tc-99 Source under the Equilibrium

Desorption Condition with No Dispersion.

3.4 Comparison of RESRAD-OFFSITE and DUST-MS Results for Case I Concerning Release from a 0.3-m Tc-99 Source under the Uniform

Release Condition with No Dispersion....

3.5 Comparison of RESRAD-OFFSITE and DUST-MS Results for Case I

Concerning Release from a 0.3-m Tc-99 Source under the Equilibrium

Desorption Condition with a Dispersivity of $0.03 \mathrm{~m}$

3.6 Comparison of RESRAD-OFFSITE and DUST-MS Results for Case I

Concerning Release from a 0.3-m Tc-99 Source under the Equilibrium

Desorption Condition with a Dispersivity of $0.1 \mathrm{~m}$

3.7 Comparison of RESRAD-OFFSITE and DUST-MS Results for Case I

Concerning Release from a 0.3-m Tc-99 Source under the Uniform

Release Condition with a Dispersivity of $0.03 \mathrm{~m}$

3.8 Comparison of RESRAD-OFFSITE and DUST-MS Results for Case I

Concerning Release from a 0.3-m Tc-99 Source under the Uniform

Release Condition with a Dispersivity of $0.1 \mathrm{~m}$

3.9 Comparison of RESRAD-OFFSITE and DUST-MS Results for Case II

Concerning Release from a 0.3-m Cs-137 Source under the Equilibrium

Desorption Condition with No Dispersion.

3.10 Comparison of RESRAD-OFFSITE and DUST-MS Results for Case II

Concerning Release from a 0.3-m Cs-137 Source under the Uniform

Release Condition with No Dispersion....

3.11 Comparison of RESRAD-OFFSITE and DUST-MS Results for Case II

Concerning Release from a 0.3-m Cs-137 Source under the Equilibrium

Desorption Condition with a Dispersivity of $0.03 \mathrm{~m}$ 


\section{Figures (Continued)}

3.12 Comparison of RESRAD-OFFSITE and DUST-MS Results for Case II Concerning Release from a 0.3-m Cs-137 Source under the Uniform

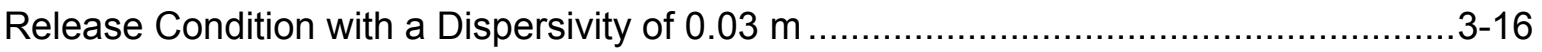

3.13 Comparison of RESRAD-OFFSITE and DUST-MS Results for Case III Concerning Release of U-234 from a 0.3-m U-234 Source with No Dispersion .............3-18

3.14 Comparison of RESRAD-OFFSITE and DUST-MS Results for Case III Concerning Release of Th-230 from a 0.3-m U-234 Source with No Dispersion...............18

3.15 Comparison of RESRAD-OFFSITE and DUST-MS Results for Case III Concerning Release of Ra-226 from a 0.3-m U-234 Source with No Dispersion ..............19

3.16 Comparison of RESRAD-OFFSITE and DUST-MS Results for Case III Concerning Release of Pb-210 from a 0.3-m U-234 Source with No Dispersion .............20

3.17 Comparison of RESRAD-OFFSITE and DUST-MS Results for Case III Concerning Release of Po-210 from a 0.3-m U-234 Source with No Dispersion ..............20

3.18 Comparison of RESRAD-OFFSITE and DUST-MS Results for Case III Concerning Release of U-234 from a 0.3-m U-234 Source with a Dispersivity of $0.03 \mathrm{~m}$

3.19 Comparison of RESRAD-OFFSITE and DUST-MS Results for Case III Concerning Release of Th-230 from a 0.3-m U-234 Source with a Dispersivity of $0.03 \mathrm{~m}$

3.20 Comparison of RESRAD-OFFSITE and DUST-MS Results for Case III Concerning Release of Ra-226 from a 0.3-m U-234 Source with a Dispersivity of $0.03 \mathrm{~m}$.....

3.21 Comparison of RESRAD-OFFSITE and DUST-MS Results for Case III Concerning Release of $\mathrm{Pb}-210$ from a 0.3-m U-234 Source with a Dispersivity of $0.03 \mathrm{~m}$.....

3.22 Comparison of RESRAD-OFFSITE and DUST-MS Results for Case III Concerning Release of Po-210 from a 0.3-m U-234 Source with a Dispersivity of $0.03 \mathrm{~m}$

3.23 Comparison of RESRAD-OFFSITE and DUST-MS Results for Case IV Concerning Release from a 3-m Tc-99 Source under the Equilibrium

Desorption Condition with No Dispersion.

3.24 Comparison of RESRAD-OFFSITE and DUST-MS Results for Case IV Concerning Release from a 3-m Tc-99 Source under the Uniform Release Condition with No Dispersion

3.25 Comparison of RESRAD-OFFSITE and DUST-MS Results for Case IV

Concerning Release from a 3-m Tc-99 Source under the Equilibrium Desorption Condition with a Dispersivity of $0.3 \mathrm{~m}$ 


\section{Figures (Continued)}

3.26 Comparison of RESRAD-OFFSITE and DUST-MS Results for Case IV Concerning Release from a 3-m Tc-99 Source under the Equilibrium

Desorption Condition with a Dispersivity of $1 \mathrm{~m}$.

3.27 Comparison of RESRAD-OFFSITE and DUST-MS Results for Case IV

Concerning Release from a 3-m Tc-99 Source under the Uniform Release

Condition with a Dispersivity of $0.3 \mathrm{~m}$

3.28 Comparison of RESRAD-OFFSITE and DUST-MS Results for Case IV

Concerning Release from a 3-m Tc-99 Source under the Uniform Release

Condition with a Dispersivity of $1 \mathrm{~m}$.....

3.29 Comparison of RESRAD-OFFSITE and DUST-MS Results for Case V

Concerning Release of U-234 from a 3-m U-234 Source with No Dispersion

3.30 Comparison of RESRAD-OFFSITE and DUST-MS Results for Case V

Concerning Release of Th-230 from a 3-m U-234 Source with No Dispersion.

3.31 Comparison of RESRAD-OFFSITE and DUST-MS Results for Case V

Concerning Release of Ra-226 from a 3-m U-234 Source with No Dispersion

3.32 Comparison of RESRAD-OFFSITE and DUST-MS Results for Case V

Concerning Release of Pb-210 from a 3-m U-234 Source with No Dispersion

3.33 Comparison of RESRAD-OFFSITE and DUST-MS Results for Case V

Concerning Release of Po-210 from a 3-m U-234 Source with No Dispersion

3.34 Comparison of RESRAD-OFFSITE and DUST-MS Results for Case V

Concerning Release of U-234 from a 3-m U-234 Source with a Dispersivity

of $0.3 \mathrm{~m}$.....

3.35 Comparison of RESRAD-OFFSITE and DUST-MS Results for Case V

Concerning Release of Th-230 from a 3-m U-234 Source with a Dispersivity

of $0.3 \mathrm{~m}$.

3.36 Comparison of RESRAD-OFFSITE and DUST-MS Results for Case V

Concerning Release of Ra-226 from a 3-m U-234 Source with a Dispersivity

of $0.3 \mathrm{~m}$

3.37 Comparison of RESRAD-OFFSITE and DUST-MS Results for Case V

Concerning Release of Pb-210 from a 3-m U-234 Source with a Dispersivity

of $0.3 \mathrm{~m}$

3.38 Comparison of RESRAD-OFFSITE and DUST-MS Results for Case V

Concerning Release of Po-210 from a 3-m U-234 Source with a Dispersivity of $0.3 \mathrm{~m}$.

4.1 Layout of the Hypothetical Site

4.2 The Cross Section of the Primary Contamination and the Soil beneath It 


\section{Figures (Continued)}

4.3 Specifying the Lower and Higher Values to be Used for an Input Selected for Sensitivity Analysis.

4.4 Resetting the Sensitivity Analysis Range to Avoid Exceeding the Bounds on the Inputs

4.5 Temporal Variation of Dose with the Volume of the Surface Water Body Showing a Range of $48.3 \mathrm{mrem} /$ year for the Peak Dose from 38.5 to $86.8 \mathrm{mrem} /$ year

4.6 Temporal Variation of Dose with the Mean Residence Time of Water in the Surface Water Body showing a Range of $47.4 \mathrm{mrem} / \mathrm{year}$ for the Peak Dose from 38.7 to $86.1 \mathrm{mrem} /$ year.

4.7 Temporal Variation of Dose with the Duration of Release of ${ }^{226} \mathrm{Ra}$ Showing a Range of 20.4 mrem/year for the Peak Dose from 48.8 to $69.2 \mathrm{mrem} / \mathrm{year}$

4.8 Temporal Variation of Dose with the Density of the Primary Contamination Showing a Range of $18.8 \mathrm{mrem} /$ year for the Peak Dose from 49.3 to $68.1 \mathrm{mrem} /$ year

4.9 Temporal Variation of Dose with the Depth of Aquifer Contributing to the Surface Water Body Showing a Range of $13.2 \mathrm{mrem} / \mathrm{year}$ for the Peak Dose from 49.0 to $62.2 \mathrm{mrem} / \mathrm{year}$

4.10 Temporal Variation of Dose with the Distribution Coefficient of ${ }^{210} \mathrm{~Pb}$ in the First Unsaturated Zone Showing a Range of $12.9 \mathrm{mrem} / \mathrm{year}$ for the Peak Dose from 51.5 to $64.4 \mathrm{mrem} /$ year...

4.11 Temporal Variation of Dose with the Distribution Coefficient of ${ }^{226} \mathrm{Ra}$ in the First Unsaturated Zone Showing a Range of $12.2 \mathrm{mrem} /$ year for the Peak Dose from 52.2 to $64.4 \mathrm{mrem} /$ year

4.12 Temporal Variation of Dose with the Evapotranspiration Coefficient Showing a Range of $11.5 \mathrm{mrem} /$ year for the Peak Dose from 47.6 to $59.1 \mathrm{mrem} / \mathrm{year}$

4.13 Temporal Variation of Dose with the Fraction of Fruit, Nonleafy Vegetables, and Grain from the Contaminated Region Showing a Range of $9.8 \mathrm{mrem} /$ year for the Peak Dose from 53.9 to $63.7 \mathrm{mrem} /$ year.

4.14 Temporal Variation of Dose with the Irrigation Applied per Year to the Fruit, Nonleafy Vegetables, and Grain Growing Areas Showing a Range of $9.7 \mathrm{mrem} /$ year for the Peak Dose from 53.9 to $63.6 \mathrm{mrem} /$ year....

4.15 Temporal Variation of Dose with the Wet Weight Crop Yield of Fruit, Nonleafy Vegetables, and Grain in the Growing Areas Showing a Range of $9.6 \mathrm{mrem} /$ year for the Peak Dose from 54.0 to $63.6 \mathrm{mrem} /$ year 


\section{Figures (Continued)}

4.16 Temporal Variation of Dose with the Translocation Factor for Fruit, Nonleafy Vegetables, and Grain Showing a Range of $9.6 \mathrm{mrem} /$ year for the Peak

Dose from 54.0 to $63.6 \mathrm{mrem} / \mathrm{year}$

4.17 Temporal Variation of Dose with the Foliar Interception Factor for Irrigation in the Fruit, Nonleafy Vegetables, and Grain Growing Area Showing a Range of $9.6 \mathrm{mrem} / \mathrm{year}$ for the Peak Dose from 54.0 to $63.6 \mathrm{mrem} / \mathrm{year}$

4.18 Temporal Variation of Dose with the $x$ Dimension of the Primary Contamination Showing a Range of $9.1 \mathrm{mrem} / \mathrm{year}$ for the Peak Dose from 53.3 to $62.4 \mathrm{mrem} /$ year.

4.19 Temporal Variation of Dose with the y Dimension of the Primary Contamination Showing a Range of $9.1 \mathrm{mrem} / \mathrm{year}$ for the Peak Dose from 53.3 to $62.4 \mathrm{mrem} /$ year.

4.20 Temporal Variation of Dose with the Thickness of the Primary Contamination Showing a Range of $8.2 \mathrm{mrem} /$ year for the Peak Dose from 53.9 to $62.1 \mathrm{mrem} /$ year

4.21 Temporal Variation of Dose with the Duration of the Growing Season of Fruit, Nonleafy Vegetables, and Grain Showing a Range of $8.1 \mathrm{mrem} /$ year for the Peak Dose from 54.2 to $62.3 \mathrm{mrem} /$ year

4.22 Temporal Variation of Dose with the Weathering Removal Constant for Fruit, Nonleafy Vegetables, and Grain Showing a Range of $8.1 \mathrm{mrem} / \mathrm{year}$ for the Peak Dose from 54.2 to $62.3 \mathrm{mrem} /$ year

4.23 Temporal Variation of Dose with the Weathering Removal Constant for Fruit, Nonleafy Vegetables, and Grain Showing a Range of $6.9 \mathrm{mrem} / \mathrm{year}$ for the Peak Dose from 54.5 to $61.4 \mathrm{mrem} /$ year

4.24 Temporal Variation of Dose with the Erodibility Factor of Soil in the Primary Contamination Showing a Range of 5.0 mrem/year for the Peak Dose from 55.6 to $60.6 \mathrm{mrem} /$ year

4.25 Temporal Variation of Dose with the Irrigation Applied per Year to the Pasture and the Silage Growing Areas Showing a Range of $4.0 \mathrm{mrem} / \mathrm{year}$ for the Peak Dose from 56.2 to 60.2 mrem/year

4.26 Temporal Variation of Dose with the Wet Weight Crop Yield from the Pasture and the Silage Growing Areas Showing a Range of $4.0 \mathrm{mrem} / \mathrm{year}$ for the Peak Dose from 56.2 to $60.2 \mathrm{mrem} /$ year .

4.27 Temporal Variation of Dose with the Grain Intake by Cattle Raised for Meat Showing a Range of $0.8 \mathrm{mrem} / \mathrm{year}$ for the Peak Dose from 57.5 to $58.3 \mathrm{mrem} / \mathrm{year}$ 


\section{Figures (Continued)}

4.28 Temporal Variation of Dose with the Longitudinal Dispersivity in the Saturated Zone to the Surface Water Body Showing a Range of $0.0 \mathrm{mrem} / \mathrm{year}$ for the Peak Dose from 57.8 to $57.8 \mathrm{mrem} /$ year

4.29 Temporal Variation of Dose with the Dry Bulk Density of Soil in the Fruit, Nonleafy Vegetables, and Grain Growing Area Showing a Range of $0.0 \mathrm{mrem} /$ year for the Peak Dose from 57.8 to $57.8 \mathrm{mrem} / \mathrm{year}$.

4.30 Temporal Variation of Dose with the Effective Porosity of the Primary Contamination Showing a Range of 2.5 mrem/year for the Peak Dose from 56.2 to $58.7 \mathrm{mrem} /$ year

4.31 Temporal Variation of Dose with the Total Porosity of the Primary Contamination Showing a Range of $2.2 \mathrm{mrem} / \mathrm{year}$ for the Peak Dose from 56.5 to $58.7 \mathrm{mrem} /$ year.

4.32 Temporal Variation of Dose with the Field Capacity of the Primary Contamination Showing a Range of 0.0 mrem/year for the Peak Dose from 57.8 to $57.8 \mathrm{mrem} /$ year

4.33 Temporal Variation of Dose with the Leach Rate of ${ }^{226} \mathrm{Ra}$ in Primary Contamination for the First Order Release Example Scenario

4.34 Temporal Variation of Dose with the Distribution Coefficient of ${ }^{226} \mathrm{Ra}$ in Primary Contamination for the Equilibrium Desorption Release Example Scenario

4.35 Step-by-step Analysis Tab of the Uncertainty and Probabilistic Analysis Form

4.36 Specifying the Range for an Input Selected for Multiple Input Sensitivity Analysis.

4.37 Specified Range Adjusted to Conform to Bounds Built into the Code $4-48$

4.38 Scatter Plot of Field Capacity against Total Porosity to Show the Effect of Specifying a Correlation of 0.9 between Them

4.39 Specifying Correlation between Inputs.

4.40 Specifying Relationships between Inputs.

4.41 Scatter Plot of the Length Parallel to Aquifer Flow against the $\mathrm{x}$ Dimension of the Primary Contamination

4.42 Cumulative Distribution Function of Peak Dose from the Identification Run of the Preliminary Sensitivity Analysis.

4.43 Regression Output of Preliminary Sensitivity Analysis with Inputs Sorted in Descending Order of Influence.

4.44 Standardized Regression Coefficients of the Top 63 Inputs Sorted in Descending Order of Importance 


\section{Figures (Continued)}

4.45 Standardized Regression Coefficients of All 222 Inputs Sorted

in Descending Order of Importance ..........................................................4-56

4.46 Regression Output of Preliminary Sensitivity Analysis with Inputs Sorted

in Descending Order of Influence .................................................................... $4-57$

4.47 Cumulative Distribution Function of Peak Dose from the 30-input

Verification Run of the Preliminary Sensitivity Analysis .......................................4-58

4.48 Cumulative Distribution Function of Peak Dose from the 17-input

Verification Run of the Preliminary Sensitivity Analysis ........................................4-59

4.49 Cumulative Distribution Function of Peak Dose from the 5-input

Verification Run of the Preliminary Sensitivity Analysis ...........................................4-60

4.50 Cumulative Distribution Function of Peak Dose from Identification and

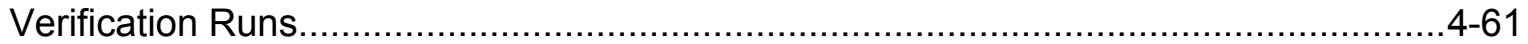

B.1 Modeling of a First Order Release with GoldSim .................................................. B-3

B.2 Modeling of an Equilibrium Desorption Release with GoldSim ................................. B-4

B.3 Comparison of RESRAD-OFFSITE and GoldSim Results for Case I

Concerning Release from a 0.3-m Tc-99 Source under the Equilibrium

Desorption Condition with No Dispersion

B.4 Comparison of RESRAD-OFFSITE and GoldSim Results for Case I

Concerning Release from a 0.3-m Tc-99 Source under the Uniform

Release Condition with No Dispersion

B.5 Comparison of RESRAD-OFFSITE and GoldSim Results for Case I

Concerning Release from a 0.3-m Tc-99 Source under the First Order

Release Condition with No Dispersion

B.6 Comparison of RESRAD-OFFSITE and GoldSim Results for Case I

Concerning Release from a 0.3-m Tc-99 Source under the Equilibrium

Desorption Condition with a Dispersivity of $0.03 \mathrm{~m}$

B.7 Comparison of RESRAD-OFFSITE and GoldSim Results for Case I

Concerning Release from a 0.3-m Tc-99 Source Under the Equilibrium

Desorption Condition with a Dispersivity of $0.1 \mathrm{~m}$

B.8 Comparison of RESRAD-OFFSITE and GoldSim Results for Case I

Concerning Release from a 0.3-m Cs-137 Source under the Equilibrium

Desorption Condition with No Dispersion

B.9 Comparison of RESRAD-OFFSITE and GoldSim Results for Case I

Concerning Release from a 0.3-m Cs-137 Source under the Uniform

Release Condition with No Dispersion 


\section{Figures (Continued)}

B.10 Comparison of RESRAD-OFFSITE and GoldSim Results for Case I

Concerning Release from a 0.3-m Cs-137 Source under the First

Order Release Condition with No Dispersion

B-14

B.11 Comparison of RESRAD-OFFSITE and GoldSim Results for Case III

Concerning Release of U-234 from a 0.3-m U-234 Source with No Dispersion

B.12 Comparison of RESRAD-OFFSITE and GoldSim Results for Case III

Concerning Release of Th-230 from a 0.3-m U-234 Source with No Dispersion ......... B-16

B.13 Comparison of RESRAD-OFFSITE and GoldSim Results for Case III

Concerning Release of Ra-226 from a 0.3-m U-234 Source with No Dispersion......... B-17

B.14 Comparison of RESRAD-OFFSITE and GoldSim Results for Case III

Concerning Release of Pb-210 from a 0.3-m U-234 Source with No Dispersion ......... B-18

B.15 Comparison of RESRAD-OFFSITE and GoldSim Results for Case III

Concerning Release of Po-210 from a 0.3-m U-234 Source with No Dispersion ......... B-19

C.1 Specifying Three-point Single-input Sensitivity Analysis for the Input of Interest ........... C-6

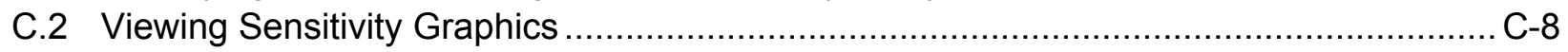

C.3 Identifying Significant Exposure Pathways for the Base Case .................................... C-8

C.4 Sensitivity of Predicted Dose from Drinking Water ............................................... C-9

C.5 Sensitivity of Predicted Dose from Ingestion of Vegetables....................................... C-9

C.6 Sensitivity of Predicted Dose from Ingestion of Meat............................................. C-10

C.7 Sensitivity of Predicted Dose from Ingestion of Milk ............................................. C-10

C.8 Sensitivity of Predicted Dose from Direct Exposure to Waterborne

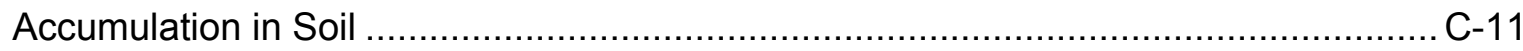

C.9 Sensitivity of Predicted Dose from Ingestion of Fish ............................................ C-11

C.10 Specifying Distributed Sensitivity Analysis on an Input............................................ C-15

C.11 Viewing the Output of a Distributed Sensitivity Analysis on an Input ........................... C-17

C.12 Variation of Peak Predicted Dose with Hydraulic Conductivity

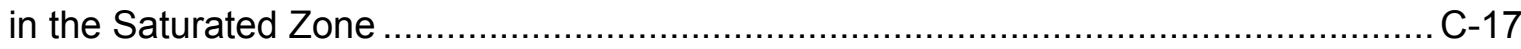

C.13 Variation of Peak Predicted Dose from Drinking Water with

Hydraulic Conductivity in the Saturated Zone........................................................ C-18

C.14 Variation of Peak Predicted Dose from Ingestion of Vegetables

with Hydraulic Conductivity in the Saturated Zone .............................................. C-18

C.15 Variation of Peak Predicted Dose from Ingestion of Meat with

Hydraulic Conductivity in the Saturated Zone.......................................................... C-19

C.16 Variation of Peak Predicted Dose from Ingestion of Milk with

Hydraulic Conductivity in the Saturated Zone................................................... C-19

C.17 Variation of Peak Predicted Dose from Direct Exposure to Waterborne

Accumulation in Soil with Hydraulic Conductivity in the Saturated Zone 


\section{Figures (Continued)}

C.18 Variation of Peak Predicted Dose from Ingestion of Fish with Hydraulic

Conductivity in the Saturated Zone.

C.19 Option Box to be Checked to Flag the Code to Produce a Full Suite of

Temporal Plots for Each Probabilistic Simulation

C.20 Relative Contributions of Exposure Pathways at 1984 m/yr Hydraulic

Conductivity ....

C.21 Total Dose at a Hydraulic Conductivity of $1984 \mathrm{~m} / \mathrm{yr}$ in Saturated Zone. C-26

C.22 Relative Contributions of Exposure Pathways at $752.1 \mathrm{~m} / \mathrm{yr}$ Hydraulic Conductivity . C-27

C.23 Total Dose at a Hydraulic Conductivity of $752.1 \mathrm{~m} / \mathrm{yr}$ in Saturated Zone C-27

C.24 Relative Contributions of Exposure Pathways at $732.1 \mathrm{~m} / \mathrm{yr}$ Hydraulic Conductivity C-28

C.25 Total Dose at a Hydraulic Conductivity of $732.1 \mathrm{~m} / \mathrm{yr}$ in Saturated Zone C-28

C.26 Relative Contributions of Exposure Pathways at 709.8 m/yr Hydraulic Conductivity .

C.27 Total Dose at a Hydraulic Conductivity of $709.8 \mathrm{~m} / \mathrm{yr}$ in Saturated Zone C-29

C.28 Relative Contributions of Exposure Pathways at $299.2 \mathrm{~m} / \mathrm{yr}$ Hydraulic Conductivity. C-30

C.29 Total Dose at a Hydraulic Conductivity of $299.2 \mathrm{~m} / \mathrm{yr}$ in Saturated Zone... C-30

C.30 Relative Contributions of Exposure Pathways at 258 m/yr Hydraulic Conductivity. C-31

C.31 Total Dose at a Hydraulic Conductivity of $258 \mathrm{~m} / \mathrm{yr}$ in Saturated Zone. C-31

C.32 Relative Contributions of Exposure Pathways at $213.7 \mathrm{~m} / \mathrm{yr}$ Hydraulic Conductivity .

C.33 Total Dose at a Hydraulic Conductivity of $213.7 \mathrm{~m} / \mathrm{yr}$ in Saturated Zone. C-32

C.34 Rate of Change of Peak Predicted Dose with Hydraulic Conductivity C-34

C.35 Fractional Rate of Change of Peak Predicted Dose with Hydraulic Conductivity

C.36 Shape of $y=x^{k}$ for select values of $k$. C-38

C.37 Dimensions of Plume Prior to Lateral Dispersion and Dimension of Saturated Zone Contributing to the Surface Water Body

C.38 and C.39 Distributions of Radionuclides in the Transverse Plane at the Location of the Surface Water Body. C-42

C.40 Dimensions of the Initial Plume in the Saturated Zone and the Dimensions of the Aquifer Contributing to the Well for Five Hydraulic Conductivities in the Saturated Zone C-44

C.41 Variation in Dilution Factor with Hydraulic Conductivity in the Saturated Zone C-46 


\section{Figures (Continued)}

C.42 and C.43 Distributions of Radionuclides in the Transverse Plane at the Well............... C-46

C.44 Specifying Relationships between Inputs........................................................ C-47

C.45 Specifying the Dependent Input of a Relationship ................................................ -48

C.46 Adding an Independent Input to the Relationship ............................................... C-48

C.47 Adding an Arithmetic Operator and Selecting the Second Independent Input

to the Relationship ................................................................................ -49

C.48 Completing the Specification of a Relationship and Transferring It to the

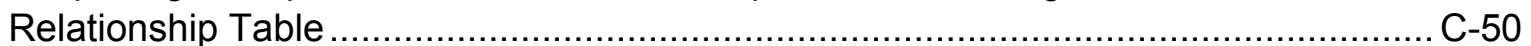

C.49 Inputting Numerical Constants in a Relationship .................................................. -50

C.50 Using a Related Input in the Relationship of Another Input ..................................... -52

C.51 Indirect Introduction of [ ] Using Placeholder Inputs, Enabling Creation

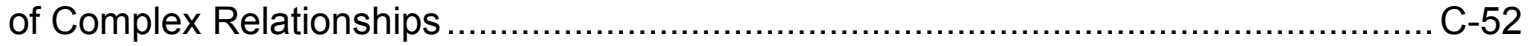

C.52 Relationship between Mean Residence Time of Water in the Surface

Water Body and the Hydraulic Conductivity in the Saturated Zone........................... C-53

C.53 Variation with Hydraulic Conductivity of Peak Predicted Dose from

Ingestion of Fish When a Relationship Is Specified between Hydraulic

Conductivity and Mean Residence Time of Water in the Surface Water Body............ C-54

C.54 Variation with Hydraulic Conductivity of Peak Predicted Dose from All

Pathways When a Relationship Is Specified between Hydraulic

Conductivity and Mean Residence Time of Water in the Surface Water Body............ C-55

C.55 Specifying a Discrete Distribution on an Input ....................................................... C-57

C.56 Pairing of Samples in a Two-input Probabilistic Analysis Where One Input

Has a Continuous Uniform Distribution and the Other a Discrete

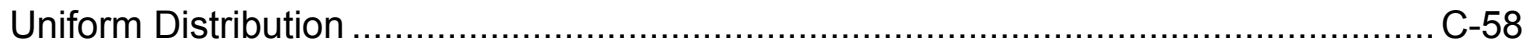

C.57 Variation of Peak Predicted Dose with Hydraulic Conductivity at Different

Discrete Values of Depth of Aquifer Contributing to the Well .................................. C-59

C.58 Specifying a Continuous Linear Distribution ........................................................ C-60

C.59 Samples from a Continuous Linear Distribution Showing a Greater

Concentration of Samples from One Part of the Range and Sparse

Sampling over the Rest of the Range.....

C.60 Variation of Peak Predicted Dose with Hydraulic Conductivity at Discrete

Values for Depth of Aquifer Contributing to the Well

C.61 Variation of Peak Predicted Dose with Hydraulic Conductivity in the

Range 200 to 800 m/yr at Discrete Values for Depth of Aquifer

Contributing to the Well

C.62 Range of Peak Predicted Dose at Different Discrete Values for Depth of

Aquifer Contributing to the Well Caused by Variation of Hydraulic Conductivity 


\section{Figures (Continued)}

C.63 Variation of Peak Predicted Dose with Hydraulic Conductivity-the Scatter Shows the Effect of Sampling the Depth of Aquifer Contributing to the Well and the Evapotranspiration Coefficient.

C.64 Peak Predicted Dose at Three Values of Evapotranspiration Coefficientthe Range of Peak Predicted Dose Is Due to Sampling the Depth of Aquifer Contributing to the Well and the Hydraulic Conductivity.

C.65 Pairing of Samples in a Two-input Probabilistic Analysis Where Both Inputs Have Continuous Distributions.

C.66 Variation of Peak Predicted Dose with Hydraulic Conductivity-the Scatter Shows the Effect of Sampling the Depth of Aquifer Contributing to the Well

C.67 Variation of Peak Predicted Dose with Depth of Aquifer Contributing to the Well-the Scatter Shows the Effect of Sampling the Hydraulic Conductivity

C.68 Estimating the Range of Peak Predicted Doses at Any Given Value of $x$-coordinate

C.69 Linear Regression on Standardized Raw and Ranked Data for the Two-input Continuous Distribution Example.

C.70 Linear Regression on Standardized Raw and Ranked Data for the Second Two-input Continuous Distribution Example

C.71 Variation of Peak Predicted Dose with Hydraulic Conductivity-the Scatter Shows the Effect of Sampling the Depth of Aquifer Contributing to the Well for the Second Example Case.

C.72 Variation of Peak Predicted Dose with Depth of Aquifer Contributing to the Well-the Scatter Shows the Effect of Sampling the Hydraulic Conductivity for the Second Case

C.73 Linear Regression on Standardized Raw and Ranked Data for the Three-input Continuous Distribution Example

C.74 Variation of Peak Predicted Dose with Hydraulic Conductivity-the Scatter Shows the Effect of Sampling the Depth of Aquifer Contributing to the Well and the Evapotranspiration Coefficient.

C.75 Variation of Peak Predicted Dose with Depth of Aquifer Contributing to the Well - the Scatter Shows the Effect of Sampling the Hydraulic Conductivity and the Evapotranspiration Coefficient

C.76 Variation of Peak Predicted Dose with Evapotranspiration Coefficientthe Scatter Shows the Effect of Sampling the Hydraulic Conductivity and the Depth of Aquifer Contributing to the Well 


\section{Figures (Continued)}

C.77 Scatter Plot of a Linear Relationship for a Case Involving Only a

Single Variable

C.78 Scatter Plot of the Dependent Variable and the First Independent Variable

for a Case Involving a Linear Relationship with Two Independent Variables

C.79 Scatter Plot of the Dependent Variable and the Second Independent

Variable for a Case Involving a Linear Relationship with

Two Independent Variables

C.80 Scatter Plot of the Dependent Variable and the First Independent

Variable for a Case Involving Linear and Nonlinear Relationships with Three Independent Variables

C.81 Scatter Plot of the Dependent Variable and the Second Independent

Variable for a Case Involving Linear and Nonlinear Relationships

with Three Independent Variables

C.82 Scatter Plot of the Dependent Variable and the Third Independent

Variable for a Case Involving Linear and Nonlinear Relationships with

Three Independent Variables

C.83 Scatter Plot of the Rank of the Dependent Variable and the Rank of the

First Independent Variable for a Case Involving Linear and Nonlinear

Relationships with Three Independent Variables

C.84 Scatter Plot of the Rank of the Dependent Variable and the Rank of the

Second Independent Variable for a Case Involving Linear and Nonlinear

Relationships with Three Independent Variables

C.85 Scatter Plot of the Rank of the Dependent Variable and the Rank of the

Third Independent Variable for a Case Involving Linear and Nonlinear

Relationships with Three Independent Variables

C.86 Cumulative Distribution Function of One of the Inputs

C.87 Cumulative Distribution Function of the Output, the Peak Predicted Dose

C.88 Regression Report

C.89 Plot of the Standardized Regression Coefficients from the Regression Report

C-92

C.90 Scatter Plots of Peak Predicted Dose against Inputs Identified as the

Four Most Significant by the Regression Analysis.

C.91 Scatter Plots of Peak Predicted Dose against Inputs Identified as the

Second Set of Four Most Significant by the Regression Analysis

C.92 Scatter Plots of Peak Predicted Dose against Inputs Identified as the

Third Set of Four Most Significant by the Regression Analysis

C.93 Scatter Plots of Peak Predicted Dose against Inputs Identified as the

Fourth Set of Four Most Significant by the Regression Analysis 


\section{Figures (Continued)}

C.94 Scatter Plots of Peak Predicted Dose against Inputs Identified as the Fifth Set of Four Most Significant by the Regression Analysis .............................. C-97

C.95 Scatter Plots of Peak Predicted Dose against Inputs Identified as the Sixth Set of Four Most Significant by the Regression Analysis ............................. C-98

C.96 Scatter Plots of Peak Predicted Dose against Inputs Identified as the Seventh Set of Four Most Significant by the Regression Analysis ......................... C-99

C.97 Scatter Plots of Peak Predicted Dose against Inputs Identified as the Eighth Set of Four Most Significant by the Regression Analysis .......................... C-100

C.98 Scatter Plots of Peak Predicted Dose against Inputs Identified as the Ninth Set of Four Most Significant by the Regression Analysis

C.99 Scatter Plots of Peak Predicted Dose against Inputs Identified as the 10th Set of Four Most Significant by the Regression Analysis

C.100 Cumulative Distribution Function Plots from the Three Multi-input Sensitivity Analyses

C.101 Cumulative Distribution Function of the Output, the Peak Predicted Dose, When National Distributions Are Used to Obtain 500 Samples of Each of the 203 Inputs

C.102 Plot of the Standardized Regression Coefficients from the Regression Report of Sensitivity Analysis Using 500 Observations on National Distribution................. C-108

C.103 Cumulative Distribution Function of Peak Predicted Dose with 5000 Samples, When National Distributions Are Used for the Inputs C-109

C.104 Plot of the Standardized Regression Coefficients from the Regression Report of Sensitivity Analysis Using 5000 Observations on National Distributions ............. C-110

C.105 Regression Report of Sensitivity Analysis Using 5000 Observations on National Distributions

C.106 Cumulative Distribution Function of Peak Predicted Dose with 5000 Samples, When National Distributions Are Used for the Inputs after Depressing

Erosion Release to Surface Water Body and Release to Atmosphere.

C.107 Regression Report of Sensitivity Analysis Using 5000 Observations on National Distributions Inputs after Depressing Erosion Release to Surface Water Body and Release to Atmosphere

C.108 Cumulative Distribution Function Plot of the Input Samples for the Thickness of the Unsaturated Zone.... C-116

C.109 Cumulative Distribution Function Plot of the Input Samples for the Distribution Coefficient for Radium in the Unsaturated Zone.....

C.110 Cumulative Distribution Function Plot of the Input Samples for the Hydraulic Gradient of the Saturated Zone to the Surface Water Body C-118 


\section{Figures (Continued)}

C.111 Cumulative Distribution Function Plot of the Input Samples for the Hydraulic

Conductivity of the Saturated Zone to the Surface Water Body ............................ -119

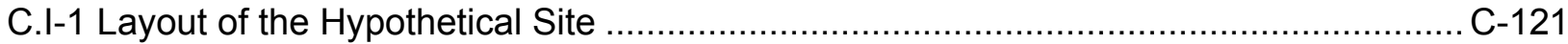

C.I-2 The Cross Section of the Primary Contamination and the Soil beneath It ................. C-122

D.1 Specifying Inputs for the Version 2 Release Methodology ........................................ D-1

D.2 Specifying Inputs for First-Order, Rate-Controlled Release of Material

That Initially Is Entirely in the Releasable Form ................................................ D-14

D.3 Comparison of Pathway Doses Predicted by Version 3.1 with the

Doses Predicted by the Previously Benchmarked 3.0 Beta Version of

RESRAD-OFFSITE for a First-Order, Rate-Controlled Release ........................... D-15

D.4 Specifying Inputs for Equilibrium Desorption Release of Material

That Initially Is Entirely in the Releasable Form ................................................. -17

D.5 Comparison of Pathway Doses Predicted by Version 3.1 with the

Doses Predicted by the Previously Benchmarked 3.0 Beta Version of

RESRAD-OFFSITE for an Equilibrium Desorption Release ................................. D-18

D.6 Specifying Inputs for Uniform Release of Material That Initially Is

Entirely in the Releasable Form................................................................. D-20

D.7 Comparison of Pathway Doses Predicted by Version 3.1 with the

Doses Predicted by the Previously Benchmarked 3.0 Beta Version

of RESRAD-OFFSITE for a Uniform Release .................................................. D-21

D.8 A First-Order Release with Radionuclide-Bearing Material Becoming Available

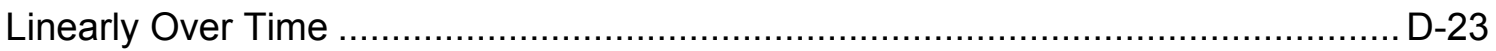

D.9 A First-Order Release with Radionuclide-Bearing Material Becoming Available

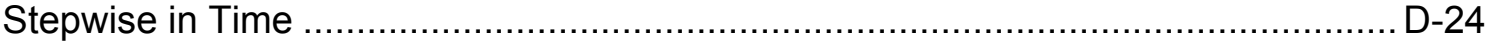

D.10 An Instantaneous Equilibrium Desorption Release in Which

Radionuclide-Bearing Material Becomes Available for Release Linearly in Time ..... D-25

D.11 An Instantaneous Equilibrium Desorption Release in Which

Radionuclide-bearing Material Becomes Available for Release

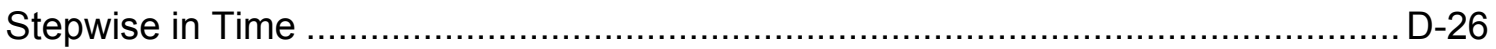

E.1 Area Factors Form - Default Options ........................................................... E-2

E.2 Area Factors Form - Option to Specify the Range of the Y Dimension of the Small Area of Elevated Contamination ........................................................... E-3

E.3 Distribution of Area of the Small Area of Elevated Contamination under Three Distributions Options for Sampling the Dimensions of the Small Area of Elevated Contamination: triangular skewed to the high end, uniform, and triangular skewed to the low end

E.4 Area Factors Form - Generate Dose-Area Plot Command Button Activated ................. E-7 


\section{Figures (Continued)}

E.5 Scatter Plot of Dose against Area of Contamination for Cs-137 Where the Direct External Exposure Is the Dominant Pathway

E.6 Scatter Plot of Dose against Area of Contamination for Sr-90 for Which

Groundwater Transport Is the Dominant Route of Exposure.... E-10

\section{Tables}

2.1 Input Files Used to Specify the Source Characteristics and Releases to the Code.......2-38

3.1 Assumptions for the Release Sources in Different Comparison Cases .........................3-6

B.1 Properties of the Radioactive Species Considered in GoldSim Modeling...................... B-5

C.1 Peak Predicted Doses and Times of Peak Predicted Doses From Different Exposure Pathways ........................................................................ C-21

C.2 Effect of the Choice of the Representative Normalizing Value on the Computed Fractional Rate of Change ............................................................... C-36

C.3 Relationship between Fractional Rate of Change and the Shape of the Curve of Peak Predicted Dose and the Input

C.4 One Hundred Samples of Data Used in Section 3.6.1 to Illustrate Linear Regression on Raw Values C-80 
This page is intentionally left blank. 


\section{Executive Summary}

The RESRAD-OFFSITE computer code evaluates the radiological dose and excess cancer risk to an individual who is exposed while located within or outside the area of initial (primary) contamination. The RESRAD-OFFSITE code Version 2 was developed by adding offsite transport and offsite accumulation modules to the original and well-established RESRAD (onsite) code, which was designed for the evaluation of radiological doses to an onsite receptor from exposure to RESidual RADioactive materials in soil. The source release to groundwater model used in Version 2 (i.e., the first-order, rate-controlled leaching model, sometimes referred to as the exponential leaching model) is largely the same as the one used in the RESRAD (onsite) code. This model is retained in RESRAD-OFFSITE Version 3, which adds three new source release mechanisms.

The RESRAD-OFFSITE code Version 3 was improved by the addition of three release mechanisms to groundwater. The transport of the radionuclides in the primary contamination is modeled for all three new release options. The first release option, that is, the "first-order, release-with-transport" option, assumed that the release of the radionuclide is proportional to the inventory in the primary contamination; the user-specified leach rate is the proportionality constant. The waste forms/materials that resemble the "first order release with transport" release option include dewatered sludge and ion-exchange resins. The second release option, that is, the "equilibrium desorption release" option, assumed that the release is controlled by the linear equilibrium partitioning between the solid and aqueous phase; the user specifies the distribution coefficient which quantifies the partitioning of the radionuclide between the solid and aqueous phases. The waste forms/materials that fit this specification include compacted lab trash, such as clothes or glove boxes, as well as small gadgets or tools. Under the third release option, that is, the "uniform release" option, the radionuclides are released from a constant fraction of the initially contaminated material during each time interval; the user specifies the duration over which the radionuclides are released. An example of waste forms/materials that may fit these descriptions is activated metal, which would corrode in the environment and release the imbedded radionuclides. The preexisting release methodology of the Version 2 code is also retained; it is a first-order, release-without-transport option where the user may specify the leach rate or ask the code to estimate a leach rate from the specified distribution coefficient. The user-specified leach rate has to meet a non-negative distribution coefficient check.

Two of the new source release models (i.e., equilibrium desorption release and uniform release) are comparable to two of the release mechanisms simulated by DUST-MS, a computer code designed for evaluating radionuclide releases from underground waste disposal units. The uniform release condition would result if the waste materials undergo constant dissolution upon 
contact with water, then release radionuclides contained within the materials. The equilibrium desorption release condition would result if radionuclides are distributed on the surface of the waste materials, then dissolve to water when the waste materials are rinsed by water. In the effort to benchmark the new source term models with DUST-MS, radionuclide release rates, which were obtained from the bottom of a contaminated zone/disposal unit and calculated under similar release conditions, were compared. The comparison with DUST-MS involved the development of five different cases that consider different sources containing different radionuclides, assuming different dimensions, and experiencing different water infiltration rates. Overall, the agreement between the RESRAD-OFFSITE results and DUST-MS results is very good, with both codes predicting the same or very similar profiles over time. Numerical dispersion in the DUST-MS results was identified as potentially contributing to the disagreement in the release rates when no dispersion was considered. The mobility of radionuclides in the soil column and the radioactive decay half-life of radionuclides might also affect the agreement, with greater discrepancy in the release rates found for short-lived, fast-moving radionuclides than found for long-lived, slow-moving radionuclides.

The new source term model in RESRAD-OFFSITE code Version 3 was also compared with the Contaminant Transport Module for Radionuclides (RT Module) in GoldSim. To simulate the first order and uniform release, mass was introduced through the source element in the cell pathway. The waste material without any barrier was assumed to be contained in the source element and was assumed to degrade with a constant rate in the case of first-order release and with specified lifetime in the case of uniform release. The contaminants released from the source element were mixed in the cell element and were carried to the pipe element with the infiltrating water. The pipe element was assumed to be soil as the infill medium with known density and porosity. The contaminants were assumed to partition between the solid and liquid phases and transported through the advection process in the pipe element. The contaminant's outflow from the pipe element was linked to a sink element from which the release rates were obtained. To simulate the release of radionuclides under equilibrium desorption conditions, the initial inventory of radionuclides was uniformly distributed in the pipe (or aquifer, in the case of no dispersion) element. In the limited comparisons conducted, the results obtained with RESRAD-OFFSITE and GoldSim were, in general, in excellent agreement for all three source release options.

Multi-input probabilistic analysis and single input sensitivity analysis can be performed on the inputs that quantify the release. Examples of single input analysis and multiple input analysis are provided to illustrate how to perform these sensitivity analyses and to illustrate the sensitivity outputs that are available in the code. Appendix $C$ discusses the sensitivity analyses in greater detail.

The revisions and additions to Version 3.1 of the code extend the idea of a uniform release, where the radionuclides in equal fractions of the contaminated material become available for 
release at equal time intervals to both release mechanisms, that is, first-order rate controlled and instantaneous desorption equilibrium. The revisions and additions also make it possible to model time-delay scenarios where the radionuclides become available for release at some time in the future. This delayed and time-distributed release option allows modeling of situations where the radionuclides are initially in an immobilized state due to engineered barriers or release-resistant waste forms, but might become available for release over a future period of time as the engineered barriers and/or waste forms deteriorate with time.

RESRAD-OFFSITE code Version 3.1 automates the calculation of area factors for offsite exposure scenarios using the probabilistic feature of the code. The area factor is defined as the ratio of the calculated dose from the large area of primary contamination divided by the dose from a small area of elevated activity (hot spot) for the same exposure scenario. Area factors can be used in conjunction with the Multi-Agency Radiation Survey and Site Investigation Manual (MARSSIM) to quickly determine cleanup criteria for small areas of elevated contamination. 


\section{Acknowledgments}

The authors would like to recognize John Randall and Wendy Reed, the U.S. Nuclear Regulatory Commission (NRC) Project Managers, for their effective project technical direction and helpful guidance in ensuring the high quality and timeliness of the work. We would like to thank Adam Schwartzman, Phil Reed, and Boby Eid, all of NRC, for reviewing and providing constructive comments on the draft report. We would also like to thank Dr. David LePoire of Argonne National Laboratory (Argonne) for his technical peer review of the final report and his constructive comments during the development of the new source term models. We would like to thank Terry Sullivan of Brookhaven National Laboratory for providing the DUST-MS source and executable codes and for helpful discussions on the source release mechanisms implemented in the DUST code. We would like to thank Mark Nutt of Argonne for his interest and support on the application of GoldSim. We also thank Andrea Manning and Suzanne Williams, technical editors at Argonne, and Linda Conlin and Vicki Skonicki of the Document Processing Center of Argonne's Communications, Education, and Public Affairs Division for preparing the manuscript. 


\section{Introduction}

The RESRAD-OFFSITE Version 2 computer code was released in June 2007 (Yu et al. 2007). It is designed to evaluate the radiological dose and excess cancer risk to an individual who is exposed while located within or outside of the area of initial (primary) contamination. The primary contamination, which is the source of all of the releases modeled by the code, is assumed to be a layer of soil material. Just as with the RESRAD (onsite) code (Yu et al. 2001), the RESRAD-OFFSITE code Version 2 models the release from the primary contamination using a first-order exponential leaching model to estimate the release of contaminants. It then models the movement of contaminants from the area of primary contamination to agricultural areas, pastures, a dwelling area, a well, and a surface water body. The RESRAD-OFFSITE code also models the accumulation of the contaminants at those locations where appropriate. Any contribution of the contaminants from the water sources to the land-based locations is also modeled.

\subsection{Overview of the Source Release Model in RESRAD-OFFSITE Version 2}

The source release model used in RESRAD-OFFSITE Version 2 assumes a homogeneous distribution of radionuclides within an initially contaminated soil layer of uniform thickness. It accounts for radiological transformations (decay and ingrowth). The leaching of radionuclides in the contaminated layer by infiltration is modeled as a first-order, rate-controlled process occurring over the depth of the contamination. The code does not model the transport of the release within the contaminated zone; all of the material that is leached at any time is assumed to leave at the bottom of the contamination at that time. It also considers mixing in a surface layer. The concentration in the mixing layer is affected by erosion release to runoff and by leaching. Erosion is modeled by using the Universal Soil Loss Equation. Release to the atmosphere is computed by assuming that the clean dust that settles on the primary contamination is balanced by the release of an equal amount of contaminated dust.

RESRAD-OFFSITE has been used by the U.S. Nuclear Regulatory Commission (NRC) and its licensees; the U.S. Department of Energy (DOE) and its contractors; and other Federal agencies, health physicists, and risk assessors for the evaluation of contaminated sites, remediation alternatives, derivation of cleanup criteria, and performance assessments of waste disposal facilities. Recent decommissioning activities have involved evaluating the exposure to materials other than soil (e.g., slag, concrete, etc.). The current source term model (i.e., the first-order exponential leaching model) implemented in the RESRAD-OFFSITE code needs to be expanded to properly model various source release mechanisms. These release mechanisms may include surface wash off or surface rinse, diffusion, dissolution, and ion- 
exchange. A proposal in the form of a Letter Report on expanding the source term model used in the RESRAD-OFFSITE code was submitted to NRC. This Letter Report is included in Appendix A. The new source release mechanisms/models are developed referencing the models used in the DUST-MS (Disposal Unit Source Term-Multiple Species) code (Sullivan 2001a). The existing contaminant transport models and the assumptions and algorithms of the RESRAD-OFFSITE code are taken into account when developing the new source term models. Some of the considerations are discussed in the next section.

\subsection{Considerations for Expanding the Source Term Model}

The source term model implemented in RESRAD-OFFSITE Version 2 computes three releases from the contaminated zone, as well as the concentration and distribution of the radionuclides within the contaminated zone. The model calculates the atmospheric release of particulates due to resuspension and that of volatiles due to diffusion and evapotranspiration, the surface water release due to erosion by runoff water, and the groundwater release due to leaching by infiltrating water. The release rates are used by the code to compute the transport of contaminants and exposure at offsite locations, whereas the concentration and distribution of radionuclides within the contaminated zone are used to compute the direct external exposure from the primary contamination, as well as any exposure from onsite activities. The newly developed source term models consider these releases and distribution in the primary contaminated zone.

Another consideration is the radionuclide decay and ingrowth chain. The new source term models consider not just the parent radionuclide but all radionuclides in the decay chain. The RESRAD-OFFSITE code Version 2 uses the radionuclide information in International Commission on Radiological Protection Publication 38 (ICRP-38) to construct the decay sequences of the radionuclide being analyzed. The codes in the RESRAD family are being modified to use the radionuclide information in ICRP-107. The user of the code specifies a cutoff half-life. The fate and transport of all transformation products that are of a half-life greater than or equal to the user-specified cutoff are modeled explicitly. Any transformation product that has a half-life that is less than that of the cutoff is assumed to be in secular equilibrium with its immediate parent whose half-life is greater than that of the cutoff.

In addition, it is desirable to be able to perform sensitivity analysis and probabilistic analysis on the input parameters of the new source term models. The newly implemented source term models do, in fact, allow users to perform both sensitivity and probabilistic analyses on new inputs. However, the probabilistic distribution functions for some input parameters under various conditions will still need to be developed. 


\subsection{The Proposed Approaches and the Implemented Source Term Models}

Three approaches were proposed to NRC for adding the new release source options to the RESRAD-OFFSITE code (see Appendix A). The first is to derive the formulations for the desired mechanisms of release on the basis of DUST-MS code and implement the formulations in RESRAD-OFFSITE. The second is to link the DUST-MS code (executables) to RESRAD-OFFSITE. The third approach is to incorporate parts of the source code from DUST-MS regarding the desired source release mechanisms into the source code of RESRAD-OFFSITE. The pros and cons of these three approaches are discussed in detail in Appendix $A$; summaries of the advantages and disadvantages of each follow.

Approach 1, which involves including the formulations of the various release options to uncontained contamination, will enhance the code while preserving all of the current capabilities of RESRAD-OFFSITE. This approach will provide more options for calculating release to groundwater while evaluating exposure from all of the current exposure pathways in RESRAD-OFFSITE. It will be possible to perform probabilistic and sensitivity analyses on all of the new inputs.

Approach 2, which involves the linking of the one-dimensional, finite-difference DUST code (executables) to RESRAD-OFFSITE, is expected to be the easiest to implement. All three approaches will require coding to the user interface to allow specification of the additional inputs. In addition, this approach will require coding to prepare the input file for DUST from the interface and coding to reformat the output of DUST to a form that is useable by RESRAD OFFSITE. Drawbacks include the inability to perform sensitivity and probabilistic analyses and the ability to model only the exposure from the release to groundwater.

Approach 3, which involves incorporating the analytical formulations for container degradation and release into RESRAD-OFFSITE, will be more difficult to implement than Approach 2. It will be necessary to understand the coding to properly combine it with the transport formulations in RESRAD-OFFSITE so as to make the necessary changes to ensure that the imported coding is compatible with the FORTRAN complier used in RESRAD-OFFSITE. While it will be possible to perform sensitivity and probabilistic analyses, this approach will also be limited to modeling exposure from the release to groundwater.

Although these three approaches are not mutually exclusive, different tasks need to be performed and efforts made to implement the different approaches for expanding the existing source term model. It would be preferable to include more than one approach to allow users the flexibility of choosing the option appropriate for their applications. Of the proposed approaches, 
the preferred task was to implement Approach 1 (provide more release options for uncontained contamination in soil).

Approach 1 has the advantages of allowing users to perform both sensitivity and probabilistic analyses on the new input parameters, and it is the approach approved by NRC and implemented in RESRAD-OFFSITE Version 3.

The new source release mechanisms implemented in RESRAD-OFFSITE are described in detail in Chapter 2. These new release mechanisms are not limited to treating soil materials as the source (primary contamination). The release mechanisms are also not limited to uncontainerized sources because the new source release models assume that the releases are from a distinct phase of source material; once released into surrounding soils, they are transported in the primary contamination. The new source release models implemented in RESRAD-OFFSITE are compared with DUST-MS code, and the results are presented in Chapter 3. Additional comparisons were also conducted later with the GoldSim software, and these results are presented in Appendix B. Appendix $C$ contains the detailed description of and examples on how to conduct sensitivity and probabilistic analyses using RESRAD-OFFSITE code. The results of using sensitivity and probabilistic analyses on the new source release input parameters are presented in Chapter 4. This report is available from the RESRAD Web site in the Documents section (http://web.ead.anl.gov/resrad/documents/). It is also included in the RESRAD-OFFSITE Version 3 code in the Help button. The RESRAD-OFFSITE code Version 3 can be downloaded from the RESRAD Web site: http://www.evs.anl.gov/resrad.

Additional source term options were added to RESRAD-OFFSITE code Version 3.1 to consider the time-delay feature of source release mechanisms. These options consider two forms of contaminated material, one that is susceptible to releasing the radionuclides and the other that is not. Therefore, two time-delay periods are considered: (1) a period of time of no releases, and (2) a time period for the transition of contaminated material from one form to another. These additions were made after the preparation of the main body of this report and are described in Appendix D. Appendix D also includes some limited comparisons of the outputs of the Version 3.0 beta release options and the Version 3.1 release options.

\subsection{Area Factors for Offsite Exposure Scenarios}

Another new feature added to RESRAD-OFFSITE code Version 3.1 is the calculation of area factors for offsite exposure scenarios. The area factor is defined as the ratio of the calculated dose from the large area of primary contamination divided by the dose from a small area of elevated activity (hot spot) for the same exposure scenario. Area factors can be used in conjunction with the Multi-Agency Radiation Survey and Site Investigation Manual (MARSSIM) to quickly determine cleanup criteria for small areas of elevated contamination. The area 
factors are calculated using the probabilistic feature of the code. The interface of the code automates the calculation of air transport distances and directions, groundwater transport distances, and direct exposure distances for a number of small areas of elevated contamination sampled according to a number of user-specified conditions. The doses calculated for each of these small areas of elevated contamination are displayed graphically in the probabilistic output interface and are used to compute a table of area factors for each radionuclide in the primary contamination. The detailed users' guide in Appendix E describes the area factor. 
This page is intentionally left blank. 


\section{Primary Contamination and the Source Term}

This chapter consists of five sections. The first (Section 2.1) describes the manner in which RESRAD-OFFSITE conceptualizes the primary contamination, specifically its physical dimensions; the concentration of radionuclides within it; and the releases to the atmosphere, to surface erosion, and to groundwater as a function of time. Section 2.2 derives the expressions that result from the conceptual model, and Section 2.3 deals with the implementation of the solution or the evaluation of these expressions in the computational code. Section 2.4 describes the input forms, the input, and how the inputs are used in the code to compute the releases. The last subsection (2.5) describes the method to override the source term model of the RESRAD-OFFSITE code. These formulations were extended further in RESRAD-OFFSITE Version 3.1 by adding a time-delay feature to the source release mechanism as described in Appendix $D$ after the writing of the main body of this report.

\subsection{Conceptualization of the Primary Contamination}

The initial contamination is assumed to be distributed uniformly throughout a soil layer. This contaminated layer is of uniform thickness and can have a clean cover of uniform thickness above it. The clean cover and the primary contamination may be eroded by surface runoff, and their thicknesses can decrease over time. Erosion occurs from a well-mixed surface layer. The thickness of the primary contamination is not affected until the thickness of the cover erodes to the thickness of this surface layer. The primary contamination is situated above the water table; it can be just above and in contact with the water table, or there can be up to five different intervening, partially saturated soil layers. The assumptions about the shape of the conceptual primary contamination vary across exposure and transport models. The atmospheric and groundwater transport modules assume rectangular shapes, although the shape specified for use by the two transport modes need not be identical. The external exposure module assumes either a circular shape or a polygonal shape for the primary contamination.

The current version of RESRAD-OFFSITE contains three new options to compute the release to groundwater. The "First Order Release with Transport" option is similar to the release mechanism that was available in the preceding versions of the code. The other two new options that are currently in the code are the "Uniform Release" and the "Equilibrium Desorption Release." The preexisting release methodology is being retained for backward compatibility, and it is also the default release option for the new code, Version 3.

Under the First Order Release with Transport option, the transfer to groundwater at any time is proportional to the radionuclide inventory at that time and occurs uniformly over the thickness of the primary contamination. Thus, the transfer to groundwater affects the concentration in the 
primary contamination but not its physical dimensions; the vertical concentration profile remains uniform over time. The proportionality constant, the leach rate, has to be specified by the user if this option is chosen.

The Uniform Release is the other rate-controlled option in the code. This option models a situation where the source weathers at a uniform rate; the radionuclides and their progeny contained within a constant fraction of the initial contamination are transferred to the soil moisture in a unit of time. This transfer also occurs uniformly over the thickness of the primary contamination. The vertical concentration profile remains uniform over time, and the dimensions of the contamination are not affected by the transfer. The concentration in the primary contamination varies linearly with time because of this transfer, and exponentially with time because of radiological transformations. The user must specify the duration of the release if this option is chosen.

The infiltration that comes into contact with the contamination will become more contaminated as it passes through the primary contamination and will eventually attain the equilibrium concentration of the radionuclides. How long it takes to reach equilibrium and hence how deep it needs to travel before it reaches equilibrium depend on the rate at which the radionuclides are released from the soil solids into the infiltrating water and the rate at which the radionuclides sorb or precipitate back on the solids in the soil from the infiltrating water. The code uses the assumption of instantaneous equilibrium, one in which the forward and backward reaction rates are so fast that equilibrium is attained instantaneously, as soon as the clean infiltration contacts the top of the contamination. Under this assumption, the radionuclides are removed from the top of the contamination. In the Equilibrium Desorption Release model, the concentration in the infiltrating water is determined by the distribution coefficient and the concentration in the soil. The distribution coefficient also determines the rate at which the radionuclides are transported by the infiltration down through the primary contamination. The user must specify the distribution coefficient if this option is chosen. This situation is most easily and quickly modeled by treating it as an instantaneous release of radionuclide over the initial depth of contamination and by applying the groundwater transport model. The groundwater transport model gives the flux out of the bottom of the primary contamination. It is also possible to apply the groundwater transport model independently to compute the vertical concentration profile of each parent and progeny in the surface soil; however, this functionality has not been coded. Thus, the code computes only the release to groundwater under this option; it does not compute releases to the atmosphere nor erosion release by surface runoff.

The radionuclides are released to groundwater uniformly over the depth of the primary contamination under the rate-controlled release options-first-order release and uniform release. Thus, the release to groundwater affects the concentration in the primary contamination but not its physical dimensions. In instantaneous equilibrium-controlled groundwater release (i.e., solubility or adsorption/desorption) models, contaminants are 
removed from the top of the layer, leading to nonuniform concentration profiles in the vertical direction. Currently, the code computes the release to the atmosphere and to surface runoff only for the rate-controlled releases. The additional computations of concentration profile for the equilibrium desorption release will consume significant computation time; these can be coded to be performed only if the surface soil will become contaminated. The release of dust to the atmosphere and the release of contaminated soil to surface runoff occur from the well-mixed surface layer at the top of the primary contamination. Thus, these releases lower the total quantity of the radionuclides in primary contamination but not the concentration in the primary contamination below the surface layer. The release of $\mathrm{H}-3$ and $\mathrm{C}-14$ by evasion can occur from over the whole depth of the contamination; it is modeled in RESRAD-OFFSITE as being uniform over the entire depth of the primary contamination and affects the concentration in the primary contamination but not its physical dimensions.

Transport of the radionuclides within the contaminated zone by the movement of infiltrating water is modeled for all three releases. If appropriate, the user can specify the distribution coefficient in the primary contamination to model the interaction of the radionuclide with the soil even when the release is not controlled by equilibrium desorption/adsorption in the soil.

\subsection{Derivation of Mathematical Expressions for the Conceptual Primary Contamination Model}

The conceptual model has to be translated into mathematical expressions before it can be used in the computational code. The idealized descriptions of the previous section are expressed in mathematical terms in this section.

\subsubsection{Thickness of the Primary Contamination}

The thickness of the primary contamination is computed as a function of time on the basis of the values of the initial thicknesses and erosion rates of the cover and the primary contamination as follows:

$$
\begin{gathered}
T_{p c}(t)=T_{p c}(0) \text { when } t \leq t_{c v}, \text { and } \\
T_{p c}(t)=T_{p c}(0)-\varepsilon_{p c}\left(t-t_{c v}\right) \text { when } t>t_{c v},
\end{gathered}
$$


where

$$
\begin{aligned}
T_{p c}(t) & =\text { thickness of the primary contamination at time } t(\mathrm{~m}), \\
T_{p c}(0) & =\text { initial thickness of the primary contamination }(\mathrm{m}), \\
t & =\text { time since the site was characterized }(\mathrm{yr}), \\
t_{C V} & =T_{C V}(0) / \varepsilon_{C V}=\text { time to erode the cover }(\mathrm{yr}), \\
T_{C V}(0) & =\text { initial thickness of the cover }(\mathrm{m}), \\
\varepsilon_{C V} & =\text { rate at which the cover is eroded }\left(\mathrm{m} \mathrm{yr}^{-1}\right), \text { and } \\
\varepsilon_{p c} & =\text { rate at which the primary contamination is eroded }\left(\mathrm{m} \mathrm{yr}^{-1}\right) .
\end{aligned}
$$

The erosion rate is computed using the Universal Soil Loss Equation. Section 12.10 of the Handbook of Hydrology (Shen and Julien 1993) has figures and tables for the first five factors in the following expression for erosion rate,

$$
\varepsilon=224 \times R \times K \times L S \times C \times P /\left(\rho \times 10^{6}\right)
$$

where

$$
\begin{aligned}
\varepsilon= & \text { erosion rate }\left(\mathrm{m} \mathrm{yr}^{-1}\right), \\
\mathrm{R}= & \text { annual rainfall erosion index, the rainfall erosivity factor, or the rainfall and runoff } \\
& \text { factor }\left(\mathrm{yr}^{-1}\right), \\
\mathrm{K}= & \text { soil erodibility factor (tons/acre) } \\
\mathrm{LS}= & \text { slope length-steepness factor (dimensionless) } \\
\mathrm{C}= & \text { cropping-management factor or the cover and management factor } \\
& \text { (dimensionless), } \\
\mathrm{P}= & \text { conservation practice factor or the support practice factor (dimensionless), } \\
224= & \text { to convert tons per acre to gram per square meter } \left.\left(\mathrm{g} \mathrm{m}^{-2} \text { [tons/acre] }\right]^{-1}\right), \\
\rho= & \text { dry bulk density of the soil }\left(\mathrm{g} \mathrm{cm}^{-3}\right), \text { and } \\
10^{6}= & \text { to convert per cubic centimeter to per cubic meter }\left(\mathrm{cm}^{3} \mathrm{~m}^{-3}\right) .
\end{aligned}
$$




\subsubsection{Concentration of Radionuclides in the Primary Contamination}

Radiological transformations and the release to infiltrating water diminish the concentration of radionuclides in soil in the primary contamination over time. The concentration of progeny radionuclides can increase over time if the transformation of the parent radionuclide produces more progeny radionuclides than are lost to the infiltrating water and by radiological transformations of the progeny. The vertical concentration profile of the radionuclides in the primary contamination for each release mechanism is derived in this sub-section. The implementation of the algorithms to compute the concentration profile is described in Section 2.3.

\subsubsection{Concentration of Radionuclides in the Primary Contamination under the First Order Release Model}

The transfer to the infiltrating water at any time is proportional to the concentration at that time. Because both rate-controlled leaching and radiological transformations are proportional to the concentration or inventory at that time, both of these processes will occur uniformly over the entire depth of the primary contamination if the initial concentration profile is uniform. Thus, the activity concentration of the radionuclide remains uniform over the primary contamination under the conceptual model of RESRAD-OFFSITE. Under these conditions, the activity concentrations of the radionuclide that is present initially and its progeny are obtained by solving the following series of equations,

$$
\begin{gathered}
\frac{d A_{1}}{d t}=-\left(\lambda_{1}+\mu_{1}\right) A_{1} \text {, and } \\
\frac{d A_{k}}{d t}=-\left(\lambda_{k}+\mu_{k}\right) A_{k}+\lambda_{k} A_{k-1} \text { for } 2 \leq k \leq n,
\end{gathered}
$$

where

$$
\begin{aligned}
A_{k}(t) & =\text { activity concentration of the } k^{\text {th }} \text { radionuclide of the transformation chain }\left(\mathrm{pCi}^{-1}\right), \\
t & =\text { time since the site was characterized }(\mathrm{yr}) \\
\lambda_{k} & =\text { radiological transformation constant of the } k^{\text {th }} \text { radionuclide }\left(\mathrm{yr}^{-1}\right), \text { and } \\
\mu_{k} & =\text { leach rate constant of the } k^{\text {th }} \text { radionuclide }\left(\mathrm{yr}^{-1}\right) .
\end{aligned}
$$

The term on the left is the change in activity concentration, and the terms on the right are the reductions of activity concentration due to radiological transformations and leaching, respectively, and the gain in activity concentration due to radiological transformations of the 
parent. The solutions obtained by multiplying the equation by $e^{\left(\lambda_{k}+\mu_{k}\right) t}$ and evaluating the integral $\int_{0}^{t} e^{\left(\lambda_{k}+\mu_{k}\right) t} A_{k-1}(t) d t$, are of the form,

$$
A_{k}(t)=\sum_{i=1}^{k} a_{k, i} \exp \left(-\lambda_{i} t-\mu_{i} t\right)
$$

where

$$
\begin{aligned}
& a_{k, i}=\text { set of coefficients defined by } a_{1,1}=A_{1}(0), \\
& a_{k, i}=\frac{\lambda_{k} a_{k-1, i}}{\lambda_{k}+\mu_{k}-\lambda_{i}-\mu_{i}} \text { for all } 1 \leq i<k, \text { and } \\
& a_{k, k}=-\sum_{i=1}^{k-1} a_{k, i} .
\end{aligned}
$$

For $\mathrm{H}-3$ and $\mathrm{C}-14$, the activity concentration is given by the equation,

$$
A(t)=A(0) \exp (-\lambda t-\mu t-\varepsilon v(t) t)
$$

where

$$
\begin{aligned}
\varepsilon v(t)= & \text { evasion rate at time } t\left(\mathrm{yr}^{-1}\right) \text { (see Appendix } L \text { of the User's Manual for RESRAD } \\
& \text { Version } 6 \text { [Yu et al. 2001] for the time-dependent evasion rate and for a } \\
& \text { discussion of the } \mathrm{H}-3 \text { and } \mathrm{C}-14 \text { models). }
\end{aligned}
$$

\subsubsection{Concentration of Radionuclides in the Primary Contamination under the Uniform Release Model}

The release to the infiltrating water at any time is the product of two independent processes, one dependent and the other independent of time. The incremental volume of source from which radionuclides are transferred in each unit of time remains constant over the release duration; this process occurs uniformly over the depth of the primary contamination.

Radiological transformations are proportional to the concentration or inventory at that time; this process will occur uniformly over the entire depth of the primary contamination if the initial concentration profile is uniform. Thus, the activity concentration of the radionuclide remains uniform over the primary contamination. Under these conditions, the activity concentrations of the radionuclide that is present initially and its progeny are obtained by solving the following series of equations, 


$$
\begin{gathered}
\frac{d A_{1}}{d t}=-\lambda_{1} A_{1} \text {, and } \\
\frac{d A_{k}}{d t}=\lambda_{k} A_{k-1}-\lambda_{k} A_{k} \text { for } 2 \leq k \leq n,
\end{gathered}
$$

where

$$
\begin{aligned}
A_{k}(t) & =\text { activity concentration of the } k^{\text {th }} \text { radionuclide of the transformation chain }\left(\mathrm{pCi} \mathrm{g}^{-1}\right), \\
t & =\text { time since the site was characterized }(\mathrm{yr}) \\
\lambda_{k} & =\text { radiological transformation constant of the } k^{\text {th }} \text { radionuclide }\left(\mathrm{yr}^{-1}\right)
\end{aligned}
$$

and then accounting for the release to groundwater.

The solutions obtained by multiplying the equation by $e^{\lambda_{k} t}$ and evaluating the integral $\int_{0}^{t} e^{\lambda_{k} t} A_{k-1}(t) d t$, are of the form,

$$
A_{k}(t)=\left(1-\frac{t}{T_{\text {release }}}\right) \sum_{i=1}^{k} a_{k, i} \exp \left(-\lambda_{i} t\right)
$$

where

$$
\begin{aligned}
T_{\text {release }} & =\text { is the duration of the release (years), } \\
a_{k, i} & =\text { set of coefficients defined by } a_{1,1}=A_{1}(0), \\
a_{k, i} & =\frac{\lambda_{k} a_{k-1, i}}{\lambda_{k}-\lambda_{i}} \text { for all } 1 \leq i<k, \text { and } \\
a_{k, k} & =-\sum_{i=1}^{k-1} a_{k, i} .
\end{aligned}
$$

For $\mathrm{H}-3$ and $\mathrm{C}-14$, the activity concentration is given by the equation,

$$
A_{k}(t)=\left(1-\frac{t}{T_{\text {release }}}\right) A(0) \exp (-\lambda t-\varepsilon v(t) t)
$$


where

$$
\begin{aligned}
\varepsilon v(t)= & \text { evasion rate at time } t\left(\mathrm{yr}^{-1}\right) \text { (see Appendix } L \text { of the User's Manual for RESRAD } \\
& \text { Version } 6 \text { [Yu et al. 2001] for the time-dependent evasion rate and for a } \\
& \text { discussion of the } \mathrm{H}-3 \text { and } \mathrm{C}-14 \text { models). }
\end{aligned}
$$

\subsubsection{Concentration of Radionuclides in the Primary Contamination under the Equilibrium Desorption Model}

The infiltrating water attains the equilibrium concentration of the radionuclide as soon as it comes into contact with the contaminated soil. Thus, the radionuclides will be removed from the leading edge of the primary contamination, and the thickness of the contamination will change with time. Because the different radionuclides can have different equilibrium distribution coefficients, the profile of the progeny may not be uniform over the thickness; a rapidly moving parent will produce more progeny at the lower part of the primary contamination than at the upper regions. The release to groundwater under this release mechanism is computed by modeling an instantaneous release of material uniformly over the thickness of the primary contamination at time zero and then letting the groundwater transport formulation compute the flux at all subsequent times. The concentration profile of the radionuclide over the thickness of the contamination at different times is not needed for the computation of the flux released to groundwater. The current draft code does not compute the concentration profile in the primary contamination at various times for this release mechanism. The concentration profile over the thickness of the primary contamination needs to be computed if direct exposure from the primary contamination or onsite exposure-or both-are to be computed. The concentration profile over the thickness of the primary contamination that eventually gets incorporated into the mixing layer will need to be computed if release to the atmosphere or release to surface runoff is to be modeled. While the concentration profile can be computed by using the formulations in the groundwater transport section of the RESRAD-OFFSITE manual (Sections 3.2.4 and 3.2.5), this step is not currently performed in the code. The calculations will be both memory- and computation time-intensive and will need to be coded so that they are performed only when necessary and so that memory and computation time requirements are minimized.

\subsubsection{Surface Soil Mixing Model}

The releases to the atmosphere and to surface runoff occur from the surface soil layer. The concentration of radionuclides in surface soil differs from the concentration in the primary contamination because of mixing with any uncontaminated cover or with soil below the primary contamination. Because the contaminant concentrations are expressed in terms of mass of soil (and not in terms of the volume of soil), it is necessary to account for any differences in density among the different layers of soil. The formulations in this section apply for the two 
rate-controlled release mechanisms. The thickness of the primary contamination is dependent on the radionuclide and also varies with time as the radionuclides are released from the top to the infiltrating water for equilibrium-based releases. These processes need to be incorporated into the surface soil mixing model for the equilibrium desorption release mechanism.

\subsubsection{Density of Soil in the Mixing Zone}

The density of the mixing zone is computed by assuming that mixing occurs continuously (over time) over the specified mixing depth. If the thickness of the cover exceeds the depth of mixing, the mixing zone will be uncontaminated, and the density is not computed for this condition. The cover can erode with time, and the mixing zone will then penetrate the primary contamination. The density of the mixing zone for this condition is computed as follows.

When $T_{c v}(t)<d_{m i x} \leq T_{c v}(t)+T_{p c}(t)$, the density is obtained by solving the equation $d_{m i x} \frac{d \rho_{m i x}}{d t}=\varepsilon\left(\rho_{p c}-\rho_{m i x}\right)$ with the appropriate initial condition. The term on the left is the change in mass within the mixing zone, the terms on the right are, in order, the mass entering the mixing zone from the primary contamination and the mass leaving the mixing zone due to erosion.

The initial conditions are:

$$
\begin{gathered}
\rho_{m i x}(0)=\rho_{c v} \text { if } T_{c v}(0) \geq d_{m i x} \text {, and } \\
\rho_{m i x}(0)=\rho_{p c}+\frac{T_{c v}(0)}{d_{m i x}}\left(\rho_{c v}-\rho_{p c}\right) \text { if } T_{c v}(0)<d_{m i x} .
\end{gathered}
$$

The solution is easier to understand and is more compact when expressed as a function of the depth of penetration of the mixing zone into the primary contamination since initial mixing, rather than as a function of time. The expression for the density of the mixing zone is:

$$
\rho_{\text {mix }}\left(d_{p c}\right)=\rho_{p c}+\left(\rho_{m i x}(0)-\rho_{p c}\right) \exp \left(-d_{p c} / d_{m i x}\right),
$$

where

$$
\begin{aligned}
T_{C V}(t) & =\text { thickness of the cover after time } t(\mathrm{~m}), \\
d_{m i x} & =\text { depth of the mixing zone }(\mathrm{m}), \\
\rho_{\text {mix }}\left(d_{p c}\right) & =\text { density of the mixing zone }\left(\mathrm{g} \mathrm{cm}^{-3}\right),
\end{aligned}
$$




$$
\begin{aligned}
\rho_{p C}= & \text { density of the primary contamination }\left(\mathrm{g} \mathrm{cm}^{-3}\right) \\
\rho_{C V}= & \text { density of the clean cover }\left(\mathrm{g} \mathrm{cm}^{-3}\right), \text { and } \\
d_{p c}= & \text { depth of penetration of the mixing zone into the primary contamination since } \\
& \text { initial mixing }(\mathrm{m}) .
\end{aligned}
$$

Eventually, the cover and primary contamination will be eroded so that their combined thickness will be less than the depth of the mixing zone. Then the soil underlying the primary contamination will enter the mixing zone. Because it is too cumbersome to track the layers (any unsaturated zones first and then the saturated zone) that can enter the mixing zone over time, density changes are not modeled after the mixing zone penetrates the bottom of the primary contamination. However, if the initial thicknesses of the clean cover and the primary contamination are less than the post-release mixing depth, the code does compute the density at initial mixing, including consideration of the densities of the underlying layers that enter the mixing zone at time zero.

\subsubsection{Volume Fraction of Soil from the Primary Contamination in the Mixing Zone}

The quantity of radionuclides in the mixing zone is directly proportional to the volume of soil in the mixing zone that originated from the primary contamination. The mixing zone is uncontaminated as long as its depth is less than the thickness of the clean cover. The volume fraction of soil from the primary contamination in the mixing zone is evaluated when the depth of the mixing zone exceeds the thickness of the clean cover. It is evaluated by assuming that mixing occurs continuously (over time) over the specified mixing depth and that the volume fraction in the eroded soil is the same as the volume fraction in surface soil.

When $T_{c v}(t)<d_{m i x} \leq T_{c v}(t)+T_{p c}(t)$, the volume fraction of primary contamination in the mixing zone is obtained by solving the equation $d_{\min } \frac{d f_{v m}}{d t}=\varepsilon\left(1-f_{v m}\right)$, with the appropriate initial condition. The term on the left is the change in the volume of soil from the primary contamination in the mixing zone, and the terms on the right are, in order, the volume of soil that enters the mixing zone from the primary contamination and the volume of soil from the primary contamination that leaves the mixing zone due to erosion. The initial conditions are:

$$
\begin{gathered}
f_{v m}(0)=0 \text { if } T_{c v}(0) \geq d_{m i x} \text {, and } \\
f_{v m}(0)=1-\frac{T_{c v}(0)}{d_{m i x}} \text { if } T_{c v}(0)<d_{m i x} .
\end{gathered}
$$


As in the case with the density in the preceding section (Section 2.2.3.1), the solution is easier to understand and is more compact when expressed as a function of the depth of penetration of the mixing zone into the primary contamination since initial mixing, rather than as a function of time. The expression for the volume fraction of primary contamination in the mixing zone is:

$$
f_{v m}\left(d_{p c}\right)=1-\left(1-f_{v m}(0)\right) \exp \left(-d_{p c} / d_{m i x}\right) \text {, }
$$

where

$$
f_{v m}\left(d_{p c}\right)=\text { volume fraction of primary contamination in the mixing zone. }
$$

As the cover erodes, the mixing layer penetrates deeper into the primary contamination, and the volume fraction increases asymptotically toward unity as long as the bottom of the mixing zone stays within the primary contamination.

When the bottom of the mixing zone moves out of the primary contamination and into the underlying layers, the volume fraction will decrease. The volume fraction is computed under the assumption that the underlying soil is uncontaminated. ${ }^{1}$

When $d_{m i x}>T_{c v}(t)+T_{p c}(t)$, the volume fraction of contamination in the mixing layer is obtained by solving the equation $d_{m i x} \frac{d f_{v m}}{d t}=-\varepsilon f_{v m}$ with the appropriate initial condition. The initial conditions are:

$$
\begin{gathered}
f_{v m}^{p}=1-\exp \left(-T_{p c}(0) / d_{m i x}\right) \text { if } T_{c v}(0) \geq d_{m i x}, \\
f_{v m}^{p}=1-\frac{T_{c v}(0)}{d_{m i x}} \exp \left(-\frac{T_{c v}(0)+T_{p c}(0)-d_{m i x}}{d_{m i x}}\right) \text { if } T_{c v}(0)<d_{m i x}<T_{c v}(0)+T_{p c}(0), \text { and } \\
f_{v m}^{p}=\frac{T_{p c}(0)}{d_{m i x}} \text { if } d_{m i x} \geq T_{c v}(0)+T_{p c}(0)
\end{gathered}
$$

1 Because the code calculates only the flux of contaminants across the partially saturated zone boundaries and across the water table, and not the concentration profile in those layers, it is not possible to account for the contaminants in the underlying layer in this calculation and in the calculation of external direct radiation. 
The expression for the volume fraction of primary contamination in the mixing zone, now expressed in terms of the depth of penetration below the primary contamination for clarity and simplicity, is:

$$
f_{v m}\left(d_{u p c}\right)=f_{v m}^{p} \exp \left(-d_{u p c} / d_{m i x}\right)
$$

where

$$
\begin{aligned}
d_{u p c}= & \text { depth of penetration of the mixing zone into the layers underlying the primary } \\
& \text { contamination since initial mixing }(\mathrm{m}) .
\end{aligned}
$$

The algorithms in the code can accommodate a situation where there is no mixing layer. In such a situation, the combined modification factor is zero while there is a cover and unity when there is no cover.

\subsubsection{Concentration of Radionuclides in the Surface Soil above the Primary Contamination}

The concentration of radionuclides in surface soil is computed by applying two modification factors to the concentration in the primary contamination: the first considers the volumetric mixing within the mixing layer, and the other accounts for the differences in density. These modification factors are independent of the concentration in the primary contamination and can be treated separately:

$$
A_{s c}(t)=f_{v m}(t) A_{p c}(t) \rho_{p c} / \rho_{m i x}(t),
$$

where

$$
\begin{aligned}
& A_{S C}(t)=\text { activity concentration in surface soil after time } t\left(\mathrm{pCi} \mathrm{g}^{-1}\right), \text { and } \\
& A_{p c}(t)=\text { activity concentration in primary contamination after time } t\left(\mathrm{pCi} \mathrm{g}^{-1}\right) .
\end{aligned}
$$

\subsubsection{Three-Layer Model}

The mixing model conceptualizes three layers for the source: (1) a clean cover, (2) the unmixed portion of the initial primary contamination, and (3) a mixing layer that is contaminated to a lesser extent than is the primary contamination. From the preceding sections (2.2.3.1, 2.2.3.2, and 2.2.3.3) that discuss the surface layer mixing model, it can be seen that no more than two of these layers can exist at any particular time. As long as the depth of the mixing zone is less than the depth of the clean cover, there will not be a contaminated mixing zone. Conversely, 
after the cover thickness decreases to the depth of the mixing zone, there will be no clean cover. With time, erosion could also bring about a situation in which the mixing zone breaks through the layer of initial contamination; then there would not be any initial contamination that is unmixed. These conditions are summarized below.

$$
\begin{gathered}
\text { When } T_{c v}(t) \geq d_{m i x} \text {, then } \\
T_{c v}^{c}(t)=T_{c v}(t), T_{m i x}^{c}(t)=0 \text {, and } T_{p c}^{u m}(t)=T_{p c}(0),
\end{gathered}
$$

where

$$
\begin{aligned}
T_{c v}^{c}(t) & =\text { thickness of the clean cover }(\mathrm{m}), \\
T_{m i x}^{c}(t) & =\text { thickness of the contaminated mixing layer }(\mathrm{m}), \text { and } \\
T_{p c}^{u m}(t) & =\text { thickness of the unmixed portion of the primary contamination }(\mathrm{m}) .
\end{aligned}
$$

When $T_{c v}(t)<d_{m i x} \leq T_{c v}(t)+T_{p c}(t)$, then

$$
\begin{gathered}
T_{c v}^{c}(t)=0, T_{m i x}^{c}(t)=d_{m i x} \text {, and } T_{p c}^{u m}(t)=T_{p c}(t)+T_{c v}(t)-d_{m i x} . \\
\text { When } T_{c v}(t)+T_{p c}(t)<d_{m i x} \text {, then } \\
T_{c v}^{c}(t)=0, T_{m i x}^{c}(t)=d_{m i x} \text {, and } T_{p c}^{u m}(t)=0 .
\end{gathered}
$$

\subsubsection{Release by Surface Runoff}

The activity of radionuclide released to surface water by the erosion of the surface soil above the primary contamination per unit of time is given by the product of the activity concentration in surface soil and the surface erosion rate. It is more easily computed as the product of the mass of primary contamination that is eroded per unit of time and the activity concentration in the primary contamination. The rate $\left(\mathrm{g} \mathrm{yr}^{-1}\right)$ at which soil from the primary contamination is eroded is given by:

$$
\left.m_{p c}(t)=\varepsilon A f_{v m}(t) \rho_{p c} 10^{6}\right)
$$

where

$$
\begin{aligned}
A & =\text { area of the primary contamination }\left(\mathrm{m}^{2}\right), \text { and } \\
10^{6} & =\text { to convert per cubic centimeter to per cubic meter }\left(\mathrm{cm}^{3} \mathrm{~m}^{-3}\right) .
\end{aligned}
$$


Then

$$
R_{k}^{s r}(t)=m_{p c}(t) A_{k}(t)
$$

where

$$
\begin{aligned}
R_{k}^{s r}(t)= & \text { rate }(\text { activity per time }) \text { at which the } k^{\text {th }} \text { radionuclide of the transformation chain is } \\
& \text { released by erosion }\left(\mathrm{pCi} \mathrm{yr}^{-1}\right) .
\end{aligned}
$$

\subsubsection{Release to Groundwater}

The flux of radionuclides out of the primary contamination depends on two processes: the transfer of radionuclides from the solid phase of the soil to the soil moisture followed by the transport of the radionuclides in soil moisture from the point of the transfer to the bottom of the primary contamination. The transfer of the radionuclides to the soil moisture is first computed by using the activity concentrations in the soil for the two rate-controlled release options. This computation is followed by modeling the transport of the radionuclides in soil moisture. Both processes are modeled together for the equilibrium desorption release option.

\subsubsection{Transfer of Radionuclides to Soil Moisture under the First Order Release Model}

The activity of radionuclide transferred by rate-controlled leaching to soil moisture per unit of time is given by the product of the total activity in soil and the first order leach rate. The transfer to groundwater under this release mechanism is given by:

$$
T_{k}^{g w}(t)=\mu_{k} A_{k}(t) \rho_{p c} A\left(f_{v m} T_{m i x}^{c}(t)+T_{p c}^{u m}(t)\right) 10^{6},
$$

where

$$
\begin{aligned}
& T_{k}^{g w}(t)=\text { rate (activity per time) at which the } k^{\text {th }} \text { radionuclide of the transformation chain is } \\
& 10^{6}=\text { to convert per cubic centimeter to per cubic meter }\left(\mathrm{cm}^{3} \mathrm{~m}^{-3}\right) \text {. }
\end{aligned}
$$


where

$$
\begin{aligned}
& a_{k, i}=\text { set of coefficients defined by } a_{1,1}=A_{1}(0), \\
& a_{k, i}=\frac{\lambda_{k} a_{k-1, i}}{\lambda_{k}+\mu_{k}-\lambda_{i}-\mu_{i}} \text { for all } 1 \leq i<k, \text { and } \\
& a_{k, k}=-\sum_{i=1}^{k-1} a_{k, i} .
\end{aligned}
$$

\subsubsection{Transfer of Radionuclides to Soil Moisture under the Uniform Release Model}

The activity of radionuclides transferred under this release mechanism to soil moisture per unit of time is the activity in incremental volume of soil that became susceptible to release during that time period. The release to groundwater under this release mechanism is given by:

$$
T_{k}^{g w}(t)=\frac{1}{T_{r e l}} A_{k}^{\prime}(t) \rho_{p c} A\left(f_{v m} T_{m i x}^{c}(t)+T_{p c}^{u m}(t)\right) 10^{6}
$$

with $A_{k}^{\prime}(t)=\sum_{i=1}^{k} a_{k, i} \exp \left(-\lambda_{i} t\right)$,

where

$$
\begin{aligned}
& a_{k, i}=\text { set of coefficients defined by } a_{1,1}=A_{1}(0), \\
& a_{k, i}=\frac{\lambda_{k} a_{k-1, i}}{\lambda_{k}-\lambda_{i}} \text { for all } 1 \leq i<k, \text { and } \\
& a_{k, k}=-\sum_{i=1}^{k-1} a_{k, i} .
\end{aligned}
$$




\subsubsection{Transport of Radionuclides from the Point of Transfer to the Bottom of the Primary Contamination for the Rate-Controlled Release Options}

The transport of the radionuclide from within the primary contamination is modeled using a modified form of the equations in Sections 3.2.4 and 3.2.5 of the RESRAD-OFFSITE manual. The quantity transferred from the original waste form to the soil is available in the previous two subsections (Sections 2.2.5.1 and 2.2.5.2). These transfers occur uniformly over the thickness of the primary contamination. This process is analogous to the uniform input pulse of contaminants into the water table below the length of the primary contamination as conceptualized in the groundwater transport models in Sections 3.2.4 and 3.2.5 of the RESRAD-OFFSITE manual. Those formulations are thus applicable with some modification to account for the different orientation and location and the fact that only longitudinal dispersion is modeled in the primary contamination-not lateral dispersion.

\subsubsection{Release of Radionuclides to Groundwater under the Equilibrium Desorption Model}

The flux to groundwater under this release mechanism is given by the formulations in Sections 3.2.4 and 3.2.5 of the RESRAD-OFFSITE manual for an instantaneous pulse input. For a parent radionuclide, this rate is given by a modified form of equation 3.39 of the RESRADOFFSITE manual,

$$
\begin{gathered}
R_{k}^{g w}(t)=A_{k}(0) \rho_{p c} A T_{p c}(0) 10^{6} \frac{V_{c} e^{-\lambda t}}{2 T_{c z}}\left[\operatorname{erf}\left(\frac{T_{c z}-V_{c} t}{\sqrt{4 D_{z}^{c} t}}\right)-e r f\left(\frac{-V_{c} t}{\sqrt{4 D_{z}^{c} t}}\right)\right] \\
-A_{k}(0) \rho_{p c} A T_{p c}(0) 10^{6} \sqrt{\frac{D_{z}^{c}}{4 \pi t}} \frac{e^{-\lambda t}}{T_{c z}}\left[e^{-\frac{\left(T_{c z}-V_{c} t\right)^{2}}{4 D_{z}^{c} t}}-e^{-\frac{\left(-V_{c} t\right)^{2}}{4 D_{z}^{c} t}}\right] .
\end{gathered}
$$

where

$$
\begin{aligned}
R_{k}^{g w}(t)= & \text { rate }(\text { activity per time }) \text { at which the } k^{\text {th }} \text { radionuclide of the transformation chain is } \\
& \text { released to groundwater }\left(\mathrm{pCi} \mathrm{yr} r^{-1}\right), \\
V_{c}= & \text { is the contaminant transport velocity }\left(\mathrm{m} \mathrm{yr}^{-1}\right), \text { and } \\
D_{z}^{c}= & \text { is the contaminant dispersion coefficient }\left(\mathrm{m}^{2} \mathrm{yr}^{-1}\right) .
\end{aligned}
$$

The expressions for the flux of the progeny produced in the primary contamination are more complicated and are obtained by using the formulations in Section 3.2.5. 


\subsubsection{Release to the Atmosphere in the Form of Dust}

The activity of radionuclide released to the atmosphere per unit of time is given by the product of the activity concentration in surface soil and the rate at which dust is released from the area of primary contamination. The conceptual model assumes that there is no net change in the mass loading of dust above the region of primary contamination. Under this assumption, the rate at which dust is released from the primary contamination is equal to the rate at which dust settles out of the air onto the region of primary contamination:

$$
R_{k}^{d u}(t)=f_{v m}(t) \frac{\rho_{p c}}{\rho_{m i x}(t)} A_{k}(t) m_{d u} A v_{d u} 3.15576 \times 10^{7},
$$

where

$$
\begin{aligned}
& R_{k}^{d u}(t)=\text { rate (activity per time) at which the } k^{\text {th }} \text { radionuclide of the transformation chain is }
\end{aligned}
$$

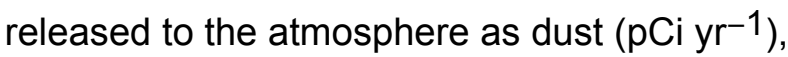

$$
\begin{aligned}
& m_{d u}=\text { concentration of dust in the air above the area of primary contamination }\left(\mathrm{g} \mathrm{m}^{-3}\right) \text {, } \\
& v_{d u}=\text { deposition velocity of dust in the area of primary contamination }\left(\mathrm{m} \mathrm{s}^{-1}\right) \text {, and } \\
& 3.15576 \times 10^{7} \text { is to convert per second to per year }\left(\mathrm{s} \mathrm{yr}^{-1}\right) \text {. }
\end{aligned}
$$

\subsection{Implementation of the Models for the Primary Contamination}

This section describes how the conceptual model (Section 2.1) or the expressions derived from the conceptual model (Section 2.2) are implemented in the computational code.

\subsubsection{Shape and Dimensions of the Primary Contamination}

The shape of the conceptual primary contamination in the horizontal plane depends on the exposure or transport model. The dimensions and orientation of the rectangular shape for the atmospheric transport model are specified in the site layout form or the map interface as described in Sections 4.3 and 4.4 of the user's guide. The area of primary contamination is computed by the computational code as the product of the two dimensions.

The groundwater transport model assumes that the primary contamination is rectangular in shape and with one pair of sides parallel to the direction of groundwater flow. The length of one of these sides is specified in the primary contamination form as described in Section 4.14 of the 
user's guide. The length of the other pair of sides is computed by the computational code using the area of the primary contamination.

Either a circular shape or a polygonal shape can be specified for the calculation of direct external radiation from the primary contamination, as described in Section 4.27 of the user's guide. The polygon can have as many sides as necessary to approximate the shape of the actual contamination. Polygonal-shaped areas of primary contamination and circular-shaped ones involving a nonconcentric receptor are analyzed by finding the fractions of the areas of a set of 16 annular regions, concentric with the receptor, that are covered by the primary contamination. The computation of the fraction of the annular regions that contain the primary contamination is performed in the interface and not in the computational code.

\subsubsection{Thicknesses of the Primary Contamination, Cover, Clean Cover, Contaminated Mixing Zone, and Undisturbed Primary Contamination}

The thickness of the cover is computed at each intermediate time on the basis of the values of the initial thickness and erosion rate of the cover as follows:

$$
\begin{gathered}
T_{c v}(t)=T_{c v}(0)-\varepsilon_{c v} t \text { when } t \leq t_{c v}=T_{c v}(0) / \varepsilon_{c v} \text {, and } \\
T_{c v}(t)=0 \text { when } t>t_{c v} .
\end{gathered}
$$

The thickness of the primary contamination is computed at each intermediate time on the basis of the values of the initial thicknesses and erosion rates of the cover and the primary contamination as follows:

$$
\begin{gathered}
T_{p c}(t)=T_{p c}(0) \text { when } t \leq t_{c v}, \\
T_{p c}(t)=T_{p c}(0)-\varepsilon_{p c}\left(t-t_{c v}\right) \text { when } t_{c v}<t \leq t_{c v}+t_{p c}=T_{c v}(0) / \varepsilon_{c v}+T_{p c}(0) / \varepsilon_{p c} \text {, and } \\
T_{p c}(t)=0 \text { when } t>t_{c v}+t_{p c} .
\end{gathered}
$$


The thicknesses of the clean cover, the unmixed portion of the initial primary contamination, and the contaminated mixing zone are computed at each intermediate time on the basis of the thicknesses of the cover and the primary contamination at that time and the depth of the mixing zone, as follows:

$$
\begin{gathered}
T_{c v}^{c}(t)=T_{c v}(t), T_{m i x}^{c}(t)=0, T_{p c}^{u m}(t)=T_{p c}(0) \text { when } T_{c v}(t) \geq d_{m i x}, \\
T_{c v}^{c}(t)=0, T_{m i x}^{c}(t)=d_{m i x} \text {, and } T_{p c}^{u m}(t)=T_{p c}(t)+T_{c v}(t)-d_{m i x} \\
\text { when } T_{c v}(t)<d_{m i x} \leq T_{c v}(t)+T_{p c}(t) \text {, and } \\
T_{c v}^{c}(t)=0, T_{m i x}^{c}(t)=d_{m i x} \text { and } T_{p c}^{u m}(t)=0 \text { when } T_{c v}(t)+T_{p c}(t)<d_{m i x} .
\end{gathered}
$$

\subsubsection{Concentration of Radionuclides in the Primary Contamination}

The activity concentrations of the radionuclides under the two rate-controlled release options are computed at each of the intermediate time points on the basis of the initial activity of the parent radionuclides and on the release rate constants and transformation constants of the radionuclides in the transformation chain.

\subsubsection{Concentration of Radionuclides in the Primary Contamination under the First-Order Release Model}

The activity concentrations of the radionuclides under this release option are computed using the following analytical expression:

$$
A_{k}(t)=\sum_{i=1}^{k} a_{k, i} \exp \left(-\lambda_{i} t-\mu_{i} t\right),
$$

where

$$
\begin{aligned}
& a_{k, i}=\text { set of coefficients defined by } a_{1,1}=A_{1}(0), \\
& a_{k, i}=\frac{\lambda_{k} a_{k-1, i}}{\lambda_{k}+\mu_{k}-\lambda_{i}-\mu_{i}} \text { for all } 1 \leq i<k, \text { and } \\
& a_{k, k}=-\sum_{i=1}^{k-1} a_{k, i} .
\end{aligned}
$$




\subsubsection{Concentration of Radionuclides in the Primary Contamination under the Uniform Release Model}

The activity concentrations of the radionuclides under this release option are computed using the following analytical expression:

$$
A_{k}(t)=\left(1-\frac{t}{T_{\text {release }}}\right) \sum_{i=1}^{k} a_{k, i} \exp \left(-\lambda_{i} t\right) \text { if } t<T_{\text {release }}
$$

and

$$
A_{k}(t)=0 \text { if } t \geq T_{\text {release }}
$$

where

$$
\begin{aligned}
T_{\text {release }} & =\text { is the duration of the release (years), } \\
a_{k, i} & =\text { set of coefficients defined by } a_{1,1}=A_{1}(0), \\
a_{k, i} & =\frac{\lambda_{k} a_{k-1, i}}{\lambda_{k}-\lambda_{i}} \text { for all } 1 \leq i<k, \text { and } \\
a_{k, k} & =-\sum_{i=1}^{k-1} a_{k, i}
\end{aligned}
$$

\subsubsection{Depth of Penetration of the Mixing Zone}

The expressions developed in Section 2.2 for the density of the mixing layer and for the volume fraction of soil from the primary contamination in the mixing zone were compact and easier to understand when stated in terms of the depth of penetration of the mixing zone, instead of in terms of time. The depth of penetration into the primary contamination following initial mixing is computed at each intermediate time as the difference between the sums of the thicknesses of the cover and primary contamination at the time of initial mixing and at the intermediate time. This algorithm is simple and has to deal with only two conditions:

$$
d_{p c}(t)=T_{c v}\left(t_{i m}\right)+T_{p c}\left(t_{i m}\right)-\left[T_{c v}(t)+T_{p c}(t)\right] \geq 0 \text {, while } d_{m} \leq T_{c v}(t)+T_{p c}(t),
$$

where

$$
\begin{aligned}
& T_{c v}\left(t_{i m}\right)+T_{p c}\left(t_{i m}\right)=T_{p c}(0)+d_{m} \text { if } d_{m} \leq T_{c v}(0), \\
& T_{c v}\left(t_{i m}\right)+T_{p c}\left(t_{i m}\right)=T_{p c}(0)+T_{c v}(0) \text { if } d_{m}>T_{c v}(0), \text { and }
\end{aligned}
$$




$$
\begin{aligned}
t_{i m}= & \text { denotes the time of initial mixing, which does not need to be determined but is } \\
& \text { used for identification purposes. }
\end{aligned}
$$

The depth of penetration of the mixing zone below the primary contamination is also computed in a similar manner using the following algorithm:

$$
d_{u p c}(t)=T_{c v}\left(t_{p m}\right)+T_{p c}\left(t_{p m}\right)-\left[T_{c v}(t)+T_{p c}(t)\right] \text { when } d_{m}>T_{c v}(t)+T_{p c}(t) \text {, }
$$

where

$$
\begin{aligned}
& T_{c v}\left(t_{p m}\right)+T_{p c}\left(t_{p m}\right)=d_{m} \text { if } d_{m} \leq T_{c v}(0)+T_{p c}(0), \\
& T_{c v}\left(t_{p m}\right)+T_{p c}\left(t_{p m}\right)=T_{p c}(0)+T_{c v}(0) \text { if } d_{m}>T_{c v}(0)+T_{p c}(0), \text { and } \\
& t_{p m}=\text { time at which the mixing layer contains the highest amount of soil from the } \\
& \text { primary contamination, which does not need to be determined but is used for } \\
& \text { identification purposes. }
\end{aligned}
$$

\subsubsection{Density of Soil in the Mixing Zone}

The algorithms in the code consider two possible initial conditions for the mixing zone.

Typically, the depth (or thickness) of the mixing zone will be smaller than the thickness of the clean cover; the mixing zone has the same properties as the cover under these conditions. If the depth of the mixing zone is specified to be greater than the depth of the clean cover, the code assumes immediate mixing of the material within the mixing zone at time zero. The initial density (at time zero) is calculated as follows:

$$
\begin{gathered}
\rho_{\text {mix }}(0)=\rho_{c v} \text { if } T_{c v}(0) \geq d_{m i x}, \\
\rho_{\text {mix }}(0)=\rho_{p c}+\frac{T_{c v}(0)}{d_{m i x}}\left(\rho_{c v}-\rho_{p c}\right) \text { if } T_{c v}(0)<d_{m i x} \leq T_{c v}(0)+T_{p c}(0), \text { and } \\
\rho_{\text {mix }}(0)=\rho_{p c}+\frac{T_{c v}(0)}{d_{m i x}}\left(\rho_{c v}-\rho_{p c}\right)+\sum_{i s}^{N_{s}} \frac{H_{d m}(i s)}{d_{m i x}}\left(\rho_{u s}(i s)-\rho_{p c}\right)+\frac{T_{d m}^{s a t}}{d_{m i x}}\left(\rho_{s a t}-\rho_{p c}\right) \\
\text { if } T_{c v}(0)+T_{p c}(0)<d_{m i x},
\end{gathered}
$$


where

$$
\begin{aligned}
N_{s}= & \text { number of partially saturated zones, } \\
H_{d m}(i s)= & \text { thickness of each partially saturated zone that is within the mixing zone at time } \\
& \text { zero }(\mathrm{m}), \\
\rho_{u s}(i s)= & \text { dry bulk density of the partially saturated zone }\left(\mathrm{g} \mathrm{cm}^{-3}\right), \\
T_{d m}^{s a t}= & \text { thickness of the saturated zone that is within the mixing layer at time zero }(\mathrm{m}), \\
& \text { and } \\
\rho_{s a t}= & \text { dry bulk density of the saturated zone }\left(\mathrm{g} \mathrm{cm}^{-3}\right) .
\end{aligned}
$$

The density of the mixing layer is computed at each intermediate time; the algorithm used was greatly simplified by the use of the depth of penetration instead of time as the independent variable. The density of the mixing layer is computed by using the following algorithm:

$$
\rho_{\text {mix }}\left(d_{p c}\right)=\rho_{p c}+\left(\rho_{m i x}(0)-\rho_{p c}\right) \exp \left(-d_{p c} / d_{m i x}\right) \text { while } d_{p c} \leq T_{c v}(t)+T_{p c}(t) .
$$

\subsubsection{Volume Fraction of Soil from the Primary Contamination in the Mixing Zone}

The algorithms in the code consider two possible initial conditions for the mixing zone. If, as is the typical case, the depth (or thickness) of the mixing zone is less than the thickness of the clean cover, the mixing zone has the same properties as the cover. If the depth of the mixing zone is specified to be greater than that of the clean cover, the code assumes immediate mixing of the material within the mixing zone at time zero. The initial volume fraction of soil from the primary contamination in the mixing zone is calculated as follows:

$$
\begin{gathered}
f_{v m}(0)=0 \text { if } T_{c v}(0) \geq d_{m i x}, \\
f_{v m}(0)=1-\frac{T_{c v}(0)}{d_{m i x}} \text { if } T_{c v}(0)<d_{m i x} \leq T_{c v}(0)+T_{p c}(0), \text { and } \\
f_{v m}(0)=\frac{T_{p c}(0)}{d_{m i x}} \text { if } T_{c v}(0)+T_{p c}(0)<d_{m i x} .
\end{gathered}
$$


The volume fraction of soil from the primary contamination in the mixing zone is computed with the following algorithms at each intermediate time point:

$$
\begin{gathered}
f_{v m}\left(d_{p c}\right)=1-\left(1-f_{v m}(0)\right) \exp \left(-d_{p c} / d_{m i x}\right) \text { when } d_{m i x} \leq T_{c v}(t)+T_{p c}(t) \text {, and } \\
f_{v m}\left(d_{u p c}\right)=f_{v m}^{p} \exp \left(-d_{u p c} / d_{m i x}\right) \text { when } d_{m i x}>T_{c v}(t)+T_{p c}(t),
\end{gathered}
$$

where

$$
\begin{aligned}
& f_{v m}^{p}=1-\exp \left(-T_{p c}(0) / d_{m i x}\right) \text { if } T_{c v}(0) \geq d_{m i x}, \\
& f_{v m}^{p}=1-\frac{T_{c v}(0)}{d_{m i x}} \exp \left(-\frac{T_{c v}(0)+T_{p c}(0)-d_{m i x}}{d_{m i x}}\right) \text { if } T_{c v}(0)<d_{m i x} \leq T_{c v}(0)+T_{p c}(0), \text { and } \\
& f_{v m}^{p}=\frac{T_{p c}(0)}{d_{m i x}} \text { if } T_{c v}(0)+T_{p c}(0)<d_{m i x} .
\end{aligned}
$$

The algorithms in the code can also accommodate a situation where there is no mixing zone. In such a situation, the combined modification factor is zero as long as there is a cover and unity when there is no cover.

\subsubsection{Concentration of Radionuclides in the Surface Soil above the Primary Contamination}

The concentration of radionuclides in surface soil is computed at each intermediate time by using the concentration in the primary contamination, the volume fraction of soil from the primary contamination in the mixing zone, and the dry bulk densities of the mixing zone and the primary contamination:

$$
A_{s c}(t)=f_{v m}(t) A_{p c}(t) \rho_{p c} / \rho_{m i x}(t) .
$$

\subsubsection{Release by Surface Runoff}

The rate at which soil from the primary contamination is eroded is computed at each intermediate time as the product of the rate of erosion of the surface soil, the area of the primary contamination, the volume fraction of soil from the primary contamination in the mixing zone, and the dry bulk density of the primary contamination:

$$
m_{p c}(t)=\varepsilon A f_{v m}(t) \rho_{p c} 10^{6}
$$


where

$$
\begin{aligned}
& \varepsilon=\varepsilon_{C V} \text { when } T_{C V}(t)>0, \text { and } \\
& \varepsilon=\varepsilon_{p c} \text { when } T_{C V}(t)=0 .
\end{aligned}
$$

The rate at which the radionuclide is released to surface water by the erosion of the surface soil above the primary contamination is computed at each intermediate time as the product of the mass of primary contamination that is eroded per unit of time and the activity concentration in the primary contamination:

$$
R_{k}^{s r}(t)=m_{p c}(t) A_{k}(t)
$$

\subsubsection{Transfer to Groundwater under the First-Order Release Model}

The rate at which the radionuclide is transferred to groundwater by rate-controlled leaching is computed at each intermediate time as the product of the total activity in soil and the release rate:

$$
T_{k}^{g w}(t)=\mu_{k} A_{k}(t) \rho_{p c} A\left(f_{v m} T_{m i x}^{c}(t)+T_{p c}^{u m}(t)\right) 10^{6} .
$$

\subsubsection{Transfer to Groundwater under the Uniform Release Model}

The rate at which the radionuclide is transferred to groundwater under the uniform release is computed at each intermediate time as the activity in incremental volume of soil that became susceptible to release during that time period:

$$
T_{k}^{g w}(t)=\frac{1}{T_{r e l}} A_{k}^{\prime}(t) \rho_{p c} A\left(f_{v m} T_{m i x}^{c}(t)+T_{p c}^{u m}(t)\right) 10^{6}
$$

\subsubsection{Release to Groundwater under the Two Rate-Controlled Release Options}

The transfer rate from the preceding two subsections is convolved with the transport equations in Sections 3.2.4 and 3.2.5 of the RESRAD-OFFSITE manual to obtain the release at the bottom of the primary contamination. The transport section of the code has been updated to allow subdivision of the primary contamination. If the primary contamination is subdivided, the number of intermediate time points used for the calculations must be sufficient to capture the variation in the fluxes across the boundaries of the subzones. This step can add appreciably to the computation time needed. The primary contamination should only be subdivided when necessary, that is, when both longitudinal dispersion and the different transport rates of the parent and the progeny have a significant impact on the progeny flux and when this progeny flux 
affects the dose to the receptor. Considering that the release is uniform over the thickness of the primary contamination, dispersion in general is not likely to be significant.

\subsubsection{Release to Groundwater under the Instantaneous Desorption Equilibrium Release}

Under the instantaneous desorption equilibrium release, the flux of the parent radionuclide at the bottom of the primary contamination is computed at each intermediate time using the instantaneous release expression:

$$
\begin{aligned}
R_{k}^{g w}(t)= & A_{k}(0) \rho_{p c} A T_{p c}(0) 10^{6} \frac{V_{c} e^{-\lambda t}}{2 T_{c z}}\left[\operatorname{erf}\left(\frac{T_{c z}-V_{c} t}{\sqrt{4 D_{z}^{c} t}}\right)-e r f\left(\frac{-V_{c} t}{\sqrt{4 D_{z}^{c} t}}\right)\right] \\
& -A_{k}(0) \rho_{p c} A T_{p c}(0) 10^{6} \sqrt{\frac{D_{z}^{c}}{4 \pi t}} \frac{e^{-\lambda t}}{T_{c z}}\left[e^{-\frac{\left(T_{c z}-V_{c} t\right)^{2}}{4 D_{z}^{c} t}}-e^{-\frac{\left(-V_{c} t\right)^{2}}{4 D_{z}^{c} t}}\right] .
\end{aligned}
$$

The expression used for the progeny depends on whether the longitudinal dispersion or the radionuclide-specific transport rates dominate the transport. Section 3.2.5.1 of the RESRADOFFSITE manual deals with dispersion-dominant transport, and Section 3.2.5.2 addresses transport dominated by the different transport rates of the parent and progeny. The transport zone can be subdivided if both processes have a significant effect on the transport. If the primary contamination is subdivided, the number of intermediate time points must be sufficient to capture the variation of the fluxes across the boundaries of the subzones.

\subsubsection{Release to the Atmosphere in the Form of Dust}

The rate at which the radionuclide is released to the atmosphere is given by the product of the activity concentration in surface soil and the rate at which dust is released from the area of primary contamination:

$$
R_{k}^{d u}(t)=f_{v m}(t) \frac{\rho_{p c}}{\rho_{\text {mix }}(t)} A_{k}(t) m_{d u} A v_{d u} 3.15576 \times 10^{7}
$$




\subsection{Users Guide to the Release Options in the RESRAD-OFFSITE Source Term Model}

This section describes the forms in the RESRAD-OFFSITE interface where the release options can be specified and forms in which some of the inputs that affect the release are located.

\subsubsection{Source Release and Deposition Velocity Form ${ }^{2}$}

The release option for each radionuclide is specified in this form (Figure 2.1). This version of the code (Version 3) contains four release options:

1. "Version 2 Release Methodology";

2. "First-Order Release with Transport";

3. "Equilibrium Desorption Release"; and

4. "Uniform Release."

Each of these is discussed in the following subsections.

\subsubsection{Version 2 Release Methodology}

This option is provided to allow input files created using the previous release version of the code to be read and executed and for users who wish to continue to use the Version 2 (and RESRAD) release methodology. This option is chosen by checking the "Version 2 Release Methodology" check box on the form.

2 Due to the addition of a time-delay feature to the source release mechanism, the input forms shown in Figures 2.1 and 2.2 have been replaced by the more detailed input form shown in the figures of Appendix D. 


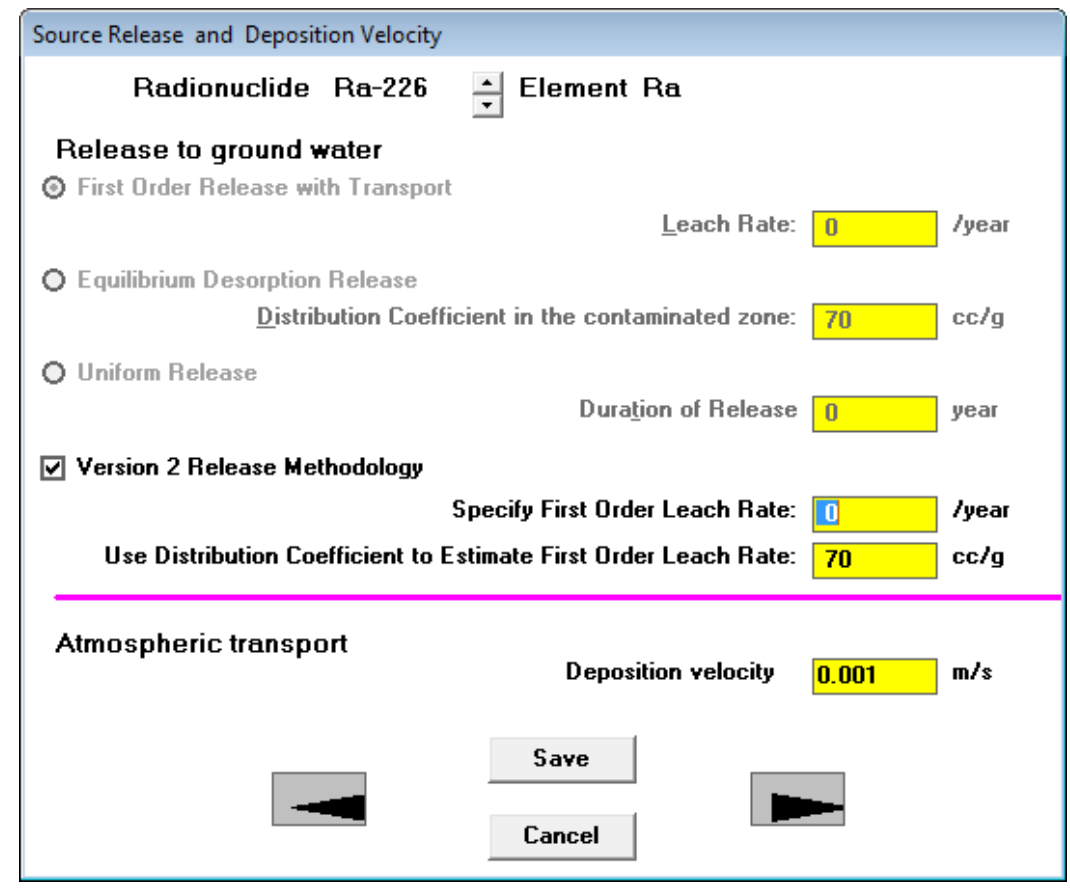

\section{FIGURE 2.1 Source Release and Deposition Velocity Form - Version 2 Option}

The user can specify one or both of the inputs that become active when this option is chosen; "Specify First Order Leach Rate" and "Use Distribution Coefficient to Estimate First Order Leach Rate." If the Specify First Order Leach Rate input is left unchanged at zero, the code will estimate that leach rate by using the expression:

$$
\mu=\frac{1}{\theta_{p c}+K_{d} \rho_{b}} \frac{I}{T_{p c}(0)} .
$$

where

$\mu$ is the first-order leach rate $\left(\mathrm{yr}^{-1}\right)$,

$I$ is the infiltration rate $\left(\mathrm{m} \mathrm{yr}^{-1}\right)$,

$\theta_{p c}$ is the total moisture content of the primary contamination,

$K_{d}$ is the distribution coefficient $\left(\mathrm{cm}^{3} \mathrm{~g}^{-1}\right)$,

$\rho_{b}$ is the bulk density of the primary contamination $\left(\mathrm{g} \mathrm{cm}^{-3}\right)$, and

$T_{p c}(0)$ is the initial thickness of the primary contamination ( $\left.\mathrm{m}\right)$. 
If a nonzero value is specified for the leach rate, the code will use it provided that the condition,

$$
K_{d}=\frac{I-\mu T_{p c} \theta_{p c}}{\mu T_{p c} \rho_{b}} \geq 0 \text { or } \mu \leq \frac{I}{T_{p c} \theta_{p c}}
$$

is satisfied. If not, the input for the leach rate is ignored, and the leach rate is estimated as in the case of an input of zero. Transport of the radionuclides within the primary contamination is not modeled when this release option is chosen.

\subsubsection{First-Order Release with Transport}

This option is chosen by first unchecking the "Version 2 release methodology" check box and then clicking the option button titled "First Order Release with Transport" on the form (Figure 2.2). This option is different from the Version 2 release methodology. The user must specify the leach rate if this option is chosen. The leach rate specified in the leach rate input box will be used directly by the code and is not subject to a calculated upper limit as in the case of the Version 2 methodology; a zero input for leach rate is taken at face value-no releaseand is not a flag for estimating leach rate.

The radionuclides leach out into the soil moisture over the entire thickness of the primary contamination. The radionuclides that were leached out near the top of the contamination will need to travel almost the entire thickness of the contamination, whereas those that were leached out deeper in the contaminated zone will have to travel over shorter distances before they are released from the primary contamination into the soil below. The groundwater transport code is used to model this transport, and it accounts for the advective and dispersive transport in soil moisture, the partitioning of radionuclides between the solid and aqueous phase of soil, and for radiological transformations. The inputs that specify this transport are discussed in Section 2.4.1.6. 


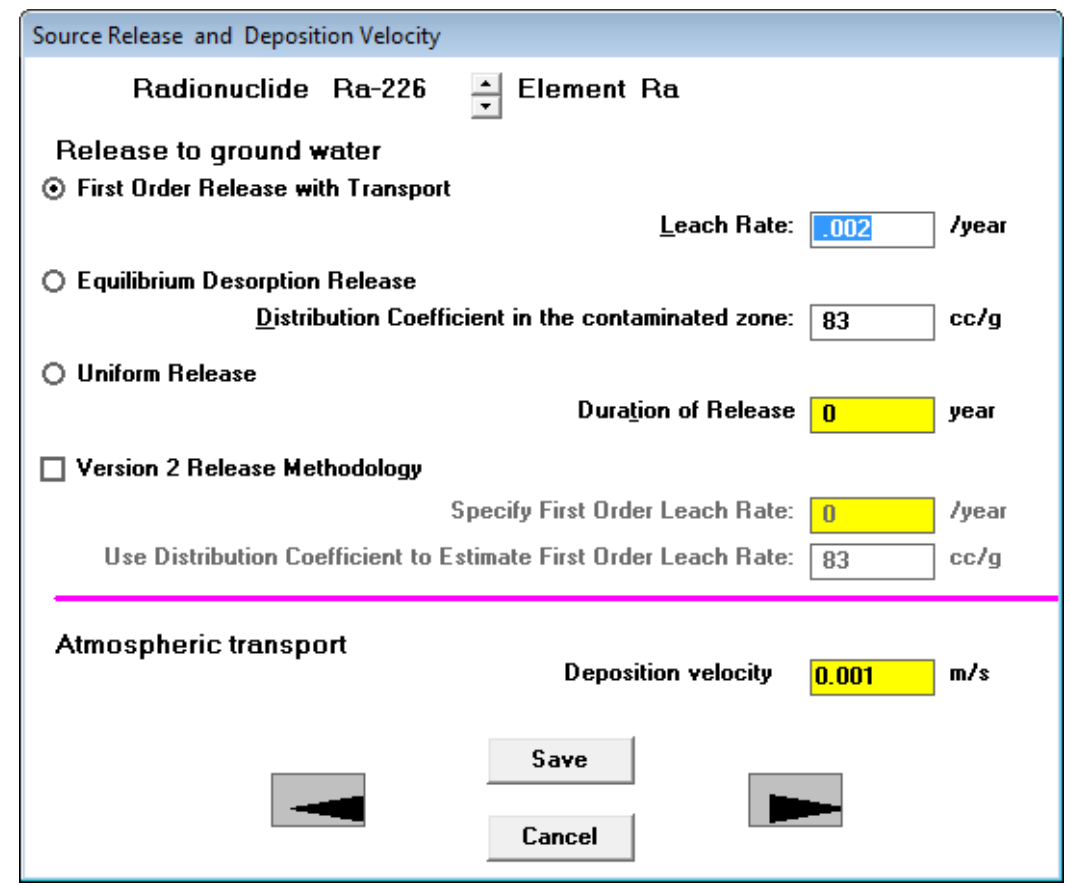

\section{FIGURE 2.2 Source Release and Deposition Velocity} Form - New Release Options

\subsubsection{Equilibrium Desorption Release}

This option is chosen by first unchecking the "Version 2 release methodology" check box and then clicking the option button titled "Equilibrium Desorption Release" on the form (Figure 2.2). The user must specify the distribution coefficient, in either the source release form or the distribution coefficients form (Figures 2.2 and 2.3), if this option is chosen. All of the radionuclides in the soil are immediately available for release; however, how soon they are released from the bottom of the primary contamination depends on how quickly they can be transported to the bottom of the primary contamination. 


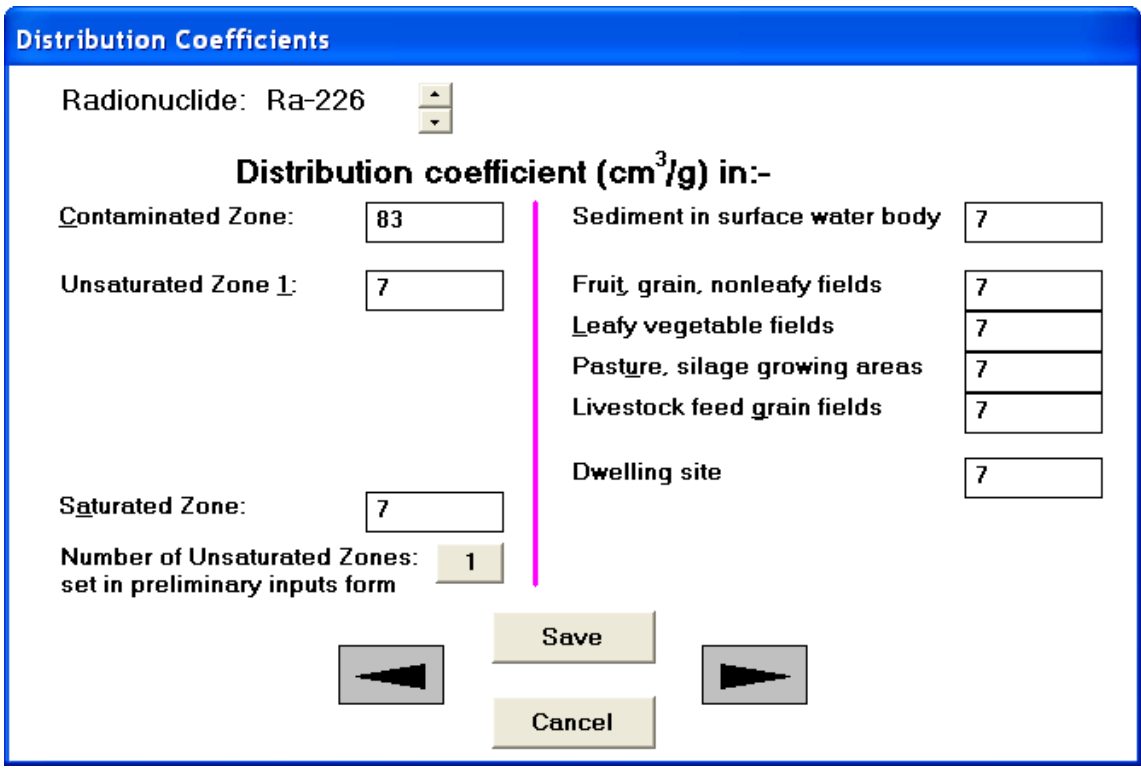

FIGURE 2.3 Distribution Coefficients Form

\subsubsection{Uniform Release}

This option is chosen by first unchecking the "Version 2 release methodology" check box and then clicking the option button titled "Uniform Release" on the form (Figure 2.2). The user must specify the duration of the release if this option is chosen. An instantaneous release will be modeled if a duration of release of zero is specified, as this selection would be identical to the equilibrium desorption release.

\subsubsection{Release of Progeny Produced by Radiological Transformations of an Initially Present Parent}

The release mechanism specified for the initially present parent is applied for the release of the progeny radionuclides that are produced in the primary contamination by the transformation of that parent. If the uniform release option is specified for the parent, then the release duration specified for the parent is used for the ingrowth progeny radionuclides, as well. If the parent is released by desorption equilibrium, then the distribution coefficient specified for the progeny will be used to determine the release of the ingrowth progeny. If the parent radionuclides are released by a first-order leach rate mechanism, the release of the ingrowth progeny will also be controlled by first-order leaching, and the leach rate specified for each progeny will be used to compute the progeny that is transferred to the soil moisture. Thus, it may be necessary to input values for leach rate, distribution coefficient, and the release duration for the same radionuclide if different release mechanisms are chosen for the radionuclide and its parent radionuclide. 


\subsubsection{Transport of the Radionuclides within the Primary Contamination}

The transport of the radionuclides from the location where they enter the soil moisture to the bottom of the primary contamination is modeled for all three new release options. The distribution coefficient, which determines how quickly the radionuclides move, can be specified in the source release form or in the distribution coefficients form; changes made in one form will appear on the other form if both are open at the same time. Two inputs that only affect the modeling of the transport in the primary contamination, the longitudinal dispersivity in the primary contamination and the effective porosity of the primary contamination, can be specified in the primary contamination form (Figure 2.4).

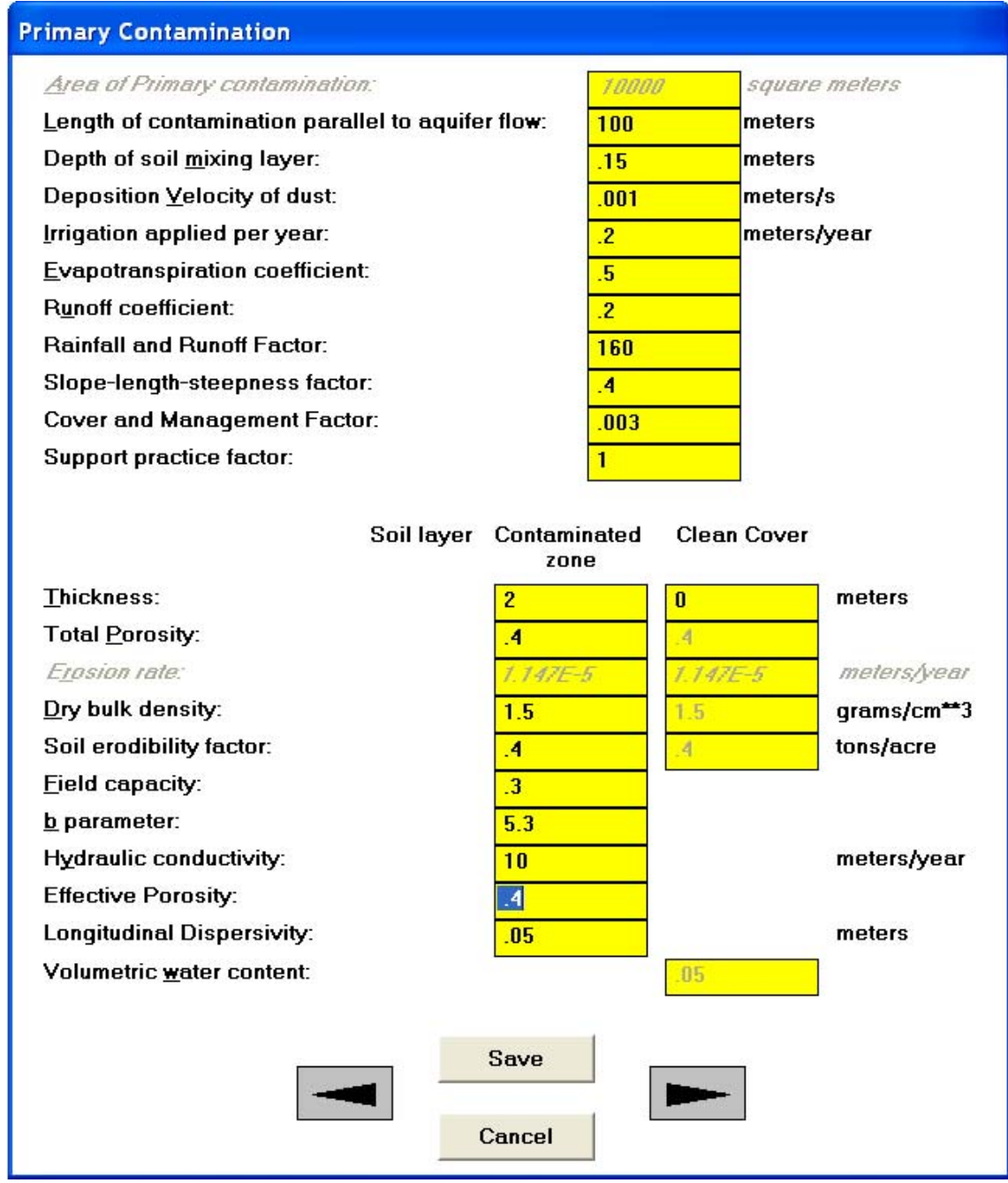

FIGURE 2.4 Primary Contamination Form 


\subsubsection{Temporal Plots of the Different Release Options}

Figures 2.5 through 2.8 show the temporal variations of the flux out of the primary contamination for the different release options with various types of transport. While these options add to the versatility of the code, the user has to make more decisions, not just about which release option to use but also about what values to use for the inputs that affect the release. To aid in this decision-making process, the code has the functionality to perform sensitivity analysis (both probabilistic sensitivity analysis and "one input at a time-three-point" deterministic sensitivity analysis) on all of the new inputs that affect release. 


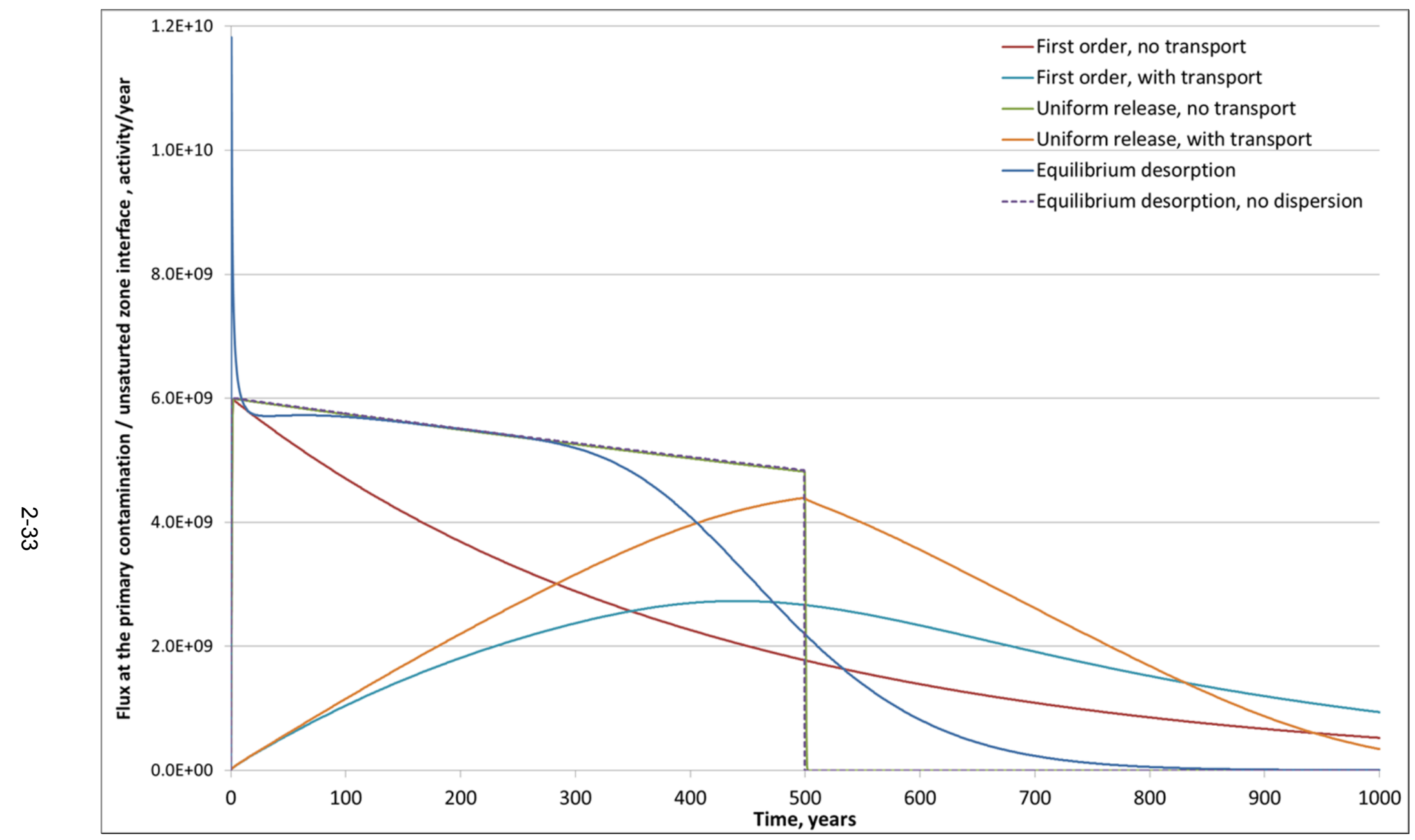

FIGURE 2.5 Temporal Profiles of Flux Out of the Primary Contamination for Different Release Options 


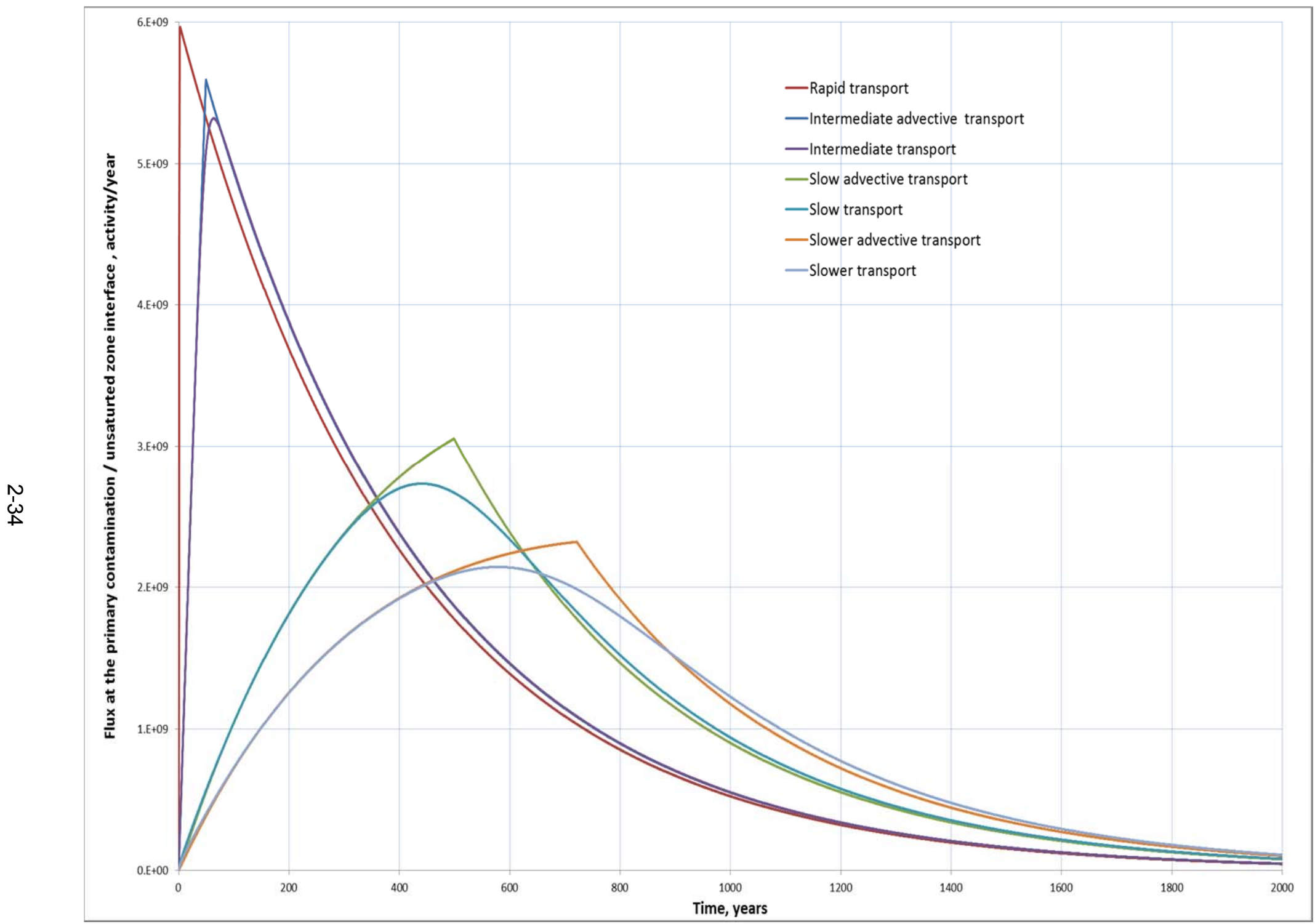

FIGURE 2.6 Temporal Profiles of Flux Out of the Primary Contamination for the First-Order Release Option with Different Rates of Transport 


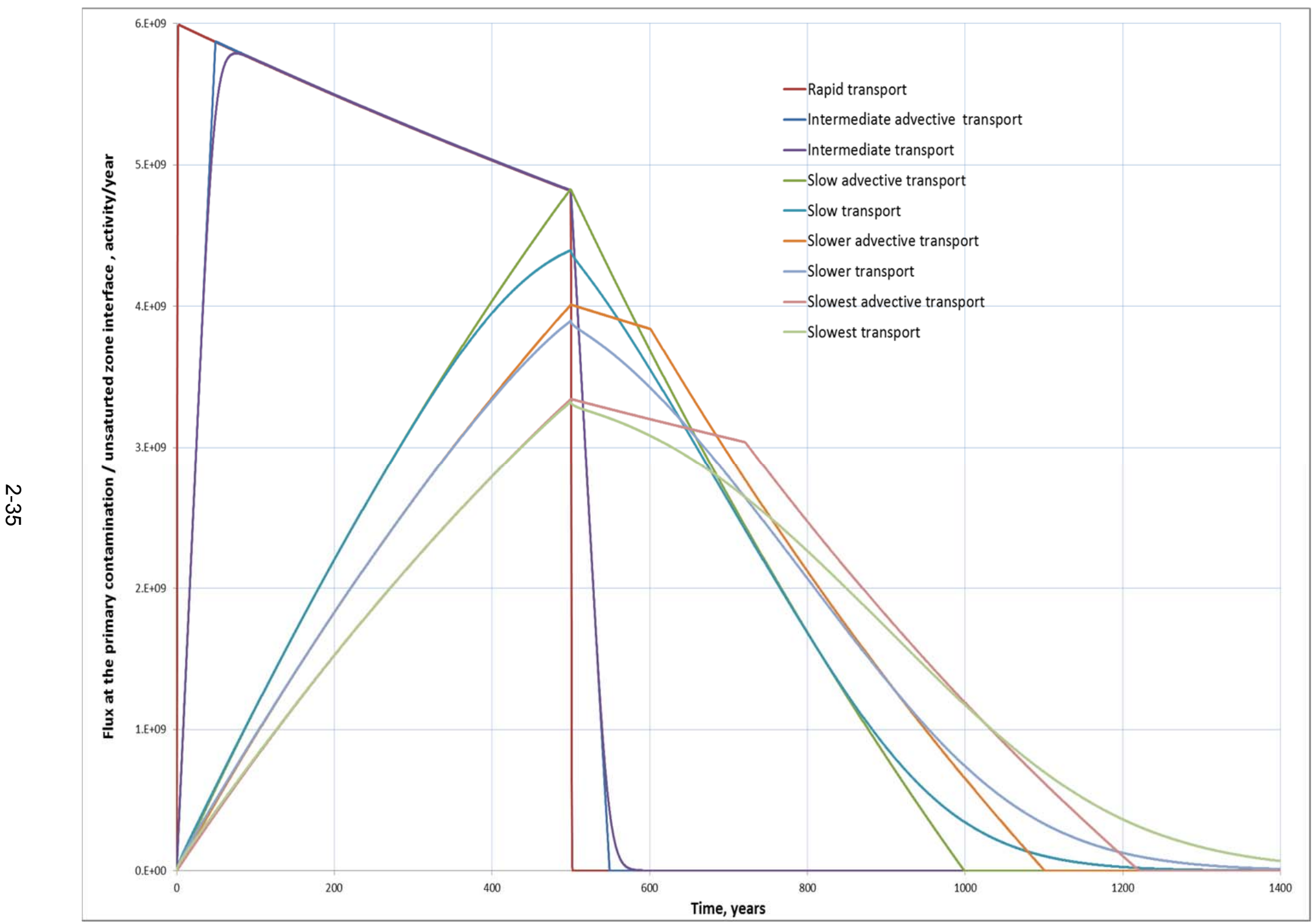

FIGURE 2.7 Temporal Profiles of Flux Out of the Primary Contamination for the Uniform Release Option with Different Rates of Transport 


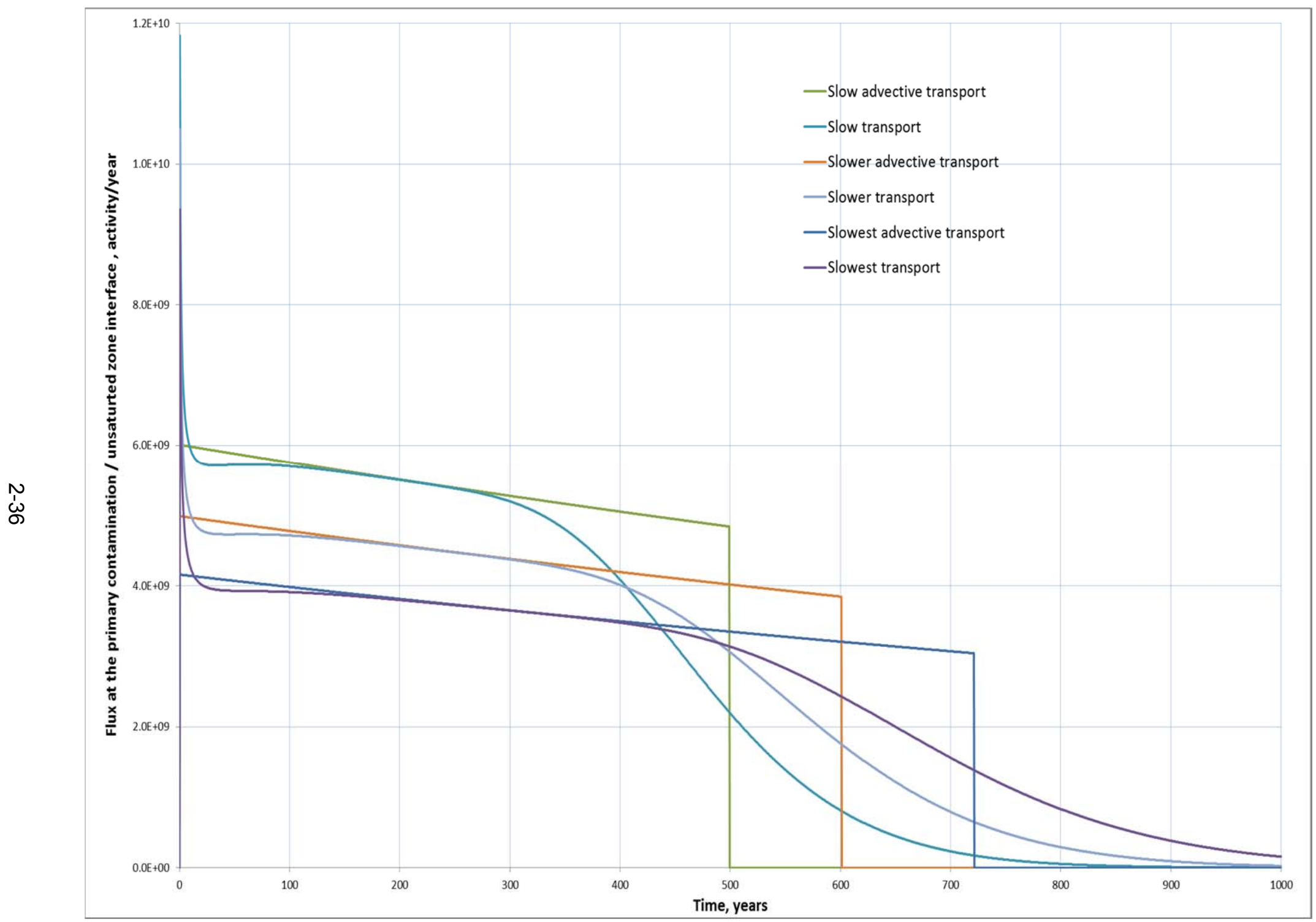

FIGURE 2.8 Temporal Profiles of Flux Out of the Primary Contamination for the Equilibrium Desorption Release Option with Different Rates of Transport 


\subsection{Overriding the RESRAD-OFFSITE Source Term Model}

If all of the information that is computed by the RESRAD-OFFSITE source term model is available either from a more sophisticated model or from a series of measurements, the computational code can be flagged to suppress its source term module and to read in the time series of the information. The temporal series of source term and release information has to be in the format that is useable by the RESRAD-OFFSITE computational code. The names of the files containing the temporal source term and release data and their contents are described in Table 2.1.

The data files are structured on the basis of the number of parent-progeny combinations at the site. They contain a column of data for each parent-progeny combination. The order of the columns is determined as follows. The radionuclides that are initially present are sorted first alphabetically by their chemical symbol and then by the nominal atomic weight in the case of isotopes. The first column of data pertains to the first radionuclide in the sorted list. If that radionuclide has principal radionuclide progeny, there must be a column of data for each progeny in the order in which they occur in the transformation chain. If the radionuclide has more than one transformation thread, there must be additional columns of data for each transformation thread. Then there must be a column of data for the second initially present radionuclide in the sorted list, followed by a column each for its progeny in the order in which they occur in its transformation chain and so on for each radionuclide in the sorted list. The number of times that data are available determines the number of rows in the different filesthere must be a row for each time that data are available.

The computational code uses a linear interpolation between the specified times when performing the calculations. The input interface does not at present have a form to bypass the source module and accept these inputs because of the complexity of the format required for these input files. 
TABLE 2.1 Input Files Used to Specify the Source Characteristics and Releases to the Code

\begin{tabular}{|c|c|}
\hline File name & Contents \\
\hline SFSIN.DAT & $\begin{array}{l}\text { Temporal data of the concentration (Section } 2.2 .2 \text { ), in } \mathrm{pCi}^{-1} \text {, of each } \\
\text { initially present radionuclide and its principal radionuclide progeny in the } \\
\text { unmixed region of the primary contamination. }\end{array}$ \\
\hline CZTHICK3.DAT & $\begin{array}{l}\text { Temporal data of the composite modification factor for the concentration } \\
\text { of radionuclides in the mixing zone (Section } 2.2 .3 .3 \text { ) and of the } \\
\text { thicknesses, in m, of clean cover, contaminated mixing zone, and the } \\
\text { unmixed portion of the contaminated zone (Section } 2.2 .3 .4 \text { ). }\end{array}$ \\
\hline AQFLUXIN.DAT & $\begin{array}{l}\text { Temporal data of the flux, in } \mathrm{pCi} \mathrm{yr}^{-1} \text {, of each initially present radionuclide } \\
\text { and its principal radionuclide progeny, to the groundwater pathway. }\end{array}$ \\
\hline SWFLUXIN.DAT & $\begin{array}{l}\text { Temporal data of the eroded flux, in } \mathrm{pCi} \mathrm{yr}^{-1} \text {, of each initially present } \\
\text { radionuclide and its principal radionuclide progeny and the mass of } \\
\text { eroded soil, in } \mathrm{g} \mathrm{year}^{-1} \text {, to surface runoff. }\end{array}$ \\
\hline AIFLUXIN.DAT & $\begin{array}{l}\text { Temporal data of the flux, in } \mathrm{pCi} \mathrm{yr}^{-1} \text {, of each initially present radionuclide } \\
\text { and its principal radionuclide progeny, to the atmosphere. }\end{array}$ \\
\hline
\end{tabular}




\section{Benchmarking of Radionuclide Release Rates Calculated by RESRAD-OFFSITE Version 3 and DUST-MS}

This chapter documents the comparison of radionuclide release rates calculated by Version 3 of RESRAD-OFFSITE vs. DUST-MS. The focus of comparison is on the release rates of radionuclides from the bottom of the contaminated zone that was assumed to contain radioactive source materials buried in soil. The newly developed source term model incorporated into the RESRAD-OFFSITE code Version 3 was used for the calculations.

The DUST-MS code was developed by Brookhaven National Laboratory more than a decade ago. Its predecessor, DUST, which stands for Disposal Unit Source Term, was first available in 1993 (Sullivan 1993). Later on, DUST was expanded to evaluate multiple species and was issued as DUST-MS in 2001 (Sullivan 2001a). Another version, DUSTMS-D, which allows consideration of distributed failure of waste containers, was also issued in the same year (Sullivan 2001b). The DUST codes (DUST, DUST-MS, and DUSTMS-D) are mostly used for evaluating radionuclide release rates from disposal units buried underground; the release rates then can be input to a groundwater transport model for further evaluation of potential groundwater contamination. The DUST code that was used for comparison with RESRADOFFSITE results was DUST-MS.

This benchmarking focused on the contaminant release rates; the transport of released contaminants outside of the primary contaminated zone is beyond the scope of this study. The comparison of release rates was performed for various sources with different dimensions and containing different radionuclides.

\subsection{Conceptualization of Radioactive Sources within the Contaminated Zone for Release and Transport Modeling}

Radioactive sources within the contaminated zone could consist of waste materials such as sludge that was dispersed directly within soils, or waste materials such as activated metals that were contained by drums or canisters and buried underground. The radionuclides contained or imbedded in the waste materials have the potential of dissolving in water when water infiltrates soils or enters the waste containers through cracks or holes and makes contact with the waste materials. Upon dissolving in water, the radionuclides could be either carried directly downward through the soil column or indirectly carried outside of the containers and then downward through the soil column, and then transported to the bottom of the contaminated zone where release rates of radionuclides were calculated for this comparison. Both RESRAD-OFFSITE 
Version 3 and DUST-MS model the vertical transport of radionuclides in soils within the contaminated zone.

To model the release of radionuclides from waste materials, RESRAD-OFFSITE conceptualizes the waste materials as being distributed evenly over the depth of the contaminated zone. Figure 3.1 is a schematic presentation of the conceptualization. The release rates of radionuclides would depend on the interaction between the contaminants, waste materials, and water; therefore, the release rates would be different for different types of waste materials. Although currently the release rates of radionuclides to the surrounding soil are calculated starting at time 0 , meaning that immediate breaching of the waste containers occurs, a container integrity time period during which no release would occur can be considered by adjusting the initial radionuclide concentrations with ingrowth and decay factors that correspond to the container integrity time period, and then inputting the adjusted concentrations to RESRAD-OFFSITE code Version 3.

Unlike RESRAD-OFFSITE, which analytically solves the mathematical equations describing the transport of radionuclides in soils, DUST-MS implements a numerical analysis method called finite difference (FD) to solve the same radionuclide transport equations. To use the FD method, users are required to subdivide the contaminated zone with a number of grids, which can be associated with release from radioactive waste materials. The distribution of the waste materials into these grids is determined by the users, with the amount over the grids adding up to the total inventory. Upon leaving the waste materials, radionuclides are assumed to partition between solid and liquid phases in equilibrium in the surrounding soil and to transport downward from one grid to another. The schematic presentation for the conceptualization of radioactive sources within the contaminated zone by DUST-MS is also shown in Figure 3.1.

The transition of radionuclide concentration profile with the RESRAD-OFFSITE modeling is continuous over the depth of the contaminated zone, whereas the transition of the concentration profile with the DUST-MS modeling is step-wise. 


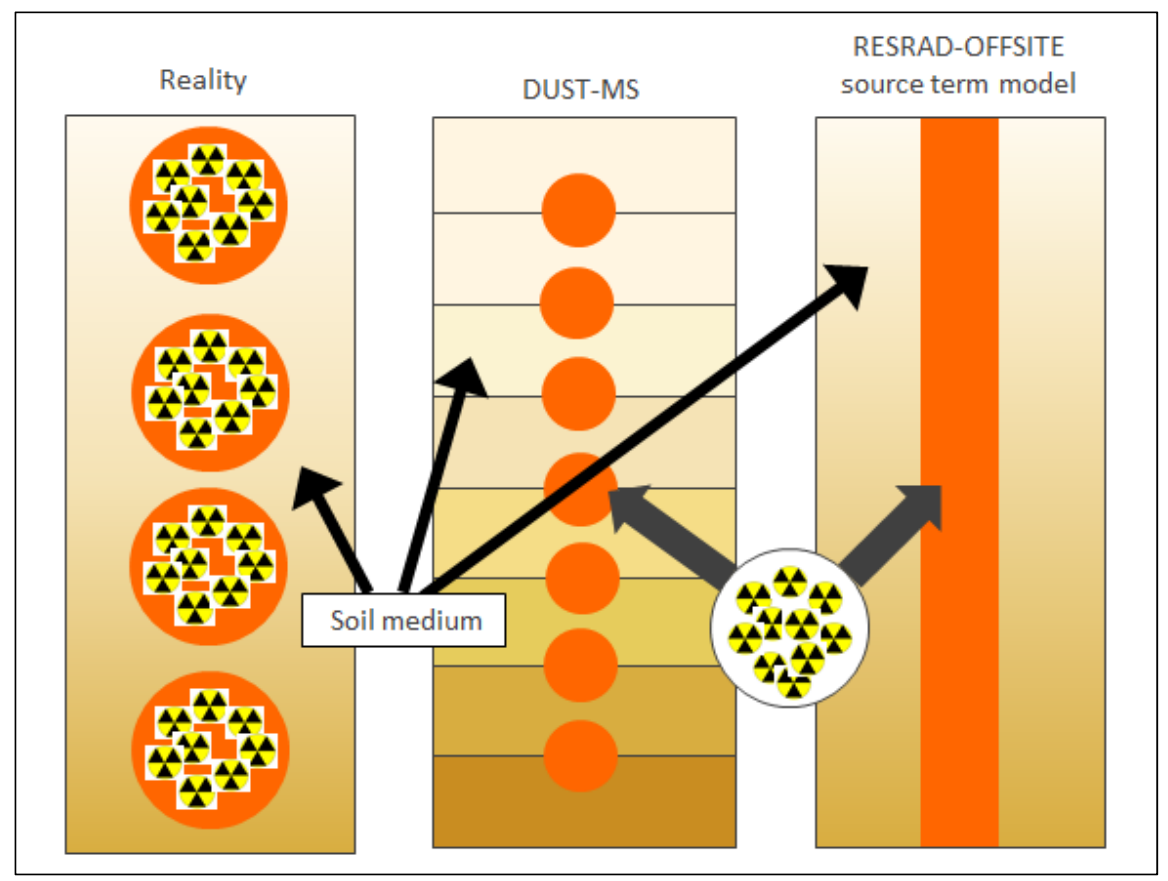

FIGURE 3.1 Conceptualization of Radioactive Sources Within the Contaminated Zone

\subsection{Release Options/Mechanisms}

As the release rates of radionuclides from the source materials to the surrounding soils would be different for different materials, RESRAD-OFFSITE Version 3 and DUST-MS each provide users with different options for calculating the release rates.

\subsubsection{RESRAD-OFFSITE Version 3}

RESRAD-OFFSITE Version 3 provides three new options for estimating the release rates of radionuclides from waste materials to the surrounding soils-first-order release, equilibrium desorption release, and uniform release.

The first-order release considers the release rate of each radionuclide to be proportional to the remaining inventory of that radionuclide in the waste materials. The proportionality constant is characterized by a leach rate specified by the user. Waste materials that resemble this type of release may include dewatered sludge or resins used for ion-exchange.

The equilibrium desorption release assumes that all radionuclides would distribute between the solid and liquid phases of the surrounding soils from the beginning of simulation on the basis of 
equilibrium desorption. This assumption implies that radionuclides would be available for release from the waste materials and dissolve in water immediately when they are in contact with water. For this to happen, radionuclides would have to attach to the waste materials loosely and, most likely, on the surface. Waste materials that fit this specification may include compacted lab trash, such as clothes or glove boxes, as well as small gadgets or tools.

The uniform release option considers the dissolution of a constant fraction of the initial radionuclide inventory over time. However, because of the adjustment for radiological ingrowth and decay, the actual radioactivity released may be different as time progresses. Assuming the distribution of radionuclides throughout the waste materials is homogeneous, to be eligible for this option, a fixed portion of the waste materials would need to dissolve in water or disintegrate upon contact with water each year. This dissolution or disintegration of waste materials would then release the radionuclides contained or imbedded within. An example of waste materials that may fit these descriptions is activated metal, which would corrode in the environment and release the imbedded radionuclides.

\subsubsection{DUST-MS}

DUST-MS considers the mechanism by which radionuclides are released from the waste materials. Three mechanisms are provided for consideration-dissolution, rinse release, and diffusion. In addition to the three mechanisms, solubility can be specified to limit the amount of radionuclides dissolved in water.

The dissolution mechanism accounts for constant dissolution of the waste materials, resulting in release of radionuclides. It is characterized by a fractional release rate, which is the inverse of the release duration used by RESRAD-OFFSITE for the uniform release option. The dissolution mechanism considered by DUST-MS is similar to the uniform release option considered by RESRAD-OFFSITE.

The rinse release mechanism assumes that radionuclides are distributed on the surface of the waste materials and would partition to and dissolve in water upon rinsing by water. This release mechanism is the same as that considered by RESRAD-OFFSITE for the equilibrium desorption option.

The diffusion mechanism assumes that release of radionuclides is controlled by diffusion, which applies to radionuclides dispersed in the bulk of porous waste materials. An example of waste materials that may assume this mechanism to release radionuclides is cement-solidified waste. 


\subsubsection{Options/Mechanisms Selected for Comparison}

On the basis of the above discussions, the release rates corresponding to the uniform release option of RESRAD-OFFSITE were calculated for comparison with those calculated by DUST-MS and corresponding to the dissolution mechanism, whereas the release rates corresponding to the equilibrium desorption option of RESRAD-OFFSITE were calculated for comparison with those calculated by DUST-MS and corresponding to the surface rinse mechanism. To match the assumption used by RESRAD-OFFSITE for the equilibrium desorption option, the partitioning factors of radionuclides within the waste materials was set to $0 \mathrm{~cm}^{3} / \mathrm{g}$ for the DUST-MS calculations.

Because there is no comparable match for the first-order release option of RESRAD-OFFSITE with the release mechanisms considered by DUST-MS, and the diffusion release mechanism considered by DUST-MS has not been incorporated into RESRAD-OFFSITE, comparison of the release rates associated with these two release options/mechanisms was not attempted.

\subsection{Assumptions for Source Materials}

For the comparison of radionuclide release rates, five different cases involving different source materials, as described in the following sections, were developed. The source materials could be of any specific waste forms. In the following sections, the terms "source materials," "release sources," and "waste forms" are used interchangeably.

\subsubsection{Release Sources in Different Comparison Cases}

The first three comparison cases, designated as Cases I, II, and III, involve radionuclides in the source material with a thickness of $0.3 \mathrm{~m}$. For Case I, the source was assumed to contain Tc-99. For Case II, the source was assumed to contain Cs-137, which has a much shorter halflife than that of Tc-99. For the third case, Case III, the source was assumed to contain U-234, which would subsequently decay to Th-230, Ra-226, Pb-210, and Po-210. For Cases IV and V, the source was assumed to be 3-m thick; in Case IV, the source contains Tc-99, and in Case V, the source contains U-234. Because RESRAD-OFFSITE and DUST-MS simulate transport of radionuclides only in the vertical direction, the size of the cross sectional area would not affect the concentration profile of radionuclides over the thickness. Therefore, an area of $1 \mathrm{~m}^{2}$ was assumed for all of the release sources. The initial concentration of radionuclide in each source was assumed to be $100 \mathrm{pCi} / \mathrm{g}$. In addition to the dimensions and initial concentration, the density of the waste form was assumed to be $1.5 \mathrm{~g} / \mathrm{cm}^{3}$, resulting in an initial total radioactivity of $4.5 \times 10^{-5} \mathrm{Ci}$ for Cases I, II, and III, and $4.5 \times 10^{-4} \mathrm{Ci}$ for Cases IV and V. 
All of the waste forms were assumed to start releasing radionuclides at time 0 as a result of water infiltration. A water infiltration rate of $0.4 \mathrm{~m} / \mathrm{yr}$ was assumed for Cases I and II, whereas a smaller water infiltration rate of $0.1 \mathrm{~m} / \mathrm{yr}$ was assumed for Cases III, IV, and V.

Table 3.1 summarizes the assumptions made for the release sources in the five comparison cases.

\section{TABLE 3.1 Assumptions for the Release Sources in Different Comparison Cases}

\begin{tabular}{lccccc}
\hline Parameter & Case I & Case II & Case III & Case IV & Case V \\
\hline Area $\left(\mathrm{m}^{2}\right)$ & 1 & 1 & 1 & 1 & 1 \\
Thickness $(\mathrm{m})$ & 0.3 & 0.3 & 0.3 & 3 & 3 \\
Density $\left(\mathrm{g} / \mathrm{cm}^{3}\right)$ & 1.5 & 1.5 & 1.5 & 1.5 & 1.5 \\
Initial radionuclide & Tc-99 & Cs-137 & U-234 & Tc-99 & U-234 \\
Initial concentration $(\mathrm{pCi} / \mathrm{g})$ & 100 & 100 & 100 & 100 & 100 \\
Water infiltration rate $(\mathrm{m} / \mathrm{yr})$ & 0.4 & 0.4 & 0.1 & 0.1 & 0.1 \\
\hline
\end{tabular}

\subsubsection{Input Parameters Used for RESRAD-OFFSITE}

To obtain a water infiltration rate of $0.4 \mathrm{~m}$ for Cases I and II, the following input parameters were assumed: (1) the precipitation rate was $1 \mathrm{~m} / \mathrm{yr}$; (2) the irrigation rate was $0 \mathrm{~m} / \mathrm{yr}$; (3) the evapotranspiration coefficient was 0.5 ; and (4) the runoff coefficient was 0.2 . To obtain an infiltration rate of $0.1 \mathrm{~m}$ for Cases III, IV, and V, the above settings were maintained, except for the runoff coefficient, which was increased to 0.8 . All of the other parameters assumed the default values.

The parameter values specified for the contaminated zone were for the surrounding soil through which radionuclides transport toward the underlying groundwater table. A soil erodibility factor of 0 ton/acre was used to eliminate soil erosion over time. Both the total porosity and effective porosity were assumed to be 0.4 . Although the value for the length of contamination parallel to aquifer flow parameter would not affect the calculated radionuclide release rates at the bottom of the contaminated zone, a value of $1 \mathrm{~m}$ was specified to reflect the assumption that the cross sectional area for the source was $1 \mathrm{~m}^{2}$. 
The RESRAD-OFFSITE calculations were executed for a time frame of 500 years for Cases I and II, and a time frame of 10,000 years for Cases III, IV, and V. The number of graphic points varied from 512 for Cases I and II to 2,048 for Cases III, IV, and V. An exposure duration of 12 years was specified for the first two cases, and the radionuclide release rates were reported every year. For the later cases, an exposure duration of 240 years was specified to obtain radionuclide release rate results every 5 years.

Various dispersivity values, along with various soil Kd values, were specified to obtain multiple sets of radionuclide release rates for comparison with DUST-MS results.

The calculated release rates of radionuclides from the contaminated zone were recorded in an output file, AQFLUXIN.DAT, which was opened to retrieve data after each execution.

\subsubsection{Input Parameters Used for DUST-MS}

DUST-MS considers three release mechanisms along with solubility limits. The three release mechanisms can be considered simultaneously with designated fractions that specify the contribution of each mechanism to the overall radionuclide release rate; however, to simplify the comparison with RESRAD-OFFSITE results, only one release mechanism was considered in each execution of the code. Furthermore, only the dissolution and the rinse release mechanisms were used for comparison, because release of radionuclides through the diffusion mechanism has not been incorporated into the RESRAD-OFFSITE source term model.

Because of the implementation of the FD method to solve the transport equations for radionuclides, the domain of analysis has to be subdivided into smaller grids to improve the precision in the calculation results. In addition, the initial condition for the entire domain and the conditions, which are applicable at any time period during the analysis for the upper and lower boundary, have to be specified. The DUST-MS analysis involves solving the dissolved radionuclide concentrations in the soil column of transport; therefore, the initial concentration for the entire domain was set to $0 \mathrm{pCi} / \mathrm{L}$. The condition for the upper boundary was set such that no radionuclide would transport upward across the boundary, that is, the flux of radionuclide was $0 \mathrm{pCi} / \mathrm{m}^{2} / \mathrm{sec}$. The condition for the lower boundary was selected such that the lower boundary is far away so that no radionuclide would reach that boundary, that is, the dissolved concentration of any radionuclide would be $0 \mathrm{pCi} / \mathrm{L}$. To make the lower boundary condition feasible, an unsaturated zone having the same properties as the contaminated zone but with a much greater thickness than that of the contaminated zone was added and included as part of the domain for analysis.

Considering that the way the domain of analysis was subdivided into smaller grids might affect the precision of the calculation results, five different subdivisions, each with a different total number of grids, were designed. The first design was used for a 0.3-m contaminated zone, 
which was subdivided into 10 grids, with $10 \mathrm{~m}$ of unsaturated zone underlying it. The unsaturated zone was divided into three subzones, which were subdivided into 5, 5, and 96 grids, respectively. In total, the first design has 116 grids, which were separated by 117 nodes (one node at the top of the domain and another at the bottom of the domain). The second, third, and fourth designs were also for a 0.3-m contaminated zone with a $10-\mathrm{m}$ unsaturated zone but with different numbers of grids and nodes. The total numbers of nodes for these three designs were 223,465 , and 481, respectively. The last design was for a 3-m source, along with a 100-m unsaturated zone. The distribution of nodes was the same as that of the third design, with a total of 465 nodes, except that the interval between two nodes was 10 times that of the third design. Figure 3.2 depicts the domain of analysis and the distribution of grids and nodes for the various designs. The maximum number of nodes allowed by DUST-MS is 500 .

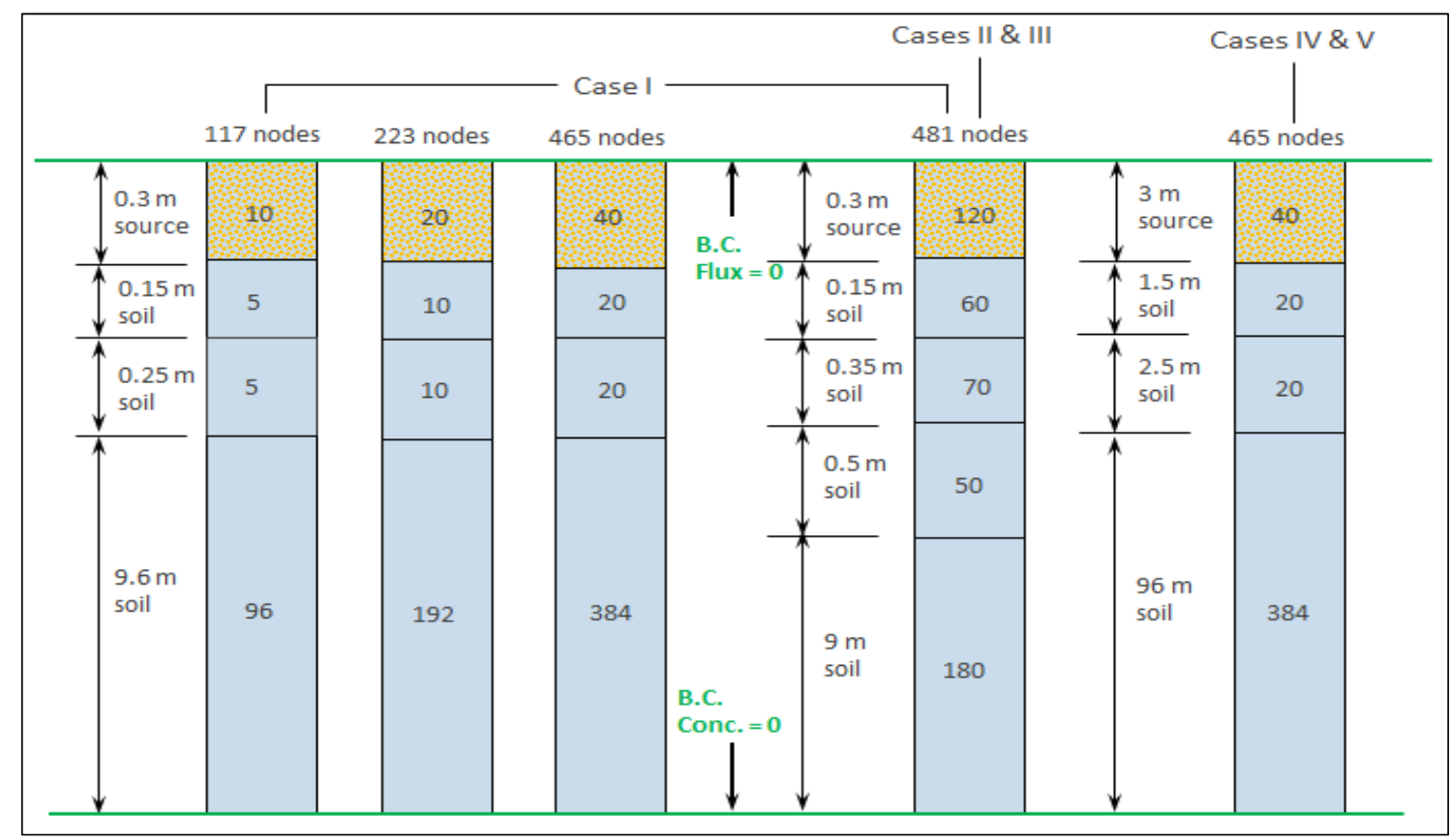

\section{FIGURE 3.2 Various Designs for Subdividing the Domain of Analysis for DUST-MS Calculation}

Input parameters required to run DUST-MS were selected so that they either matched or corresponded to the values used to run RESRAD-OFFSITE for this comparison. For Cases I and II, on the basis of RESRAD-OFFSITE input values, the corresponding moisture content in the soil column was 0.3157 . For Cases III, IV, and V, the corresponding moisture content was 0.2851. For Cases I and II, the DUST-MS calculated release rates were recorded every year for 500 years. For Cases III, IV, and V, the release rates were recorded every 5 years for 10,000 years. 


\subsection{Results of Comparison}

\subsubsection{Case I - $0.3 \mathrm{~m}$ of Tc-99}

Case I considers releases from a 0.3-m Tc-99 source. Annual release rates of Tc-99 in terms of $\mathrm{pCi} / \mathrm{yr}$ were calculated over 500 years. The release rates were calculated with no dispersion, as well as with different levels of dispersion in the soil column.

\subsubsection{No Dispersion}

Equilibrium desorption. Figure 3.3 compares the RESRAD-OFFSITE results obtained with the equilibrium desorption option with the DUST-MS results obtained with the rinse release mechanism. No dispersion in the soil column was involved. The partitioning factor of Tc-99 in the source material was set to $0 \mathrm{~cm}^{3} / \mathrm{g}$ when the DUST-MS code was executed, so that all of the Tc-99 radionuclides in the source would dissolve in water when the source material was rinsed by water.

In Figure 3.3, RESRAD-OFFSITE results were plotted with solid lines, and DUST-MS results were plotted with dashed lines. For each soil Kd value, four sets of results were obtained with DUST-MS, corresponding to the different grid designs for the domain of analysis as discussed in Section 3.3.3. The comparison shows that regardless of the soil Kd value, the DUST-MS results approach RESRAD-OFFSITE results as the number of grids increases. The discrepancy between the two could be caused by numerical dispersion in the DUST-MS results because no dispersion was assumed in this case (and DUST-MS showed dispersion in the results). As the number of grids increases, the numerical dispersion decreases in this case.

Uniform release. Figure 3.4 compares the RESRAD-OFFSITE results obtained with the uniform release option with the DUST-MS results obtained with the dissolution mechanism. The waste form was assumed to disintegrate uniformly with a disintegration (dissolution) fraction of $0.01 / y r$ for 100 years.

Consistent with the observation made using the equilibrium desorption option, the DUST-MS results approach the RESRAD-OFFSITE results as the number of grids increases. The cause of discrepancy could also be explained by numerical dispersion in DUST-MS results. 


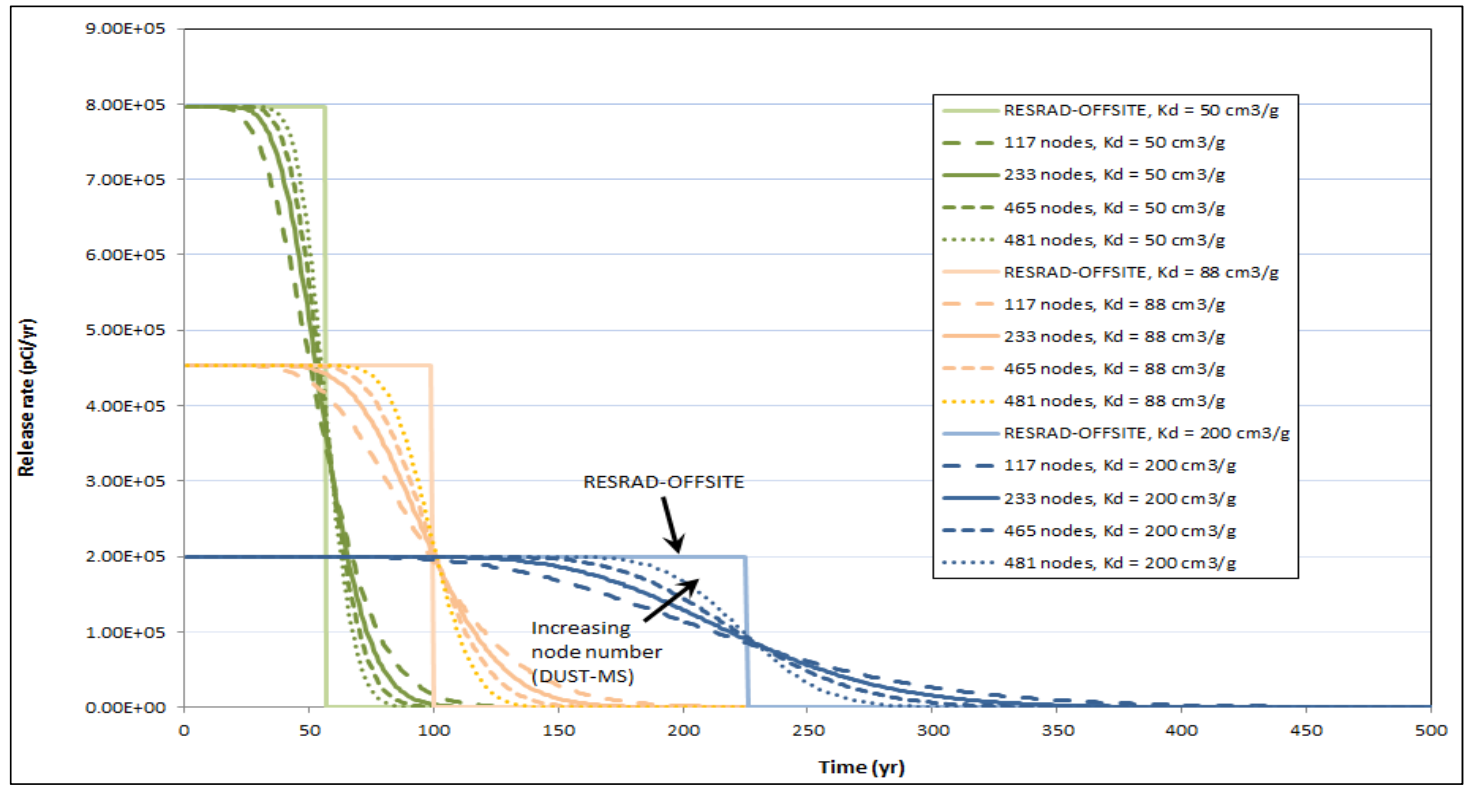

FIGURE 3.3 Comparison of RESRAD-OFFSITE and DUST-MS Results for Case I Concerning Release from a 0.3-m Tc-99 Source under the Equilibrium Desorption Condition with No Dispersion

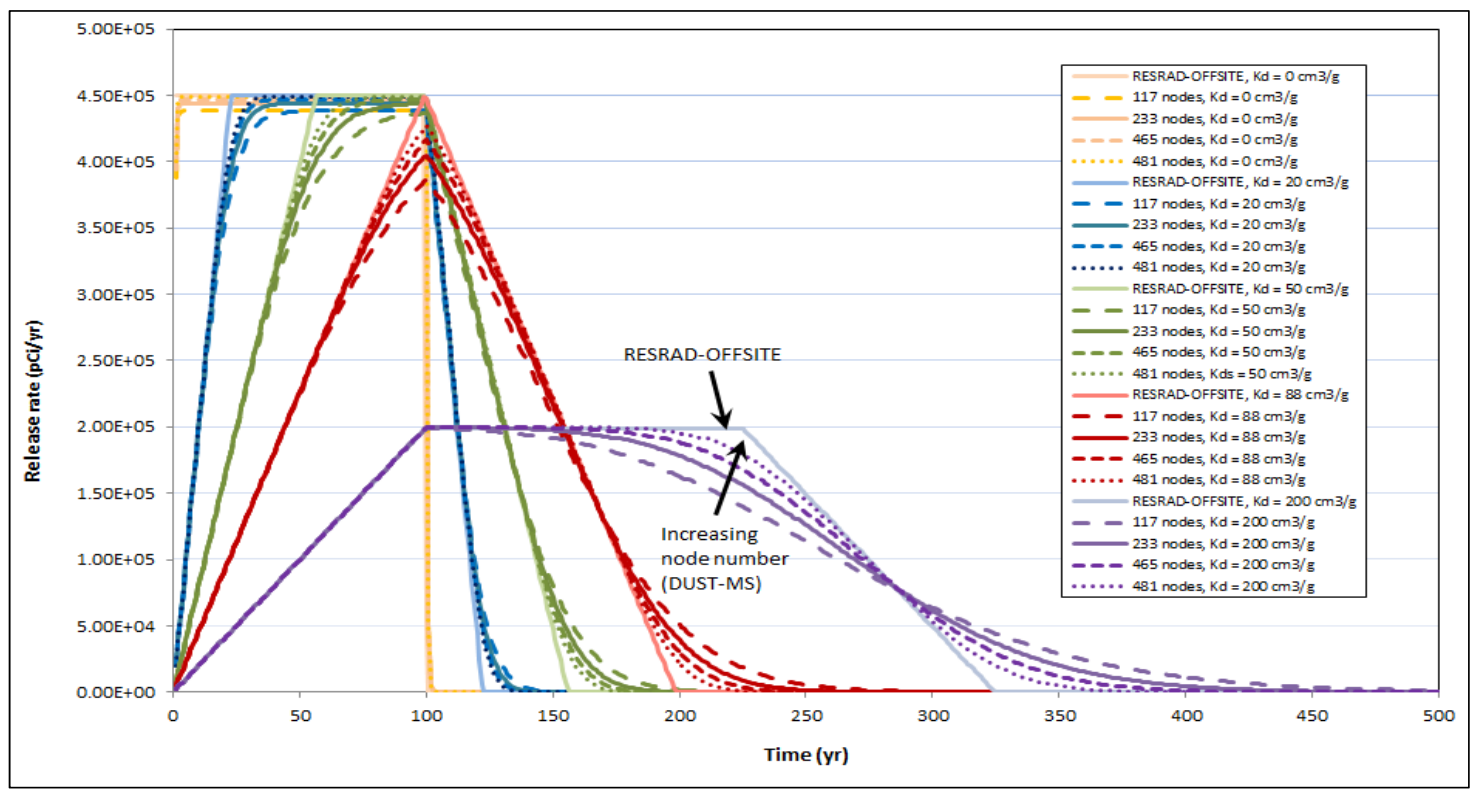

FIGURE 3.4 Comparison of RESRAD-OFFSITE and DUST-MS Results for Case I Concerning Release from a 0.3-m Tc-99 Source under the Uniform Release Condition with No Dispersion 


\subsubsection{With Dispersion}

Two dispersion levels were considered for the comparison, one with a dispersivity of $0.03 \mathrm{~m}$, that is, $1 / 10$ of the thickness of contaminated zone, and the other with a dispersivity of $0.1 \mathrm{~m}$. The DUST-MS results were obtained with the subdivision design of 481 nodes.

Equilibrium desorption. Figure 3.5 shows the comparison of RESRAD-OFFSITE results obtained with the equilibrium desorption option with DUST-MS results obtained with the rinse release mechanism for a dispersivity of $0.03 \mathrm{~m}$. The RESRAD-OFFSITE results and DUST-MS results agree very well with each other for all of the Kd values used. By increasing the dispersivity to $0.1 \mathrm{~m}$, the RESRAD-OFFSITE results still agree fairly well with the DUST-MS results (see Figure 3.6), although the agreement slips a little.

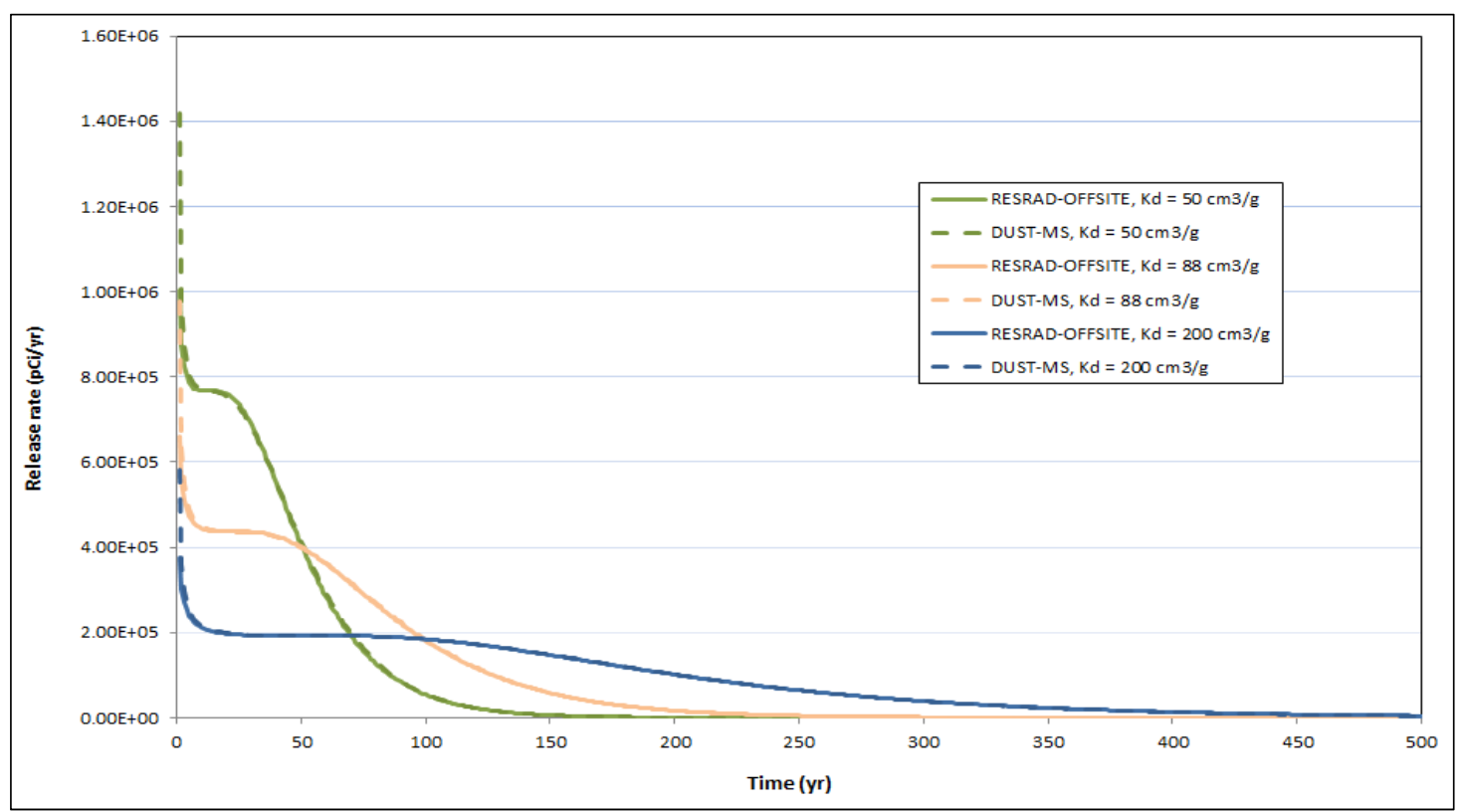

FIGURE 3.5 Comparison of RESRAD-OFFSITE and DUST-MS Results for Case I Concerning Release from a 0.3-m Tc-99 Source under the Equilibrium Desorption Condition with a Dispersivity of $0.03 \mathrm{~m}$ 


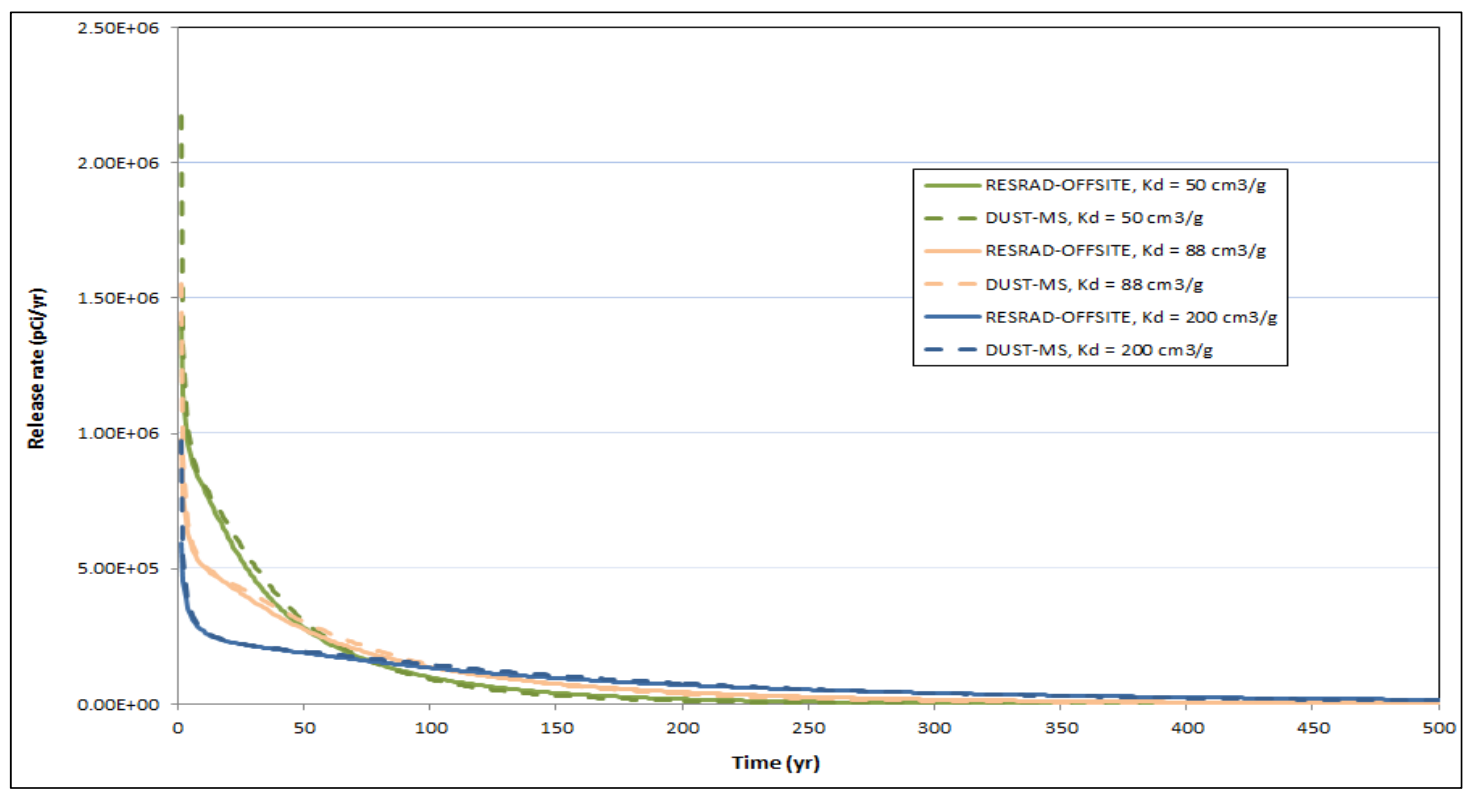

FIGURE 3.6 Comparison of RESRAD-OFFSITE and DUST-MS Results for Case I Concerning Release from a 0.3-m Tc-99 Source under the Equilibrium Desorption Condition with a Dispersivity of $0.1 \mathrm{~m}$

Uniform release. As observed under the equilibrium desorption condition, under the constant release condition, the RESRAD-OFFSITE results agree very well with the DUST-MS results when the dispersivity is $0.03 \mathrm{~m}$ (see Figure 3.7). The agreement slips a little when the dispersivity is increased to $0.1 \mathrm{~m}$ (Figure 3.8); however, the agreement is still considered fairly well.

\subsubsection{Case II $-0.3 \mathrm{~m}$ of Cs-137}

Case II considers the release of Cs-137 from a 0.3-m source. Because Cs-137 has a much shorter decay half-life (30 years) as compared to that of Tc-99 $\left(2.13 \times 10^{5}\right.$ years), the influence of radioactive decay on the release rates would be more pronounced for Cs-137 than for Tc-99. The DUST-MS results were obtained with the grid design that has 481 nodes distributed in the domain of analysis. 


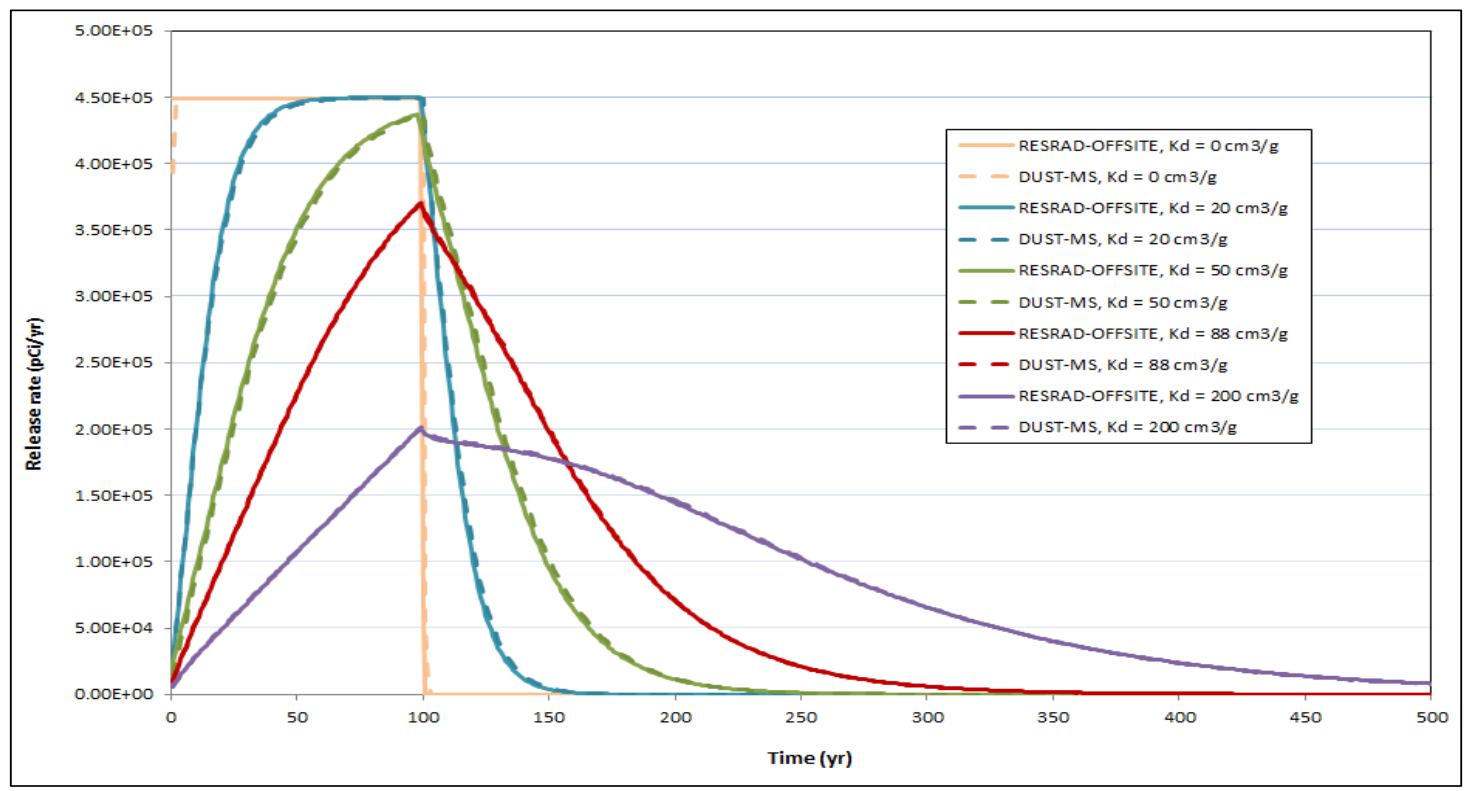

FIGURE 3.7 Comparison of RESRAD-OFFSITE and DUST-MS Results for Case I Concerning Release from a 0.3-m Tc-99 Source under the Uniform Release Condition with a Dispersivity of $0.03 \mathrm{~m}$

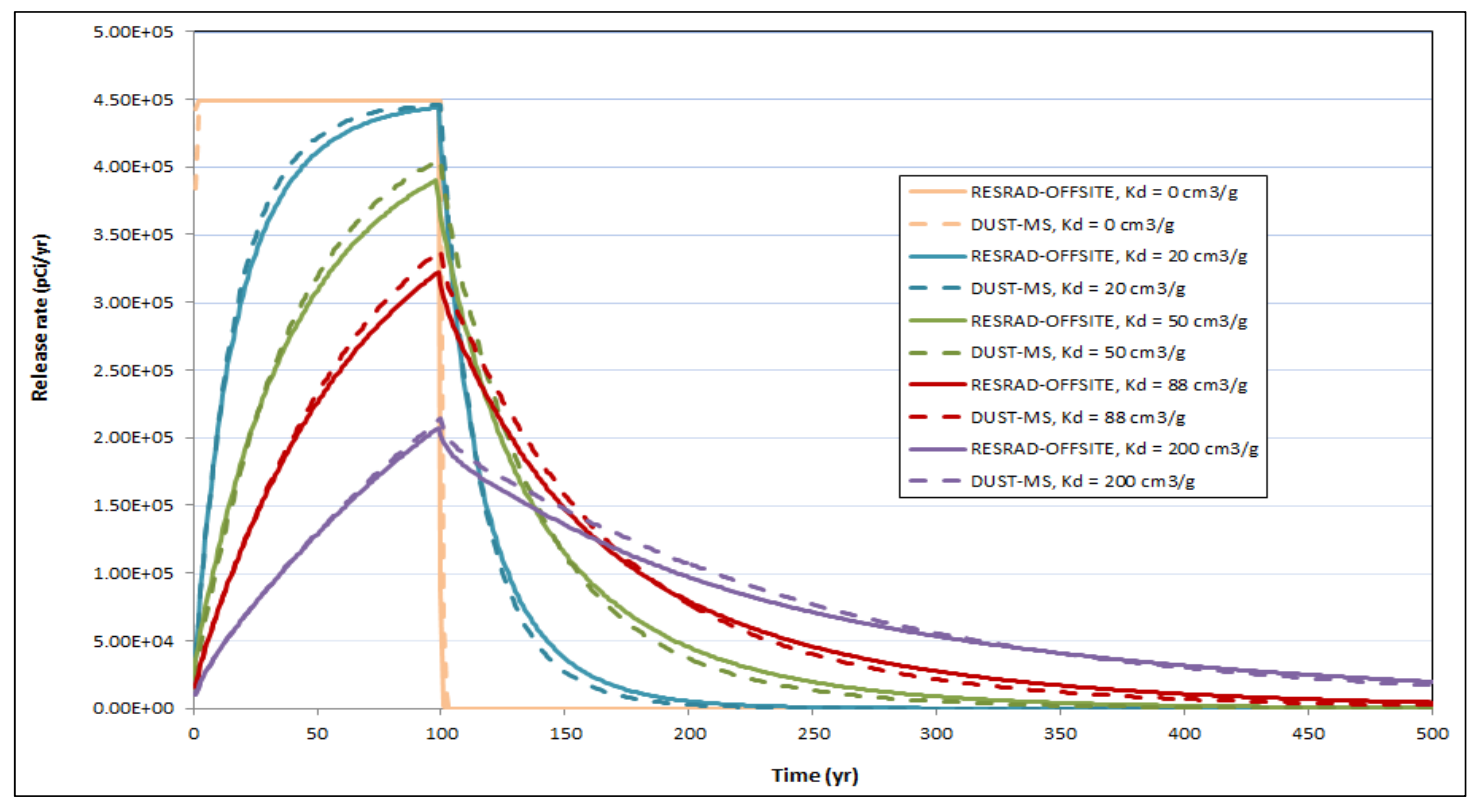

FIGURE 3.8 Comparison of RESRAD-OFFSITE and DUST-MS Results for Case I Concerning Release from a 0.3-m Tc-99 Source under the Uniform Release Condition with a Dispersivity of $0.1 \mathrm{~m}$ 


\subsubsection{No Dispersion}

The comparison of RESRAD-OFFSITE results with the DUST-MS results starts with the condition when there is no dispersion of Cs-137 in the soil column.

Equilibrium desorption. Figure 3.9 shows the comparison between the RESRAD-OFFSITE equilibrium desorption results versus the DUST-MS rinse release results. The discrepancies between the two were considered to be caused by the numerical dispersion associated with the DUST-MS results.

Without radioactive decay during transport, the release rate of radionuclides would stay constant for a period of time, that is, the time required for the radionuclides to transport from the top of the contaminated zone to the bottom of the contaminated zone, as seen in Figure 3.3 with the RESRAD-OFFSITE results for Tc-99. However, because of the significant radioactive decay of Cs-137, the release rate at the bottom of the contaminated zone cannot be maintained at the initial level, and the release rate would decrease over time until all Cs-137 radionuclides leave the contaminated zone, as shown in Figure 3.9.

Uniform release. The comparison of RESRAD-OFFSITE results and the DUST-MS results is shown in Figure 3.10 for a uniform release of $0.01 / \mathrm{yr}$ for 100 years. Again, because of the significant radioactive decay of $\mathrm{Cs}-137$, the radionuclide release rate is not maintained at a constant level, even when a $\mathrm{Kd}$ value of $0 \mathrm{~cm}^{3} / \mathrm{g}$ was assumed. Except for around the time of peak release rate with a $\mathrm{Kd}$ of 0 or $20 \mathrm{~cm}^{3} / \mathrm{g}$, the DUST-MS results are almost the same as those of RESRAD-OFFSITE.

\subsubsection{With Dispersion}

Equilibrium desorption. Figure 3.11 shows the comparison of RESRAD-OFFSITE results versus the DUST-MS results for an equilibrium desorption release. The two codes predict almost the same release rates over time when the dispersivity is $0.03 \mathrm{~m}$.

Uniform release. Figure 3.12 shows the comparison of RESRAD-OFFSITE results versus the DUST-MS results for a uniform release. As in the comparison for the equilibrium desorption condition, the comparison for the uniform release condition shows that RESRAD-OFFSITE results and DUST-MS results are almost identical. 


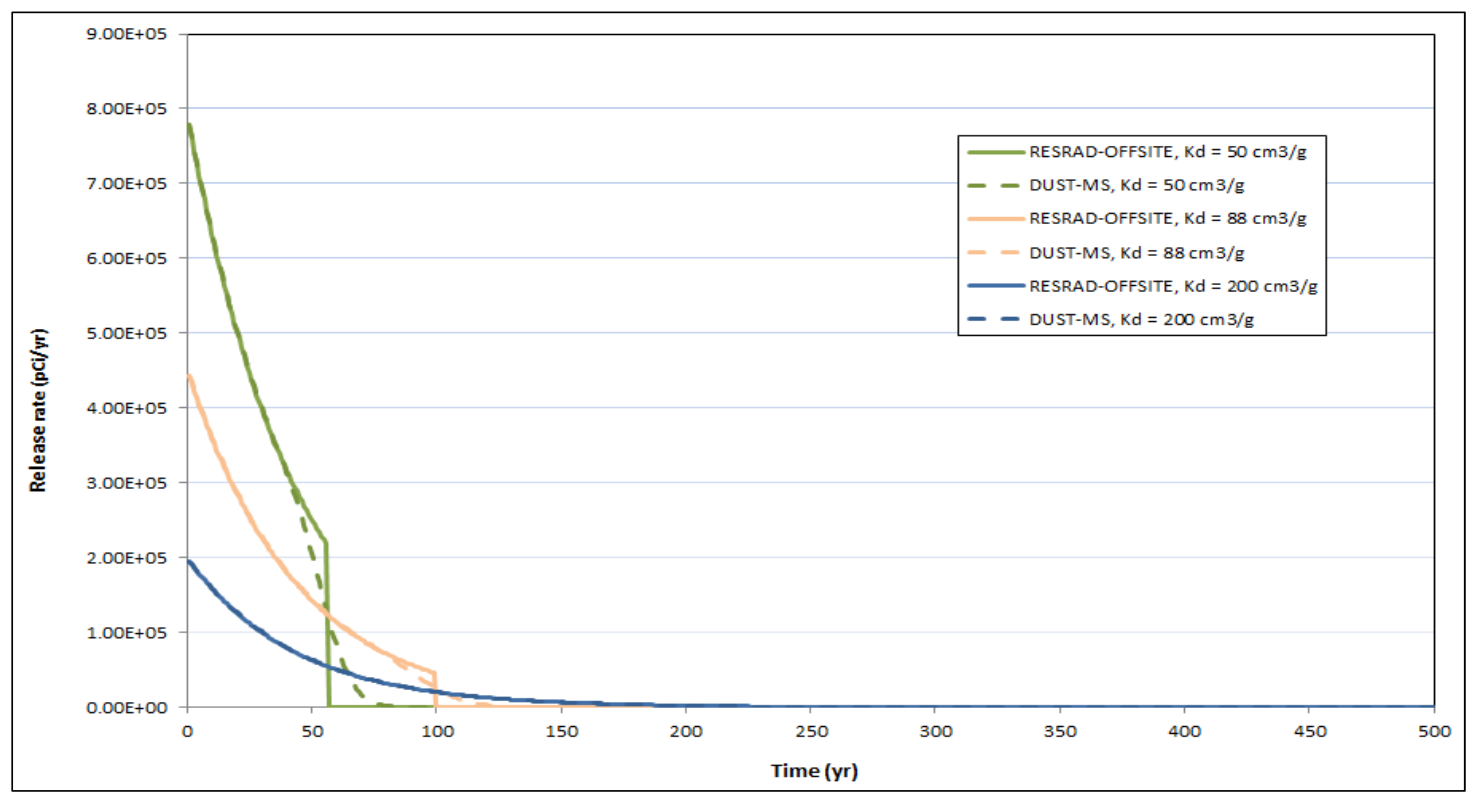

FIGURE 3.9 Comparison of RESRAD-OFFSITE and DUST-MS Results for Case II Concerning Release from a 0.3-m Cs-137 Source under the Equilibrium Desorption Condition with No Dispersion

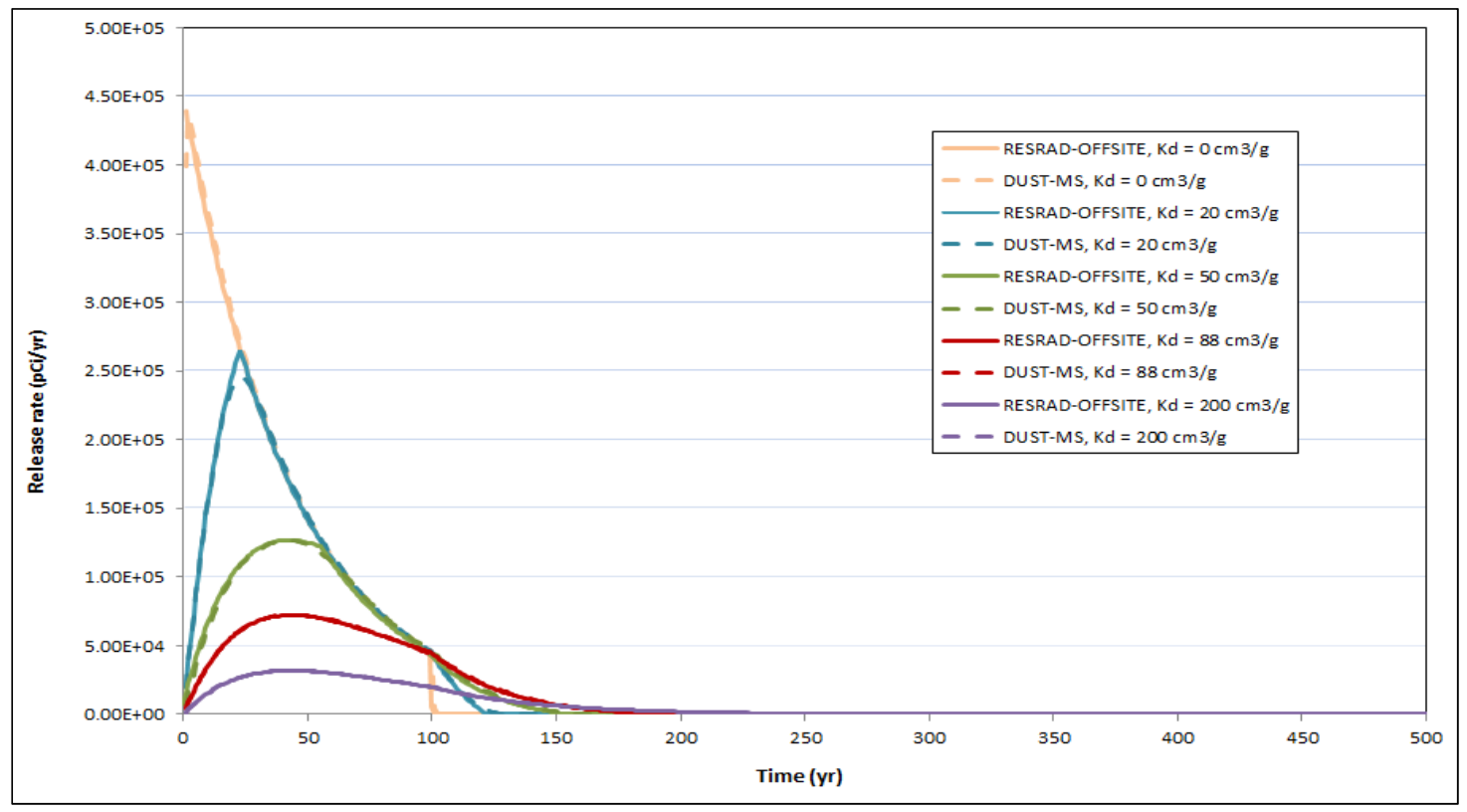

FIGURE 3.10 Comparison of RESRAD-OFFSITE and DUST-MS Results for Case II Concerning Release from a 0.3-m Cs-137 Source under the Uniform Release Condition with No Dispersion 


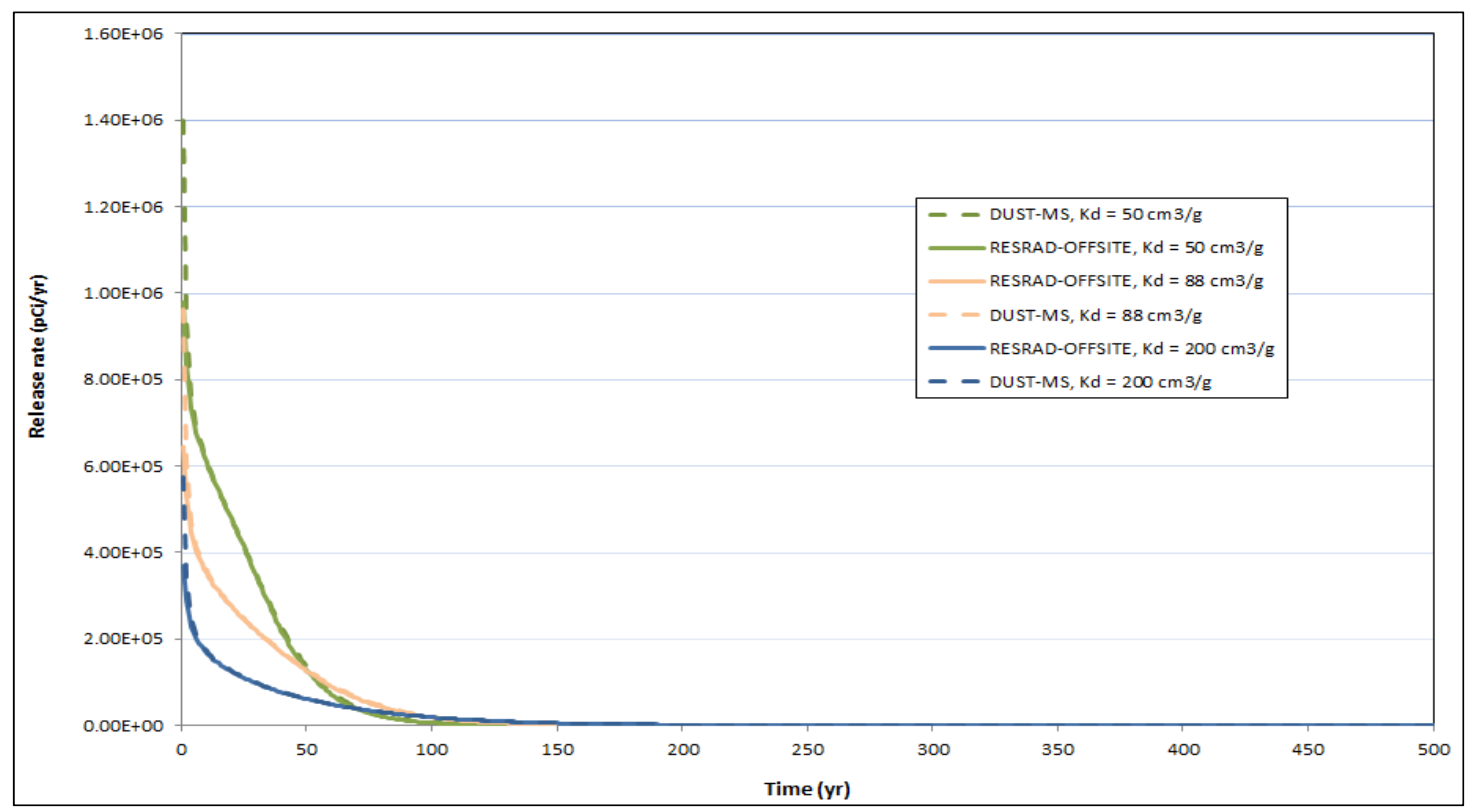

FIGURE 3.11 Comparison of RESRAD-OFFSITE and DUST-MS Results for Case II Concerning Release from a 0.3-m Cs-137 Source under the Equilibrium Desorption Condition with a Dispersivity of $0.03 \mathrm{~m}$

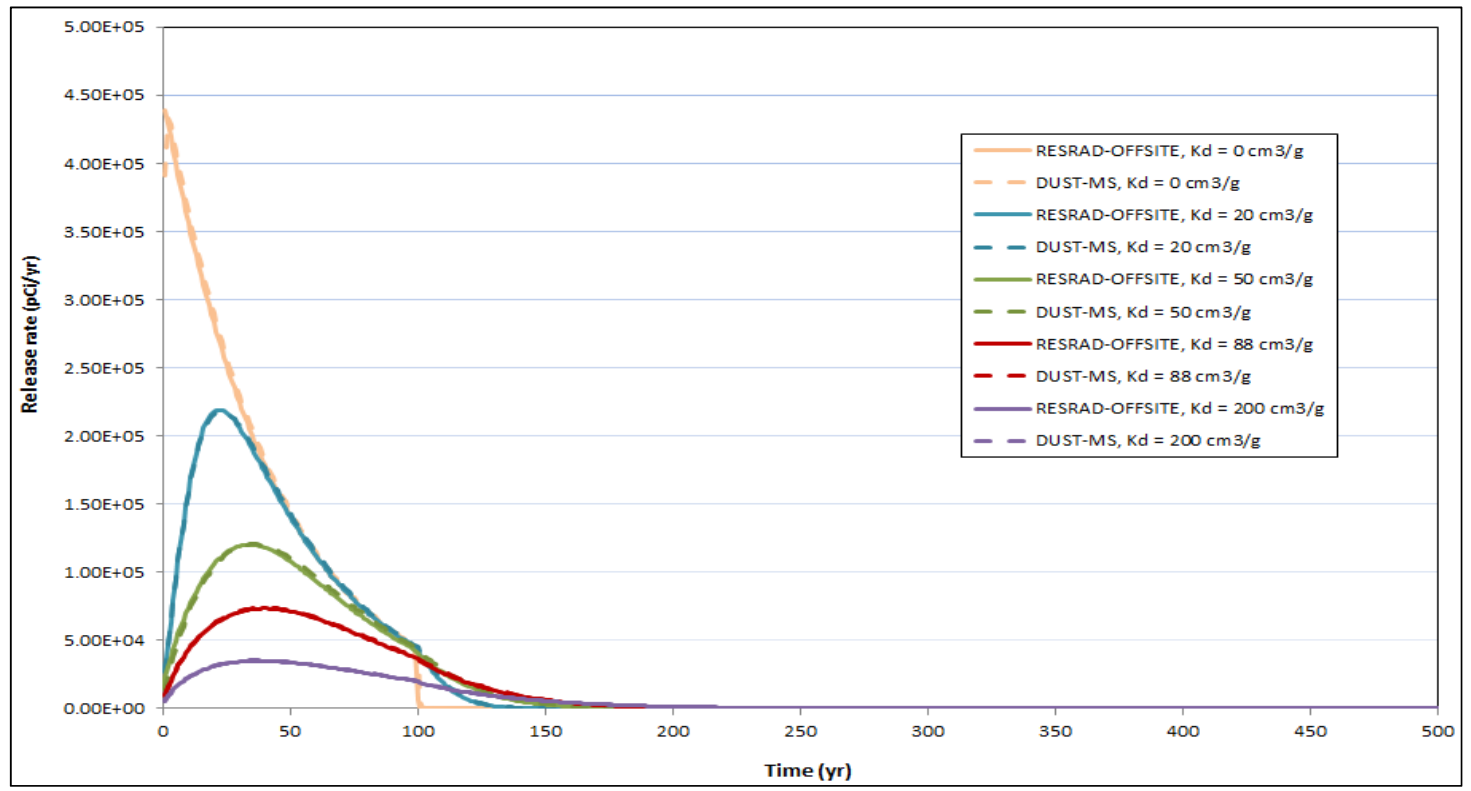

FIGURE 3.12 Comparison of RESRAD-OFFSITE and DUST-MS Results for Case II Concerning Release from a 0.3-m Cs-137 Source under the Uniform Release Condition with a Dispersivity of $0.03 \mathrm{~m}$ 


\subsubsection{Case III $-0.3 \mathrm{~m}$ of U-234}

The comparison of release rates from a waste form containing U-234 involves comparing not only the release rate of $\mathrm{U}-234$, but also the release rates of $\mathrm{Th}-230, \mathrm{Ra}-226, \mathrm{~Pb}-210$, and Po-210. The progeny radionuclides would be formed not only within the waste form, but also in the soil column during the transport of $\mathrm{U}-234$. They may assume different $\mathrm{Kd}$ values from $\mathrm{U}$ 234 and thereby transport with different speeds toward the bottom of the contaminated zone. The $\mathrm{Kd}$ values assumed for U-234 and its progeny radionuclides were $200,6,000,70,100$, and $10 \mathrm{~cm}^{3} / \mathrm{g}$, respectively.

When progeny radionuclides are formed within the waste form, they are assumed to be released through the same mechanism as is their parent radionuclide to the surrounding soils. To allow for more ingrowth of progeny radionuclides within the waste form, the water infiltration rate was reduced from $0.4 \mathrm{~m} / \mathrm{yr}$ as used in Cases I and II to $0.1 \mathrm{~m} / \mathrm{yr}$. Furthermore, when calculating radionuclide release rates under the uniform release condition, a uniform release of $0.00111 / y r$ for 900 years was assumed.

The DUST-MS results were obtained with the grid design of 481 nodes.

\subsubsection{No Dispersion}

Figure 3.13 compares the release rates of U-234. Numerical dispersion might be the cause of discrepancies between the RESRAD-OFFSITE results and DUST-MS results.

Figure 3.14 compares the release rates of Th-230. The RESRAD-OFFSITE results and the DUST-MS results are almost identical. The release rates increase starting at time 0 as Th-230 radionuclides are formed because of the decay of $U-234$. The Th-230 radionuclides formed would largely adsorb to soil particles and would stay in the soil column longer than would U-234 radionuclides, because the $\mathrm{Kd}$ value assumed for Th-230 was much greater than that used for $\mathrm{U}-234$. The adsorbed Th-230 radionuclides feed into the continuous release of Th-230 radionuclides long after all U-234 radionuclides have left the source.

Ra-226 radionuclides are formed as Th-230 radionuclides undergo radioactive decay. Figure 3.15 compares the release rates of Ra-226 calculated by RESRAD-OFFSITE vs. DUST-MS. The calculations show that the release rates of Ra-226 would increase from the beginning and reach maximum levels shortly after the release rates of U-234 peaked. After reaching the maximum levels, the release rates of Ra-226 would have decreased quickly, as the release rates of $U-234$, had there not been $T h-230$ radionuclides adsorbing to the soil particles and undergoing radioactive decay. 


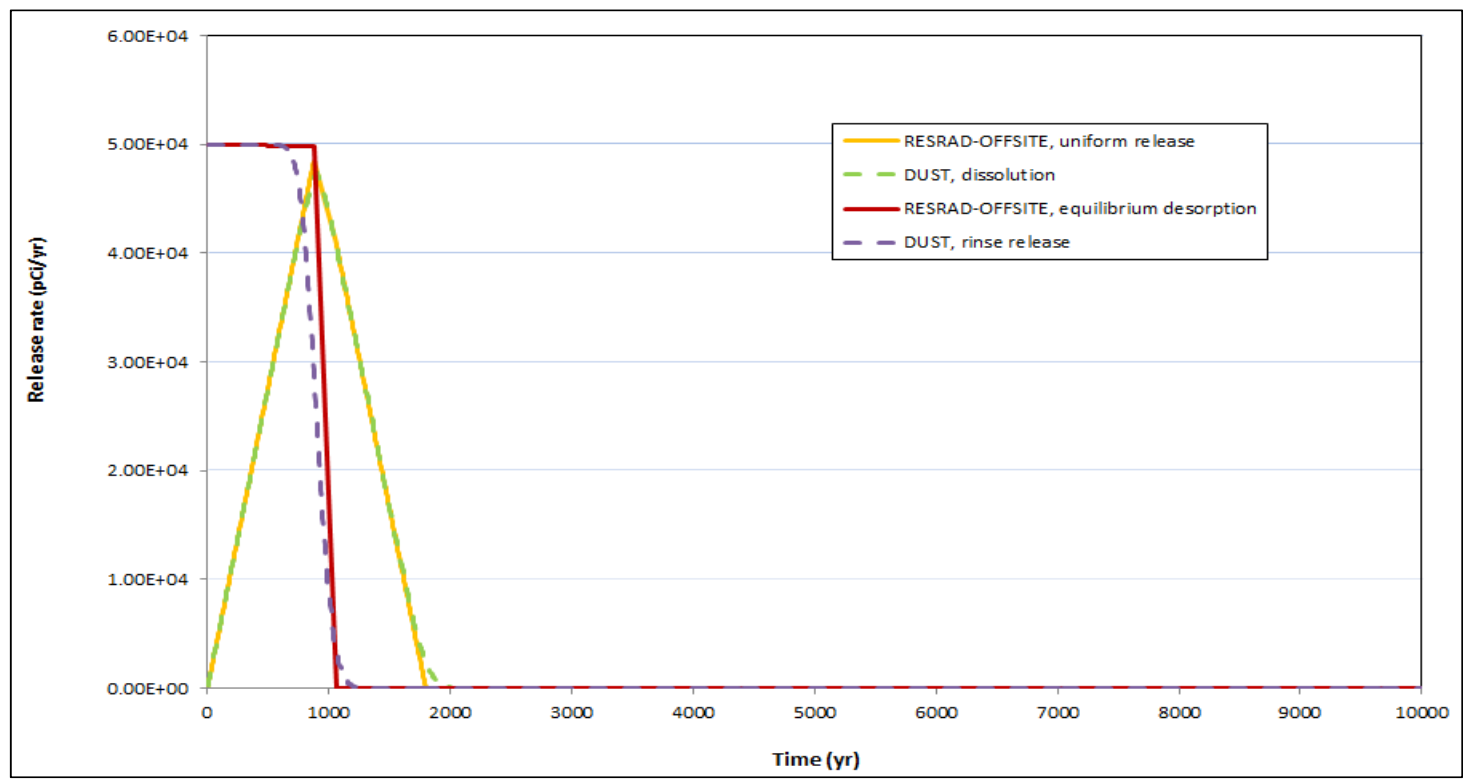

FIGURE 3.13 Comparison of RESRAD-OFFSITE and DUST-MS Results for Case III Concerning Release of U-234 from a 0.3-m U-234 Source with No Dispersion

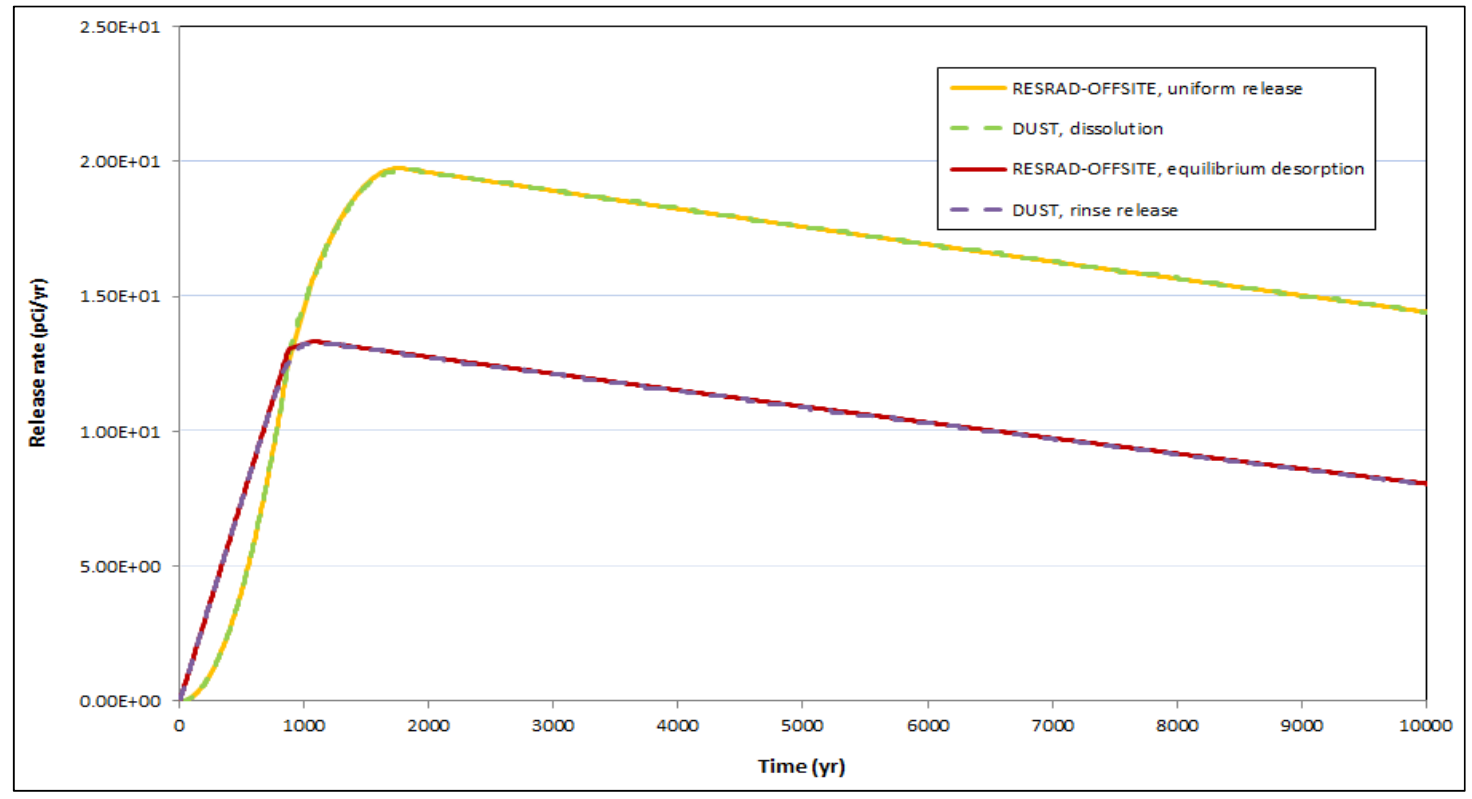

FIGURE 3.14 Comparison of RESRAD-OFFSITE and DUST-MS Results for Case III Concerning Release of Th-230 from a 0.3-m U-234 Source with No Dispersion 


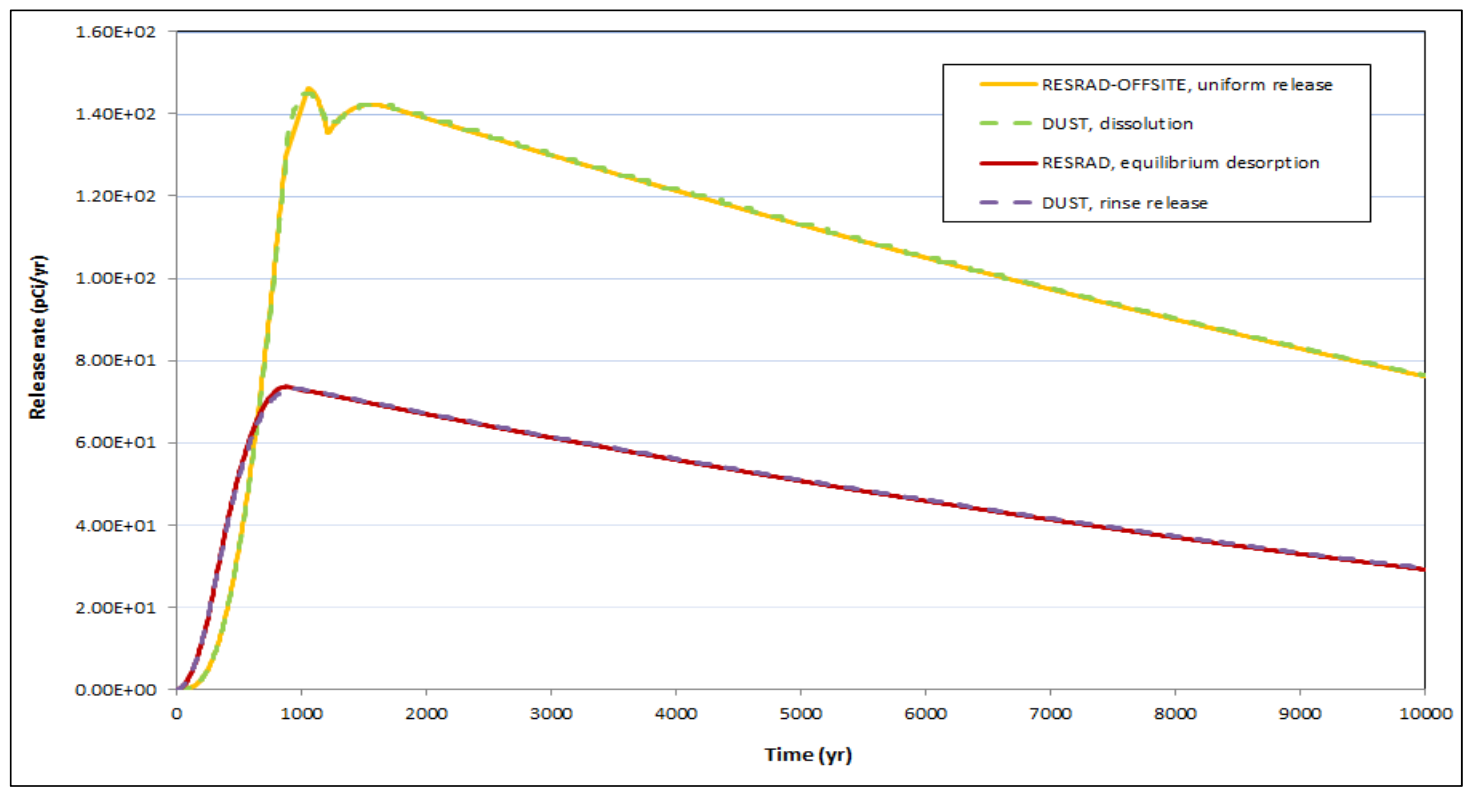

\section{FIGURE 3.15 Comparison of RESRAD-OFFSITE and DUST-MS Results for Case III Concerning Release of Ra-226 from a 0.3-m U-234 Source with No Dispersion}

The decay of Ra-226 generates $\mathrm{Pb}-210$. Because the Kd value assumed for $\mathrm{Pb}-210$, $100 \mathrm{~cm}^{3} / \mathrm{g}$, is close to the $\mathrm{Kd}$ value of $70 \mathrm{~cm}^{3} / \mathrm{g}$ assumed for Ra-226, the release rate profile of $\mathrm{Pb}-210$ would be similar to the release rate profile of Ra-226, as shown in Figure 3.16.

The last radioactive radionuclide in the decay chain of U-234 is Po-210, which has a short halflife of 0.38 year. Because of the short half-life, the total radioactivity level of Po-210 at any depth of the soil column is expected to be about the same as that of $\mathrm{Pb}-210$. On the other hand, because Po-210 has a Kd value $\left(10 \mathrm{~cm}^{3} / \mathrm{g}\right) 10$ times smaller than that of $\mathrm{Pb}-210$, the dissolution of Po-210 in the pore water is expected to be about 10 times greater than that $\mathrm{Pb}-210$, and so is the release rate of $\mathrm{Po}-210$ versus the release rate of $\mathrm{Pb}-210$. Comparing the release rates of $\mathrm{Po}-210$ in Figure 3.17 with those of $\mathrm{Pb}-210$ in Figure 3.16 confirms the above expectations. 


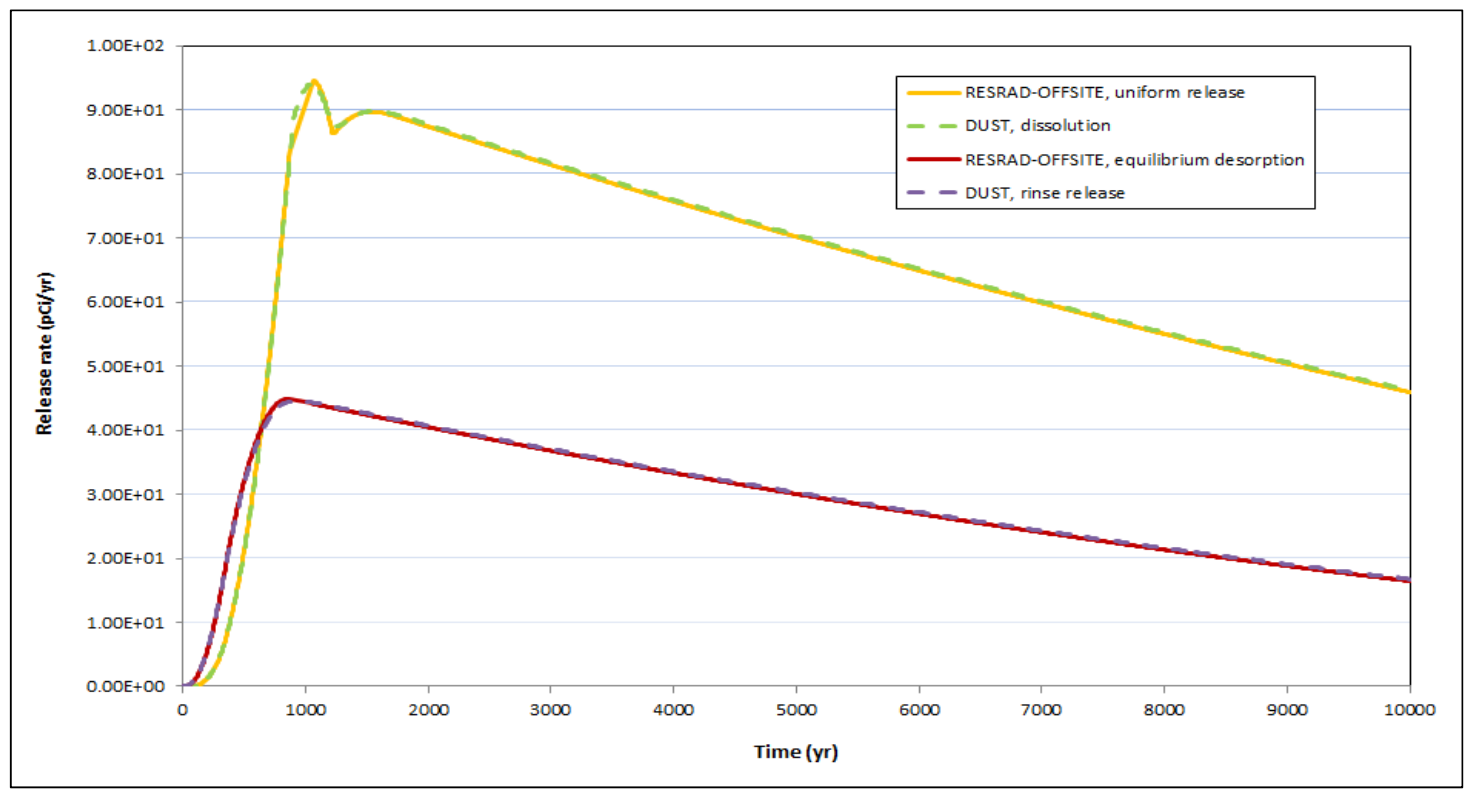

FIGURE 3.16 Comparison of RESRAD-OFFSITE and DUST-MS Results for Case III Concerning Release of Pb-210 from a 0.3-m U-234 Source with No Dispersion

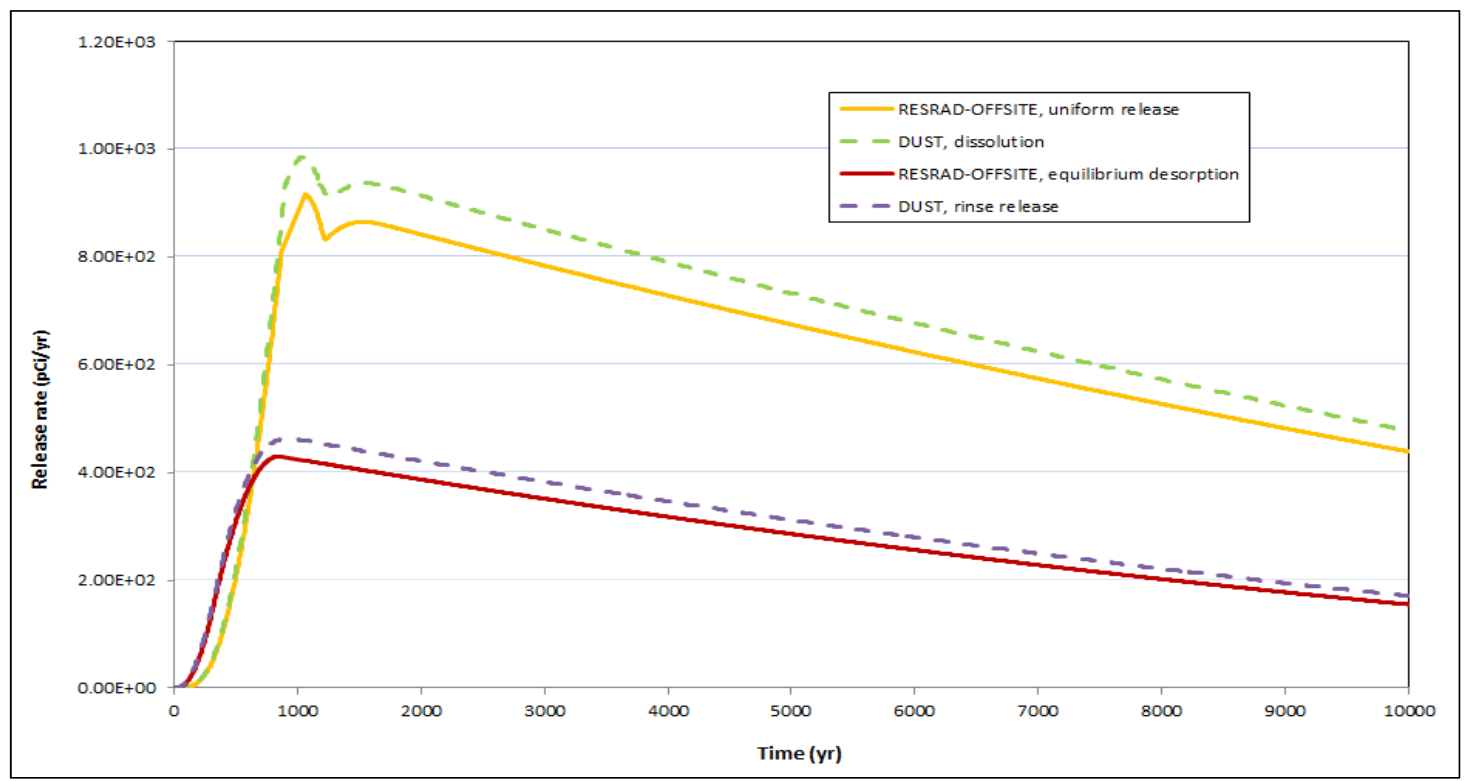

FIGURE 3.17 Comparison of RESRAD-OFFSITE and DUST-MS Results for Case III Concerning Release of Po-210 from a 0.3-m U-234 Source with No Dispersion 
In general, the release rates calculated by RESRAD-OFFSITE agree very well with those calculated by DUST-MS except for the release rate of Po-210. For Po-210, the profiles of release rates calculated by RESRAD-OFFSITE and DUST-MS are the same; however, the magnitudes vary slightly. The exact reason for this discrepancy is not known; perhaps it is related to the short half-life of Po-210, which could result in more numerical dispersion in the calculation results when using the same time step and grid design as for other radionuclides. Further benchmarking results presented in Appendix B seems confirm this point.

\subsubsection{With Dispersion}

Figures 3.18 through 3.22 compare the RESRAD-OFFSITE and DUST-MS results by considering a dispersivity of $0.03 \mathrm{~m}$ for all radionuclides in the soil column. Discrepancy is observed starting with the first progeny, Th-230, and continuing for the rest of the decay chain. However, the release rate profiles are similar and the agreement is considered acceptable, given the fact that the simulations were carried out for an extended time period of 10,000 years. Further benchmarking results of this case are presented in Appendix B.

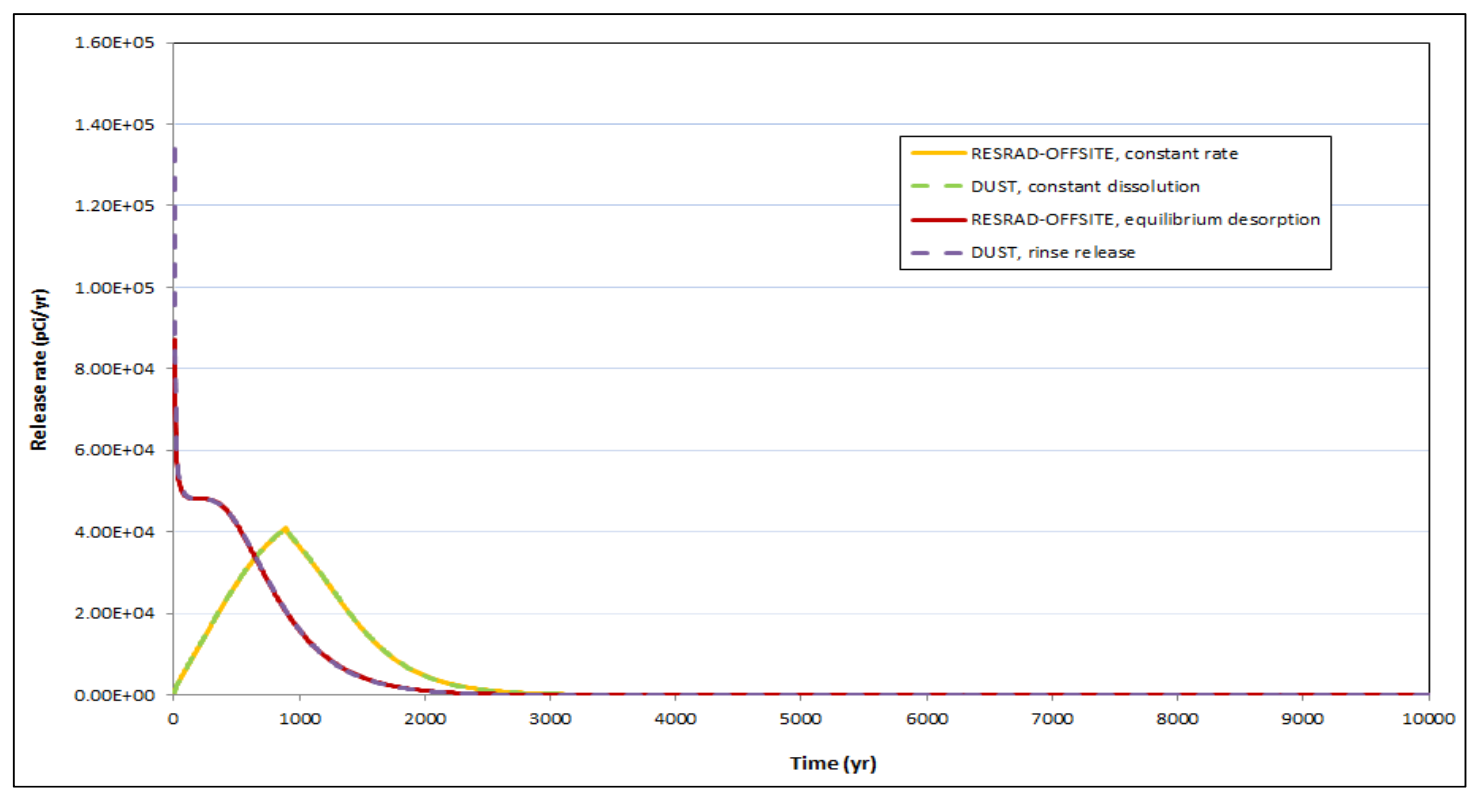

FIGURE 3.18 Comparison of RESRAD-OFFSITE and DUST-MS Results for Case III Concerning Release of U-234 from a 0.3-m U-234 Source with a Dispersivity of $0.03 \mathrm{~m}$ 


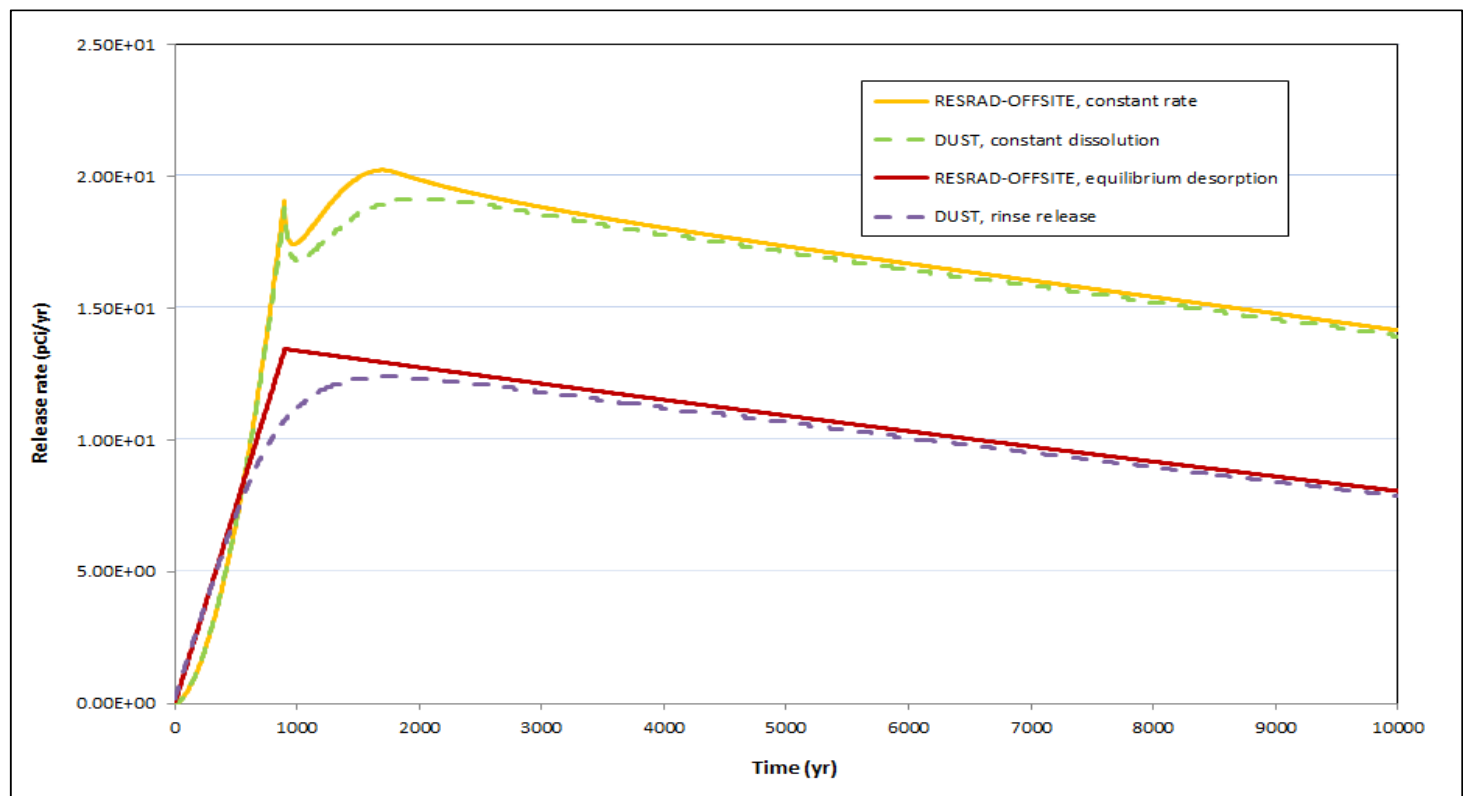

FIGURE 3.19 Comparison of RESRAD-OFFSITE and DUST-MS Results for Case III Concerning Release of Th-230 from a 0.3-m U-234 Source with a Dispersivity of $0.03 \mathrm{~m}$

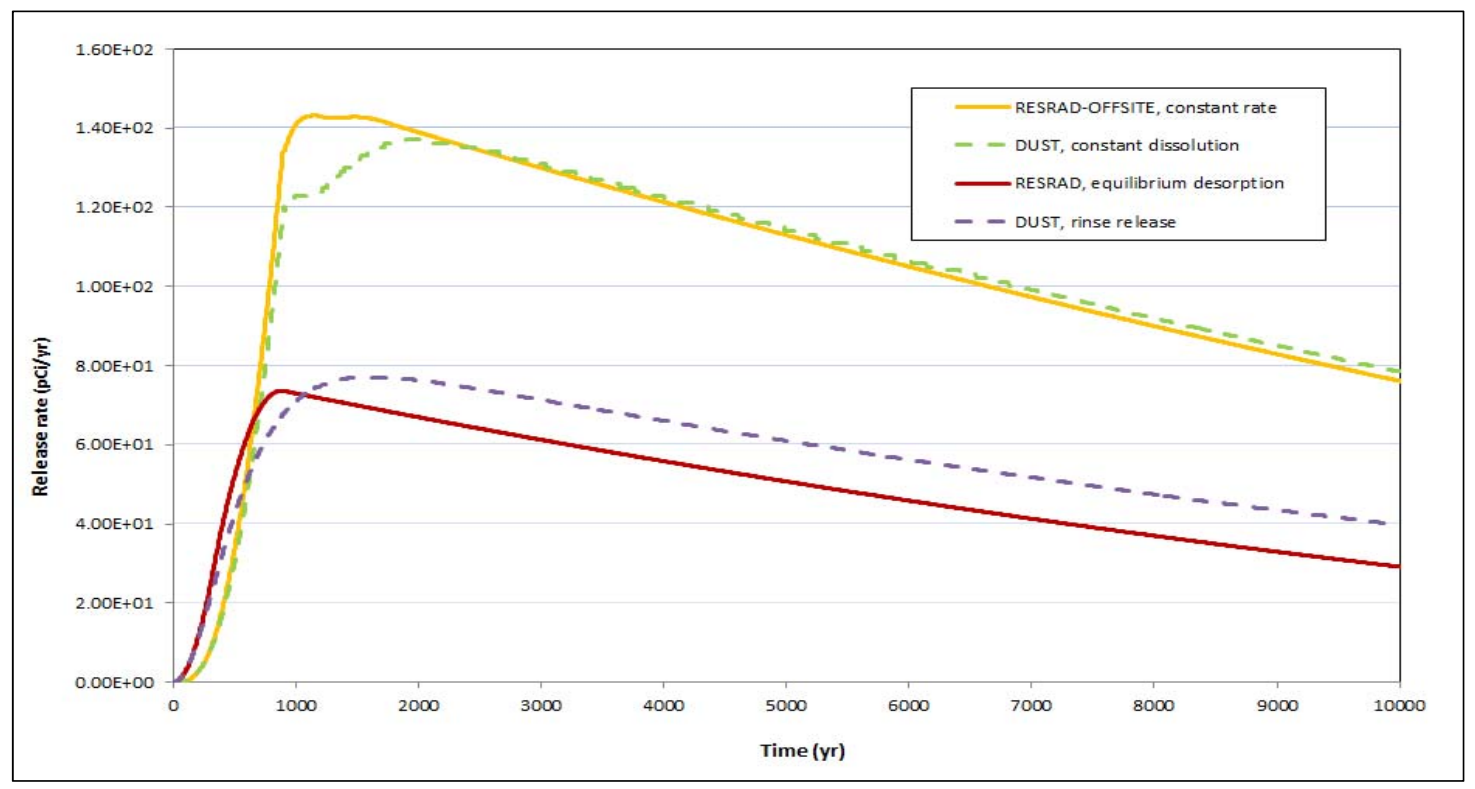

FIGURE 3.20 Comparison of RESRAD-OFFSITE and DUST-MS Results for Case III Concerning Release of Ra-226 from a 0.3-m U-234 Source with a Dispersivity of $0.03 \mathrm{~m}$ 


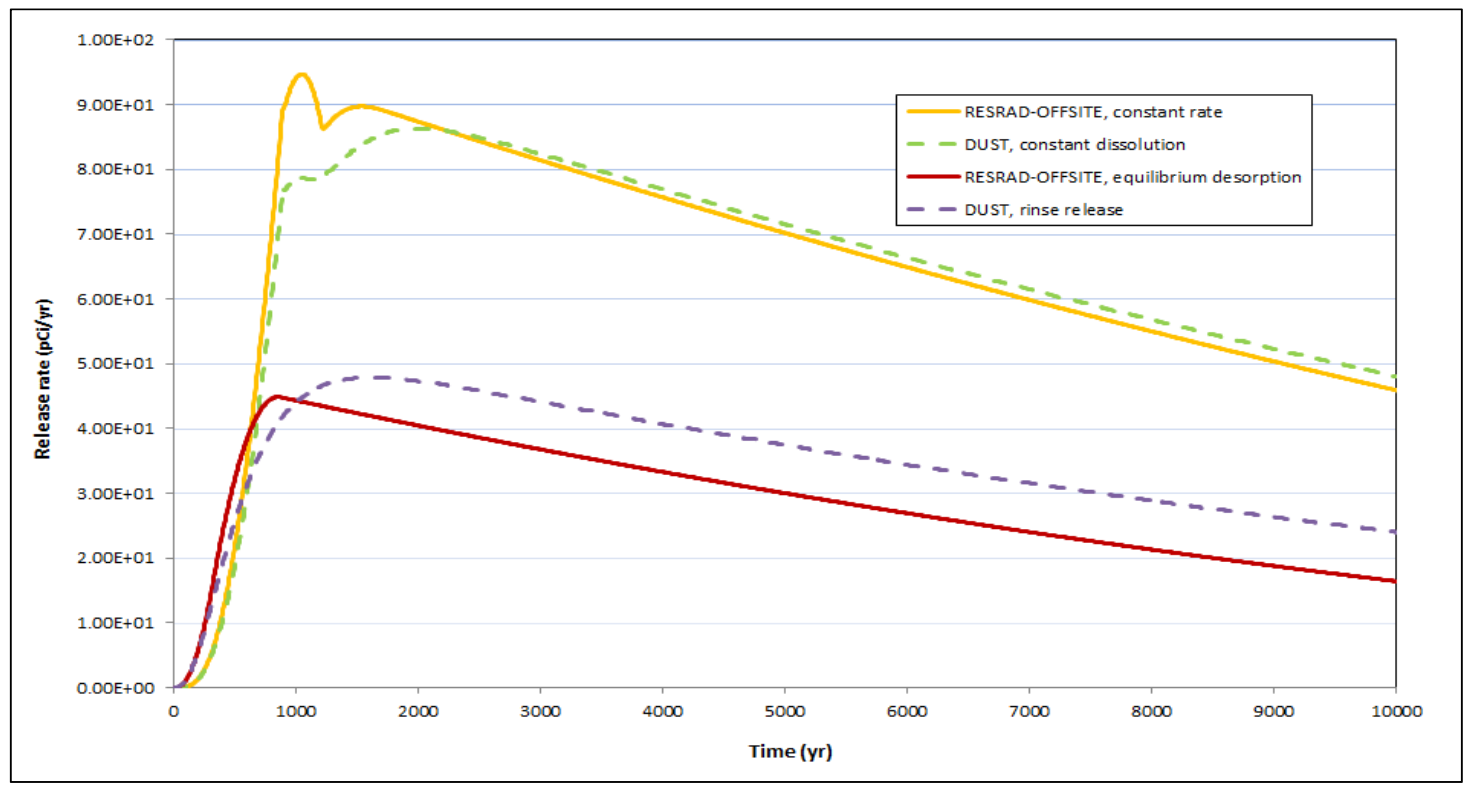

FIGURE 3.21 Comparison of RESRAD-OFFSITE and DUST-MS Results for Case III Concerning Release of Pb-210 from a 0.3-m U-234 Source with a Dispersivity of $0.03 \mathrm{~m}$

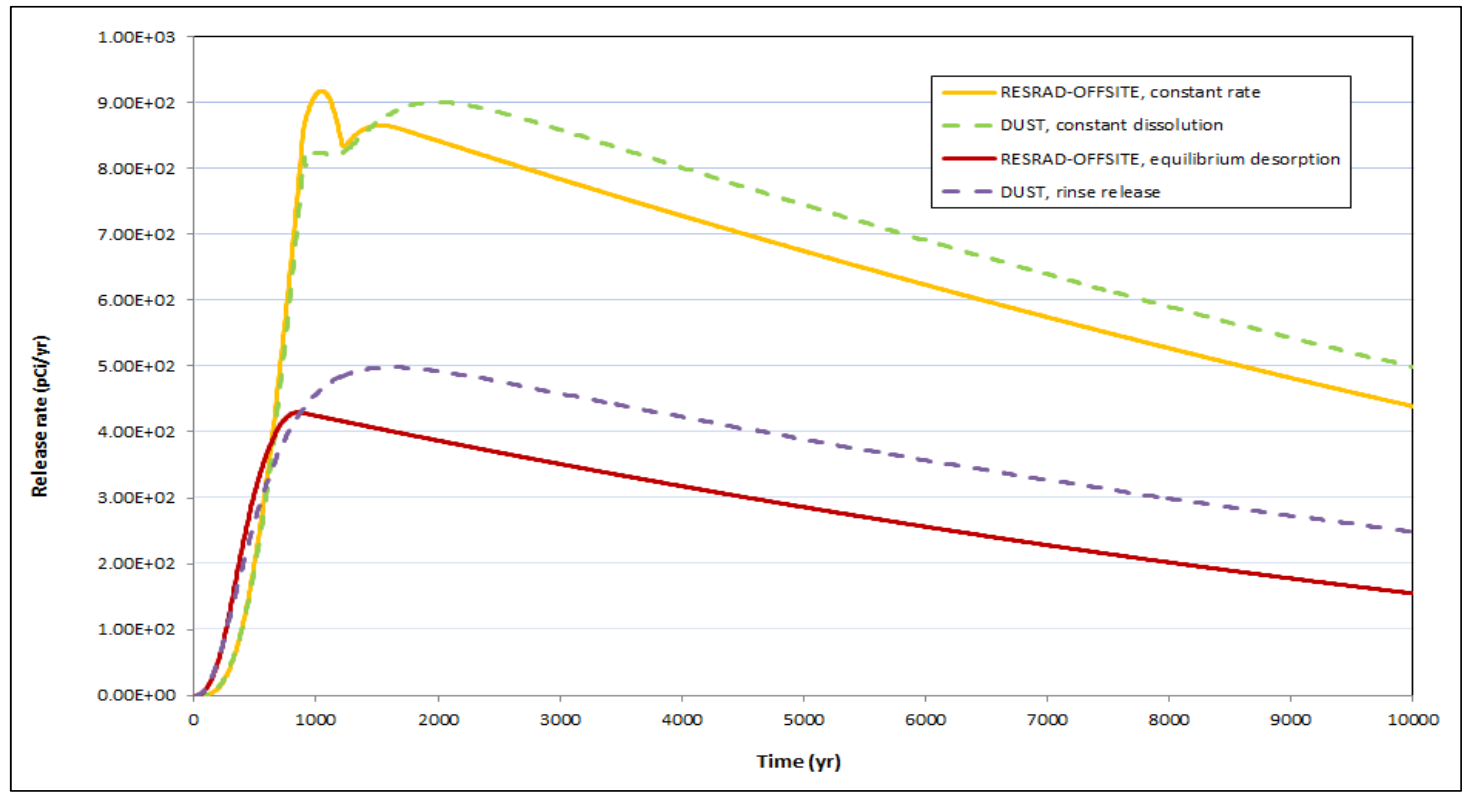

FIGURE 3.22 Comparison of RESRAD-OFFSITE and DUST-MS Results for Case III Concerning Release of Po-210 from a 0.3-m U-234 Source with a Dispersivity of $0.03 \mathrm{~m}$ 


\subsubsection{Case IV $-3 \mathrm{~m}$ of Tc-99}

Cases I, II, and III consider a release source with a thickness of $0.3 \mathrm{~m}$. In Case IV, a release source with 10 times that thickness was considered. Because of the greater thickness, transport of radionuclides within the soil column would take a longer time to reach the bottom of the contaminated zone. In addition, a smaller water infiltration rate of $0.1 \mathrm{~m} / \mathrm{yr}$, less than the $0.4 \mathrm{~m} / \mathrm{yr}$ assumed for Cases I and II, was used. The smaller water infiltration rate would carry less radionuclides leaving the contaminated zone within the same period of time. As a result, the release of radionuclides from the bottom of the contaminated zone would last much longer in Case IV than in previous cases.

The release source for Case IV was assumed to contain Tc-99. The DUST-MS calculations were performed with the last grid design discussed in Section 3.3.3, which includes a 100-m unsaturated zone beneath the contaminated zone to make the assumed lower boundary condition, $0 \mathrm{pCi} / \mathrm{L}$ of dissolved radionuclide concentration, valid at all times. The grid design has 465 nodes.

Both the equilibrium desorption condition and the uniform release condition were simulated. When the uniform release condition was simulated, the waste form was assumed to disintegrate with a rate of $0.001 / \mathrm{yr}$ for 1,000 years.

\subsubsection{No Dispersion}

Equilibrium desorption. Figure 3.23 compares the RESRAD-OFFSITE results corresponding to the equilibrium desorption option with the DUST-MS results corresponding to the rinse release mechanism. In general, the agreement is good with the peak release rates calculated by both codes matching each other. The discrepancy is considered to result from the numerical dispersion in DUST-MS results, which was studied and discussed in Section 3.4.1.1.

Uniform release. Figure 3.24 compares the RESRAD-OFFSITE results corresponding to the uniform release option with the DUST-MS results corresponding to the dissolution mechanism. The peak release rates match each other. The discrepancy is considered also to be attributable to the numerical dispersion in DUST-MS results. 


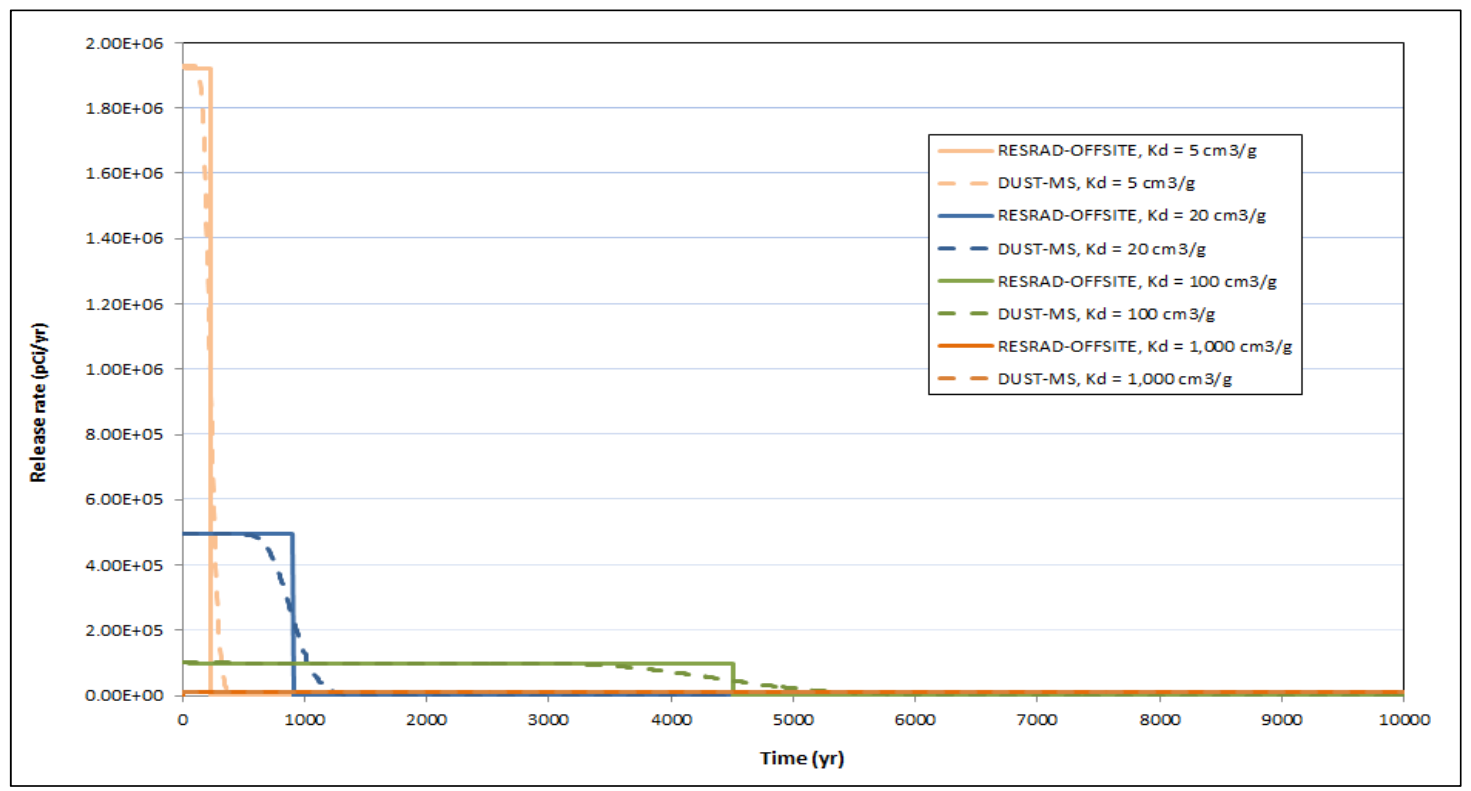

FIGURE 3.23 Comparison of RESRAD-OFFSITE and DUST-MS Results for Case IV Concerning Release from a 3-m Tc-99 Source under the Equilibrium Desorption Condition with No Dispersion

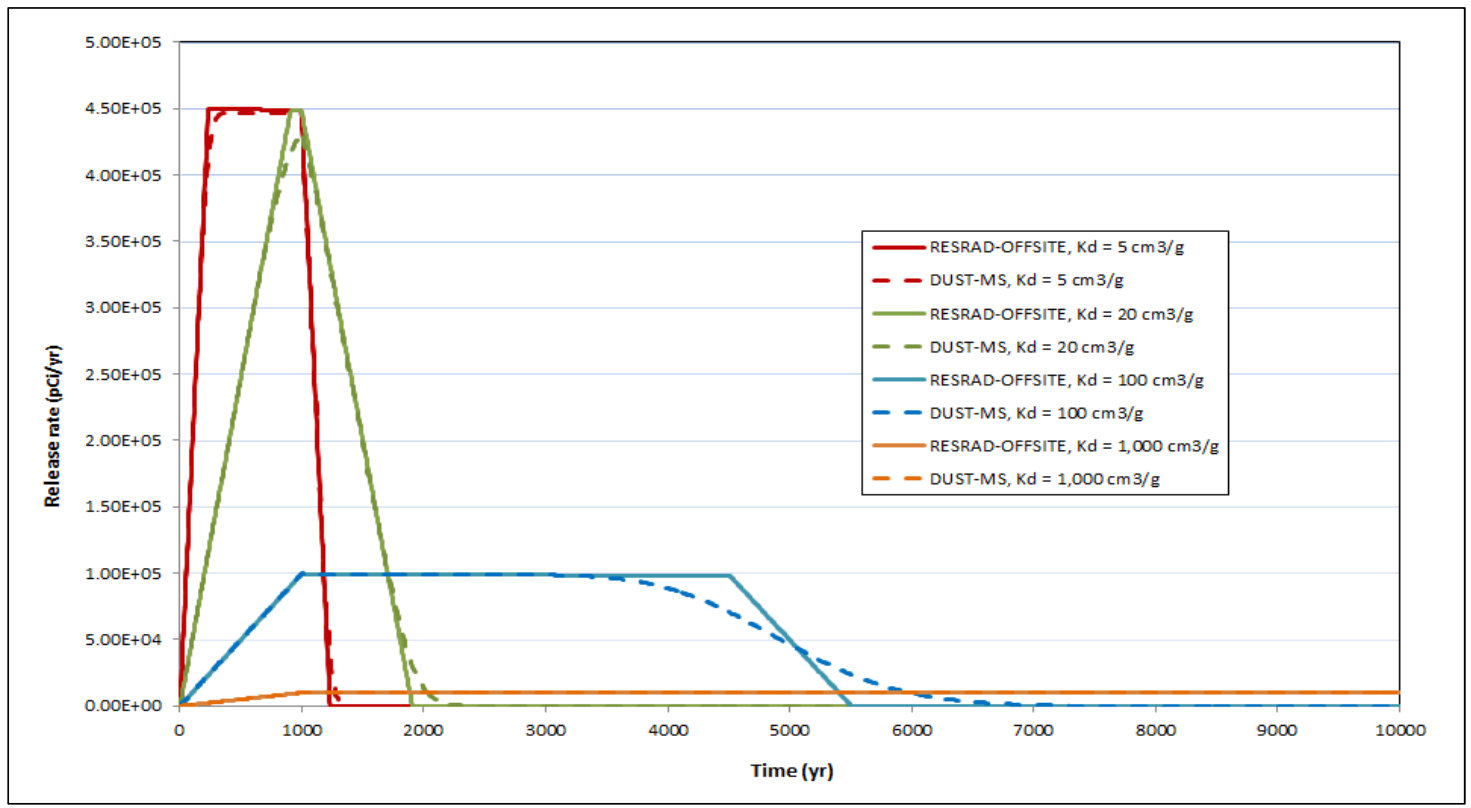

FIGURE 3.24 Comparison of RESRAD-OFFSITE and DUST-MS Results for Case IV Concerning Release from a 3-m Tc-99 Source under the Uniform Release Condition with No Dispersion 


\subsubsection{With Dispersion}

Equilibrium desorption. When there is dispersion in the soil column, agreement between the RESRAD-OFFSITE results and DUST-MS results improves as compared to when there is no dispersion. See Figure 3.25 for the comparison of RESRAD-OFFSITE and DUST-MS results with a dispersivity of $0.3 \mathrm{~m}$, and Figure 3.26 for the comparison of RESRAD-OFFSITE and DUST-MS results with a dispersivity of $1 \mathrm{~m}$.

Uniform release. The improvement in agreement between the RESRAD-OFFSITE and DUST-MS results with the consideration of dispersion was also observed under the uniform release condition. This result is illustrated by comparing Figure 3.27 (with a dispersivity of $0.3 \mathrm{~m}$ ) and Figure 3.28 (with a dispersivity of $1 \mathrm{~m}$ ) with Figure 3.24 (with no dispersion).

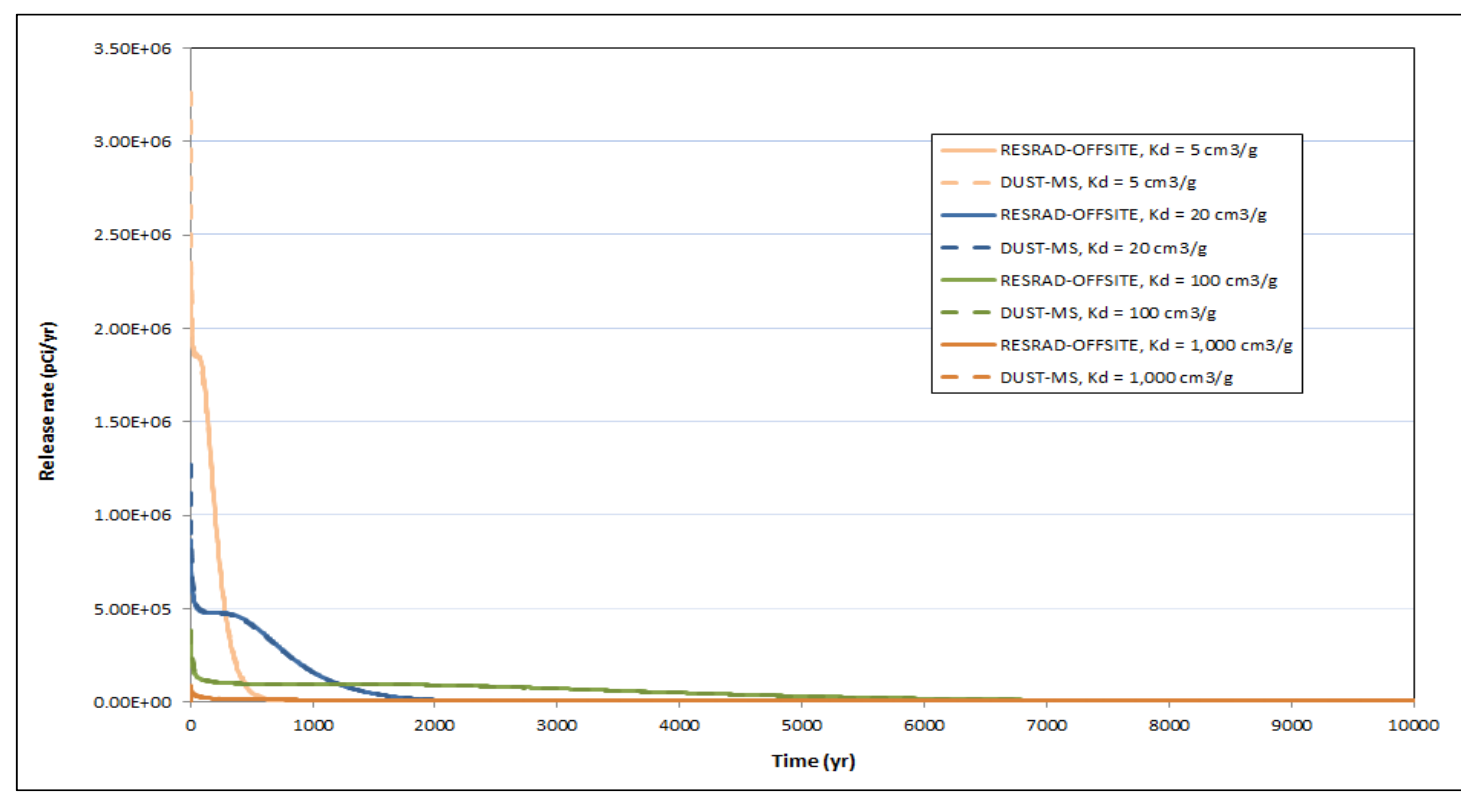

FIGURE 3.25 Comparison of RESRAD-OFFSITE and DUST-MS Results for Case IV Concerning Release from a 3-m Tc-99 Source under the Equilibrium Desorption Condition with a Dispersivity of $0.3 \mathrm{~m}$ 


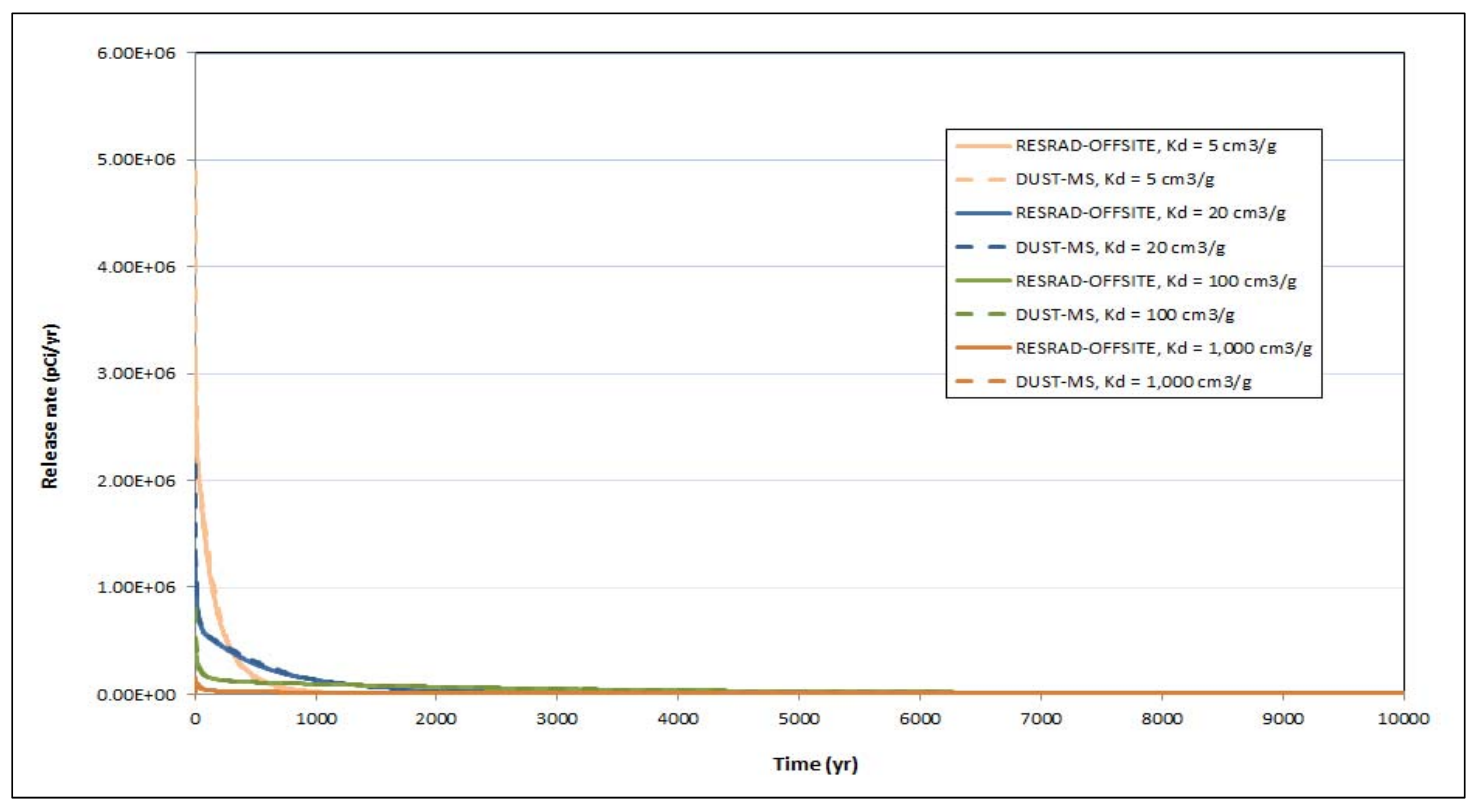

FIGURE 3.26 Comparison of RESRAD-OFFSITE and DUST-MS Results for Case IV Concerning Release from a 3-m Tc-99 Source under the Equilibrium Desorption Condition with a Dispersivity of $1 \mathrm{~m}$

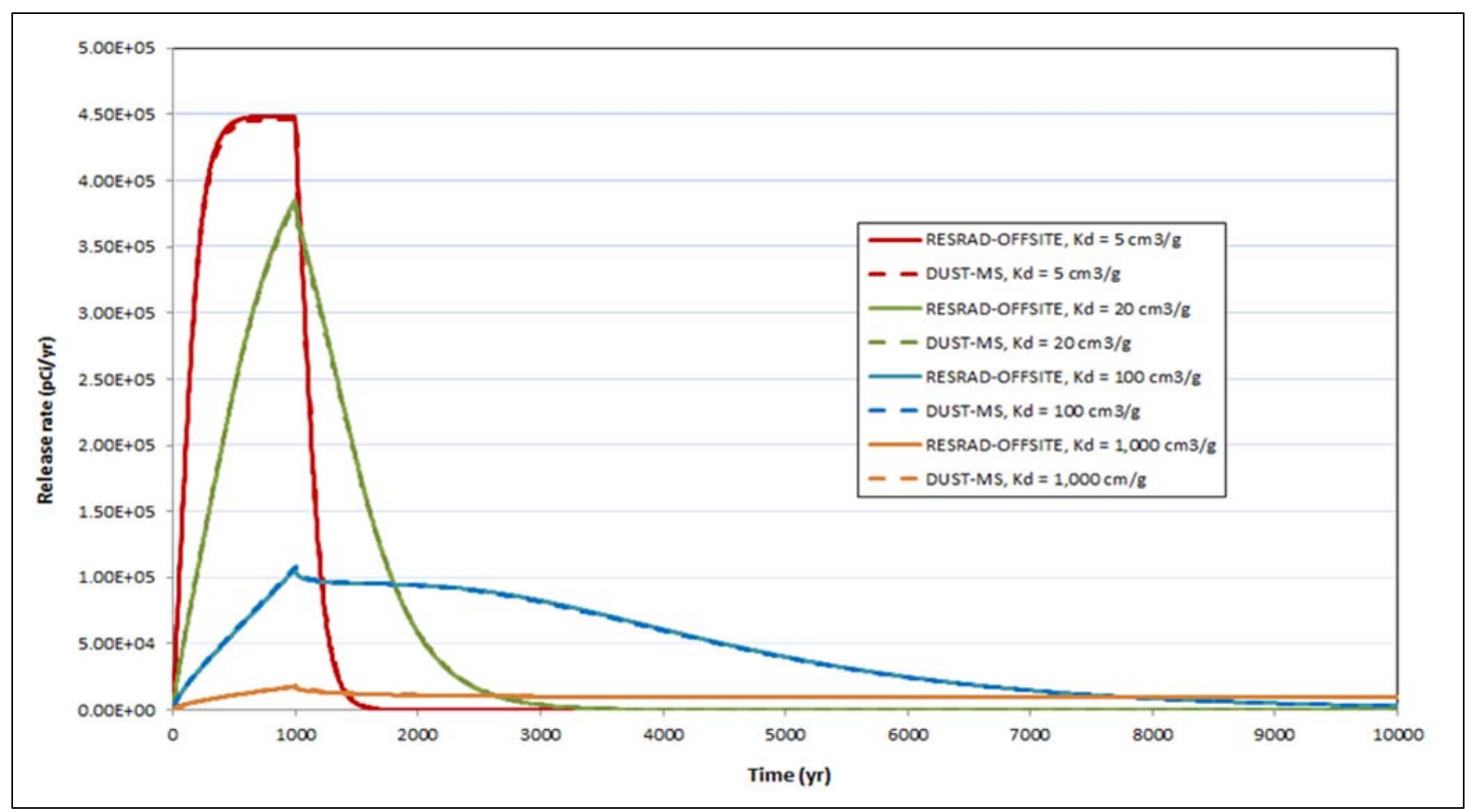

FIGURE 3.27 Comparison of RESRAD-OFFSITE and DUST-MS Results for Case IV Concerning Release from a 3-m Tc-99 Source under the Uniform Release Condition with a Dispersivity of $0.3 \mathrm{~m}$ 


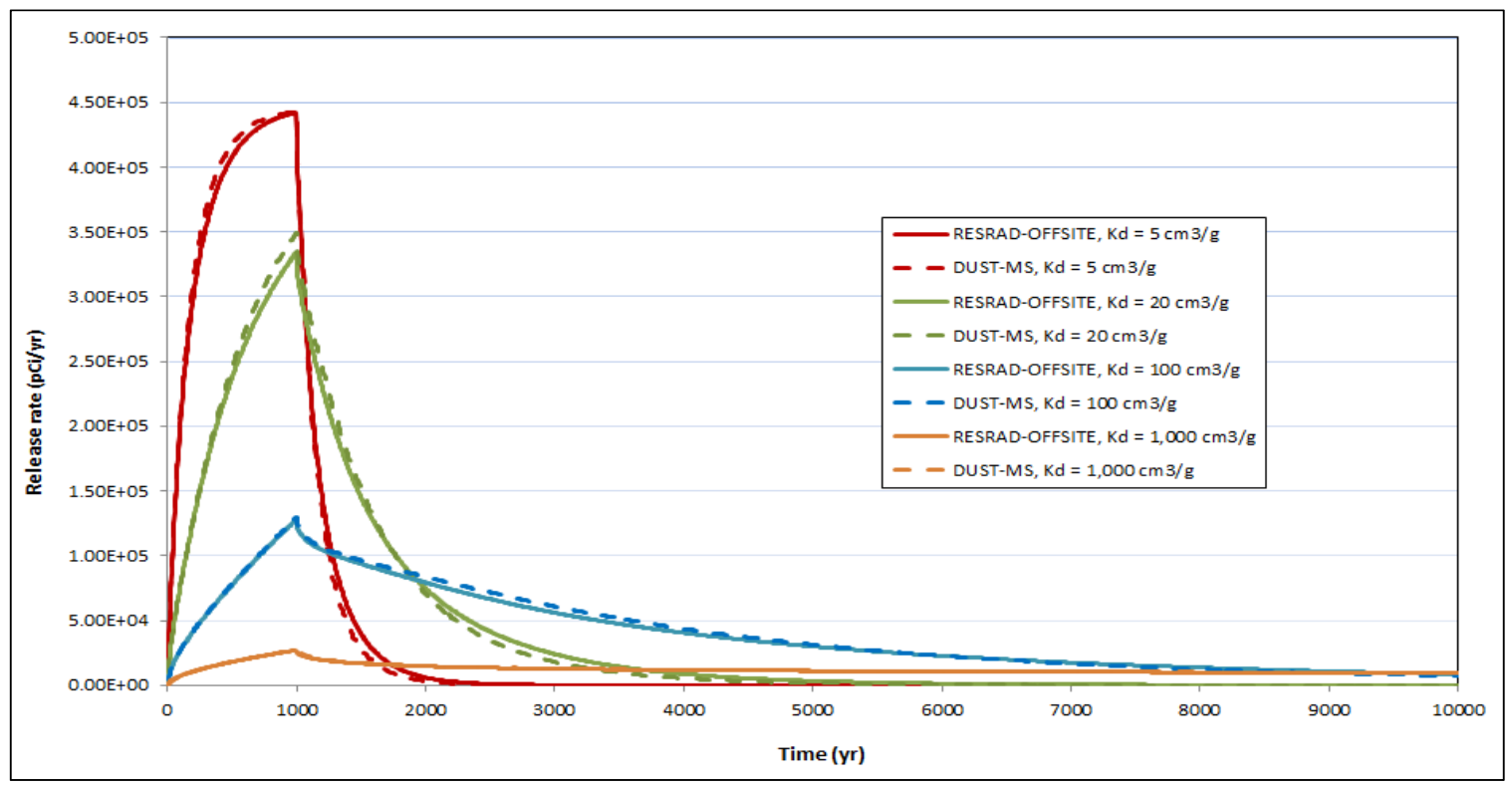

FIGURE 3.28 Comparison of RESRAD-OFFSITE and DUST-MS Results for Case IV Concerning Release from a 3-m Tc-99 Source under the Uniform Release Condition with a Dispersivity of $1 \mathrm{~m}$

\subsubsection{Case V - $3 \mathrm{~m}$ of U-234}

Case $\mathrm{V}$ concerns a 3-m release source containing $\mathrm{U}-234$. The release source was assumed to disintegrate with a rate of $0.001 / y r$ for 1,000 years under the uniform release condition. A water infiltration rate of $0.1 \mathrm{~m} / \mathrm{yr}$ was assumed. The Kd values assumed for U-234, Th-230, Ra-226, $\mathrm{Pb}-210$, and Po-210 in soils were $50,6,000,70,100$, and $10 \mathrm{~cm}^{3} / \mathrm{g}$, respectively. The DUST-MS results were obtained with a grid design of 465 nodes.

\subsubsection{No Dispersion}

Figures 3.29 to 3.33 compare the RESRAD-OFFSITE and DUST-MS results when there is no dispersion in the soil column. As the comparison shows, the agreement for progeny release rates, except for Po-210, between RESRAD-OFFSITE and DUST-MS is very good. Some discrepancy is observed with the $U-234$ and Po-210 release rates. The former is thought to be attributable to numerical dispersion in the DUST-MS results, which could be reduced if the domain of analysis is subdivided into additional grids. However, the maximum number of nodes accepted by DUST-MS is 500 . The actual reason for the discrepancy with Po-210 release rates is not yet clear. It could be related to the short decay half-life of Po-210, as well as the small Kd value that allows Po-210 radionuclides to transport faster in the soil column as compared to other radionuclides in the same decay chain. 


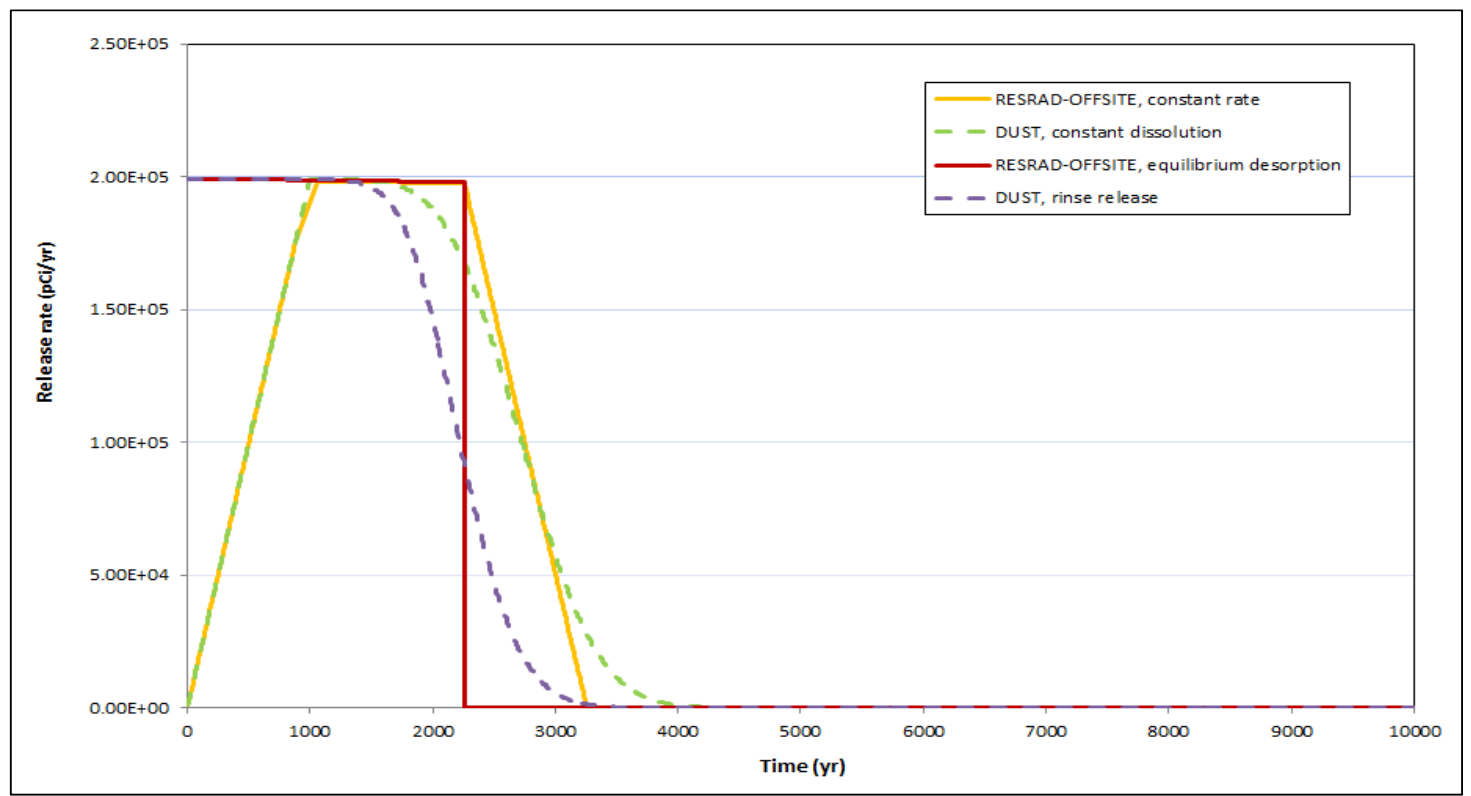

FIGURE 3.29 Comparison of RESRAD-OFFSITE and DUST-MS Results for Case V Concerning Release of U-234 from a 3-m U-234 Source with No Dispersion

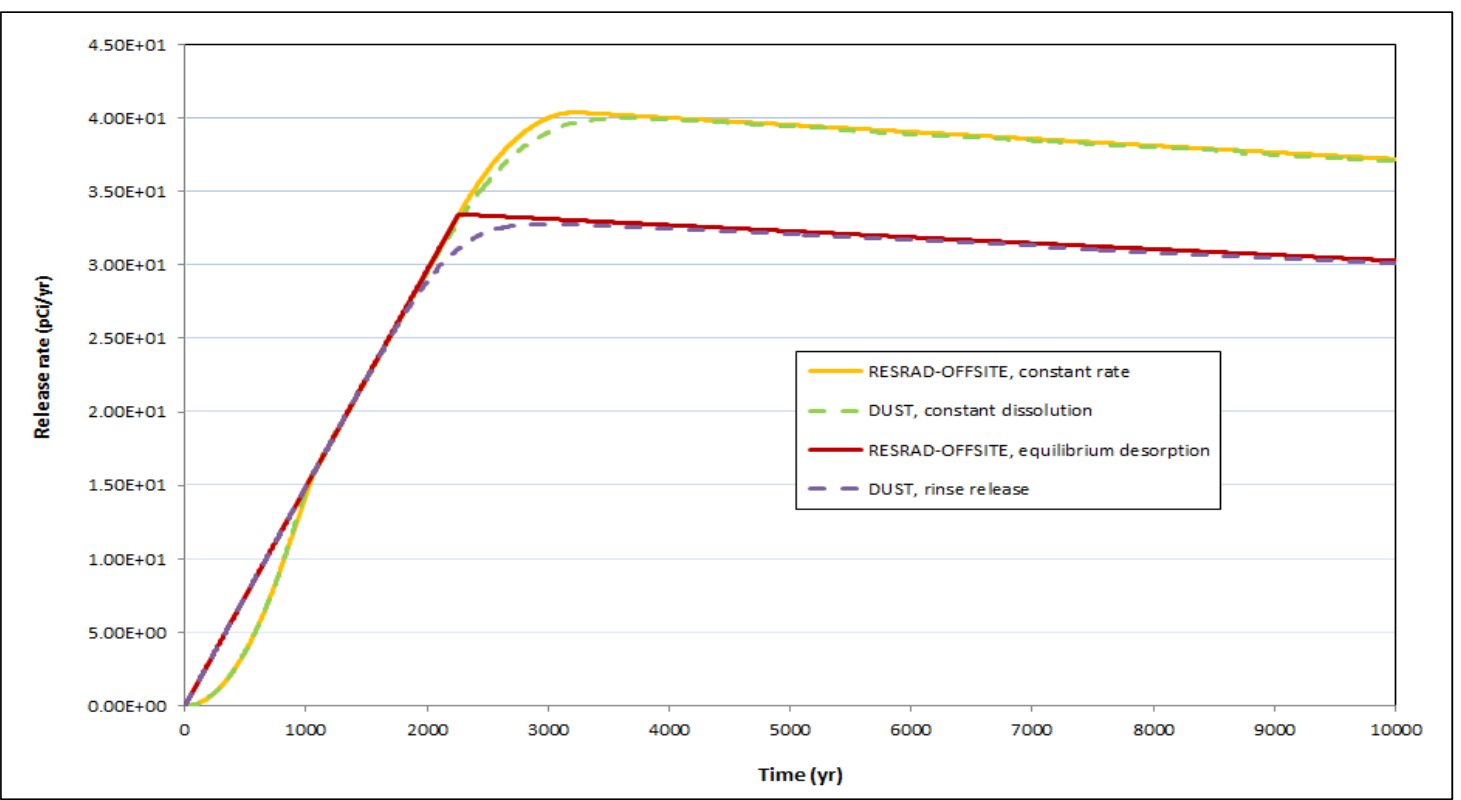

FIGURE 3.30 Comparison of RESRAD-OFFSITE and DUST-MS Results for Case V Concerning Release of Th-230 from a 3-m U-234 Source with No Dispersion 


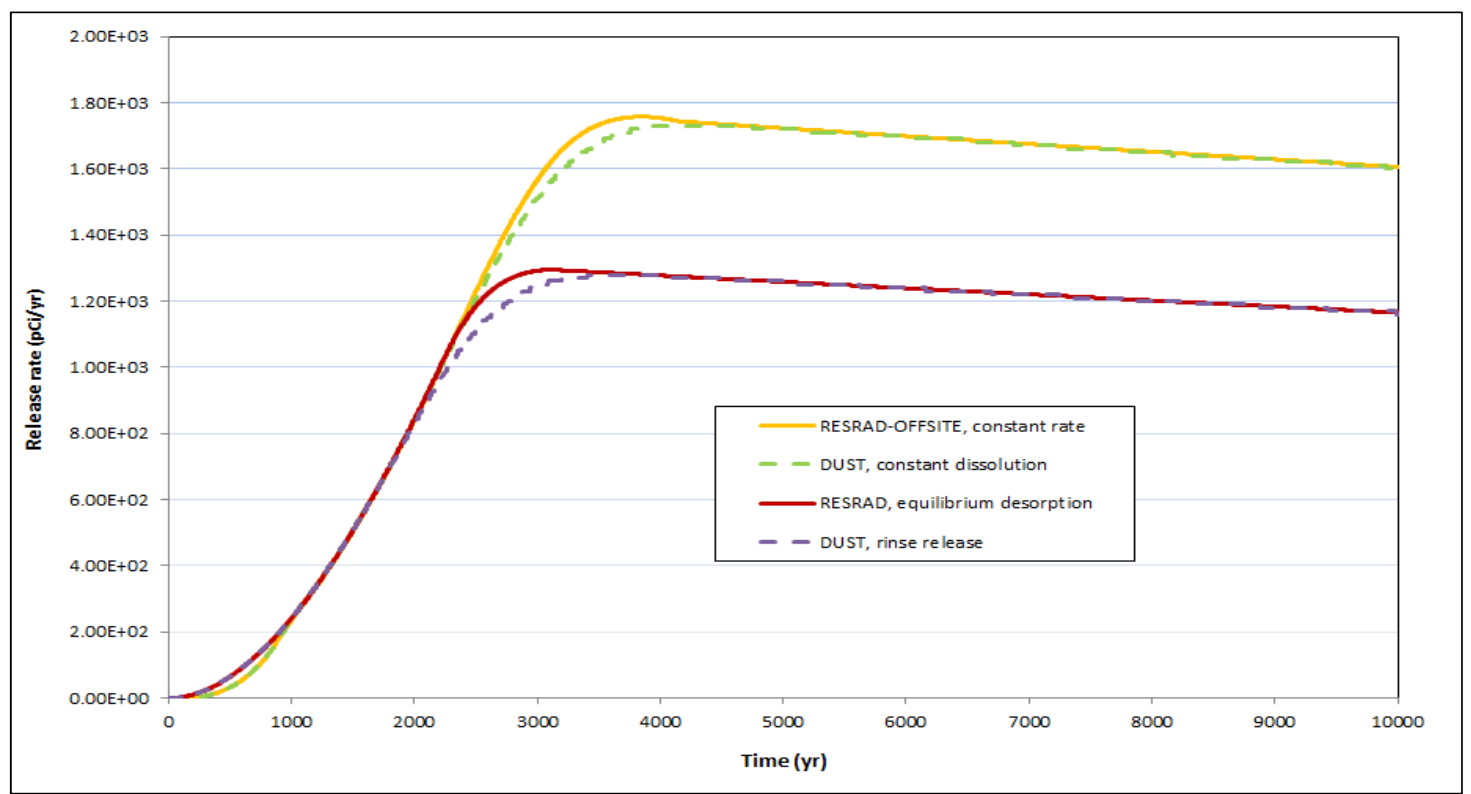

FIGURE 3.31 Comparison of RESRAD-OFFSITE and DUST-MS Results for Case V Concerning Release of Ra-226 from a 3-m U-234 Source with No Dispersion

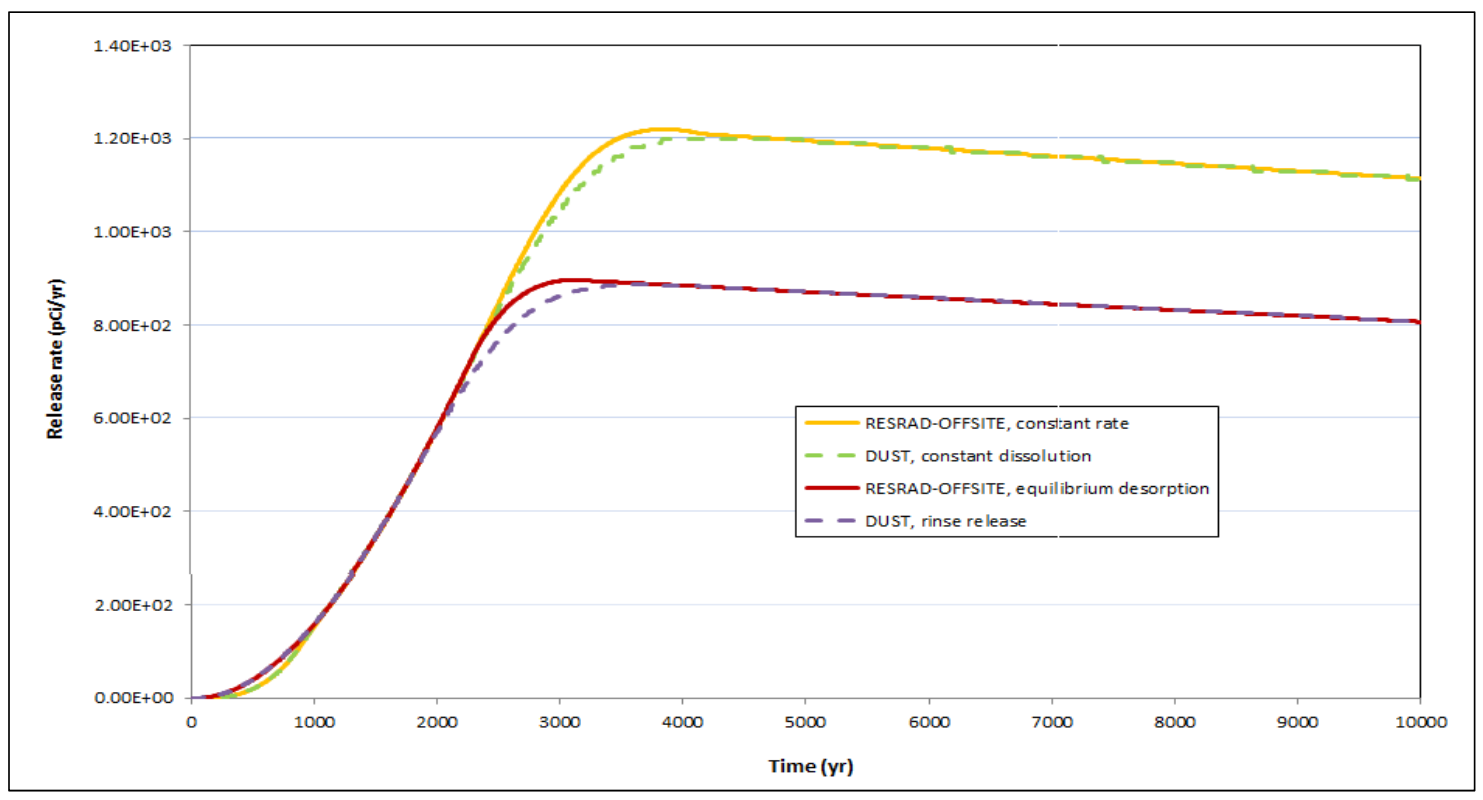

FIGURE 3.32 Comparison of RESRAD-OFFSITE and DUST-MS Results for Case V Concerning Release of Pb-210 from a 3-m U-234 Source with No Dispersion 


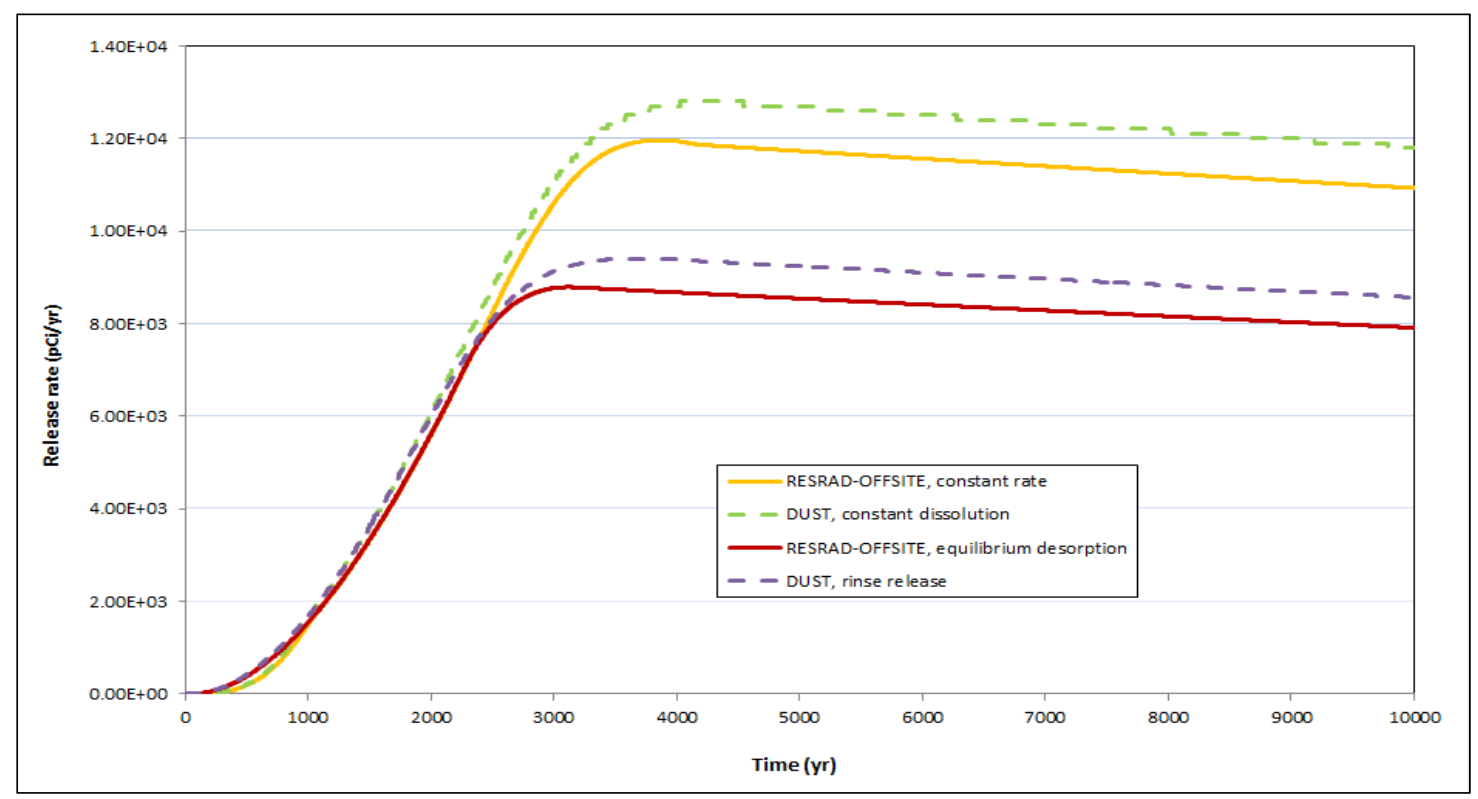

FIGURE 3.33 Comparison of RESRAD-OFFSITE and DUST-MS Results for Case V Concerning Release of Po-210 from a 3-m U-234 Source with No Dispersion

\subsubsection{With Dispersion}

Figures 3.34 to 3.38 compare the RESRAD-OFFSITE and DUST-MS results when dispersion in the soil column is considered. With a dispersivity of $0.3 \mathrm{~m}$, the agreement with $\mathrm{U}-234$ release rates is better than it is without dispersion. However, a greater discrepancy is observed with the release rates of Th-230, Ra-226, and $\mathrm{Pb}-210$ when dispersion takes place. The agreement with the Po-210 release rates remains at about the same level.

\subsection{Summary of Comparison}

Comparisons of radionuclide release rates calculated by RESRAD-OFFSITE and DUST-MS were conducted for five different cases involving different source dimensions, radionuclides, water infiltration rates, and release mechanisms. Overall, the agreement between the RESRADOFFSITE results and DUST-MS results is very good, with both codes predicting the same or very similar profiles over time.

The cause of disagreement in release rates of parent radionuclides under the no-dispersion condition was studied with a $0.3-\mathrm{m}$ Tc-99 source. The study showed that numerical dispersion in the DUST-MS results was responsible for the disagreement. The disagreement can be reduced by increasing node density when subdividing the domain of analysis for the DUST-MS calculations. 


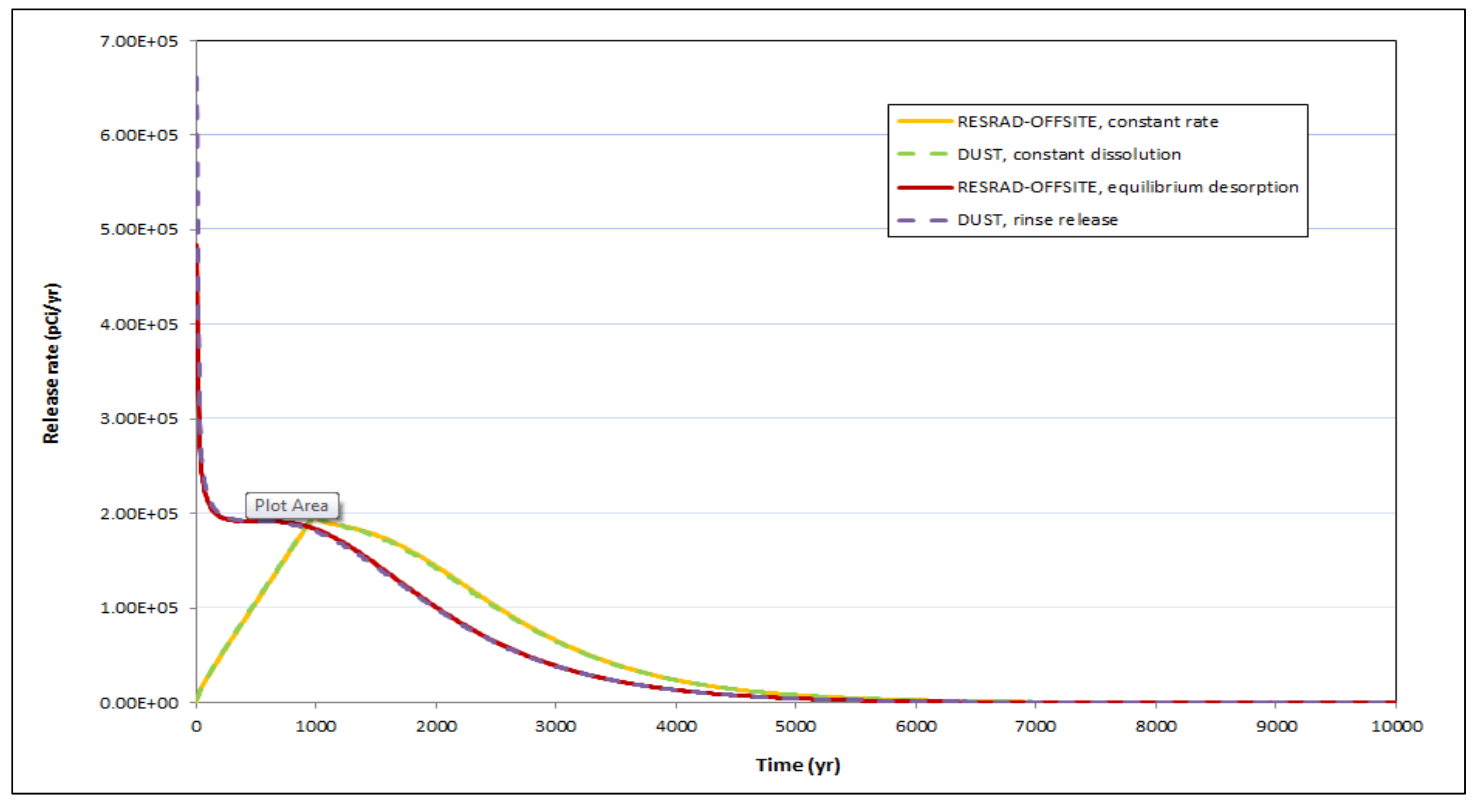

FIGURE 3.34 Comparison of RESRAD-OFFSITE and DUST-MS Results for Case V Concerning Release of U-234 from a 3-m U-234 Source with a Dispersivity of $0.3 \mathrm{~m}$

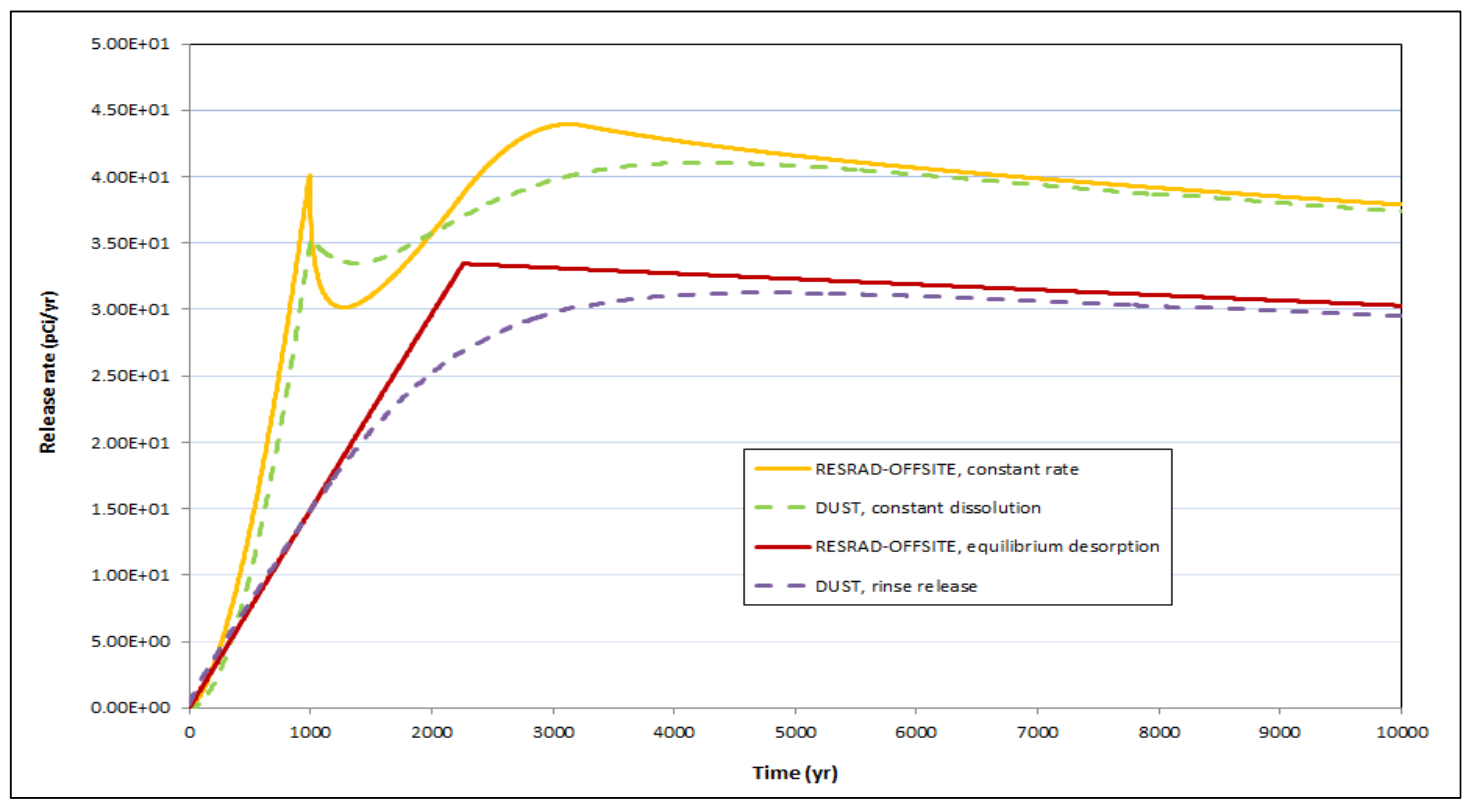

FIGURE 3.35 Comparison of RESRAD-OFFSITE and DUST-MS Results for Case V Concerning Release of Th-230 from a 3-m U-234 Source with a Dispersivity of $0.3 \mathrm{~m}$ 


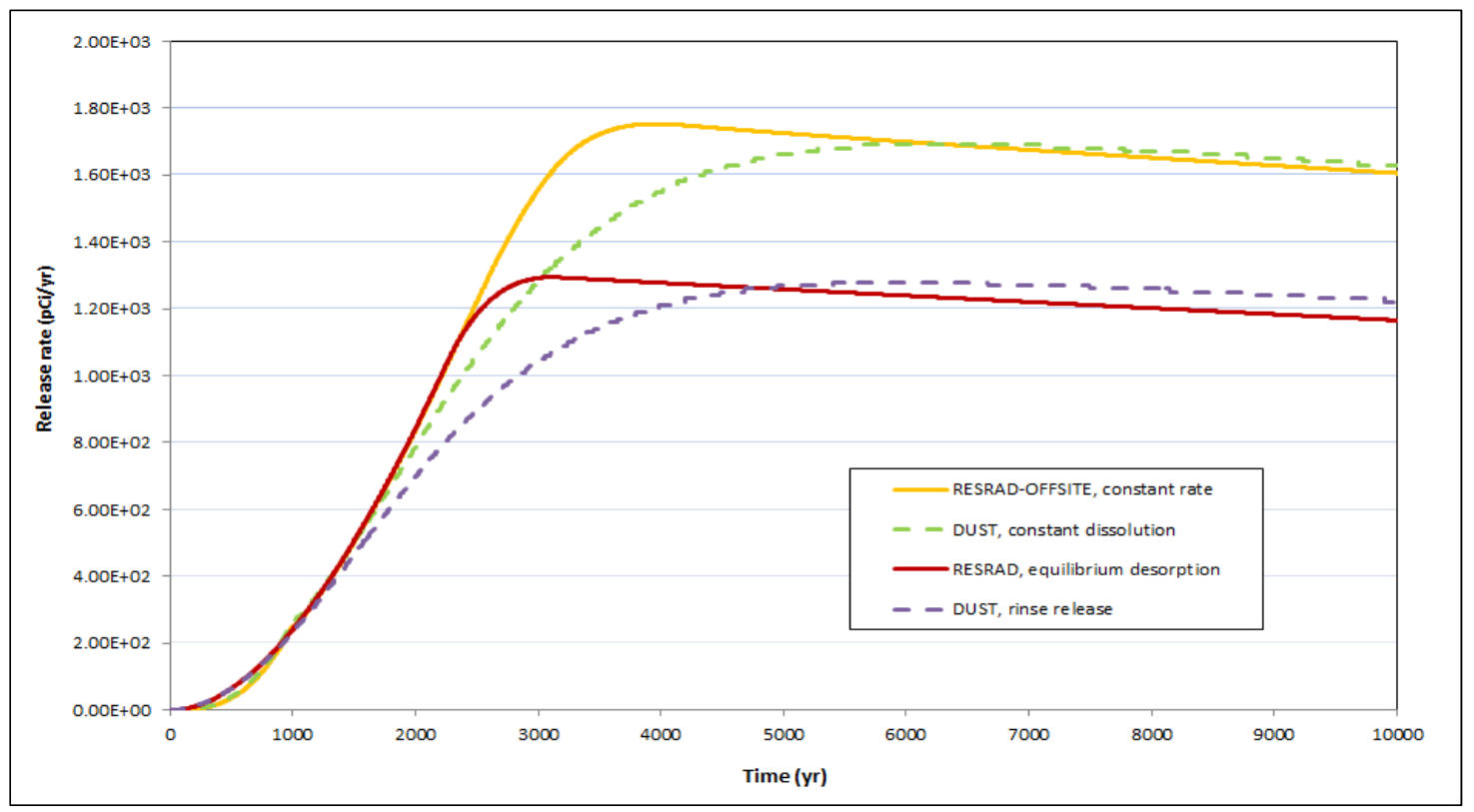

FIGURE 3.36 Comparison of RESRAD-OFFSITE and DUST-MS Results for Case V Concerning Release of Ra-226 from a 3-m U-234 Source with a Dispersivity of $0.3 \mathrm{~m}$

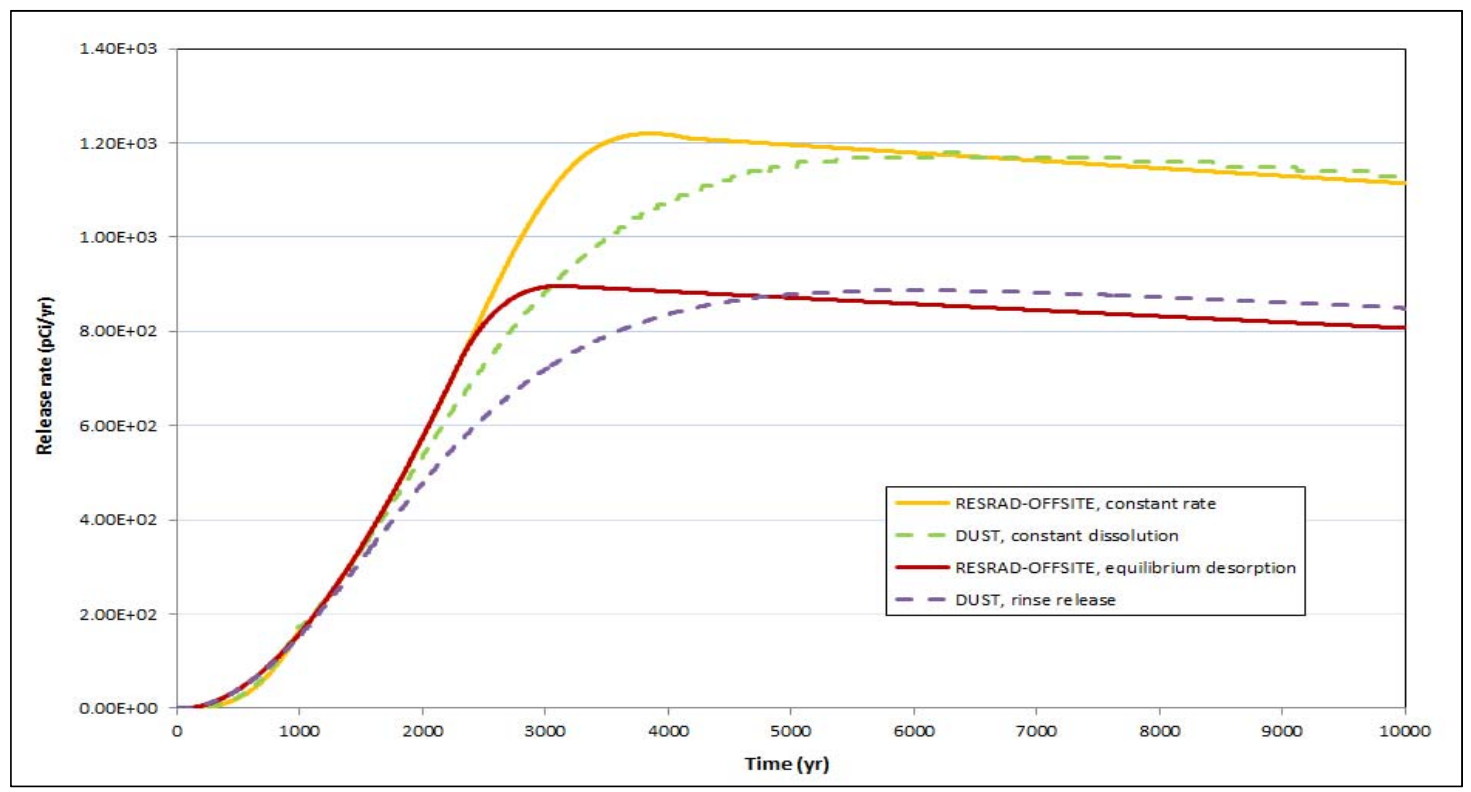

FIGURE 3.37 Comparison of RESRAD-OFFSITE and DUST-MS Results for Case V Concerning Release of Pb-210 from a 3-m U-234 Source with a Dispersivity of $0.3 \mathrm{~m}$ 


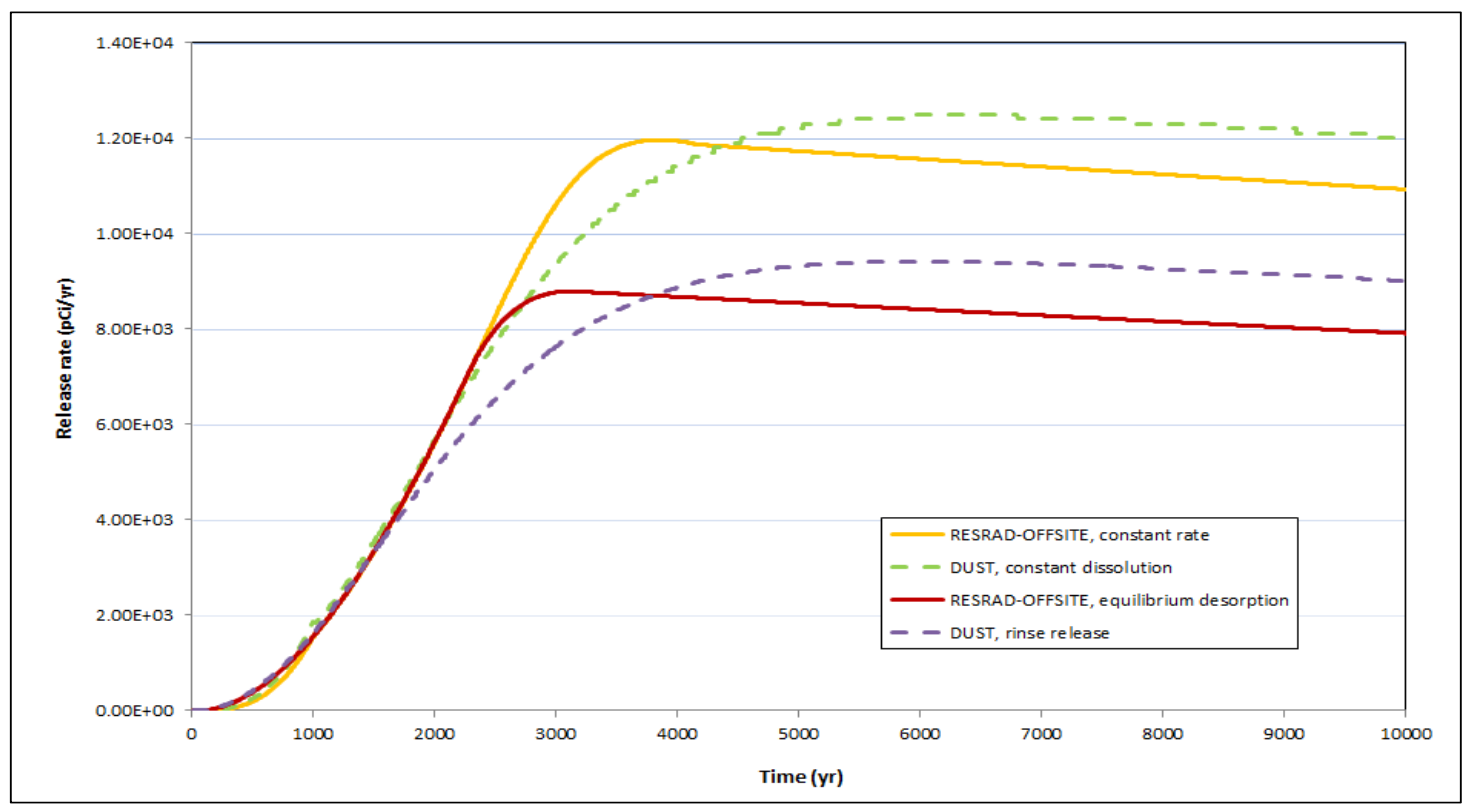

\section{FIGURE 3.38 Comparison of RESRAD-OFFSITE and DUST-MS Results for Case V Concerning Release of Po-210 from a 3-m U-234 Source with a Dispersivity of $0.3 \mathrm{~m}$}

The agreement between the RESRAD-OFFSITE results and DUST-MS results for parent radionuclides improves when dispersion in the soil column was considered. This improvement is observed with all of the comparison cases.

When the release source contains $U-234$, which decays to multiple progenies, the comparison shows that agreement between the RESRAD-OFFSITE results and DUST-MS results is better for the longer-lived progenies (Th-230, Ra-226, and Pb-210) than for the shorter-lived progeny (Po-210). The exact cause of this difference in agreement is not clear, but is thought to be related to the combination of a short half-life and greater mobility in the soil column. Even with some discrepancy, the agreement with the Po-210 release rates between RESRAD-OFFSITE and DUST-MS is still considered well acceptable.

The comparisons demonstrate that the RESRAD-OFFSITE source term model calculates radionuclide release rates comparable to those calculated by DUST-MS under the release conditions that it is equipped to consider. Additional benchmarking results presented in Appendix B confirm this observation. 


\section{Sensitivity Analysis to Identify Influential Inputs}

This chapter provides examples of identifying the inputs that contribute to the uncertainty of the radiological dose due to the uncertainty or variability of the inputs. Appendix $\mathrm{C}$ describes the three different methods available in RESRAD-OFFSITE to identify the influential inputs, the advantages and disadvantages of each of the three methods, and how each method can be used. The hypothetical scenario used in Appendix $C$ to illustrate the identification of influential inputs is expanded in this chapter to include the new source release options available in the RESRAD-OFFSITE code Version 3.

\subsection{Hypothetical Scenario to Illustrate Identification of Influential Inputs}

\subsubsection{Site Layout and Dimensions}

The layout of the hypothetical site and the section through the contaminated layer are depicted in Figures 4.1 and 4.2.

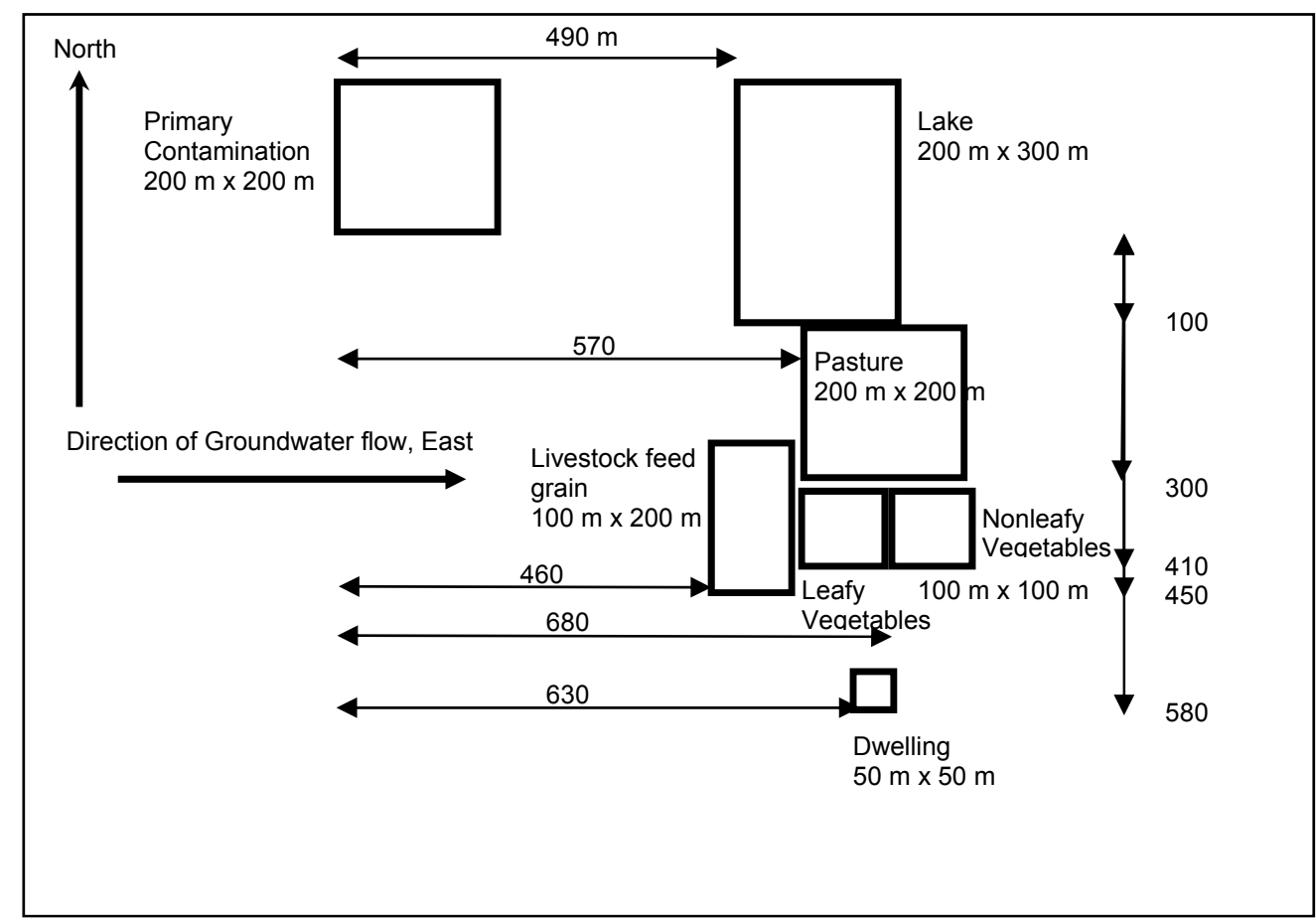

FIGURE 4.1 Layout of the Hypothetical Site 


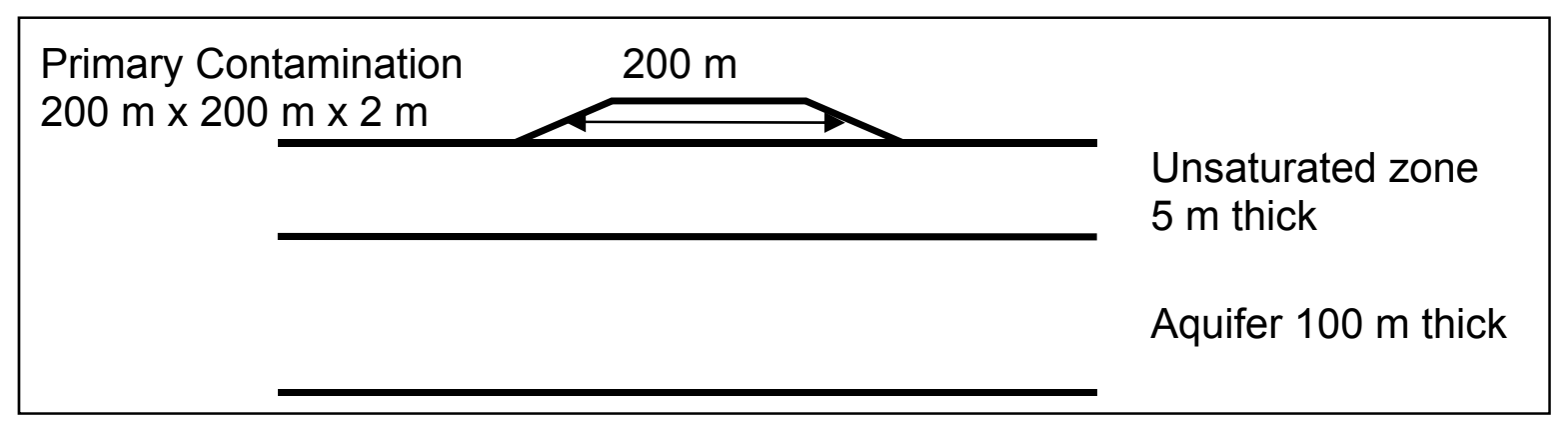

FIGURE 4.2 The Cross Section of the Primary Contamination and the Soil beneath It

\subsubsection{Radionuclides in the Primary Contamination}

The radionuclides in the primary contamination and their concentrations are listed below:

${ }^{226} \mathrm{Ra}: 35 \mathrm{pCi} / \mathrm{g}$
${ }^{210} \mathrm{~Pb}: 35 \mathrm{pCi} / \mathrm{g}$
${ }^{210} \mathrm{Po}: 35 \mathrm{pCi} / \mathrm{g}$

\subsubsection{Preliminary Data for the Hypothetical Site}

Preliminary data developed for this site are as given below. All other inputs are assumed to be at the preloaded (default) RESRAD-OFFSITE values for this preliminary analysis.

It is assumed that there is no cover on the pile. The preliminary data indicate that the release of the radionuclides from the waste pile is controlled by the rate of dissolution of the solids in which the radionuclides are embedded; the release duration is 10,000 years for all radionuclides. Alternatively, if a first order release option is to be used, leach rates for all radionuclides are 0.0001 per year. If the release is to be modeled as being controlled by equilibrium desorption, a value of $1000 \mathrm{~cm}^{3} / \mathrm{g}$ will need to be used for the distribution coefficients of all radionuclides in the contaminated zone.

The distribution coefficient of the radionuclides for partitioning between the solid phase and the moisture are as follows for the soil and conditions at the site:

$$
\begin{aligned}
& { }^{226} \mathrm{Ra}: 22 \mathrm{~cm}^{3} / \mathrm{g} \\
& { }^{210} \mathrm{~Pb}: 5 \mathrm{~cm}^{3} / \mathrm{g} \\
& { }^{210} \mathrm{Po}: 5 \mathrm{~cm}^{3} / \mathrm{g}
\end{aligned}
$$

These distribution coefficients will be used for all transport zones and offsite locations for the preliminary analysis. 
The average annual precipitation at this location is $1.25 \mathrm{~m} / \mathrm{yr}$. The primary contamination is not cultivated and is not irrigated. The preloaded (default) RESRAD-OFFSITE values for rainfall and runoff factor (160), slope length steepness factor (0.4), and support practice factor (1) are appropriate for this site. The cover management factor for the primary contamination is 0.04 , and the runoff coefficient is 0.5 . This value corresponds to an area that is $60 \%$ covered with weeds, short brush, and grass.

The leafy vegetables are grown in a field that has $20 \%$ slope and is farmed along the contour; thus, a support practice factor of 0.9 , a cover and management factor of 0.08 , and a slope length steepness factor of 4 are appropriate.

The fruit, grain, nonleafy vegetables, and livestock feed grain are grown in relatively flat land; thus, slope length steepness factors of 0.4 and support practice factors of 1 are appropriate for both areas. The cover management factor is 0.04 for these two areas. A slope length steepness factor of 0.4 and a support practice factor of 1 are also appropriate for the pasture.

Assume for atmospheric transport purposes that this hypothetical site is located in Peoria, Illinois. The topographical map indicates that the ground level of the livestock feed grain growing area is $15 \mathrm{~m}$ above the ground level at the location of primary contamination. The corresponding differences in elevation for the leafy vegetable growing area and the dwelling site are 20 and 40 meters. All the other offsite locations are at approximately the same elevation as the ground in the vicinity of the primary contamination. A preliminary sensitivity analysis on the grid spacing for this site shows that a grid spacing of $125 \mathrm{~m}$ is adequate for the desired accuracy.

The hydraulic gradient of the groundwater is 0.004 across the entire site, and the hydraulic conductivity is $10^{5} \mathrm{~m} / \mathrm{yr}$. The longitudinal, horizontal lateral, and vertical lateral dispersivities to the surface water body are estimated to be $8,0.8$, and $0.05 \mathrm{~m}$, respectively.

The surface water body is the source of all water used in this scenario. For groundwater transport purposes, the surface water body is $290 \mathrm{~m}$ along the groundwater flow line, from the down gradient edge of the primary contamination. The right and left edges of the surface water body are at 200 and $100 \mathrm{~m}$ from the groundwater flow line through the center of the primary contamination. This surface water body does not have any edible crustacea or fish.

The individual spends 0.5 of the time inside the dwelling, 0.2 of the time outdoors in the vicinity of the dwelling, 0.08 of the time in each of the vegetable plots and in the livestock grain field, and 0.01 of the time in the pasture. The remainder of time is spent away from the area. 
A preliminary (deterministic) analysis of this scenario confirms that the long-term dose from this site results from the ${ }^{226} \mathrm{Ra}$ that was at the site because of the short half-life of the other two radionuclides that are initially present at the site. The ${ }^{210} \mathrm{~Pb}$ and ${ }^{210} \mathrm{Po}$ that were initially present would have transformed away at the time of the peak in the dose. Thus, these two radionuclides are left out of the probabilistic sensitivity analysis in order to avoid spending computation time on computations that do not affect the final results.

Users should perform a sensitivity analysis to find the inputs that have a significant impact on the dose so that the data-gathering effort can be focused on those inputs. Reasonable initial distributions or ranges for the inputs should be used, and any relationships or correlations between the inputs should be considered, if possible.

\subsubsection{Initial Ranges or Distributions of Inputs}

The values identified in the hypothetical scenario for the inputs are initial estimates. The actual value of the input is expected to lie within some range of this initial estimate. As the inputs vary across their ranges of values, the resulting predicted peak dose will take a range of values, as well. A majority of the inputs will have an insignificant influence on the dose; the dose will remain essentially the same regardless of what value these inputs take over their probable ranges. The remaining variables will have an influence on the dose; the dose will change noticeably as the values of these inputs change over their probable range. The purpose of a preliminary sensitivity analysis is to identify the inputs that cause the dose to vary over its range. It will usually be possible to break these inputs into groups on the basis of how much influence they exert on the variability of the dose. This analysis will allow resources to be focused on the more influential inputs to find more site-specific and (hopefully) narrower ranges or distributions for them. The initial estimates of the value and range of each input has to be found by using a small portion of the available resource. The initial estimates are usually based on the more easily obtainable site characteristics, for example, the soil type, climate, topography, and vegetation. The initial distributions will usually be uniform over the typical range appropriate for the easily obtainable site characteristics.

The uncertainty of the value of an input is likely to be lower if an initial value was developed for it based on site characteristics rather than if it were left at the preloaded value in RESRADOFFSITE. Thus, if an initial estimate is given in this example scenario, a range of $10 \%$ on either side of the initial value will be used in most cases. However, there will be inputs for which it will be appropriate to use larger ranges, of $25 \%$ or even $50 \%$ on either side of the initial estimate, especially for inputs that span orders of magnitude, or where there is uncertainty about the conceptualization. For these reasons, $25 \%$ ranges will be specified for the release duration and the distribution coefficients in the transport zones and in the offsite locations; $50 \%$ ranges will be specified for the leach rates and, when modeling this as an equilibrium desorption release, for the distributions coefficient in the contaminated zone. In this preliminary sensitivity analysis 
example, a range of $50 \%$ will be used on either side of a preloaded RESRAD-OFFSITE value unless a smaller range is indicated by the nature of that input. Smaller ranges were specified for inputs that describe the standard receptor (10\% range), the density or porosity of soil (25\% range), and the ambient temperature (10\% range).

\subsubsection{Relationships or Correlations between Inputs}

The RESRAD-OFFSITE inputs are not all independent; some of the inputs are correlated with others, and there may even be exact relationships between some of the inputs under certain circumstances. In this example scenario, the groundwater is shown to flow from west to east along the $x$ dimension of the primary contamination. Thus, in this example, the length of contamination parallel to the aquifer flow is equal to the $x$ dimension of the primary contamination-an exact relationship. In this example, the total porosity and the effective (in transport) porosity of the primary contamination are assigned the same initial preloaded RESRAD-OFFSITE value. They will also have identical ranges and distributions in this example scenario according to the discussion in Section 4.1.2. If these distributions are sampled independently, there will be many samples where the value for the effective porosity will exceed the value for total porosity. The only way to prevent this result is to specify that the effective porosity must be equal to total porosity in the contaminated zone: an exact relationship.

There are many other inputs in RESRAD-OFFSITE which, while not related, are correlated. For example, consider the four inputs, dry bulk density, total porosity, effective (in transport) porosity, and field capacity of a soil layer. The field capacity, the volume fraction of pores that can hold water against gravity flow, has to be lower than the total porosity. Considering the initial values, 0.3 for field capacity and 0.4 total porosity, and the initial distributions of 0.225 to 0.375 for field capacity and 0.3 to 0.5 for total porosity, it will be necessary to specify a positive correlation between these two inputs to ensure that the value used for field capacity is lower than the value used for total porosity in any of the simulations. Not all of the pores are effective in conducting moisture through the soil; thus, the effective porosity cannot exceed the total porosity. It is also likely that high total porosities should lead to high effective porosities. Hence, a positive correlation should be specified between total porosity and effective porosity. If total porosity is positively correlated with both field capacity and effective porosity, then field capacity and effective porosity must also be positively correlated. The more porous the soil, the lower its dry bulk density; these two inputs need to be inversely or negatively correlated. The dry bulk density would have to be negatively correlated with field capacity and effective porosity, as well, because they are positively correlated with total porosity. 


\subsection{Three-Point Single Input Sensitivity Analysis}

Section C. 2 of Appendix $C$ discusses the three-point single input sensitivity analysis and describes how to perform this analysis in RESRAD-OFFSITE. The sensitivity analysis is performed on a single input parameter at a time. The sensitivity analysis involves three simulations; the first with all of the inputs, including the one selected for sensitivity analysis, at their initially estimated values or at the preloaded RESRAD-OFFSITE values if initial estimates have not yet been developed for them; the second with a higher value for the input selected for sensitivity analysis while all of the other inputs are at the value used for the first simulation; and the third with a lower value for the input selected for sensitivity analysis while all of the other inputs are at the value used for the first simulation. The differences in the predicted dose among the three simulations are directly attributable to the three different values used for the selected input in the simulations. If the higher and lower values used in the second and third simulation for the input selected for sensitivity analysis are chosen to represent the probable range of that input, then the difference between the doses predicted for the two simulations is a measure of the influence of that input on dose. When an input is selected for sensitivity analysis (using the F9 key for example), the set sensitivity analysis range pops up as shown in Figure 4.3. The user can specify the factor (1.1 in Figure 4.3), which determines the lower and higher values to be used for that input in the two sensitivity analysis simulations. The lower value (181.81 in Figure 4.3) is obtained by dividing the base value, which is the value used for the input in the first simulation (200 in Figure 4.3), by the specified factor. The higher value (220 in Figure 4.3) is obtained by multiplying the base value by the specified factor. Factors of $1.1,1.25$, and 1.5 were specified to simulate the $10 \%, 25 \%$, and $50 \%$ ranges on either side of the base value. These factors would have produced the desired values at the upper end, but the lower values would have been approximately $9 \%, 20 \%$, and $33 \%$ below the base value instead of the desired $10 \%, 25 \%$, and $50 \%$; however, this slate of values would not affect the conclusions. The lower and higher (or upper) values that will be used in the two sensitivity analysis simulations are displayed on the Set Sensitivity Analysis Range form. 


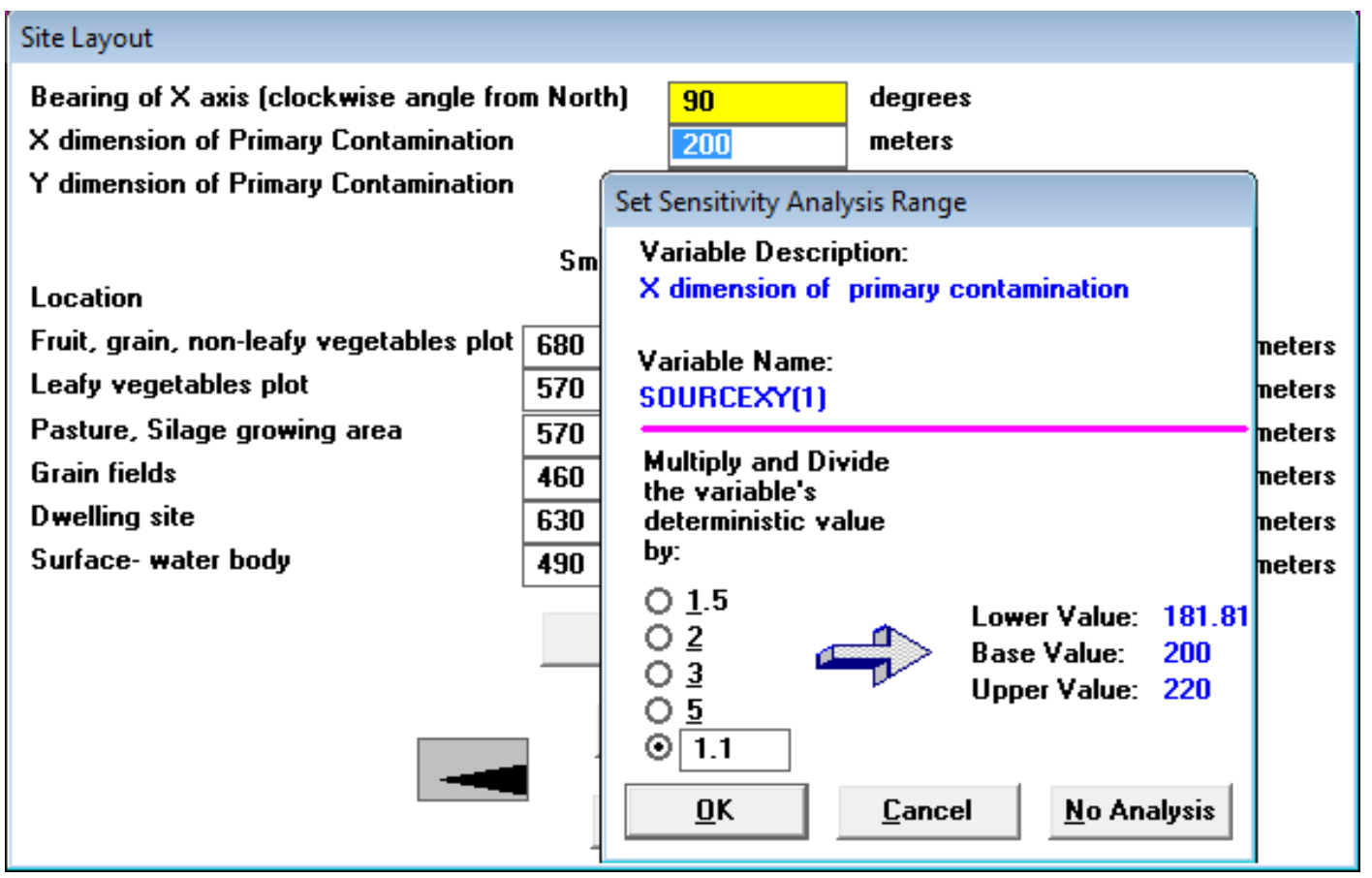

FIGURE 4.3 Specifying the Lower and Higher Values to be Used for an Input Selected for Sensitivity Analysis

The interface checks to ensure that the two values computed for the sensitivity analysis simulations are within the upper and lower bounds that are in the code. If either the lower or higher values, computed by using the specified factor and the base value, violate the lower or upper bounds in the code for that input, another form pops up allowing the user to make a choice that satisfies the bounds, as shown in Figure 4.4.

Up to 25 inputs can be selected for sensitivity analysis in an input file. When this file is executed by the code, it first performs a simulation with all of the inputs set to the base value. It then performs a pair of simulations for each input selected for sensitivity analysis. An input file was created by using the data set out in Section 4.1 for the uniform release option. Twenty-five of these inputs were selected for sensitivity analysis. The temporal sensitivity plots and the difference between the peak doses for the pair of sensitivity simulations for each input selected are shown in Figures 4.5 through 4.29. The figures are arranged in decreasing order of influence based on the range of the peak dose. A second input file with 25 other inputs selected for sensitivity analysis was also created. Three of those plots are shown in Figures 4.30, 4.31, and 4.32. There are at least 224 inputs in this example scenario that are candidates for sensitivity analysis, so 9 input files would be needed to perform sensitivity analysis on all of them. Figures 4.7, 4.33, and 4.34 illustrate that sensitivity analysis can be performed on all three inputs that characterize the release mechanisms available in RESRAD-OFFSITE. 


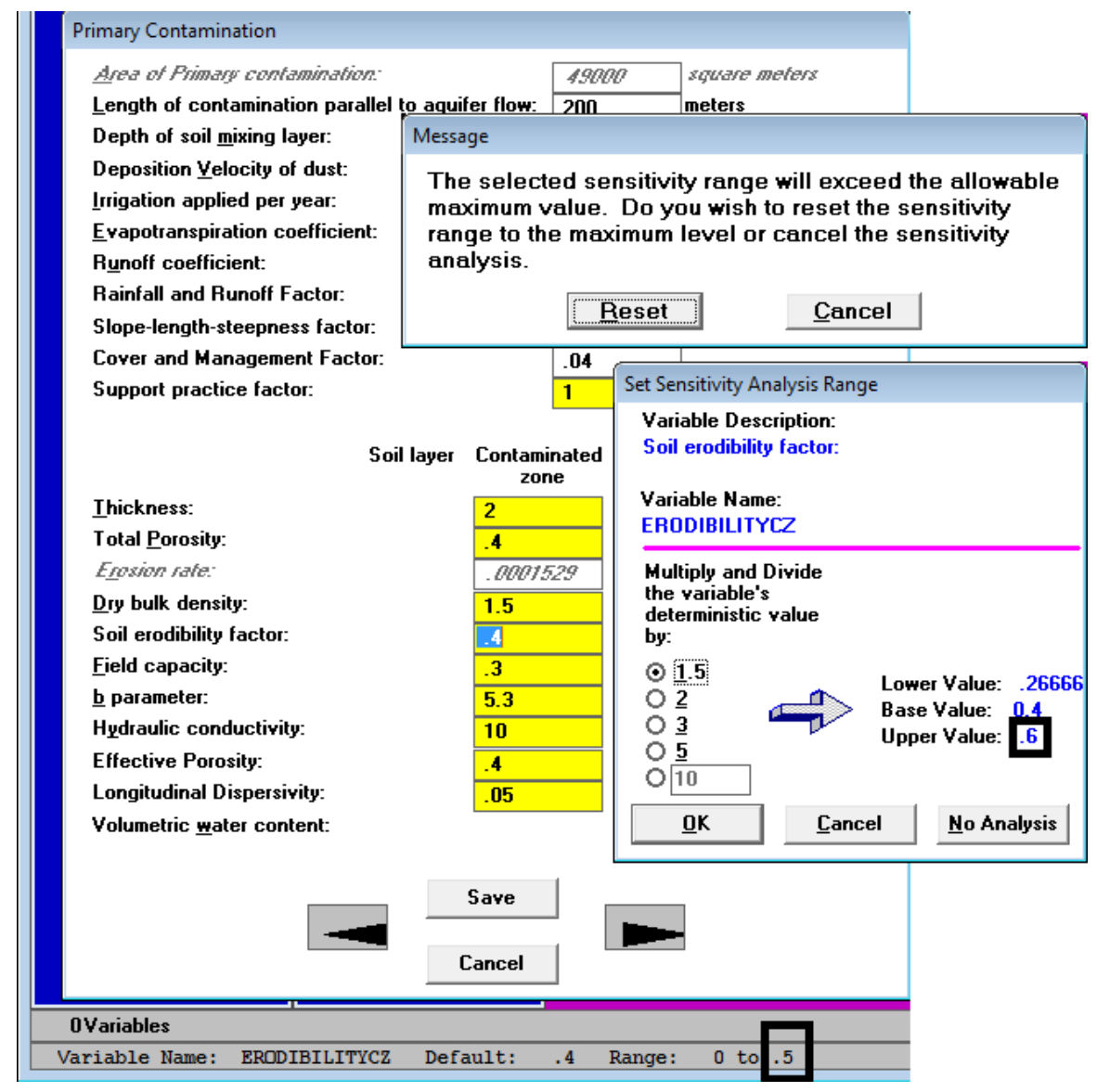

\section{FIGURE 4.4 Resetting the Sensitivity Analysis Range to Avoid Exceeding the Bounds on the Inputs}

\subsubsection{Advantages and Disadvantages of Three-point Single-input Sensitivity Analysis}

This sensitivity analysis is very easy to understand. Two simulations are performed for each input selected for sensitivity analysis; only the value of the selected input changes between the two simulations, whereas the values of all of the other inputs remain the same. The difference between the predicted doses for the two simulations is the direct result of the change in the value of the selected input. If the two values specified for the selected input are the limits of the likely range of that input for this scenario, the difference in the peak doses is a measure of the uncertainty in the peak dose due to the uncertainty in the value of the selected input. The influence of the selected input on dose can be seen from the pair of temporal dose curves from the two simulations plus the curve from the initial base value simulation. More effort is required to obtain a quantitative measure of this influence; specifically, users must view data and 
manually find the peak doses for the two simulations or export the data to a spreadsheet and then find the peaks.

The process of viewing the plots and then computing the difference between the peak doses of each pair of simulations is tedious when there are a large number of inputs as in this example scenario and therefore limits the usefulness of this method. This method would be easier to use if the code were to compute the differences between the peak doses of each pair of simulations and were to produce an ordered list of inputs based on that difference. If this automated process of ranking the inputs by their influence on the peak dose is implemented in the code, then it would also be necessary to remove the limit on the number of inputs that can be selected for three-point single-input sensitivity analysis.

The fact that only the value of the selected input is changed in the pair of simulations allows the effect of that input to be seen easily in the temporal plots. This clarity turns into a disadvantage, however, when there is some relationship between the inputs or if there is interaction between the inputs. Figures 4.30 and 4.31 illustrate the influence of the total porosity and the effective porosity of the soil in the primary contamination on dose. According to Figure 4.30, the peak dose decreases as the effective porosity in the primary contamination increases. Figure 4.31 shows that the peak dose increases as the total porosity in the primary contamination increases. The influence of the two inputs appears to be similar in magnitude but opposite in direction. Recall that the effective porosity is the part of the total porosity of soil that is effective in the transport of water in soil. If there is uncertainty in these two porosities, it is reasonable to assume that the uncertainties will be correlated. In other words, if the total porosity were to be higher than the initially assigned value, it is likely that the effective porosity will also be higher than the initially assigned value. So when we change total porosity for the two simulations, we should also change effective porosity; they will have opposing effects on the peak dose, and the net result is likely to be a small change in the peak dose. However, we can only change the value of a single input in this sensitivity analysis. Sections C.3.2 and C.3.3 of Appendix C illustrate the interaction between inputs: changes to input $A$ can cause a large change in the dose for certain values of inputs $B, C$, and $D$; whereas the same change in input $A$ will have very little effect on the doses for other values of inputs $B, C$, and $D$. Thus, if there is uncertainty in inputs $A, B, C$, and $D$, then determining the effect of changes in the value of input $A$ at a single combination of values of inputs $B, C$, and $D$ only provides a partial picture of the effect of input $A$ on the dose. 


\subsubsection{Conclusion about the Use of 3-point Single-input Sensitivity Analysis}

This sensitivity analysis is easy to understand and requires a small number of simulations, one more than twice the number of inputs being studied. Even though it does not consider relationships between inputs and the interactions between inputs, it is a useful tool especially in the preliminary stages of a sensitivity analysis when there might not be very much information about the relationships between the inputs. The main limitation to the usefulness of this method is the effort needed to view each plot in turn to find inputs that have an influence on the dose and then to calculate the difference between the peak doses in each pair of simulations to quantify the influence. If the following conditions were met, namely, that (1) there were no limits on the number of inputs that can be selected for sensitivity analysis, and (2) if the code were to compute the difference in the peak dose between pairs of simulations and (3) were to produce an ordered list of inputs on the basis of their influence, the three-point, single-input sensitivity analysis could be a more useful tool to identify the influential inputs in RESRAD-OFFSITE. 


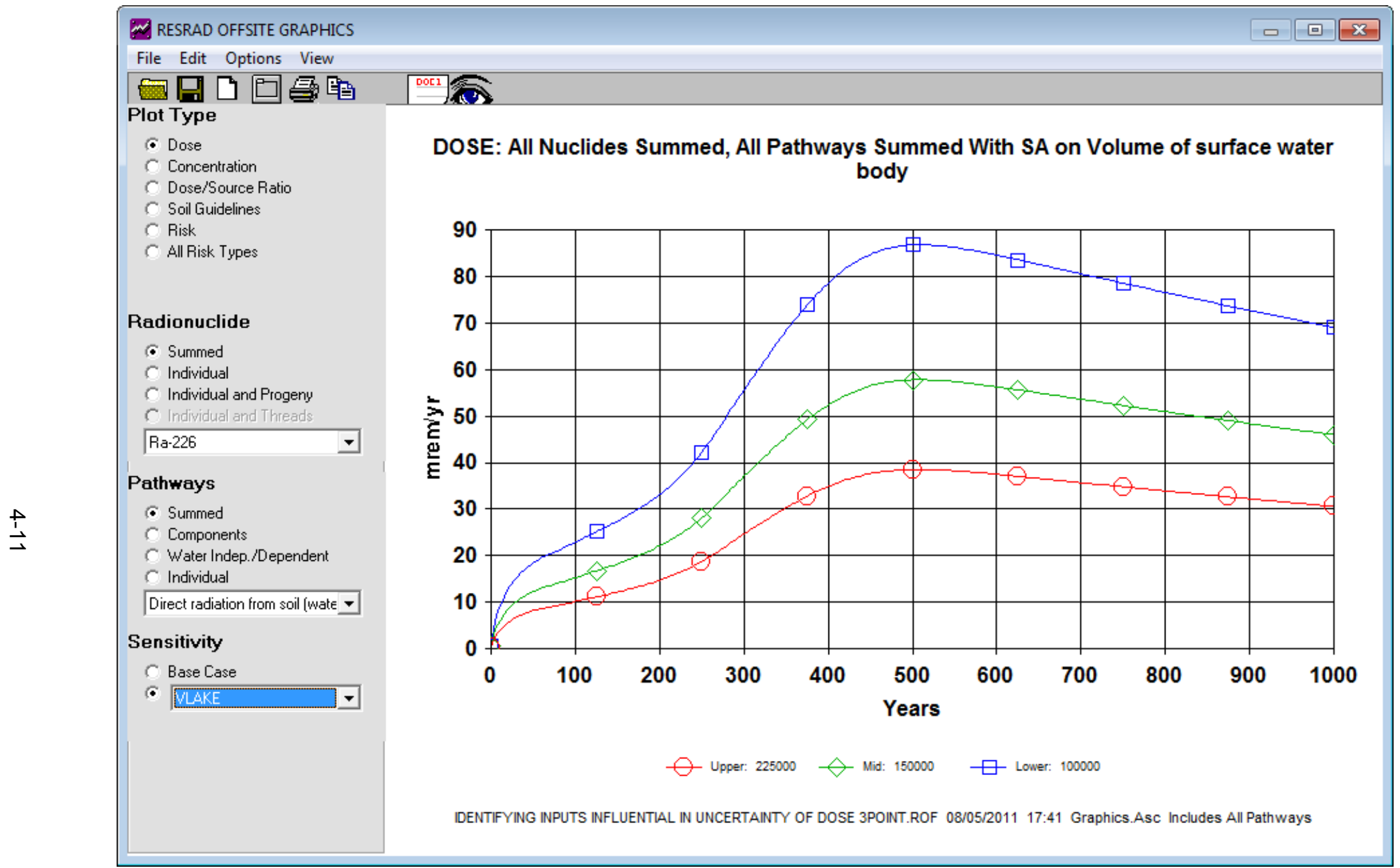

FIGURE 4.5 Temporal Variation of Dose with the Volume of the Surface Water Body Showing a Range of $48.3 \mathrm{mrem} / \mathrm{year}$ for the Peak Dose from 38.5 to $86.8 \mathrm{mrem} / \mathrm{year}$ 


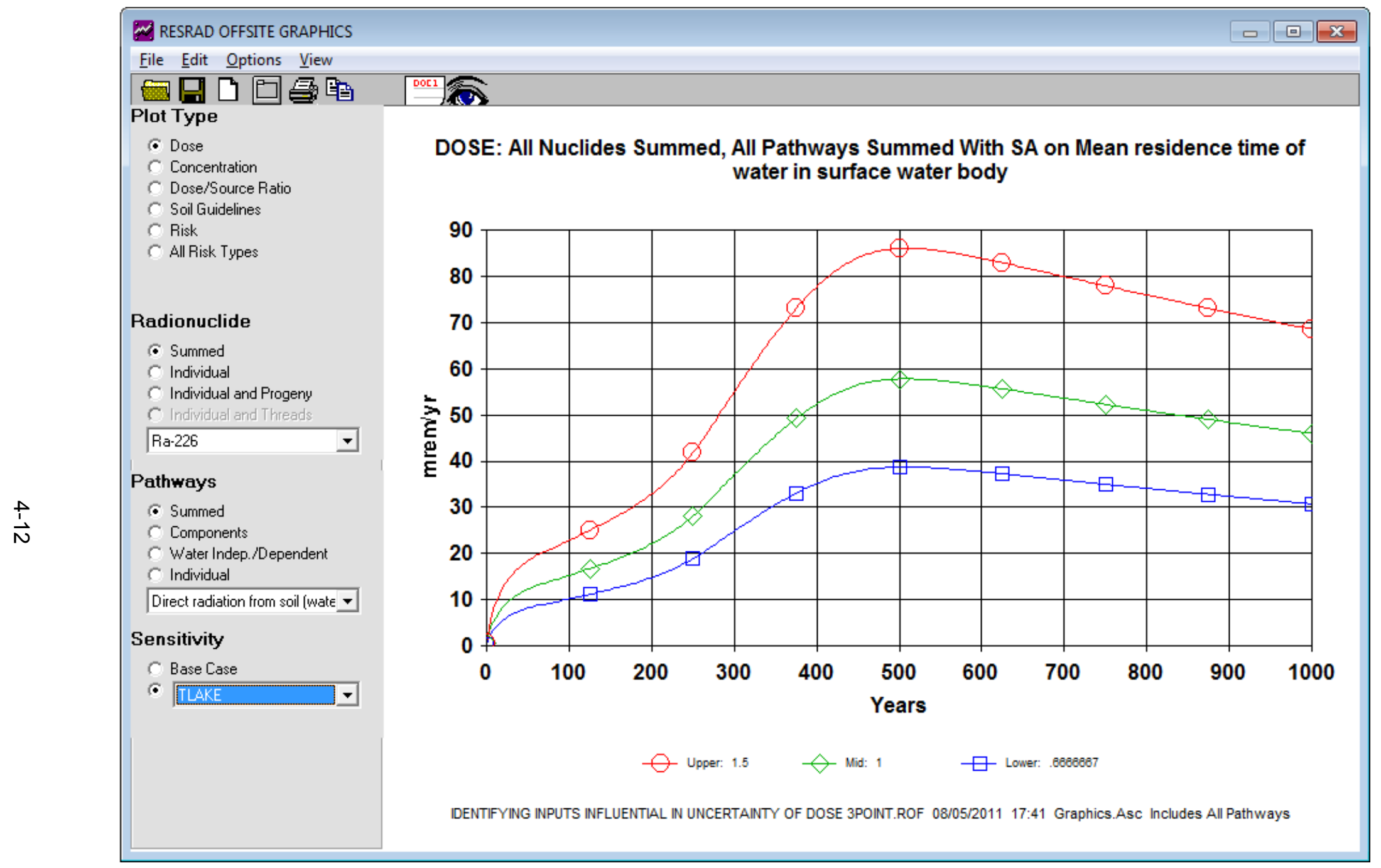

FIGURE 4.6 Temporal Variation of Dose with the Mean Residence Time of Water in the Surface Water Body Showing a Range of $\mathbf{4 7 . 4}$ mrem/year for the Peak Dose from 38.7 to $86.1 \mathrm{mrem} / \mathrm{year}$ 


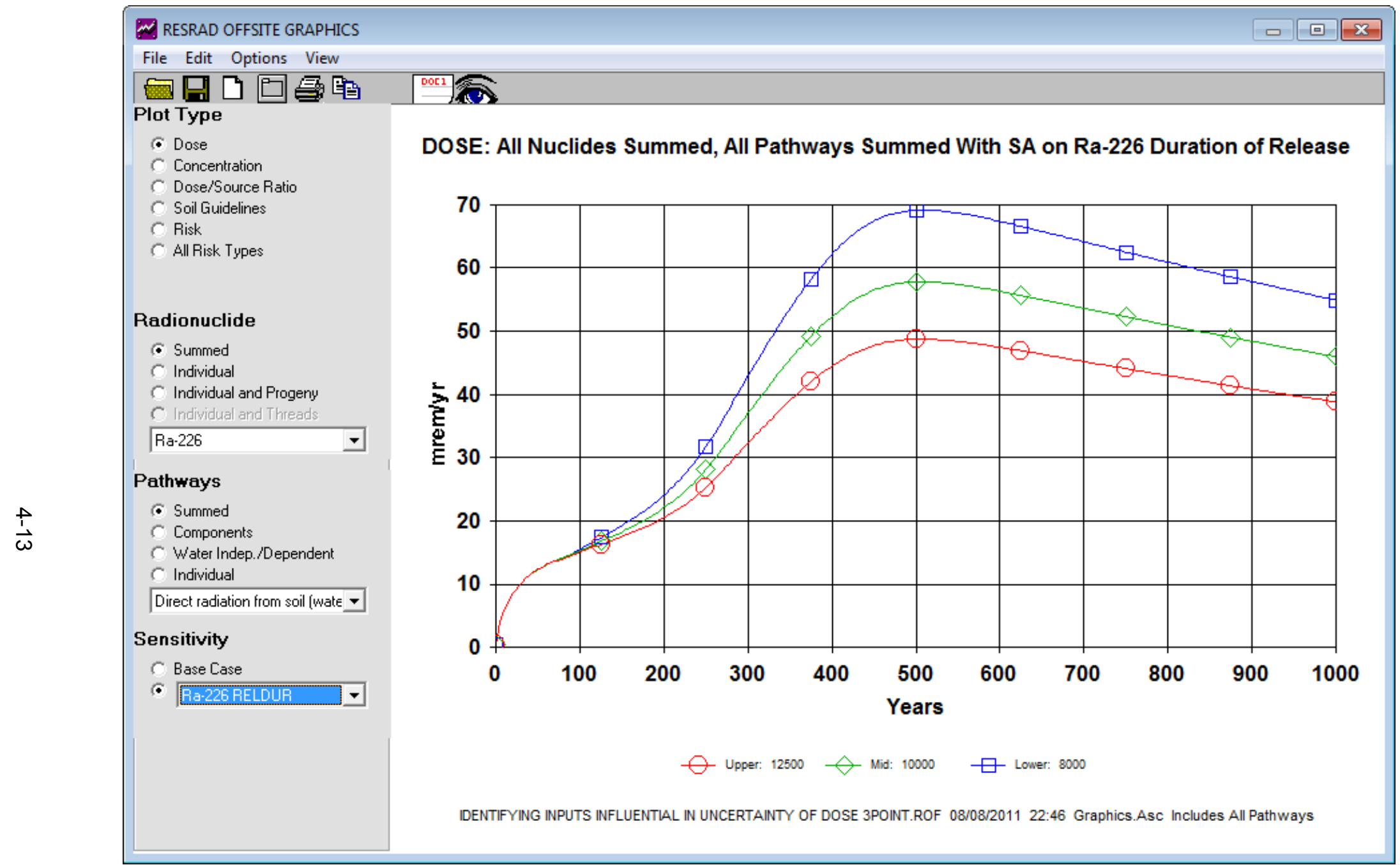

FIGURE 4.7 Temporal Variation of Dose with the Duration of Release of ${ }^{226} \mathrm{Ra}$ Showing a Range of $20.4 \mathrm{mrem} / \mathrm{year}$ for the Peak Dose from 48.8 to $69.2 \mathrm{mrem} / \mathrm{year}$ 


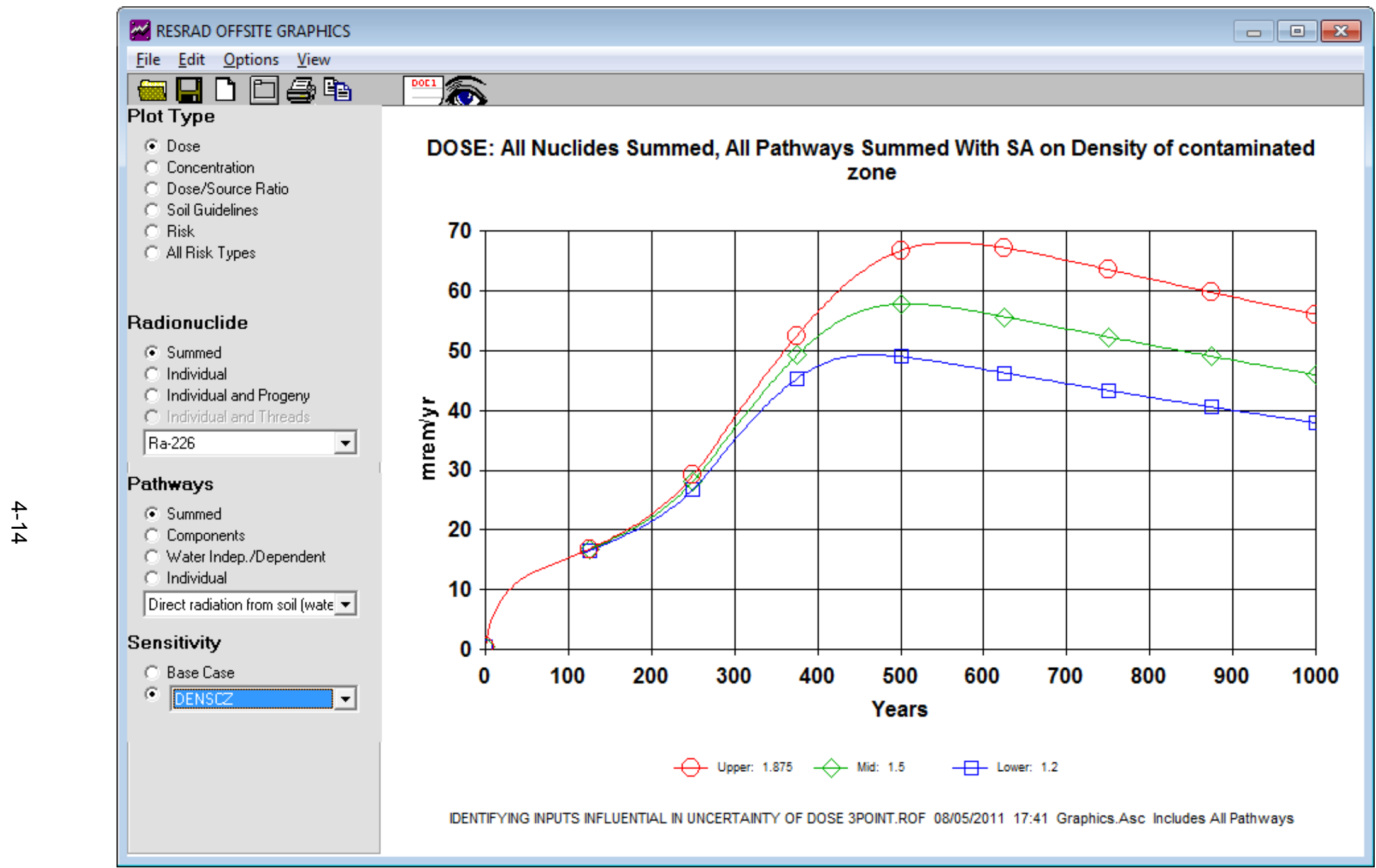

FIGURE 4.8 Temporal Variation of Dose with the Density of the Primary Contamination Showing a Range of $18.8 \mathrm{mrem} / \mathrm{year}$ for the Peak Dose from 49.3 to $68.1 \mathrm{mrem} / \mathrm{year}$ 


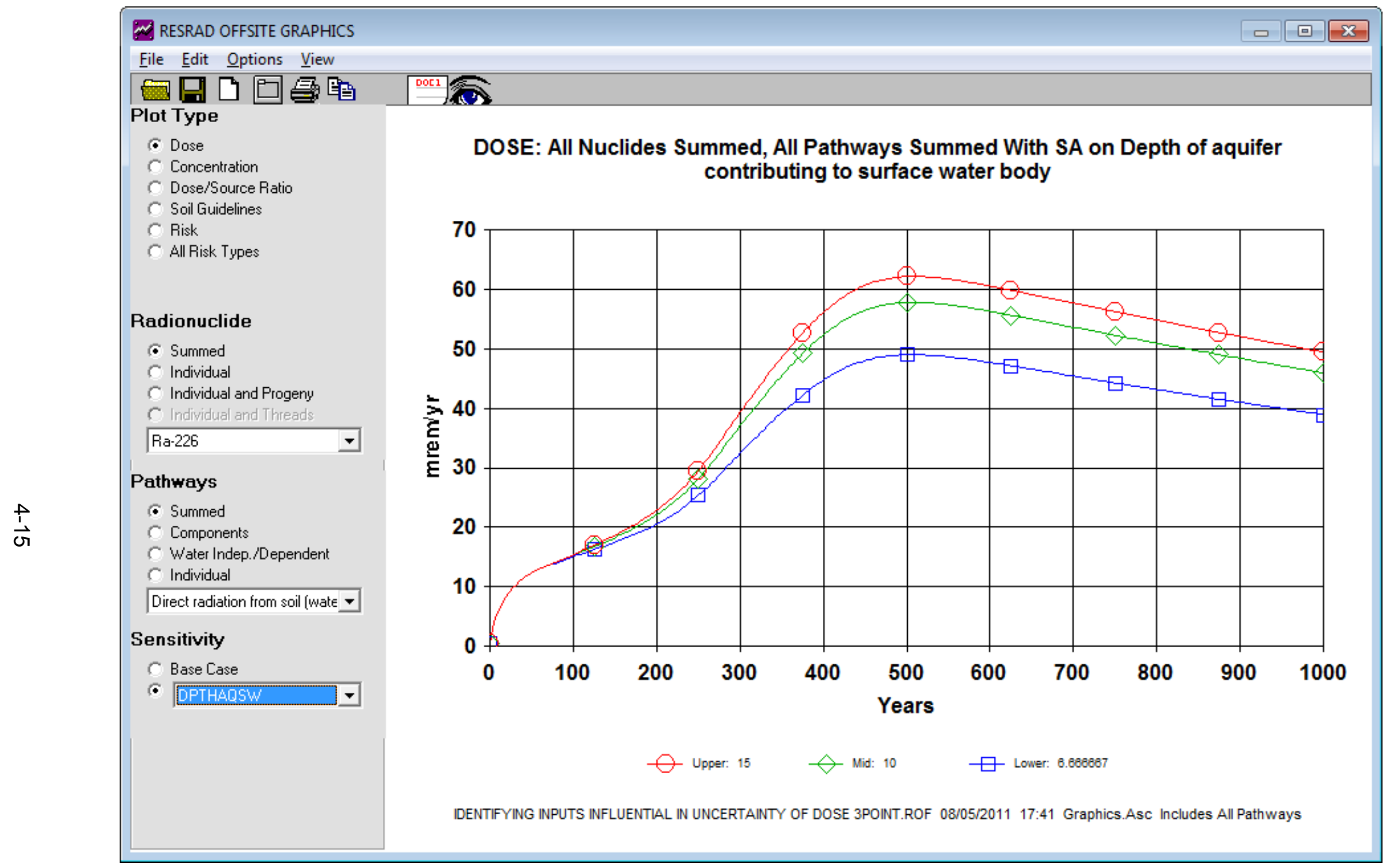

FIGURE 4.9 Temporal Variation of Dose with the Depth of Aquifer Contributing to the Surface Water Body Showing a Range of $\mathbf{1 3 . 2} \mathrm{mrem} / \mathrm{year}$ for the Peak Dose from 49.0 to $62.2 \mathrm{mrem} / \mathrm{year}$ 


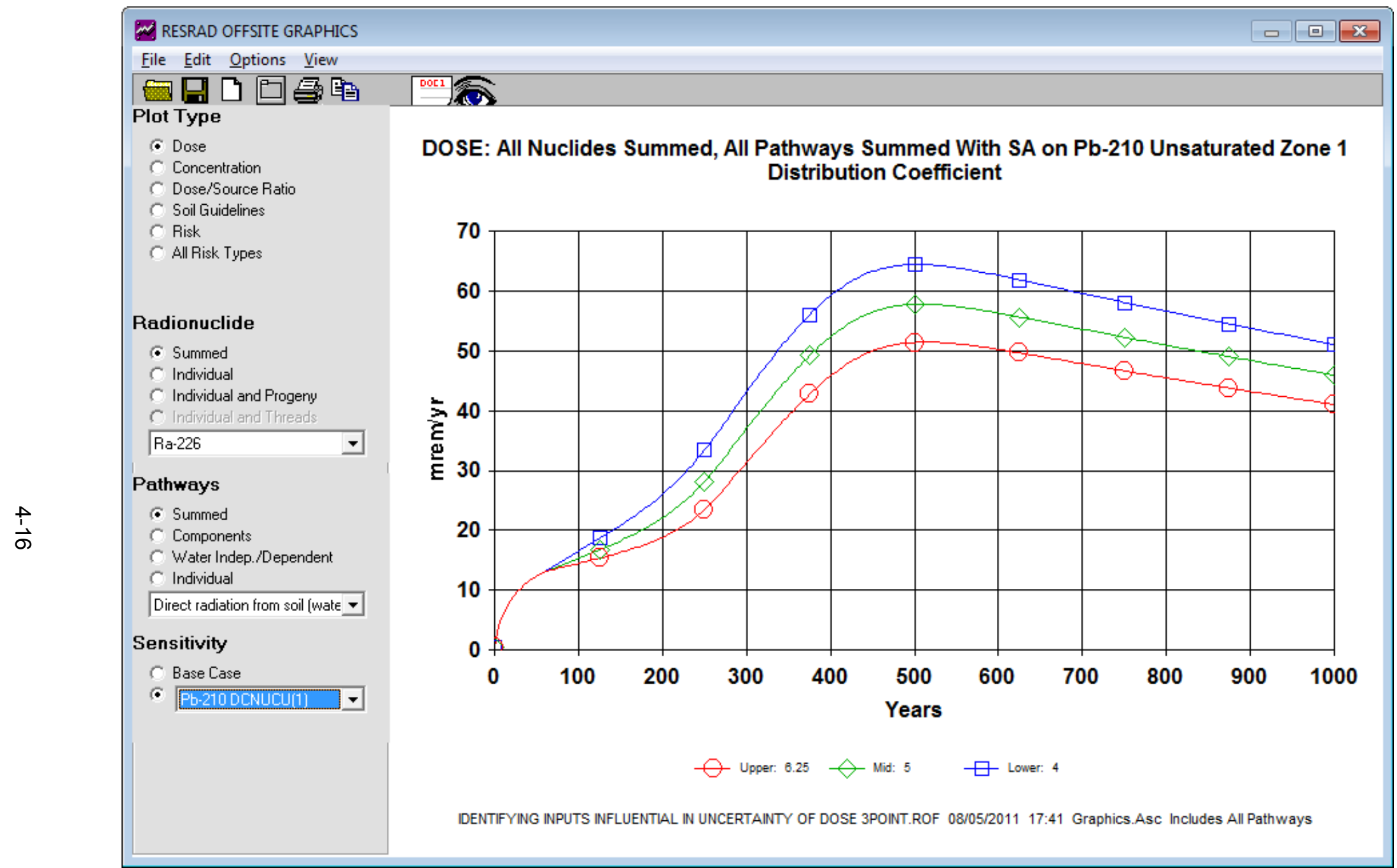

FIGURE 4.10 Temporal Variation of Dose with the Distribution Coefficient of ${ }^{210} \mathrm{~Pb}$ in the First Unsaturated Zone Showing a Range of $\mathbf{1 2 . 9}$ mrem/year for the Peak Dose from 51.5 to 64.4 mrem/year 


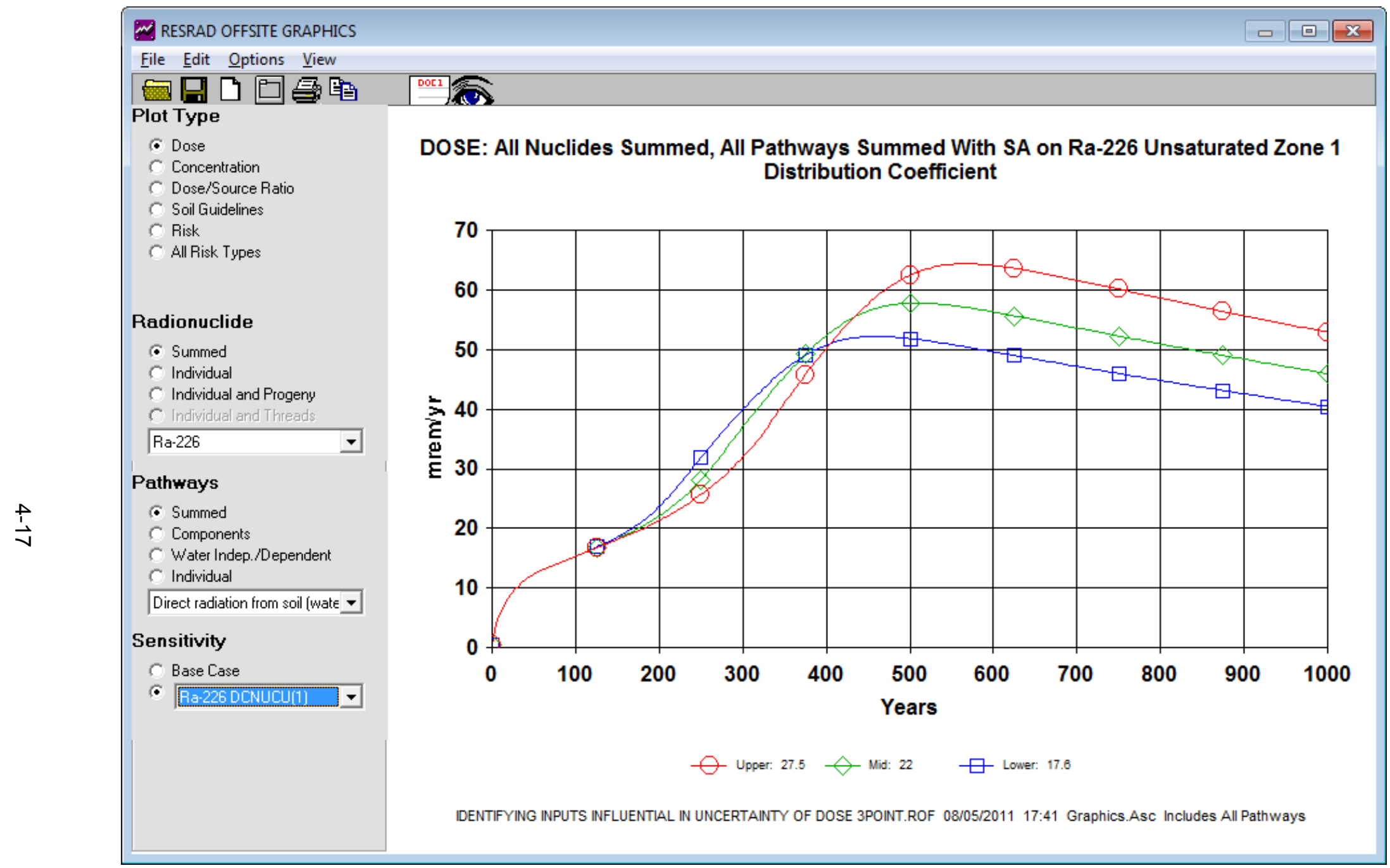

FIGURE 4.11 Temporal Variation of Dose with the Distribution Coefficient of ${ }^{226} \mathrm{Ra}$ in the First Unsaturated Zone Showing a Range of 12.2 mrem/year for the Peak Dose from 52.2 to $64.4 \mathrm{mrem} / \mathrm{year}$ 


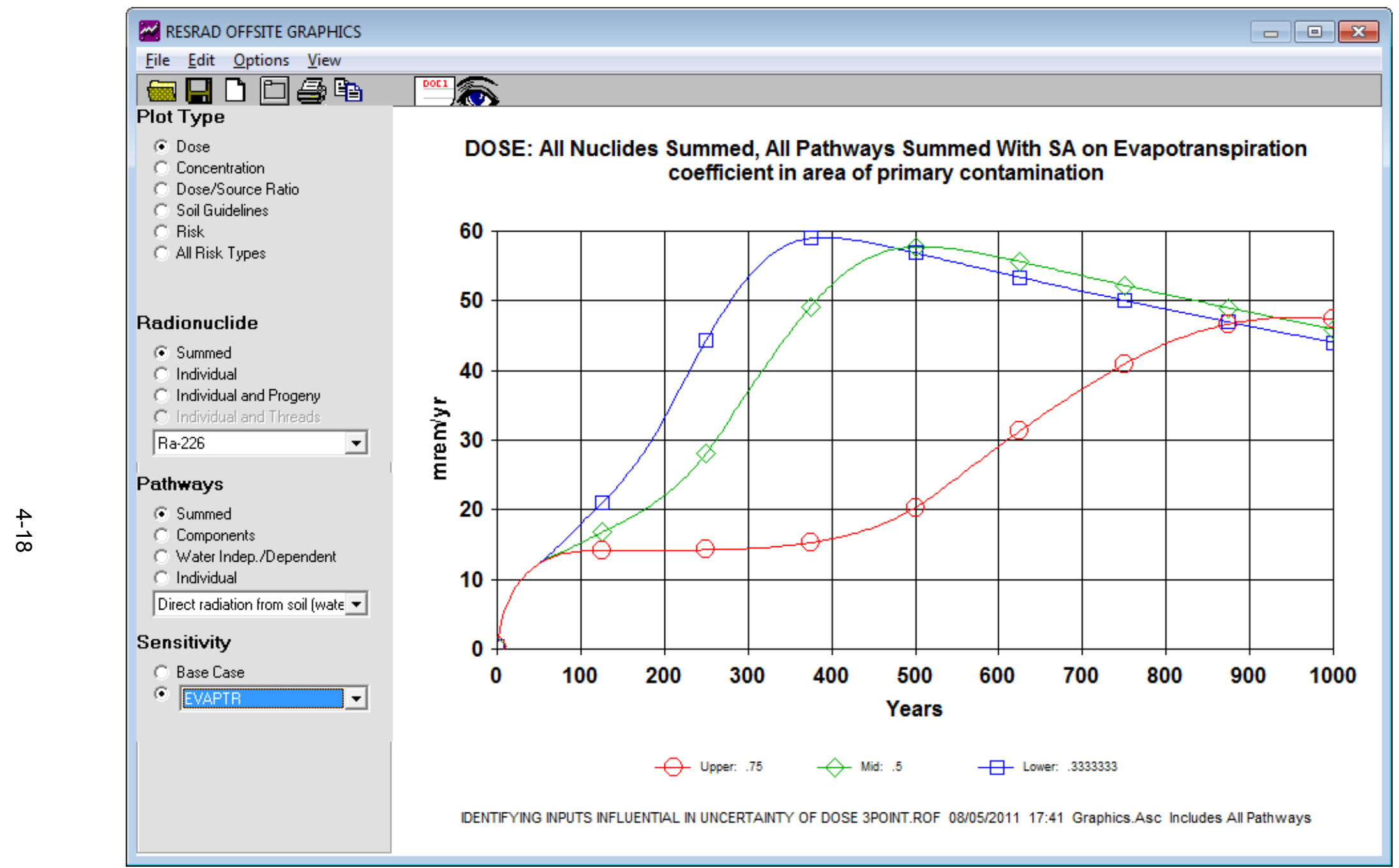

FIGURE 4.12 Temporal Variation of Dose with the Evapotranspiration Coefficient Showing a Range of $11.5 \mathrm{mrem} / \mathrm{year}$ for the Peak Dose from 47.6 to $59.1 \mathrm{mrem} / \mathrm{year}$ 


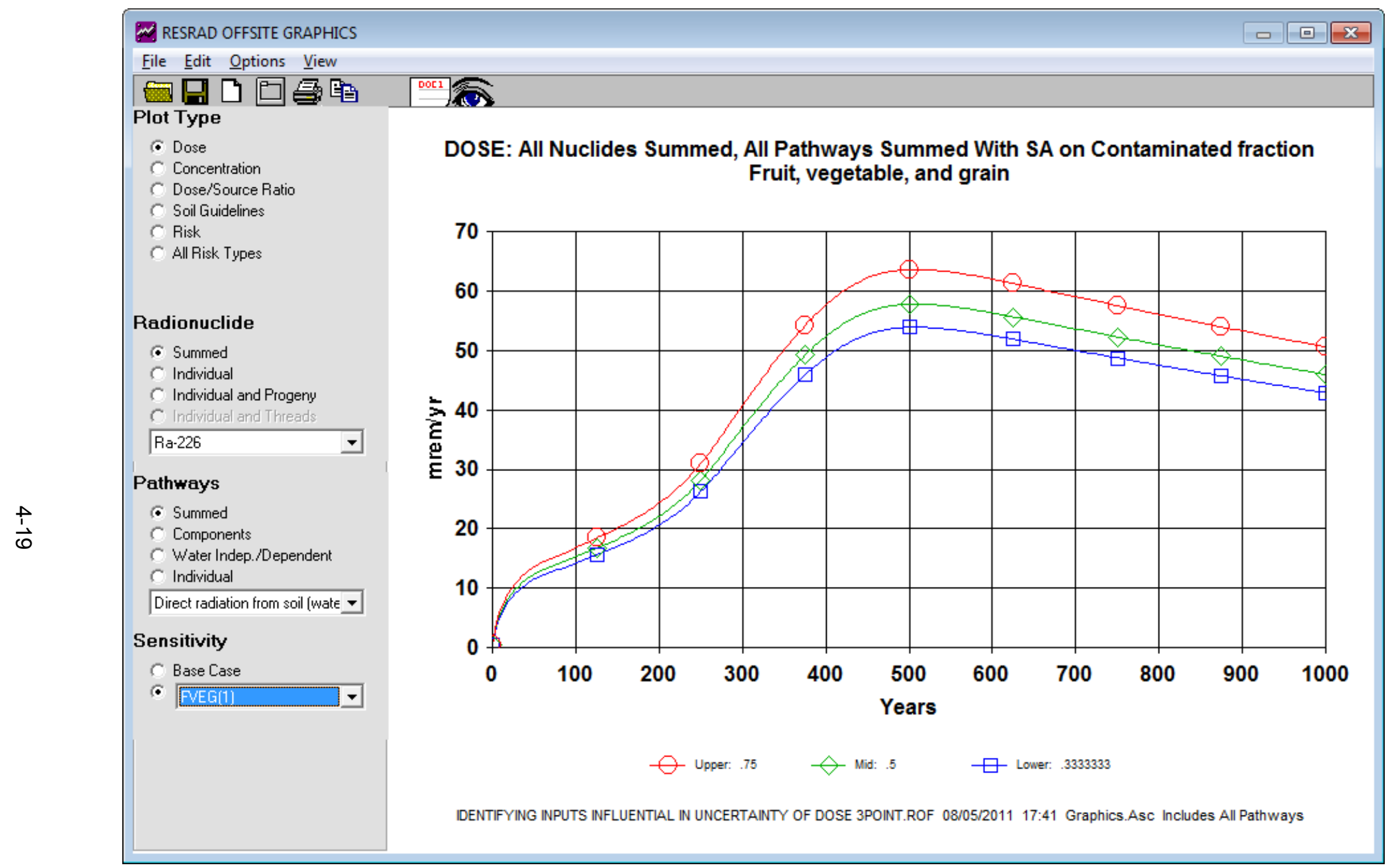

FIGURE 4.13 Temporal Variation of Dose with the Fraction of Fruit, Nonleafy Vegetables, and Grain from the Contaminated Region Showing a Range of $9.8 \mathrm{mrem} / \mathrm{year}$ for the Peak Dose from 53.9 to $63.7 \mathrm{mrem} / \mathrm{year}$ 


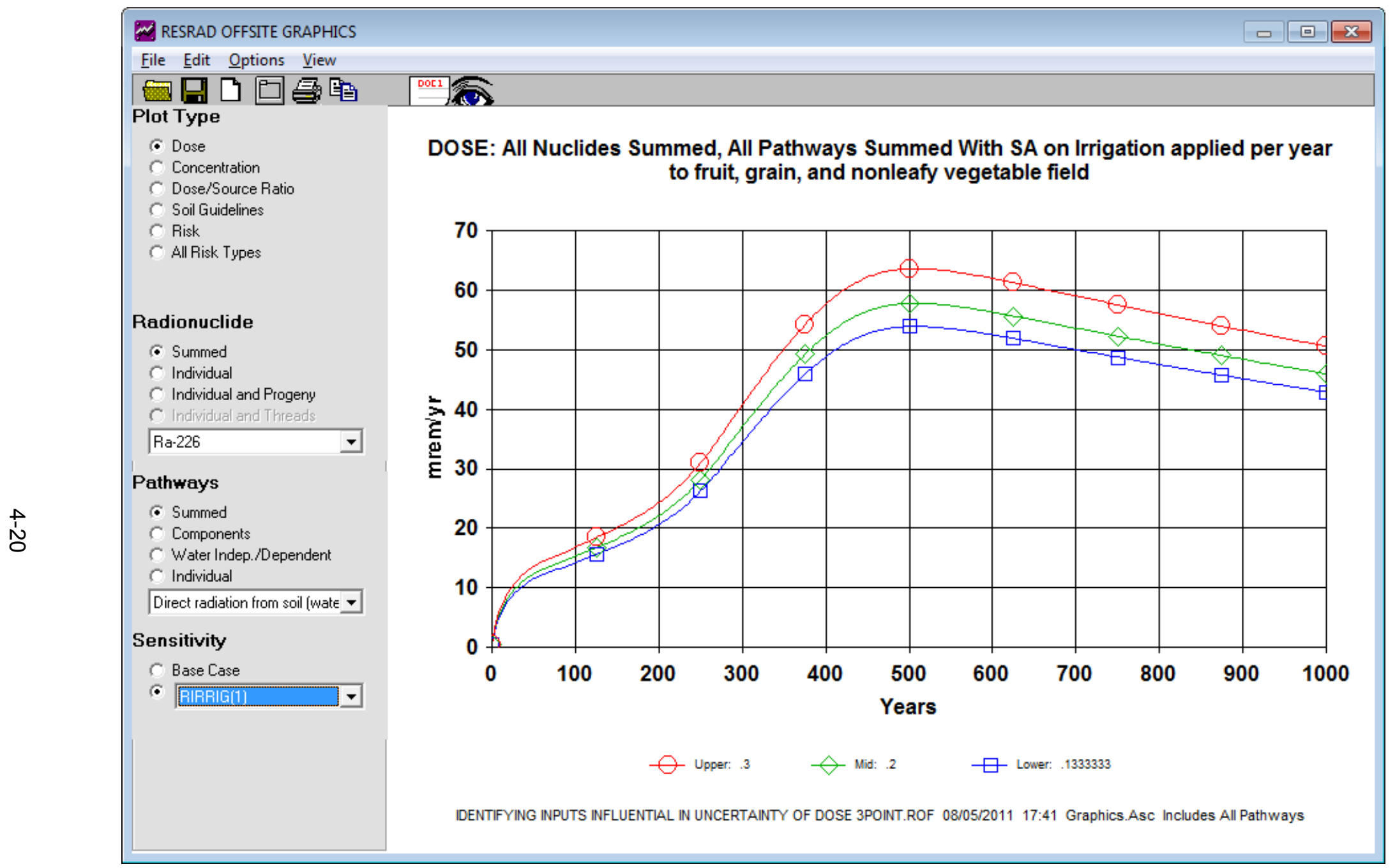

FIGURE 4.14 Temporal Variation of Dose with the Irrigation Applied per Year to the Fruit, Nonleafy Vegetables, and Grain Growing Areas Showing a Range of 9.7 mrem/year for the Peak Dose from 53.9 to $63.6 \mathrm{mrem} / \mathrm{year}$ 


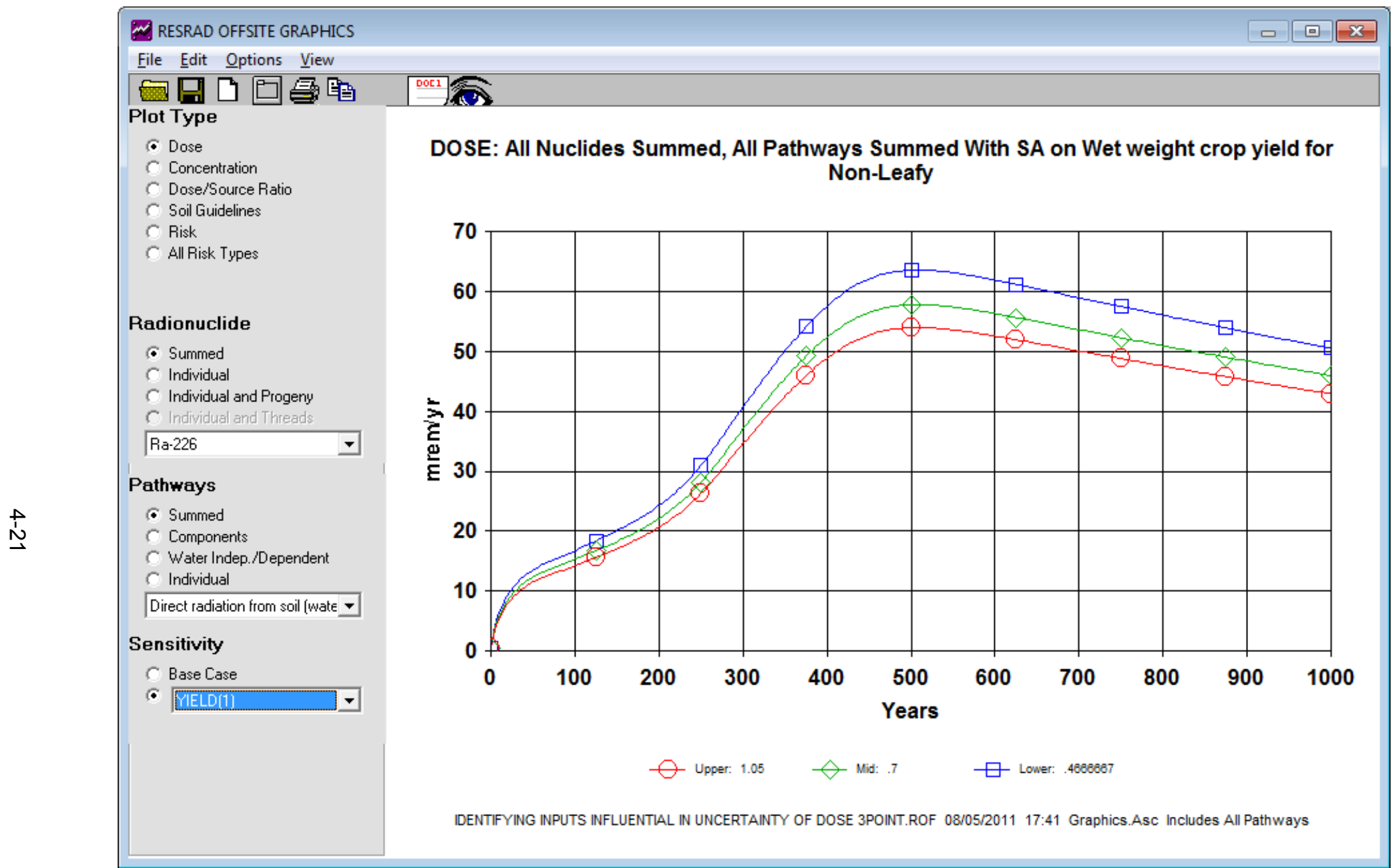

FIGURE 4.15 Temporal Variation of Dose with the Wet Weight Crop Yield of Fruit, Nonleafy Vegetables, and Grain in the Growing Areas Showing a Range of $9.6 \mathrm{mrem} / y e a r$ for the Peak Dose from 54.0 to $63.6 \mathrm{mrem} / \mathrm{year}$ 


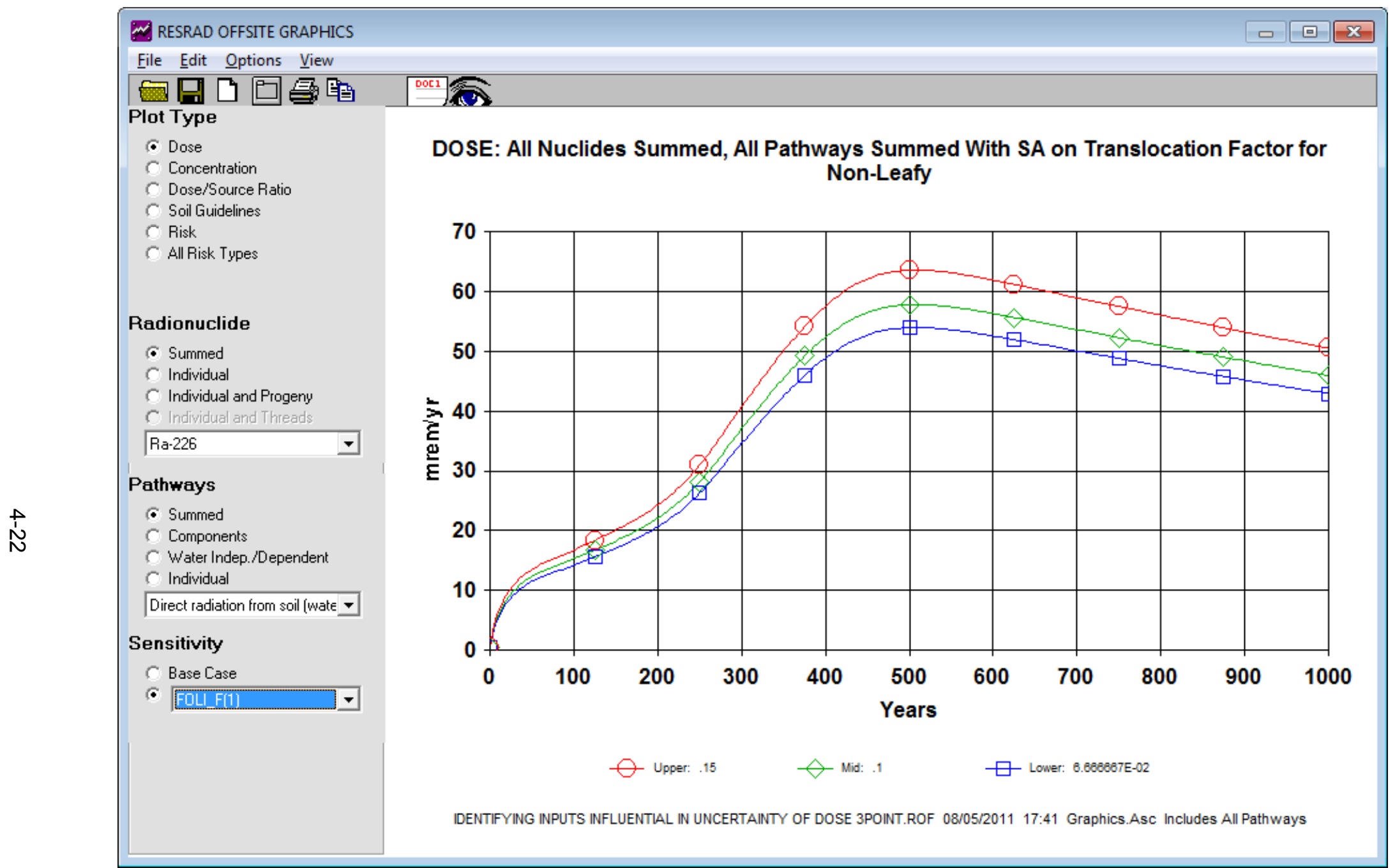

FIGURE 4.16 Temporal Variation of Dose with the Translocation Factor for Fruit, Nonleafy Vegetables, and Grain Showing a Range of 9.6 mrem/year for the Peak Dose from 54.0 to $63.6 \mathrm{mrem} / \mathrm{year}$ 


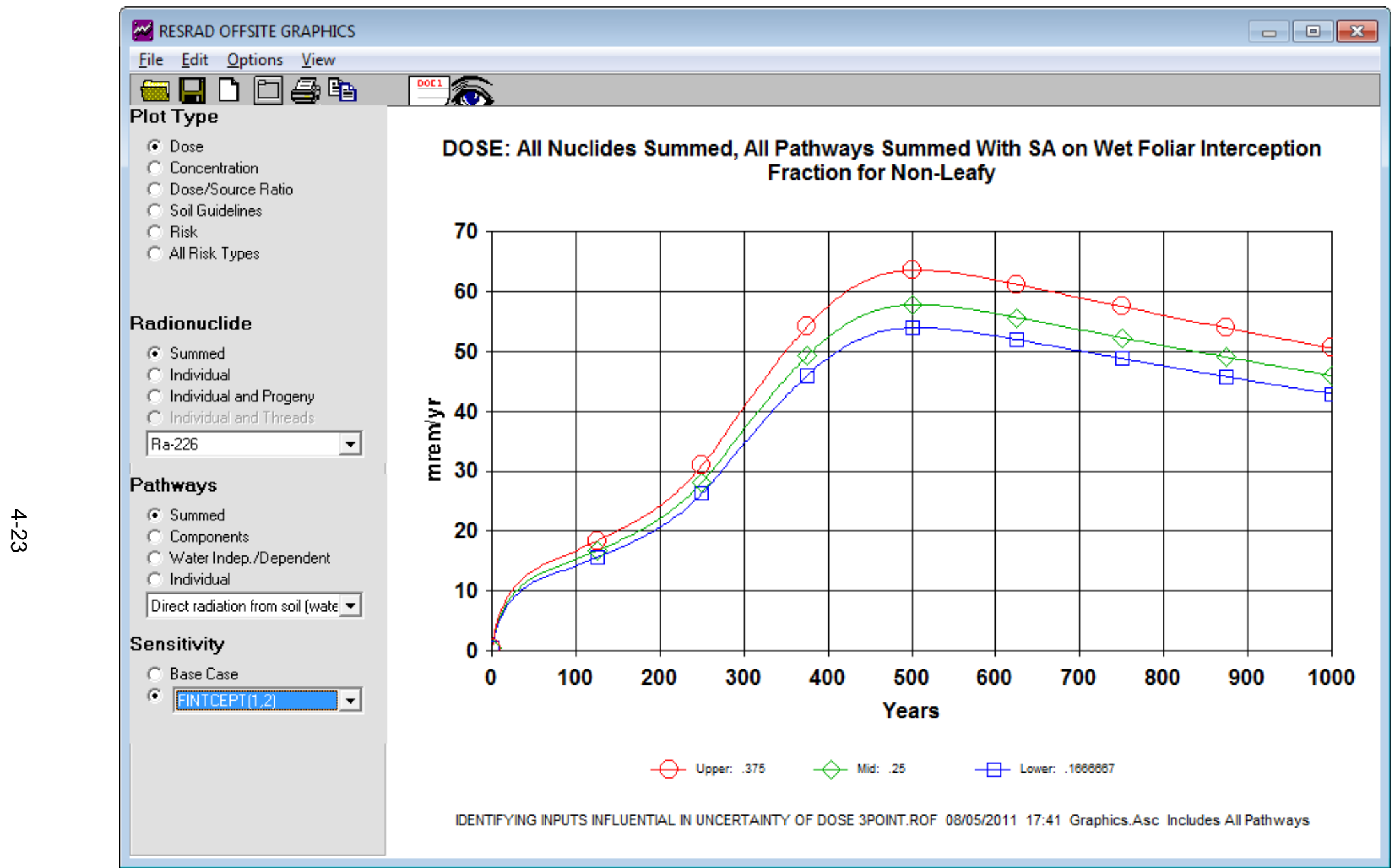

FIGURE 4.17 Temporal Variation of Dose with the Foliar Interception Factor for Irrigation in the Fruit, Nonleafy Vegetables, and Grain Growing Area Showing a Range of $9.6 \mathrm{mrem} / \mathrm{year}$ for the Peak Dose from 54.0 to $63.6 \mathrm{mrem} / \mathrm{year}$ 


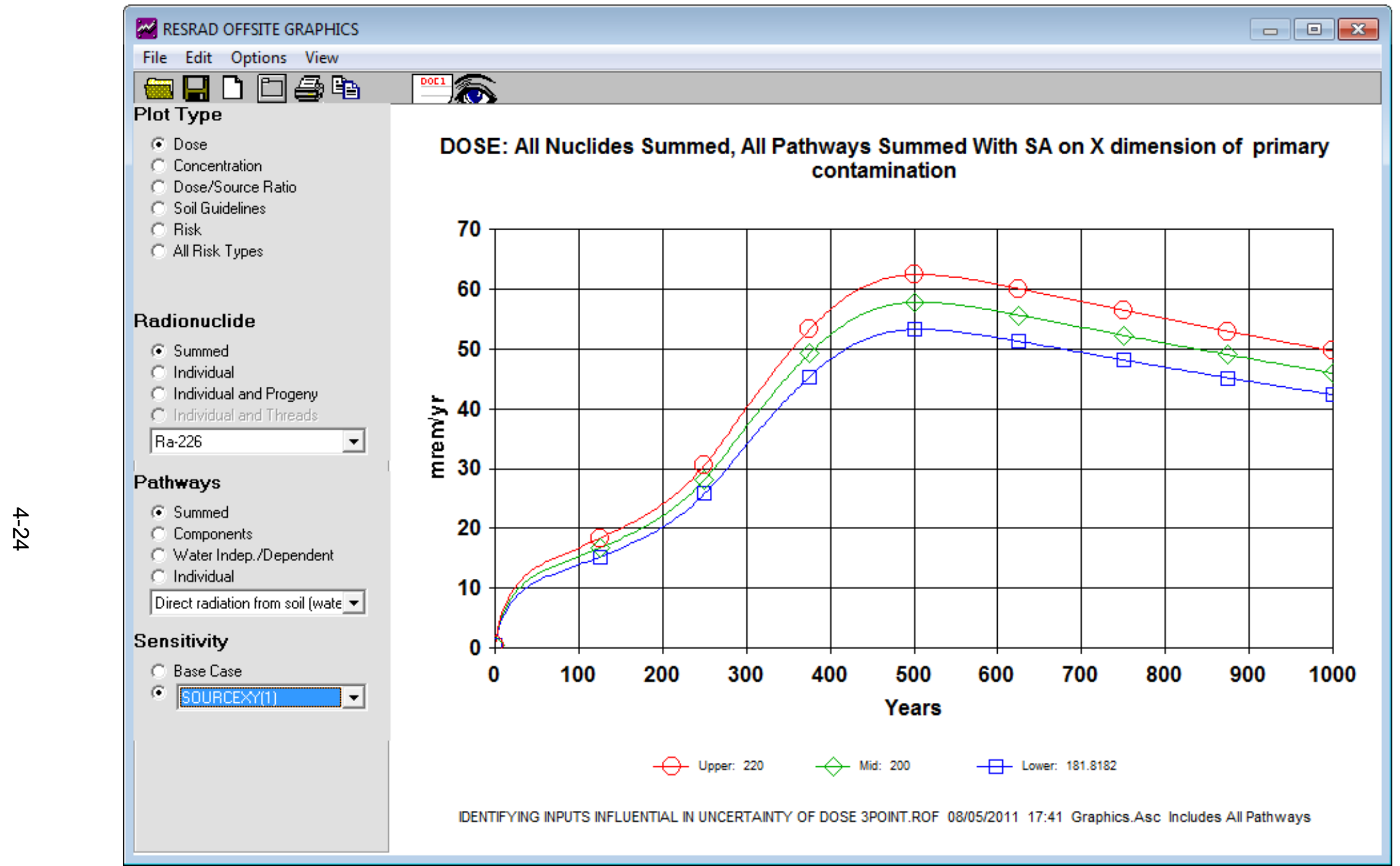

FIGURE 4.18 Temporal Variation of Dose with the $x$ Dimension of the Primary Contamination Showing a Range of $9.1 \mathrm{mrem} / \mathrm{year}$ for the Peak Dose from 53.3 to $62.4 \mathrm{mrem} / \mathrm{year}$ 


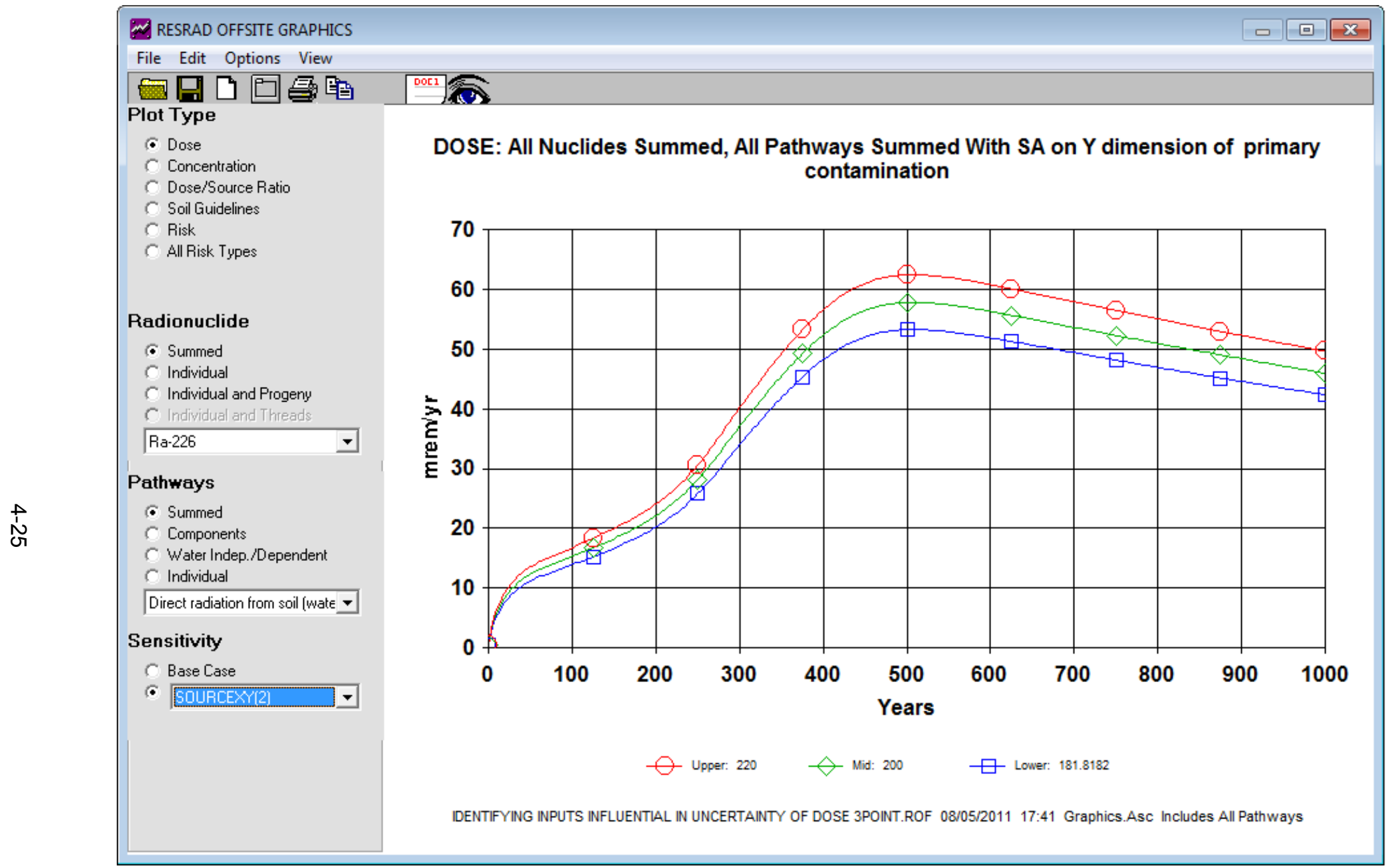

FIGURE 4.19 Temporal Variation of Dose with the $y$ Dimension of the Primary Contamination Showing a Range of $9.1 \mathrm{mrem} / \mathrm{year}$ for the Peak Dose from 53.3 to $62.4 \mathrm{mrem} / \mathrm{year}$ 


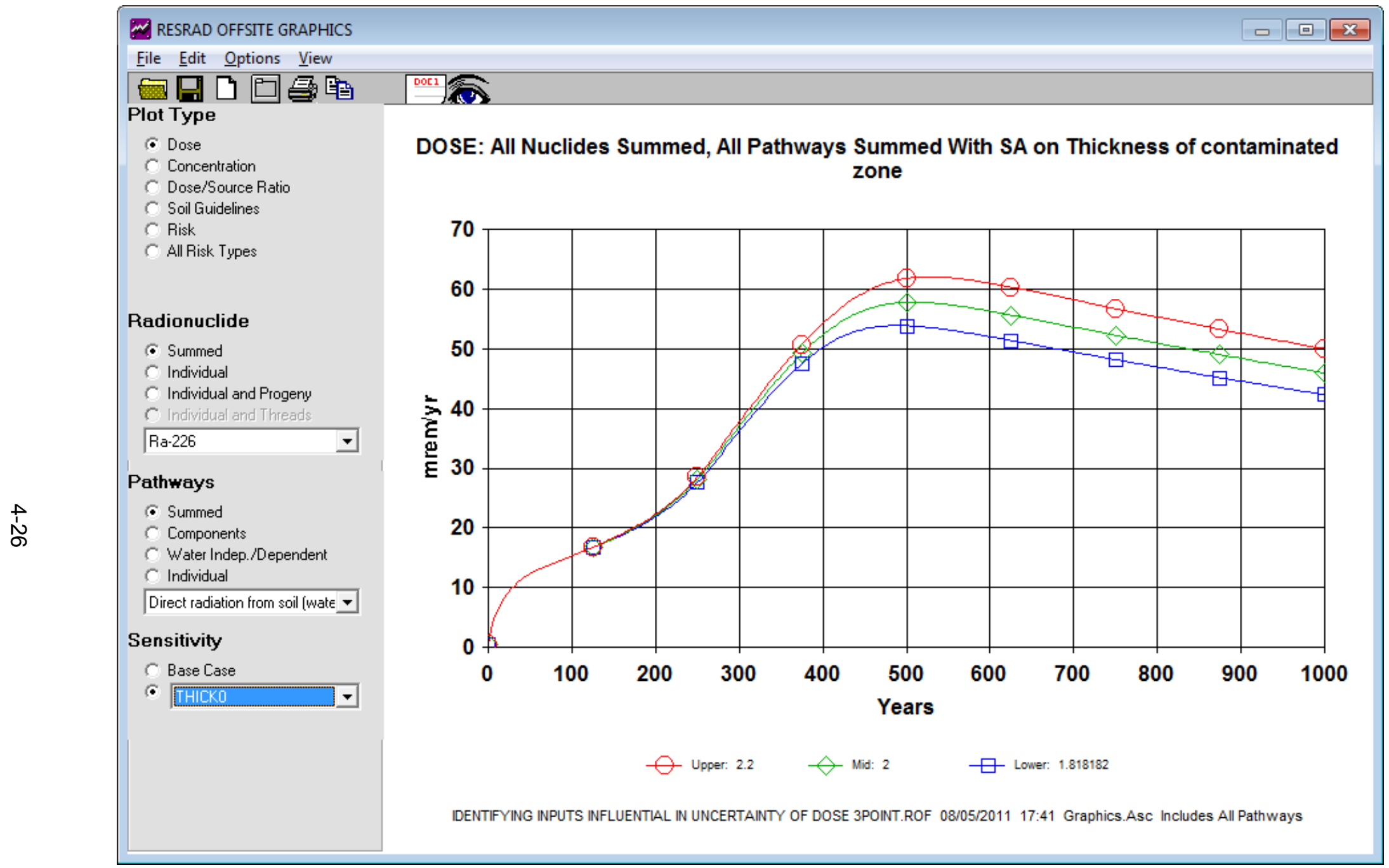

FIGURE 4.20 Temporal Variation of Dose with the Thickness of the Primary Contamination Showing a Range of $8.2 \mathrm{mrem} / \mathrm{year}$ for the Peak Dose from 53.9 to $62.1 \mathrm{mrem} / \mathrm{year}$ 


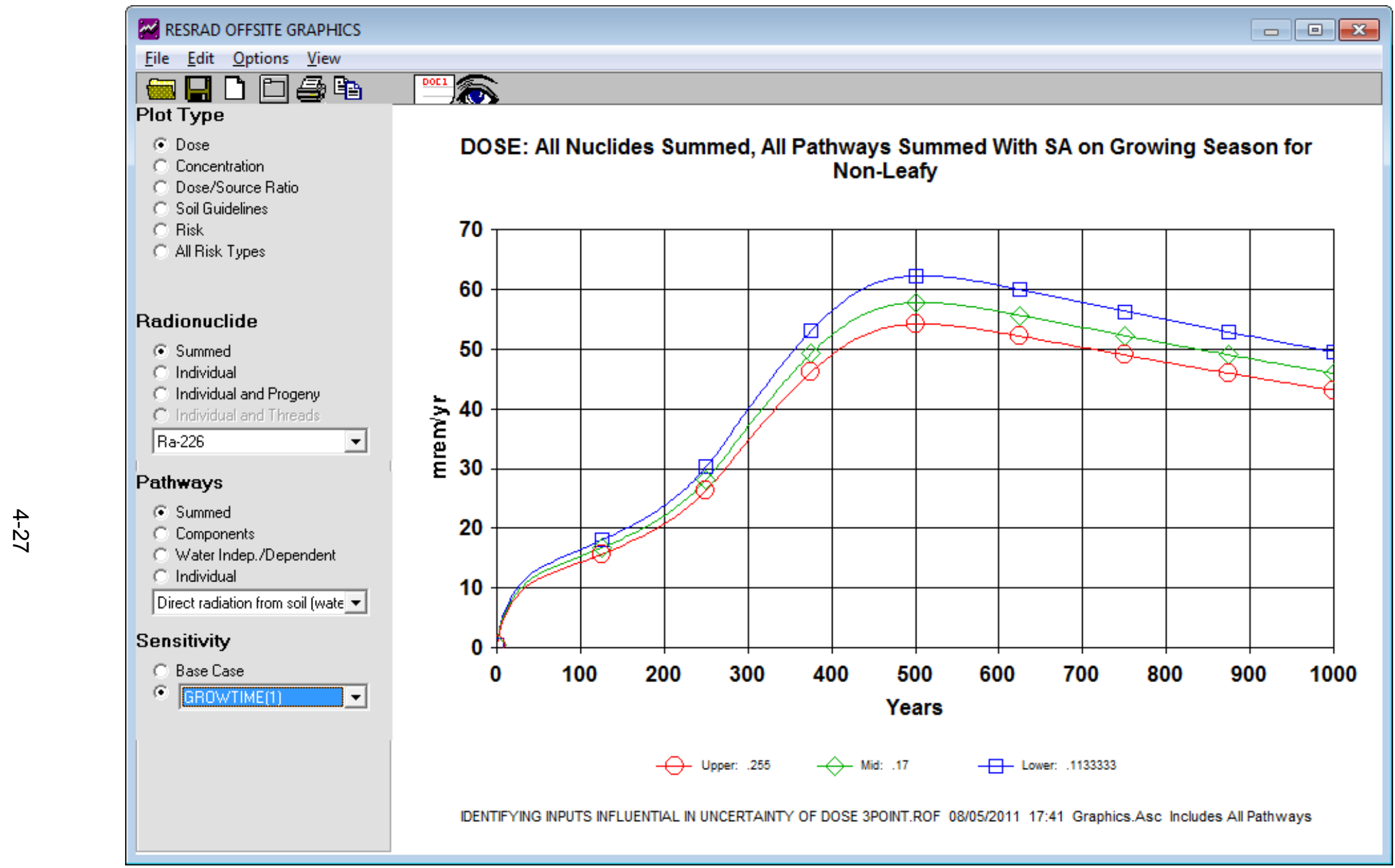

FIGURE 4.21 Temporal Variation of Dose with the Duration of the Growing Season of Fruit, Nonleafy Vegetables, and Grain Showing a Range of $8.1 \mathrm{mrem} / \mathrm{year}$ for the Peak Dose from 54.2 to $62.3 \mathrm{mrem} / \mathrm{year}$ 


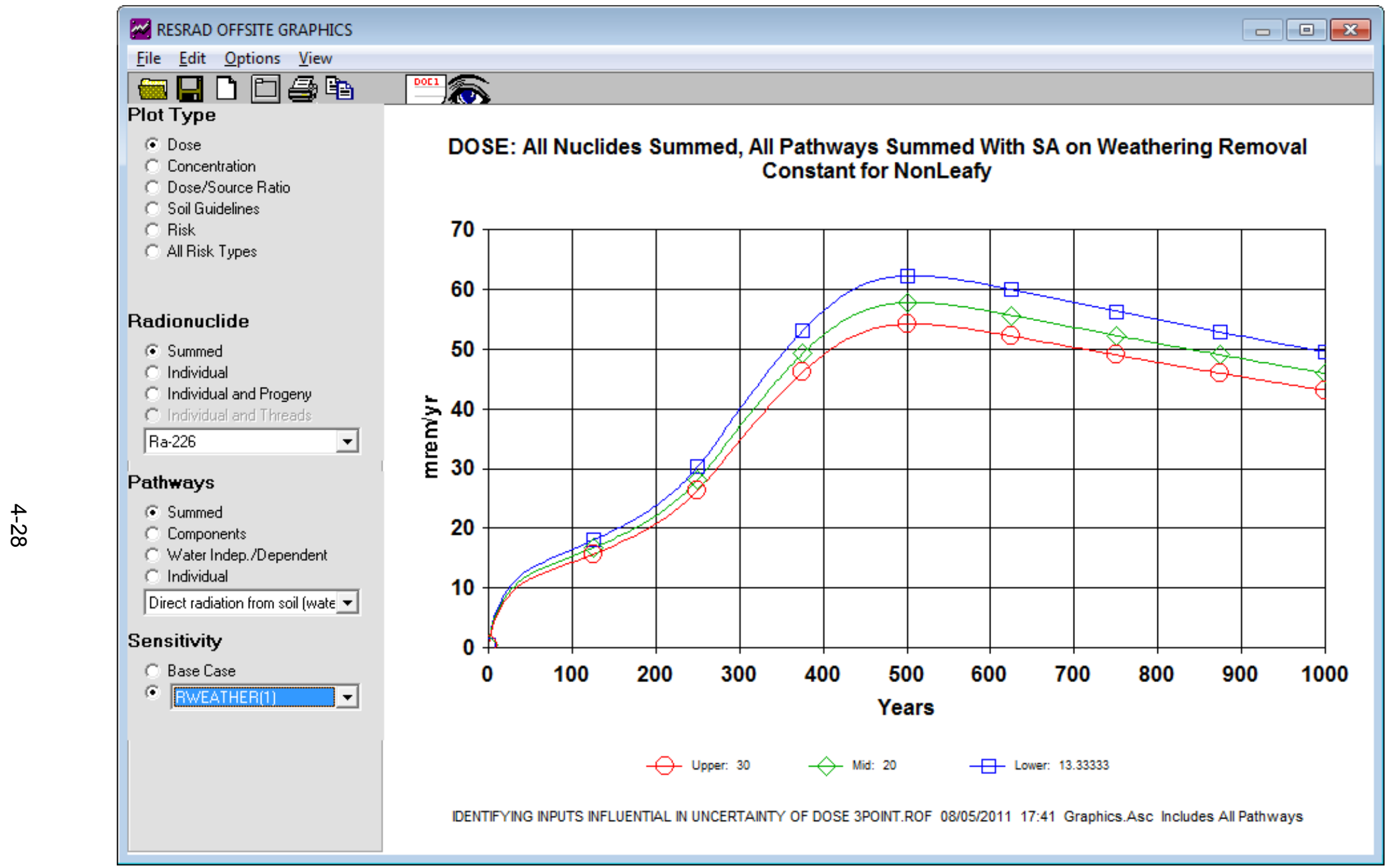

FIGURE 4.22 Temporal Variation of Dose with the Weathering Removal Constant for Fruit, Nonleafy Vegetables, and Grain Showing a Range of $8.1 \mathrm{mrem} / \mathrm{year}$ for the Peak Dose from 54.2 to $62.3 \mathrm{mrem} / \mathrm{year}$ 


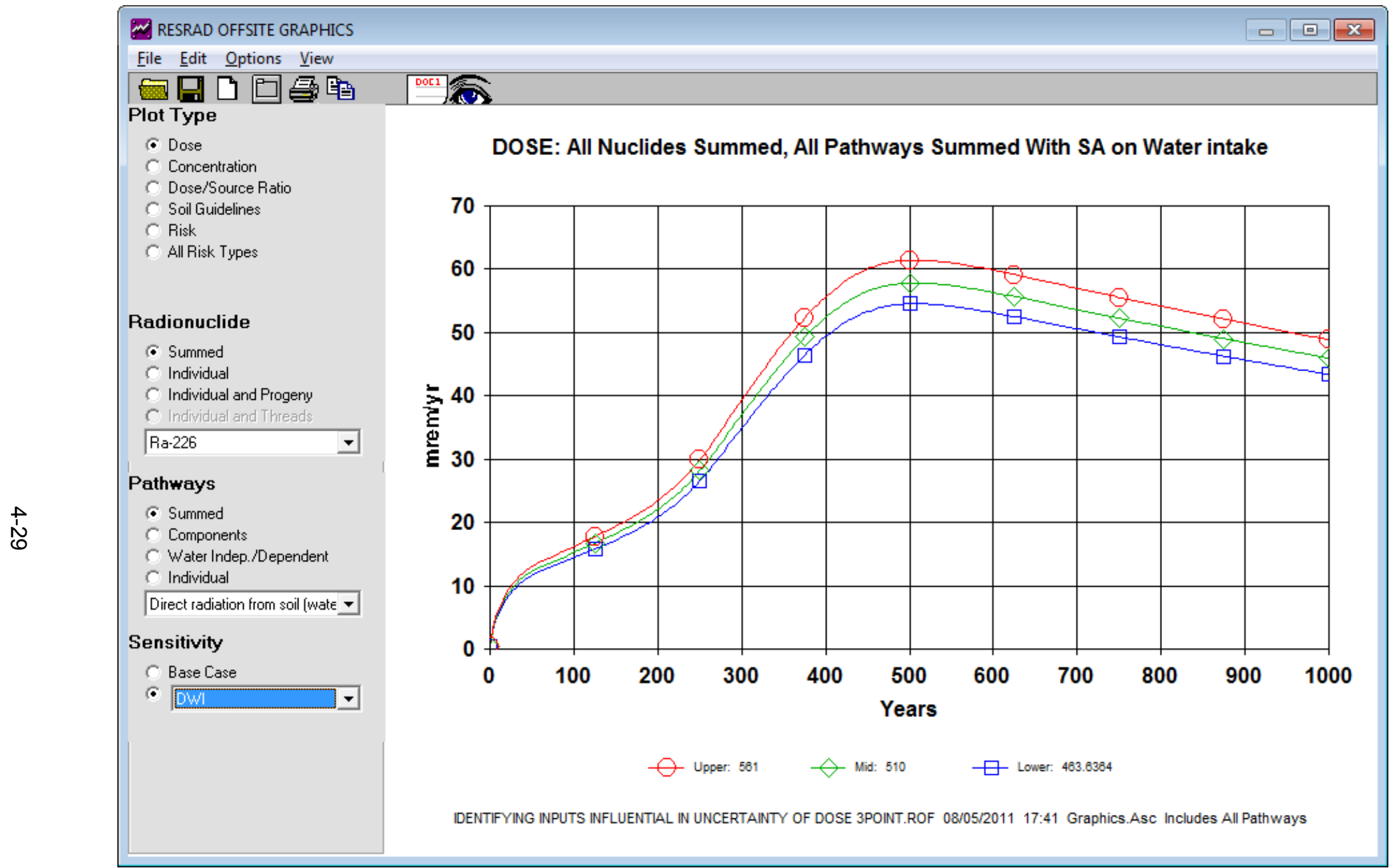

FIGURE 4.23 Temporal Variation of Dose with the Weathering Removal Constant for Fruit, Nonleafy Vegetables, and Grain Showing a Range of 6.9 mrem/year for the Peak Dose from 54.5 to 61.4 mrem/year 


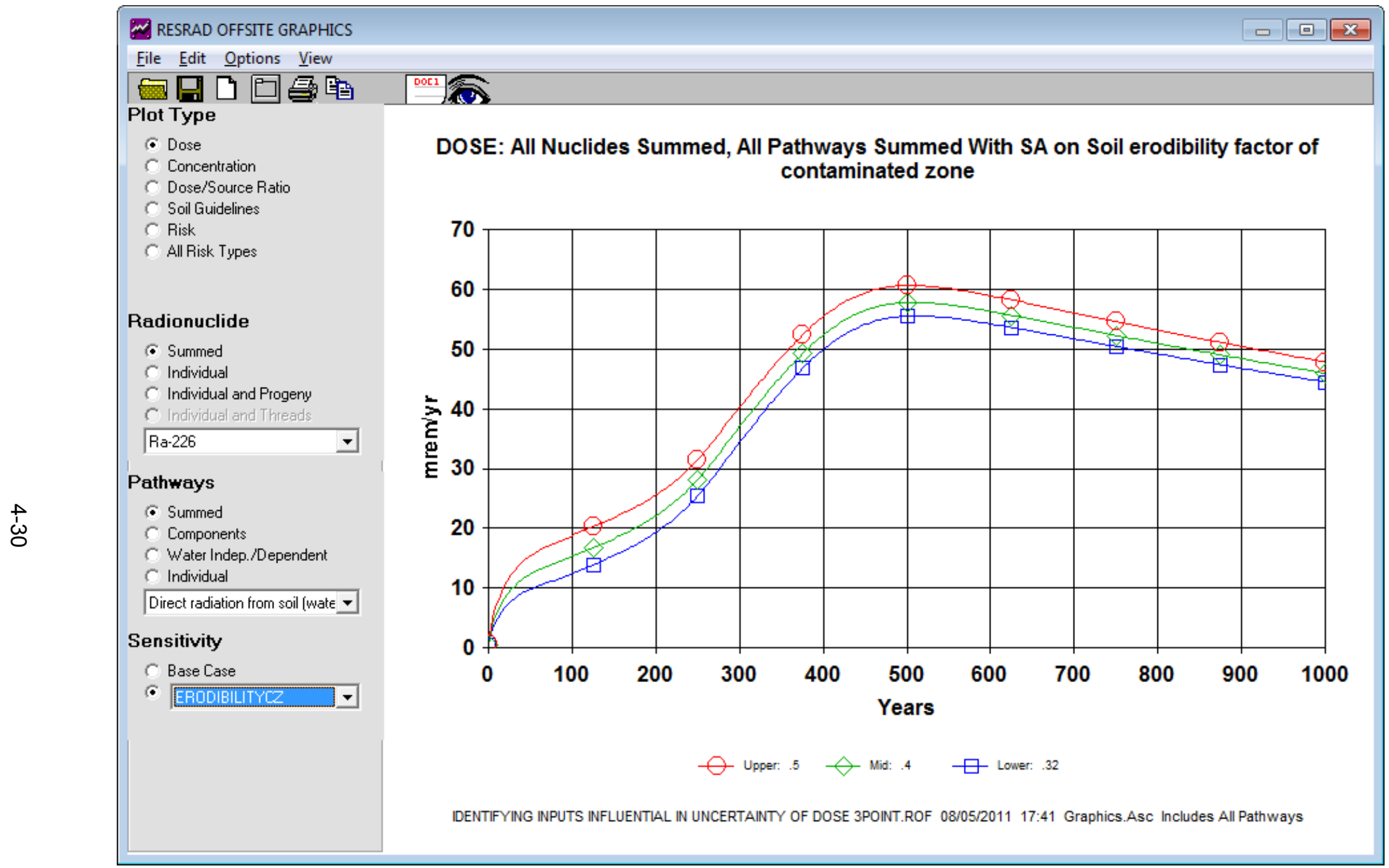

FIGURE 4.24 Temporal Variation of Dose with the Erodibility Factor of Soil in the Primary Contamination Showing a Range of 5.0 mrem/year for the Peak Dose from 55.6 to 60.6 mrem/year 


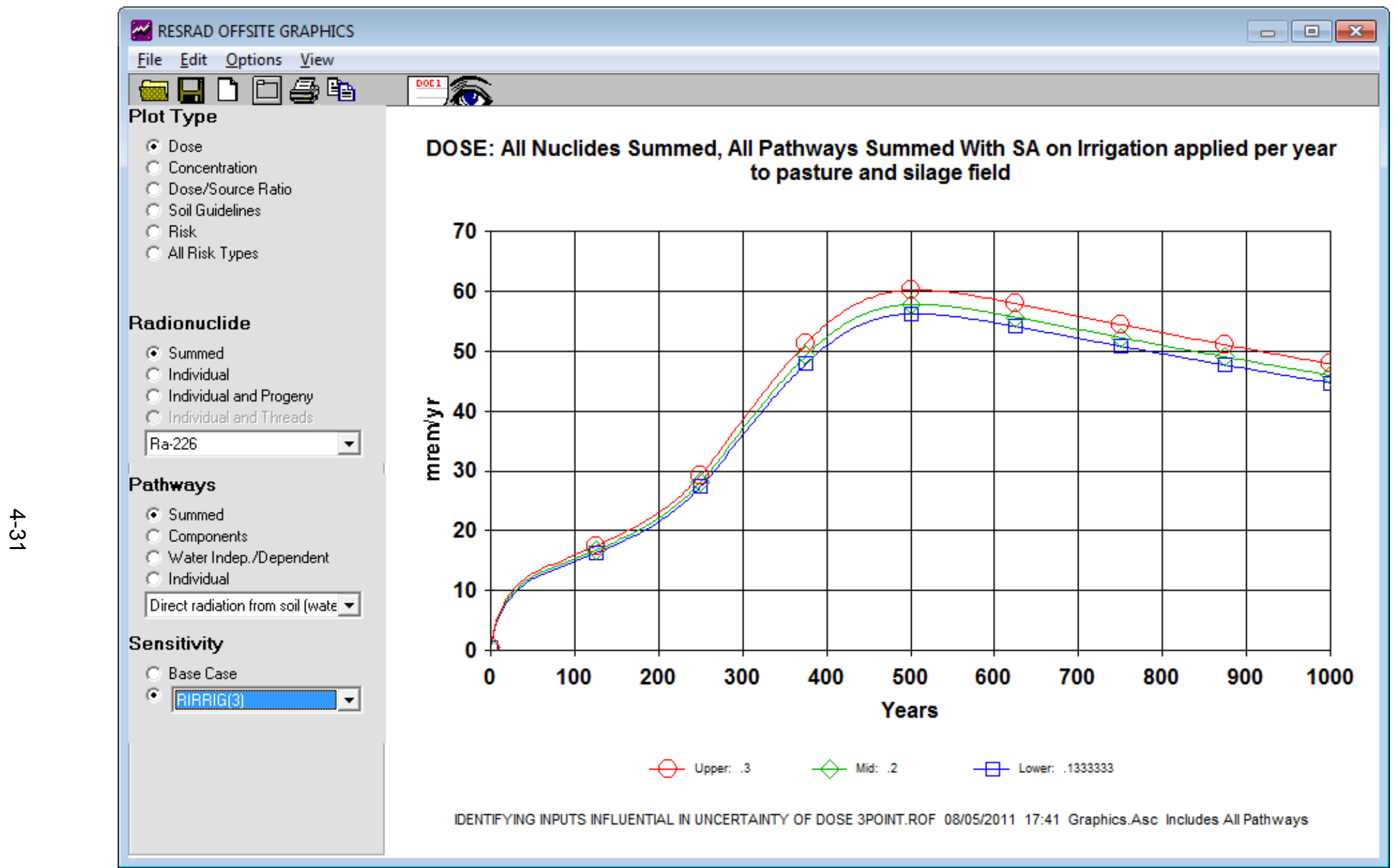

FIGURE 4.25 Temporal Variation of Dose with the Irrigation Applied per Year to the Pasture and the Silage Growing Areas Showing a Range of 4.0 mrem/year for the Peak Dose from 56.2 to $60.2 \mathrm{mrem} / \mathrm{year}$ 


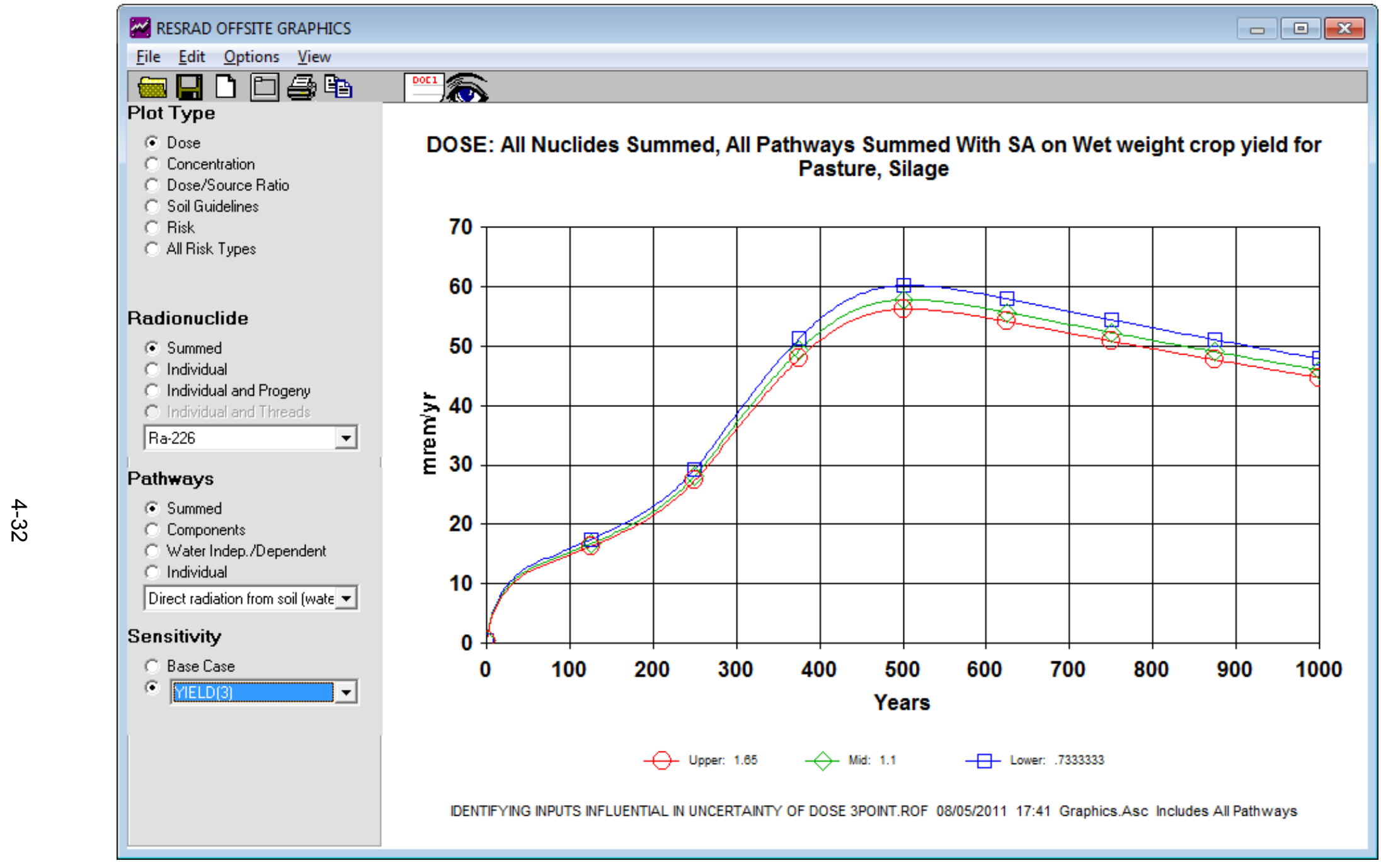

FIGURE 4.26 Temporal Variation of Dose with the Wet Weight Crop Yield from the Pasture and the Silage Growing Areas Showing a Range of 4.0 mrem/year for the Peak Dose from 56.2 to $60.2 \mathrm{mrem} / \mathrm{year}$ 


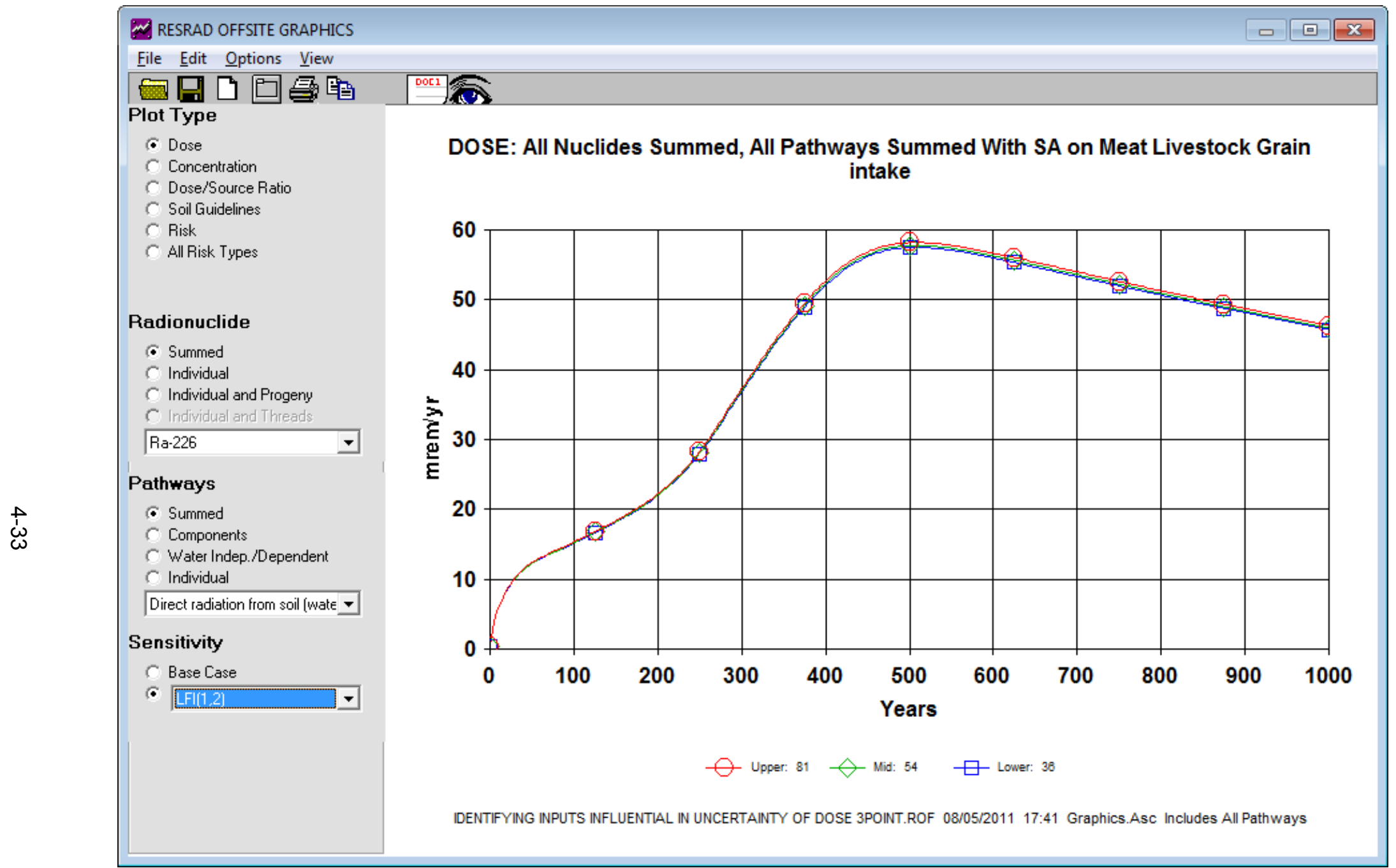

FIGURE 4.27 Temporal Variation of Dose with the Grain Intake by Cattle Raised for Meat Showing a Range of $0.8 \mathrm{mrem} / y e a r$ for the Peak Dose from 57.5 to $58.3 \mathrm{mrem} / \mathrm{year}$ 


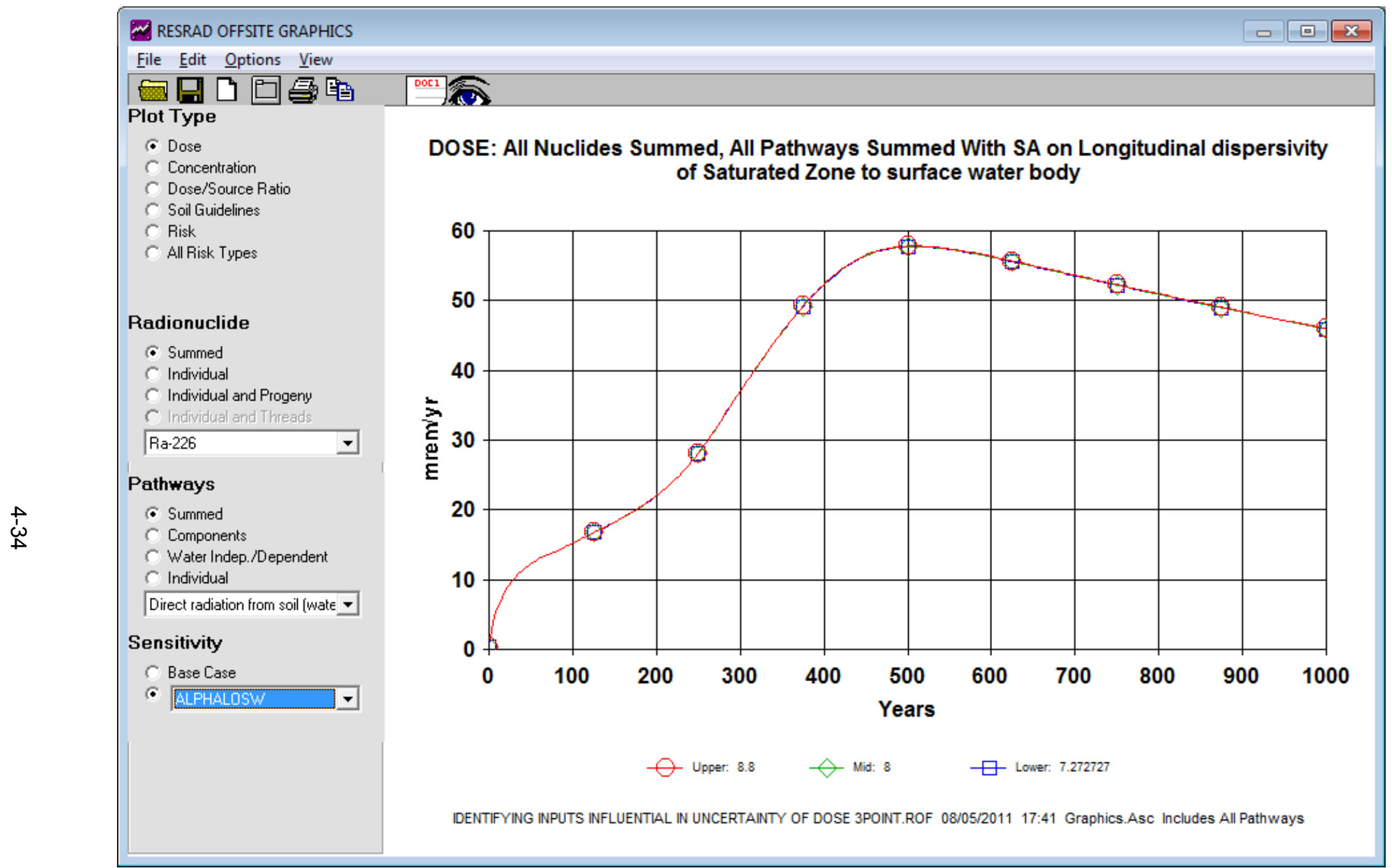

FIGURE 4.28 Temporal Variation of Dose with the Longitudinal Dispersivity in the Saturated Zone to the Surface Water Body Showing a Range of $0.0 \mathrm{mrem} / \mathrm{year}$ for the Peak Dose from 57.8 to $57.8 \mathrm{mrem} / \mathrm{year}$ 


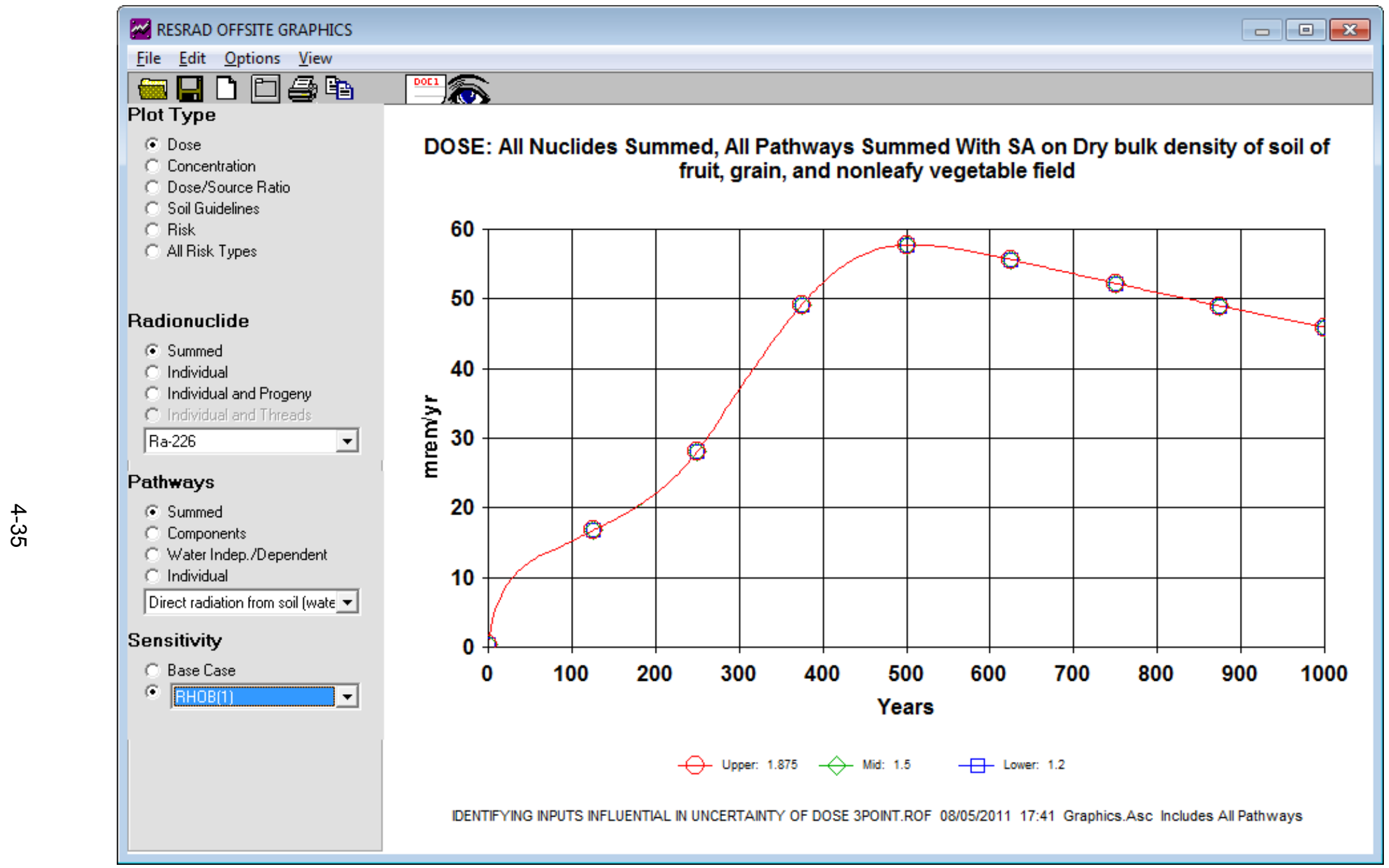

FIGURE 4.29 Temporal Variation of Dose with the Dry Bulk Density of Soil in the Fruit, Nonleafy Vegetables, and Grain Growing Area Showing a Range of $0.0 \mathrm{mrem} / \mathrm{year}$ for the Peak Dose from 57.8 to $57.8 \mathrm{mrem} / \mathrm{year}$ 


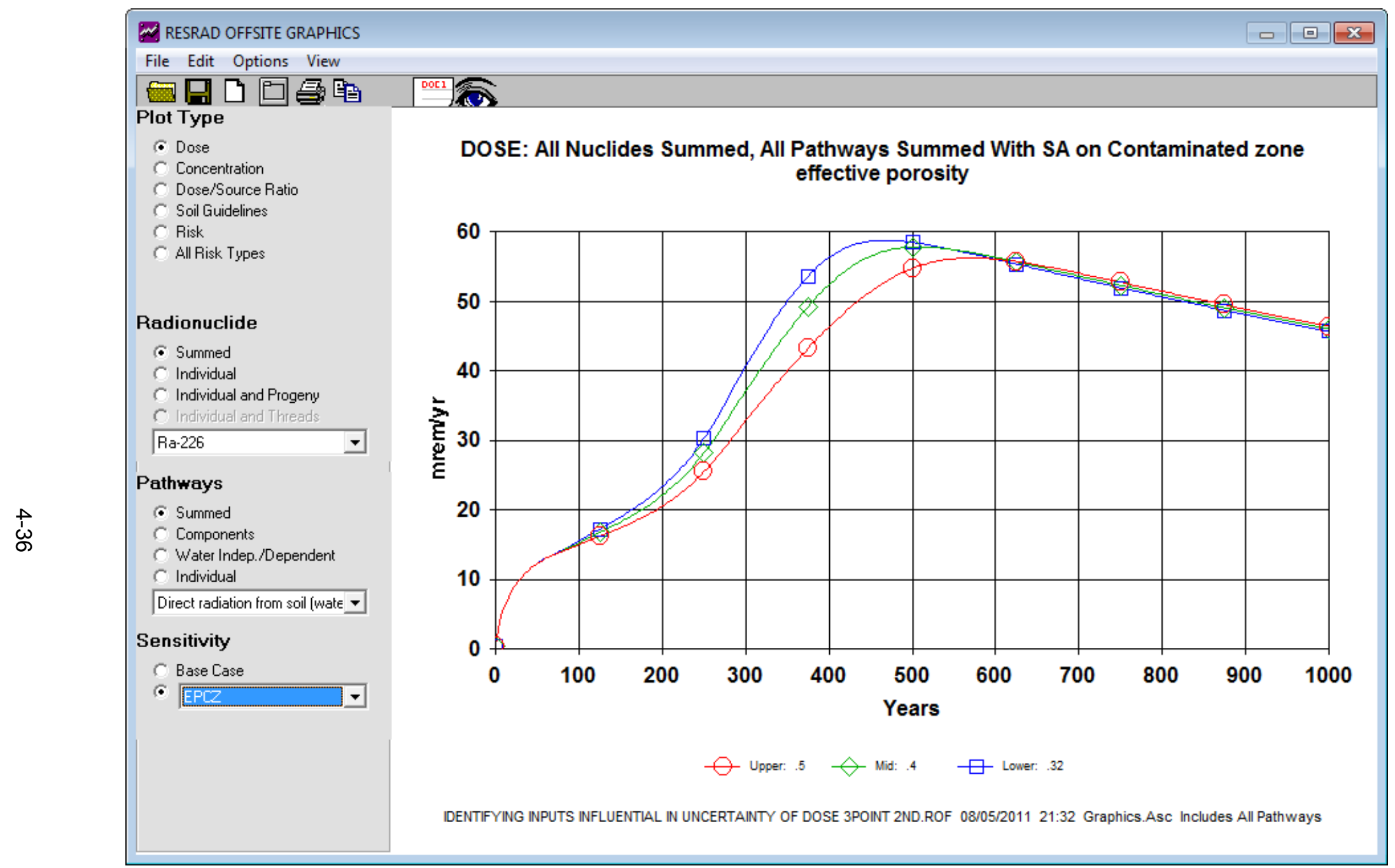

FIGURE 4.30 Temporal Variation of Dose with the Effective Porosity of the Primary Contamination Showing a Range of $2.5 \mathrm{mrem} / \mathrm{year}$ for the Peak Dose from $\mathbf{5 6 . 2}$ to $58.7 \mathrm{mrem} / \mathrm{year}$ 


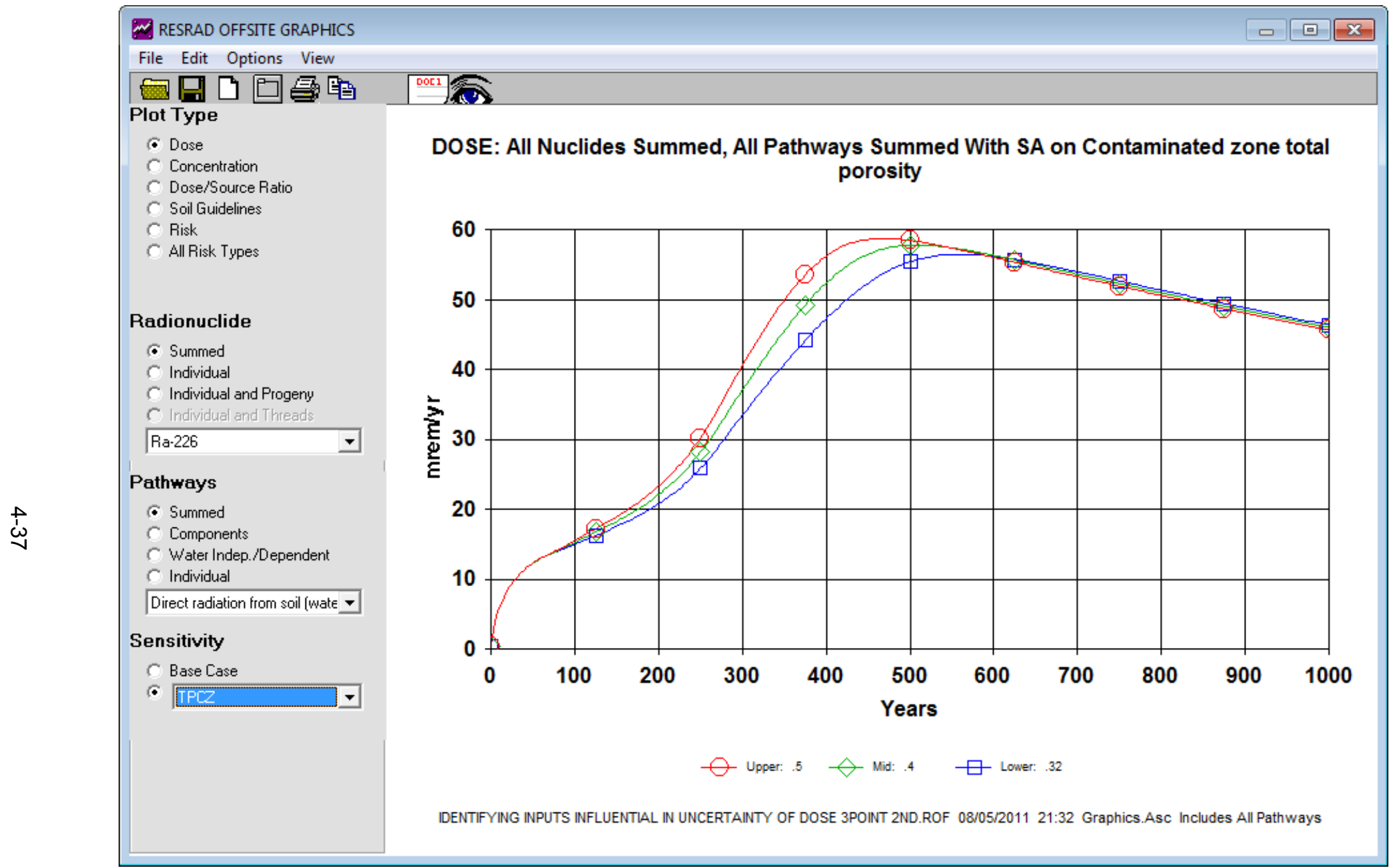

FIGURE 4.31 Temporal Variation of Dose with the Total Porosity of the Primary Contamination Showing a Range of $2.2 \mathrm{mrem} / \mathrm{year}$ for the Peak Dose from 56.5 to $58.7 \mathrm{mrem} / \mathrm{year}$ 


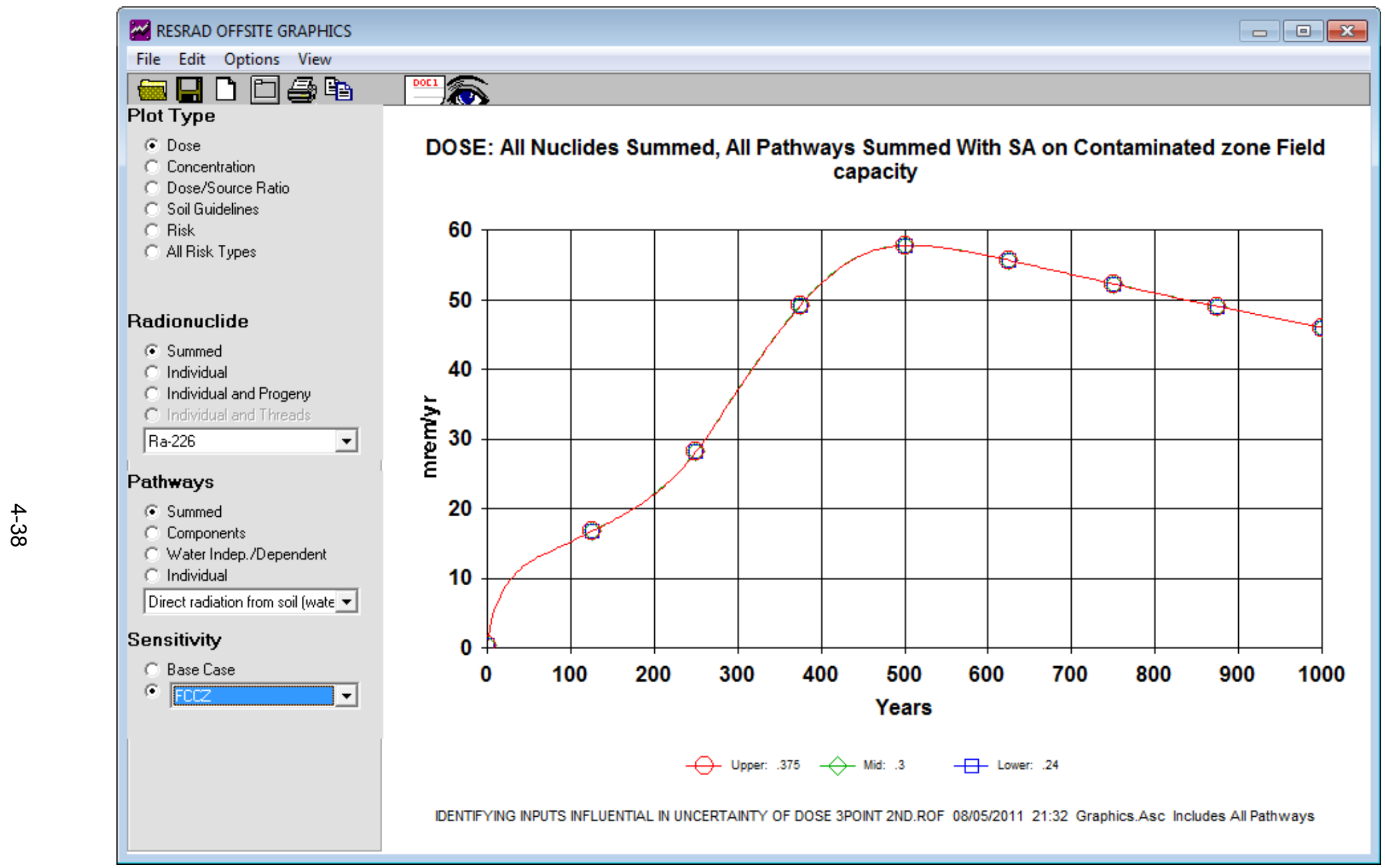

FIGURE 4.32 Temporal Variation of Dose with the Field Capacity of the Primary Contamination Showing a Range of $\mathbf{0 . 0} \mathrm{mrem} / \mathrm{year}$ for the Peak Dose from $\mathbf{5 7 . 8}$ to $\mathbf{5 7 . 8} \mathrm{mrem} / \mathrm{year}$ 


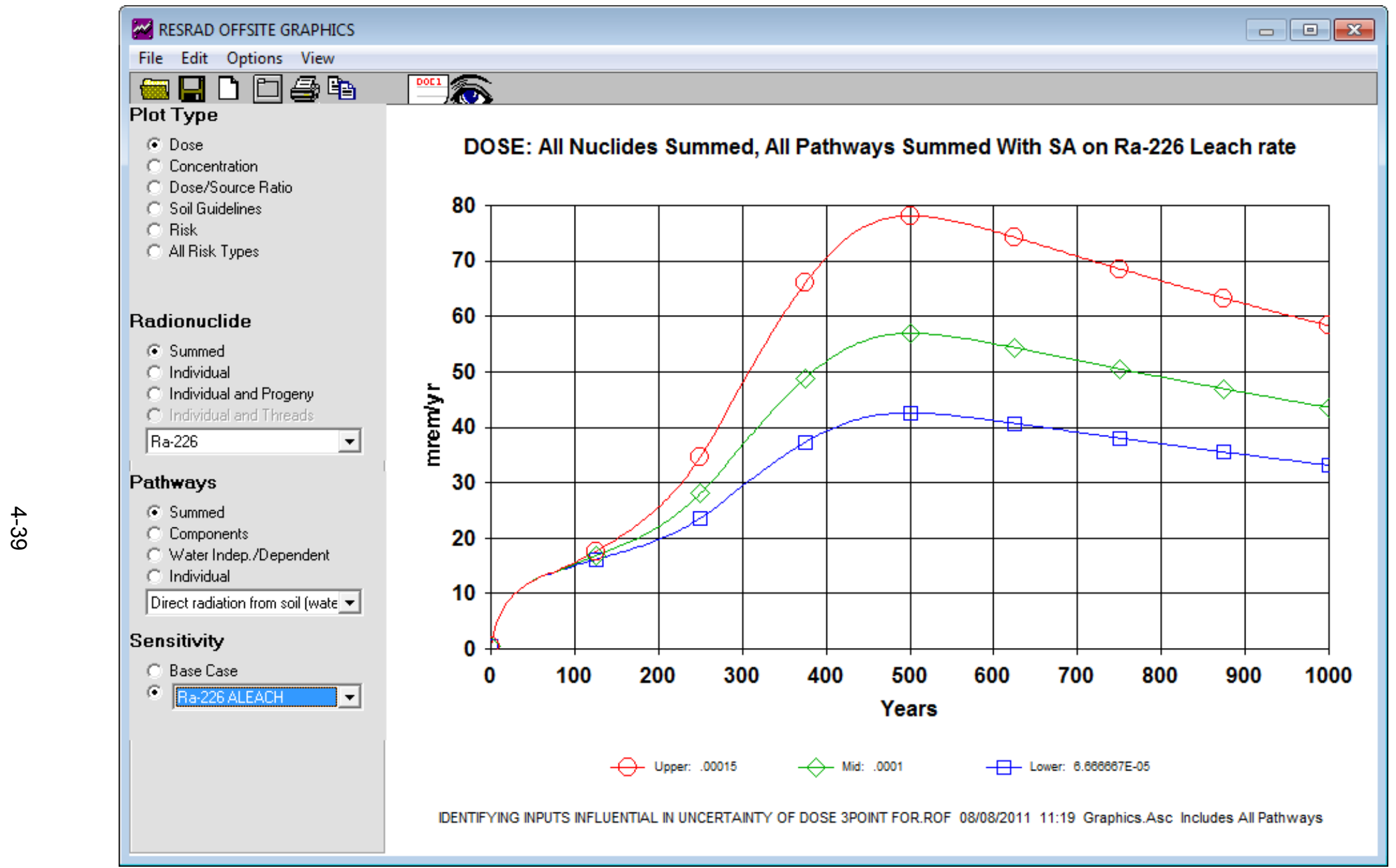

FIGURE 4.33 Temporal Variation of Dose with the Leach Rate of ${ }^{226} \mathrm{Ra}$ in Primary Contamination for the First Order Release Example Scenario 


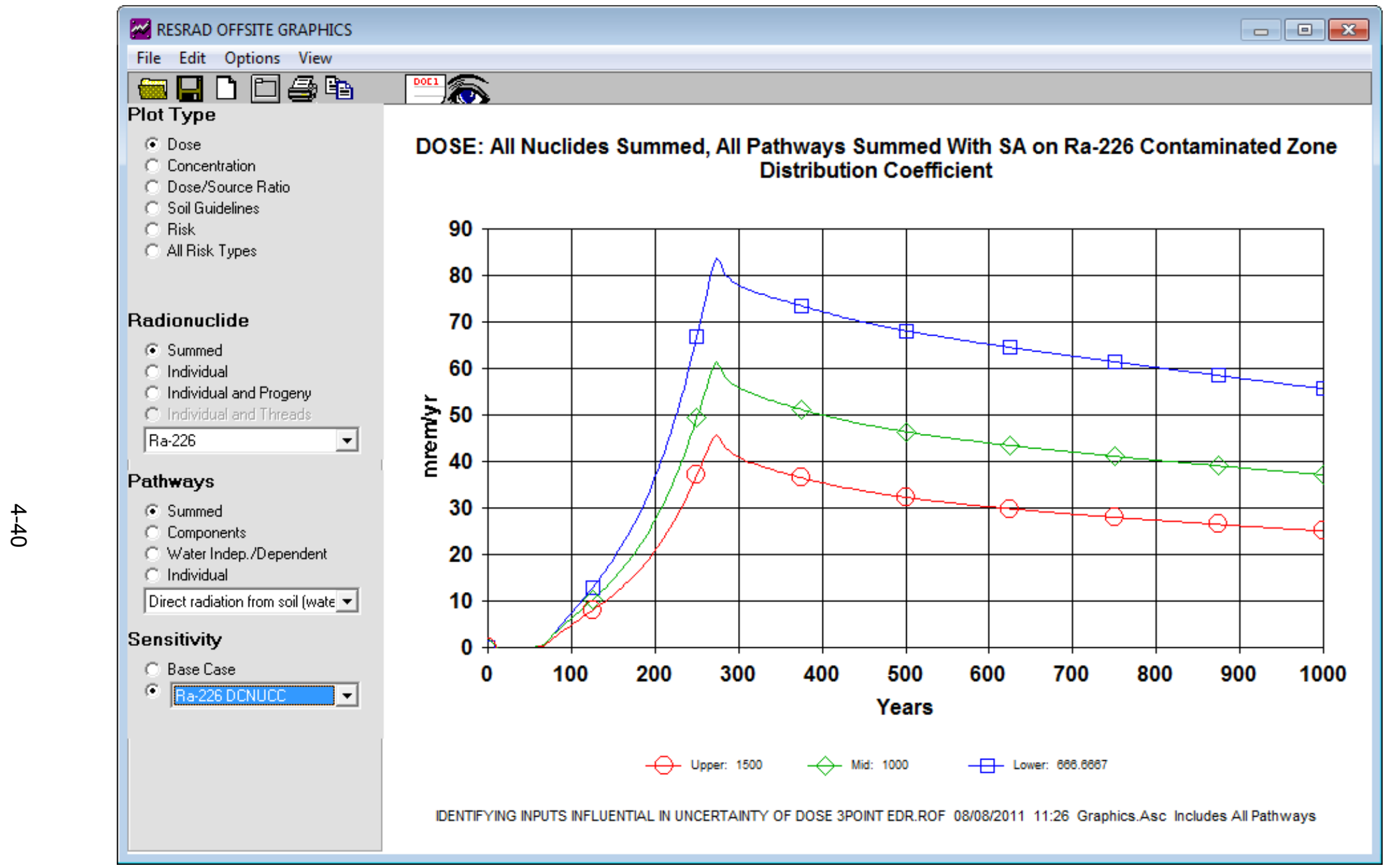

FIGURE 4.34 Temporal Variation of Dose with the Distribution Coefficient of ${ }^{226} \mathrm{Ra}$ in Primary Contamination for the Equilibrium Desorption Release Example Scenario 


\subsection{Multiple Input Sensitivity Analysis}

Section C.4 of Appendix C discusses the multiple input sensitivity analysis and describes how to perform this analysis in RESRAD-OFFSITE. The method involves specifying distributions-and not just ranges but probabilities associated with different parts of the range-for the inputs that are selected for sensitivity analysis. The code samples each distribution a specified number of times and then combines a sample from each of the distributions to produce a number of sets of inputs. Each sample is used in only one set of inputs, so the number of sets of inputs is equal to the number of times each distribution is sampled. If correlations are specified between inputs, the code takes those into account when it combines the samples from the distributions of those inputs to form the sets of inputs. Exact relations can also be specified between inputs in the step-by-step analysis mode (Figure 4.35). The code then performs the specified number of simulations using those sets of inputs. It is difficult visually to see the effect of an input on the dose in a scatter plot because all of the inputs change from simulation to simulation, unlike in the case of the single input sensitivity analysis. Even if it were possible to see the effect in the plots, viewing that many plots would be a tedious process. The code performs multivariable linear regression analysis between the peak dose of each simulation (the dependent variable) and the inputs ("independent" variables) and outputs a list of inputs in decreasing order of importance. The regression can be performed on the actual values of peak dose and inputs (raw), or it can be performed on the ranks assigned to the dose and the inputs. The ranks are simply the positions of the samples of input in a sorted list; thus, the smallest sample of each input would have a rank of 1 , the second smallest sample of each input would have a rank of 2 , and so on. The predicted peak dose also would be ranked from the least to the greatest. The linear regression on the ranks is useful if the relationship between the peak dose and the inputs is not linear but monotonic. The linear regression on the raw data can be used if the relationship is close to linear.

When an input is selected for multiple input sensitivity analysis using the Shift-F8 key, the "Uncertainty and Probabilistic Analysis" form pops up with a uniform distribution about the initial value of the input (Figure 4.36). The user can select from among ranges of $10 \%, 25 \%$, or $50 \%$ on either side of the initial value; alternatively, the user can specify other distributions. If the present ranges $(10 \%, 25 \%$, or $50 \%)$ are chosen, the interface will check to ensure that the ranges remain within the bounds of the input. Thus, for example, a $50 \%$ range on either side of a 0.4 value for the erodibility input would require a range of 0.2 to 0.6 . The upper bound for erodibility is set to 0.5 in the code. Thus, the interface sets a range of 0.2 to 0.5 when a $50 \%$ range is selected on a base value of 0.4 for the erodibility of soil (Figure 4.37). A total of 222 inputs were selected for sensitivity analysis using the ranges in Section 4.1.2; a 10\% range was specified for 42 of them, a $25 \%$ range was specified for 50 of them, and a $50 \%$ range was specified for the remaining 130 inputs. 
The correlations between inputs can be specified by a correlation coefficient between the ranks of the data. Possible correlations between inputs of this example scenario were discussed in Section 4.1.3. Information will probably not be available about the extent of correlation at the stage of a preliminary analysis. One guide to estimating the degree of correlation is the physical constraint. The field capacity cannot exceed the total porosity; this constraint appears to have been met with a rank correlation coefficient of 0.9 between the two inputs, as can be seen in Figure 4.38. This figure contains 12,000 points; 4000 samples were obtained from each distribution, and the process was repeated three times. The points from the different repetitions are shown in different colors. The specification of correlation between inputs is shown in Figure 4.39. The inputs to be correlated have to be identified by the variable name. The variable name is displayed in the variable information bar at the bottom of the RESRADOFFSITE interface when the cursor is placed in an input box. When the variable is selected from the dropdown box, its descriptive title appears below the dropdown box. Pairwise correlations for all possible pairs were specified for a group of correlated inputs. For example, there are four properties of the first unsaturated zone that were correlated: dry bulk density, effective porosity, field capacity, and total porosity. It is possible to form six pairs from this group of four inputs: dry bulk density - effective porosity, dry bulk density - field capacity, dry bulk density - total porosity, effective porosity - field capacity, effective porosity - total porosity, and field capacity - total porosity. When correlations are specified between inputs, it is best to generate the samples and view the scatter plots between the correlated inputs.

The two relationships discussed in Section 4.1.3 are specified in the related inputs tab (Figure 4.40) after generating the input samples. The variable name for the length of contamination parallel to the aquifer flow is LCZPAQ, and SOURCEXY(1) is the variable name of the $x$ dimension of the primary contamination. The first expression in the table specifies that the value of length of contamination parallel to aquifer flow must be set to the sample value of the $x$ dimension of the primary contamination for every simulation. Figure 4.41 confirms that this relationship is being used to generate the values for the length of primary contamination parallel to the aquifer flow.

After all of the input samples have been generated, the next step is to perform the probabilistic simulation on the code to generate the output samples. A total of 12,000 simulations were performed in this example scenario; three repetitions each containing 4000 samples. The cumulative distribution function of peak dose is shown in Figure 4.42. The figure contains three curves, one for each repetition. The predicted peak dose ranges from 9 to $480 \mathrm{mrem} / \mathrm{year}$ and has a mean of $63 \mathrm{mrem} / \mathrm{year}$. This range of uncertainty is a consequence of the uncertainty in the 224 inputs. The purpose of the sensitivity analysis is to identify the inputs that cause this uncertainty in the peak dose.

Linear regression can be performed between the output samples of peak dose and the input samples, and the resulting standardized regression coefficients can be used to identify the 
inputs that cause the uncertainty in the peak dose. The code uses the standardized regression coefficients to sort the inputs in the order of influence from the most influential to the least influential. The list includes all 222 inputs selected for sensitivity analysis; the related inputs are not included in this list. A partial listing showing the top 50 inputs for the uniform release example scenario is shown in Figure 4.43. A bar chart of the standardized regression coefficients of the top 63 inputs is shown in Figure 4.44, while those of all 222 inputs are shown in Figure 4.45. The inputs at the top of the list have a large influence on the range of the peak dose. The impact of the input on the range of the peak dose decreases as we move down the list. We need to identify the point on the list below which the inputs have a negligible effect on the range of the dose. This identification will subsequently be confirmed by the comparison of the cumulative distribution function plots of two runs as discussed later in this section. The first 5 inputs clearly have a very large influence on the uncertainty of the peak dose, and the next 12 have a moderate influence (Figures 4.44 and 4.46). The last 160 inputs are judged not to contribute to the uncertainty in the peak dose: the standardized regression coefficient from at least two of the repetitions is 0.00 , and that from the third repetition is $0.01,0.00$, or -0.01 (Figure 4.45). Somewhere between the top 17 inputs and the bottom 160 inputs is a point below which the inputs have only a negligible effect on the uncertainty in the peak dose. The judgment regarding where this cut-off point falls will vary from analyst to analyst, but it needs to be defensible. For this illustration, the top 30 inputs (Figure 4.46) are judged to have some effect on the uncertainty of the predicted peak dose; the next 32 are judged to make a negligible contribution to the uncertainty of the predicted peak dose. The standardized regression coefficients from the three repetitions of any of the top 30 inputs are consistent, as they are within 0.01 of their mean values. The difference between the values from the three repetitions is not significant as compared to the mean of the three values. The mean of the standardized regression coefficients of the 30th input is 0.03 , and a difference of 0.01 between the three values was considered insignificant especially in light of the fact that the standardized regression coefficients in the list have been rounded to a one-hundredth. Most importantly, this identification is supported by the close agreement between the cumulative distribution functions of two runs, the identification run, and the verification run. The identification run was the first run where all 222 inputs had the distributions specified in Section 4.1.2 together with the correlations and relationships developed in Section 4.1.3. For the verification run, the top 30 inputs and the two inputs that were correlated to one of them retained the same distributions as in the identification run; the correlations and relations were also retained. The range of the uniform distribution of the 190 inputs that were judged to have either a negligible influence or no influence on the uncertainty of the predicted peak dose were reduced to $0.1 \%$ of the preliminary value; thus, the samples for these inputs ranged from 0.999 to 1.001 of their preliminary value (essentially the preliminary value). The reason for retaining the very narrow distributions for the190 insignificant inputs is to ensure that the samples of the 30 influential inputs and, more importantly, the grouping of those samples into sets of inputs in the verification run is the same as that in the identification run. The cumulative distribution function of the peak dose from the verification run of the 30 most significant inputs in Figure 4.47 is very similar to the cumulative 
distribution function of the 222-input identification run. The predicted peak doses for the verification run with the 30 most significant inputs range from 10 to $480 \mathrm{mrem} / \mathrm{year}$ and have a mean of $64 \mathrm{mrem} / \mathrm{year}$, which is quite close to the values of 9,480 , and 63 obtained from the identification run. Thus, the top 30 inputs in this example scenario can be identified justifiably as the most significant, and available resources can be spent on gathering more site-specific values and distributions for these inputs. During this data-gathering process, it is likely that more site-specific values and distributions will also be found for some of the inputs that are related to or associated with the significant inputs.

The sensitivity analysis would then have to be repeated with the newly gathered distributions or point values for the 30 significant inputs and for any of the other associated inputs; the remaining inputs would retain their values and distributions from the preliminary sensitivity analysis. This step will lead to the identification of another set of significant inputs, some of which might be different from those identified from the preliminary sensitivity analysis. More resources will need to be spent identifying site-specific values and distributions for the newly identified significant inputs. This iterative process is repeated until either the uncertainty in the peak dose of the last sensitivity analysis run is acceptable, or all inputs identified as being significant by the last sensitivity run already have site-specific distributions indicating that the uncertainty in peak dose cannot be reduced further.

The cumulative distribution function plots with the top 17 inputs and with the top 5 inputs are shown in Figures 4.48 and 4.49. The cumulative distribution functions of all 4 runs are plotted together in Figure 4.50. The cumulative distribution function plots of the 222-input identification run and the 30-input verification run are essentially indistinguishable. Even the curves from the 17 -input verification run and the 5-input verification run are remarkably close to that of the identification run. Two of the top 5 inputs had a range of $25 \%$, and the other three had a $50 \%$ range. Three of the top 17 inputs were of the $10 \%$ range, and four were of the $25 \%$-range variety. On the other end, 96 of the 130 inputs for which the $50 \%$ range was specified ended up in the "no influence" category. Thus, the use of different ranges do not necessarily skew the results; the range specified for each input needs to be the probable range for each input.

\subsubsection{Advantages and Disadvantages of Multiple Input Sensitivity Analysis}

This sensitivity analysis is more complex than that of the three-point sensitivity analysis. Distributions need to be specified for each input selected for sensitivity analysis, instead of just the endpoints of the ranges. The code samples each distribution many times; thus, the analyst has to specify how many times the distribution is to be sampled. The results will not be reliable if too few samples are chosen; this will be evident by the large differences between the results of the repetitions. If there are large differences between the results of the repetitions, the sensitivity analysis has to be repeated with a larger number of samples. Relations and correlations between the inputs can be specified. These are taken into account when forming 
the sets of inputs. A set of inputs is made up of one sample from each of these distributions; each sample is only used once. Thus, there are as many sets of inputs as there are samples. The code produces a sample of output, peak dose, for each set of inputs, so there are as many samples of output as there are for each input. The values of all of the inputs change from simulation to simulation. As a result, it is not easy to see the effect of an input on the variability in the peak dose from a scatter plot of the two; whereas an experienced analyst might be able to pick out many (but not all) of the important inputs from the scatter plots, most analysts would not be able to visually identify more than a few important inputs. Linear regression has to be performed between the output and the inputs in order to pick out all of the significant inputs. The linear regression output sorts the inputs in descending order of importance, thereby facilitating the identification of the significant inputs. Cumulative distribution function plots of the identification and verification runs can be compared to confirm the selection of the significant inputs. The multiple input sensitivity analysis requires a longer time to execute the code as compared to that required by the three-point sensitivity analysis. One the other hand, the significant inputs can be picked more easily from the sorted list of the regression output instead of having to view each figure in turn, as in the case of the three-point sensitivity analysis; this advantage is temporary until the code is able produce a sorted list for the three-point sensitivity analysis as well. The uncertainty of the peak dose can be obtained from the multiple input sensitivity analysis but not from the three-point, single-input sensitivity analysis.

\subsubsection{Conclusion about the Use of Sensitivity Analysis}

This sensitivity analysis is difficult to understand and requires a large number of simulations, the number of which has to be decided upon by the analyst. It considers relationships between inputs and the interactions between inputs. Although a significant execution time may be needed to generate the samples of peak dose, the sorted regression output allows the analyst to quickly judge the influential outputs. The process of verifying the selection of influential inputs can be made easier if the code were to narrow the distributions of the insignificant inputs.

The inputs included in the first input file for the three-point, single-input sensitivity analysis were the top 22 from the list generated by the regression report, and the other three were from the no-influence category. There is good agreement between the ranking of the inputs by the two methods of sensitivity analysis. However, there are some significant differences, as well. Foliar interception factor for irrigation of fruit, grain, and nonleafy vegetables is judged to have no influence in the multiple input sensitivity analysis; it is 13th out of 25 in the three-point, singleinput sensitivity analysis file. Grain (kg/day) intake of meat livestock was judged to have some influence on the uncertainty in the dose by the multiple input sensitivity analysis, whereas it shows only a small effect on the temporal dose in the three-point, single-input sensitivity analysis. 




FIGURE 4.35 Step-by-step Analysis Tab of the Uncertainty and Probabilistic Analysis Form 


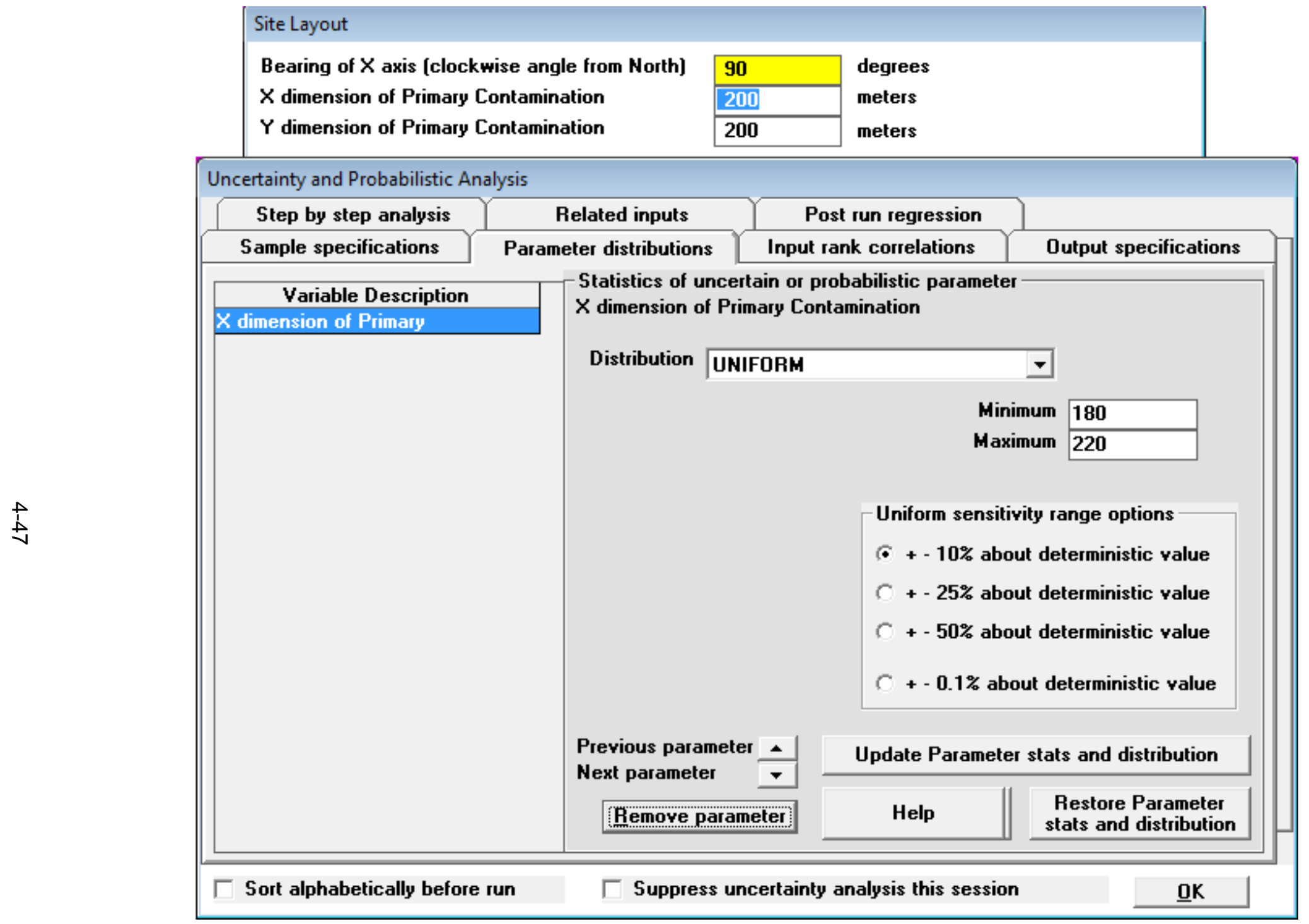

FIGURE 4.36 Specifying the Range for an Input Selected for Multiple Input Sensitivity Analysis 


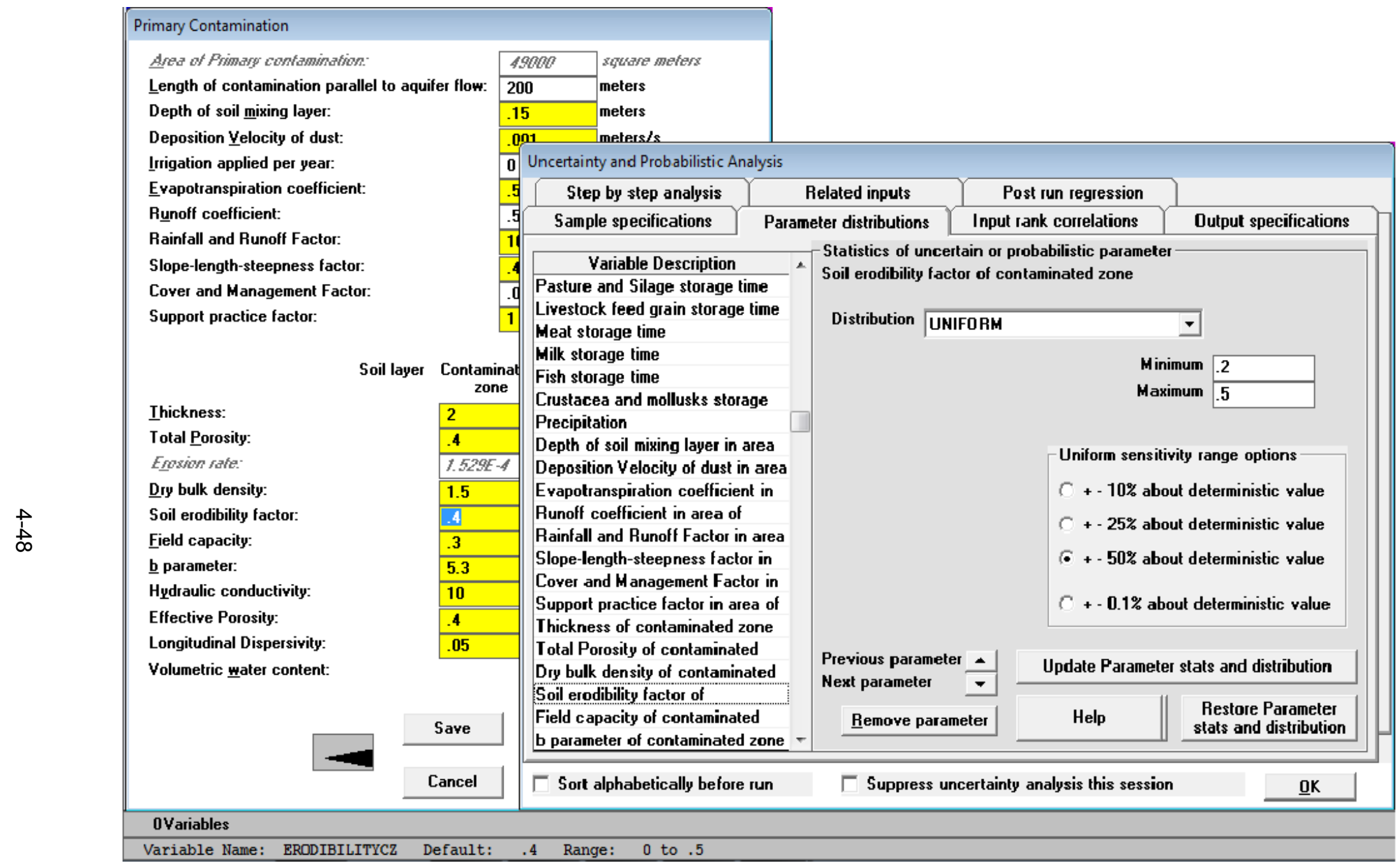

FIGURE 4.37 Specified Range Adjusted to Conform to Bounds Built into the Code 


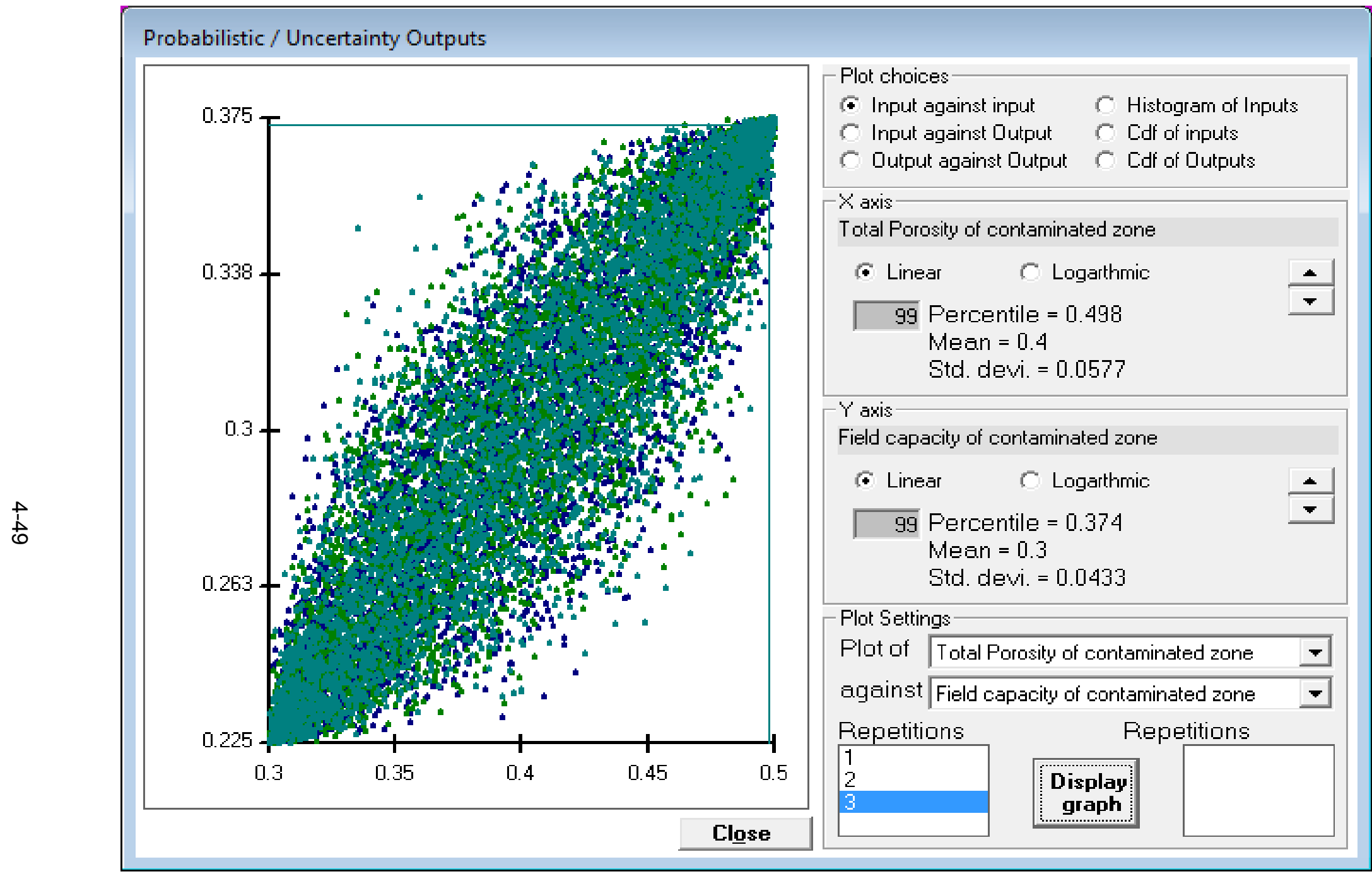

FIGURE 4.38 Scatter Plot of Field Capacity against Total Porosity to Show the Effect of Specifying a Correlation of 0.9 between Them 


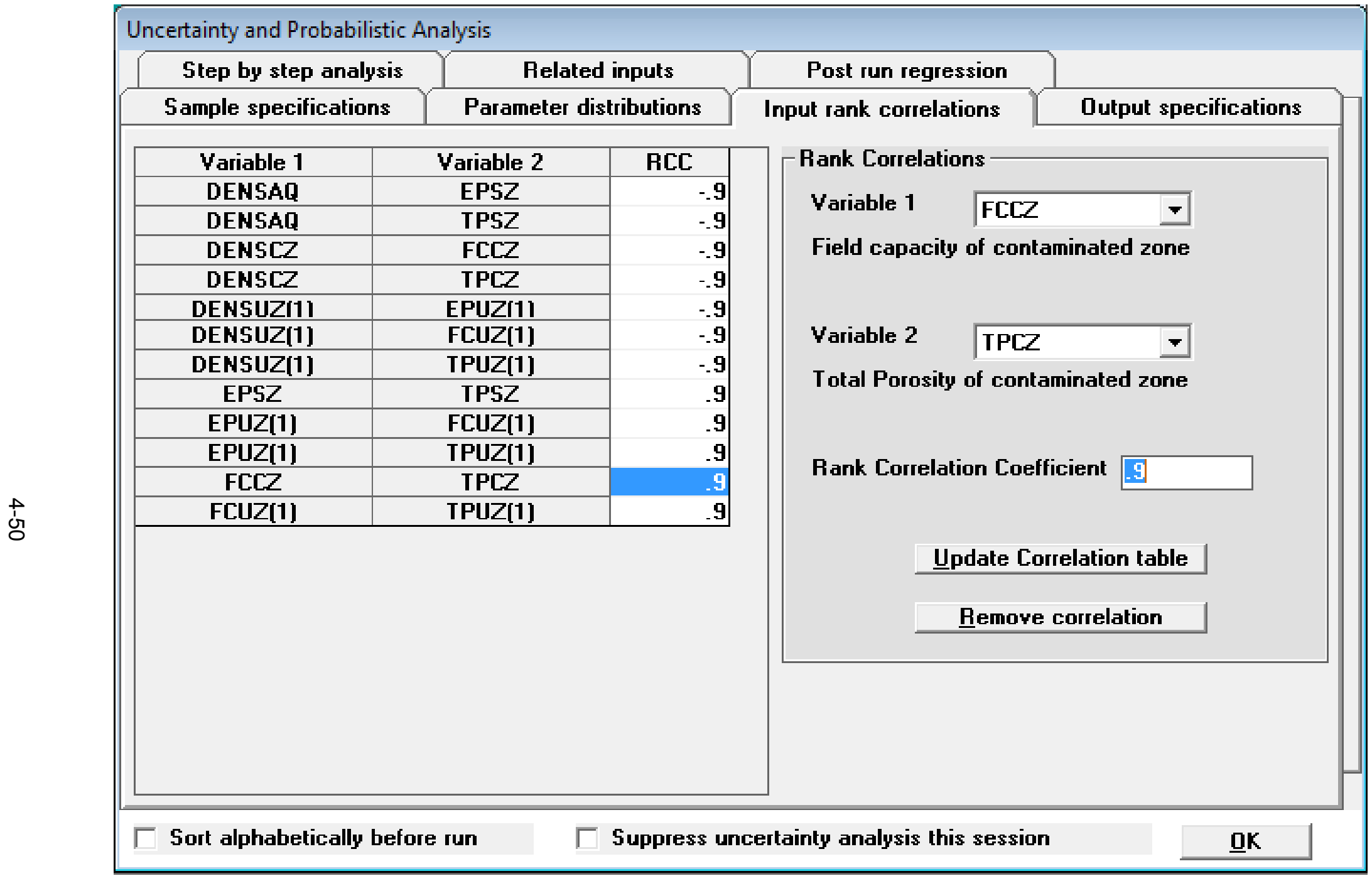

FIGURE 4.39 Specifying Correlation between Inputs 


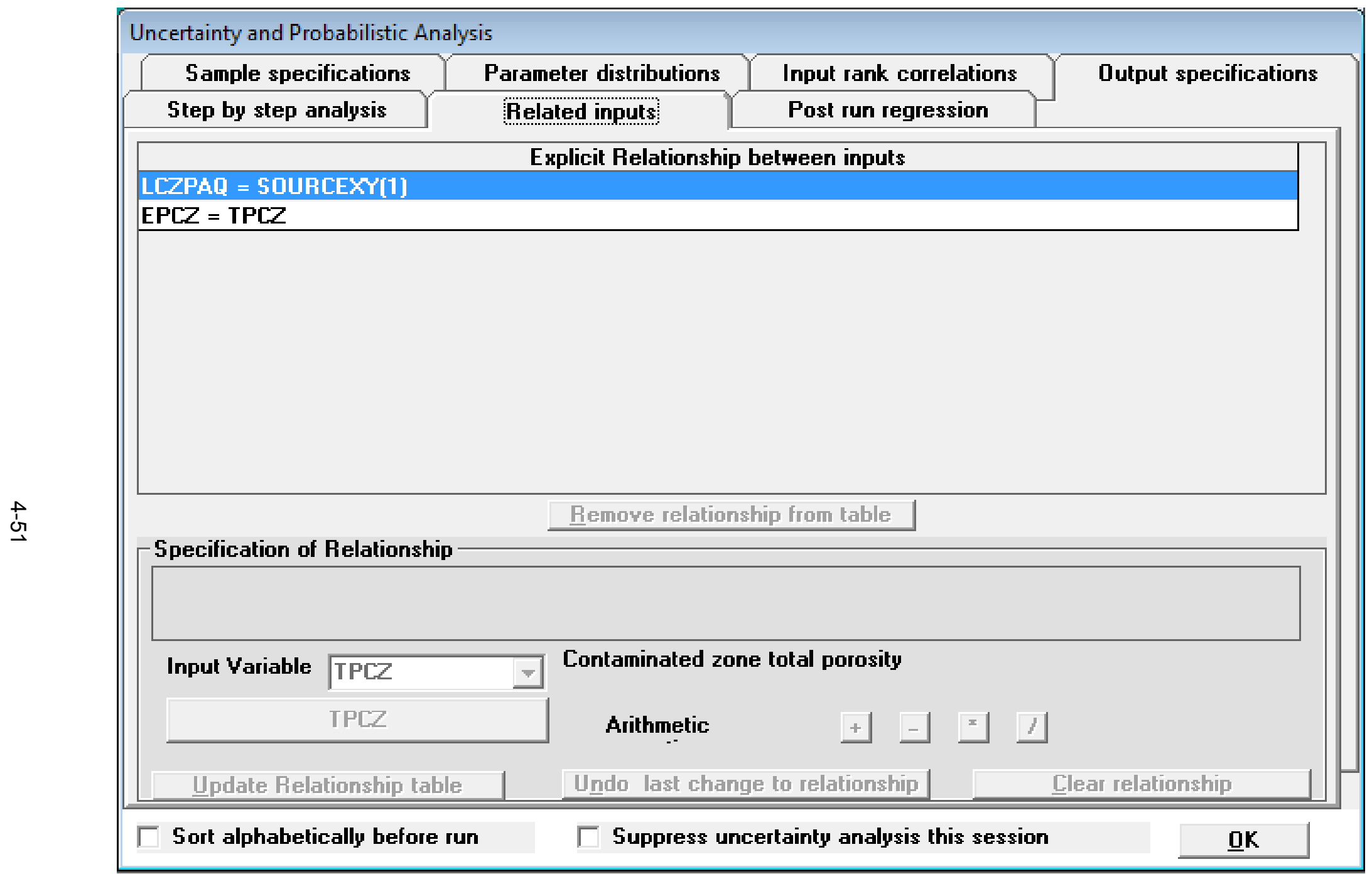

FIGURE 4.40 Specifying Relationships between Inputs 


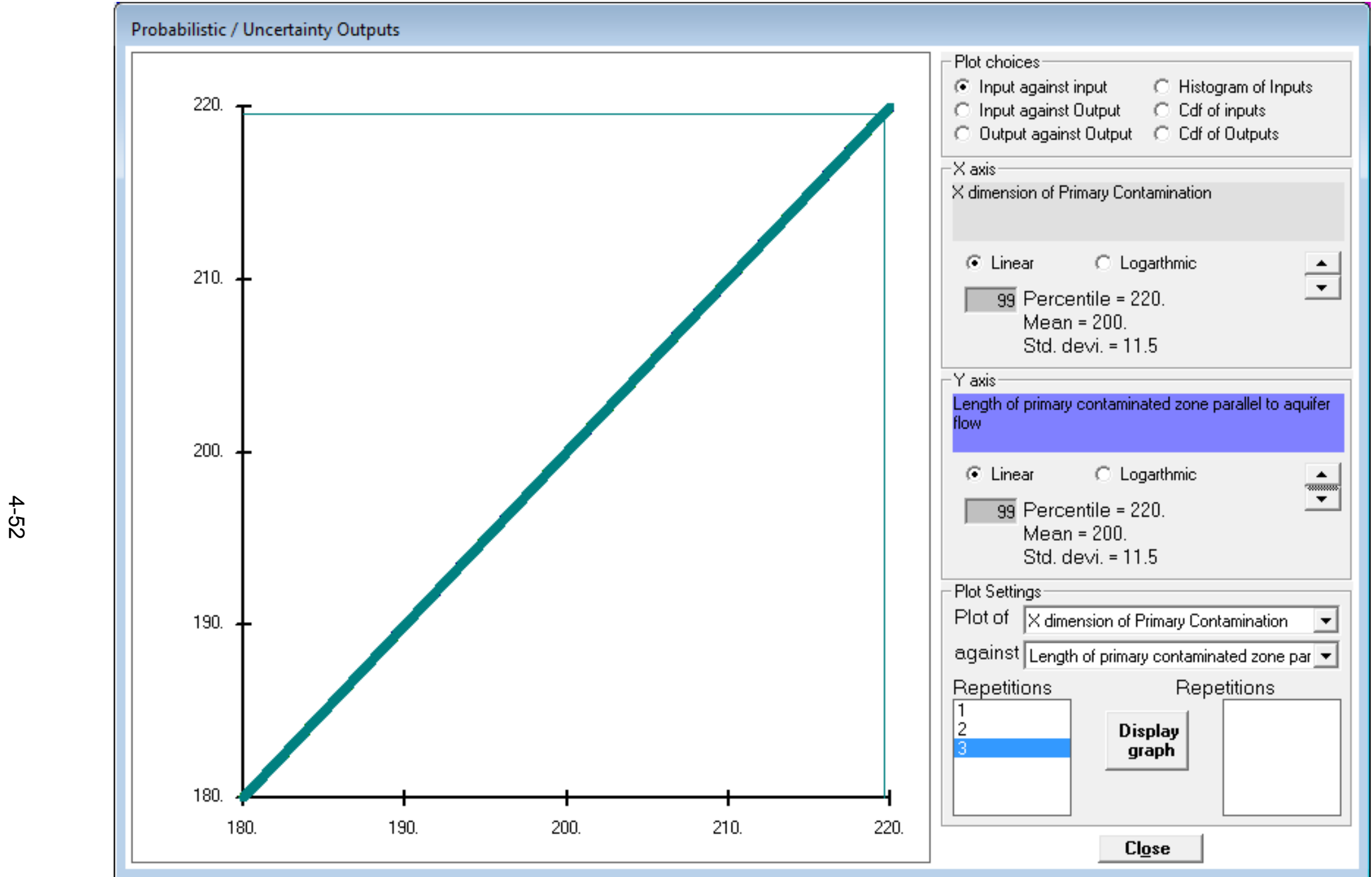

FIGURE 4.41 Scatter Plot of the Length Parallel to Aquifer Flow against the $x$ Dimension of the Primary Contamination 


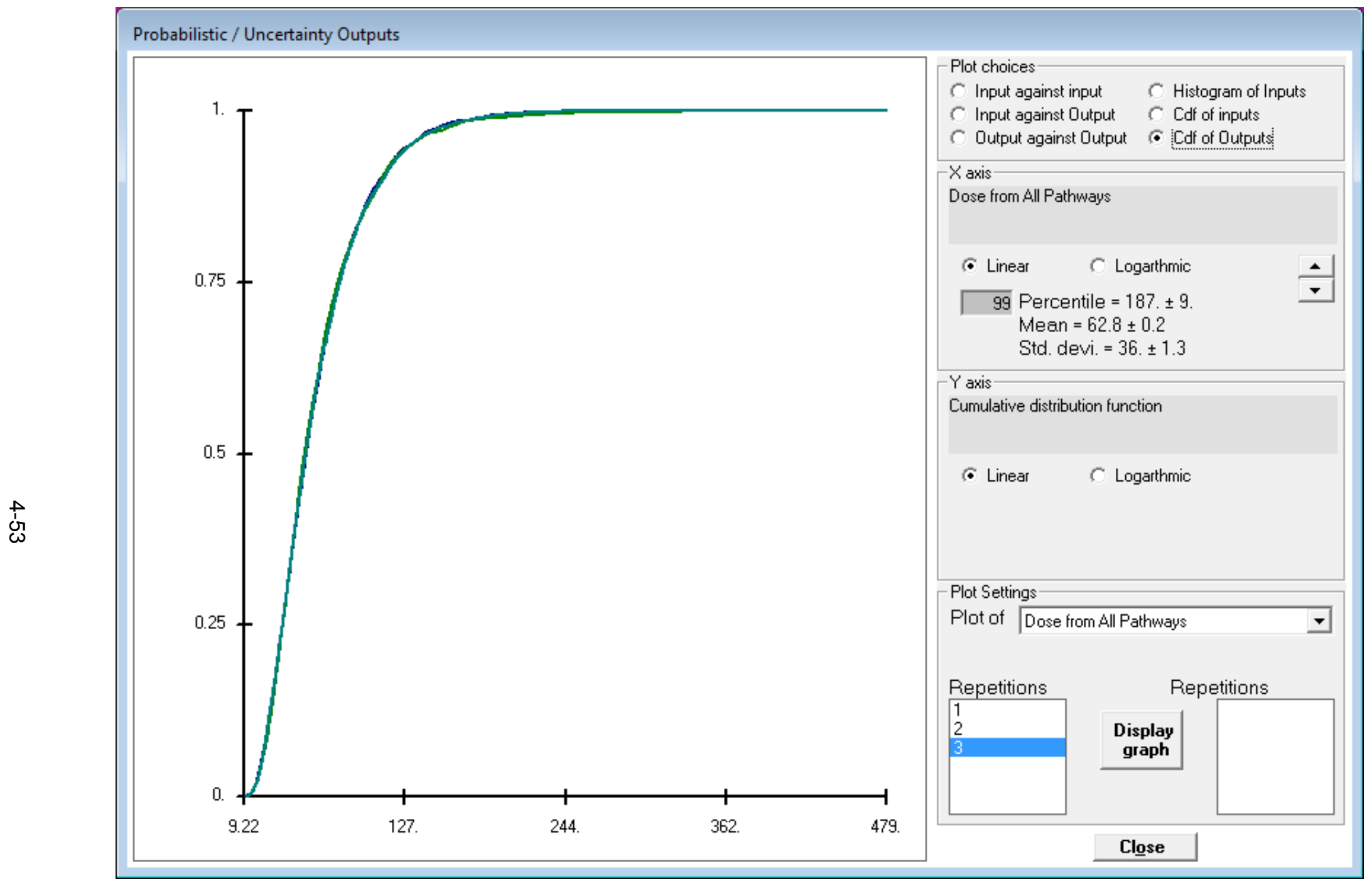

FIGURE 4.42 Cumulative Distribution Function of Peak Dose from the Identification Run of the Preliminary Sensitivity Analysis 


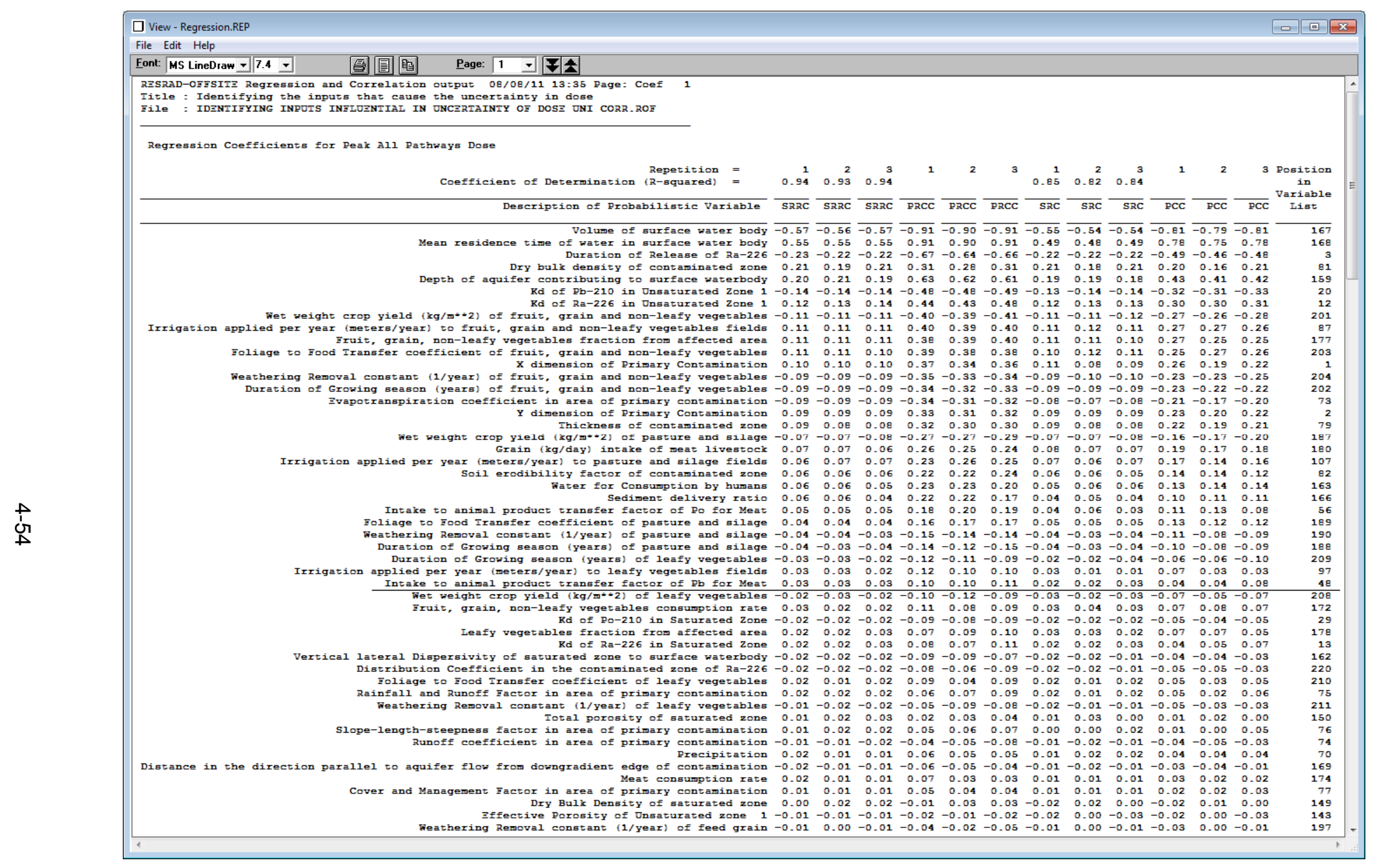

FIGURE 4.43 Regression Output of Preliminary Sensitivity Analysis with Inputs Sorted in Descending Order of Influence 


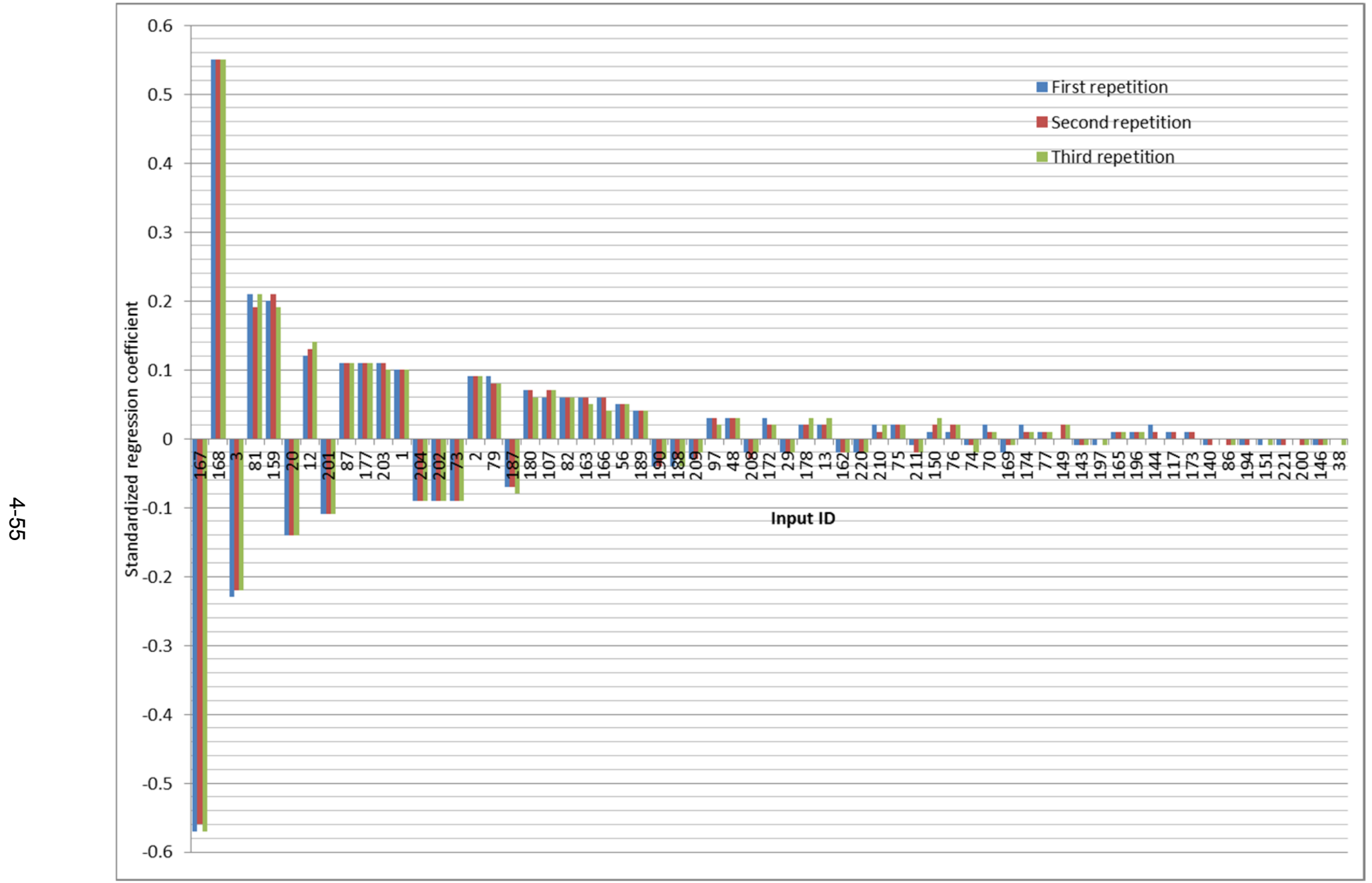

FIGURE 4.44 Standardized Regression Coefficients of the Top 63 Inputs Sorted in Descending Order of Importance 


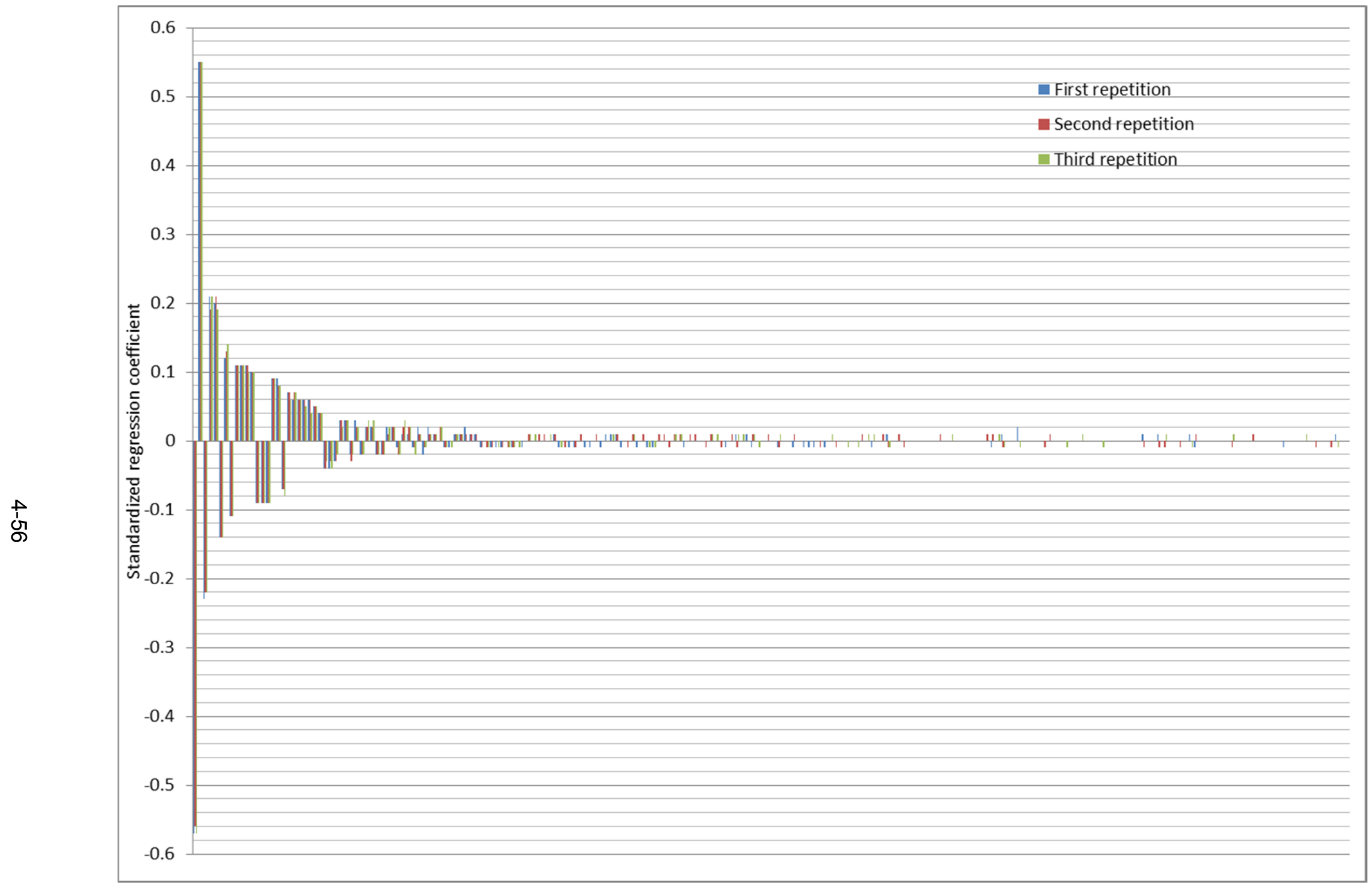

FIGURE 4.45 Standardized Regression Coefficients of All 222 Inputs Sorted in Descending Order of Importance 


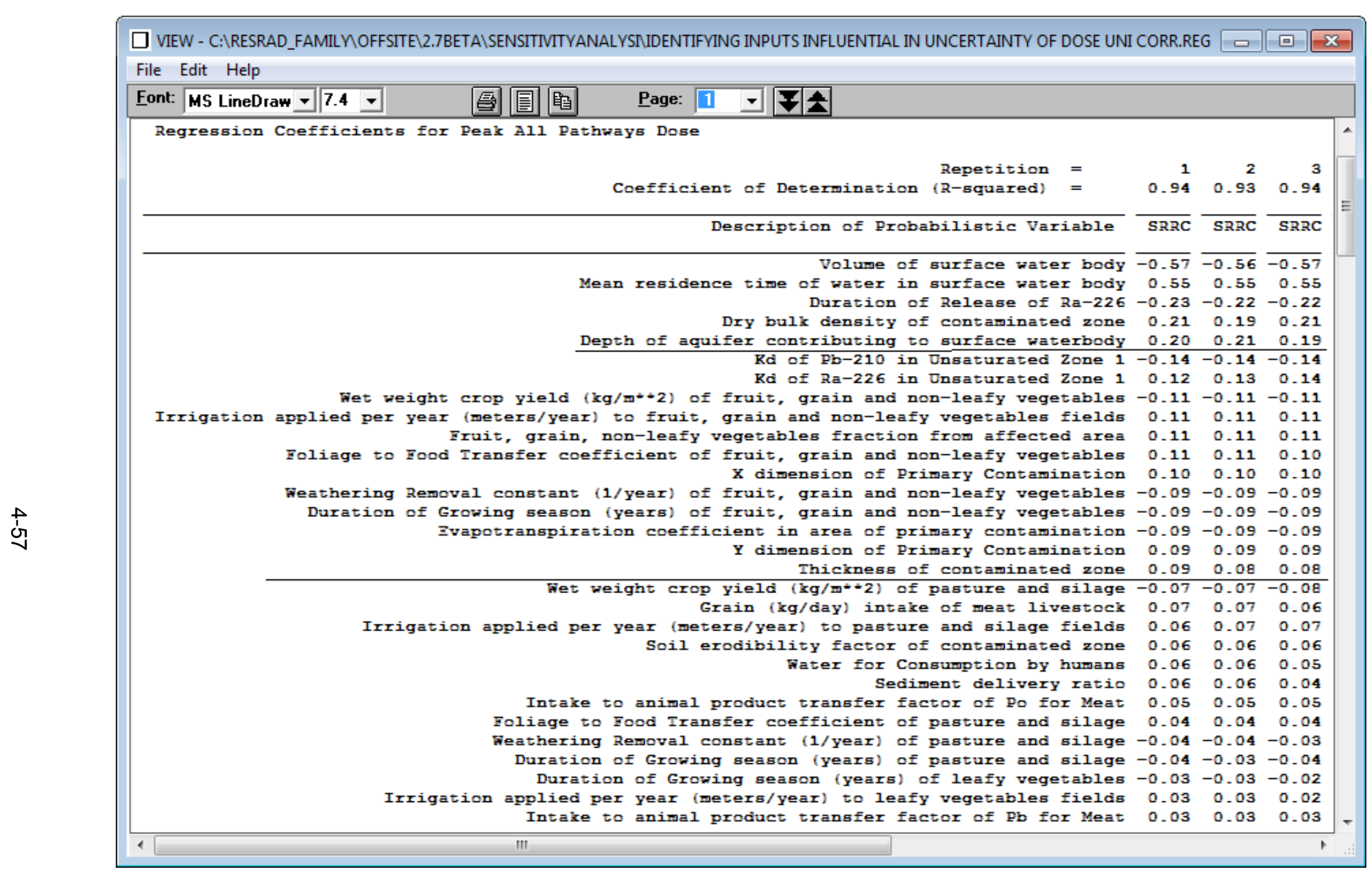

\section{FIGURE 4.46 Regression Output of Preliminary Sensitivity Analysis with Inputs Sorted in Descending Order of Influence}




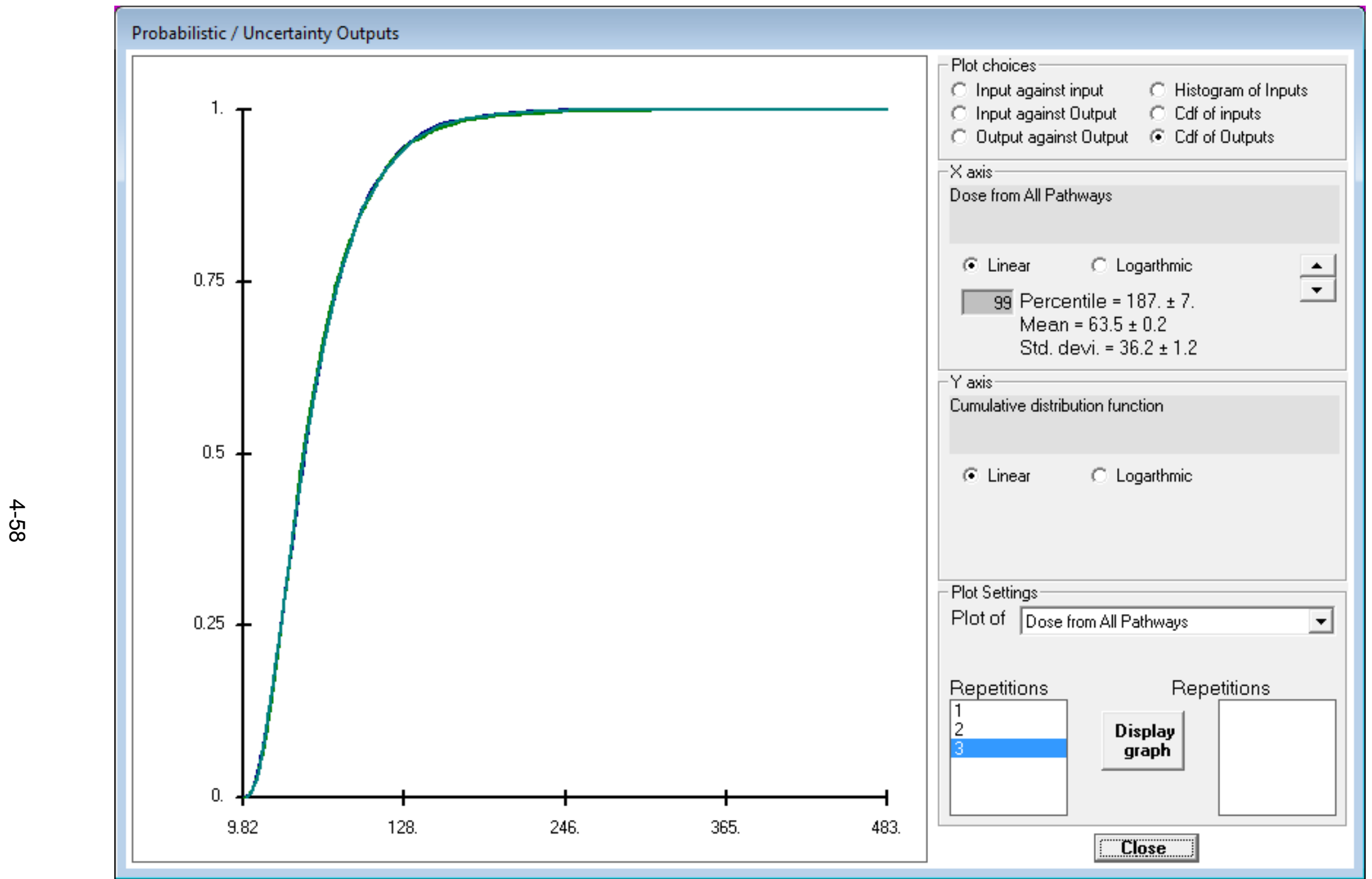

FIGURE 4.47 Cumulative Distribution Function of Peak Dose from the 30-input Verification Run of the Preliminary Sensitivity Analysis 


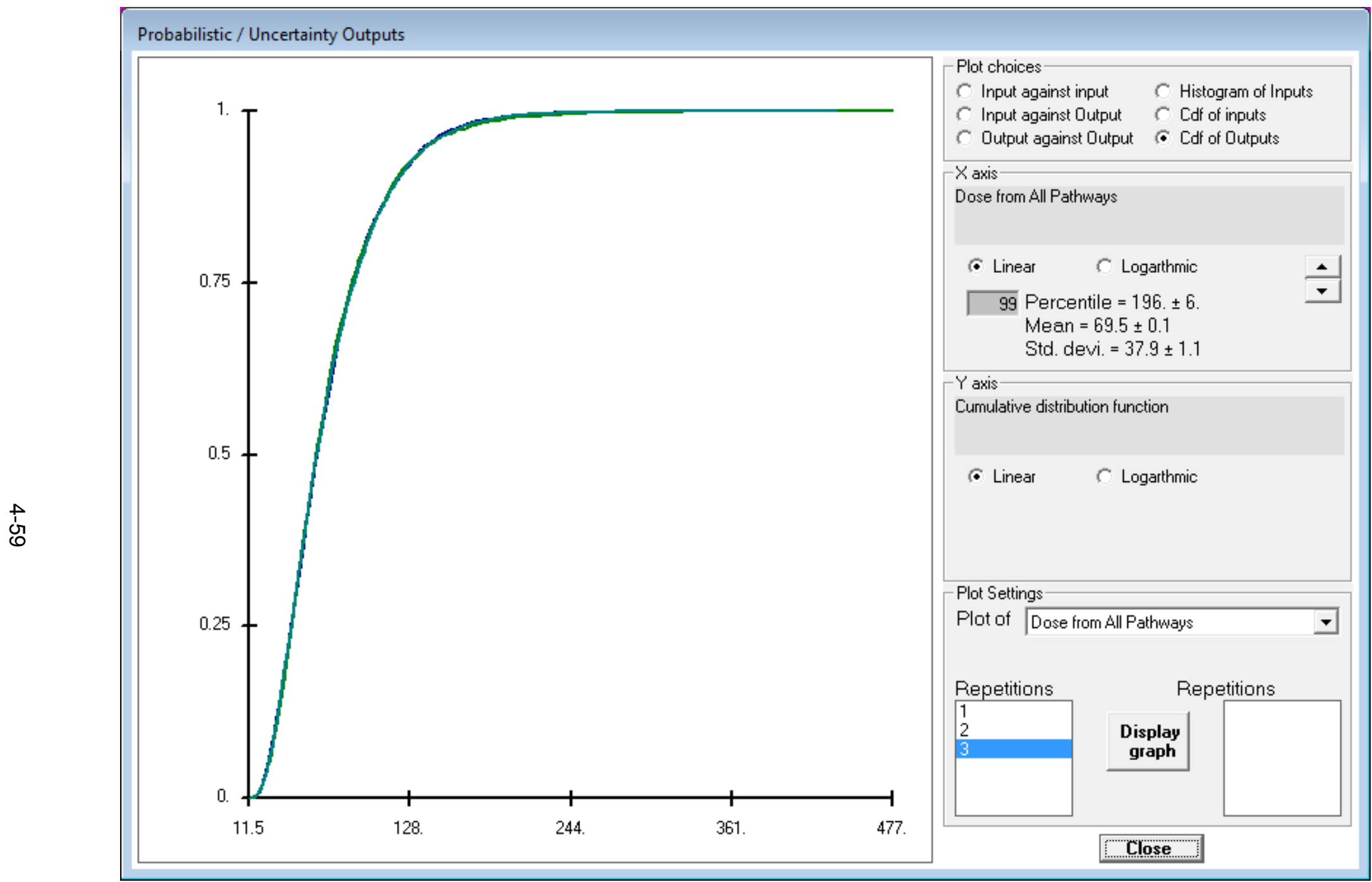

FIGURE 4.48 Cumulative Distribution Function of Peak Dose from the 17-input Verification Run of the Preliminary Sensitivity Analysis 


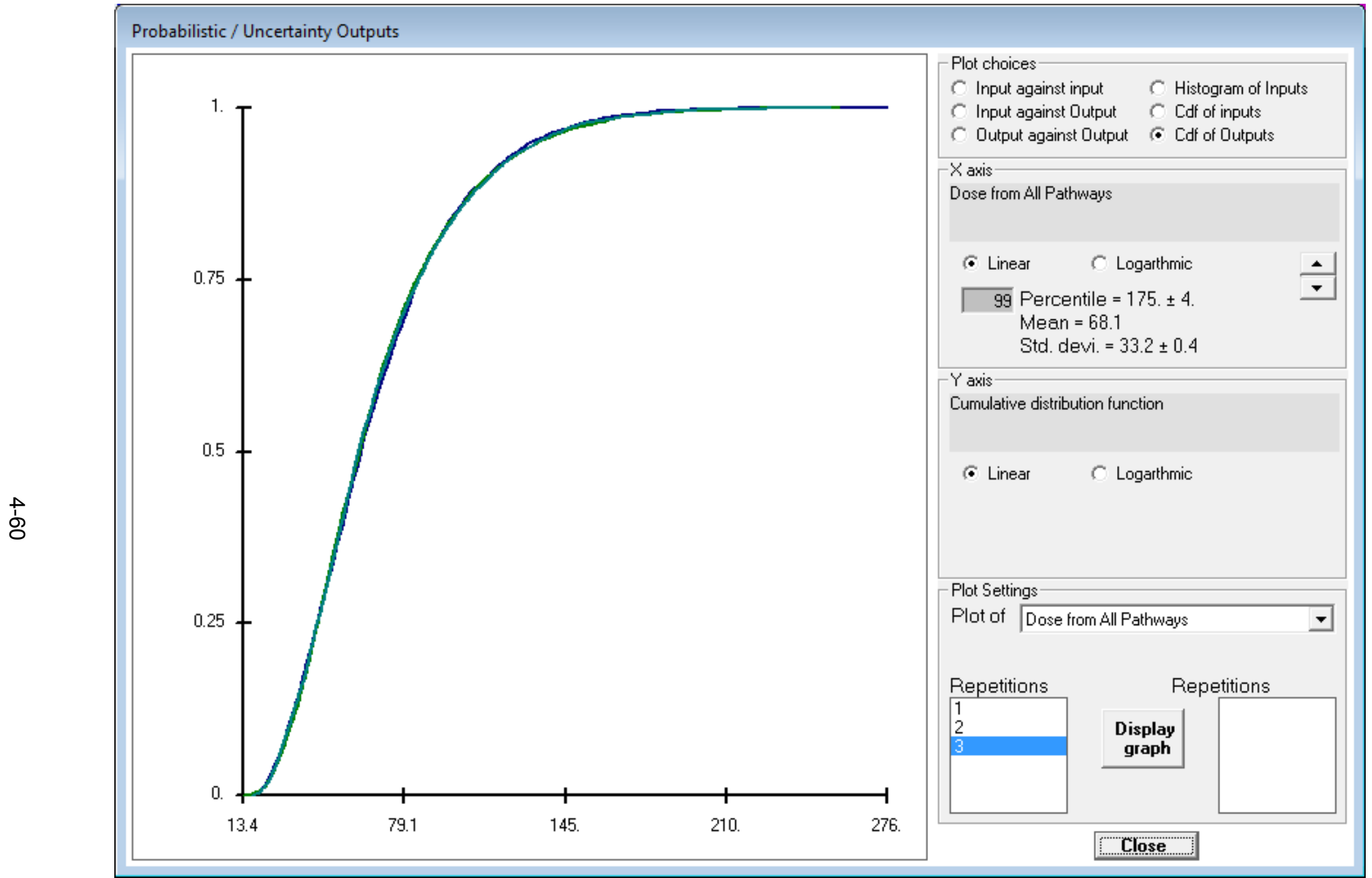

FIGURE 4.49 Cumulative Distribution Function of Peak Dose from the 5-input Verification Run of the Preliminary Sensitivity Analysis 


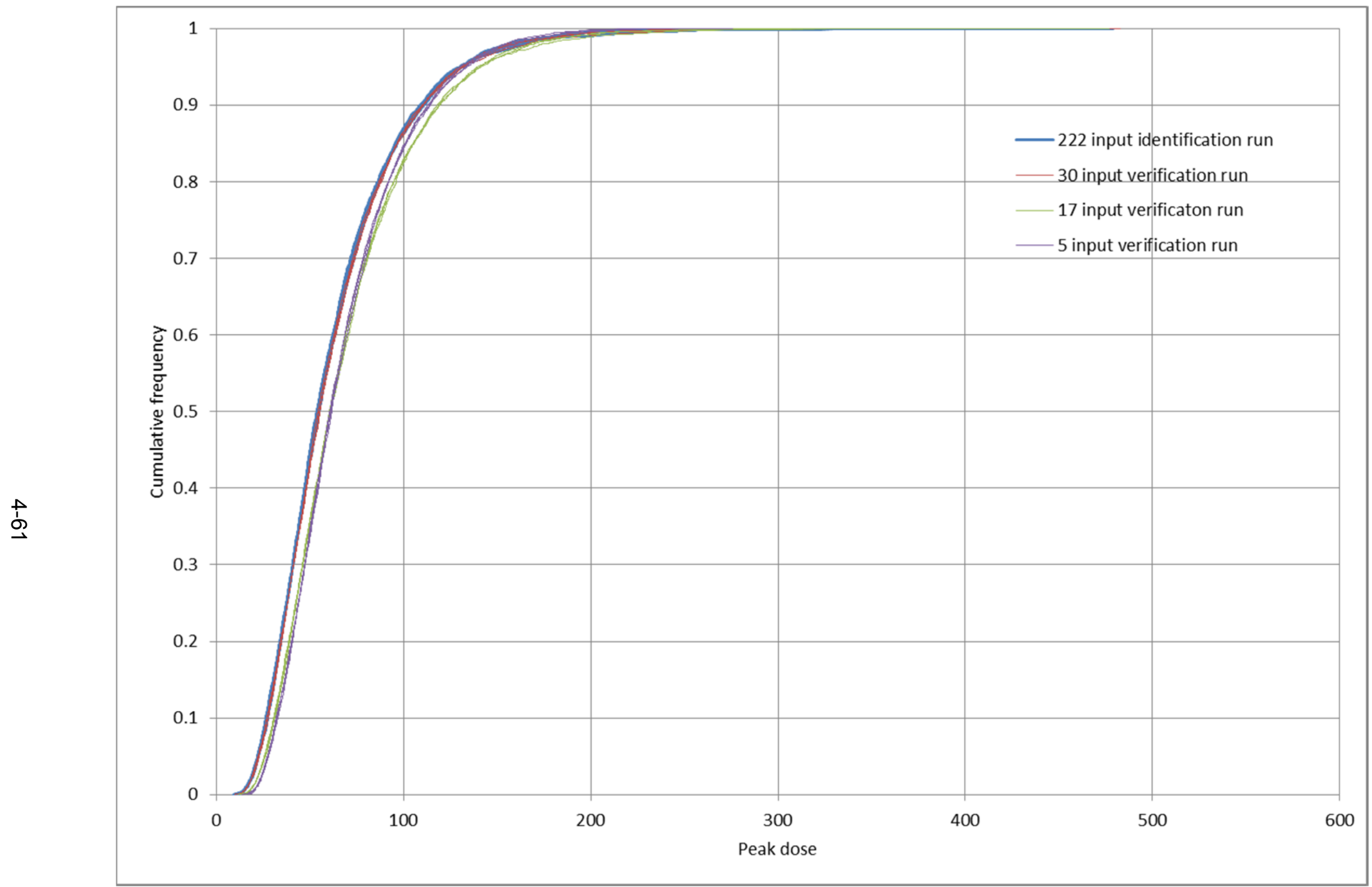

FIGURE 4.50 Cumulative Distribution Function of Peak Dose from Identification and Verification Runs 
This page is intentionally left blank. 


\section{References}

MARSSIM Manual, 2000, Multi-Agency Radiation Survey and Site Investigation Manual (MARSSIM), NUREG-1575, Rev. 1, EPA 402-R-97-016, Rev. 1, DOE/EH-0624, Rev. 1. , August.

Shen, H.S., and P.Y. Julien, 1993, "Erosion and Sediment Transport," in Handbook of Hydrology, Chapter 12, ed. D.R. Maidment, 12.1-12.61, New York: McGraw-Hill.

Sullivan, T.M., 1993, Disposal Unit Source Term (DUST) Data Input Guide, NUREG/CR-6041, BNL-NUREG-52375, prepared for the U.S. Nuclear Regulatory Commission, Brookhaven National Laboratory, Upton, N.Y., April.

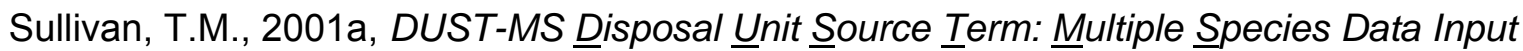
Guide, Brookhaven National Laboratory, Upton, New York, Jan.

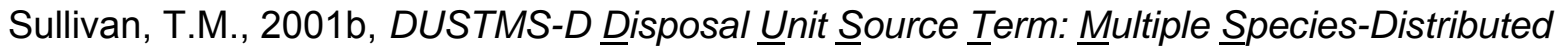
Failure Data Input Guide, Brookhaven National Laboratory, Upton, N.Y., Jan.

Yu, C., et al., 2001, User's Manual for RESRAD Version 6, ANL/EAD-4, Argonne National Laboratory, Argonne, III., July.

Yu, C., et al., 2007, User's Manual for RESRAD-OFFSITE Version 2, ANL/EVS/TM/07-1, Argonne National Laboratory, Argonne, III., June. 
This page is intentionally left blank. 


\section{Appendix A}

\section{Letter Report - Proposed Approach for the Task of Expanding the Source Term Model for RESRAD-OFFSITE}

January 22, 2010 



\section{Appendix A}

\section{Letter Report - Proposed Approach for the Task of Expanding the Source Term Model for RESRAD-OFFSITE}

\section{A.1 Background}

RESRAD-OFFSITE inherits and expands upon the objective of RESRAD (onsite) code for modeling the environmental fate and transport of radionuclides in contaminated soil and evaluating subsequent human radiation exposures through consideration of multiple exposure pathways. Several enhancements were incorporated into RESRAD-OFFSITE to extend the location of human receptors beyond the contaminated area, where receptors considered by RESRAD (onsite) would be confined. These enhancements include a source mass balance and mixing model, an air dispersion model, a one-dimensional advection with three-dimensional dispersion groundwater transport model, an offsite soil accumulation model, and a surface water mixing and dilution model. In addition to the enhancement of fate and transport models, RESRAD-OFFSITE was also equipped with the option to specify the temporal fluxes of radionuclide releases to air, to surface runoff, and to groundwater. This feature bypasses the calculation of release rates by RESRAD-OFFSITE so that in addition to releases from contaminated soils, releases from other sources (e.g., waste disposed in soils, emissions from effluent stacks, or discharges from wastewater pipelines, etc.) can be evaluated as well.

The approach proposed in this letter report has three objectives: (1) to provide more release mechanisms for the user to choose from; (2) to allow specification of the fraction of inventory that will be released by each mechanism; and (3) to model situations where the initial contamination is in containers, in addition to the current conceptualization of the initial contamination being in soil. If all the proposed approaches are adopted, the code will be able to model the long-term performance of waste disposal facilities and be applied to evaluate different disposal methods.

Two computer codes, DUST (Disposal Unit Source Term) and BLT (Breach, Leach, and Transport), designed to estimate radionuclide release rates from disposal facilities, provide the technical background for these additions. A brief overview of these two computer codes is presented in this letter report, with an emphasis on their methodologies for calculating source release rates. 


\section{A.2 Description of the Task Assignment}

The task proposal approved by the U.S. Nuclear Regulatory Commission (NRC) for the task of expanding the source term model for RESRAD-OFFSITE is reproduced as follows:

\section{Task 3 - Expand the source-term model for RESRAD-OFFSITE}

RESRAD-OFFSITE uses a pathway analysis method to relate radionuclide concentrations in soil to doses received by a member of the critical population group. Recent decommissioning activities have involved evaluating the exposure to materials other than soil (e.g., slags). In this task the contractor shall expand the current source term model to incorporate other source term release mechanisms or constraints such as:

- Solubility-Limits

- Surface-Wash Off or Surface Rinse

- Diffusion

- Dissolution / Uniform Dissolution

- Ion-Exchange

- Complex, non-uniform sources with variable size, depth, content, concentrations, and degradability

This task may be accomplished by incorporating previously developed codes (e.g., DUST, BLT), modifying the current source-term model within RESRAD-OFFSITE, or some other method suggested by the contractor and agreed upon by NRC. NRC will provide the source codes (e.g., DUST, BLT) to the contractor for incorporation into the RESRAD-OFFSITE code.

Subtask A - The contractor shall develop an approach for incorporating the mechanisms and constraints listed above into the current RESRAD-OFFSITE code.

Subtask B - Upon review and approval by NRC staff, the contractor shall incorporate the agreed upon approach for expanding the source term model into the RESRAD-OFFSITE software.

Subtask C - The contractor shall perform verification testing on RESRAD-OFFSITE by developing test cases to evaluate the expanded source term capabilities. This includes entering input data, running the code, and verifying that the mathematical formulations are captured in the code and that the output correctly reflects these formulations.

Subtask D - The contractor shall submit a report for publication as a NUREG/CR consisting of the technical basis for the source term model, a User's Guide, and documentation of the verification. 
Subtask E - The contractor shall benchmark the expanded source term model against other codes that evaluate similar release mechanisms.

The anticipated level of effort for this task is 1,200 staff hours (or 8.0 staff months). This estimate is higher than the original NRC estimate of 600 staff hours (per NRC's Statement of Work of September 12, 2008) based on the following rationale:

1. This task contains a substantial amount of effort (Subtask A through $E$ ),

2. This task entails a major increment in development to the existing RESRAD-OFFSITE Code; new modules are required to be developed to accommodate the requirements,

3. Interfacing with external codes (e.g., DUST, BLT) is needed to facilitate the task performance,

4. Additional code testing is required to ensure code functionality and maintain proper QA procedures.

In the case that the selected source term models or codes require significant modification of other parts of the RESRAD-OFFSITE code (e.g., the transport model needs to be changed from analytical solution to numerical solution), then significant increase in effort will be required.

The draft NUREG/CR report of this task (Task 3 ) is scheduled to appear 12 months after approval by NRC of the proposed approach (this letter report).

\section{A.3 Considerations for Expanding the Source Term Model}

The source term model currently implemented in RESRAD-OFFSITE computes three releases from the contaminated zone and also the concentration and distribution of the radionuclides within the contaminated zone. The model calculates the atmospheric release of particulates due to resuspension and volatiles due to diffusion and evapotranspiration, the surface water release due to erosion by runoff water, and the groundwater release due to leaching by infiltrating water. The release rates are used by the code to compute the exposure at offsite locations, while the concentration and distribution of radionuclides within the contaminated zone are used to compute the direct external exposure from the primary contamination as well as any exposure from onsite activities.

The integration and implementation of the new methodology to improve the current source term model involve the consideration of preserving the functions of the current model. While the DUST and BLT codes provide methodologies to simulate groundwater releases from radioactive wastes disposal in soils with more realistic considerations of container disintegrations over time and transport of radionuclides within the contaminated zone (disposal cell), incorporating the methodologies entirely in RESRAD-OFFSITE would disable the functions of calculating atmospheric and surface water releases. If release from containerized contamination is to be modeled in RESRAD-OFFSITE, the code will need to have two options in order to preserve 
such functions. The first option is to use the RESRAD formulations for uniform contamination in uncontained soil and the second option is to consider the release to groundwater from containerized contamination.

Another issue involved in the integration and implementation of the new methodology involves consistency and compatibility with the existing model. RESRAD-OFFSITE has transport models for both the unsaturated and saturated zones. These models are different from the ones included in the DUST and BLT codes. RESRAD-OFFSITE also implements a complicated methodology to track ingrowth and decay of radionuclides having multiple progeny radionuclides with different decay branches. Because the capability of handling radioactive decay and tracking parent and progeny radionuclides during environmental transport are critical in the evaluation of subsequent radiation exposures, it is desirable to preserve these functions while adding new capabilities to the code.

Brief overviews and comparisons of the current source term model in RESRAD-OFFSITE and those used in DUST and BLT are presented in the following sections. After the overviews and comparisons, the pros and cons of the two different approaches (linking vs. inclusion) of expanding the current source term model are discussed, along with the proposal of tasks required for implementing these approaches.

\section{A.4 Overview of the Current Source Release Model in RESRAD-OFFSITE}

The current source term model assumes a homogeneous distribution of radionuclides within an initially contaminated soil layer of uniform thickness. It accounts for radiological transformations (decay and ingrowth). The leaching of radionuclides in the contaminated layer by infiltration is modeled as a first order rate-controlled process occurring over the depth of the contamination. The code does not model the transport of the release within the contaminated zone; all of the material that is leached at any time is assumed to leave at the bottom of the contamination at that time. It also considers mixing in a surface layer. The concentration in the mixing layer is affected by erosion release to runoff and by leaching. Erosion is modeled using the Universal Soil Loss Equation. Release to the atmosphere is computed assuming that the clean dust that settles on the primary contamination is balanced by the release of an equal amount of contaminated dust. 


\section{A.5 Treatment of Radiological Transformations in RESRAD-OFFSITE}

The code currently uses the information in International Commission on Radiological Protection Publication 38 (ICRP-38) to construct the decay sequences of the radionuclide being analyzed. The codes in the RESRAD family are being modified to use the information in ICRP-107. The user of the code specifies a cutoff half-life. The fate and transport of all transformation products that are of a half-life greater than or equal to the user-specified cutoff are modeled explicitly. Any transformation product that has a half-life that is less than the cutoff is assumed to be in secular equilibrium with its immediate parent whose half-life is greater than the cutoff. Depending on the radionuclide being analyzed and the choice of the cutoff half-life, the transformation products may form a single chain. If the radionuclide being analyzed or any of its progeny transform into multiple radionuclides, then, depending on the cutoff half-life, there is the possibility that the transformation sequence forms a complicated mesh or net rather than a linear chain. In this event, the RESRAD-OFFSITE code enumerates all of the potential transformation threads; each transformation thread starts with the radionuclide being analyzed and ends at one of the stable transformation products. The thread fraction associated with each transformation thread describes the fraction of the quantity of the radionuclide being analyzed that follows that transformation thread. The computational code in RESRAD-OFFSITE computes the radiological consequences of each of the transformation threads and combines them to model the exposure from the radionuclide being analyzed.

\section{A.6 Impact of Some of the Desired Source Release-to- groundwater Mechanisms and Processes on the Conceptual Model for the Contaminated Soil Layer and on Other Releases}

Some of the desired processes and mechanisms for release from a contaminated soil layer to groundwater are: (1) advective and diffusive transport by water in the contaminated zone, (2) solubility equilibrium release, (3) solubility rate-controlled release, (4) adsorption-desorption equilibrium release, and (5) desorption rate-controlled release.

The different groundwater release mechanisms and processes lead to different concentration profiles over the depth of the contaminated soil layer. Thus, they also affect the other two releases in RESRAD-OFFSITE (release to the atmosphere and release by runoff to a surface water body) and the exposure from the primary contamination.

The impact of the above five desired release mechanisms on the contaminated soil layer is discussed as follows: 
(1) Advective and diffusive transport by water in the contaminated zone. The unsaturated zone transport model can be used to model advective and diffusive transport in the contaminated zone. All of the properties required for the unsaturated zone will have to be input for the contaminated zone as well.

(2) Solubility equilibrium release. The equilibrium solubility concentration of all isotopes (both stable and radioactive) of an element will change as the chemical conditions of the contaminated zone change over the time period of analysis. Information about the inventory of all stable and radioactive constituents of the contaminated zone and a whole host of chemical properties of the contaminated zone would have to be fed to a chemical equilibrium model to model the chemical conditions in the contaminated zone. The chemical equilibrium model would need to be run a number of times over the period of analysis. This level of detail is not compatible with the level of detail of the rest of RESRAD-OFFSITE. Such a level of detail would require a lot of current and future information about the site, also requiring a considerable computer resource to model the situation. A simple solubility equilibrium release model would use a constant release concentration for the radionuclide, which would be a user input. The clean water entering at the top of the contamination would rapidly dissolve the radionuclides there to reach the equilibrium concentration. The release would therefore occur at the top of the contaminated zone and would pass through the contaminated zone without apparent interaction with the soil in that zone; a distribution coefficient of zero would be appropriate for the zone. The removal of radionuclides by dissolution from the top would create a clean cover at the top. The rate at which the clean cover is created would differ from element to element. The creation of this clean cover would have to be tracked if onsite exposure or releases to the atmosphere or erosion releases by runoff are to be modeled.

(3) Solubility rate-controlled release. If the time the infiltrating water spends in the contaminated layer is shorter in comparison to the time needed to reach solubility equilibrium, the release might be governed by the rate of dissolution. The rate of dissolution of all isotopes (both stable and radioactive) of an element will change as the chemical conditions of the contaminated zone changes over the time period of the analysis. It will also change as the concentration in the infiltrating water increases as it percolates deeper into the contaminated zone. This level of detail is not compatible with the level of detail of the rest of RESRADOFFSITE. A simple solubility rate-controlled release would use an experimentally measured or computationally estimated release rate for the radionuclide, which would be a user input. This rate is assumed to be constant over time and over the depth of the contamination. The release would therefore occur over the entire depth of the contaminated zone and would pass through the contaminated zone without apparent interaction with the soil in that zone, so a distribution coefficient of zero would be appropriate for the zone. 
(4) Adsorption-desorption equilibrium release. The adsorption-desorption equilibrium concentration of all isotopes (both stable and radioactive) of an element, in the aqueous phase, will change as the chemical conditions of the contaminated zone change over the time period of the analysis. Information about the inventory of all stable and radioactive constituents of the contaminated zone and a whole host of chemical properties of the contaminated zone would have to be fed to a chemical equilibrium model to model the chemical conditions in the contaminated zone. The chemical equilibrium model would need to be run a number of times over the period of analysis. This level of detail is not compatible with the level of detail of the rest of RESRAD-OFFSITE. Such a level of detail would require a lot of current and future information about the site and would require a considerable computer resource to model the situation. A simple adsorption-desorption equilibrium release model would use a constant distribution coefficient for the radionuclide, which would be a user input. The radionuclides at the top of the contamination would rapidly desorb into the clean water entering the contaminated zone to reach the adsorption-desorption equilibrium concentration. The release would therefore occur at the top of the contaminated zone and would pass through the contaminated zone without apparent interaction with the soil in that zone. The user-specified distribution coefficient would be applicable for the zone, but there would be no net adsorption by the rest of the contaminated zone as the concentration in the liquid phase is what would be in equilibrium with the nuclides in the solid phase. The removal of nuclides by desorption from the top would create a clean cover at the top. The rate at which the clean cover is created would differ from element to element. The creation of this clean cover would have to be tracked if onsite exposure or releases to the atmosphere or erosion releases by runoff are to be modeled.

(5) Desorption rate-controlled release. If the time the infiltrating water spends in the contaminated layer is short in comparison to the time needed to reach adsorption-desorption equilibrium, the release might be governed by the rate of desorptions. The rate of desorption of all isotopes (both stable and radioactive) of an element will change as the chemical conditions of the contaminated zone changes over the time period of the analysis. It will also change as the concentration in the infiltrating water increases as it percolates deeper into the contaminated zone. This level of detail is not compatible with the level of detail of the rest of RESRADOFFSITE. A simple desorption rate-controlled release would use an experimentally measured or computationally estimated desorption rate for the radionuclide, which would be a user input. This desorption rate is assumed to be constant over time and over the depth of the contamination. The release rate, which is the product of the desorption rate and the radionuclide concentration, will vary with time, but will be constant over depth at any time. The release would therefore occur over the entire depth of the contaminated zone and would pass through the contaminated zone without apparent interaction with the soil in that zone, so a distribution coefficient of zero would be appropriate for the zone. This would be similar to the current leach rate model, but would need to include transport through the contaminated zone. 


\section{A.7 Overview of the Methodologies in DUST and BLT}

The above discussion applies to uncontained contaminated soil. Contaminants in containers will not be released until the walls of the container deteriorate and infiltrating water is able to penetrate the container. The two candidate codes suggested by the NRC for consideration to expand the current source term model evaluate the deterioration of the containers and the subsequent release by different release mechanisms.

\section{A.7.1 DUST Model}

The DUST model was developed by Brookhaven National Laboratory (BNL) for the NRC (Sullivan 1993). It estimates contaminant release rates from the disposal facility by solving contaminant transport equations that describe mass balance in a finite volume along the depth of the disposal facility with considerations of convective flow, diffusion and dispersion, radiological decay, and release from waste containers [Eq. (1)]. Two models were implemented in DUST to obtain numerical solutions to the transport equations - one is called the Multi-Cell Mixing Cascade (MCMC) model; the other is called the Finite Difference (FD) model. The MCMC model divides the modeled domain into mixing cells along the transport path; concentration within each mixing cell is homogeneous, and each cell has the same size and transport properties. However, container performance and wasteform release may vary between mixing cells. By neglecting dispersion and diffusion, contaminant concentrations in each mixing cell are solved analytically and expressed as functions of time. 


$$
\frac{\partial}{\partial t}(R \theta C)=\frac{\partial}{\partial x}\left(\theta D \frac{\partial C}{\partial x}\right)-\frac{\partial}{\partial x}\left(V_{D} C\right)-\lambda \theta R C+q
$$

where

$R \quad=$ retardation coefficient,

$R=1+\frac{\rho K_{d}}{\theta}$

$\rho=$ bulk density of the solid;

$K_{d}=$ soil/water distribution coefficient;

$\theta \quad=$ the volumetric moisture content of the region (dimensionless);

$C \quad=$ solution concentration;

$D \quad=$ the diffusion - dispersion coefficient,

$D=D_{\text {eff }}+\frac{a_{t}\left|V_{D}\right|}{\theta}$

$D_{\text {eff }}=$ effective diffusion coefficient;

$a_{t} \quad=$ dispersivisity coefficient;

$V_{D} \quad=$ Darcy velocity;

$\lambda \quad=$ radioactive decay constant;

$S \quad$ = adsorbed concentration, the mass adsorbed per unit mass of the solid;

$q \quad=$ source/sink term used to model release from the wasteform and external sources, e.g., ingrowth due to radioactive decay of parent nuclide.

The FD model divides the modeled domain into finite regions called controlled volumes. A mass balance is performed for each controlled volume by approximating the derivative terms in Eq. (1) using finite differences. This results in a set of coupled algebraic equations that must be solved numerically to obtain contaminant concentrations. The FD model is a generalization of the MCMC model and permits differences in transport properties in each controlled volume. In addition to different transport properties, the FD model also allows the consideration of more general wasteform releases.

To solve the transport equation [Eq. (1)], information on fluid flow must be provided (to determine the Darcy velocity, $\left.V_{D}\right)$. A constant infiltration rate is restricted for the MCMC model, while for the FD model, it can be a function of time through tabular input. In some cases, the gaseous pathway may be important. To consider gas flow, it is necessary to supply an average gas advection velocity in order to calculate the gaseous release. 
The source/sink term in Eq. (1) must be evaluated before the transport equation can be solved. This involves the consideration of container degradation. In the DUST model, two types of container failure are modeled-general failure and localized failure. In a general failure, the container prevents water ingress to the waste until failure, at which time the container no longer provides a barrier to water flow. The container failure time is provided by the users; for metallic containers, it can be estimated as the thickness of the container divided by the time-averaged corrosion rate. In addition to general failure, partial/localized failure prior to general failure can also be considered. For a partial/localized failure, only a small portion of the container will permit water access to the wasteform. This reduction of water flow will impact the amount of contaminant released and available for transport. Consideration of a partial/localized failure is implemented through the calculation of the breached area with the specified number of localized failures per unit area of container, total container area, and two coefficients that are related to the soil conditions as well as container material. The partial/localized failure can be considered only when the FD model is selected.

Regarding the release of contaminants from the wasteform, four release mechanisms are considered in the DUST code. They are (1) solubility-limited, (2) surface wash-off subject to partitioning, (3) diffusion, and (4) uniform release (e.g., dissolution). A combination of the four release mechanisms can be specified with the fractional amount of mass released by each mechanism. The solubility-limited release is modeled by allowing an instantaneous release of radionuclides into solution until the limit is reached or the entire inventory is released. Users must supply the solubility limit. The surface-rinse model assumes that the radionuclides in the wasteform are available for release as soon as water contact occurs. To use this option, a partitioning factor, which is an equilibrium ratio relating the amount of contaminant on the wasteform to the amount in solution, must be assigned. The diffusion mechanism can be considered only when the FD model is selected. Diffusion-controlled release is characterized by relatively high release rates at early times, which continually decrease over time. The release rate is calculated by analytically solving the diffusion equation corrected for decay, Eq. (2). The effective diffusion coefficient is supplied by the users. The uniform release mechanism is termed the dissolution mechanism in BLT. Besides the release of activated metals, which undergo corrosion in the environment, the release of a wide variety of waste streams/wasteforms may be described by this mechanism. To use this mechanism, a fractional release rate, which is the fraction of the mass in the wasteform that is released per unit time, must be specified. 


$$
\begin{aligned}
\frac{\partial C}{\partial t} & =\nabla \cdot D \nabla C-\lambda C \\
Q(t) & =\int d S \cdot J_{S} \\
J_{S} & =-D \frac{\partial C\left(x_{s}\right)}{\partial x}
\end{aligned}
$$

where

D = the effective diffusion coefficient;

$\boldsymbol{Q}(\boldsymbol{t})=$ the mass relase per unit time;

$\boldsymbol{J}_{\boldsymbol{S}} \quad=$ the mass flux at the surface of the wasteform; and

$\boldsymbol{x}_{\boldsymbol{s}}=$ the surface of the wasteform.

As the waste containers fail, water enters through the breached area and flows out with the dissolved contaminants. The container water flow rate is calculated as the Darcy velocity multiplied by the ratio of the breached area to the total area. The failed container is treated as a mixing cell in which radionuclides released from the wasteform are uniformly mixed. The release rate from the container is the product of the water flow rate through the container and the mixing cell concentration as calculated based on the various release mechanisms.

Contaminant concentration in the water leaving the disposal area can be obtained by solving Eq. (1) with the MCMC or FD model. This concentration multiplied by the water infiltration rate gives the release rate of radionuclides from the disposal facility, which can be input to RESRADOFFSITE for subsequent evaluation of transport in the underlying soils and groundwater aquifer.

\section{A.7.2 BLT Model}

The BLT model (Suen and Sullivan 1990) is very similar to the DUST model. It was also developed by BNL for the NRC to calculate the release rates of contaminants from a shallow land burial facility. The release mechanisms considered by BLT are the same as those considered by DUST, except that the solubility limit considered in DUST is applied as a concentration limit for the surface rinse (wash-off) mechanism in BLT. While DUST employs the MCMC and FD methods to solve the governing differential equation (Eq. 1) for radionuclide transport, BLT employs a two-dimensional (2-D) finite element method to solve the equation.

The BLT model consists of four individual compartments: (1) water flow through the waste disposal structure (typically a trench), (2) corrosion and subsequent breaching of waste containers, (3) leaching of radionuclides from the wasteform, and (4) transport of radionuclides to the boundary of the disposal structure. For the first and the last compartments, two existing 
2-D finite-element codes, FEMWATER and FEMWASTE, were modified and used. The implementation of the second and third compartments was accomplished by the design of two FORTRAN subroutines, one called BREACH and the other called LEACH. Together these two subroutines generate data for the source term, $q$, in Eq. (1) needed to solve the transport equation.

The BLT code was constructed on a revised version of FEMWASTE. To use BLT, a corresponding version of FEMWATER must first be executed with the pertinent geometry, hydraulic properties, and boundary conditions. The resulting information in water flow velocity and moisture content is then written into a file and used by BLT. Based on parameters input by the user (thickness of the waste container, surface area of the container, pitting parameters, area scale factor, number of pits per container, general corrosion rate, and clay content, $\mathrm{pH}$, and aeration index of the soil) as well as the results from FEMWATER, the BREACH subroutine calculates the time and amount (in terms of breached area) of container corrosion, which are accessed by the LEACH subroutine to calculate the radionuclide release rate from the waste along with the wasteform parameters provided by the user. The BREACH and LEACH subroutines were directly incorporated into a modified version of FEMWASTE, so that the computed radionuclide release rates feed back directly to the contaminant transport portion of the code as time-dependent sources. The modified and extended code is abbreviated as BLT to distinguish it from the original FEMWASTE code.

Three parallel radionuclide release mechanisms are modeled by the subroutine LEACH. They are (1) the surface rinse process, which releases all of the radionuclides residing on the surface of the wasteform limited by their solubility; (2) the diffusion process, which accounts for the diffusional transport of radionuclides through the pore water within the wasteform; and (3) the dissolution process, which frees the radionuclides in the bulk solid by dissolving the solid phase. As mentioned previously, these three mechanisms are the same as the surface wash-off, diffusion, and uniform dissolution mechanism, respectively, considered in DUST.

\section{A.8 Comparison of the Computational Methodology for Transport in RESRAD-OFFSITE with the Computational Methodologies for Transport in DUST and BLT}

RESRAD-OFFSITE uses analytical expressions to model the transport of radionuclides in soil. Many of these analytical expressions are too complex to evaluate analytically and are evaluated numerically. This computational methodology is compatible with the one-dimensional, analytical MCMC model in DUST. Thus, the formulations and coding of MCMC would be best suited for inclusion in the RESRAD-OFFSITE code. The one-dimensional nature of the finite-difference methodology of DUST is compatible with the one-dimensional transport assumptions of RESRAD-OFFSITE in the unsaturated zone. Thus, if the codes are to be linked externally, 
either of the two methods in DUST would be compatible with RESRAD-OFFSITE. The analytical groundwater transport model that is currently in RESRAD-OFFSITE requires the contaminant input flux into the water table to be uniform over the footprint of the primary contamination. It therefore will not be able to use any spatially varying output from the 2-D finite-element formulations in BLT. If RESRAD-OFFSITE is to be linked with BLT for the release and transport from the initially contaminated area to the water table, it will also be necessary to link in a third code to model the transport in the aquifer. Thus, BLT is not a preferred candidate for direct linking with or inclusion in RESRAD-OFFSITE at this time.

\section{A.9 Pros and Cons of Linking versus including the Coding Directly into the RESRAD-OFFSITE Code}

Three approaches are proposed in the next section of this report to add the desired release options to the RESRAD-OFFSITE code. The first is to derive and implement the formulations for the desired mechanisms of release from contaminated soil. The second and third approaches involve using external codes that model these releases from contamination in containers. There are two ways of using an external code that already models the releases from containerized contamination: (1) link that code (executables) to RESRAD-OFFSITE, or (2) incorporate parts of the source code from that code into the source code of RESRADOFFSITE. The pros and cons of linking and including external codes to RESRAD-OFFSITE are discussed as follows.

(1) The RESRAD-OFFSITE interface can be expanded to gather the inputs needed for the two candidate codes for computing release. The interface can write the inputs in the format required by the candidate codes and then launch it. When the release code has finished computations, the interface would read in the output, reformat it in the form needed by RESRAD-OFFSITE, and then launch RESRAD-OFFSITE. If the candidate code does not provide the full suite of releases (groundwater release, atmospheric release, and erosion release) or if it does not provide information about the concentration profile in the primary contamination, the exposure from those will not be computed by RESRAD-OFFSITE.

A deep understanding of the formulations of the candidate codes is not necessary, if they are to be linked through the RESRAD-OFFSITE interface to the RESRAD-OFFSITE computational code. The code is likely to run slowly because of the need to write the output from the release code to a file and the need to read the information from the file into RESRAD-OFFSITE. Probabilistic analysis will not be available on the inputs that affect the release. 
(2) The source code or the formulations of the candidate code can be integrated into the RESRAD-OFFSITE computational code. The container degradation and nuclide release mechanisms in the candidate code can be integrated with the (unsaturated zone) transport mechanisms in RESRAD-OFFSITE. Parts of the candidate code can be placed with any necessary modification into the RESRAD-OFFSITE computational code. It likely will not be possible to model release to the atmosphere and the erosion release by runoff because of incompatibility between the two models.

The formulation and coding of the candidate codes must be understood in detail in order to integrate the formulations of the candidate code with the transport formulations in RESRADOFFSITE and in order to integrate the coding of the candidate code into the RESRAD-OFFSITE coding. The code will run faster than the first method (linking code) because the information about the releases and concentration profiles will be available within the code (in memory). It will be possible to perform a probabilistic analysis on the inputs that define the release.

\section{A.10 Proposed Approaches}

The three different approaches proposed here are not mutually exclusive. Different tasks need to be performed to implement the different approaches for expanding the existing source term model.

\section{A.10.1 Approach 1: Include analytical expressions for the releases to the three media (air, runoff, and infiltration) from uncontained contamination in soil and the analytical expression for the vertical concentration profile within that contamination under the various groundwater release mechanisms.}

This approach will include the derivation of analytical formulations to compute all of the releases and concentration profile information needed by RESRAD-OFFSITE for uncontained contamination in soil with respect to the release mechanisms described in pages A-5 through A-7 of this report. The RESRAD-OFFSITE code will then be modified to implement these analytical formulations. This would involve deriving formulations for the releases to atmosphere, groundwater, and surface runoff and also for the vertical concentration profile in soil and then adding code for the formulations.

The advantages and disadvantages of Approach 1 are as follows: (1) The capability of evaluating exposure from contamination in soil is enhanced. This also provides a means, if Approach 2 and/or 3 are adopted, for estimating exposure directly from the contamination in deteriorated containers and from release to the atmosphere from contamination in deteriorated containers. (2) The code will run in a reasonable amount of time on currently available personal computers. (3) The code will have the capability to perform both probabilistic and sensitivity 
analyses on the inputs that affect the release of the contamination. The probabilistic analysis will run in a tolerable amount of time. (4) None of the current capabilities of RESRAD-OFFSITE will be lost. (5) Need to derive a number of formulations. (6) Need to code the formulations.

(7) Need to find a similar code to benchmark against.

\section{A.10.2 Approach 2: Link the one-dimensional finite-difference DUST code to RESRAD-OFFSIE via the RESRAD-OFFSITE interface.}

This approach will need the executables of the one-dimensional finite-difference DUST code. The RESRAD-OFFSITE interface will be expanded to gather all of the necessary inputs, to prepare an input file for DUST, and to execute DUST to produce the release from the contaminated layer. The interface will then reformat the DUST output (of the release) to the format required by RESRAD-OFFSITE and it will run the RESRAD-OFFSITE computational code, flagging it to suppress the source module and to read in the DUST output.

The advantages and disadvantages of Approach 2 are as follows: (1) Additional coding will be needed to reformat the DUST output to the format that is required by RESRAD-OFFSITE, especially with regard to the transformation threads. (2) The execution time will likely be longer than for the third approach. (3) The code will not have the capability to perform a probabilistic or sensitivity analysis on the inputs that affect the deterioration of the container and the release of the contamination. (4) There is no need to recode the container degradation and release formulations. (5) Transport in the contaminated layer may not be modeled in a manner that is consistent with the modeling of transport in the unsaturated and saturated zones. (6) Direct exposure from the initial contamination will not be modeled. (7) There will be no release to the atmosphere and erosion release by runoff. (8) Only need to verify that the RESRAD-OFFSITE interface correctly creates the input file for DUST.

\section{A.10.3 Approach 3: Include the analytical expressions for the deterioration of the container and the subsequent release into the RESRAD-OFFSITE code.}

This approach will need the source code for the MCMC formulations from the DUST code, combine the container deterioration and release formulation/coding of DUST with the analytical groundwater transport formulations in RESRAD-OFFSITE, and incorporate it in the computational code of RESRAD-OFFSITE.

The advantages and disadvantages of Approach 3 are as follows: (1) All relevant transformation threads will be considered and combined in RESRAD-OFFSITE without the need for additional coding to keep track of the contributions of the threads. (2) The code will run in a reasonable amount of time on currently available personal computers. (3) The code will have the capability to perform both probabilistic and sensitivity analyses on the inputs that affect the 
deterioration of the container and the release of the contamination. The probabilistic analysis will run in a tolerable amount of time. (4) An informed RESRAD-OFFSITE user will be able to use this enhanced code. (5) There is no need to recode the container degradation and release formulations; it may be necessary to change some of the statements if DUST and RESRADOFFSITE were not written for the same version of FORTRAN or were not compiled with the same options. It will be necessary to code the combination for the release with the transport in the contaminated zone. (6) Transport in the contaminated layer will be modeled in a manner that is consistent with the modeling of transport in the unsaturated and saturated zones. (7) The code can be benchmarked against DUST.

If the source code of the MCMC formulations from the DUST code is not available, write the code for the analytical formulations in the references [Sullivan et al 1998, Sullivan, T.M., 2001] and combine with the groundwater transport formulations in RESRAD-OFFSITE. This likely will require more time than in the case where source codes are available.

\section{A.11 Comparison of the Three Approaches}

Approach 1, including the formulations of the various release options to uncontained contamination, will enhance the code while preserving all of the current capabilities of RESRADOFFSITE. This will provide more options for release to groundwater while evaluating exposure from all of the current exposures pathways in RESRAD-OFFSITE. It will be possible to perform probabilistic and sensitivity analyses on all of the new inputs.

Approach 2, linking of the one-dimensional finite-difference DUST code to RESRAD-OFFSITE, is expected to be the easiest to implement. All three approaches will require coding to the user interface to allow specification of the additional inputs. In addition, this approach will require coding to prepare the input file for DUST from the interface and coding to reformat the output of DUST to a form that is useable by RESRAD-OFFSITE. Drawbacks include the inability to perform sensitivity and probabilistic analyses and the ability to model only the exposure from the release to groundwater.

Approach 3, incorporating the analytical formulations for container degradation and release into RESRAD-OFFSITE, will be more difficult to implement than Approach 2. It will be necessary to understand the coding to properly combine it with the transport formulations in RESRADOFFSITE, to make the necessary changes to ensure that the imported coding is compatible with the FORTRAN complier used in RESRAD-OFFSITE. While it will be possible to perform sensitivity and probabilistic analyses, this approach will also be limited to modeling exposure from the release to groundwater. 
It would be preferable to include more than one approach to allow users the flexibility of choosing the option appropriate for their site. The preferred proposed task is to implement Approach 1 (provide more release options for uncontained contamination in soil). If it is desired to include the ability to model the release from containerized contamination in RESRAD-OFFSITE, Approach 2 (link the one-dimensional finite-difference DUST code to RESRAD-OFFSITE) can be implemented. Some additional funding may be needed to complete both Approach 1 and 2. If it is also desirable to be able to perform a sensitivity/probabilistic analysis on the new inputs, then Approach 3 will have to be implemented. However, significant effort will be required to accomplish this task.

\section{A.12 References and Bibliography for Appendix A}

Suen, C.J., and T.M. Sullivan, 1990, BLT: A Source Term Computer Code for Low-level Waste Shallow Land Burial, BNL-NUREG-43927, DOE90-008520, Brookhaven National Laboratory, Upton, N.Y.

Sullivan, T.M., 1993, Disposal Unit Source Term (DUST) Data Input Guide, NUREG/CR-6041, BNL-NUREG-52375, Brookhaven National Laboratory, Upton, N.Y.

Sullivan, T.M., 2001, DUSTMS-D Disposal Unit Source Ierm: Multiple Species-Distributed Failure Data Input Guide, Brookhaven National Laboratory, Upton, N.Y.

Sullivan, T.M., C.R. Kempf, C.J. Suen, and S.M. Mughabghab, 1988, Development of a General Model to Predict the Rate of Radionuclide Release (Source Term) from a Low-level Waste Shallow Land Burial Facility, BNL-NUREG-40880, DOE88-007047, Brookhaven National Laboratory, Upton, N.Y.

Sullivan, T.M., R.J. MacKinnon, R.R. Kinsey, A. Aronson, and M. Divadeenam, 1996, BLT-MS (Breach, Leach, and Transport-Multiple Species) DATA INPUT GUIDE, NUREG/CR-6492, BNLNUREG-52509, Brookhaven National Laboratory, Upton, N.Y. 
This page is intentionally left blank. 


\section{Appendix B}

\section{Additional Benchmarking of Radionuclide Release Rates}





\section{Appendix B}

\section{Additional Benchmarking of Radionuclide Release Rates}

In Chapter 3, the source release rates calculated by the new source term model implemented in RESRAD-OFFSITE Version 3 code are compared with those obtained by the DUST-MS code. Although the results indicated very good agreement between these two codes, there are some minor discrepancies especially when longer decay chain results were compared. In these cases, it was difficult to pinpoint for certain exactly what caused the discrepancy. Hence, a third code, GoldSim, was employed to compare the release rates using the test cases described in Chapter 3. The GoldSim code is commercially available software designed as a generalpurpose Monte Carlo simulator for modeling complex systems in business, engineering, and science (GoldSim 2010a,b). It is equipped with a graphical user interface that helps users build, link, and dynamically simulate their models. GoldSim provides several specialized extension modules that provide additional features or functionality for particular applications. One of these, the Contaminant Transport Module for Radionuclides (RT Module), allows users to simulate the transport of contaminants in the environment and was used to generate source release rates for this comparison. The GoldSim calculations were not carried out for each comparison case under different conditions as presented in Chapter 3; however, the limited comparison with GoldSim results provides additional perspective to the RESRAD-OFFSITE source term model.

\section{B.1 Release and Environmental Transport Modeling with GoldSim}

The GoldSim (2010c) RT module solves for the movement of contaminant mass through the environmental system. It simulates the fate of mass within each system. The user can create an environmental system by defining a network of transport pathways, which are connected through mass flux links. There are two major mass flux links: advective and diffusive. The advective mass flux link was used in this comparison.

In GoldSim, there are three ways to introduce mass into a system: (1) an initial mass that exists within the system from the beginning, (2) a continuous addition of mass whose rate can be directly specified to one or more pathways in the system, and (3) a discrete addition of mass that can be assigned to one or more pathways at specific times. In addition to the above three ways, the user can define sources with specific properties and, based on the properties, 
GoldSim computes the release rates of mass over time and feeds them to the pathways that are linked to/associated with the sources.

The GoldSim transport pathways represent physical components through which contaminant species can move, such as aquifers, lakes, sediments, surface soil compartments, and the atmosphere. The Contaminant Transport Module of GoldSim provides five different pathway elements for use in modeling the transport of contaminants through these components. They are (1) cell, (2) aquifer, (3) pipe, (4) external, and (5) network elements. Three of these elements (cell, aquifer, and pipe) were used to construct source release and transport models for comparison with RESRAD-OFFSITE.

The Cell element is mathematically equivalent to a mixing cell and can be used to explicitly represent partitioning of contaminants among different phases/media, with the constraint of solubility limits.

The Aquifer element is intended to represent a feature that essentially behaves as a fluid conduit. It can be used to simulate vertical transport through an unsaturated zone or horizontal transport in aquifers, rivers, channels, and pipelines. The Aquifer element can contain only a single fluid medium; however, it can also contain a solid medium that can impact transport. The Aquifer element internally uses a set of linked Cell elements during the simulation, which are subsequently removed at the end of the simulation. In other words, the simulation approach used by the Aquifer element is similar to the finite difference approach used by DUST-MS, which subdivides the domain of analysis into multiple grids and assumes homogeneity for the solid and liquid phase, respectively, within each grid.

The intention for the Pipe element is the same as that for the Aquifer element. Mass is considered to enter at one end of the conduit, and travel through and disperse within the conduit before exiting at the other end of the conduit. However, the Pipe element uses a Laplace transform approach to provide analytical solutions to the equations governing one-dimensional advection, longitudinal dispersion, retardation, decay and ingrowth, and exchanges with immobile storage zones (e.g., matrix diffusion) as the mass moves through the conduit. This simulation approach is similar to that used by RESRAD-OFFSITE, which also solves the governing equations analytically.

\section{B.1.1 First Order Release}

To simulate the first order release condition with GoldSim, a Source element was used. The Source element contains waste materials with defined properties and characteristics. The waste materials release radionuclides to the surrounding environment. 
The radionuclide inventory was assumed to be embedded in waste materials in some form (waste form) without any barrier. The waste form was assumed to degrade with a degradation rate of $0.01 / \mathrm{yr}$ ( $1 \%$ of the remaining waste form degraded every year) for Case I and Case II and with a degradation rate of 0.0011 for Case III. When the degraded waste form was exposed to infiltrating water, radionuclides were dissolved and carried to the next element through advection. This dissolving/carrying process was simulated by associating a Cell and a Pipe element with the Source. The Cell element mixes the radionuclides released from the waste form and distributes them to the Pipe element.

The properties of the Cell element include the contaminant mass input from the source element, the volume of the water medium, and the flow rate of water to the Pipe element. The length, area, source zone length (to which the radionuclides released from the Cell element were distributed), dispersivity, and infill medium (soil) associated with the Pipe element were specified.

Figure B.1 shows the model constructed for the first order release condition with GoldSim.

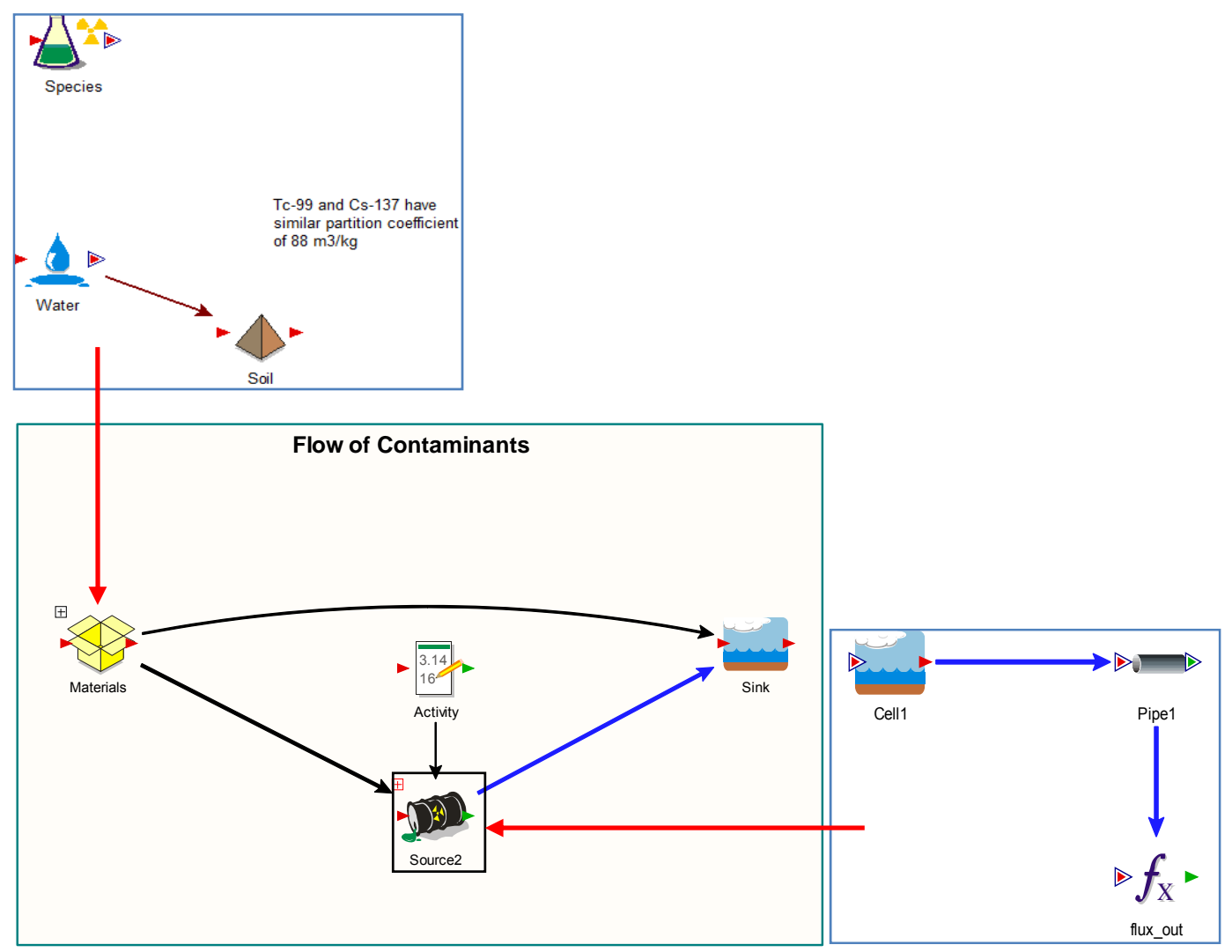

FIGURE B.1 Modeling of a First Order Release with GoldSim 


\section{B.1.2 Equilibrium Desorption Release}

To model the release of radionuclides under the equilibrium desorption condition with GoldSim, the Aquifer element was used when there is no dispersion, while the Pipe element was used when dispersion was considered. For both elements, it was assumed that initially, all radionuclides were uniformly distributed in a source zone (the initial inventory of radionuclide was specified) between the liquid and solid phases in the element. Outflow, equal to the yearly infiltration rate, was from the Aquifer or Pipe element to a Sink element, from which the release rates were obtained.

The parameters specified for an Aquifer element were about the same as those specified for a Pipe element, which included length, area, dispersivity, and infill medium. For the outflow, only advection was considered to remove radionuclides in this comparison.

Figure B.2 shows the model constructed for the equilibrium desorption release condition with GoldSim.

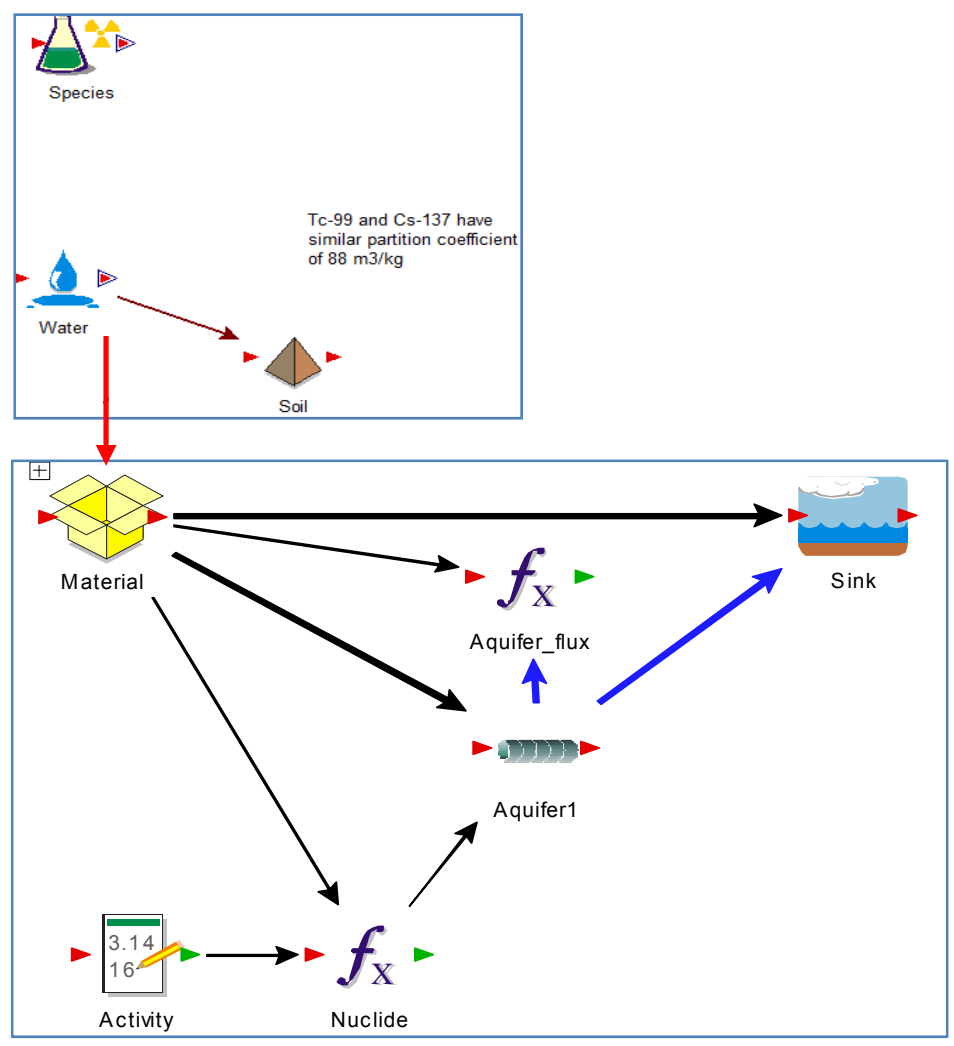

FIGURE B.2 Modeling of an Equilibrium Desorption Release with GoldSim 


\section{B.1.3 Uniform Release}

To model the uniform release condition with GoldSim, a Pipe element was used to obtain the release rates. It was assumed that a constant fraction of the initial activity, after being adjusted for radioactive ingrowth and decay over time, was released every year from a Source element. The source element was associated with the Cell and Pipe elements, just as it was in the set up for the first order release condition. Outflow from the Pipe element, equal to the yearly infiltration rate, was then linked to a Sink element from which the release rates were obtained.

\section{B.2 Input Parameters Used with GoldSim Modeling}

The GoldSim models were constructed to obtain results for three comparison cases: Case I, II, and III. Input parameters required to run the models were selected so that they either matched or corresponded to the values used to run RESRAD-OFFSITE for this comparison.

For Cases I and II, on the basis of RESRAD-OFFSITE input values, the infiltration rate was set at $0.4 \mathrm{~m} / \mathrm{yr}$; for Case III, it was kept at $0.1 \mathrm{~m} / \mathrm{yr}$. To simulate Cases I, II, and III, radioactive species-Tc-99, Cs-137, Po-210, Pb-210, Ra-226, Th-230, and U-234-were created with the properties specified in Table B.1. The half-lives and atomic weights were obtained from the RESRAD manual (Yu et al. 2001) because GoldSim does not have an inbuilt radionuclide decay database.

TABLE B.1 Properties of the Radioactive Species Considered in GoldSim Modeling

\begin{tabular}{cccc}
\hline Radionuclide & Half-life $(\mathbf{y r})^{\mathbf{1}}$ & Decay rate $\left(\mathbf{y r} \mathbf{-}^{-1}\right)$ & $\begin{array}{c}\text { Atomic weight } \\
\left(\mathbf{g} / \mathbf{m o l} \mathbf{~}^{\mathbf{1}}\right.\end{array}$ \\
\hline Tc-99 & $2.13 \mathrm{E}+05$ & $3.2542 \mathrm{E}-06$ & 98.9 \\
\hline $\mathrm{Cs}-137$ & 30 & $2.3105 \mathrm{E}-02$ & 137 \\
\hline $\mathrm{Pb}-210$ & 22.3 & $3.1083 \mathrm{E}-02$ & 210.1 \\
\hline $\mathrm{Po}-210$ & 0.37886 & $1.8296 \mathrm{E}+00$ & 210.1 \\
\hline Ra-226 & 1600 & $4.3322 \mathrm{E}-04$ & 226.1 \\
\hline Th-230 & $7.70 \mathrm{E}+04$ & $9.0019 \mathrm{E}-06$ & 230.1 \\
\hline $\mathrm{U}-234$ & $2.45 \mathrm{E}+05$ & $2.8350 \mathrm{E}-06$ & 234.1 \\
\hline
\end{tabular}

1 Half-lives and atomic weights were obtained from RESRAD manual (Yu et al. 2001). 
The soil (a solid medium specified with the material tab) was assumed to have a density of $1.5 \mathrm{~g} / \mathrm{cm}^{3}$, a porosity of 0.4 , and various $\mathrm{Kd}$ values as were used for RESRAD-OFFSITE modeling. A data element, Activity, with a total activity of $4.5 \times 10^{7} \mathrm{pCi}\left(100 \mathrm{pCi} / \mathrm{g} \times 1.5 \mathrm{~g} / \mathrm{cm}^{3} \times\right.$ $30 \mathrm{~cm} \times 10,000 \mathrm{~cm}^{2}$ ) for Tc-99, Cs-137, and U-234, respectively, was created. The initial activity for each of the U-234 progeny (Th-230, Ra-226, Pb-210, and Po-210) was set to zero. The equivalent mass of radioactive species, corresponding to the specified activity, was input as the inventory to the Source element to model the first order release and uniform release conditions.

The parameters used for the Aquifer and Pipe elements were (1) length $=0.3 \mathrm{~m}$; (2) area $=$ $1 \mathrm{~m}^{2}$; (3) dispersivity $=0.03 \mathrm{~m}$ or $0.1 \mathrm{~m}$; (4) number of cells (for the Aquifer element only) $=100$, (5) source zone length (for the Pipe element only) $=0.3 \mathrm{~m}$; and (6) infill medium $=$ soil. To obtain release rates under the equilibrium desorption condition, the initial inventory set for the Pipe/Aquifer element was equal to the total activity of $4.5 \times 10^{7} \mathrm{pCi}$ as specified for the Activity data element.

Under the uniform release condition, for Cases I and II, the input rate from the Source element to the Pipe element was set at $0.01 / \mathrm{yr}$ for 100 years, whereas for Case III, the input rate was set at $0.0011 / y r$ for 900 years. The rest of the parameters were kept at their default values.

The GoldSim simulations were carried out for a time period of 500 years for Cases I and II, and for a time period of 10,000 years for Case III. The radionuclide release rates were reported every year for Cases I and II and every five years for Case III. Various dispersivity values, along with various soil $\mathrm{Kd}$ values, were specified to obtain multiple sets of radionuclide release rates for comparison.

\section{B.3 Results of Comparison}

\section{B.3.1 Case I: $0.3 \mathrm{~m}$ of Tc-99}

Case I considers releases from a 0.3-m Tc-99 source. Annual release rates of Tc-99 in terms of $\mathrm{pCi} / \mathrm{yr}$ were calculated over 500 years. The release rates were calculated with no dispersion, as well as with different levels of dispersion in the soil column.

\section{B.3.1.1 No Dispersion}

Equilibrium desorption. Figure B.3 compares the RESRAD-OFFSITE results obtained with the equilibrium desorption option with the GoldSim results. No dispersion in the soil column was considered; however, with the GoldSim modeling, a very small dispersivity of $0.0015 \mathrm{~m}$ was automatically introduced and used in the simulation. The RESRAD-OFFSITE results were 
plotted with solid lines, whereas the GoldSim results were plotted with dashed lines. Although the comparison shows some discrepancy, the agreement between the RESRAD-OFFSITE results and the GoldSim results is considered well acceptable. The discrepancy between the two could be caused by numerical dispersion introduced in the GoldSim results, as they were obtained with an Aquifer element (GoldSim 2010c).

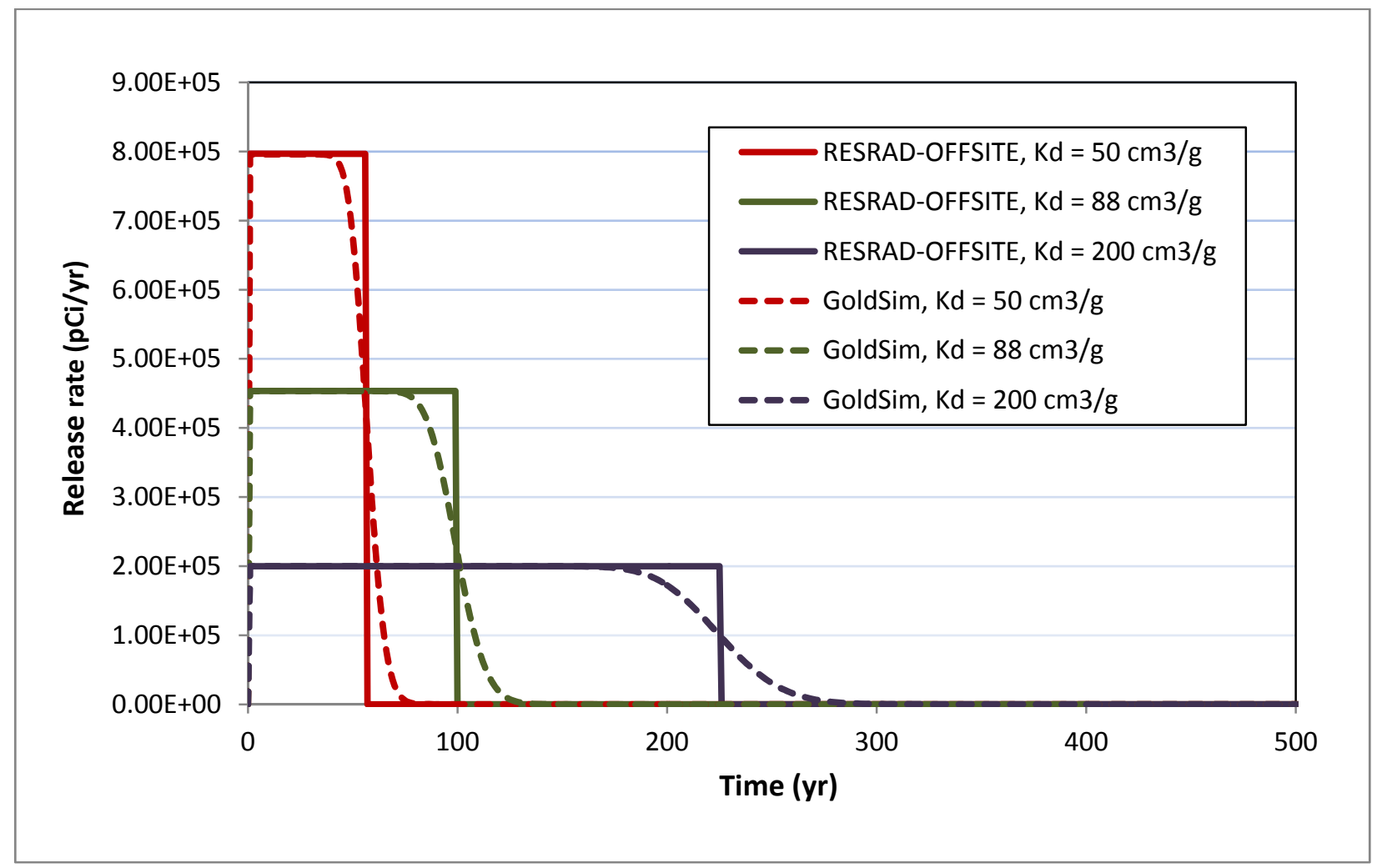

\section{FIGURE B.3 Comparison of RESRAD-OFFSITE and GoldSim Results for Case I Concerning Release from a 0.3-m Tc-99 Source under the Equilibrium Desorption Condition with No Dispersion}

Uniform release. Figure B.4 compares the RESRAD-OFFSITE results obtained with the uniform release option with the GoldSim results. The waste form was assumed to disintegrate uniformly with a disintegration fraction of $0.01 / \mathrm{yr}$ for 100 years. Good agreement was achieved between the RESRAD-OFFSITE results and the GoldSim results. The discrepancy could be caused by the small dispersivity introduced automatically to the Pipe element in the GoldSim simulation.

First Order release. Figure B.5 compares the RESRAD-OFFSITE results obtained with the first order release option with the GoldSim results. The waste form was assumed to follow a 
first order decay profile described by a rate constant of $0.01 / y r$. The GoldSim results almost match the RESRAD-OFFSITE results, except at times close to the peaks of the release profiles. Again, this discrepancy might be attributable to the small dispersivity introduced automatically to the Pipe element in the GoldSim simulation (as indicated by a Run Log warning message in GoldSim run).

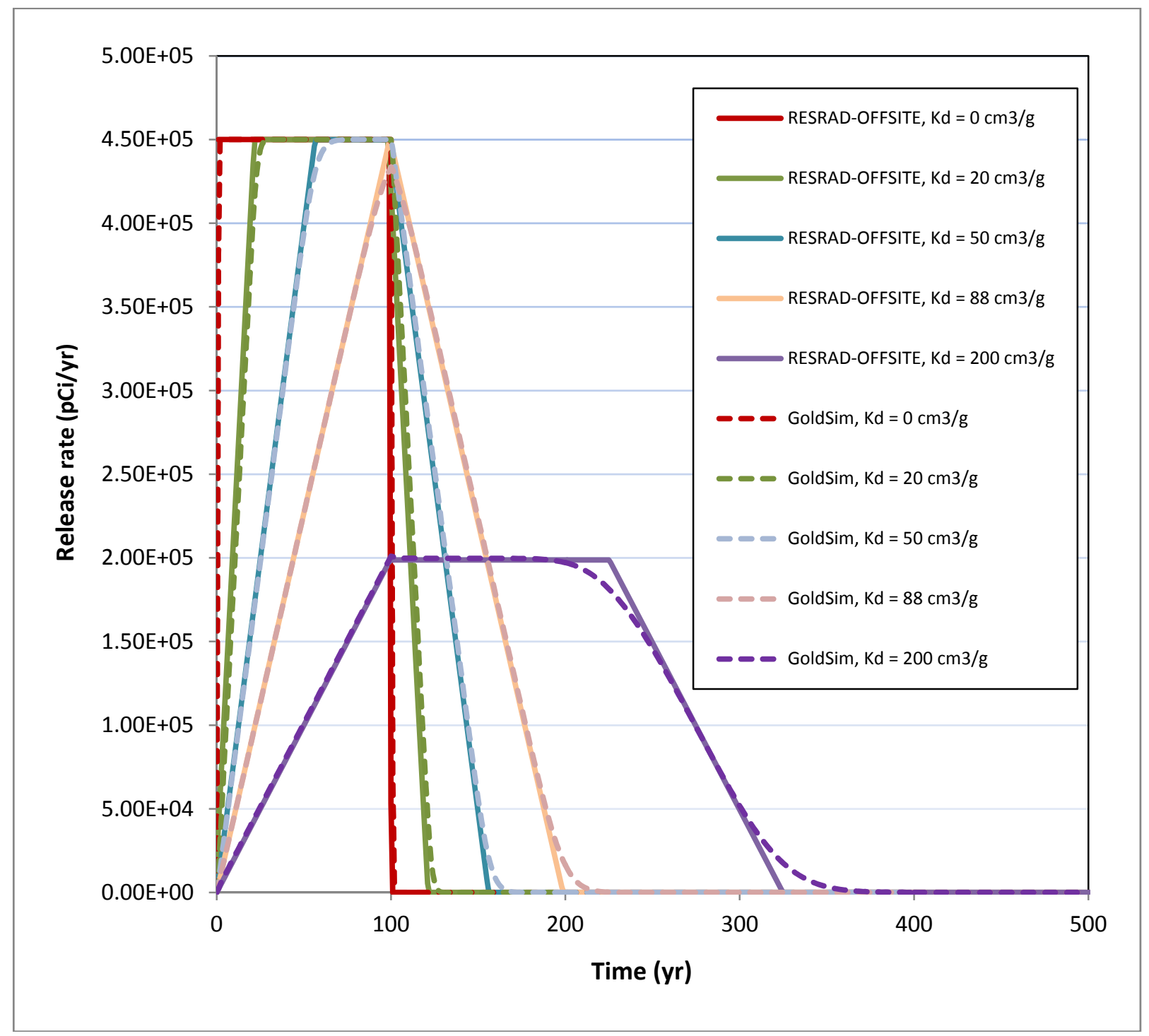

FIGURE B.4 Comparison of RESRAD-OFFSITE and GoldSim Results for Case I Concerning Release from a 0.3-m Tc-99 Source under the Uniform Release Condition with No Dispersion 


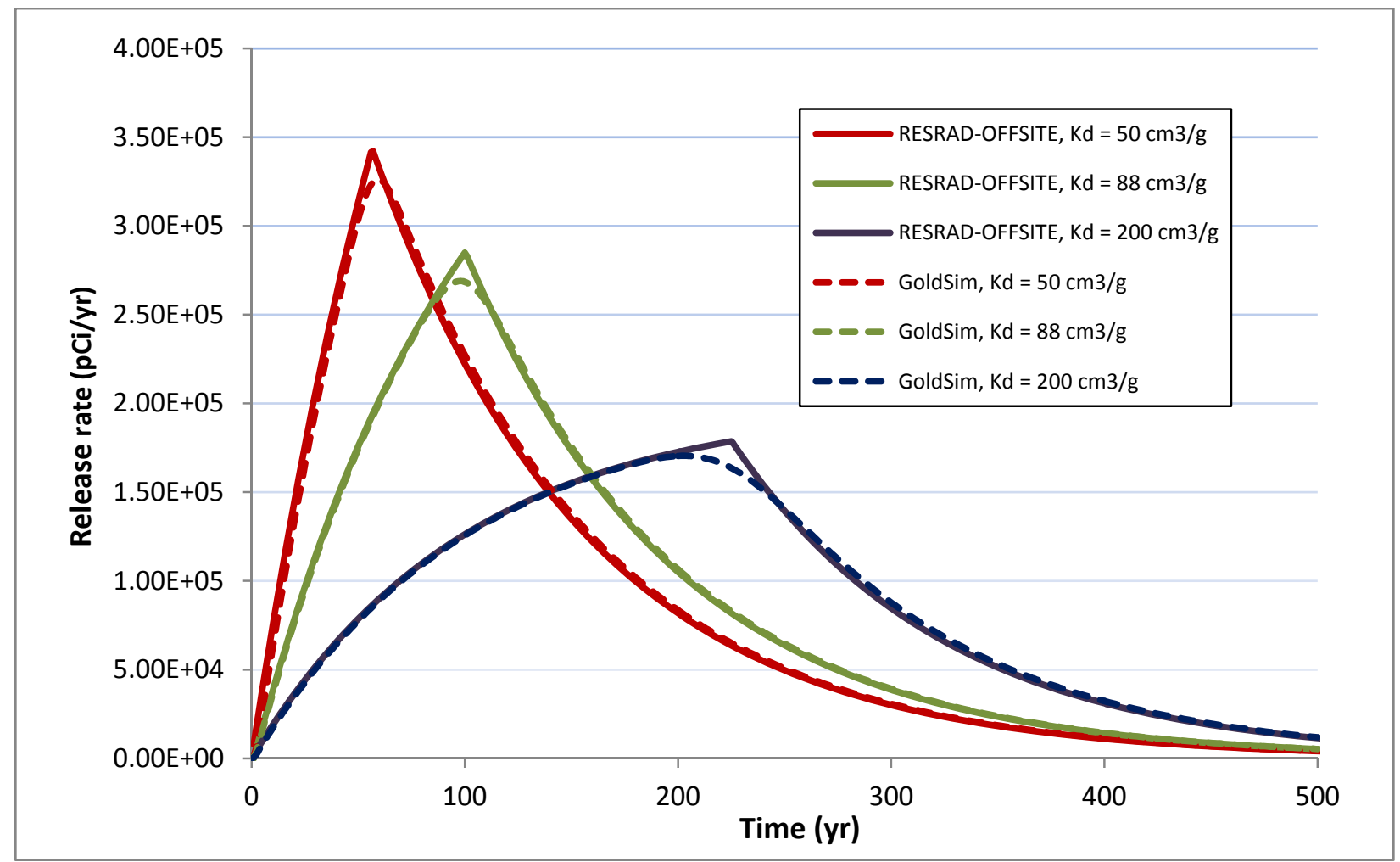

FIGURE B.5 Comparison of RESRAD-OFFSITE and GoldSim Results for Case I Concerning Release from a 0.3-m Tc-99 Source under the First Order Release Condition with No Dispersion

\section{B.3.1.2 With Dispersion}

Two dispersion levels were considered for the comparison, one with a dispersivity of $0.03 \mathrm{~m}$, that is, $1 / 10$ of the thickness of contaminated zone, the other with a dispersivity of $0.1 \mathrm{~m}$. The comparison was made only for the equilibrium desorption option.

Figure B.6 shows the comparison of RESRAD-OFFSITE results obtained with the equilibrium desorption option with GoldSim results for a dispersivity of $0.03 \mathrm{~m}$. The agreement is considered well acceptable for all of the Kd values assumed, except for the sharp rise and decline at the beginning. By increasing the dispersivity to $0.1 \mathrm{~m}$, the RESRAD-OFFSITE results still agree fairly well with the GoldSim results (see Figure B.7). 


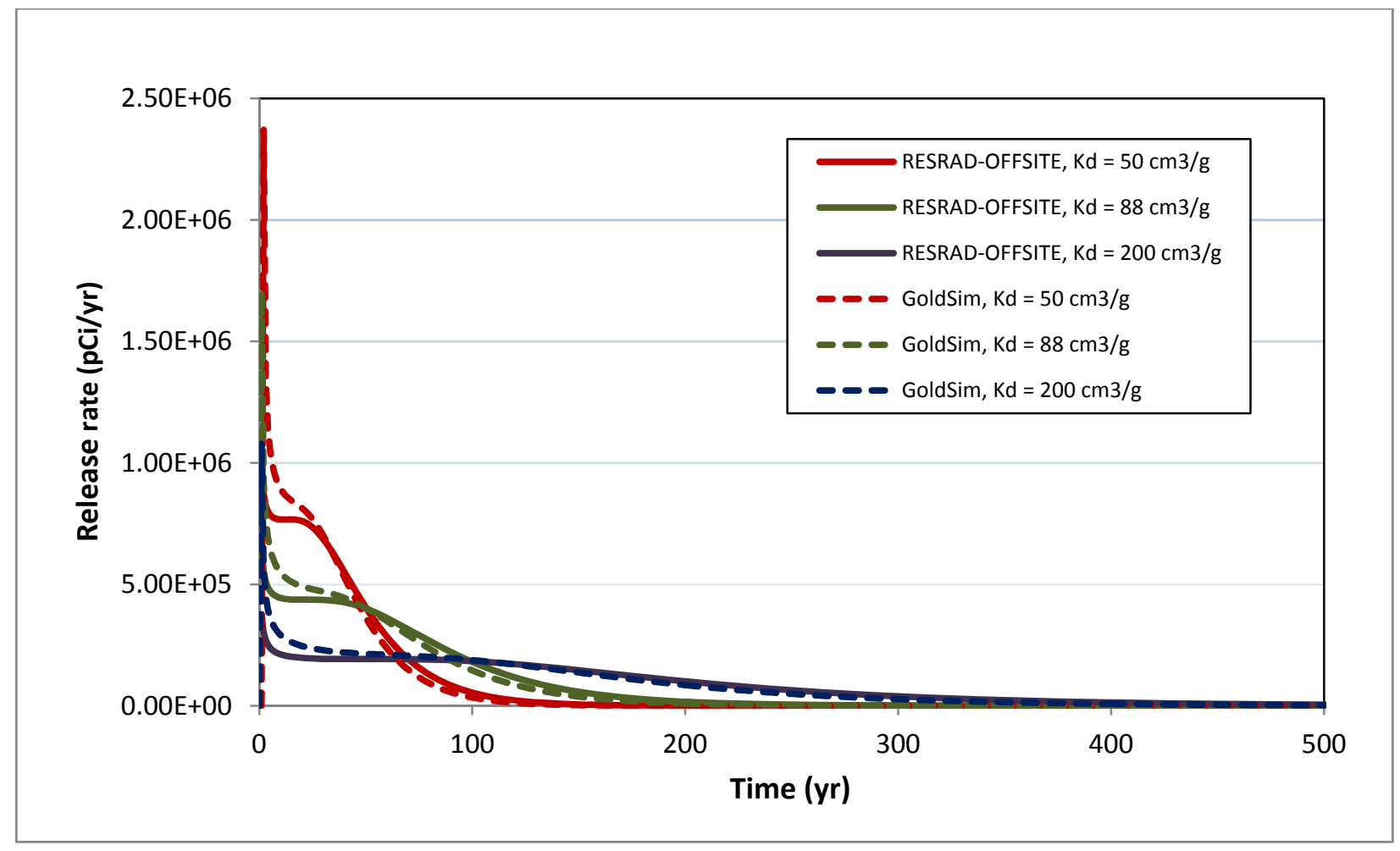

FIGURE B.6 Comparison of RESRAD-OFFSITE and GoldSim Results for Case I Concerning Release from a 0.3-m Tc-99 Source under the Equilibrium Desorption Condition with a Dispersivity of $0.03 \mathrm{~m}$ 


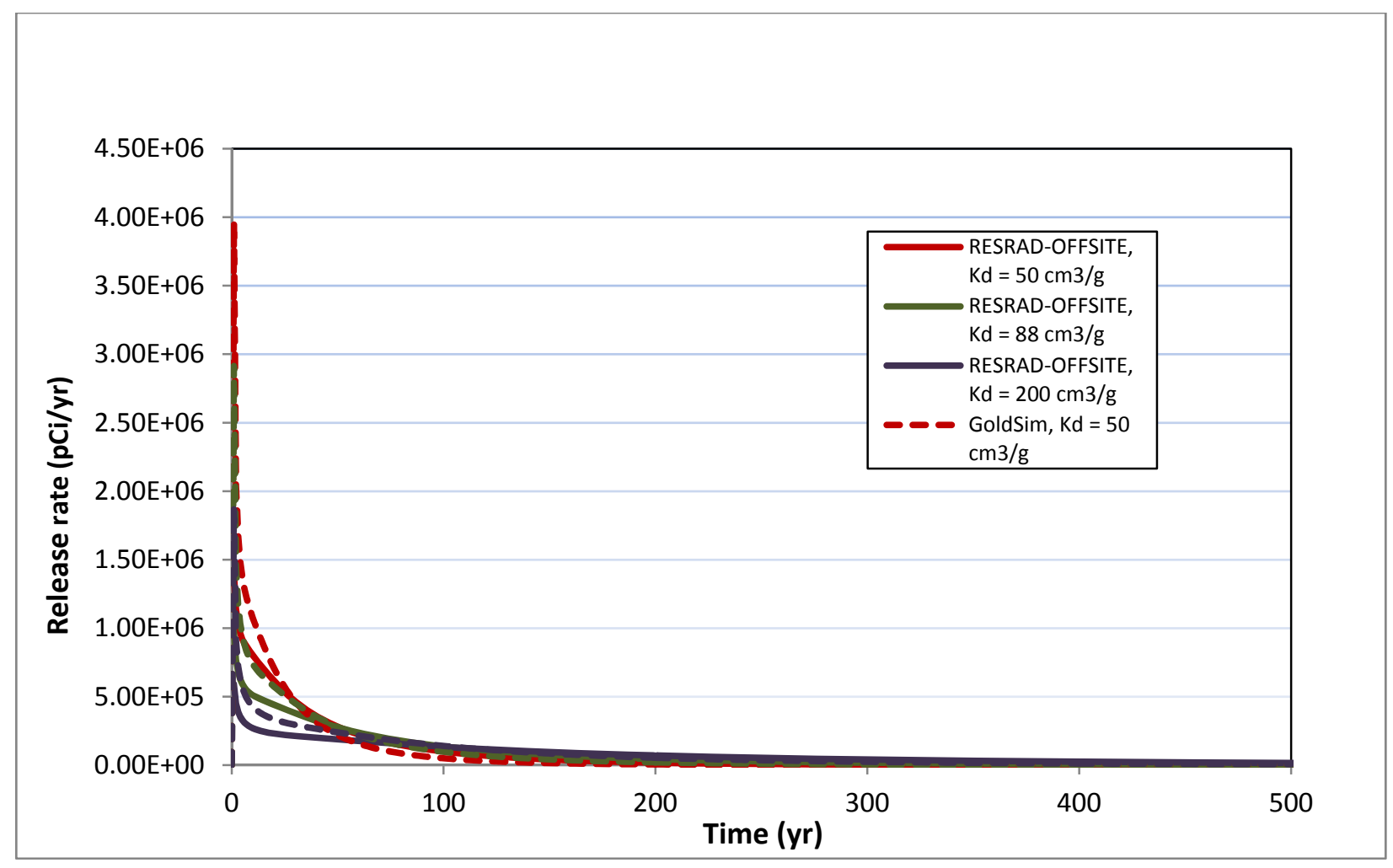

FIGURE B.7 Comparison of RESRAD-OFFSITE and GoldSim Results for Case I Concerning Release from a 0.3-m Tc-99 Source Under the Equilibrium Desorption Condition with a Dispersivity of $0.1 \mathrm{~m}$

\section{B.3.2 Case II: $0.3 \mathrm{~m}$ of $\mathrm{Cs}-137$}

Case II considers the release of Cs-137 from a 0.3-m source. Cs-137 was selected for comparison because of the shorter half-life of 30 years compared toTc-99's half-life of $2.13 \times$ $10^{5}$ years. The influence of half-life on the release rates would be more pronounced for Cs-137 compared toTc-99. The comparison was performed only for the no-dispersion condition.

Equilibrium desorption. Figure B.8 compares the RESRAD-OFFSITE results obtained with the equilibrium desorption option with the GoldSim results. No dispersion in the soil column was specified, although GoldSim automatically introduced a small dispersivity of $0.0015 \mathrm{~m}$ to the simulation (as indicated by a Run Log warning message in GoldSim run). The RESRADOFFSITE results were plotted with solid lines, while the GoldSim results were plotted with dashed lines. The discrepancy between the two could be caused by numerical dispersion introduced in the GoldSim results, which were obtained with the use of an Aquifer element. 


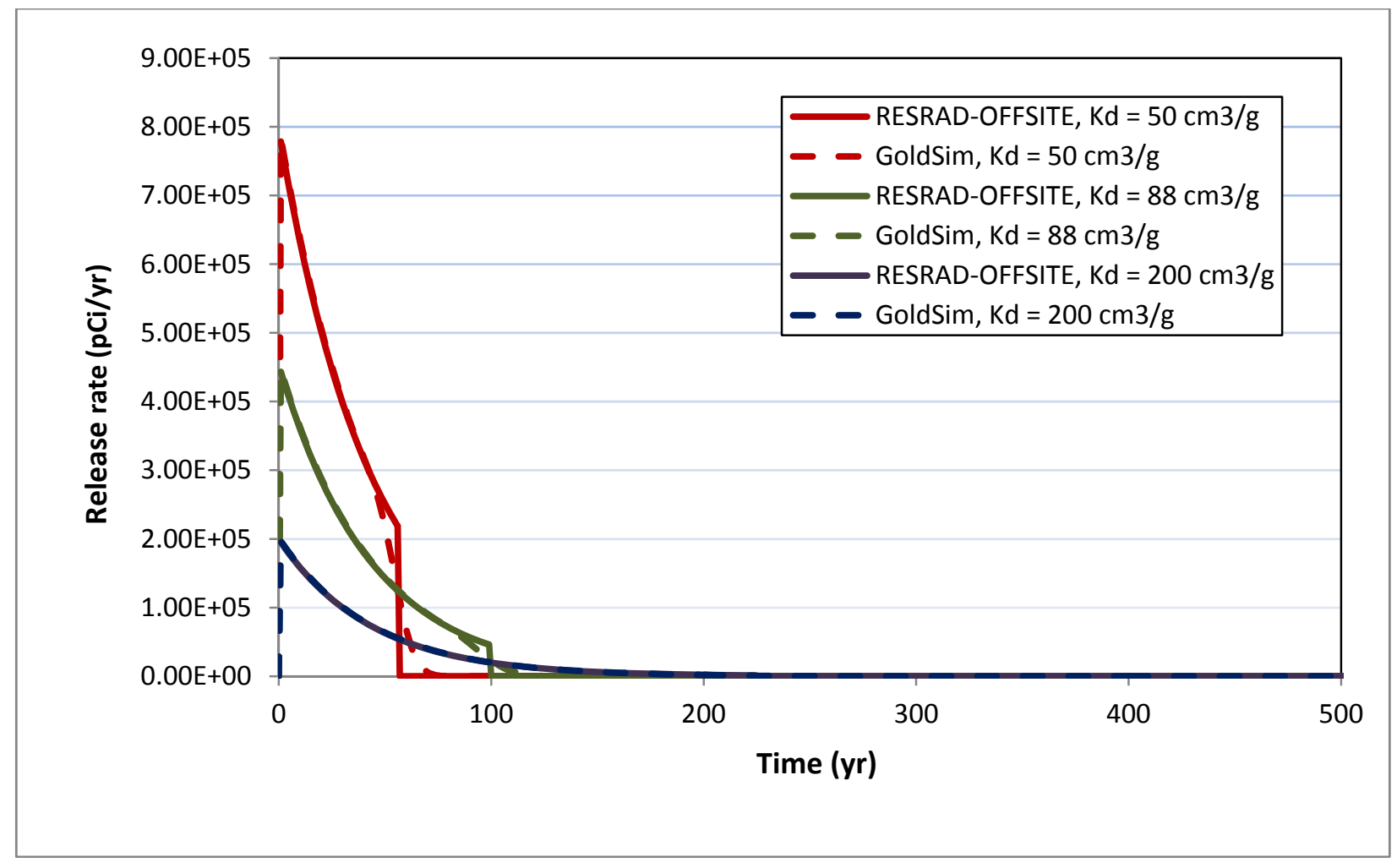

\section{FIGURE B.8 Comparison of RESRAD-OFFSITE and GoldSim Results for Case I Concerning Release from a 0.3-m Cs-137 Source under the Equilibrium Desorption Condition with No Dispersion}

Uniform release. Figure B.9 compares the RESRAD-OFFSITE results obtained with the uniform release option with the GoldSim results. The waste form was assumed to disintegrate uniformly with a disintegration (dissolution) fraction of $0.01 / \mathrm{yr}$ for 100 years. The small dispersivity introduced by GoldSim to the Pipe element might be responsible for the small discrepancy between the GoldSim results and the RESRAD-OFFSITE results.

First Order release. Figure B.10 compares the RESRAD-OFFSITE results obtained with the first order release option with the GoldSim results. The waste form was assumed to disintegrate following a first order mathematical form with a constant of $0.01 / \mathrm{yr}$. Excellent agreement was achieved between the RESRAD-OFFSITE results and the GoldSim results. 


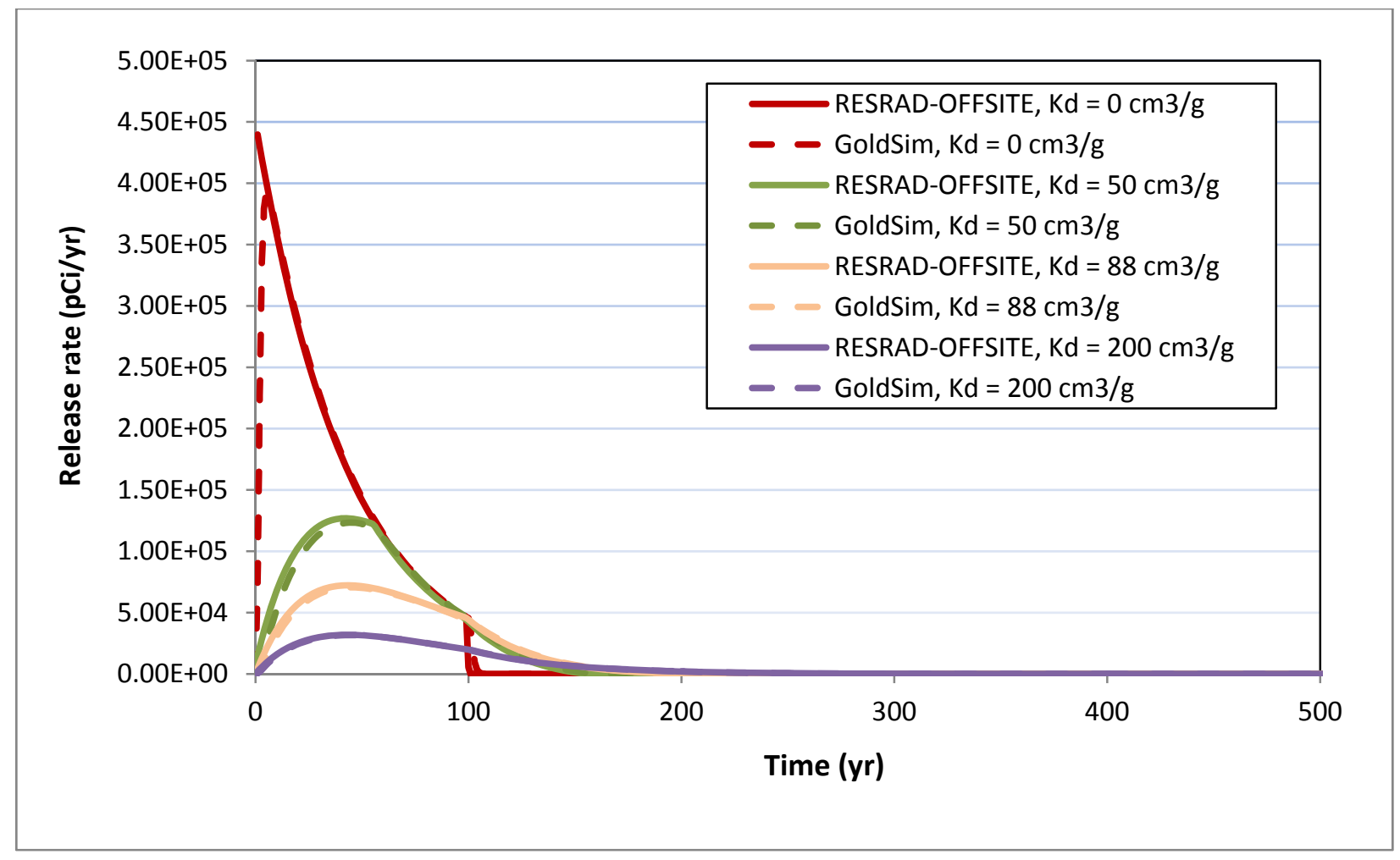

FIGURE B.9 Comparison of RESRAD-OFFSITE and GoldSim Results for Case I Concerning Release from a 0.3-m Cs-137 Source under the Uniform Release Condition with No Dispersion 


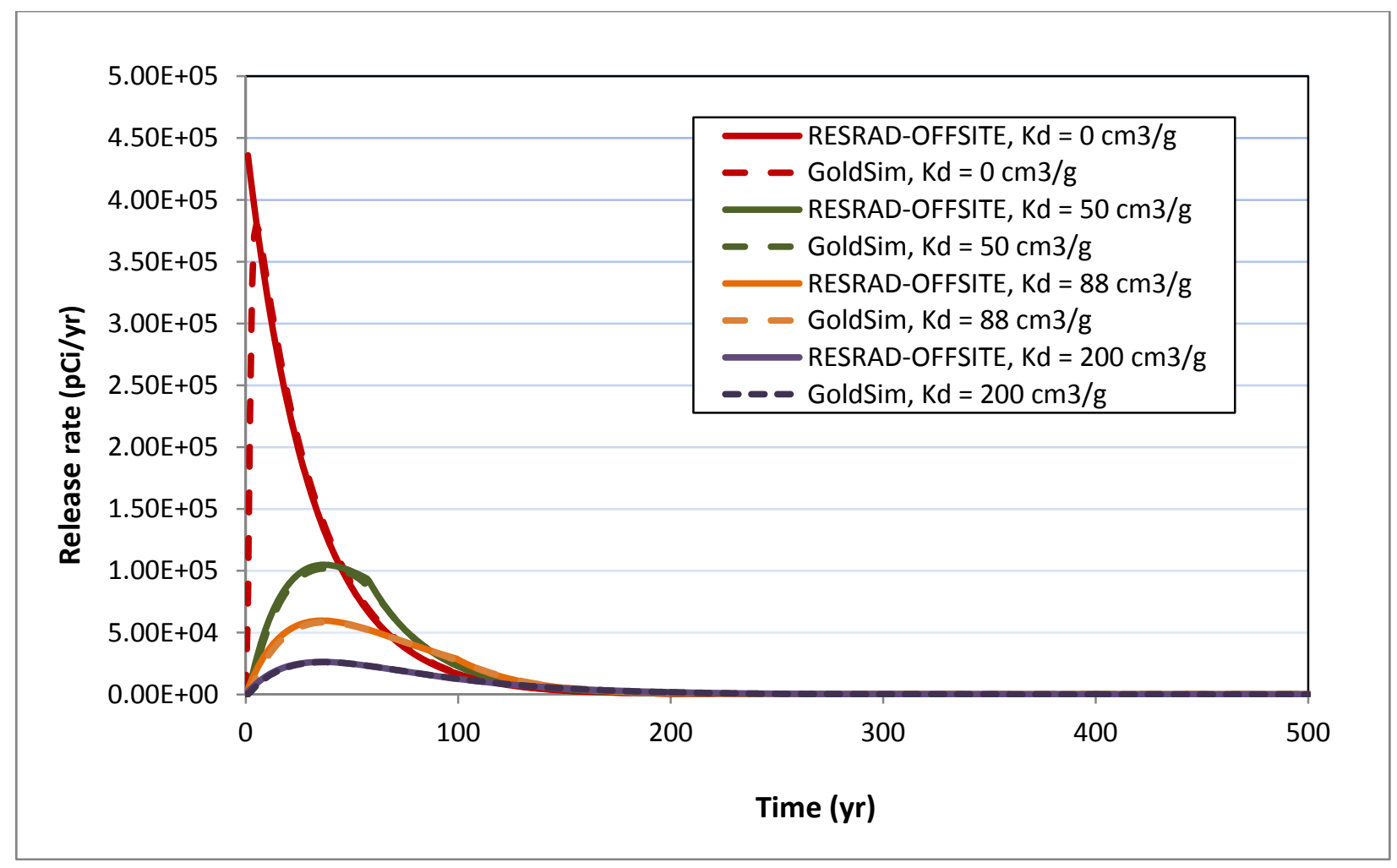

\section{FIGURE B.10 Comparison of RESRAD-OFFSITE and GoldSim Results for Case I Concerning Release from a 0.3-m Cs-137 Source under the First Order Release Condition with No Dispersion}

\section{B.3.3 Case III: $0.3 \mathrm{~m}$ of U-234}

The comparison of release rates from a waste form containing U-234 involves comparing the release rates of U-234, Th-230, Ra-226, Pb-210, and Po-210, respectively. The progeny radionuclides would be formed not only within the waste form, but also during the transport of $\mathrm{U}-234$ in the contaminated medium. The $\mathrm{Kd}$ values assumed for $\mathrm{U}-234$ and its progeny radionuclides were $200,6,000,70,100$, and $10 \mathrm{~cm}^{3} / \mathrm{g}$, respectively.

When progeny radionuclides are formed within the waste form, they are assumed to be released through the same mechanism as their parent radionuclide to the surrounding medium. In Case III, the water infiltration rate was reduced from $0.4 \mathrm{~m} / \mathrm{yr}$ as in Cases I and II to $0.1 \mathrm{~m} / \mathrm{yr}$. Furthermore, under the uniform release condition, a uniform release rate of $0.0011 / \mathrm{yr}$ for 900 years was assumed, and under the first order rate release condition, a rate constant of $0.0011 / \mathrm{yr}$ was assumed. 
Figure B.11 compares the release rates of U-234. Excellent agreement was displayed between the release rates calculated by RESRAD-OFFSITE and GoldSim under the uniform release condition and first order release condition. A slight discrepancy was observed in the release rates associated with the equilibrium desorption condition, which may be caused by numerical dispersion in the GoldSim results, because an Aquifer element was used in constructing the release model.

Figures B.12, B.13, B.14, and B.15 compare the release rates of Th-230, Ra-226, Pb-210, and Po-210, respectively. The RESRAD-OFFSITE results and the GoldSim results match very well for all of these decay progenies under all three release conditions.

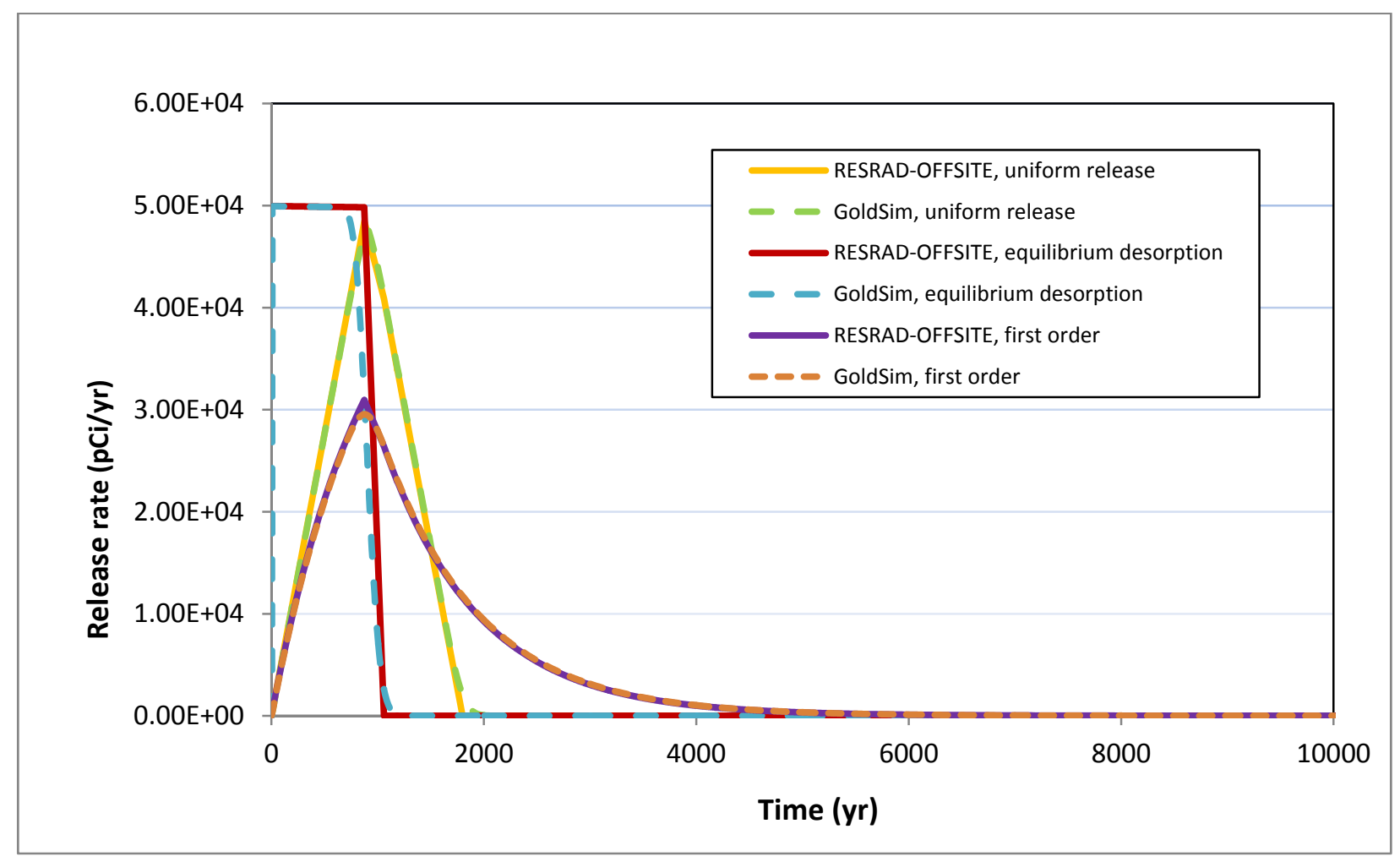

FIGURE B.11 Comparison of RESRAD-OFFSITE and GoldSim Results for Case III Concerning Release of U-234 from a 0.3-m U-234 Source with No Dispersion 


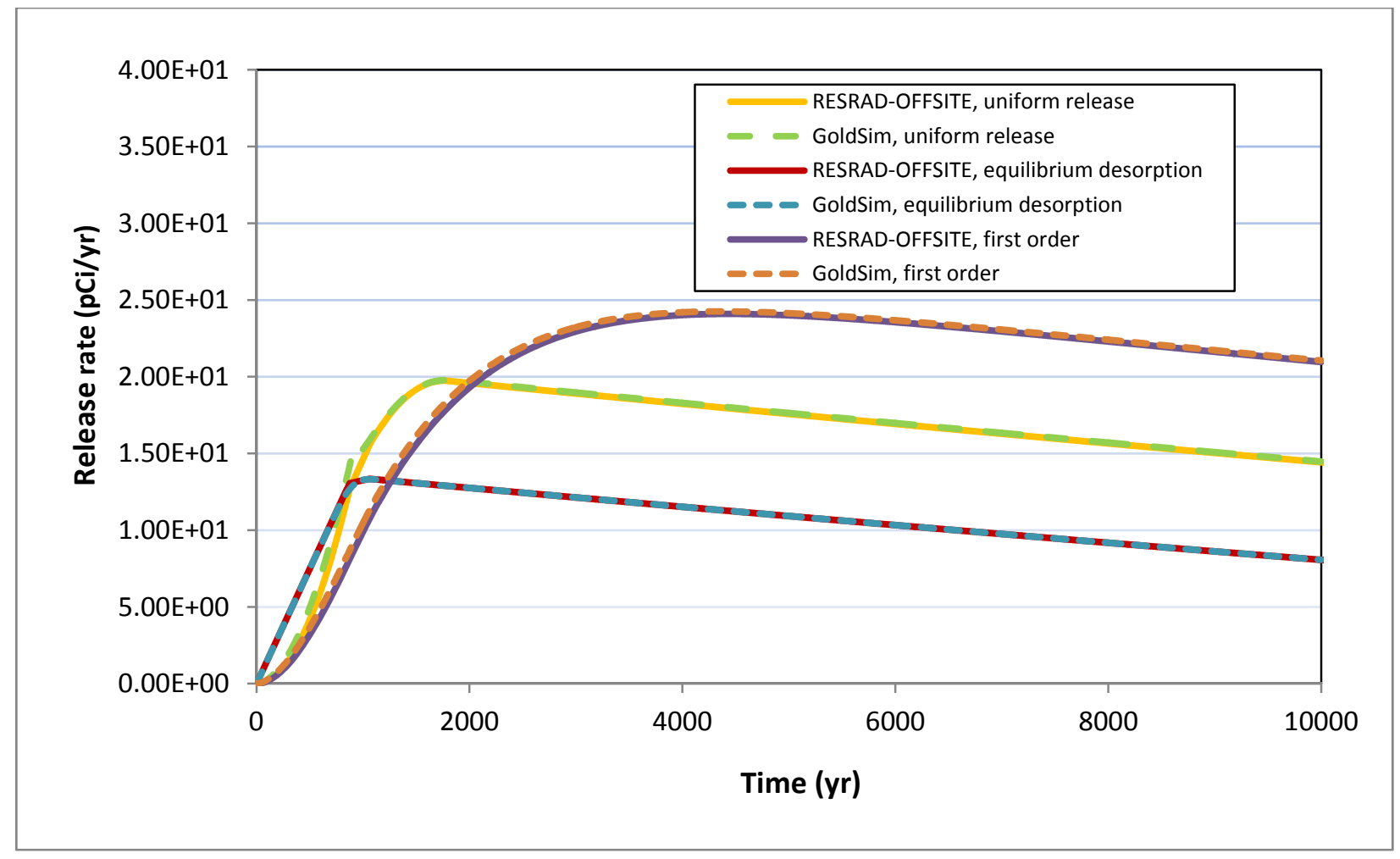

FIGURE B.12 Comparison of RESRAD-OFFSITE and GoldSim Results for Case III Concerning Release of Th-230 from a 0.3-m U-234 Source with No Dispersion 


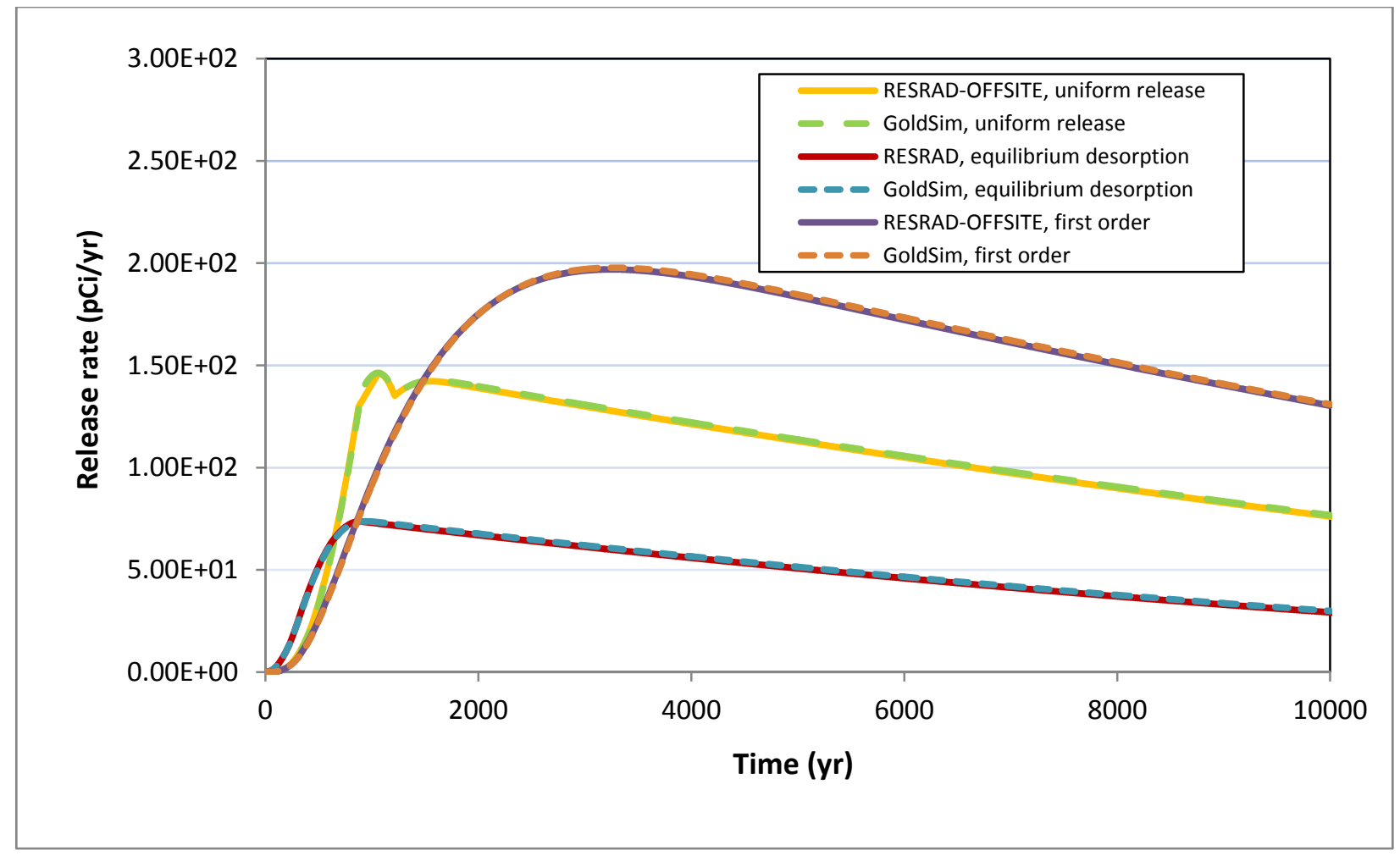

FIGURE B.13 Comparison of RESRAD-OFFSITE and GoldSim Results for Case III Concerning Release of Ra-226 from a 0.3-m U-234 Source with No Dispersion 


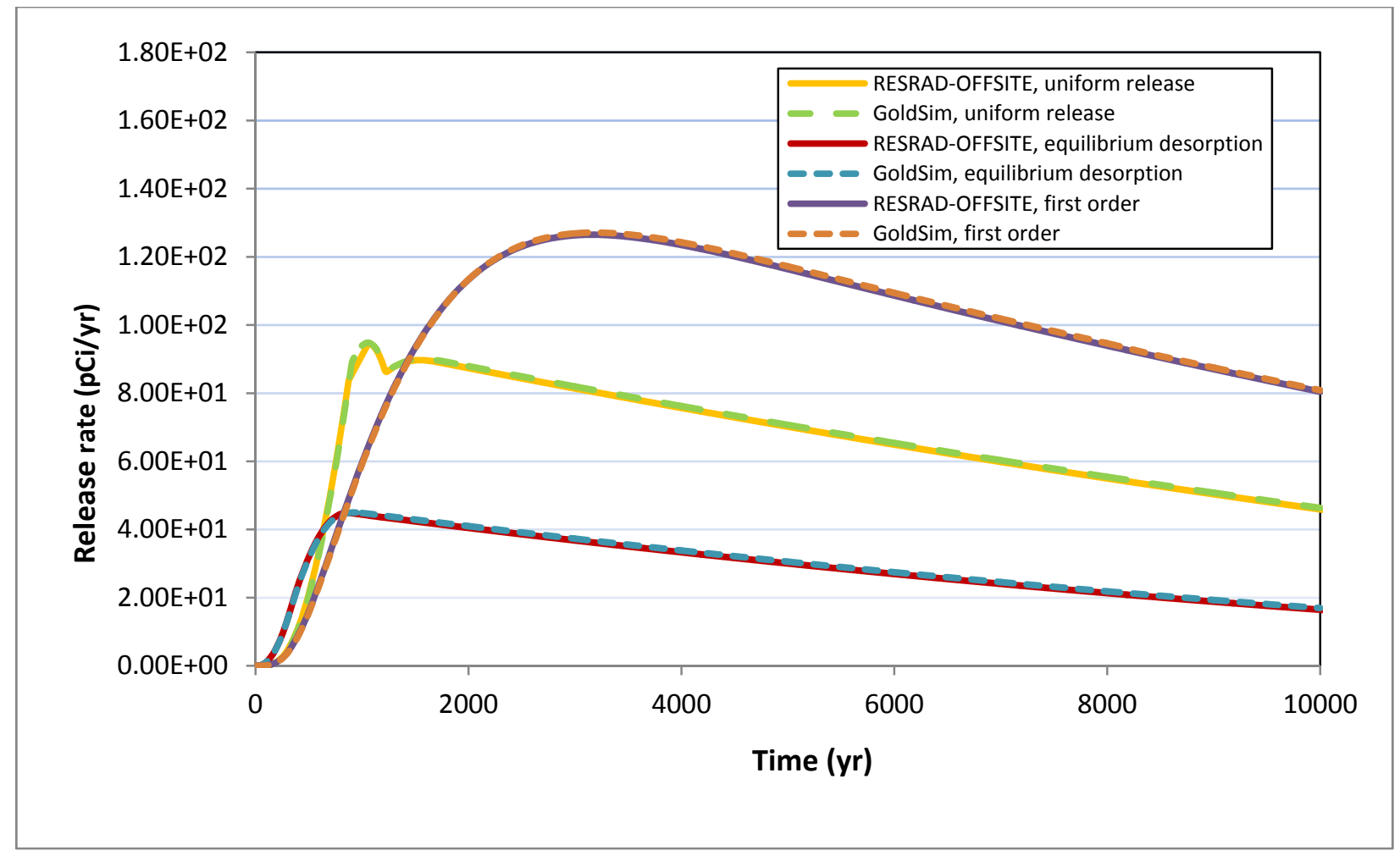

FIGURE B.14 Comparison of RESRAD-OFFSITE and GoldSim Results for Case III Concerning Release of Pb-210 from a 0.3-m U-234 Source with No Dispersion 


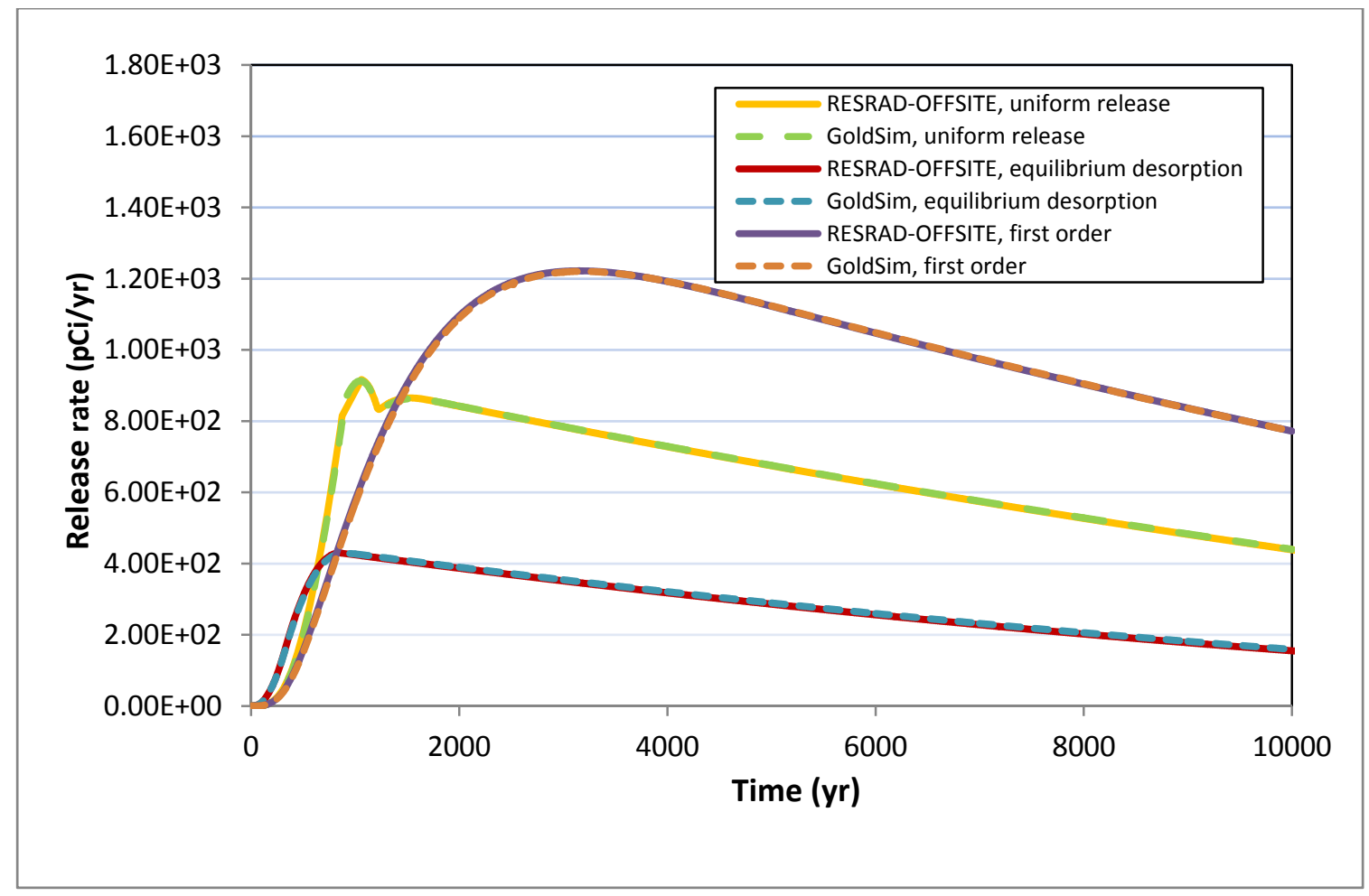

FIGURE B.15 Comparison of RESRAD-OFFSITE and GoldSim Results for Case III Concerning Release of Po-210 from a 0.3-m U-234 Source with No Dispersion

\section{B.4 References for Appendix B}

GoldSim, 2010a, Probabilistic Simulation Environment, User's Guide, Volume 1, GoldSim Technology Group, Issaquah, Wash., Version 10.5, Dec.

GoldSim, 2010b, Probabilistic Simulation Environment, User's Guide, Volume 2, GoldSim Technology Group, Issaquah, Wash., Version 10.5, Dec.

GoldSim, 2010c, Contaminant Transport Module, User's Guide, GoldSim Technology Group, Issaquah, Wash., Version 6.0, Dec.

Yu, C., et al., 2001, User's Manual for RESRAD Version 6, ANL/EAD-4, Argonne National Laboratory, Argonne, III. 
This page is intentionally left blank. 


\section{Appendix C}

\section{Sensitivity Analysis Using Probabilistic RESRAD-OFFSITE}

E. Gnanapragasam and C. Yu 



\section{Appendix C Sensitivity Analysis Using Probabilistic RESRAD-OFFSITE}

\section{C.1 Introduction}

The RESRAD-OFFSITE code is an extension of the RESRAD (onsite) code (Yu et al. 2001) ${ }^{3}$ and has similarly enhanced ability to perform sensitivity and uncertainty (probabilistic) analyses (Yu et al. 2007). 4 The sensitivity and probabilistic analyses using RESRAD (onsite) code are documented in various reports and publications, including Kamboj et al. $\left(2000,{ }^{5} 2005^{6}\right)$. This report describes in detail the different ways in which sensitivity analysis can be performed using the probabilistic RESRAD-OFFSITE code.

Sensitivity analysis attempts to quantify the influence of individual inputs on the predictions of the code. Often the influence of one parameter on the resultant predicted dose or risk is also dependent on the values of other parameters. The effect of the interaction between parameters on the sensitivity of dose to the parameters will be studied in this report.

RESRAD-OFFSITE has three methods for quantifying parameter sensitivity: the singleparameter method, the distributed single-parameter method, and the multiple-parameter method. The three methods quantify sensitivity differently and are not directly comparable. Some analyze the influence of the input on the predicted dose or risk, whereas others analyze its influence on the variability of the predicted dose or risk. Some account for the influence of other inputs.

In almost all cases, the sensitivity of a parameter depends on the values of that parameter and the other parameters. Therefore, it is imperative to use site-specific parameter values when conducting sensitivity analyses.

3 Yu, C., et al., 2001, User's Manual for RESRAD Version 6, ANL/EAD-4, Argonne National Laboratory, Argonne, III., July.

4 Yu, C., et al., 2007, User's Manual for RESRAD-OFFSITE Version 2, ANL/EVS/TM/07-1, Argonne National Laboratory, Argonne, III., June.

5 Kamboj, S., et al., 2000, "Probabilistic Dose Analysis Using Parameter Distributions Developed for RESRAD and RESRAD-BUILD Codes," NUREG/CR-6676, ANL/EAD/TM-89, prepared by Argonne National Laboratory, Argonne, III., for U.S. Nuclear Regulatory Commission, Washington, D.C.

6 Kamboj, S., et al., 2005, "Deterministic vs. Probabilistic Analyses to Identify Sensitive Parameters in Dose Assessment Using RESRAD," Operational Radiation Safety, supplement to Health Physics, pp. S104-S109, May. 
Sensitive parameters for radionuclides are sometimes dependent on the radiation type or exposure pathway. For Cs-137 and Co-60, for example, the external radiation pathway is usually the dominant pathway, unless there is a shield between the source and receptor. Hence, parameters related to the external pathway, such as the indoor and outdoor occupancy factors, are usually sensitive parameters for these radionuclides (Kamboj 2000) ${ }^{3}$. A sensitivity analysis using one of the three methods described in this report will identify the sensitive parameters.

Sensitivity analysis also helps understand how the inputs are used by RESRAD-OFFSITE to predict dose and risk, and gives insight into the different methods of quantifying sensitivity.

Sensitivity analysis is often a difficult subject for many of the professionals who perform dose or risk analysis. For this reason, this report contains many examples and is more in the form of a tutorial than a technical report. The case studies, and especially the in-depth look at the output from them, are written in first- and second-person problem-solving form rather than in the traditional style of a technical report.

There are three different ways of performing sensitivity analysis in RESRAD-OFFSITE:

- Single-input sensitivity analysis using the F9 key-referred to as the three-point singleinput sensitivity analysis because it compares code predictions for three discrete values of the input being studied (see Section C.2.1).

- $\quad$ Single-input sensitivity analysis using the Shift-F8 key-referred to as the distributed single-input sensitivity analysis because it uses the probabilistic module to generate predictions for many values of the input being studied (see Section C.2.2).

- Multiple-input sensitivity analysis using the Shift-F8 key-referred to as multiple-input sensitivity analysis using probabilistic feature of the code because it uses the probabilistic module to simultaneously analyze the sensitivity of multiple inputs (see Section C.4).

Section C.3 takes a deeper look at the case study in Section C.2. It also illustrates the methods commonly used to compare the influences of inputs. The reader is led through a natural progression from single-input sensitivity analysis to two- and three-input sensitivity analyses to multiple-input sensitivity analysis. Multiple-input sensitivity analysis is presented in Section C.4. 


\section{C.2 Single-input Sensitivity Analysis}

\section{C.2.1 Three-point Single-input Sensitivity Analysis}

In the three-point single-input method of sensitivity analysis, all inputs other than the one being studied are held at their deterministic or point values. Three simulations of the code are performed, one with the input of interest at its point value $(x)$, the second with it at a specified multiple of the original point value $(\mathrm{mx})$, and the third with it at a specified fraction of the original point value $(x / m)$. The same factor $(m)$ is used to obtain the multiple and the fraction. Temporal predictions of the three simulations are shown on the same plot. The plotting program does not compute a numerical measure of sensitivity, but the numbers needed to compute sensitivity measures are in the data used by the plotting program.

\section{C.2.1.1 Performing a Three-point Single-input Sensitivity Analysis}

The procedure for performing a three-point single-input sensitivity analysis in RESRADOFFSITE is listed below. A case study is given in Section C.2.1.2.

1. Prepare an input file that contains site-appropriate deterministic values for all inputs. Assigned values must be appropriate for the site because the sensitivity of the predicted dose to an input depends on the values of all of the other inputs.

2. Place the cursor on the input upon which you want to perform three-point single-input sensitivity analysis. Select the input to be analyzed using the Form Options menu, the sensitivity icon on the tool bar, or, most conveniently, the F9 function key on the key board. This causes the Sensitivity Analysis Range form to pop up.

3. Select or type in the factor by which you want to modify (multiply, divide) the point value of the input in the Sensitivity Analysis Range form. Then click the OK button on that form.

4. Repeat Steps 2 and 3 for the other inputs of interest. Up to 25 inputs can be selected for sensitivity analysis in an input file.

5. Run the input file. The computational code will first perform a simulation with all inputs at their deterministic point values. It then continues seamlessly to perform a pair of simulations for the first input selected for sensitivity analysis. The first simulation of the pair is performed with the input in question set to a "high" value. The high value is equal to the deterministic point value multiplied by the factor specified in Step 2. The second of the pair is performed with the input in question set to a "low" value. The low value is 
equal to the deterministic point value divided by the factor specified in Step 2. RESRADOFFSITE then resets the input of interest to its original deterministic value in preparation for the next sensitivity analysis. The computational code will continue to perform additional pairs of simulations for each input chosen.

6. A text report summarizing the deterministic doses is displayed when the simulations are completed. This text report does not contain information about the three-point singleinput sensitivity analysis. Close this report and view the deterministic graphics report. Click the Option button at the lower left side of the graphics pane to display the sensitivity output. Sensitivity of the output (predicted dose, risk, or concentration) to each of the selected inputs can be viewed in turn using the dropdown selection box that is immediately below the option button.

\section{C.2.1.2 Case Study}

This simple illustration of the procedure for three-point single-input sensitivity analysis outlined in Section C.2.1.1 will allow users to try it for themselves if they wish to. For simplicity, it is assumed that most of the preloaded RESRAD-OFFSITE input values are site-appropriate.

The simple scenario used for this case study is defined below.

- $\quad$ The preloaded values of RESRAD-OFFSITE are assumed to be appropriate for the simple hypothetical scenario, except for the following:

- Initial concentrations of $1 \mathrm{pCi} / \mathrm{g}$ for ${ }^{210} \mathrm{~Pb},{ }^{210} \mathrm{Po}$, and ${ }^{226} \mathrm{Ra}$ in the primary contamination;

- Leach rates of 0.002 per year for ${ }^{210} \mathrm{~Pb},{ }^{210} \mathrm{Po}$, and ${ }^{226} \mathrm{Ra}$;

- Distribution coefficients in the unsaturated and saturated zones of $7 \mathrm{ml} / \mathrm{g}$ for $\mathrm{Ra}$ and $10 \mathrm{ml} / \mathrm{g}$ for $\mathrm{Pb}$;

- 100-m long, 100-m wide, 2-m thick contaminated soil;

- Hydraulic conductivity of $700 \mathrm{~m} / \mathrm{yr}$ in saturated zone.

- Three-point single-input sensitivity analysis is to be performed on saturated hydraulic conductivity in the saturated zone using a modification factor of 3 .

The reader is assumed to be familiar with RESRAD-OFFSITE; therefore, we do not describe how to set up the site-appropriate point values in the code. Name this file "Three Point Single Input Sensitivity.ROF." 
The hydraulic conductivity in the saturated zone can be selected for sensitivity analysis after specifying the site-appropriate point value for that input. Press the F9 function key while the cursor is still in the input box for hydraulic conductivity in the saturated zone. The Set Sensitivity Analysis Range form pops up, as shown in Figure C.1. Click on the option button corresponding to a multiplication and division factor of 3 . The three values that will be used to perform sensitivity analysis on the hydraulic conductivity in the saturated zone will be displayed in this form.

The first simulation will use the site-appropriate values that were specified for all the inputs, including the $700 \mathrm{~m} / \mathrm{yr}$ value for saturated hydraulic conductivity in the saturated zone. The second simulation will use a value of $2100 \mathrm{~m} / \mathrm{yr}(=700 \times 3)$ for the saturated hydraulic conductivity and the site-appropriate values for all the other inputs. The third simulation will use a value of $233.3 \mathrm{~m} / \mathrm{yr}(=700 \div 3)$ for saturated hydraulic conductivity in the saturated zone and site-appropriate values for all the other inputs. All three simulations will be performed by the computational code when the "Run" command is issued. The Parent dose text report (summarizing the predicted dose at various times in the future) is displayed at the end of the run. This report does not contain results for the sensitivity analysis. Close the text report and open the deterministic graphics report to view the three-point single-input sensitivity output. 


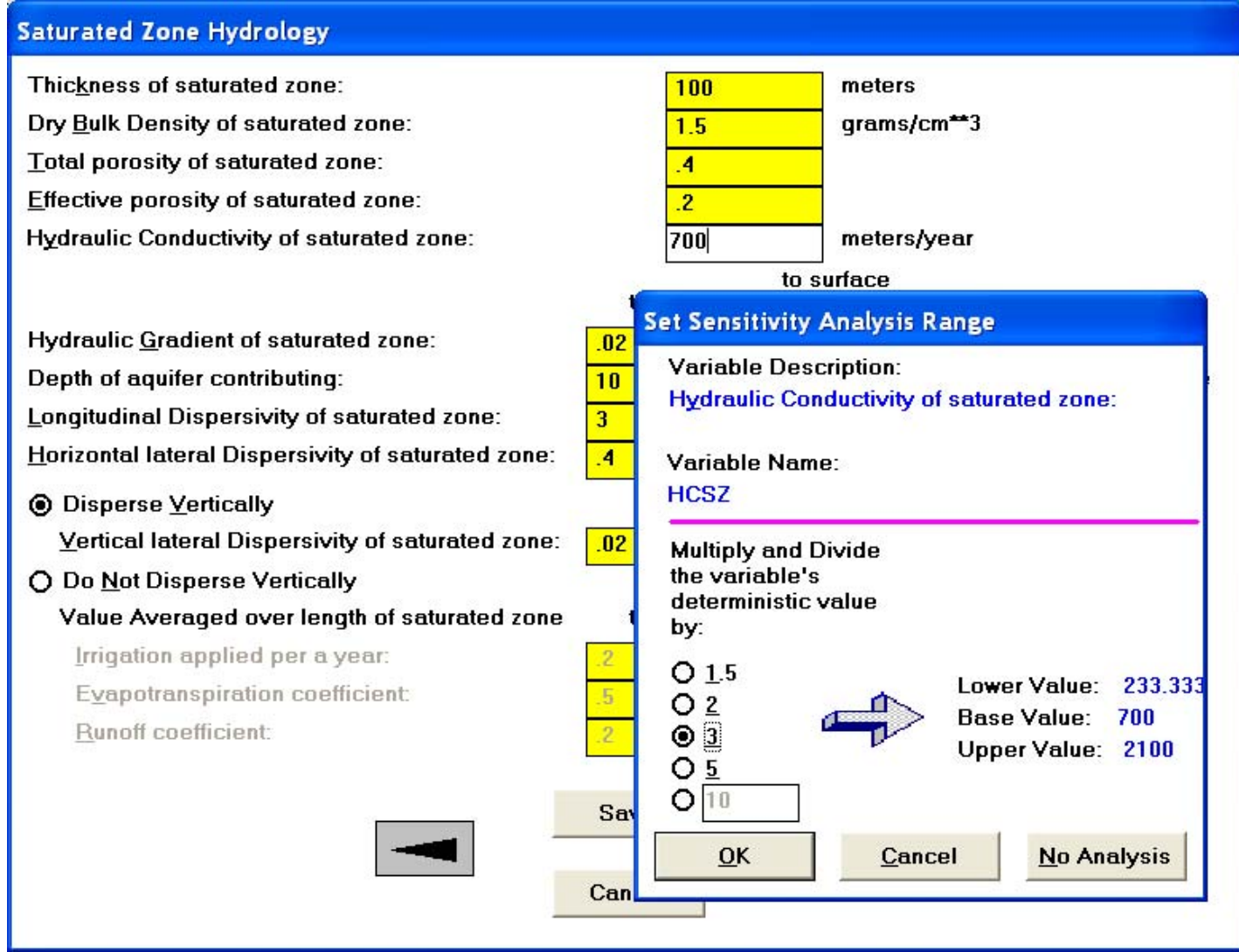

\section{FIGURE C.1 Specifying Three-point Single-input Sensitivity Analysis for the Input of Interest}

The sensitivity graphics can be viewed by clicking on the option button on the bottom left of the graphic window. The resulting screen is shown in Figure C.2. The dropdown box lists all the inputs upon which sensitivity analyses were performed. In the example shown, just one input was selected for sensitivity analysis. The three temporal plots show that the peak predicted dose increases as the hydraulic conductivity decreases from $2100 \mathrm{~m} / \mathrm{yr}$ through $700 \mathrm{~m} / \mathrm{yr}$ to $233.3 \mathrm{~m} / \mathrm{yr}$.

One measure of sensitivity is the ratio between the fractional change in peak predicted dose and the fractional change in the value of the input. The data used to plot the three curves can be saved and viewed using the menu commands or toolbar icons. The peak predicted doses for the three simulations were found to be 11.6, 21, and $41.9 \mathrm{mrem} / \mathrm{yr}$. The normalized sensitivity of predicted dose to hydraulic conductivity in the saturated zone over the range 233.33 to $2100 \mathrm{~m} / \mathrm{yr}$ is then computed as: 


$$
\text { Sensitivity }=\frac{41.9-11.6}{(41.9+11.6) / 2} \div \frac{233.3-2100}{(233.3+2100) / 2}=\frac{30.3}{26.75} \div(-1.6)=-0.71
$$

The sensitivity is negative because the predicted dose decreases as the value of the input increases.

It is also possible to compute forward and backward sensitivities. The sensitivities of predicted dose to hydraulic conductivity in the ranges 700 to $2100 \mathrm{~m} / \mathrm{yr}$ (forward sensitivity) and 233.3 to $700 \mathrm{~m} / \mathrm{yr}$ (backward sensitivity) are computed as follows:

$$
\begin{gathered}
\text { Backward Sensitivity }=\frac{41.9-21}{(41.9+21) / 2} \div \frac{233.3-700}{(233.3+700) / 2}=\frac{20.9}{31.45} \div(-1)=-0.66 \\
\text { Forward Sensitivity }=\frac{21-11.6}{(21+11.6) / 2} \div \frac{700-2100}{(700+2100) / 2}=\frac{9.4}{16.3} \div(-1)=-0.58
\end{gathered}
$$

The three sensitivity calculations show that the sensitivity of predicted dose to hydraulic conductivity in the saturated zone is not uniform over the range 233.33 to $2100 \mathrm{~m} / \mathrm{yr}$. While the results give an indication of the variability of the sensitivity, more simulations would be needed to demonstrate how the sensitivity changes over the range. This will be investigated in distributed single-input sensitivity analysis (Section C.2.2).

The temporal plots for each of the three simulations appear to be the sum of two curves that are offset in time. They are, in fact, the sum of predicted doses from 15 exposure and transport pathways, six of which make a visually significant contribution, as seen in Figure C.3. Sensitivity plots for the six exposure pathways are shown in Figures C.4 through C.9. 


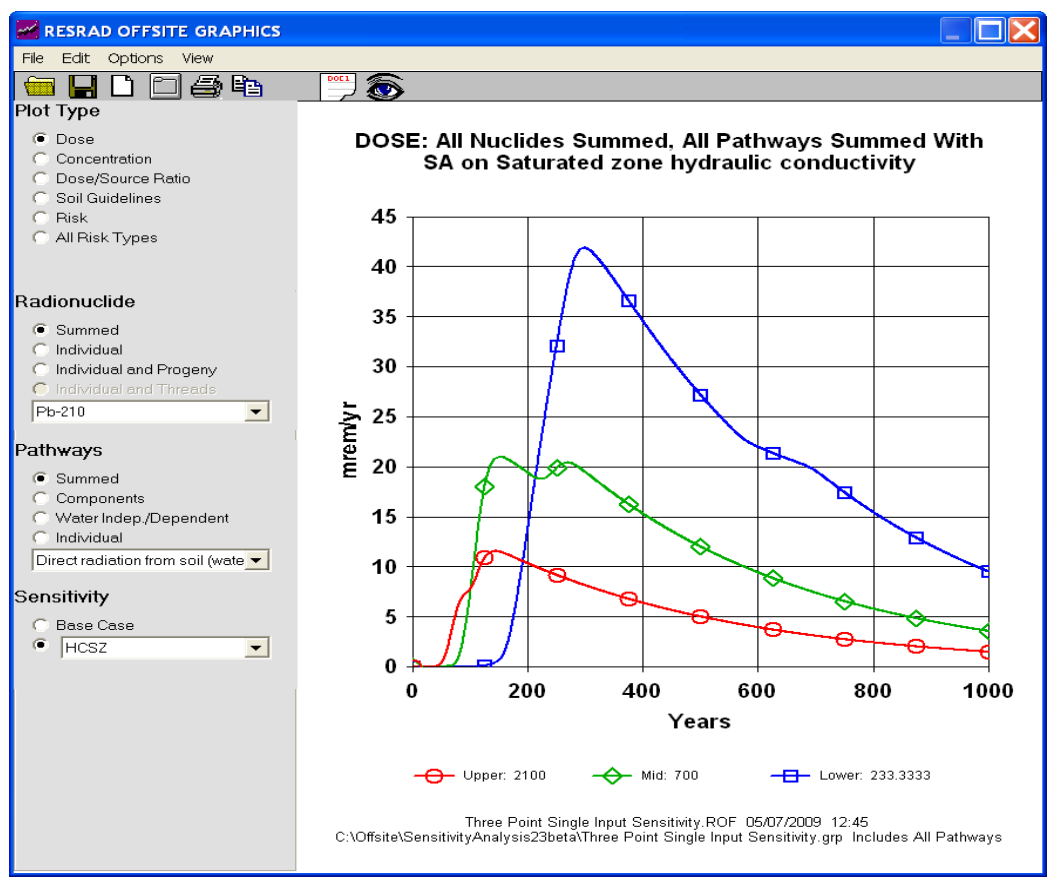

FIGURE C.2 Viewing Sensitivity Graphics

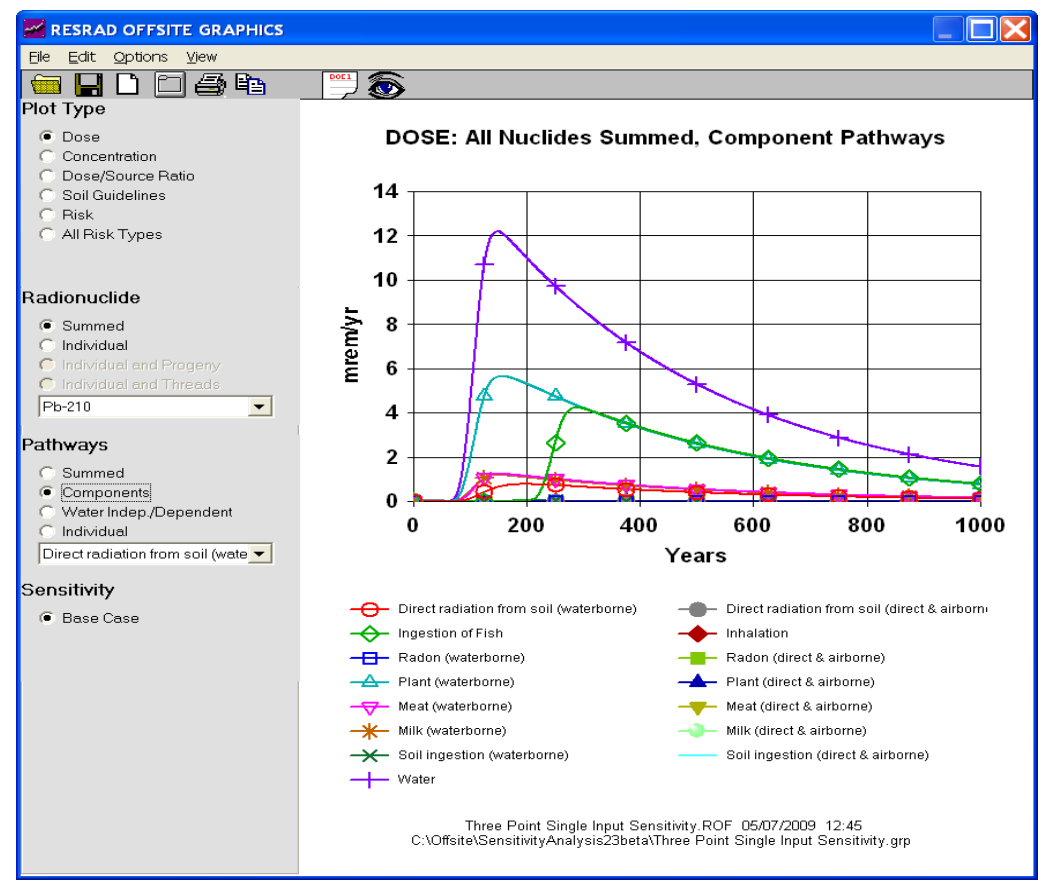

FIGURE C.3 Identifying Significant Exposure Pathways for the Base Case 


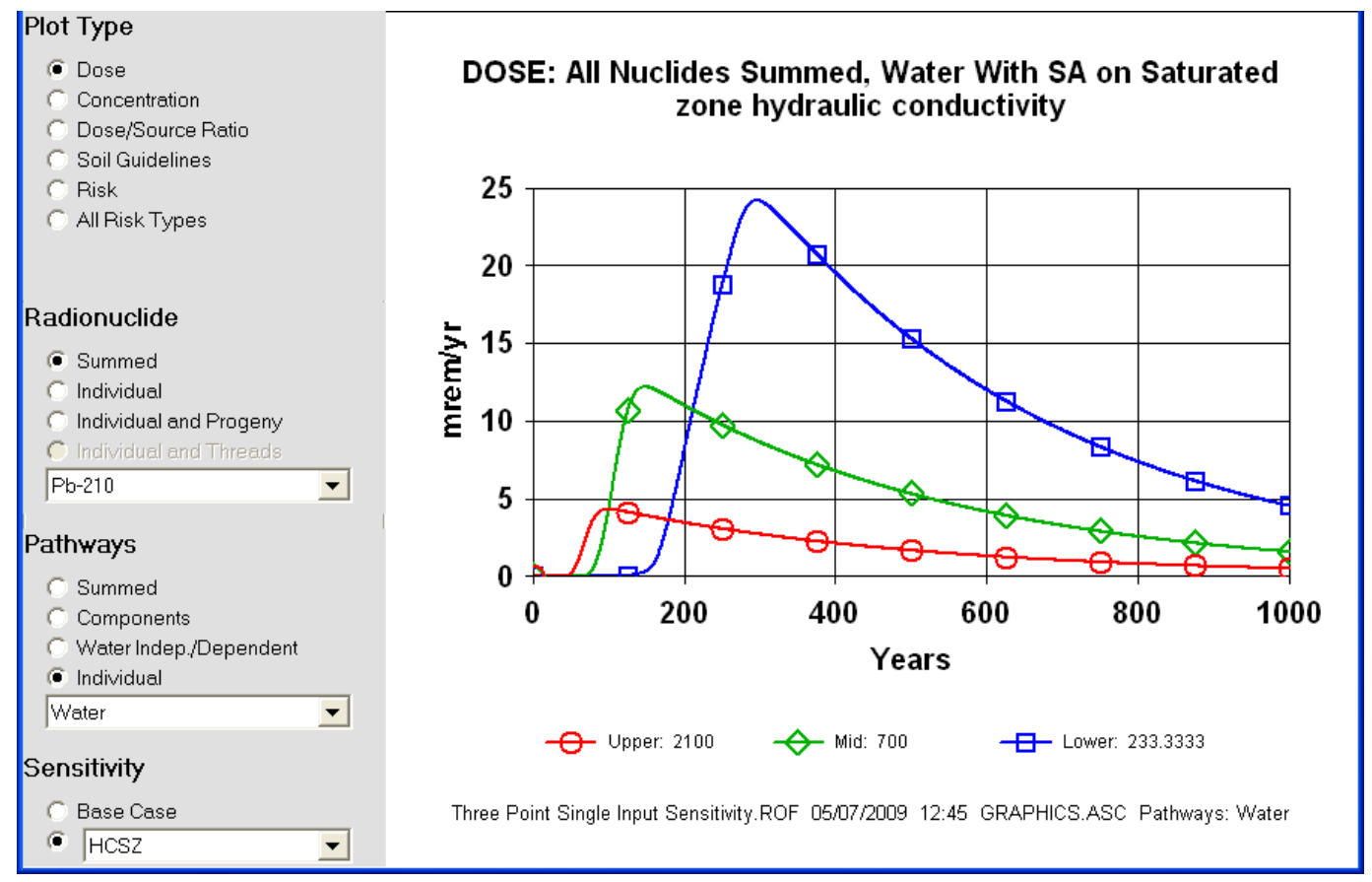

FIGURE C.4 Sensitivity of Predicted Dose from Drinking Water

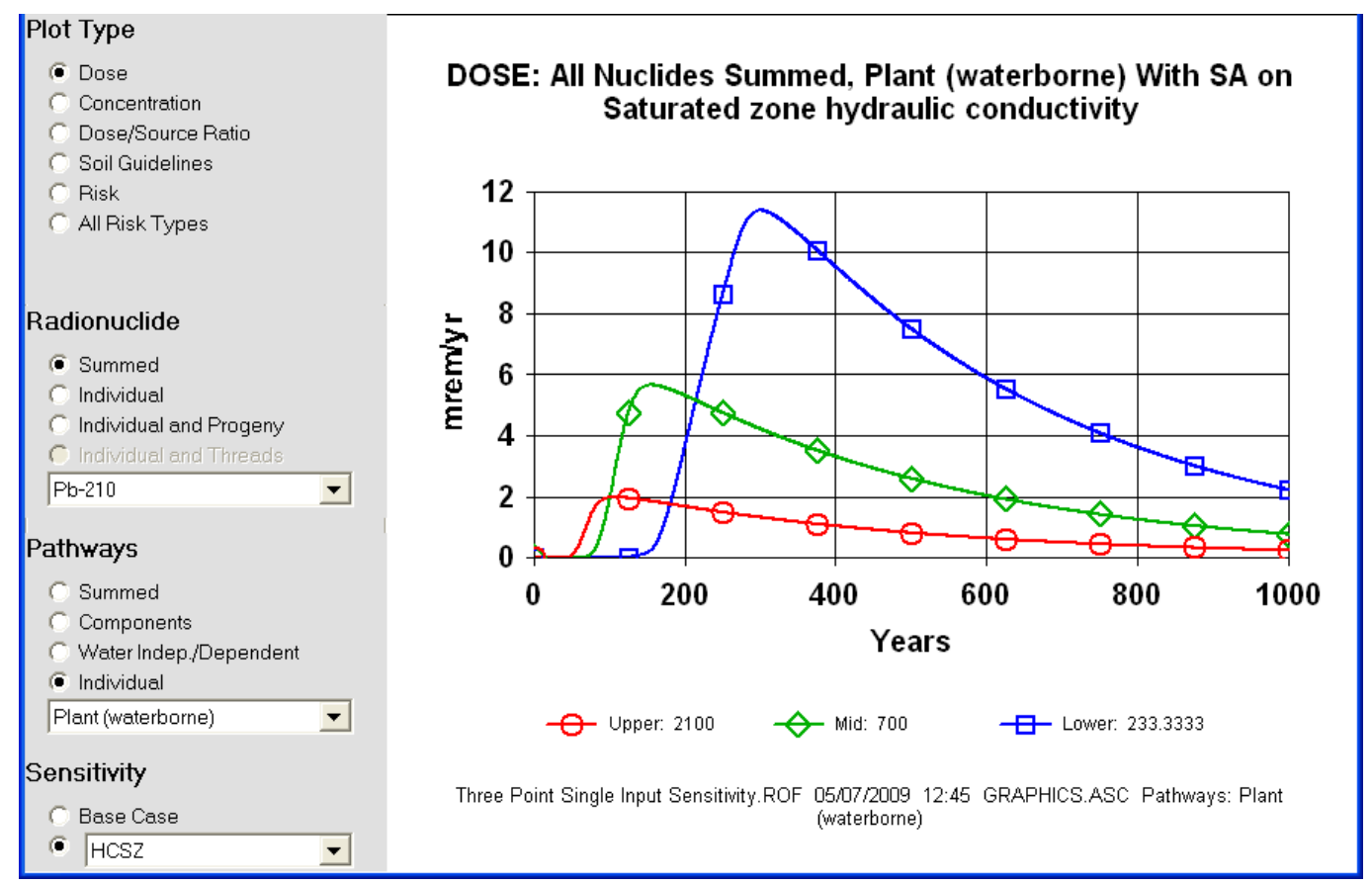

FIGURE C.5 Sensitivity of Predicted Dose from Ingestion of Vegetables (Waterborne Contamination) 


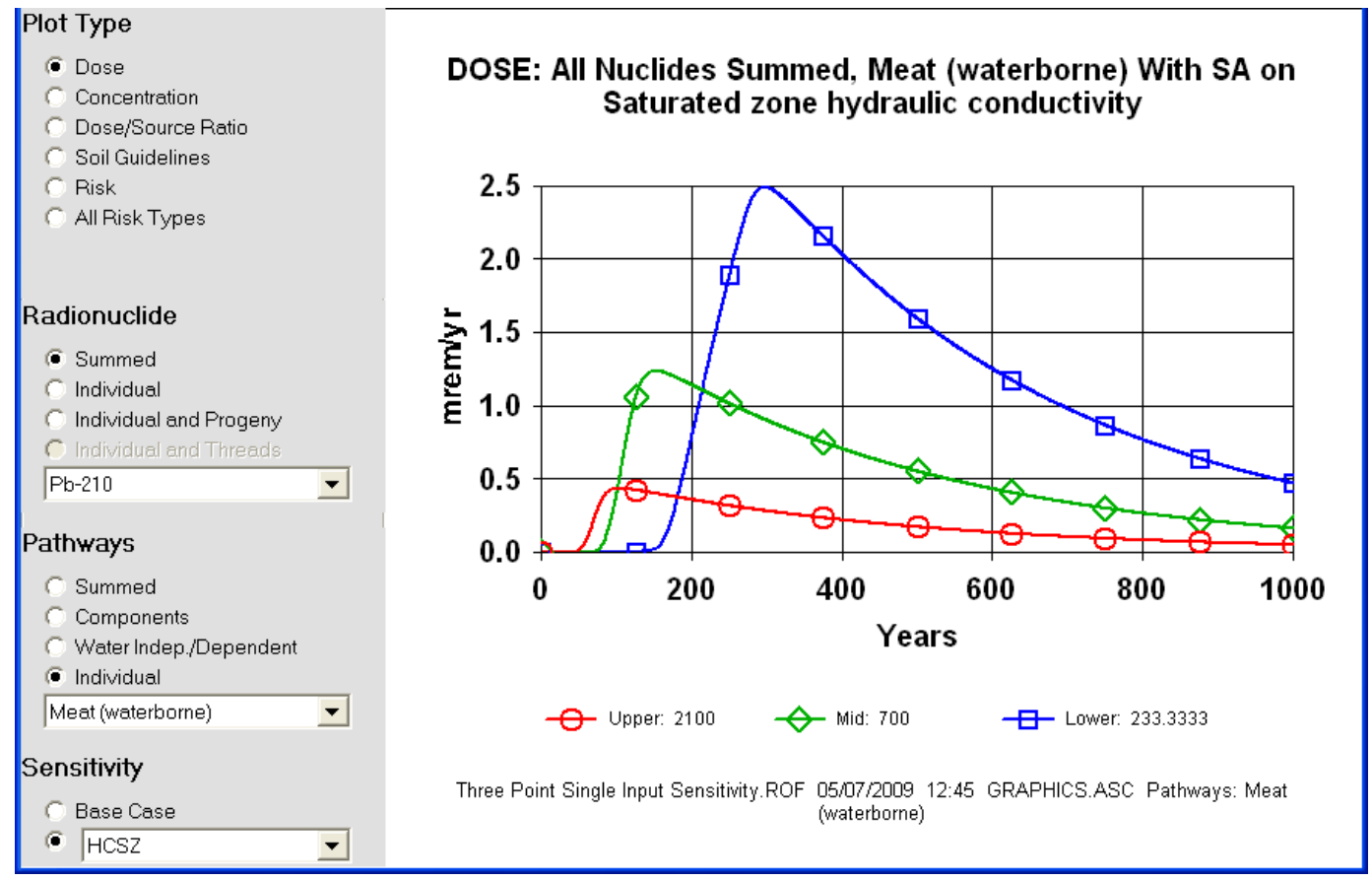

FIGURE C.6 Sensitivity of Predicted Dose from Ingestion of Meat (Waterborne Contamination)

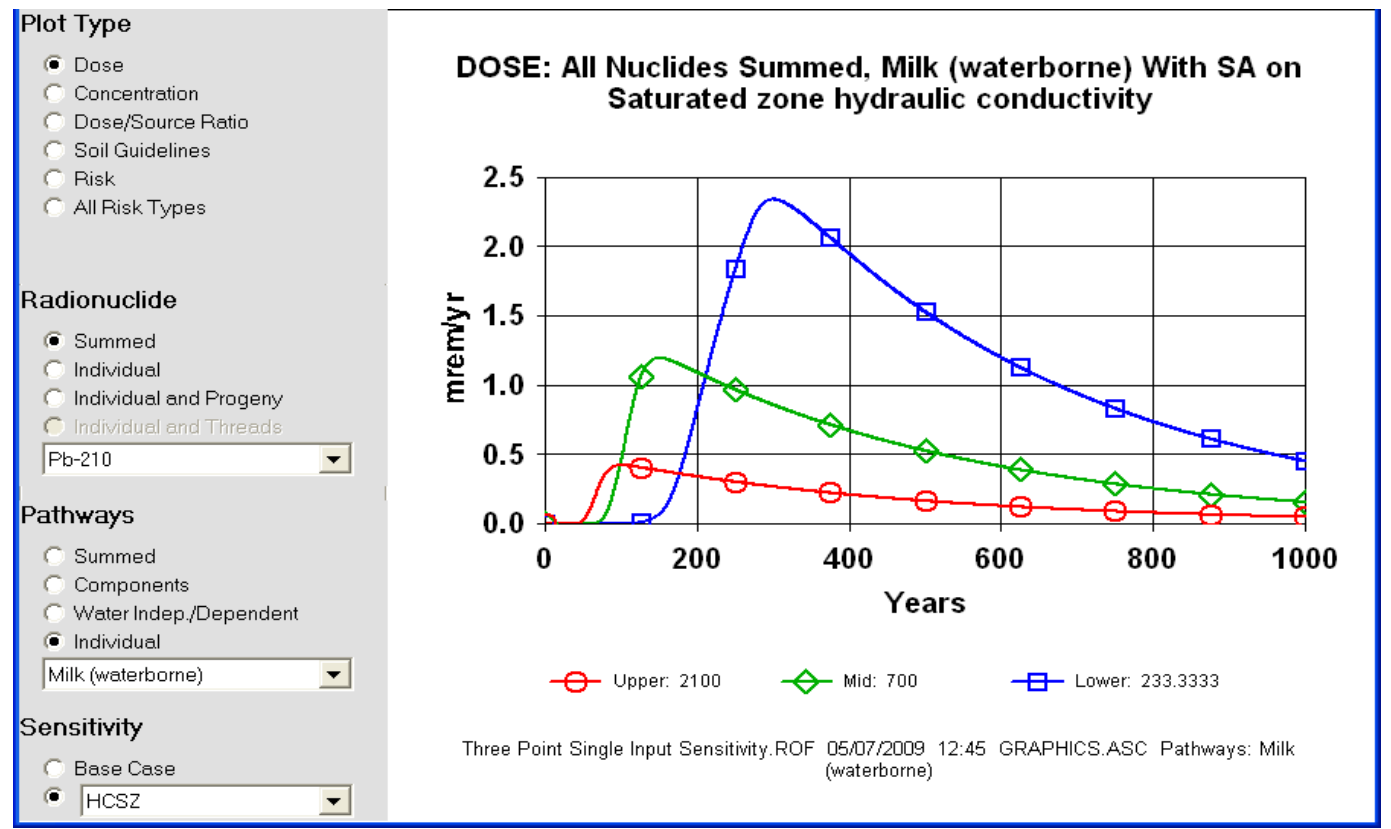

FIGURE C.7 Sensitivity of Predicted Dose from Ingestion of Milk (Waterborne Contamination) 


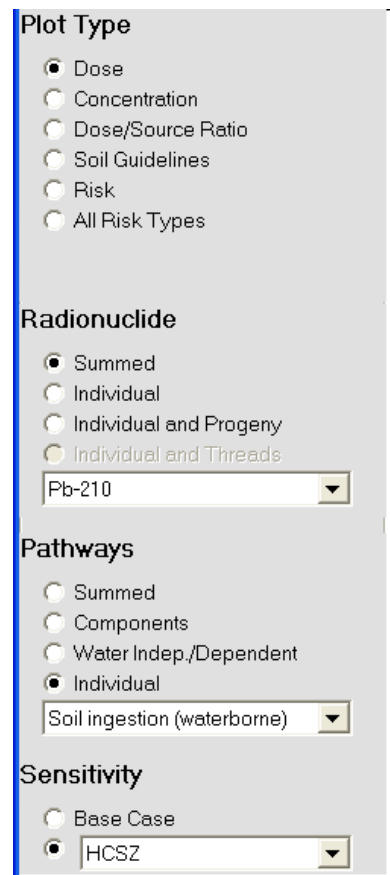

DOSE: All Nuclides Summed, Soil ingestion (waterborne) With SA on Saturated zone hydraulic conductivity

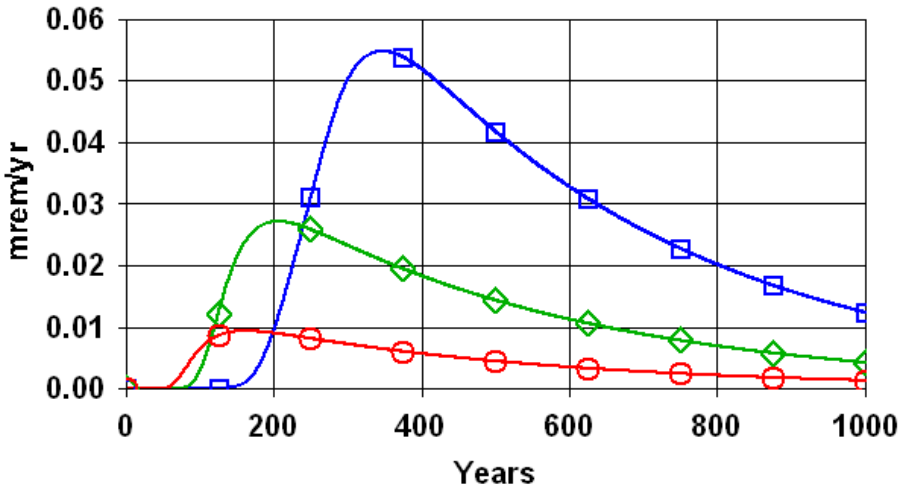

$\odot$ Upper: $2100 \quad-$ Mid: $700 \quad \square$ Lower: 233.3333

Three Point Single Input Sensitivity.ROF 05/07/2009 12:45 GRAPHICS.ASC Pathways: Soil ingestion (waterborne)

\section{FIGURE C.8 Sensitivity of Predicted Dose from Direct Exposure} to Waterborne Accumulation in Soil

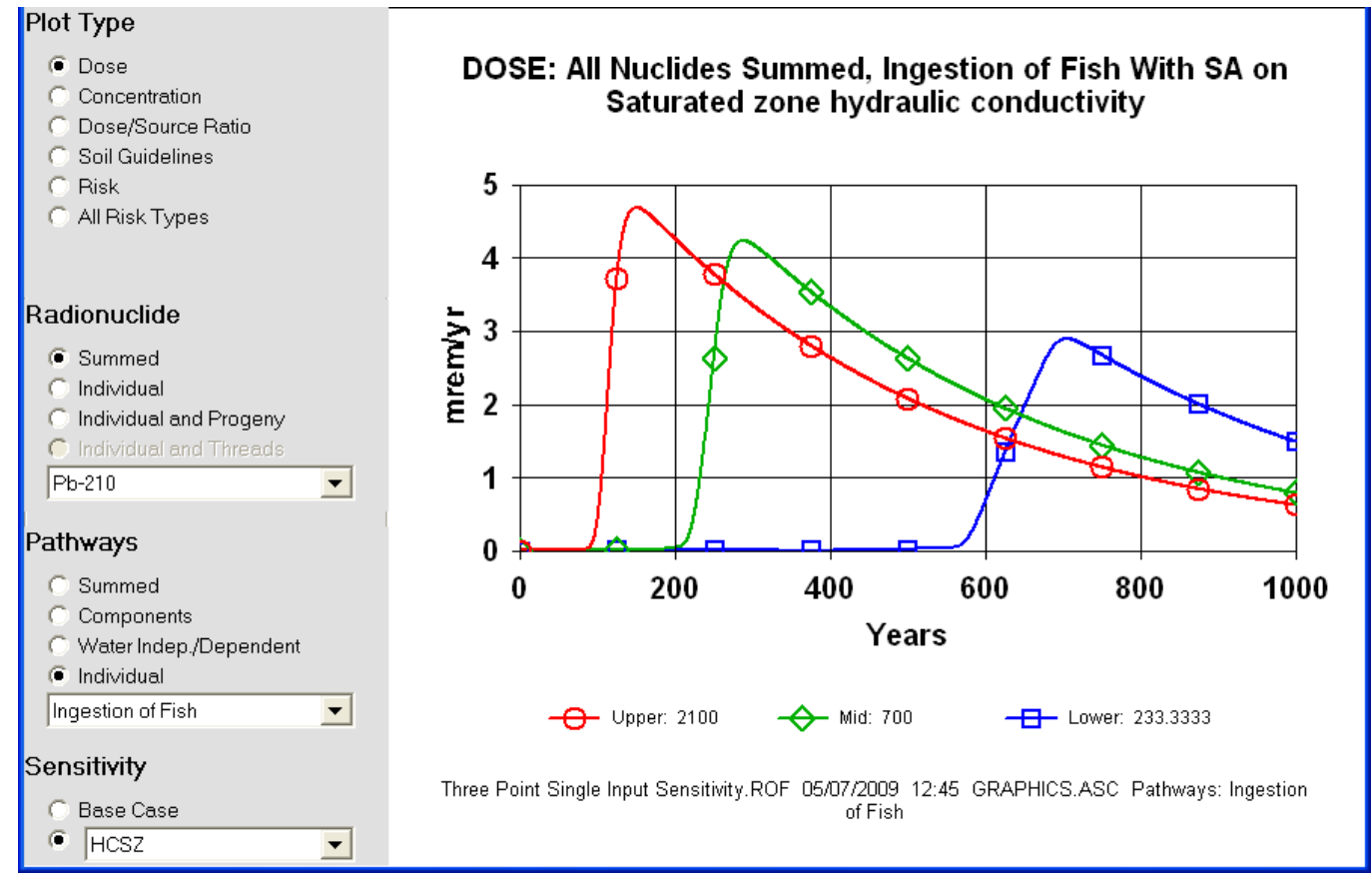

FIGURE C.9 Sensitivity of Predicted Dose from Ingestion of Fish 
The exposure pathways shown in Figures C.4 through C.8 occur as a result of the use of contaminated well water, while that shown in Figure C.9 occurs from contamination of a surface water body. The plots shown in Figures C.4 through C.8 all begin to rise at about the same time. The time of the peak predicted dose from these pathways depends on the relative contributions directly from well water and indirectly from the accumulation in soil following irrigation with well water. The predicted doses from the five pathways decrease with increasing hydraulic conductivity in the saturated zone.

The plots in Figure C.9 start rising later than those in Figures C.4 through C.8 because of the longer distance to the surface water body. The predicted dose from this pathway rises with increasing hydraulic conductivity in the saturated zone. The higher predicted dose from the ingestion of fish in conditions of high hydraulic conductivity, as contrasted with the lower predicted dose from the five well-water-based pathways in conditions of high hydraulic conductivity, is investigated in Section C.3.

\section{C.2.2 Distributed Single-input Sensitivity Analysis}

In this method of analysis, all the inputs other than the one being studied are held at its deterministic or point value. Many simulations of the code are performed, each with the input of interest set to a (different) sample value obtained from the distribution specified for that input. The analyst specifies the number of simulations to be performed. A scatter plot shows the variation of peak predicted dose (or risk) with the variable of interest. Because of the large number of simulations that are typically performed, temporal predictions for all of the simulations are not shown on the same plot. It is, however, possible to view the temporal plots of each simulation individually, though this is not typically done because of the larger number of simulations. Flag the code prior to the run to save the plot data, if you want to view the temporal plot of each simulation.

\section{C.2.2.1 Performing a Distributed Single-input Sensitivity Analysis}

The procedure for performing a distributed single-input sensitivity analysis in RESRADOFFSITE is as follows.

1. Prepare an input file that contains site-appropriate deterministic values for all inputs. Because the sensitivity of the predicted dose to an input of interest depends on the values of all of the other inputs, it is imperative that all inputs be assigned values that are appropriate for the site. 
2. Place the cursor on the input upon which you want to perform distributed single input sensitivity analysis. Select the input of interest using the Shift-F8 function key. The "Parameter distributions" tab of the Uncertainty and Probabilistic Analysis form will appear.

3. Choose a distribution type. RESRAD-OFFSITE defaults to a uniform distribution, but in some cases it might be prudent to change this. Select a log-uniform distribution if the study range spans orders of magnitude. A continuous linear distribution might also be appropriate in some circumstances (Section C.3.5.2). Specify the lower and upper limits of the study range; click the Update Parameter Stats and Distribution button to save these limits.

4. Do not select more than one input. Go to the "Sample specifications" tab of the Uncertainty and Probabilistic Analysis form and specify the desired number of observations. Set the number of repetitions to 1; repetitions are not necessary because the output of interest is a scatter plot and a distribution is being specified on only one input.

5. Run the input file. The computational code will first perform a deterministic simulation with all inputs at their deterministic point values. It then continues seamlessly to perform the specified number of probabilistic simulations for the input of interest.

6. A text report summarizing the deterministic doses is displayed when the simulations are completed. This text report does not contain information about the distributed sensitivity.

7. Close this report and view the probabilistic graphics. This displays the "Step by step analysis" tab of the Uncertainty and Probabilistic Analysis form. Select "View scatter plots of output vs. input." The scatter plot shows how the output (peak predicted dose or peak risk) varies with the selected input.

\section{C.2.2.2 Case Study}

The simple scenario from Section C.2.1.2 is continued here. For simplicity, it is assumed that most of the preloaded RESRAD-OFFSITE input values are site-appropriate. The example scenario is restated here for easy reference:

- Name the file "Distributed Single Input Sensitivity.ROF." 
- The preloaded values are assumed to be appropriate for this example scenario, except for the following:

- Initial concentration of $1 \mathrm{pCi} / \mathrm{g}$ each of $210 \mathrm{~Pb}, 210 \mathrm{Po}$, and $226 \mathrm{Ra}$ in the primary contamination;

- Leach rates of 0.002 per year for ${ }^{10} \mathrm{~Pb},{ }^{210} \mathrm{Po}$, and ${ }^{226} \mathrm{Ra}$;

- Distribution coefficients in the unsaturated and saturated zones of $7 \mathrm{ml} / \mathrm{g}$ for $\mathrm{Ra}$ and $10 \mathrm{ml} / \mathrm{g}$ for $\mathrm{Pb}$;

- 100-m long, 100-m wide, 2-m-thick contaminated soil;

- Hydraulic conductivity of $700 \mathrm{~m} / \mathrm{yr}$ in saturated zone.

- Distributed single-input sensitivity analysis is to be performed on saturated hydraulic conductivity using a uniform distribution over the range of 200 to $2000 \mathrm{~m} / \mathrm{yr}$.

The hydraulic conductivity in the saturated zone can be selected for distributed sensitivity analysis after specifying the site-appropriate point value for the input of interest. Press the Shift-F8 function key while the cursor is still in the input box for hydraulic conductivity in the saturated zone. The "Parameter distributions" tab of the Uncertainty and Probabilistic Analysis form pops up, as shown in Figure C.10.

Specify a minimum of 200 and a maximum of 2000 for limits of the uniform distribution for the hydraulic conductivity in the saturated zone; click the "Update parameter stats and distribution" button to save these limits. Go to the "Sample specification" tab of the Uncertainty and Probabilistic Analysis form. Set the number of observations to 100 and the number of repetitions to 1 , instructing the code to sample 100 values uniformly in the interval of 200 to 2000 , and to perform 100 simulations using those sample values for hydraulic conductivity in the saturated zone. Repetitions are needed when distributions are specified for more than one input or when the output of interest is the cumulative distribution function of predicted dose (or risk).

When performing a distributed single-input sensitivity analysis, the output of interest is the scatter plot between predicted dose (or risk) and the single-input for which a distribution is specified. All 100 simulations will be performed by the computational code when the "Run" command is issued. The text report (Parent dose) summarizing the deterministic dose at various times in the future is displayed at the end of the run. This report does not contain results of the distributed single-input sensitivity analysis. Close the report and view the probabilistic graphics. 


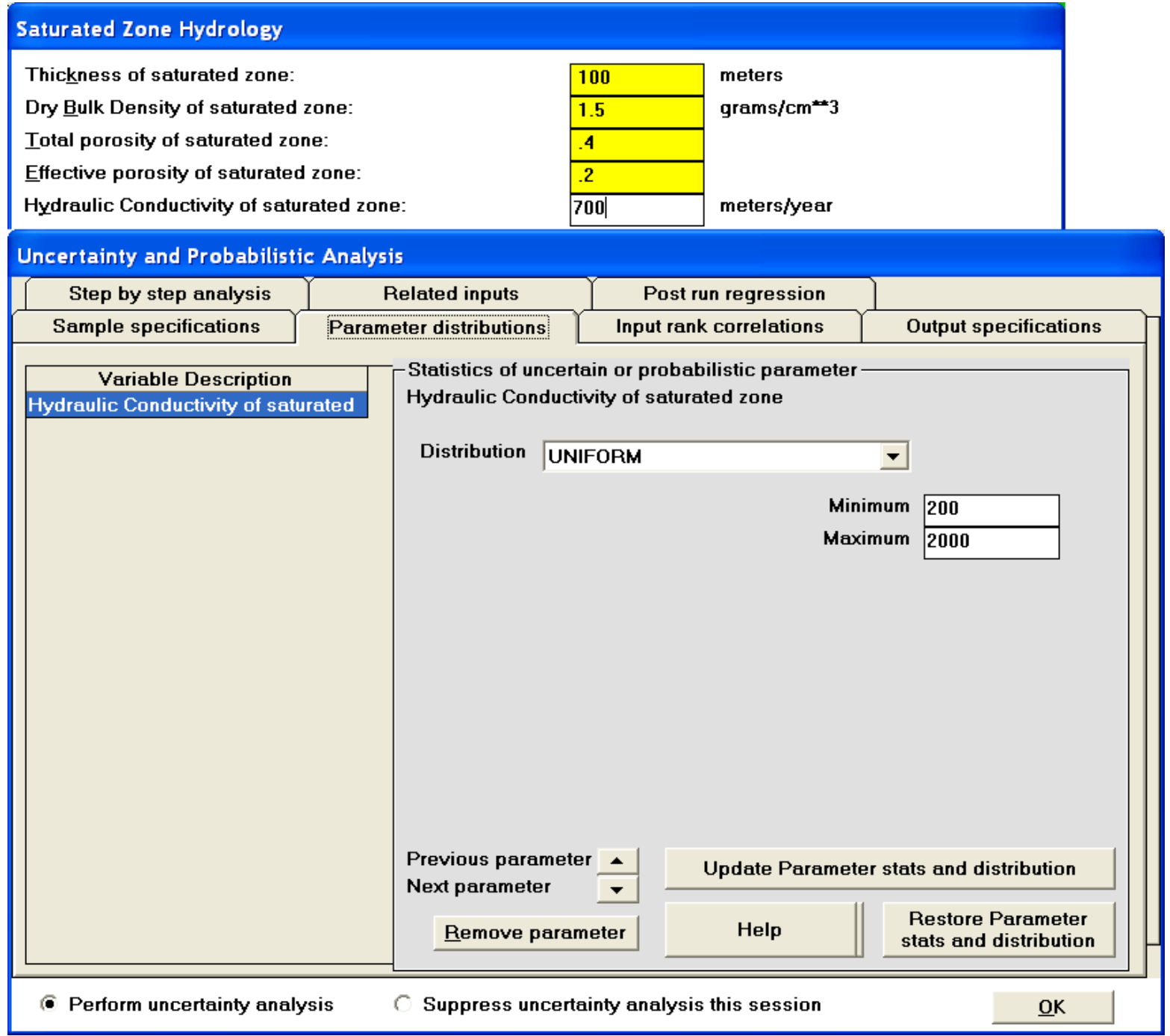

\section{FIGURE C.10 Specifying Distributed Sensitivity Analysis on an Input}

The scatter plot showing the sensitivity can be viewed by clicking on the "View scatter plots of output vs. input" button in the middle of the "Step by step analysis" tab shown in Figure C.11. The scatter plot of peak predicted dose from all pathways against hydraulic conductivity in the saturated zone for this example is shown in Figure C.12. The peak predicted dose increases as the conductivity increases from 200 to about $260 \mathrm{~m} / \mathrm{yr}$. The peak predicted dose then decreases rapidly as conductivity increases from about 260 to about $750 \mathrm{~m} / \mathrm{yr}$. A noticeable break in the curve occurs at a hydraulic conductivity of around $750 \mathrm{~m} / \mathrm{yr}$. The peak predicted dose continues to decrease as the hydraulic conductivity increases from about 750 to $2000 \mathrm{~m} / \mathrm{yr}$, but at a slower rate. The data used to plot this curve are in the peak predicted dose data file (extension .pds)—_Distributed Single-Input Sensitivity.pds" in this case. These data 
can be used to compute the sensitivity between each pair of consecutive sample points, if necessary (see Section C.2.3).

The scatter plots of peak predicted doses from the different exposure pathways against the hydraulic conductivity in the saturated zone can be viewed by clicking the up/down arrows in the probabilistic graphics output form. These are shown in Figures C.13 through C.18. The first five plots shown have similar shapes because they show the predicted doses from exposure pathways that involve contaminated well water: ingestion of well water (Figure C.13), ingestion of vegetables irrigated with well water (Figure C.14), ingestion of meat (Figure C.15) and milk (Figure C.16) from livestock that consumed well water and feed that was irrigated with well water, and external radiation from land irrigated with well water (Figure C.17). The peak predicted doses from these five pathways increase as the conductivity increases from 200 to about $260 \mathrm{~m} / \mathrm{yr}$. The predicted peak dose then decreases, rapidly at first and then at a continuously decreasing rate, as conductivity increases from about 260 to $2000 \mathrm{~m} / \mathrm{yr}$.

The plot showing the influence of hydraulic conductivity on peak predicted dose from the ingestion of fish from the surface water body (Figure C.18) has a different shape than the plots of the five exposure pathways involving contaminated well water. The peak predicted dose from the ingestion of fish increases as the hydraulic conductivity in the saturated zone increases from 200 to $2000 \mathrm{~m} / \mathrm{yr}$. The rate of increase is very rapid at first; it then diminishes as the hydraulic conductivity increases. The reasons for the differences in the shapes of the scatter plots of the pathways contaminated by the two sources of water are discussed in Section C.3.

The plot of the peak predicted dose from all pathways (Figure C.12) is similar to the plot of the peak predicted dose from the five well-water-dependent pathways in the hydraulic conductivity range of 200 to about $750 \mathrm{~m} / \mathrm{yr}$. In fact, the data in the peak predicted dose data file (with filename extension .pds) and the probabilistic input sample data file (with filename extension .pin) indicate that those five pathways peak around the same time and that the peak predicted dose from all pathways is essentially the sum of the peak predicted doses from the five pathways, when the hydraulic conductivity ranges from 200 to about $750 \mathrm{~m} / \mathrm{yr}$ (Table C.1). As the hydraulic conductivity increases beyond $750 \mathrm{~m} / \mathrm{yr}$, the peak predicted dose from all pathways exceeds the sum of the peak predicted doses from the five well-water-dependent pathways, but is less than the sum of the peak predicted doses for the six pathways listed in Table C.1. When the hydraulic conductivity is in the range 750 to $2000 \mathrm{~m} / \mathrm{yr}$, the peak predicted dose from all pathways occurs significantly later than for the five well-water-dependent pathways and is closer to the time of the peak predicted dose from the ingestion of fish. 


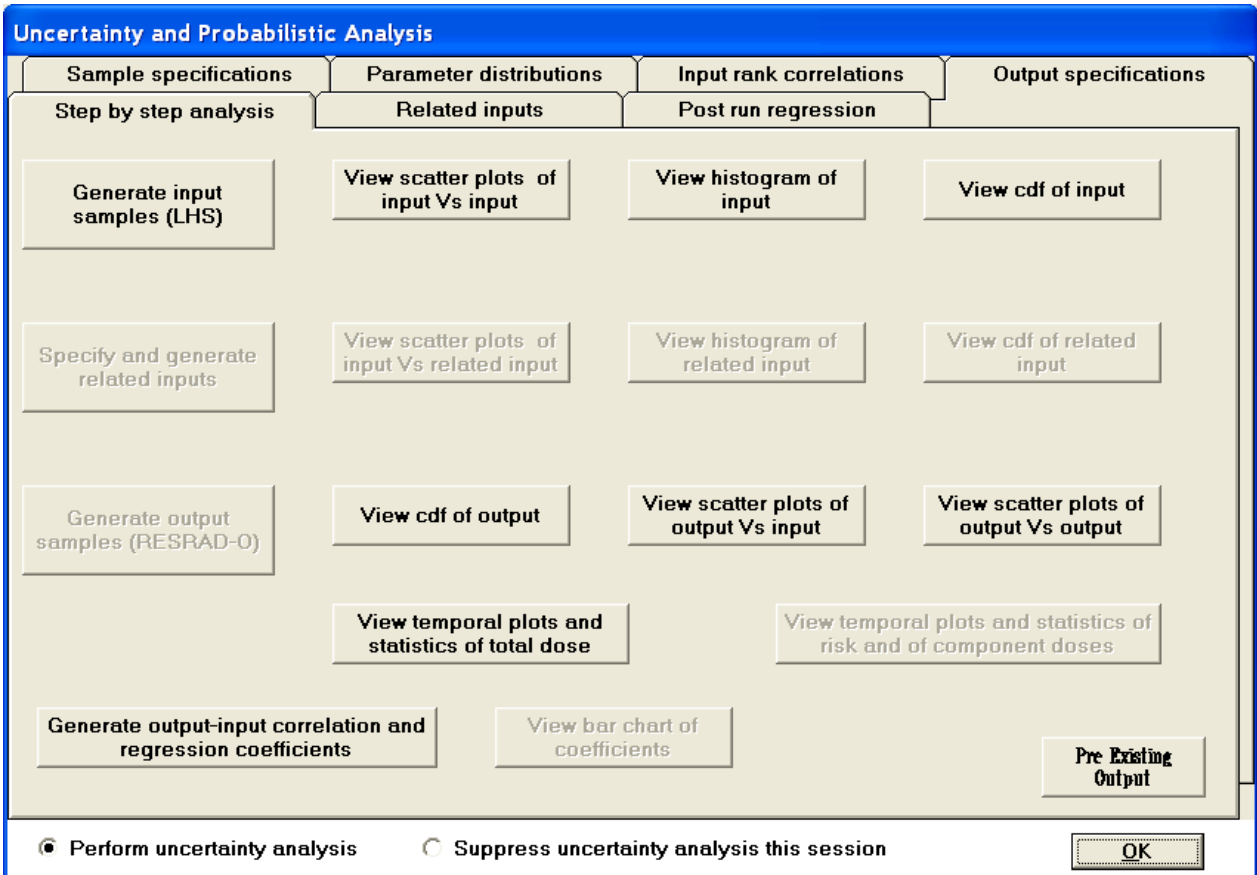

\section{FIGURE C.11 Viewing the Output of a Distributed Sensitivity Analysis on an Input}

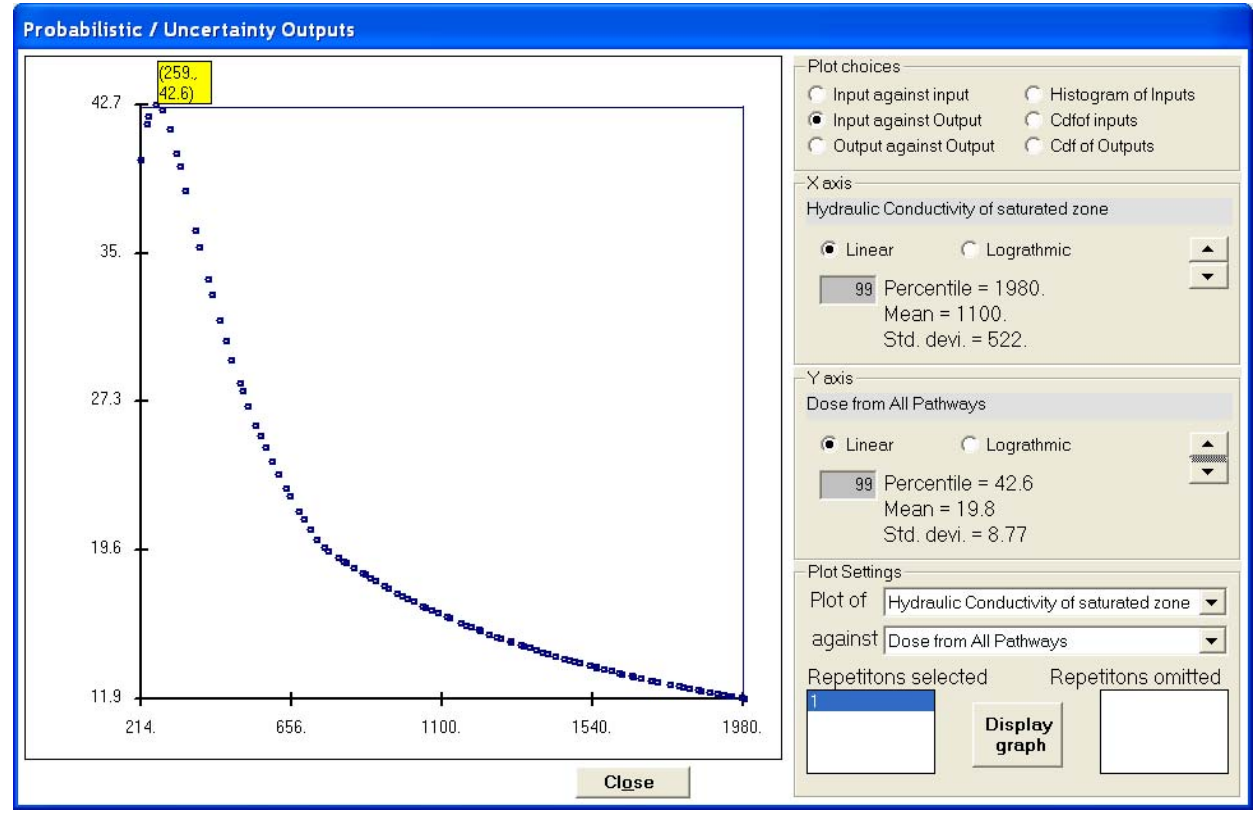

\section{FIGURE C.12 Variation of Peak Predicted Dose with Hydraulic Conductivity in the Saturated Zone}




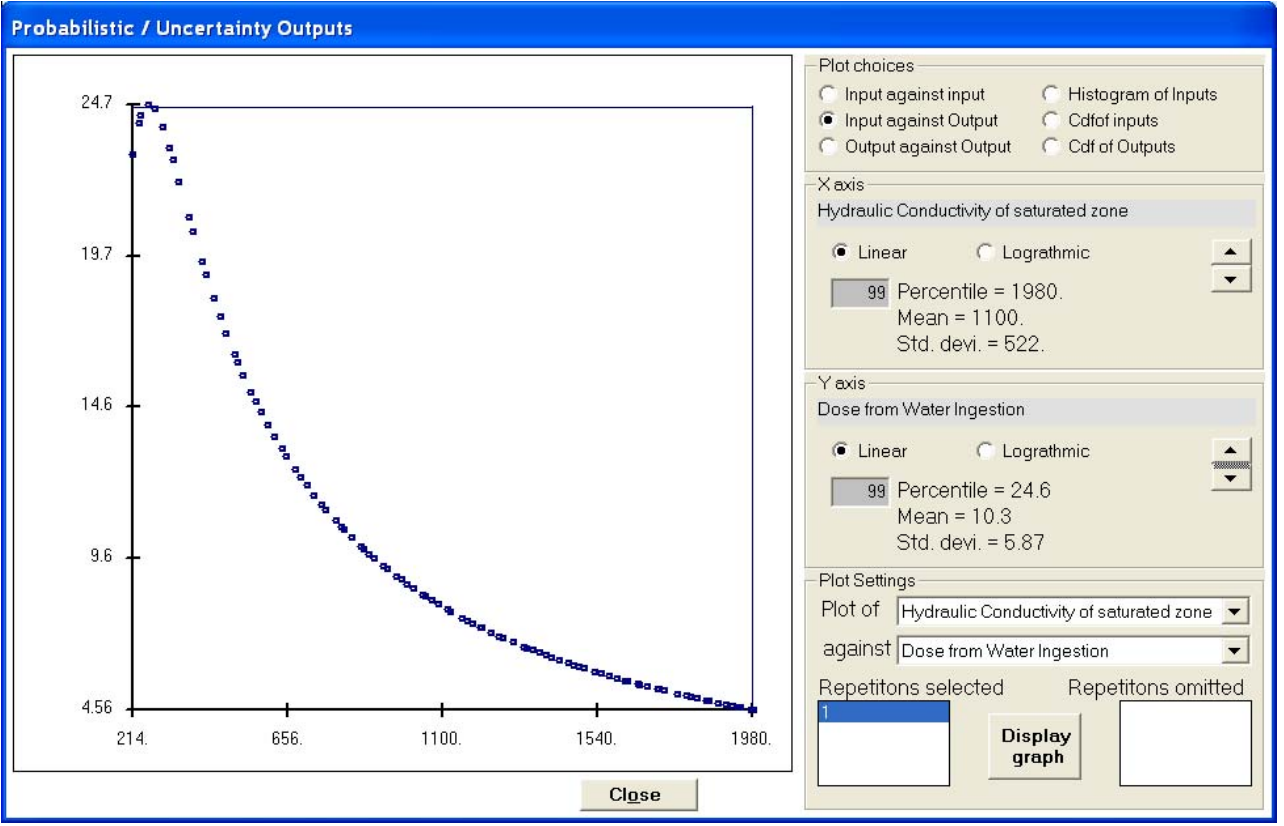

FIGURE C.13 Variation of Peak Predicted Dose from Drinking Water with Hydraulic Conductivity in the Saturated Zone

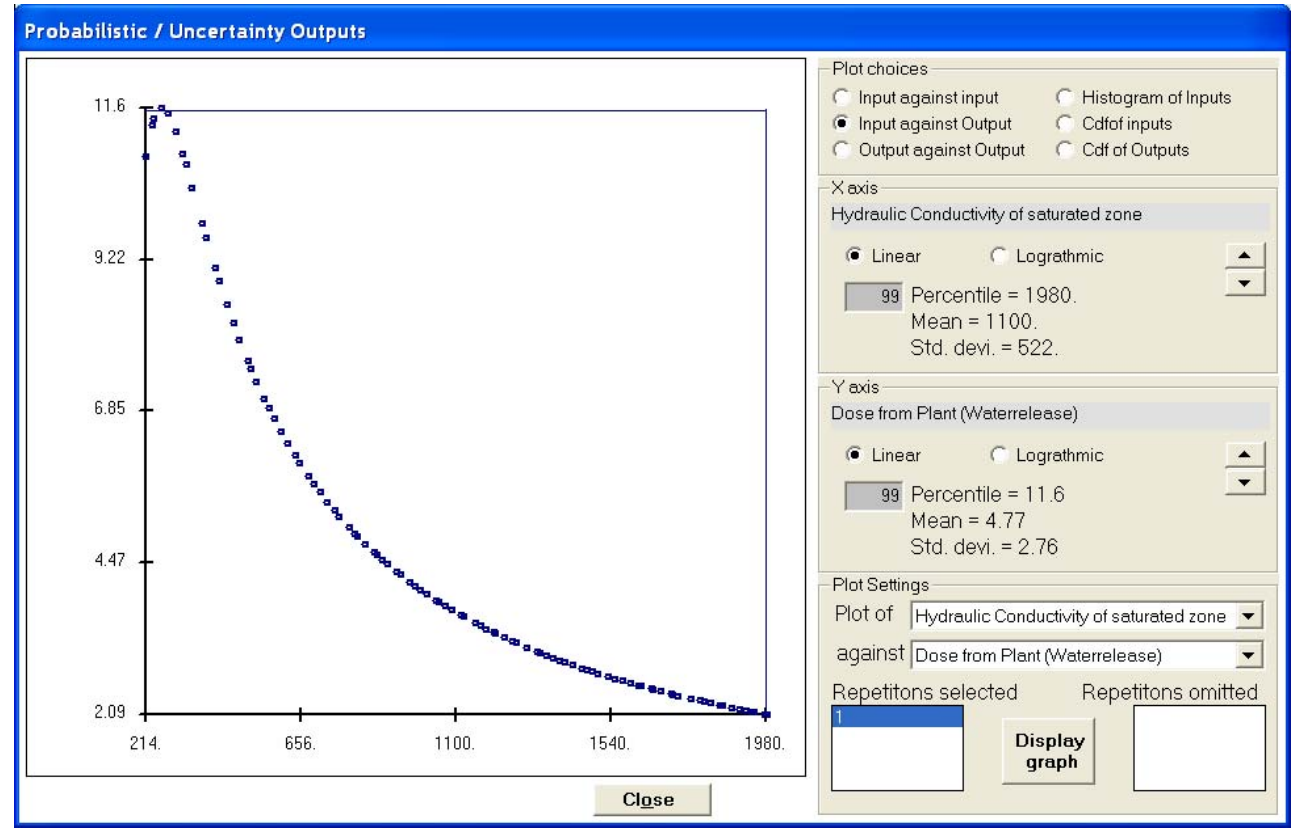

FIGURE C.14 Variation of Peak Predicted Dose from Ingestion of Vegetables (Waterborne Contamination) with Hydraulic Conductivity in the Saturated Zone 


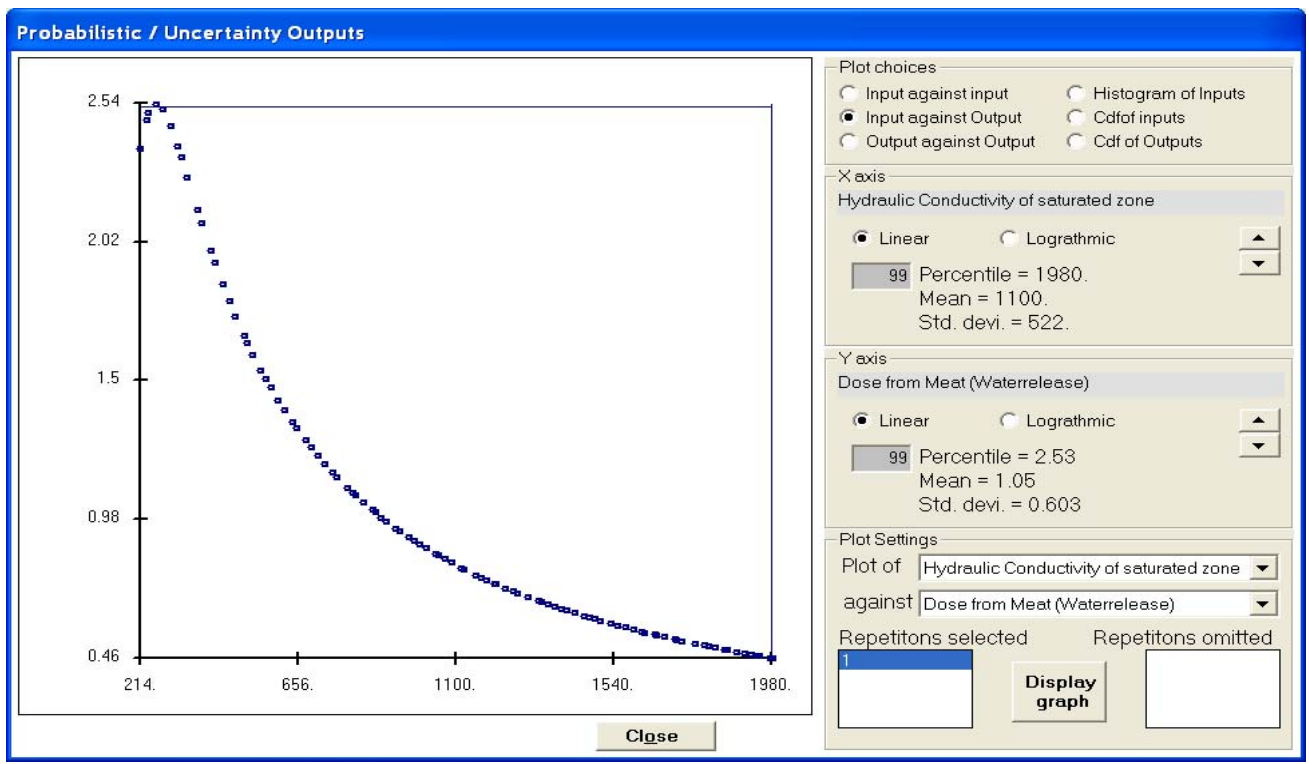

FIGURE C.15 Variation of Peak Predicted Dose from Ingestion of Meat (Waterborne Contamination) with Hydraulic Conductivity in the Saturated Zone

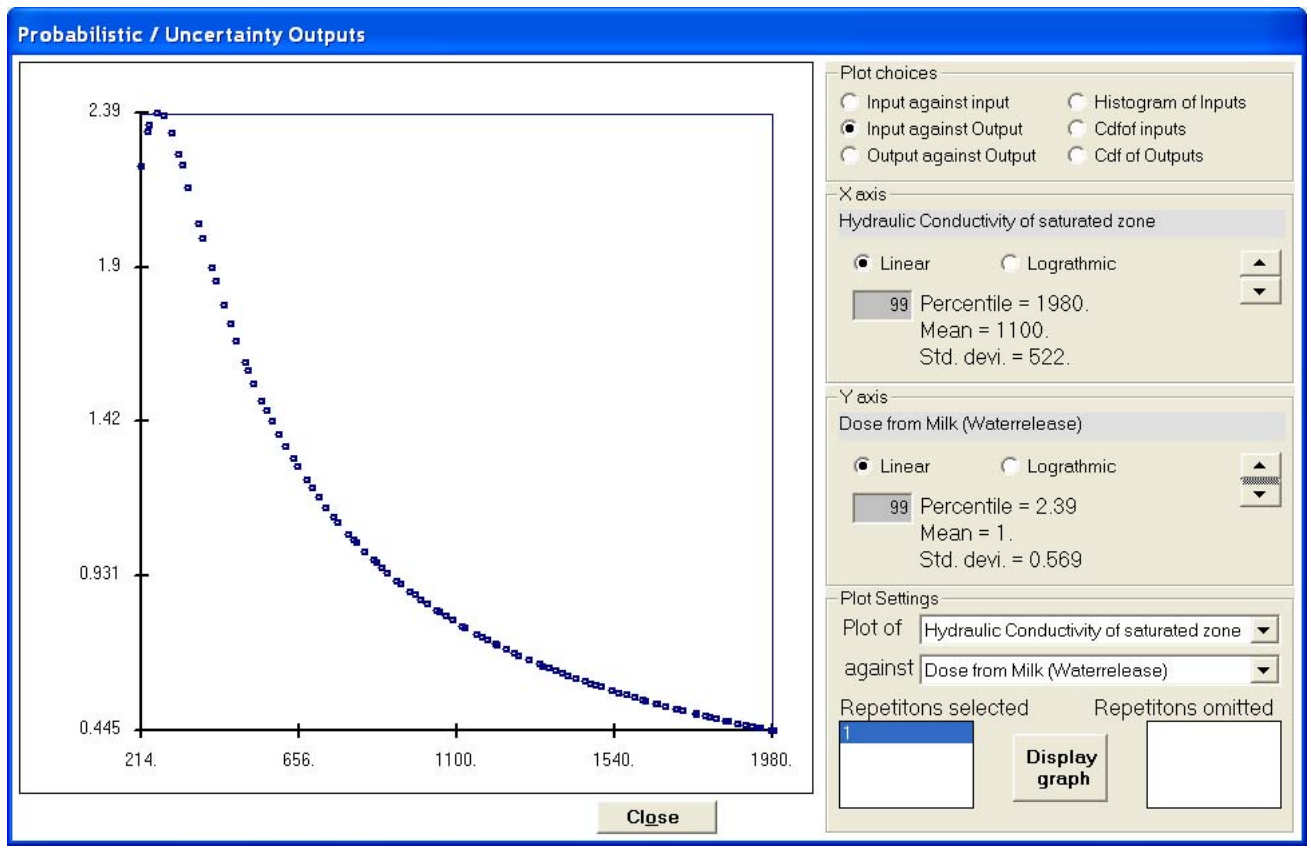

FIGURE C.16 Variation of Peak Predicted Dose from Ingestion of Milk (Waterborne Contamination) with Hydraulic Conductivity in the Saturated Zone 


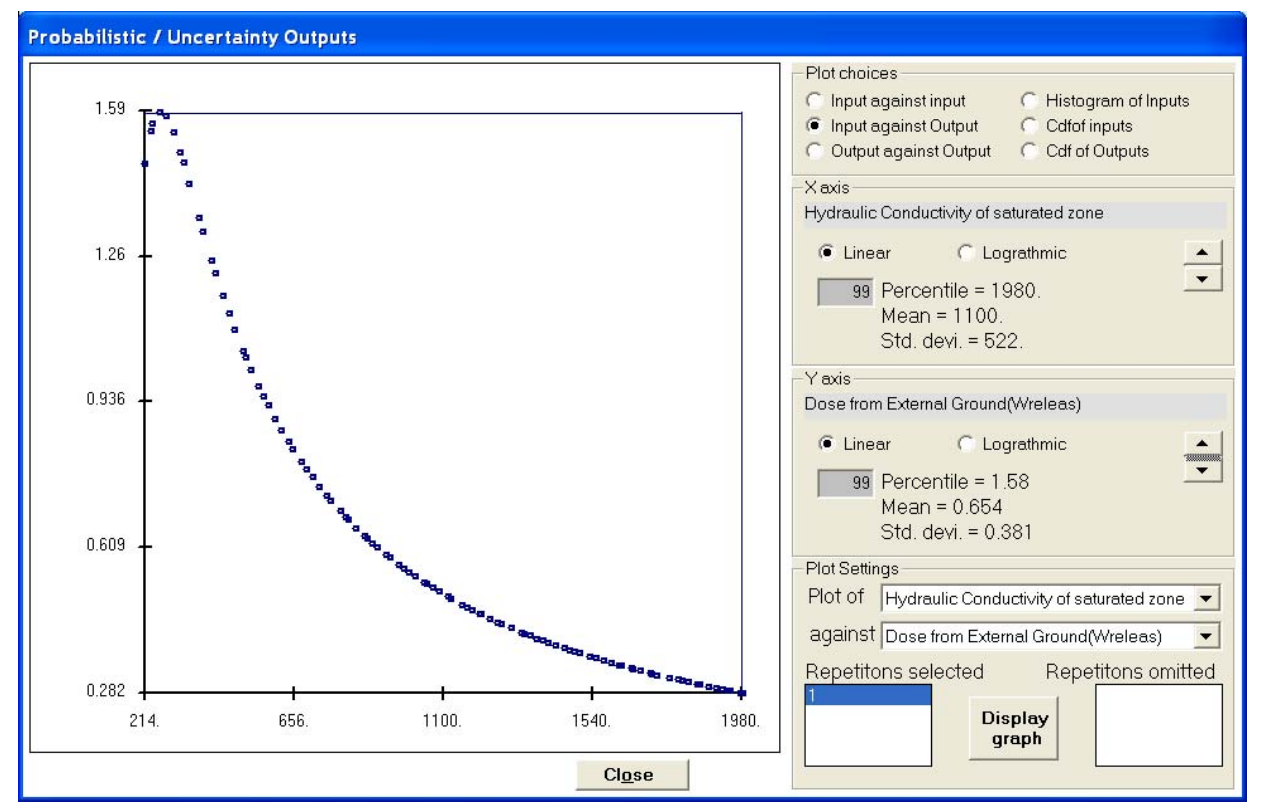

FIGURE C.17 Variation of Peak Predicted Dose from Direct Exposure to Waterborne Accumulation in Soil with Hydraulic Conductivity in the Saturated Zone

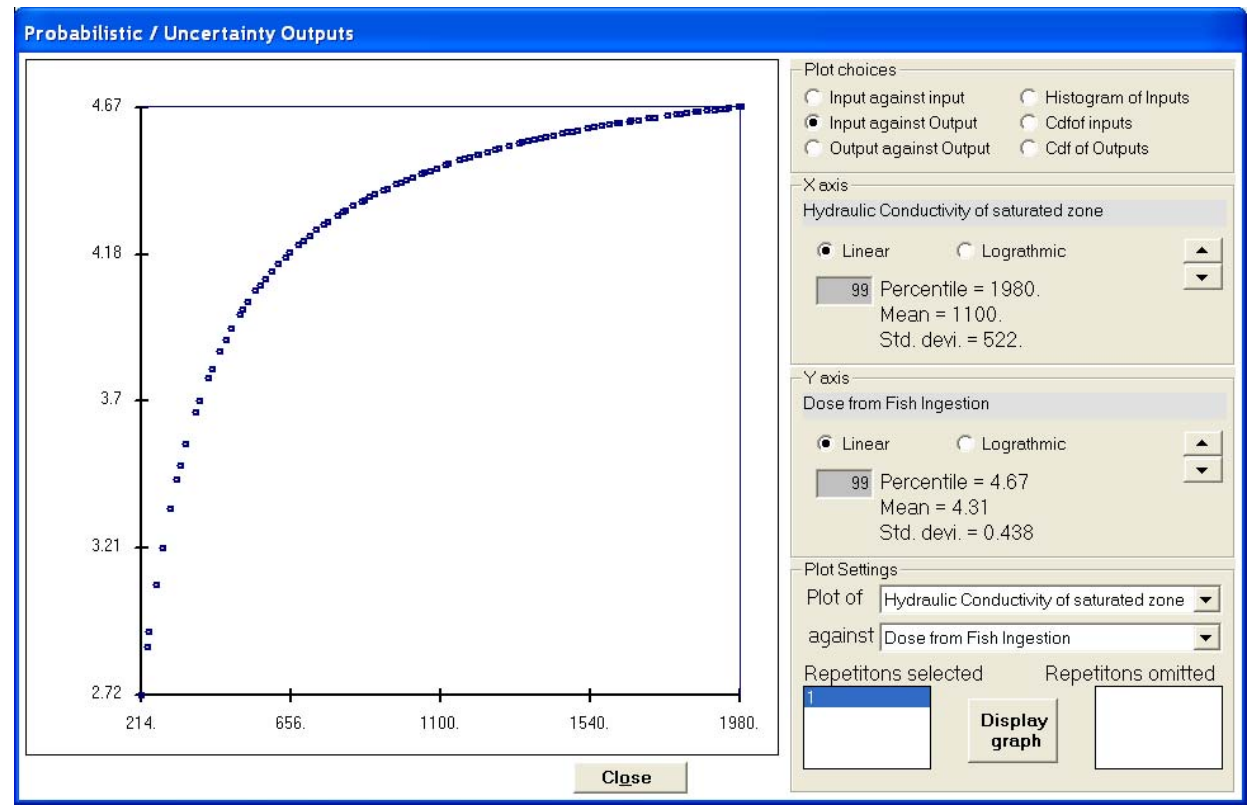

FIGURE C.18 Variation of Peak Predicted Dose from Ingestion of Fish with Hydraulic Conductivity in the Saturated Zone 
TABLE C.1 Peak Predicted Doses and Times of Peak Predicted Doses From Different Exposure Pathways

\begin{tabular}{|c|c|c|c|c|c|c|c|c|c|c|c|c|c|c|c|}
\hline $\begin{array}{l}\text { Hydraulic } \\
\text { Conduc- } \\
\text { tivity, m/yr }\end{array}$ & $\begin{array}{l}\text { Sim- } \\
\text { ula- } \\
\text { tion } \\
\text { No. }\end{array}$ & $\begin{array}{l}\text { Peak } \\
\text { dose, } \\
\text { mrem/yr }\end{array}$ & $\begin{array}{l}\text { Time of } \\
\text { Peak }\end{array}$ & $\begin{array}{l}\text { Water I } \\
\text { Peak } \\
\text { dose, } \\
\text { mrem/ } \\
\text { yr }\end{array}$ & $\begin{array}{l}\text { Time of } \\
\text { Peak }\end{array}$ & $\begin{array}{l}\text { Peak } \\
\text { dose, } \\
\text { mrem/yr }\end{array}$ & $\begin{array}{l}\text { Time of } \\
\text { Peak }\end{array}$ & $\begin{array}{l}\text { Peak } \\
\text { dose, } \\
\text { mrem/yr }\end{array}$ & $\begin{array}{l}\text { Time of } \\
\text { Peak }\end{array}$ & $\begin{array}{l}\text { Peak } \\
\text { dose, } \\
\text { mrem/yr }\end{array}$ & $\begin{array}{l}\text { Time of } \\
\text { Peak }\end{array}$ & $\begin{array}{l}\text { Peak } \\
\text { dose, } \\
\text { mrem/yr }\end{array}$ & $\begin{array}{l}\text { Time of } \\
\text { Peak }\end{array}$ & $\begin{array}{l}\text { Peak } \\
\text { dose, } \\
\text { mrem/yr }\end{array}$ & $\begin{array}{l}\text { Time of } \\
\text { Peak }\end{array}$ \\
\hline 213.727448 & 65 & 39.872 & 317.871 & 23.0338 & 315.43 & 10.8382 & 319.824 & 2.37075 & 316.895 & 2.22203 & 319.336 & 1.47254 & 368.652 & 2.72057 & 762.207 \\
\hline 231.355759 & 37 & 41.7195 & 299.805 & 24.1107 & 296.875 & 11.3347 & 301.758 & 2.47896 & 298.34 & 2.32962 & 300.781 & 1.54515 & 350.098 & 2.88096 & 709.473 \\
\hline 237.567261 & 97 & 42.1328 & 293.945 & 24.3528 & 291.016 & 11.4451 & 295.898 & 2.50294 & 292.48 & 2.35426 & 294.922 & 1.56177 & 343.75 & 2.93264 & 692.871 \\
\hline 258.008759 & 6 & 42.7195 & 276.855 & 24.7023 & 273.926 & 11.5981 & 279.297 & 2.53599 & 275.391 & 2.39195 & 277.832 & 1.58717 & 326.172 & 3.08713 & 644.043 \\
\hline 276.414276 & 17 & 42.4395 & 263.672 & 24.5488 & 260.742 & 11.5167 & 266.113 & 2.51785 & 262.207 & 2.38025 & 264.648 & 1.57916 & 312.5 & 3.20833 & 605.957 \\
\hline 299.234406 & 72 & 41.4044 & 249.512 & 23.9594 & 246.582 & 11.2296 & 251.953 & 2.45471 & 248.047 & 2.32655 & 250 & 1.54265 & 297.363 & 3.33908 & 565.43 \\
\hline 319.006073 & 80 & 40.1579 & 238.77 & 23.2454 & 235.84 & 10.8865 & 241.699 & 2.37948 & 237.793 & 2.25982 & 239.258 & 1.49729 & 286.133 & 3.43776 & 535.156 \\
\hline 328.45105 & 83 & 39.5027 & 234.375 & 22.8695 & 230.957 & 10.7066 & 236.816 & 2.34007 & 232.91 & 2.22438 & 234.375 & 1.4732 & 281.738 & 3.48073 & 521.973 \\
\hline 346.223358 & 9 & 38.2211 & 226.074 & 22.1332 & 223.145 & 10.3552 & 229.004 & 2.26314 & 225.098 & 2.15468 & 226.563 & 1.4258 & 272.949 & 3.55526 & 499.023 \\
\hline 374.722992 & 45 & 36.1491 & 214.844 & 20.9414 & 211.914 & 9.78806 & 217.773 & 2.13905 & 213.867 & 2.04121 & 214.844 & 1.34862 & 261.23 & 3.65993 & 467.285 \\
\hline 386.024292 & 25 & 35.3473 & 210.938 & 20.4799 & 207.52 & 9.56881 & 213.867 & 2.0911 & 209.961 & 1.9971 & 210.938 & 1.31863 & 257.324 & 3.69708 & 455.566 \\
\hline 411.100555 & 16 & 33.6372 & 203.125 & 19.4951 & 199.707 & 9.10153 & 206.055 & 1.98896 & 202.148 & 1.90276 & 202.148 & 1.25449 & 249.023 & 3.772 & 432.617 \\
\hline 422.747528 & 78 & 32.8804 & 199.707 & 19.0591 & 196.289 & 8.89485 & 202.637 & 1.94381 & 198.73 & 1.8609 & 198.73 & 1.22604 & 245.117 & 3.80368 & 422.852 \\
\hline 445.124115 & 95 & 31.498 & 193.359 & 18.2624 & 189.941 & 8.51745 & 196.777 & 1.86139 & 192.383 & 1.78427 & 192.383 & 1.174 & 238.77 & 3.85967 & 405.762 \\
\hline 462.823547 & 57 & 30.47 & 188.965 & 17.6697 & 185.547 & 8.23695 & 192.383 & 1.80015 & 187.988 & 1.72716 & 187.988 & 1.13525 & 234.375 & 3.89996 & 393.066 \\
\hline 481.19635 & 1 & 29.4619 & 185.059 & 17.0883 & 181.152 & 7.96205 & 187.988 & 1.74015 & 184.082 & 1.67107 & 183.594 & 1.09723 & 229.98 & 3.93853 & 381.348 \\
\hline 504.701752 & 93 & 28.2568 & 179.688 & 16.3932 & 176.27 & 7.63349 & 183.105 & 1.66846 & 179.199 & 1.60388 & 178.223 & 1.05174 & 225.098 & 3.98353 & 367.188 \\
\hline 513.378479 & 42 & 27.8342 & 178.223 & 16.1493 & 174.805 & 7.51825 & 181.641 & 1.64332 & 177.246 & 1.58028 & 176.758 & 1.03578 & 223.633 & 3.99906 & 362.305 \\
\hline 529.437073 & 77 & 27.0822 & 175.293 & 15.7153 & 171.875 & 7.31336 & 178.223 & 1.59863 & 174.316 & 1.53827 & 173.34 & 1.00739 & 220.215 & 4.02638 & 354.004 \\
\hline 551.966003 & 87 & 26.0891 & 171.387 & 15.1421 & 167.48 & 7.04288 & 174.316 & 1.53964 & 170.41 & 1.48271 & 169.434 & 0.969903 & 216.309 & 4.06188 & 342.773 \\
\hline 565.29834 & 91 & 25.5335 & 168.945 & 14.8213 & 165.527 & 6.89156 & 172.363 & 1.50664 & 168.457 & 1.45157 & 166.992 & 0.948925 & 214.355 & 4.08148 & 336.426 \\
\hline 579.406982 & 49 & 24.9692 & 166.504 & 14.4954 & 163.086 & 6.73796 & 169.922 & 1.47315 & 166.016 & 1.41994 & 164.551 & 0.927634 & 211.914 & 4.1012 & 330.566 \\
\hline 599.724426 & 64 & 24.1979 & 163.574 & 14.0498 & 160.156 & 6.52805 & 166.992 & 1.42738 & 163.086 & 1.37665 & 161.621 & 0.898532 & 208.984 & 4.12788 & 321.777 \\
\hline 618.315735 & 55 & 23.5308 & 161.133 & 13.6642 & 157.715 & 6.34658 & 164.551 & 1.3878 & 160.645 & 1.33915 & 158.691 & 0.87337 & 206.543 & 4.15072 & 314.941 \\
\hline 638.884766 & 26 & 22.8336 & 158.691 & 13.2612 & 154.785 & 6.15702 & 162.109 & 1.34646 & 158.203 & 1.29994 & 156.25 & 0.847086 & 203.613 & 4.17434 & 307.129 \\
\hline 652.01825 & 92 & 22.4087 & 156.738 & 13.0155 & 153.32 & 6.04152 & 160.645 & 1.32127 & 156.25 & 1.27601 & 154.297 & 0.831076 & 202.148 & 4.18865 & 302.734 \\
\hline 676.928101 & 11 & 21.6444 & 153.809 & 12.5733 & 150.391 & 5.83386 & 157.715 & 1.27598 & 153.809 & 1.23293 & 151.367 & 0.802277 & 199.219 & 4.21416 & 294.434 \\
\hline 692.366821 & 12 & 21.1959 & 152.344 & 12.3137 & 148.926 & 5.71202 & 155.762 & 1.2494 & 151.855 & 1.20761 & 149.902 & 0.785383 & 197.754 & 4.22906 & 290.039 \\
\hline
\end{tabular}


TABLE C.1 (Continued)

\begin{tabular}{|c|c|c|c|c|c|c|c|c|c|c|c|c|c|c|c|}
\hline \multirow[b]{2}{*}{$\begin{array}{l}\text { Hydraulic } \\
\text { Conductiv- } \\
\text { ity, m/yr }\end{array}$} & \multirow{2}{*}{$\begin{array}{l}\text { Sim- } \\
\text { ula- } \\
\text { tion } \\
\text { No. }\end{array}$} & \multicolumn{2}{|c|}{ All Pathways } & \multicolumn{2}{|c|}{$\underline{\text { Water Ingestion }}$} & \multicolumn{2}{|c|}{$\begin{array}{c}\text { Plant } \\
\text { (Water release) }\end{array}$} & \multicolumn{2}{|c|}{$\begin{array}{c}\text { Meat } \\
\text { (Water release) }\end{array}$} & \multicolumn{2}{|c|}{$\begin{array}{c}\text { Milk } \\
\text { (Water release) }\end{array}$} & \multicolumn{2}{|c|}{$\begin{array}{l}\text { External Ground } \\
\text { (Water release) }\end{array}$} & \multicolumn{2}{|c|}{ Fish Ingestion } \\
\hline & & $\begin{array}{l}\text { Peak } \\
\text { dose, } \\
\text { mrem/yr }\end{array}$ & $\begin{array}{l}\text { Time of } \\
\text { Peak }\end{array}$ & $\begin{array}{l}\text { Peak } \\
\text { dose, } \\
\text { mrem/yr }\end{array}$ & $\begin{array}{l}\text { Time of } \\
\text { Peak }\end{array}$ & $\begin{array}{l}\text { Peak } \\
\text { dose, } \\
\text { mrem/yr }\end{array}$ & $\begin{array}{l}\text { Time of } \\
\text { Peak }\end{array}$ & $\begin{array}{l}\text { Peak } \\
\text { dose, } \\
\text { mrem/yr }\end{array}$ & $\begin{array}{l}\text { Time of } \\
\text { Peak }\end{array}$ & $\begin{array}{l}\text { Peak } \\
\text { dose, } \\
\text { mrem/yr }\end{array}$ & $\begin{array}{l}\text { Time of } \\
\text { Peak }\end{array}$ & $\begin{array}{l}\text { Peak } \\
\text { dose, } \\
\text { mrem/yr }\end{array}$ & $\begin{array}{l}\text { Time of } \\
\text { Peak }\end{array}$ & $\begin{array}{l}\text { Peak } \\
\text { dose, } \\
\text { mrem/yr }\end{array}$ & $\begin{array}{l}\text { Time of } \\
\text { Peak }\end{array}$ \\
\hline 709.79541 & 68 & 20.711 & 150.391 & 12.033 & 146.973 & 5.58039 & 154.297 & 1.22068 & 150.391 & 1.18023 & 147.949 & 0.767123 & 195.801 & 4.24506 & 284.668 \\
\hline 732.122803 & 74 & 20.1215 & 148.438 & 11.6914 & 145.02 & 5.42032 & 151.855 & 1.18577 & 147.949 & 1.1469 & 145.508 & 0.744923 & 193.848 & 4.26446 & 278.809 \\
\hline 752.08 & 60 & 19.7361 & 254.883 & 11.4017 & 143.066 & 5.28466 & 150.391 & 1.15616 & 146.484 & 1.1186 & 143.555 & 0.726096 & 191.895 & 4.28083 & 273.438 \\
\hline 765.409973 & 98 & 19.568 & 251.953 & 11.2162 & 141.602 & 5.19785 & 148.926 & 1.13722 & 145.02 & 1.10047 & 142.578 & 0.714051 & 190.918 & 4.29127 & 270.02 \\
\hline 793.082581 & 30 & 19.2284 & 245.605 & 10.8496 & 139.648 & 5.02635 & 146.973 & 1.09981 & 143.066 & 1.06461 & 140.137 & 0.690241 & 188.965 & 4.3119 & 263.672 \\
\hline 809.679382 & 15 & 19.031 & 242.188 & 10.6409 & 138.184 & 4.92878 & 145.508 & 1.07852 & 141.602 & 1.04419 & 138.672 & 0.676693 & 187.5 & 4.32358 & 259.766 \\
\hline 817.123596 & 38 & 18.9441 & 240.723 & 10.5499 & 137.695 & 4.88628 & 145.02 & 1.06925 & 141.113 & 1.03528 & 138.184 & 0.670789 & 187.012 & 4.32871 & .301 \\
\hline 839.9 & 52 & 18.6834 & 236.328 & 10.2808 & 136.23 & 4.76059 & 143.555 & 1.04182 & 139.648 & 1.00893 & 136.23 & 0.653327 & 547 & 377 & 18 \\
\hline 863.278381 & 54 & 18.4245 & 231.934 & 10.0187 & 134.277 & 4.63817 & 142.09 & 1.01512 & 138.184 & 0.983236 & 134.766 & 0.636314 & 183.594 & .3585 & 249.023 \\
\hline 871.421997 & 7 & 18.3363 & 230.957 & 9.93053 & 133.789 & 4.59703 & 141.602 & 1.00614 & 695 & 0.974592 & 277 & 0.630595 & 105 & 344 & 24 \\
\hline 889.124 & 96 & 18.1482 & 227. & 9.74 & 13 & 4.51006 & 140.137 & 0.987177 & 136 & 0.956318 & 313 & 0.618502 & 129 & .37391 & 41 \\
\hline 127 & 86 & 18.0025 & 225.586 & 9.60154 & 131.836 & 4.44349 & 139.648 & 0.972656 & 135.742 & 0.942317 & 131 & 0.609 & 52 & 4.3 & 24 \\
\hline 930.153381 & 22 & 17.7299 & 221.1 & 9.33833 & 130.371 & 4.3207 & 137.695 & 0.945882 & 133.789 & 0.916476 & 130.371 & 0.59216 & 179.688 & 4.39676 & 305 \\
\hline 938.296143 & 43 & 17.6496 & 219.727 & 9.26178 & 129.883 & 4.28498 & 137.207 & 0.938094 & 133.301 & 0.908956 & 129.883 & 0.587189 & 179.199 & 4.4011 & 235.84 \\
\hline 967.092041 & 50 & 17.3734 & 215.82 & 9.00124 & 128.418 & 4.16347 & 135.742 & 0.911606 & 131.836 & 0.883348 & 128.418 & 0.570275 & 177.734 & 4.41584 & 231.445 \\
\hline 980.1 & 63 & 17.252 & 213.867 & 8.88818 & 127.441 & 4.11074 & 135.254 & 0.900114 & 131.348 & 0.872234 & 127.441 & 0.562933 & 246 & 227 & 229. \\
\hline 997.151611 & 35 & 17.0966 & 211.426 & 8.74471 & 126.953 & 4.04382 & 134.277 & 0.885532 & 130.371 & 0.85812 & 126.465 & 0.553615 & 176.27 & 4.43044 & 227.051 \\
\hline 1013.97479 & 39 & 16.9465 & 209.473 & 8.6074 & 125.977 & 3.97975 & 133.301 & 0.871568 & 129.395 & 0.844602 & 125.977 & 0.544691 & 175.781 & 4.43831 & 224.609 \\
\hline 1039.91357 & 5 & 16.7223 & 206.055 & 8.40464 & 124.512 & 3.8852 & 132.324 & 0.850973 & 128.418 & 0.824648 & 124.512 & 0.531519 & 174.316 & 4.44995 & 220.703 \\
\hline 1048.34497 & 84 & 16.6513 & 205.078 & 8.341 & 124.512 & 3.8555 & 131.836 & 0.844504 & 127.93 & 0.818377 & 124.023 & 0.527381 & 173.828 & 364 & 219.727 \\
\hline 1065 & 36 & 16.51 & 203.125 & 8.21518 & 123.535 & 3.79678 & 131.348 & 0.831716 & 127.441 & 0.805977 & 123 & 0.519 & 17 & 4.4 & 217.7 \\
\hline 1085.15088 & 70 & 16.3487 & 200.684 & 8.07269 & 122.559 & 3.73032 & 130.371 & 0.817244 & 126.465 & 0.79194 & 122.559 & 0.509939 & 172.363 & 4.46916 & 215.332 \\
\hline 1111.19568 & 76 & 16.1444 & 198.242 & 7.89407 & 121.582 & 3.64695 & 129.395 & 0.799094 & 125.488 & 0.774325 & 121.582 & 0.498322 & 171.387 & 4.47957 & 211.914 \\
\hline 1119.3811 & 8 & 16.0814 & 197.266 & 7.83935 & 121.582 & 3.62145 & 128.906 & 0.793541 & 125 & 0.768937 & 121.094 & 0.494766 & 171.387 & 4.48277 & 210.938 \\
\hline 1152.9635 & 20 & 15.8303 & 193.848 & 7.62331 & 120.117 & 3.52059 & 127.441 & 0.771584 & 123.535 & 0.74762 & 119.629 & 0.480709 & 169.922 & 4.49544 & 207.52 \\
\hline 1167.8158 & 58 & 15.7231 & 192.383 & 7.53194 & 119.629 & 3.47795 & 126.953 & 0.762305 & 123.047 & 0.738606 & 119.141 & 0.474766 & 169.434 & 4.50082 & 205.566 \\
\hline 1183.67175 & 3 & 15.6103 & 190.918 & 7.43625 & 119.141 & 3.43328 & 126.465 & 0.752588 & 122.559 & 0.729163 & 118.652 & 0.468542 & 168.945 & 4.50649 & 204.102 \\
\hline 1203.81665 & 71 & 15.4708 & 188.965 & 7.31889 & 118.164 & 3.37846 & 125.488 & 0.740656 & 122.07 & 0.717573 & 117.676 & 0.460902 & 168.457 & 4.51343 & 202.148 \\
\hline 1208.23889 & 32 & 15.4407 & 188.477 & 7.29369 & 118.164 & 3.3667 & 125.488 & 0.738099 & 121.582 & 0.715087 & 117.676 & 0.459263 & 167.969 & 4.51493 & 201.66 \\
\hline
\end{tabular}




\section{TABLE C.1 (Continued)}

\begin{tabular}{|c|c|c|c|c|c|c|c|c|c|c|c|c|c|c|c|}
\hline \multirow[b]{2}{*}{$\begin{array}{l}\text { Hydraulic } \\
\text { Conductiv- } \\
\text { ity, m/yr }\end{array}$} & \multirow[b]{2}{*}{$\begin{array}{l}\text { Sim- } \\
\text { ula- } \\
\text { tion } \\
\text { No. }\end{array}$} & \multicolumn{2}{|c|}{ All Pathways } & \multicolumn{2}{|c|}{ Water Ingestion } & \multicolumn{2}{|c|}{$\begin{array}{c}\text { Plant } \\
\text { (Water release) }\end{array}$} & \multicolumn{2}{|c|}{$\begin{array}{c}\text { Meat } \\
\text { (Water release) }\end{array}$} & \multicolumn{2}{|c|}{$\begin{array}{c}\text { Milk } \\
\text { (Water release) }\end{array}$} & \multicolumn{2}{|c|}{$\begin{array}{l}\text { External Ground } \\
\text { (Water release) }\end{array}$} & \multicolumn{2}{|c|}{ Fish Ingestion } \\
\hline & & $\begin{array}{l}\text { Peak } \\
\text { dose, } \\
\text { mrem/yr }\end{array}$ & $\begin{array}{l}\text { Time of } \\
\text { Peak }\end{array}$ & $\begin{array}{l}\text { Peak } \\
\text { dose, } \\
\text { mrem/yr }\end{array}$ & $\begin{array}{l}\text { Time of } \\
\text { Peak }\end{array}$ & $\begin{array}{l}\text { Peak } \\
\text { dose, } \\
\text { mrem/yr }\end{array}$ & $\begin{array}{l}\text { Time of } \\
\text { Peak }\end{array}$ & $\begin{array}{l}\text { Peak } \\
\text { dose, } \\
\text { mrem/yr }\end{array}$ & $\begin{array}{l}\text { Time of } \\
\text { Peak }\end{array}$ & $\begin{array}{l}\text { Peak } \\
\text { dose, } \\
\text { mrem/yr }\end{array}$ & $\begin{array}{l}\text { Time of } \\
\text { Peak }\end{array}$ & $\begin{array}{l}\text { Peak } \\
\text { dose, } \\
\text { mrem/yr }\end{array}$ & $\begin{array}{l}\text { Time of } \\
\text { Peak }\end{array}$ & $\begin{array}{l}\text { Peak } \\
\text { dose, } \\
\text { mrem/yr }\end{array}$ & $\begin{array}{l}\text { Time of } \\
\text { Peak }\end{array}$ \\
\hline 1235.7533 & 34 & 15.256 & 186.035 & 7.13964 & 117.188 & 3.29476 & 124.512 & 0.722447 & 120.605 & 0.699875 & 116.699 & 0.449238 & 167.48 & 4.52405 & 198.73 \\
\hline 1258.88123 & 14 & 15.1062 & 184.082 & 7.01574 & 116.211 & 3.2369 & 123.535 & 0.70986 & 120.117 & 0.687636 & 115.723 & 0.441177 & 166.504 & 4.53145 & 196.777 \\
\hline 1269.42834 & 21 & 15.0396 & 183.594 & 6.96089 & 116.211 & 3.21129 & 123.535 & 0.70429 & 119.629 & 0.682222 & 115.723 & 0.437609 & 166.504 & 4.53472 & 195.801 \\
\hline 1297.39136 & 31 & 14.8659 & 181.152 & 6.81885 & 115.234 & 3.14493 & 122.559 & 0.689854 & 118.652 & 0.668188 & 114.746 & 0.428365 & 165.527 & 4.54319 & 193.359 \\
\hline 1299.31299 & 28 & 14.8543 & 181.152 & 6.80941 & 115.234 & 3.14052 & 122.559 & 0.688897 & 118.652 & 0.667256 & 114.746 & 0.427752 & 165.527 & 4.54374 & 193.359 \\
\hline 1328.58472 & 10 & 14.6791 & 178.711 & 6.66741 & 114.258 & 3.07418 & 121.582 & 0.674465 & 117.676 & 0.653224 & 113.77 & 0.418512 & 164.551 & 4.55221 & 190.918 \\
\hline 1338.2 & 46 & 14.6225 & 178.223 & 6.6217 & 113.77 & 3.05283 & 121.094 & 0.66982 & 117.676 & 0.648708 & 113.281 & 0.41554 & 164.551 & 4.55495 & 189.941 \\
\hline 1353.98828 & 79 & 14.5321 & 177.246 & 6.54909 & 113.281 & 3.01891 & 120.605 & 0.662443 & 117.188 & 0.641533 & 112.793 & 0.410818 & 164.063 & 4.55926 & 188.477 \\
\hline 1374.4928 & 88 & 14.4167 & 175.781 & 6.45675 & 112.793 & 2.97576 & 120.117 & 0.653055 & 116.211 & 0.632407 & 112.305 & 0.404811 & 163.574 & 4.56479 & 187.012 \\
\hline 1391.99585 & 18 & 14.3203 & 174.316 & 6.37999 & 112.305 & 2.93989 & 119.629 & 0.645254 & 115.723 & 0.624821 & 111.816 & 0.39982 & 163.086 & 4.56934 & 185.547 \\
\hline 1406.14783 & 89 & 14.244 & 173.34 & 6.31946 & 111.816 & 2.91162 & 119.141 & 0.639107 & 115.723 & 0.618839 & 111.328 & 0.395886 & 162.598 & 4.57295 & 184.57 \\
\hline 1428.97119 & 47 & 14.1227 & 171.875 & 6.2237 & 111.328 & 2.86687 & 118.652 & 0.629372 & 114.746 & 0.609375 & 110.84 & 0.38966 & 162.109 & 4.57863 & 183.105 \\
\hline 1455.76257 & 66 & 13.9847 & 170.41 & 6.1153 & 110.84 & 2.81622 & 118.164 & 0.618359 & 114.258 & 0.598662 & 110.352 & 0.382617 & 161.621 & 4.58506 & 181.152 \\
\hline 1471.4646 & 44 & 13.9057 & 169.434 & 6.05359 & 110.352 & 2.78739 & 117.676 & 0.612086 & 113.77 & 0.592562 & 109.863 & 0.378607 & 161.133 & 4.58868 & 180.176 \\
\hline 1484.33765 & 19 & 13.8423 & 168.457 & 6.0042 & 109.863 & 2.76431 & 117.188 & 0.607064 & 113.281 & 0.58768 & 109.375 & 0.375401 & 161.133 & 4.59161 & 179.199 \\
\hline 1500.2843 & 75 & 13.7642 & 167.48 & 5.94358 & 109.375 & 2.736 & 116.699 & 0.600908 & 113.281 & 0.58169 & 109.375 & 0.371467 & 160.645 & 4.59516 & 178.223 \\
\hline 1529.224 & 48 & 13.6267 & 166.016 & 5.83731 & 108.887 & 2.68635 & 116.211 & 0.590105 & 112.305 & 0.571187 & 108.398 & 0.364566 & 160.156 & 4.6014 & 176.27 \\
\hline 1549.78137 & 100 & 13.5312 & 165.039 & 5.76387 & 108.398 & 2.65204 & 115.723 & 0.582641 & 111.816 & 0.563929 & 107.91 & 0.359801 & 159.668 & 4.60567 & 174.805 \\
\hline 1550.09094 & 90 & 13.5298 & 165.039 & 5.76282 & 108.398 & 2.65155 & 115.723 & 0.582533 & 111.816 & 0.563825 & 107.91 & 0.359732 & 159.668 & 4.60573 & 174.805 \\
\hline 1571.17676 & 82 & 13.4342 & 163.574 & 5.68955 & 107.91 & 2.61733 & 115.234 & 0.575088 & 111.328 & 0.556585 & 107.422 & 0.35498 & 159.18 & 4.60998 & 173.828 \\
\hline 1592.92505 & 33 & 13.338 & 162.598 & 5.61616 & 107.422 & 2.58305 & 114.746 & 0.567629 & 110.84 & 0.549332 & 106.934 & 0.350222 & 159.18 & 4.61424 & 172.363 \\
\hline 1616.85938 & 29 & 13.234 & 161.133 & 5.53721 & 106.934 & 2.54618 & 114.258 & 0.559606 & 110.352 & 0.541531 & 106.445 & 0.345106 & 158.691 & 4.61878 & 170.898 \\
\hline 1626.22607 & 2 & 13.1944 & 160.645 & 5.50719 & 106.934 & 2.53217 & 114.258 & 0.556557 & 110.352 & 0.538568 & 106.445 & 0.343163 & 158.203 & 4.62051 & 170.41 \\
\hline 1653.32495 & 56 & 13.0811 & 159.668 & 5.42165 & 105.957 & 2.49224 & 113.281 & 0.547863 & 109.863 & 0.530116 & 105.957 & 0.337624 & 157.715 & 4.6254 & 168.945 \\
\hline 1658.7533 & 94 & 13.0585 & 159.18 & 5.40466 & 105.957 & 2.4843 & 113.281 & 0.546136 & 109.375 & 0.528434 & 105.469 & 0.336522 & 157.715 & 4.62635 & 168.945 \\
\hline 1679.25659 & 24 & 12.9752 & 158.203 & 5.34212 & 105.469 & 2.45511 & 112.793 & 0.539781 & 108.887 & 0.522257 & 105.469 & 0.332475 & 157.715 & 4.62992 & 167.48 \\
\hline 1709.79932 & 51 & 12.8544 & 156.738 & 5.25181 & 104.98 & 2.41295 & 112.305 & 0.530604 & 108.398 & 0.513335 & 104.492 & 0.326632 & 157.227 & 4.63504 & 166.016 \\
\hline 1714.51477 & 67 & 12.8361 & 156.738 & 5.23816 & 104.98 & 2.40659 & 112.305 & 0.529217 & 108.398 & 0.511989 & 104.492 & 0.325749 & 156.738 & 4.6358 & 166.016 \\
\hline 1730.6283 & 40 & 12.774 & 155.762 & 5.19194 & 104.492 & 2.38502 & 111.816 & 0.524522 & 107.91 & 0.507422 & 104.492 & 0.322763 & 156.738 & 4.63841 & 165.039 \\
\hline
\end{tabular}


TABLE C.1 (Continued)

\begin{tabular}{|c|c|c|c|c|c|c|c|c|c|c|c|c|c|c|c|}
\hline \multirow[b]{2}{*}{$\begin{array}{l}\text { Hydraulic } \\
\text { Conductiv- } \\
\text { ity, m/yr }\end{array}$} & \multirow{2}{*}{$\begin{array}{l}\text { Sim- } \\
\text { ula- } \\
\text { tion } \\
\text { No. }\end{array}$} & \multicolumn{2}{|c|}{$\underline{\text { All Pathways }}$} & \multicolumn{2}{|c|}{$\underline{\text { Water Ingestion }}$} & \multicolumn{2}{|c|}{$\begin{array}{c}\text { Plant } \\
\text { (Water release) }\end{array}$} & \multicolumn{2}{|c|}{$\begin{array}{c}\text { Meat } \\
\text { (Water release) }\end{array}$} & \multicolumn{2}{|c|}{$\begin{array}{c}\text { Milk } \\
\text { (Water release) }\end{array}$} & \multicolumn{2}{|c|}{$\begin{array}{l}\text { External Ground } \\
\text { (Water release) }\end{array}$} & \multicolumn{2}{|c|}{ Fish Ingestion } \\
\hline & & $\begin{array}{l}\text { Peak } \\
\text { dose, } \\
\text { mrem/yr }\end{array}$ & $\begin{array}{l}\text { Time of } \\
\text { Peak }\end{array}$ & $\begin{array}{l}\text { Peak } \\
\text { dose, } \\
\text { mrem/yr }\end{array}$ & $\begin{array}{l}\text { Time of } \\
\text { Peak }\end{array}$ & $\begin{array}{l}\text { Peak } \\
\text { dose, } \\
\text { mrem/yr }\end{array}$ & $\begin{array}{l}\text { Time of } \\
\text { Peak }\end{array}$ & $\begin{array}{l}\text { Peak } \\
\text { dose, } \\
\text { mrem/yr }\end{array}$ & $\begin{array}{l}\text { Time of } \\
\text { Peak }\end{array}$ & $\begin{array}{l}\text { Peak } \\
\text { dose, } \\
\text { mrem/yr }\end{array}$ & $\begin{array}{l}\text { Time of } \\
\text { Peak }\end{array}$ & $\begin{array}{l}\text { Peak } \\
\text { dose, } \\
\text { mrem/yr }\end{array}$ & $\begin{array}{l}\text { Time of } \\
\text { Peak }\end{array}$ & $\begin{array}{l}\text { Peak } \\
\text { dose, } \\
\text { mrem/yr }\end{array}$ & $\begin{array}{l}\text { Time of } \\
\text { Peak }\end{array}$ \\
\hline 1764.87146 & 4 & 12.6449 & 154.297 & 5.09625 & 104.004 & 2.34038 & 11.328 & .514798 & 107.422 & 497973 & 103.516 & 0.316579 & 156.25 & 64376 & 163.574 \\
\hline 1768.25049 & 62 & 12.6324 & 154.297 & 5.08696 & 104.004 & 2.33604 & 111.328 & 0.513854 & 107.422 & 0.497056 & 103.516 & 0.315979 & 156.25 & 4.64428 & 163.086 \\
\hline 1791.29834 & 69 & 12.5483 & 153.32 & 5.02499 & 103.516 & 2.30714 & 110.84 & 0.507558 & 106.934 & 0.490936 & 103.027 & 0.311978 & 155.762 & 4.64775 & 162.109 \\
\hline 1803.01245 & 85 & 12.5063 & 152.832 & 4.99402 & 103.516 & 2.29271 & 110.84 & 0.504413 & 106.934 & 0.487882 & 103.027 & 0.309981 & 155.762 & 947 & 161.621 \\
\hline 1824.26672 & 53 & 12.4309 & 151.855 & 4.9387 & 103.027 & 2.26691 & 110.352 & 0.498793 & 106.445 & 0.482419 & 102.539 & 0.306411 & 155.273 & 4.65254 & 160.645 \\
\hline 1850.21289 & 73 & 12.3411 & 150.879 & 4.87307 & 102.539 & 2.23631 & 109.863 & 0.492125 & 105.957 & 0.475939 & 102.051 & 0.302178 & 154.785 & 4.65617 & 159.668 \\
\hline 1856.36206 & 99 & 12.32 & 150.879 & 4.8576 & 102.539 & 2.22911 & 109.863 & 0.490555 & 105.957 & 0.474414 & 102.051 & 0.301182 & 154.785 & 4.65701 & 159.18 \\
\hline 1883.37854 & 61 & 12.2291 & 149.902 & 4.79146 & 102.051 & 2.19828 & 109.375 & 0.483837 & 105.469 & 0.467886 & 101.563 & 0.29692 & 154.785 & 4.66067 & 158.203 \\
\hline 1903.48938 & 41 & 12.1629 & 148.926 & 4.7434 & 101.563 & 2.17588 & 108.887 & 0.478956 & 104.98 & 0.46314 & 101.074 & 0.293826 & 154.297 & 4.66329 & 157.227 \\
\hline 1924.11035 & 27 & 12.0962 & 148.438 & 4.69511 & 101.074 & 2.1534 & 108.887 & 0.474053 & 104.492 & 0.458379 & 101.074 & 0.290719 & 154.297 & 4.66596 & 156.738 \\
\hline 1938.86963 & 13 & 12.0491 & 147.949 & 4.66108 & 101.074 & 2.13754 & 108.398 & 0.470596 & 104.492 & 0.455019 & 100.586 & 0.288528 & 153.809 & 4.66782 & 156.25 \\
\hline 1946.51367 & 59 & 12.0251 & 147.461 & 4.64377 & 101.074 & 2.12949 & 108.398 & 0.468838 & 104.492 & 0.453313 & 100.586 & 0.287416 & 153.809 & 4.66879 & 155.762 \\
\hline 1976.03357 & 23 & 11.9335 & 146.484 & 4.57785 & 100.586 & 2.09879 & 107.91 & 0.462145 & 104.004 & 0.446809 & 100.098 & 0.283177 & 153.32 & 4.6724 & 154.785 \\
\hline 1983.77808 & 81 & 11.9097 & 145.996 & 4.5607 & 100.586 & 2.09083 & 107.91 & 0.460404 & 103.516 & 0.445121 & 100.098 & 0.282078 & 153.32 & 4.67335 & 154.297 \\
\hline
\end{tabular}


The interaction of the predicted doses from the well-water- and surface-water-dependent exposure pathways to predict the peak total dose can also be seen from temporal plots for each simulation. RESRAD-OFFSITE does not ordinarily save the data for the temporal plots of the individual simulations because writing the data to a file is time-consuming and most users are not likely to view the temporal plots. It is, however, possible to specify that the data be saved by checking the "Dose and risk at graphic time points" option box in the "Output specifications" tab (see Figure C.19).

The example case, "Distributed Single-Input Sensitivity.ROF," was rerun after checking the "Dose and risk at graphic time points" option box in the "Output specifications" tab. This produced 100 graphics output files-one for each of the simulations. Temporal plots of total predicted dose and component pathways for seven of the 100 simulations are shown in Figures C.20 through C.33. They correspond to (1) one point toward the upper end of the hydraulic conductivity range, (2) three points in the vicinity of the break in the curve, and (3) three points in the hydraulic conductivity range of 200 to $300 \mathrm{~m} / \mathrm{yr}$ to capture the changes near the peak of the peak predicted dose. The probabilistic input sample data file ("Distributed Single-Input Sensitivity.pin," in this case) contains the value of hydraulic conductivity used for each simulation. The first two columns in Table C.1 list the value of the hydraulic conductivity in the saturated zone and the simulation in which it was used.

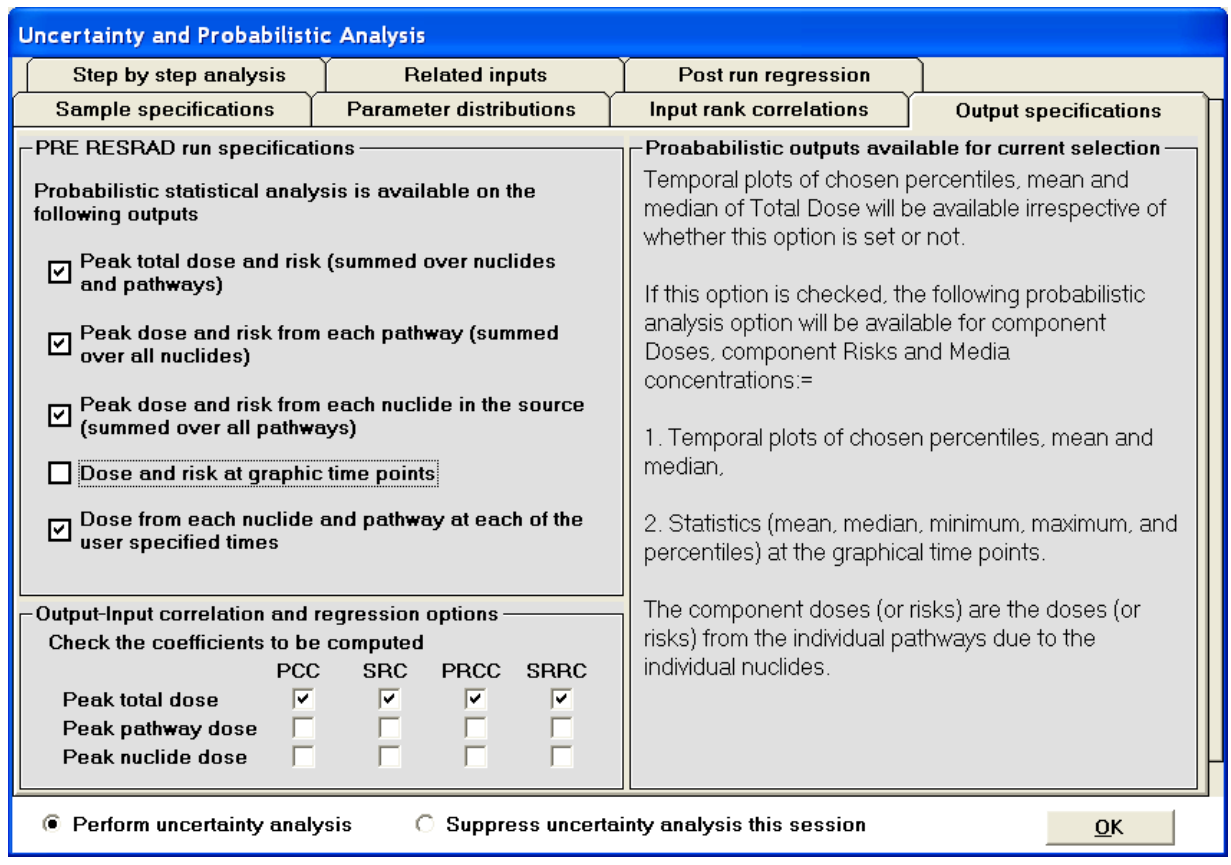

FIGURE C.19 Option Box to be Checked to Flag the Code to Produce a Full Suite of Temporal Plots for Each Probabilistic Simulation 


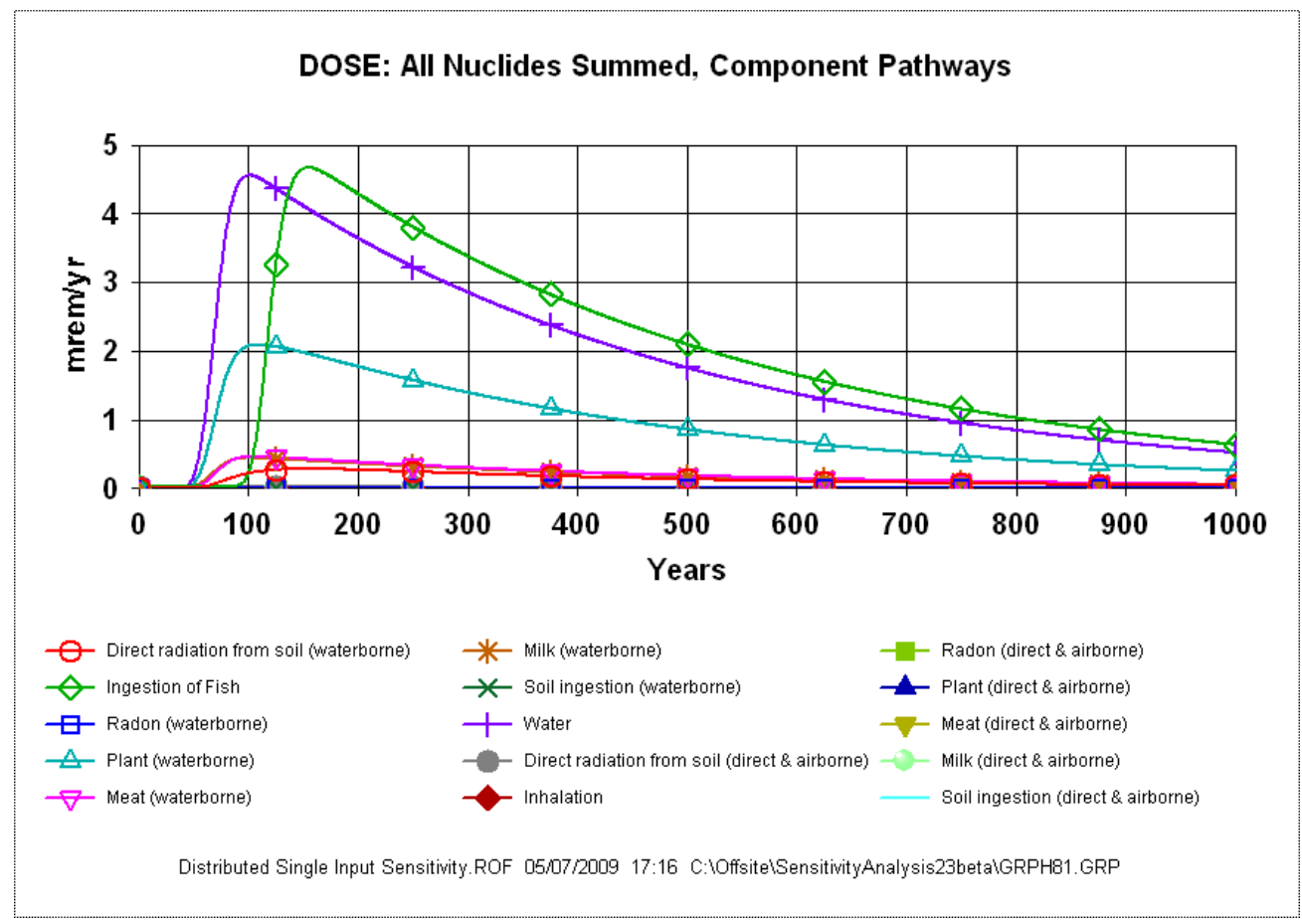

\section{FIGURE C.20 Relative Contributions of Exposure Pathways at $1984 \mathrm{~m} / \mathrm{yr}$ Hydraulic Conductivity}

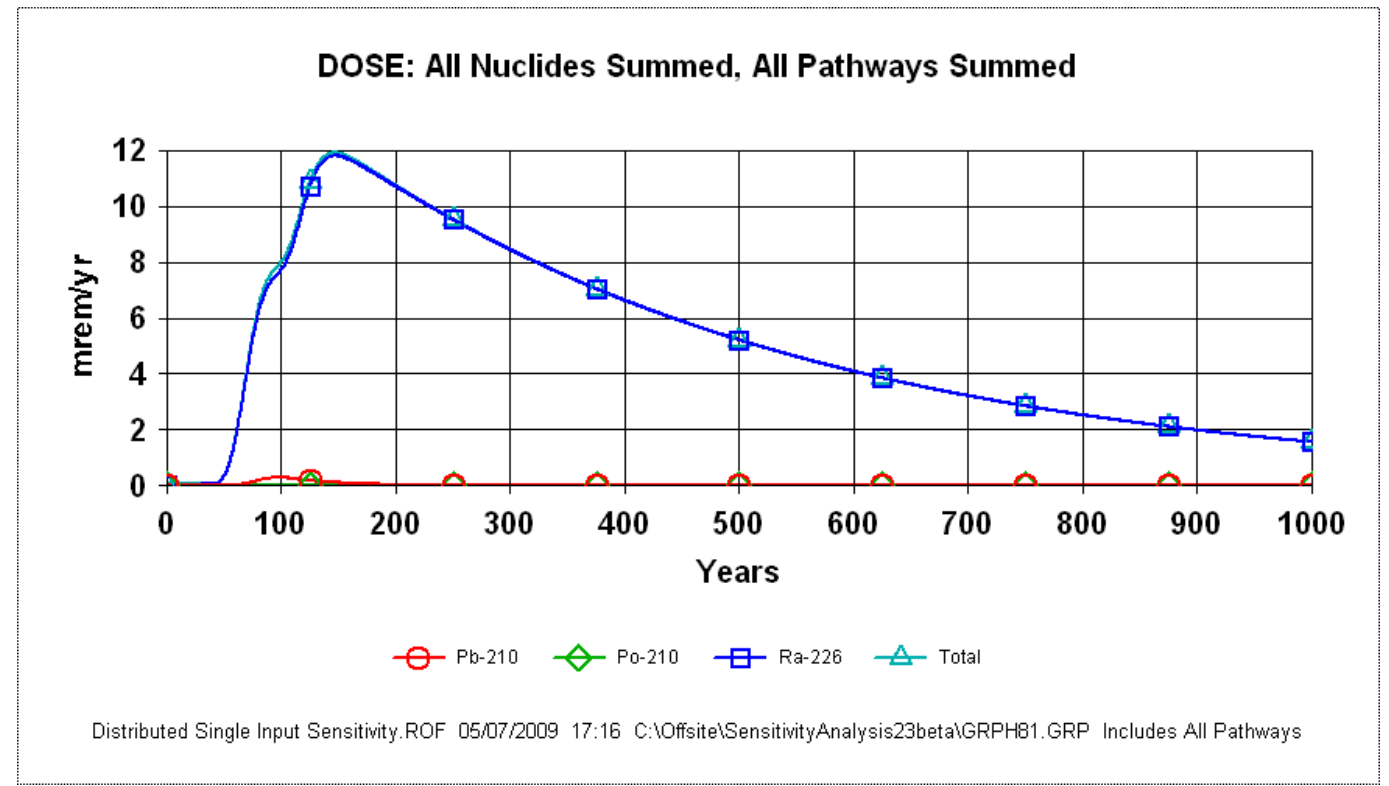

FIGURE C.21 Total Dose at a Hydraulic Conductivity of 1984 m/yr in Saturated Zone 


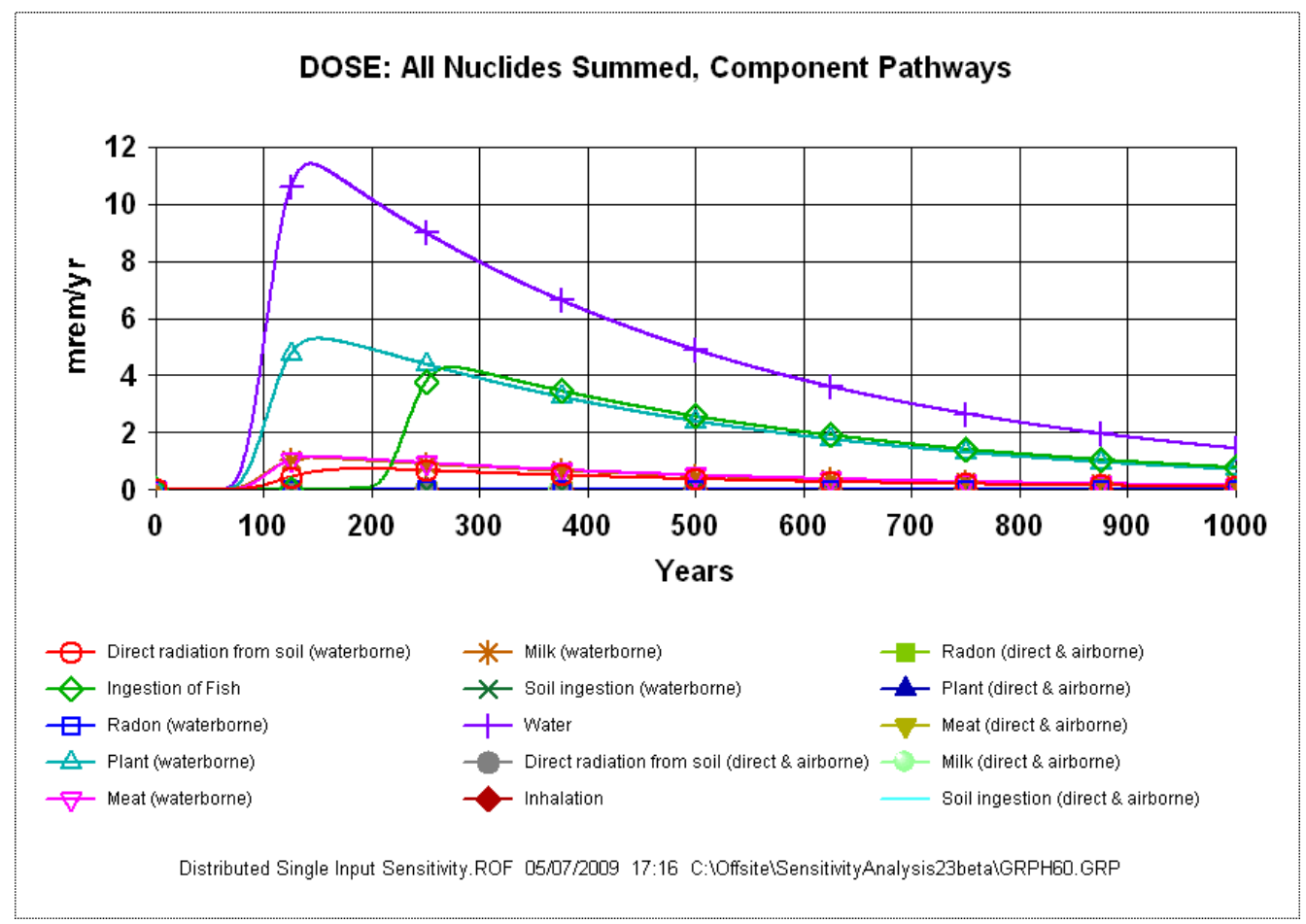

\section{FIGURE C.22 Relative Contributions of Exposure Pathways at $752.1 \mathrm{~m} / \mathrm{yr}$ Hydraulic Conductivity}

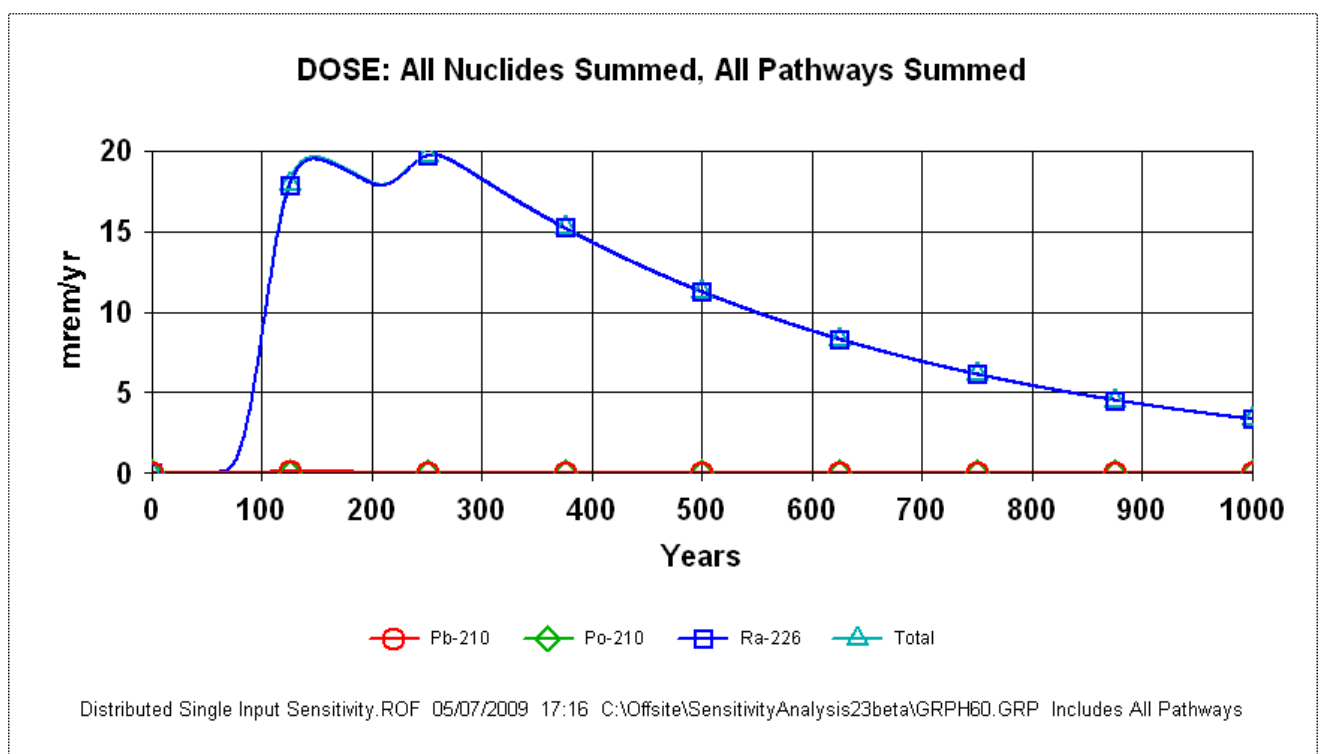

\section{FIGURE C.23 Total Dose at a Hydraulic Conductivity of $752.1 \mathrm{~m} / \mathrm{yr}$ in Saturated Zone}




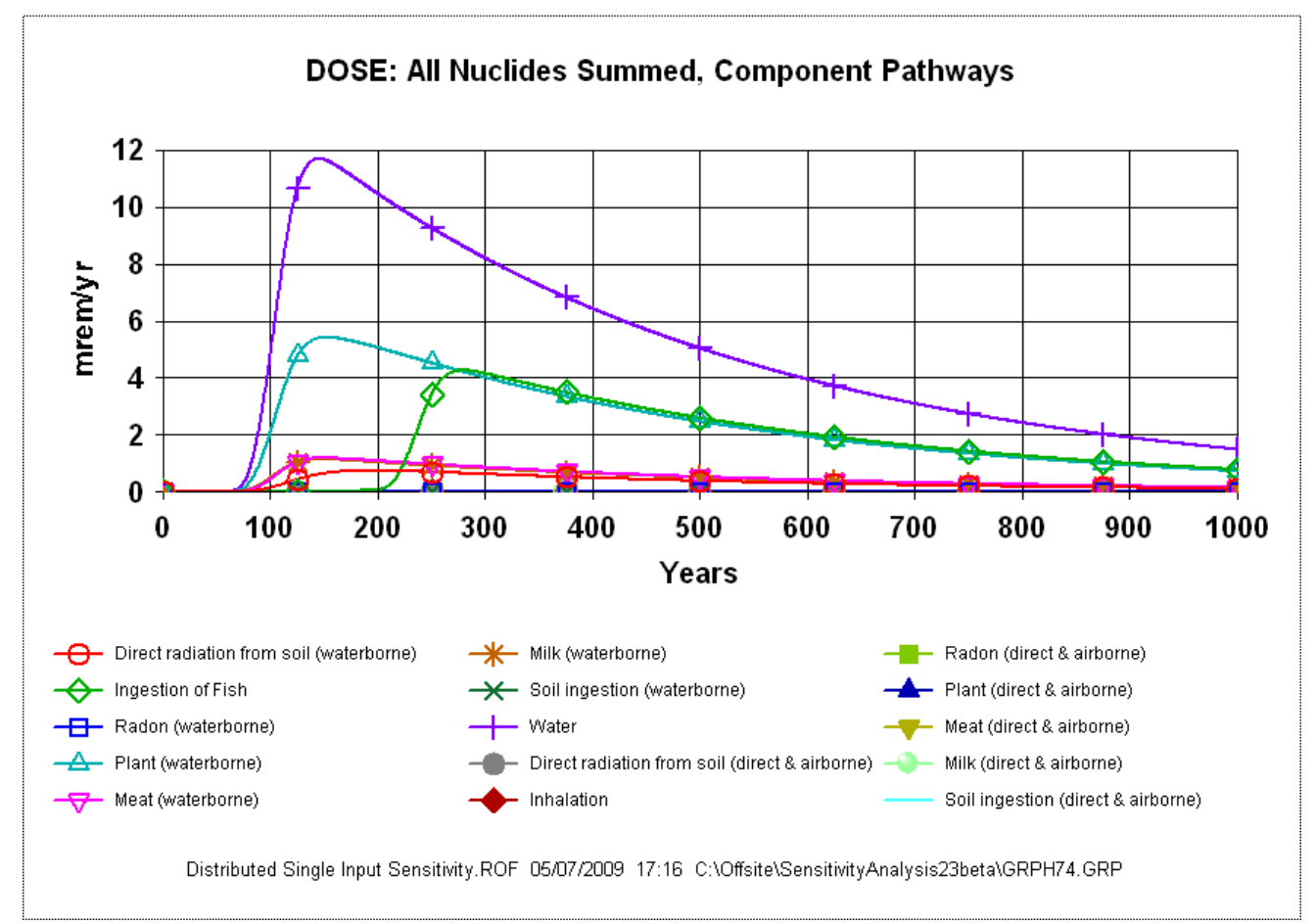
FIGURE C.24 Relative Contributions of Exposure Pathways
at $732.1 \mathrm{~m} / \mathrm{yr}$ Hydraulic Conductivity

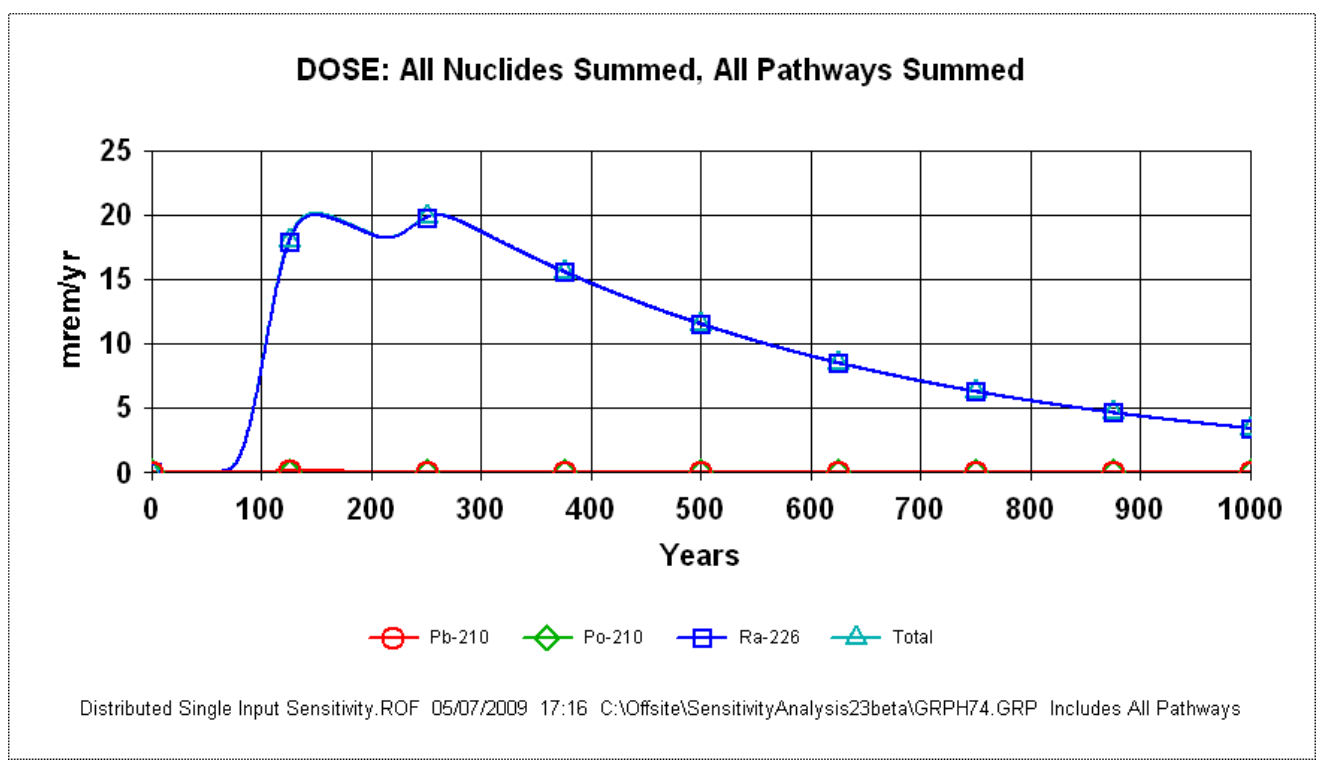

FIGURE C.25 Total Dose at a Hydraulic Conductivity of $732.1 \mathrm{~m} / \mathrm{yr}$ in Saturated Zone 


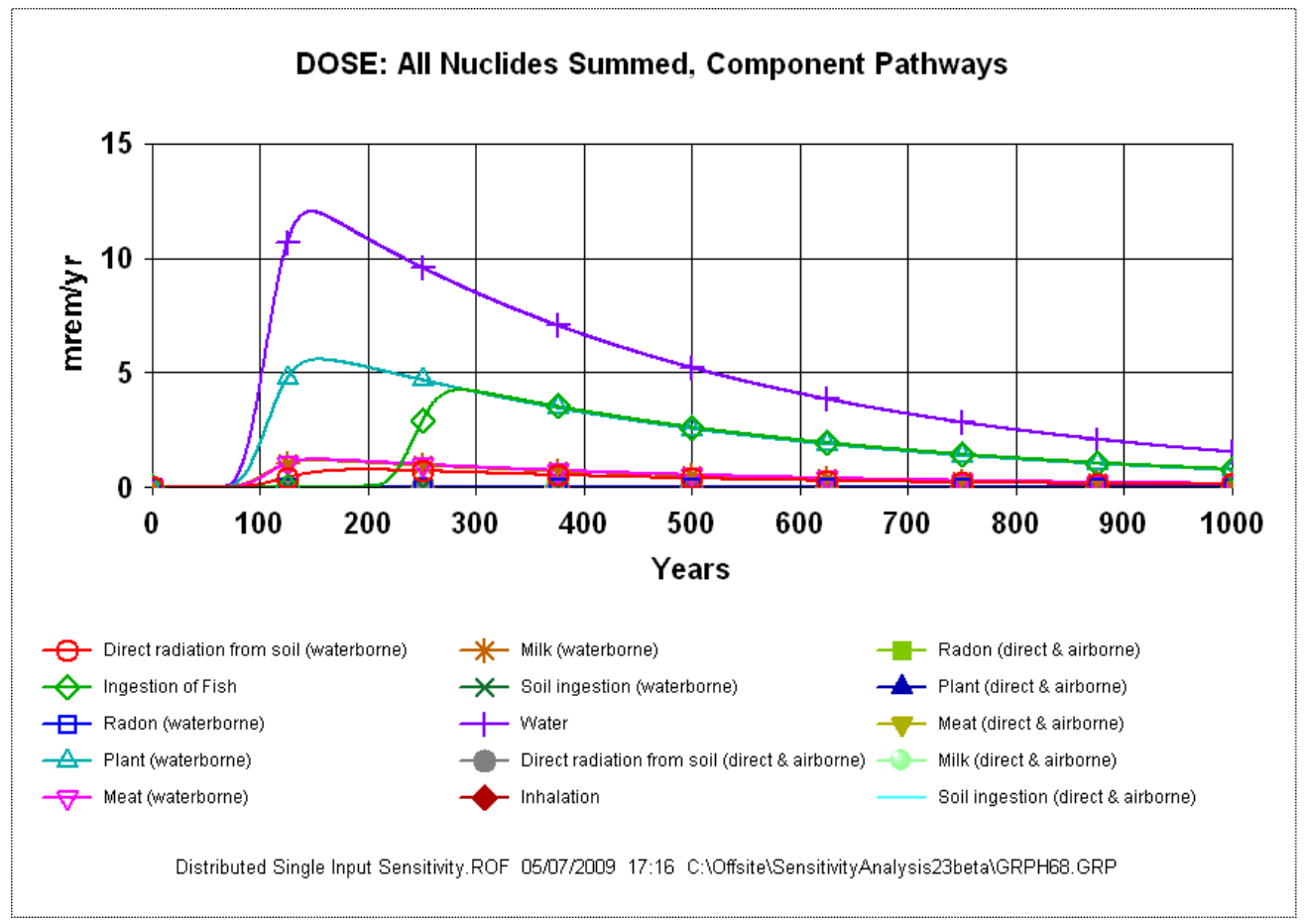

\section{FIGURE C.26 Relative Contributions of Exposure Pathways at $709.8 \mathrm{~m} / \mathrm{yr}$ Hydraulic Conductivity}

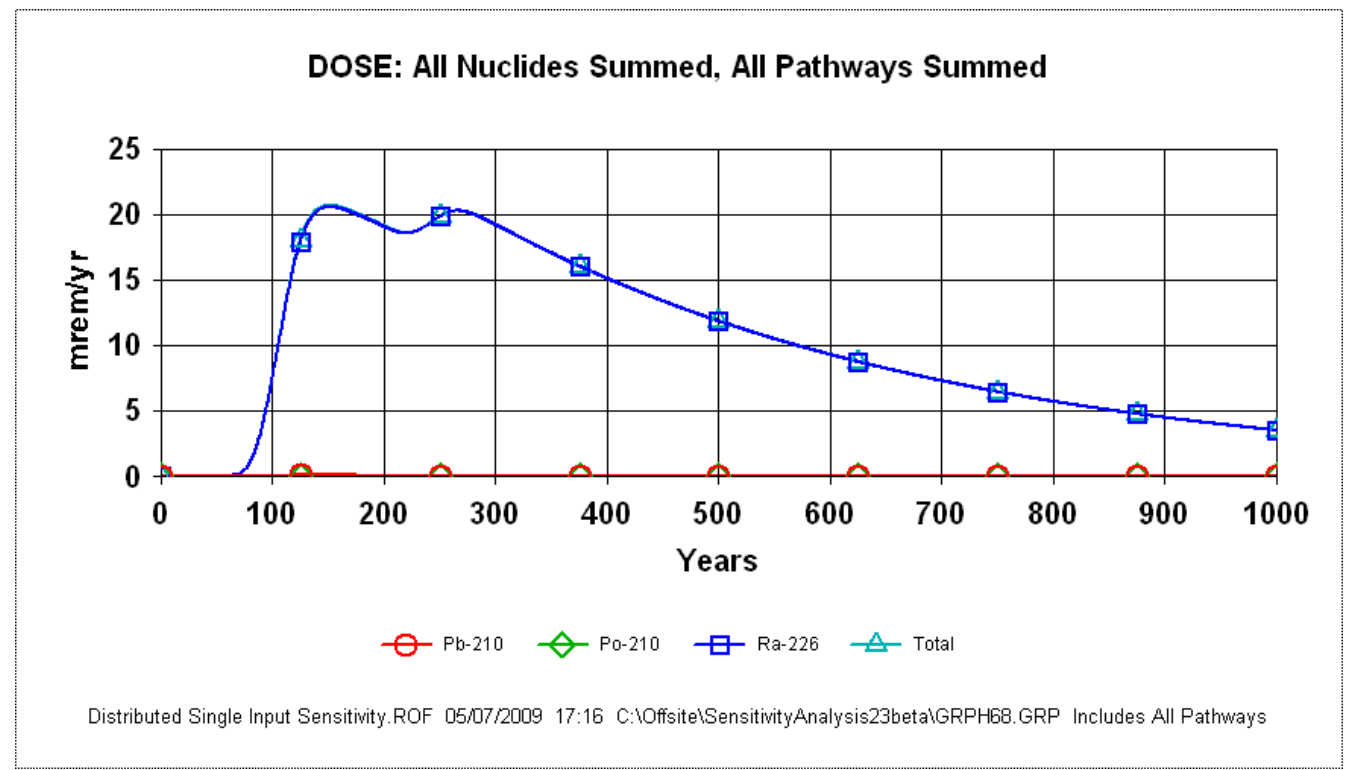

FIGURE C.27 Total Dose at a Hydraulic Conductivity of 709.8 m/yr in Saturated Zone 


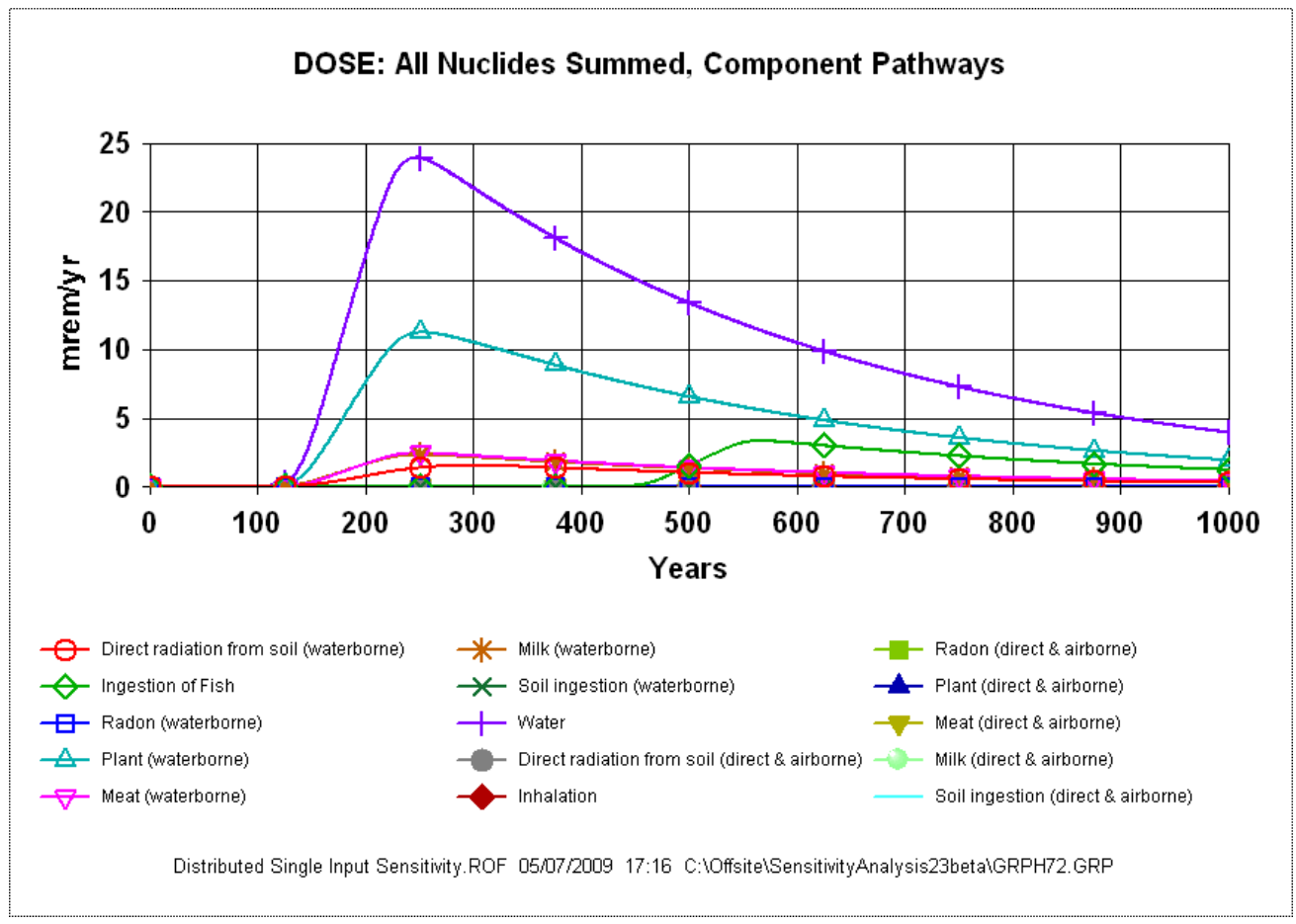

\section{FIGURE C.28 Relative Contributions of Exposure Pathways at $\mathbf{2 9 9 . 2} \mathrm{m} / \mathrm{yr}$ Hydraulic Conductivity}

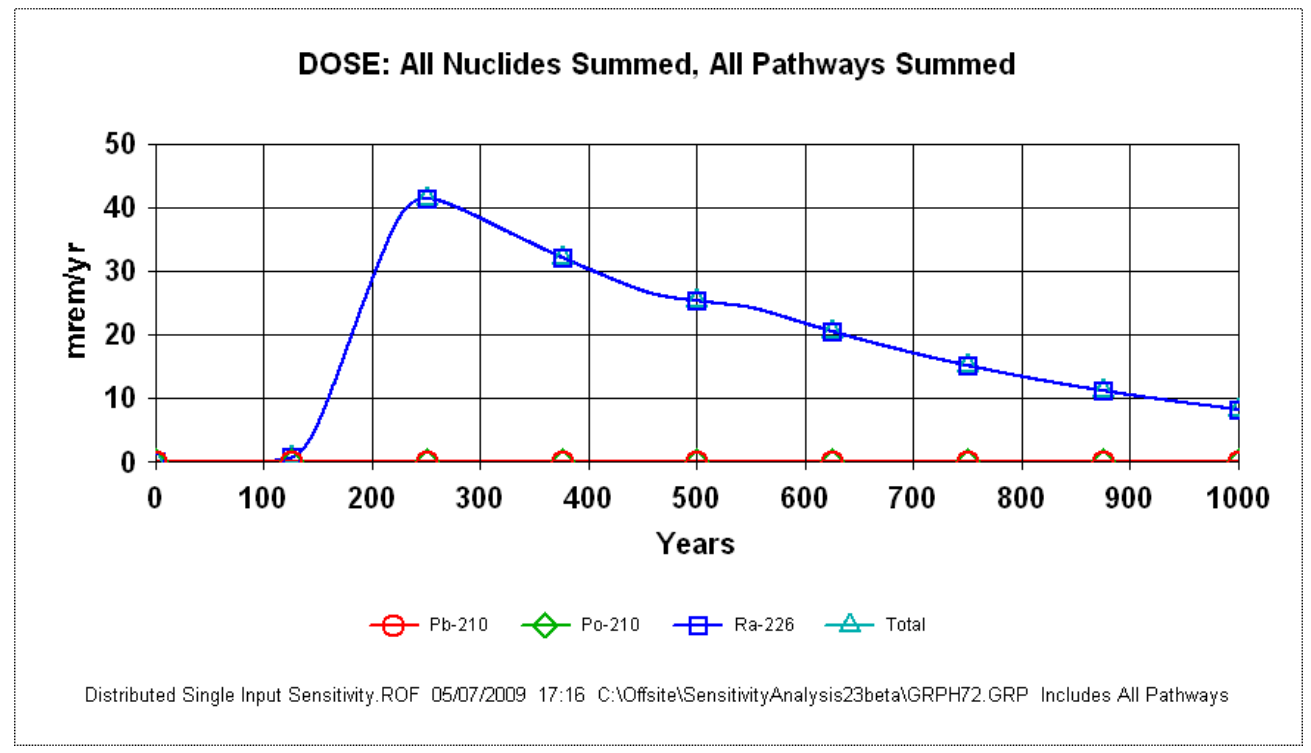

FIGURE C.29 Total Dose at a Hydraulic Conductivity of 299.2 m/yr in Saturated Zone 


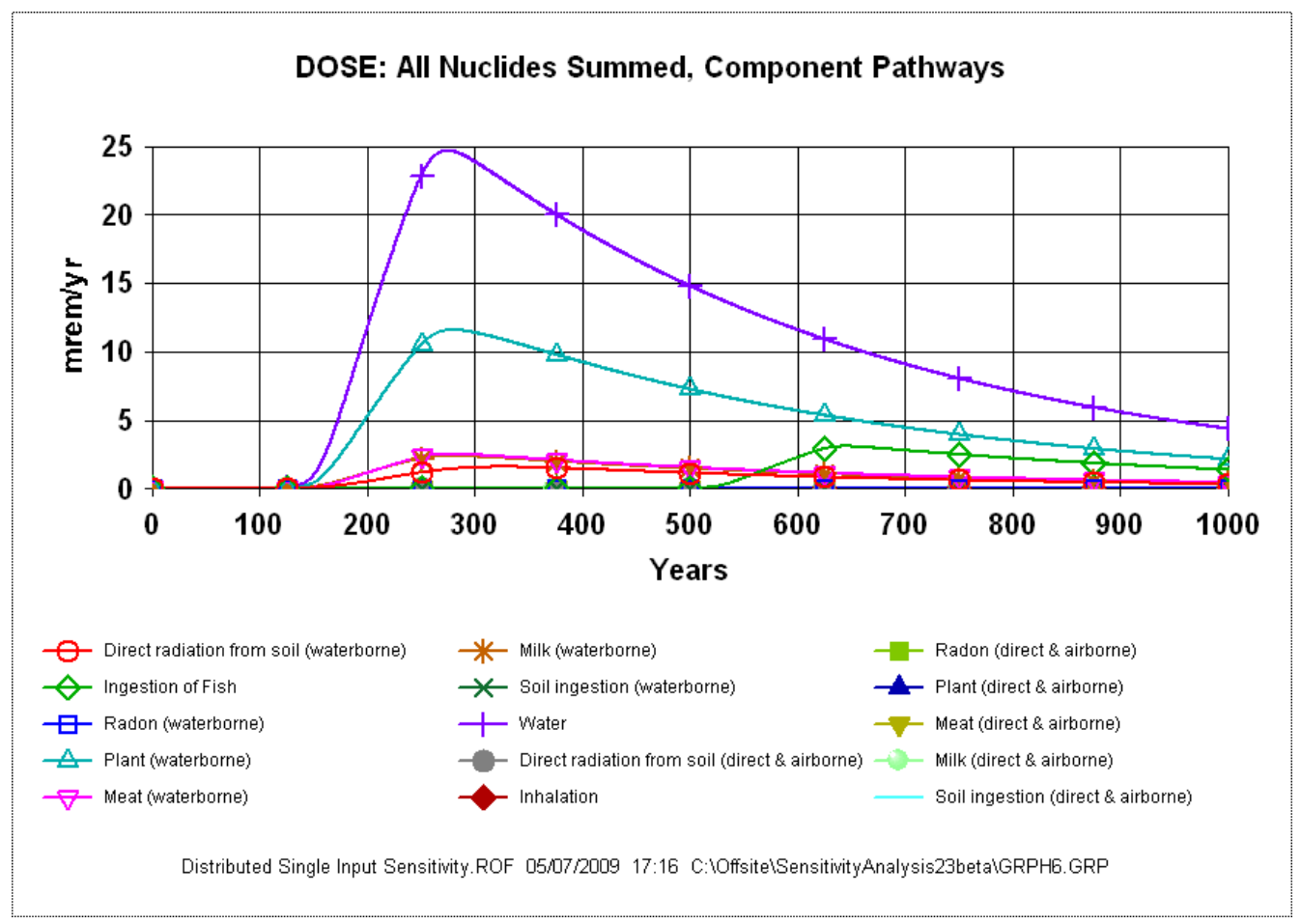

FIGURE C.30 Relative Contributions of Exposure Pathways at 258 m/yr Hydraulic Conductivity

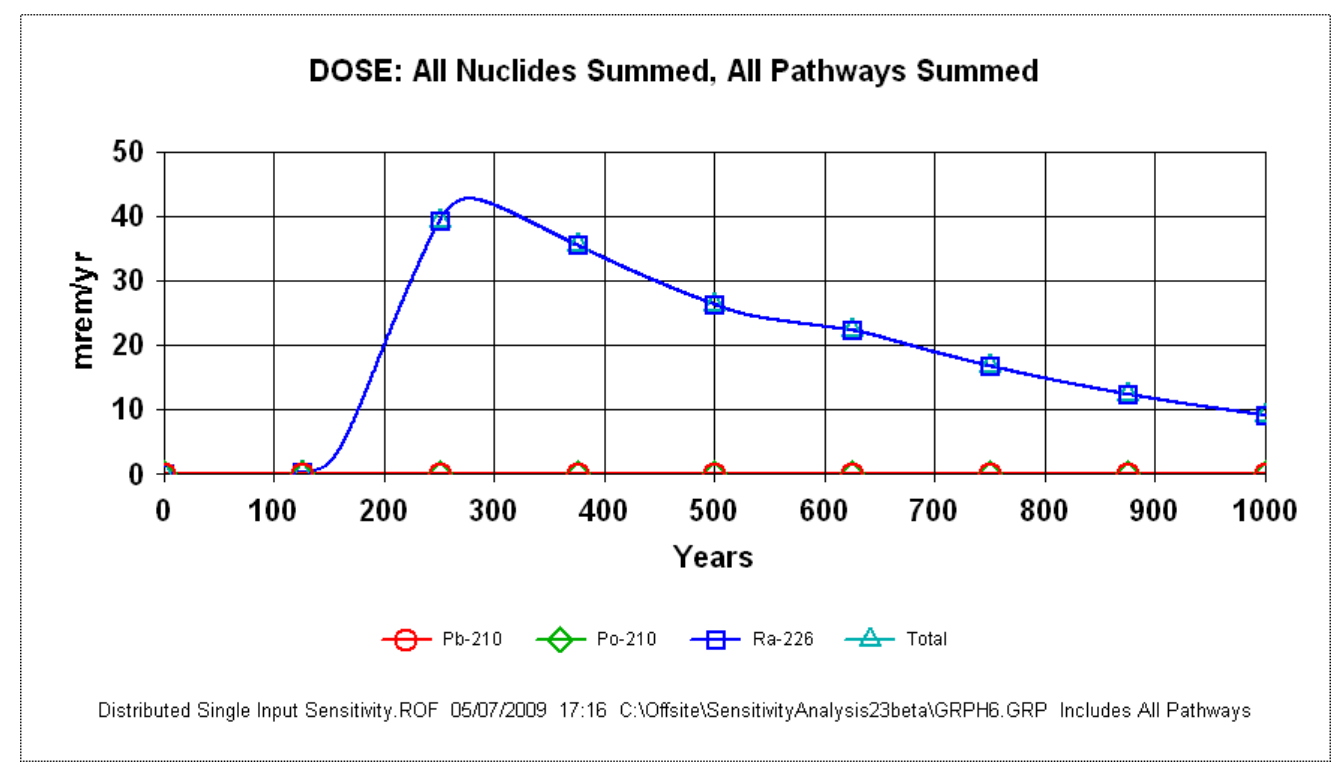

\section{FIGURE C.31 Total Dose at a Hydraulic Conductivity of 258 m/yr in Saturated Zone}




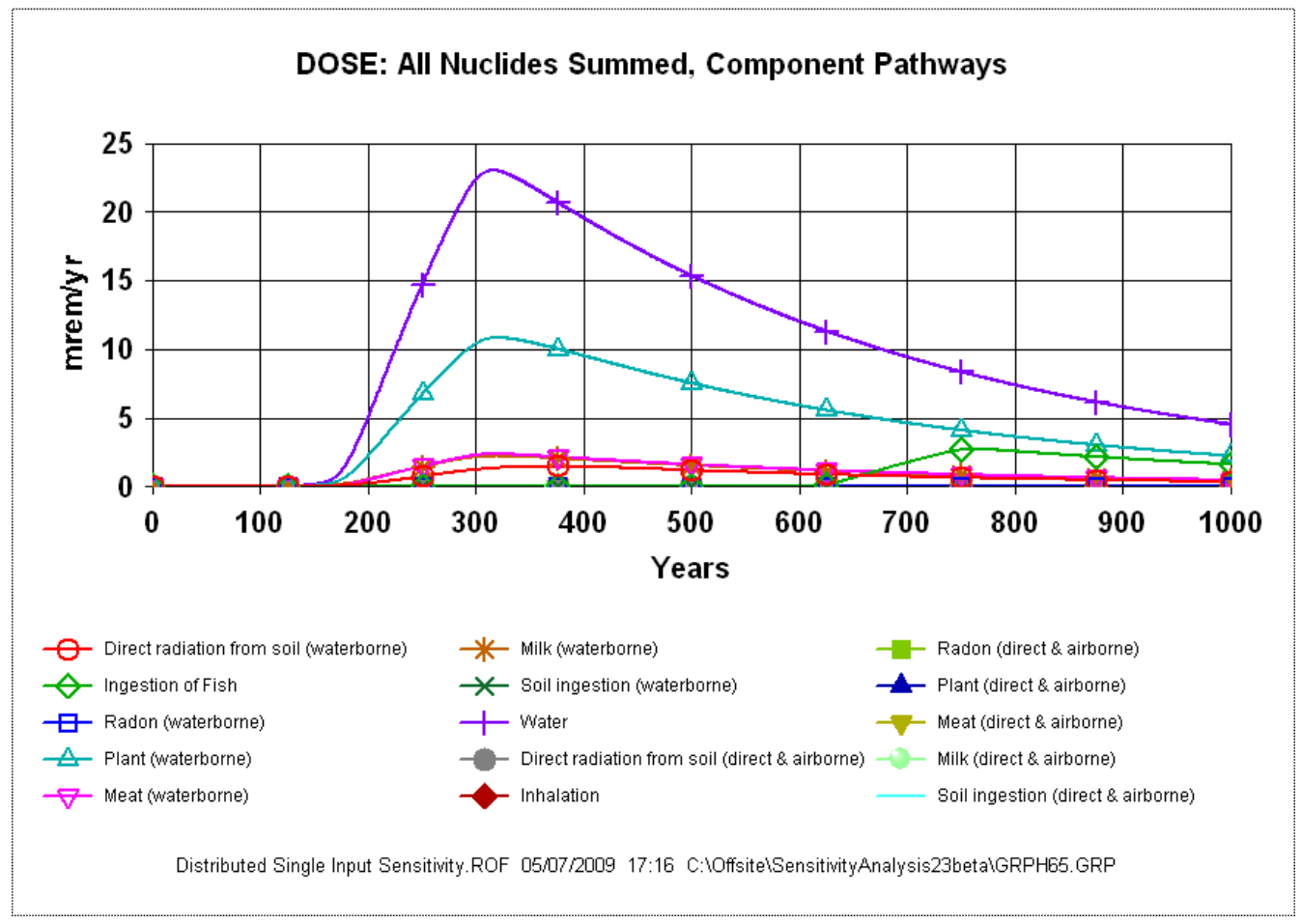

\section{FIGURE C.32 Relative Contributions of Exposure Pathways at $213.7 \mathrm{~m} / \mathrm{yr}$ Hydraulic Conductivity}

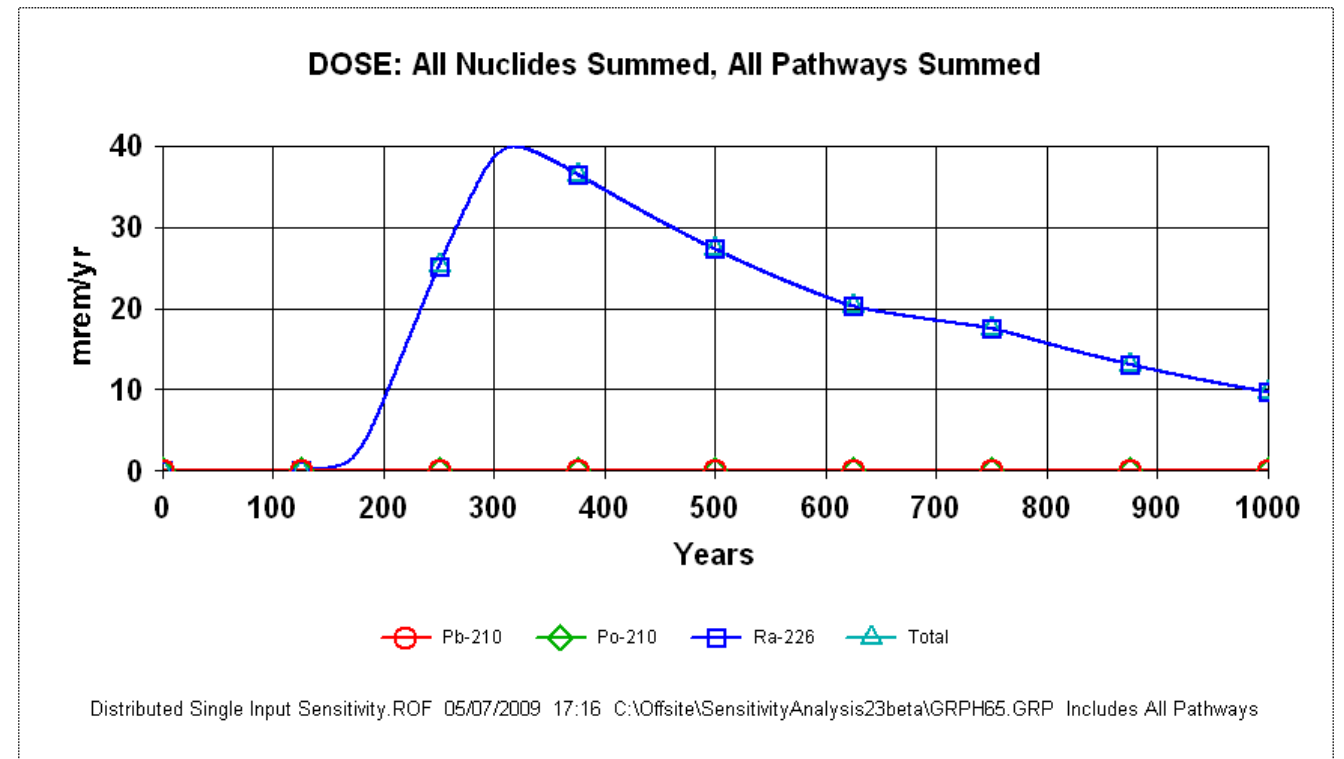

\section{FIGURE C.33 Total Dose at a Hydraulic Conductivity of $213.7 \mathrm{~m} / \mathrm{yr}$ in Saturated Zone}


When the hydraulic conductivity is $1984 \mathrm{~m} / \mathrm{yr}$, the highest value sampled in this example, the six major exposure pathways peak close together, as seen in Figure C.20. The exposure from drinking well water peaks at around 100 years, while the external radiation exposure from radionuclides in soil irrigated with well water peaks at around 150 years. The exposure pathways that receive contributions both from well water and from accumulation in soil following irrigation by well water peak at intervening times depending on the relative contributions of the two components. The exposure from the ingestion of fish from the surface water body also peaks at around 150 years, the surface water body being farther away than the well from the initial contamination. The six components combine to produce a total dose that peaks close to 150 years (Figure C.21).

As the hydraulic conductivity decreases, the additional transport time to the surface water body causes the predicted peak dose from the ingestion of fish to move away from the predicted peak doses from the five well-water-dependent pathways (Figure C.22). At a hydraulic conductivity of $752.1 \mathrm{~m} / \mathrm{yr}$, separation and relative magnitudes of the six peaks is such that the peak of the contribution from the six exposure pathways is only a little greater than the peak of the contributions from the five well-water-dependent exposure pathways (Figure C.23). At the nextlower hydraulic conductivity sampled in this case, $732.2 \mathrm{~m} / \mathrm{yr}$, the peak of the dose from all exposure pathways involves only contributions from the five well-water-dependent exposure pathways (Figures C.24 and C.25); the second peak that also involves contribution from the ingestion of fish is marginally less than the first peak. As the hydraulic conductivity decreases, the first peak becomes larger in relation to the second (Figures C.26 and C.27). The change in the exposure pathways that contribute to the peak predicted dose and the way in which these pathways vary with hydraulic conductivity in the saturated zone (Figures C.13 through C.18) are the reasons for the break in the curve, seen in Figure C.12, of peak predicted dose against hydraulic conductivity.

Figures C.28 through C.33 show the contributions for the exposure pathways to the total predicted dose in vicinity of the peak in the curve of total dose against hydraulic conductivity (Figure C.12). They do not reveal why the peak predicted dose at a hydraulic conductivity of $258 \mathrm{~m} / \mathrm{yr}$ is higher than the peak predicted doses at hydraulic conductivities of 299.2 and $213.7 \mathrm{~m} / \mathrm{yr}$. That requires a more in-depth look at the intermediate results, as described in Section C.3.

\section{C.2.3 Comparison of the Two Methods of Performing Single-input Sensitivity in RESRAD-OFFSITE}

The sensitivity of the peak predicted dose (or peak risk) to the input can be measured in many ways. This section discuss three alternatives for quantifying the sensitivity: (1) the rate of change of peak predicted dose with the input, (2) the fractional rate of change of peak predicted dose with the input, and (3) the range and distribution of the peak predicted dose when the input 
varies over its site-appropriate range of values. Each of these measures is appropriate for some purposes but not for others. The two methods of performing single-input sensitivity in RESRAD-OFFSITE are compared using these measures.

\section{C.2.3.1 The Rate of Change of Peak Predicted Dose with the Input}

The slope of the plot of peak predicted dose (or peak risk) against the input is a measure of how sensitive the peak predicted dose is to a change in the input. This will be constant over the range of input when the peak predicted dose is a linear function of the input over that range. While this is the case for some of the inputs in RESRAD-OFFSITE, the peak predicted dose is a nonlinear function of many of the inputs. Thus, the rate of change varies over the range of the input. The average rate of change over the two intervals $233.3 \mathrm{~m} / \mathrm{yr}$ to $700 \mathrm{~m} / \mathrm{yr}$ and $700 \mathrm{~m} / \mathrm{yr}$ to $2100 \mathrm{~m} / \mathrm{yr}$ can be calculated using the three-point single-input sensitivity analysis data in WRESPLOT, the deterministic graphics viewer in RESRAD-OFFSITE. These data can be saved and viewed using the menu commands or toolbar icons in WRESPLOT, after displaying the three plots. The average rate of change in the interval between each pair of adjacent samples of the distributed single-input sensitivity analysis can be computed using the data in the peak predicted dose data file (with extension .pds) and the probabilistic input sample data file (with extension .pin). These calculated values are shown in Figure C.34.

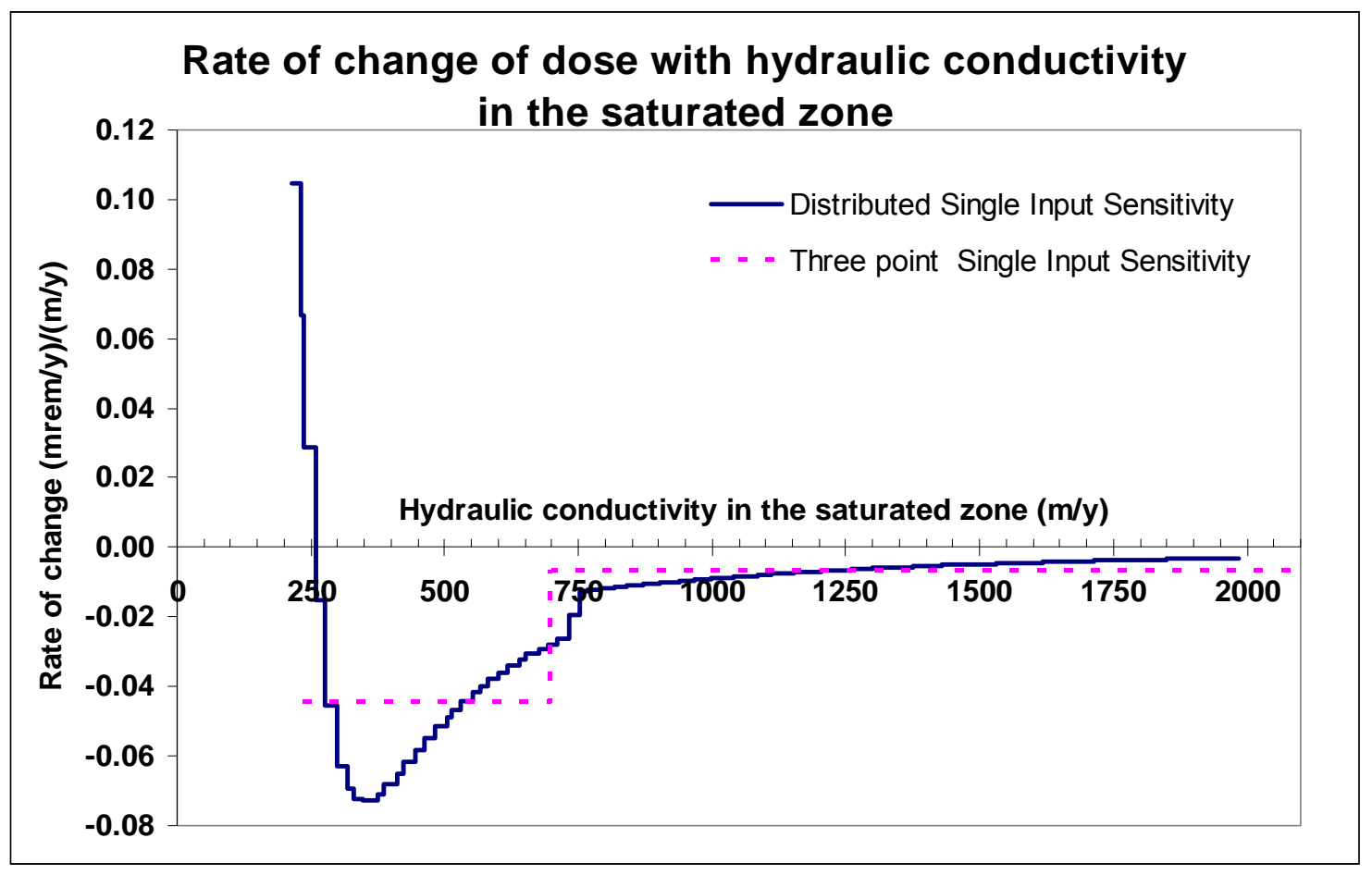

FIGURE C.34 Rate of Change of Peak Predicted Dose with Hydraulic Conductivity 
Because the interval between the samples is small (no greater than $36 \mathrm{~m} / \mathrm{yr}$ ), the values computed using the distributed single-input sensitivity analysis are expected to closely approximate the slope of the peak predicted dose-hydraulic conductivity plot. The rates of change computed using the distributed single-point sensitivity analysis data change slowly over the range of 750 through $2000 \mathrm{~m} / \mathrm{yr}$, from -0.013 to $-0.003 \mathrm{mrem} / \mathrm{m}$. The average rate of change over the range of 700 to $2100 \mathrm{~m} / \mathrm{yr}$ computed using the three-point single-input sensitivity analysis data, $-0.0067 \mathrm{mrem} / \mathrm{m}$, is in good agreement with rate of change computed using the distributed single-input sensitivity analysis data. The slope varies widely in the 200 to $750 \mathrm{~m} / \mathrm{yr}$ range. The rates of change computed using the data from the distributed single-input sensitivity analysis vary from 0.1 rapidly down to -0.073 and then gradually back up to $-0.026 \mathrm{mrem} / \mathrm{m}$. The average rate of change over the range of 233.3 to $700 \mathrm{~m} / \mathrm{yr}$, computed using the three-point single-input sensitivity analysis, is $-0.045 \mathrm{mrem} / \mathrm{m}$. Thus, the three-point single-input sensitivity analysis efficiently estimates the rate of change in the range where it is relatively constant, but does not when the slope changes rapidly or when the slope changes direction. The distributed single-input sensitivity analysis captures the variation of the rate of change over the range very well because the analyst is able to specify a larger number of simulations over the range. On the downside, the larger number of simulations requires a longer run time.

The slope of the peak predicted dose vs. input plot-that is, the rate of change of peak predicted dose with input-is a very good measure for comparing the sensitivities of peak predicted doses from different ranges of the same input. It is not an appropriate measure for comparing the sensitivity of peak predicted dose to two different inputs because there is no meaningful way to compare numbers with different units.

\section{C.2.3.2 Fractional Rate of Change of Peak Predicted Dose with Input}

A fractional rate of change-that is, the rate of change normalized by some representative value of the peak predicted dose or the input-is dimensionless and permits comparison of the sensitivities to two different inputs. Normalizing the change in peak predicted dose by a representative value of peak predicted dose for that interval make it dimensionless; likewise, normalizing the change in the input by a representative value of input for that interval make it dimensionless as well. The only issue then is what is the representative value for the range; the values at one or the other of the two ends of the range, or the mean of the two values. The choice made for the representative value does not significantly affect the computation of the fractional rate of change when the range of the interval is small; this is usually the case for the distributed single-input sensitivity analysis. The interval in the three-point single-input sensitivity analysis can in general be large. Table C. 2 illustrates how the choice of the representative normalizing value affects the computed fractional rate of change for the three-point single-input sensitivity example described in Section C.2.1.2. 
TABLE C.2 Effect of the Choice of the Representative Normalizing Value on the Computed Fractional Rate of Change

\begin{tabular}{|c|c|c|c|c|c|c|c|c|}
\hline \multicolumn{3}{|c|}{ Hydraulic conductivity, $\mathrm{m} / \mathrm{yr}$} & \multicolumn{3}{|c|}{ Peak predicted dose, mrem/yr } & \multicolumn{3}{|c|}{$\begin{array}{c}\text { Fractional rate } \\
\text { of change normalized by }\end{array}$} \\
\hline Left end & Right end & Average & Left end & Right end & Average & Left end & Right end & Average \\
\hline 233.3 & 700 & 466.7 & 41.86 & 20.98 & 31.42 & -0.25 & -1.5 & -0.66 \\
\hline 700 & 2100 & 1400 & 20.98 & 11.57 & 16.28 & -0.22 & -1.2 & -0.58 \\
\hline
\end{tabular}

The fractional rates of change computed using the averages of the interval as the normalizing values are plotted in Figure C.35. The fractional rate of change starts off at a value of 0.6 at a hydraulic conductivity of $200 \mathrm{~m} / \mathrm{yr}$ in the saturated zone. It falls rapidly at first with increasing hydraulic conductivity, levels out at a value of around -1 at a hydraulic conductivity of about $500 \mathrm{~m} / \mathrm{yr}$, and steps up to a value of about -0.5 at a hydraulic conductivity of around $750 \mathrm{~m} / \mathrm{yr}$. The fractional rate of change computed using the two methods of single-input sensitivity analysis data are in agreement in the hydraulic conductivity range of 700 to $2100 \mathrm{~m} / \mathrm{yr}$.

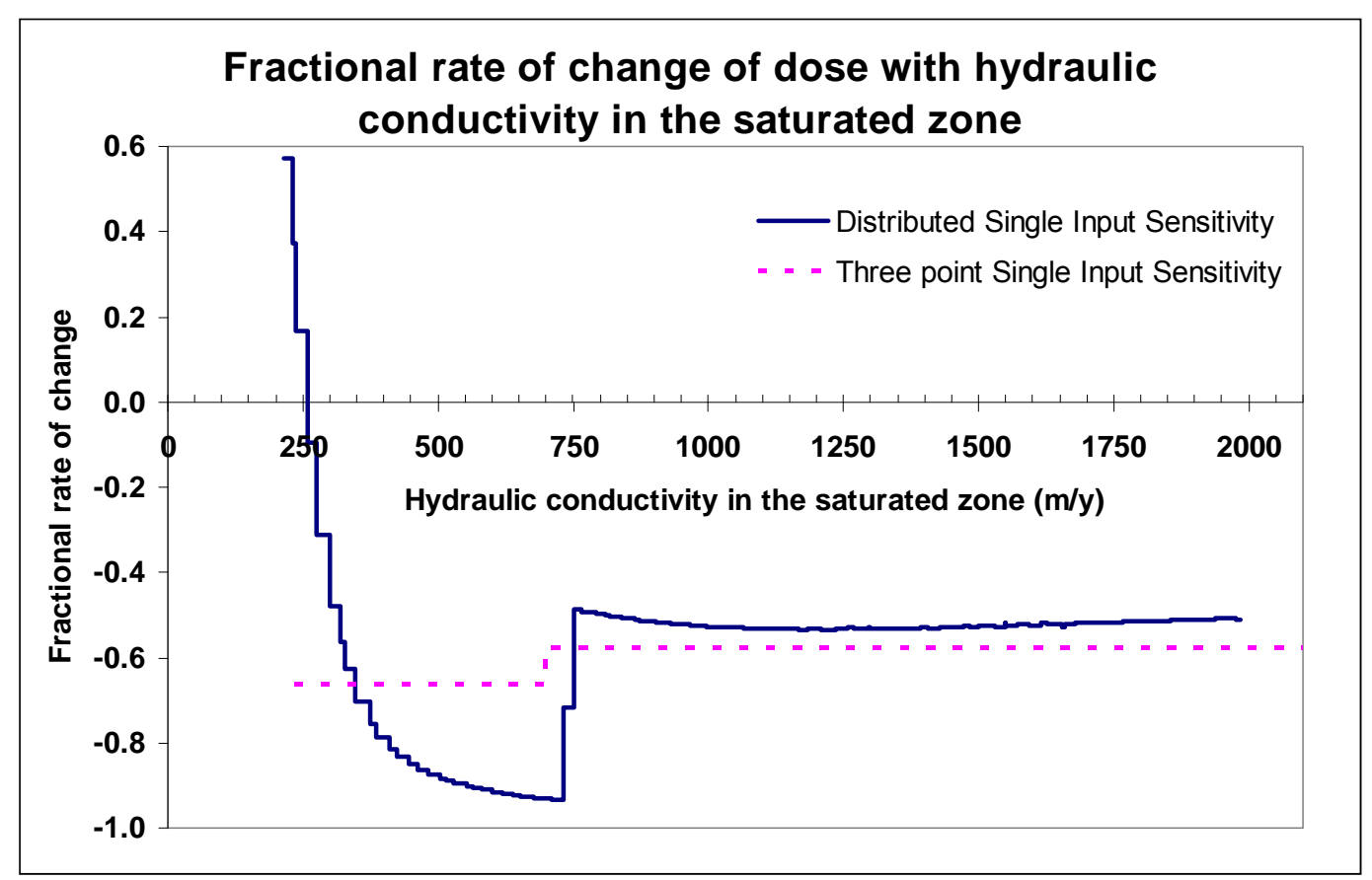

FIGURE C.35 Fractional Rate of Change of Peak Predicted Dose with
Hydraulic Conductivity 
Although normalization by a representative value makes this measure of sensitivity dimensionless and therefore a valid means of comparing the sensitivity of peak predicted dose to two or more different inputs, it also makes the interpretation of the comparison more difficult. The fractional sensitivity of peak predicted dose from the drinking well water to the hydraulic conductivity ranges from about -0.8 to -0.95 in the hydraulic conductivity range of 400 to $2000 \mathrm{~m} / \mathrm{yr}$, while the peak predicted dose from that pathway decreases rapidly in the beginning of the range and more gradually at the end of the range (Figure C.13). The fractional sensitivity to hydraulic conductivity of peak predicted dose from the ingestion of fish ranges from about 0.72 to 0.052 in the hydraulic conductivity range of $200 \mathrm{~m} / \mathrm{yr}$ to $2000 \mathrm{~m} / \mathrm{yr}$, while the peak predicted dose from that pathway increases rapidly in the beginning of the range and more gradually at the end of the range (Figure C.18).

\section{C.2.3.2.1 Interpretation of Fractional Rate of Change of Peak Predicted Dose with the Input}

This section derives the relationship between peak predicted dose and the input for a constant value of fractional rate of change. This relationship is then used to relate the value of the fractional rate of change, $k$, to the shape of the curve of peak predicted dose, $y$, against the input, $x$.

Begin with the definition of the fractional rate of change, $\frac{d y}{y} / \frac{d x}{x}=k$. Rearranging and integrating $\int \frac{d y}{y}=k \int \frac{d x}{x}$ gives $\ln y=k \ln x+c$, where $c$ is the integration constant. This is equivalent to $y=C x^{k}$, where $C=e^{c}$. Table C.3 summarizes the typical nature of this curve for various ranges of $k$.

TABLE C.3 Relationship between Fractional Rate of Change and the Shape of the Curve of Peak Predicted Dose and the Input

\begin{tabular}{|c|c|}
\hline $\begin{array}{l}\text { Fractional } \\
\text { rate of } \\
\text { change }\end{array}$ & Shape \\
\hline $1<k$ & $\begin{array}{l}\text { Peak predicted dose increases nonlinearly with increasing value of input, slowly at first and more } \\
\text { rapidly later. }\end{array}$ \\
\hline 1 & Peak predicted dose increases linearly with increasing value of input. \\
\hline $0<\mathrm{k}<1$ & $\begin{array}{l}\text { Peak predicted dose increases nonlinearly with increasing value of input, rapidly at first and more } \\
\text { gradually later. }\end{array}$ \\
\hline $\mathrm{k}<0$ & $\begin{array}{l}\text { Peak predicted dose decreases nonlinearly with increasing value of input, rapidly at first and more } \\
\text { gradually later. }\end{array}$ \\
\hline$k=-1$ & Peak predicted dose varies inversely with input; the shape is a rectangular hyperbola. \\
\hline
\end{tabular}


The curves for selected values of $k$ are shown in Figure C.36. These curves illustrate that except for unity, a constant value of the normalized rate of change over the range of the input does not signify a constant sensitivity of peak predicted dose to the input over that range. These curves also indicate that depending on the range of the input, different values of the fractional rate of change indicate greater sensitivity. For example, a $k$ of between 0 and 1 indicates a greater sensitivity at low values of the range of $x$, when compared with a $k>1$; the situation is reversed at higher values of $x$, where a $k>1$ indicates a greater sensitivity than a $k$ of 0 to 1 . Thus, the fractional rate of change is not necessarily a good indicator of the sensitivity. A more reliable indicator of sensitivity is needed.

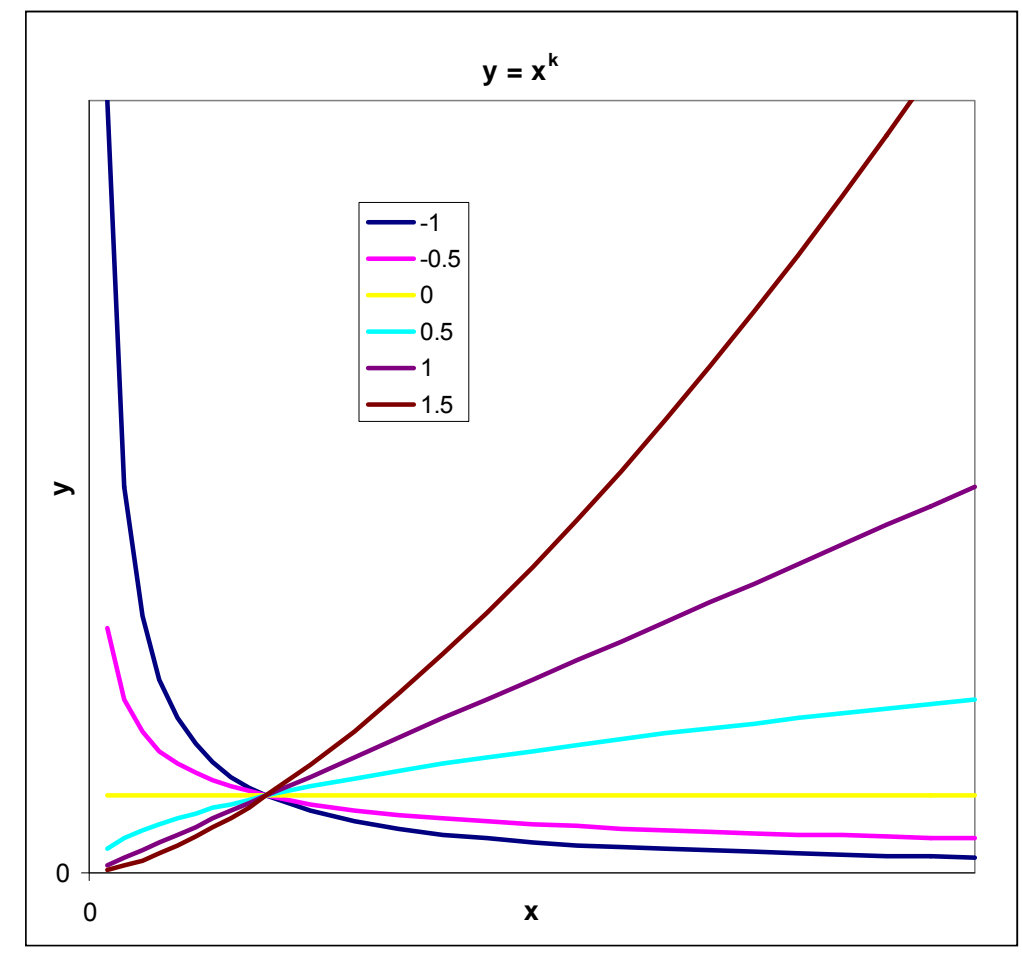

FIGURE C.36 Shape of $y=x^{k}$ for select values of $k$

\section{C.2.3.3 The Range of the Peak Predicted Dose over the Site-appropriate Range of the Input}

The discussion in Sections C.2.3.1 and C.2.3.2 indicate the difficulties of using a derived measure of sensitivity. The range of the peak predicted dose is a direct measure of sensitivity. The response of peak predicted dose to changes in one input depends on the (point) values used for many of the other inputs (Section C.3). When performing sensitivity analysis, it is therefore imperative that site-appropriate values be used for all inputs if site-specific values have not yet been identified. Three point single-input sensitivity analysis can then be performed 
on a number of inputs using either site-appropriate ranges or site-specific ranges. The ranges of the peak predicted dose that result when each input is varied over its site-appropriate or sitespecific range can be used to determine the sensitivity of peak predicted dose to that input. Alternatively distributed single-input sensitivity analysis can then be performed on a number of inputs using either site-appropriate distributions or site-specific distributions. Some measure of the distribution of the peak predicted dose that result when each input is described by its siteappropriate or site-specific distribution can be used to determine the sensitivity of peak predicted dose to that input. The standard deviation of the peak predicted dose which is composed of peak predicted dose of all the samples of the distribution, is a better measure of its distribution than the range which depends on only the two extreme predictions of peak dose.

\section{C.3 Interactions between Inputs and Their Effect on the Sensitivity to Each Input}

This section takes a deeper look at the results of the example case presented in the previous section. This in-depth look serves many purposes:

- It helps answer the questions raised in Section C.2 about the manner in which the peak predicted doses from different exposure pathways vary with hydraulic conductivity in the saturated zone.

- It provides an example of how one might go about trying to understand the response of peak predicted dose to changes in other inputs under other situations.

- It highlights the interactions between inputs and suggests that the sensitivity of dose to one input depends on the values used for other inputs.

- It points to the need for using values that are either specific or at least appropriate for the site for all the inputs when performing sensitivity analysis.

- It also shows the need for multiple-input sensitivity analysis and the need for specifying either correlations or relationships between some of the inputs.

\section{C.3.1 Anticipated Influence of Saturated Hydraulic Conductivity on Transport Time}

An increase in hydraulic conductivity in the saturated zone causes a corresponding rise in the Darcy velocity of flow in the saturated zone when the hydraulic gradient is held constant. Thus, the radionuclides are transported to the well and to the surface water body more quickly as the hydraulic conductivity increases, as is observed in the temporal plots from the three-point single-input sensitivity (Figures C.4 through C.9) and distributed single-input sensitivity analyses (Figures C.20, C.22, C.24, C.26, C.28, C.30, and C.32). The concentration of the radionuclide 
in the water can change when the difference in transport time is significant in relation to the halflife of the radionuclide. As the hydraulic conductivity increased from 200 to $2000 \mathrm{~m} / \mathrm{yr}$, the transport time to the well decreased by more than 200 years and the transport time to the surface water body fell by more than 500 years. This decrease in travel time raises the concentration of ${ }^{226} \mathrm{Ra}$ in the well by a factor of 1.1 and in the surface water body by a factor of 1.3 as the saturated hydraulic conductivity increases from 200 to $2000 \mathrm{~m} / \mathrm{yr}$.

Another consequence of a higher Darcy flow rate is that a greater quantity of water will flow through a given cross-section of the aquifer as the hydraulic conductivity increases.

Conversely, the constant-flow-rate contaminated plume entering the saturated zone will initially occupy a smaller cross-section of the aquifer. The plume will spread out in the three directions (longitudinal, horizontal lateral, and vertical lateral) as it moves away from below the primary contamination and toward the well and surface water body. The quantity and concentration of the radionuclide in the well and in the surface water body will depend on how the radionuclide is distributed over the region of the aquifer that is intercepted by each source of water. This is discussed in the next two sections.

\section{C.3.2 Influence of Saturated Hydraulic Conductivity on the Dose from Ingestion of Fish}

It is instructive to start with the dimensions of the plume in the saturated zone directly below the primary contamination prior to lateral dispersion. The width is equal to the width of the primary contamination, $100 \mathrm{~m}$ in this example. The thickness is computed by determining the thickness of the saturated zone required to convey the infiltration through the primary contamination-it is inversely proportional to the hydraulic conductivity in the saturated zone. The thicknesses and half the widths of the plumes for five hydraulic conductivities $(210,260,300,700$, and $2000 \mathrm{~m} / \mathrm{yr}$ ) are shown in Figure C.37. The dimensions of the saturated zone that feeds into the surface water body are also shown. In the absence of lateral dispersion, the entire plume would have entered the surface water body at hydraulic conductivities of $260 \mathrm{~m} / \mathrm{yr}$ or greater. 


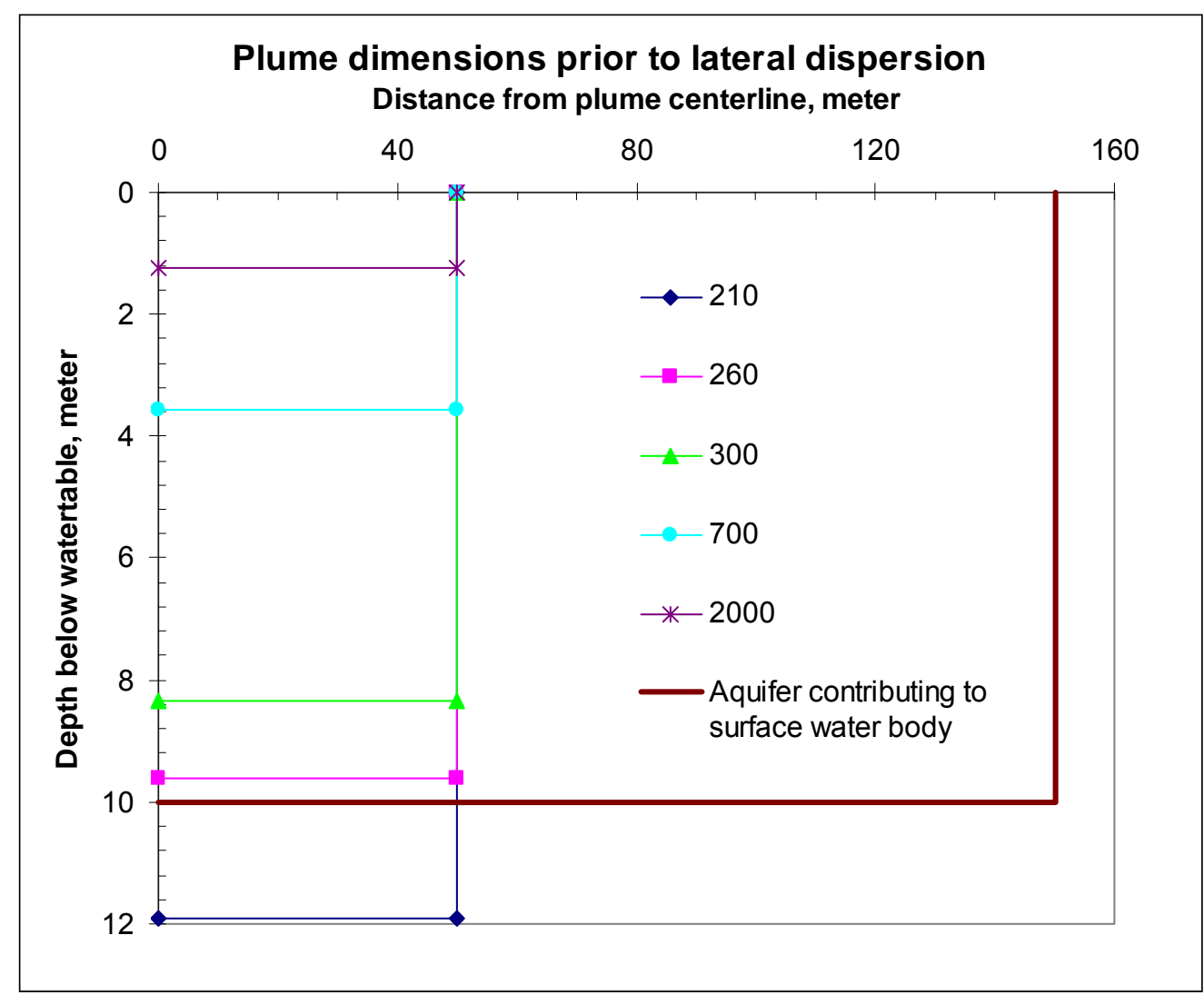

\section{FIGURE C.37 Dimensions of Plume Prior to Lateral Dispersion and Dimension of Saturated Zone Contributing to the Surface Water Body}

Longitudinal dispersion spreads the plume in the direction of flow; the effects of longitudinal dispersion can be seen in the temporal plots in WRESPLOT. Lateral dispersion spreads the plume in the vertical and horizontal directions perpendicular to the flow. Information about the transverse distribution of the radionuclides in the saturated zone at the locations of the well and the surface water body are written in the ASCIl files "PROFILEW.OUT" and "PROFILES.OUT," respectively. These files are written once for each run, so information for all the sensitivity simulations is not available. The code was run five times in the determinist mode, with the hydraulic conductivity in the saturated zone set to $210,260,300,700$, and $2000 \mathrm{~m} / \mathrm{yr}$, respectively. The horizontal and vertical distributions of ${ }^{226} \mathrm{Ra}$ in the saturated zone at the location of the surface water body for these five runs are shown in Figures C.38 and C.39. Horizontal lateral dispersion spreads the plume from its initial width of $100 \mathrm{~m}$ to a width of about $280 \mathrm{~m}$ at the surface water body. The surface water body in this example is at the plume centerline and is $300 \mathrm{~m}$ wide. Hence, horizontal lateral dispersion does not affect the quantity of radionuclides that are intercepted by the surface water body. Figure C.39 shows that vertical 
lateral dispersion has a significant effect on this quantity, however. At a hydraulic conductivity of $2000 \mathrm{~m} / \mathrm{yr}$ in the saturated zone, the plume was conceptualized to be uniformly distributed over a depth of $1.25 \mathrm{~m}$ when it was directly below the primary contamination, as shown in Figure C.37. Vertical lateral dispersion spreads the radionuclide over a depth of about $25 \mathrm{~m}$ and reduces the concentration at the water table to approximately 0.15 of the initial value.

The quantity of the radionuclides that remains within the depth of interception of the surface water body, $10 \mathrm{~m}$ in this example, can be found by integrating the area under the curves. In this example, $0.66,0.71,0.73,0.80$, and 0.81 of the plume is intercepted by the surface water body at hydraulic conductivities of $210,260,300,700$, and $2000 \mathrm{~m} / \mathrm{yr}$, respectively. This, too, leads to an increase in the peak predicted dose from the ingestion of fish with increasing hydraulic conductivity in the saturated zone-by a factor of 1.22 as the hydraulic conductivity increases from 200 to $2000 \mathrm{~m} / \mathrm{yr}$. Along with the factor of 1.3 increase discussed in Section C.3.1, it is a main reason for the shape of the plot of peak predicted dose from ingestion of fish against hydraulic conductivity (Figure C.18).
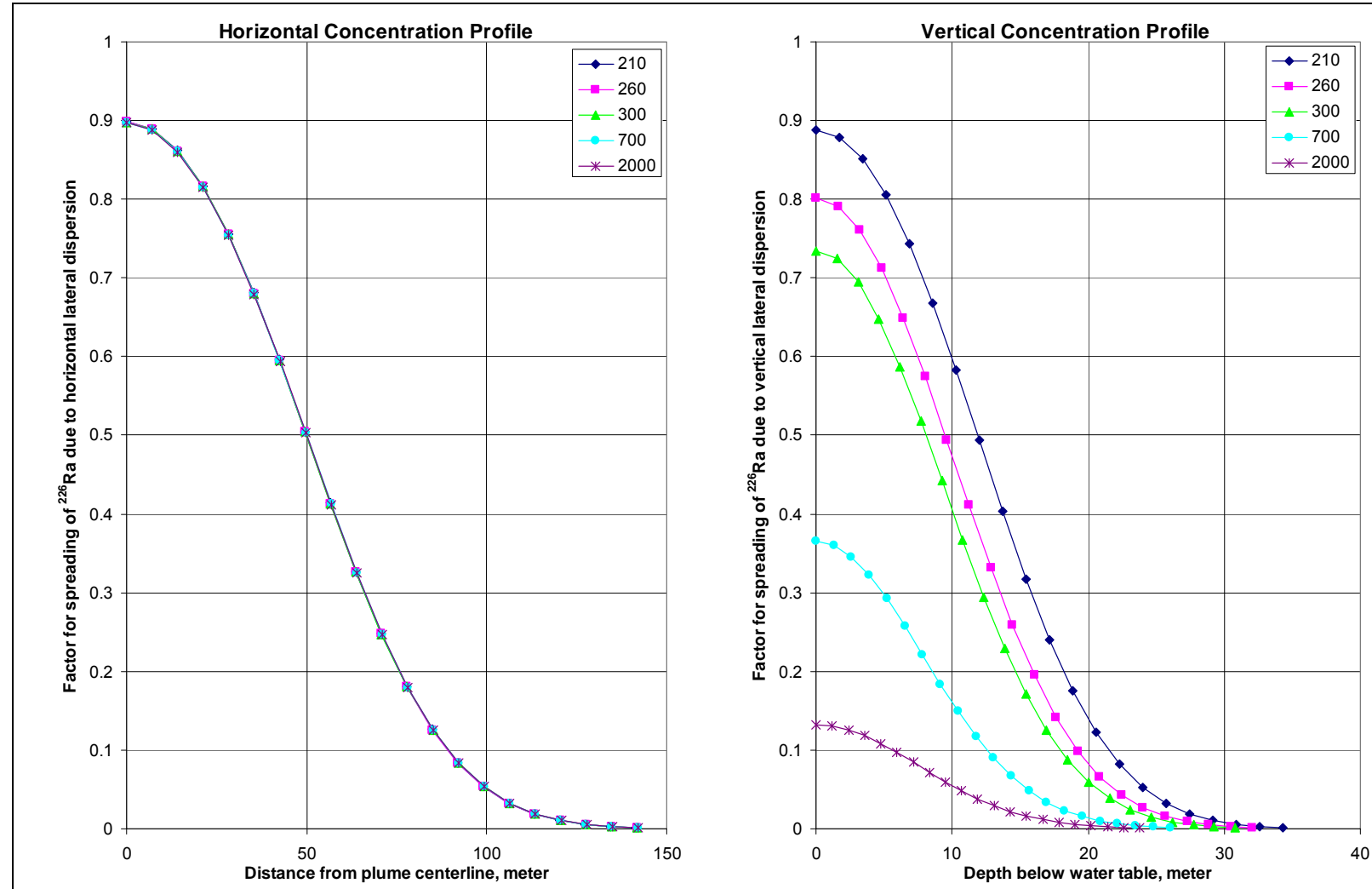

FIGURES C.38 (left) and C.39 (right) Distributions of Radionuclides in the Transverse Plane at the Location of the Surface Water Body 
The quantity of water entering the surface water body will increase in proportion to the hydraulic conductivity in the saturated zone, if the depth of interception is constant. This can be expected to affect the mean residence time of water in the surface water body. The mean residence time is an independent input in RESRAD-OFFSITE. It is not changed in the single-input sensitivity analysis on hydraulic conductivity in the saturated zone. From the discussion in this section, the sensitivity of peak predicted dose from the ingestion of fish to hydraulic conductivity can be expected to depend on the values specified for many inputs, including the mean residence time of water in the surface water body, the depth of interception and the width of the surface water body, and all the inputs that affect the quantity of water infiltrating through the primary contamination. This indicates the need for performing multiple-input sensitivity analysis and specifying a relationship between the hydraulic conductivity in the saturated zone and the mean residence time in the lake in this example. Section C.3.4 extends the example sensitivity analysis to include some of the interaction inputs discussed in this section.

\section{C.3.3 Influence of Saturated Hydraulic Conductivity on the Dose from Well-water- dependent Exposure Pathways}

It is again helpful to start by comparing the dimensions of the plume in the saturated zone directly below the primary contamination prior to lateral dispersion, with the dimensions of the aquifer contributing to the well. The thickness of the aquifer contributing to the well is the depth of the well. The width of aquifer contributing to the well is computed from the well pumping rate, the depth, and the Darcy velocity. The dimensions of the initial plume into the saturated zone are computed as described in Section C.3.2. The concentration within the initial plume is assumed to be uniform.

The dimensions of the two regions for five different hydraulic conductivities $(210,260,300,700$, and $2000 \mathrm{~m} / \mathrm{yr}$ ) are compared in Figure C.40. If the effects of lateral dispersion were to be ignored, the dilution due to mixing of the plume with the clean water would simply be the fraction of the cross-sectional area of the aquifer contributing to the well that is contaminated. At a hydraulic conductivity of $210 \mathrm{~m} / \mathrm{yr}$, the plume thickness exceeds the depth of the well while the width of the aquifer contributing to the well exceeds the width of the undispersed plume. Thus, part of the plume passes below the well, while the well draws clean water from the two sides of the plume in addition to the contaminated water from the width of the plume. As the hydraulic conductivity increases, both the thickness of the plume and the width of the aquifer contributing to the well decrease. When the width of the aquifer contributing to the well decreases, a smaller proportion of water drawn into the well will be clean. The concentration in the well and thus the peak predicted dose from well-water-dependent pathways increases until either the width of the aquifer contributing to the well becomes equal to the width of the plume or the thickness of the aquifer contributing to the well becomes equal to the thickness of the plume. The former happens at a hydraulic conductivity of $250 \mathrm{~m} / \mathrm{yr}$ in this example. The peak predicted dose 

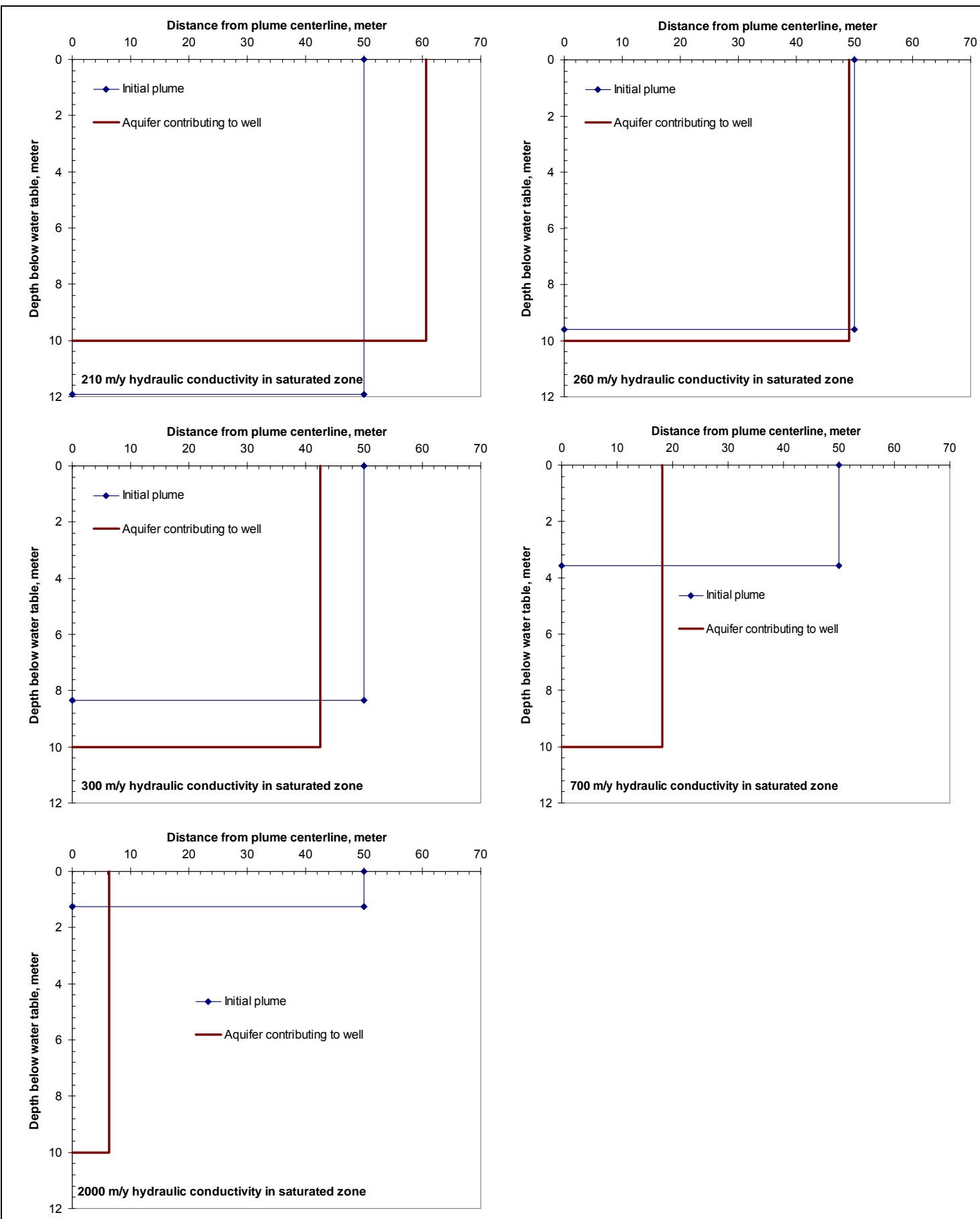

FIGURE C.40 Dimensions of the Initial Plume in the Saturated Zone and the Dimensions of the Aquifer Contributing to the Well for Five Hydraulic Conductivities in the Saturated Zone 
remains constant until the other dimension of the plume and the portion of the aquifer contributing to the well become equal.

The thickness of the plume becomes equal to the well depth at a hydraulic conductivity of $255 \mathrm{~m} / \mathrm{yr}$ in this example. As the hydraulic conductivity increases beyond this value, the thickness of the plume shrinks in comparison to the depth of the well below the water table, while the width of the plume exceeds the width of the aquifer contributing to the well. The peak predicted dose falls as clean water underlying the plume is drawn into the well while part of the plume passes around the well. The change in dilution factor with hydraulic conductivity, computed by ignoring lateral dispersion, in the saturated zone for this example is shown in Figure C.41.

Lateral dispersion spreads the plume laterally, making the concentration profile nonuniform. Information from the output file "PROFILEW.OUT" for five determinist runs with the hydraulic conductivity in the saturated zone set to 210, 260, 300,700, and $2000 \mathrm{~m} / \mathrm{yr}$ were used to plot the horizontal and vertical distributions of ${ }^{226} \mathrm{Ra}$ in the saturated zone at the well in Figures C.42 and C.43. The code computes the dilution factor by multiplying the average factor for lateral horizontal dispersion over the width of the aquifer contributing to the well by the average factor for lateral vertical dispersion over the thickness of the aquifer contributing to the well. The change in dilution factor with hydraulic conductivity is shown in Figure C.41.

When the thickness of the plume is small in comparison to the thickness of the aquifer contributing to the well and the width of the plume is large in comparison to the width of the aquifer contributing to the well, dispersion will not affect the quantity of radionuclides that reach the well. Nor will dispersion affect the quantity of radionuclides that reach the well when the thickness of the plume is large compared with the thickness of the aquifer contributing to the well and the width of the plume is small in comparison to the width of the aquifer contributing to the well. Lateral dispersion influences the peak predicted dose from well-water-related exposure pathways when one or both of the dimensions - the thicknesses or the widths of the plume and the region of aquifer contributing to the well-are of comparable magnitude. This is the case in the range of hydraulic conductivity over which the two curves in Figure C.41 deviate from each other.

From the preceding discussion, the sensitivity of peak predicted dose from well-waterdependent exposure pathways to hydraulic conductivity can be expected to depend on the values specified for many inputs, including the depth and pumping rate of the well, the lateral dispersivities, and all the inputs that affect the quantity of water infiltrating through the primary contamination. This indicates the need for performing multiple-input sensitivity analysis.

Section C.3.4 extends the example sensitivity analysis to include some of the interaction inputs discussed in this section. 


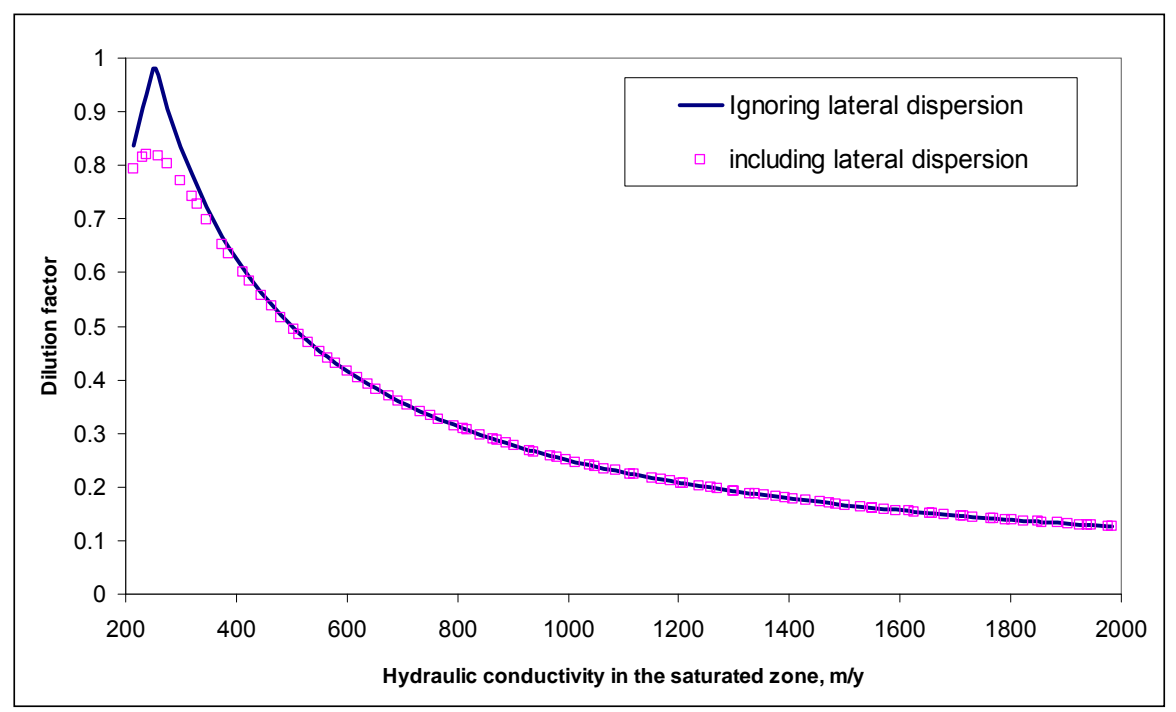

FIGURE C.41 Variation in Dilution Factor with Hydraulic Conductivity in the Saturated Zone
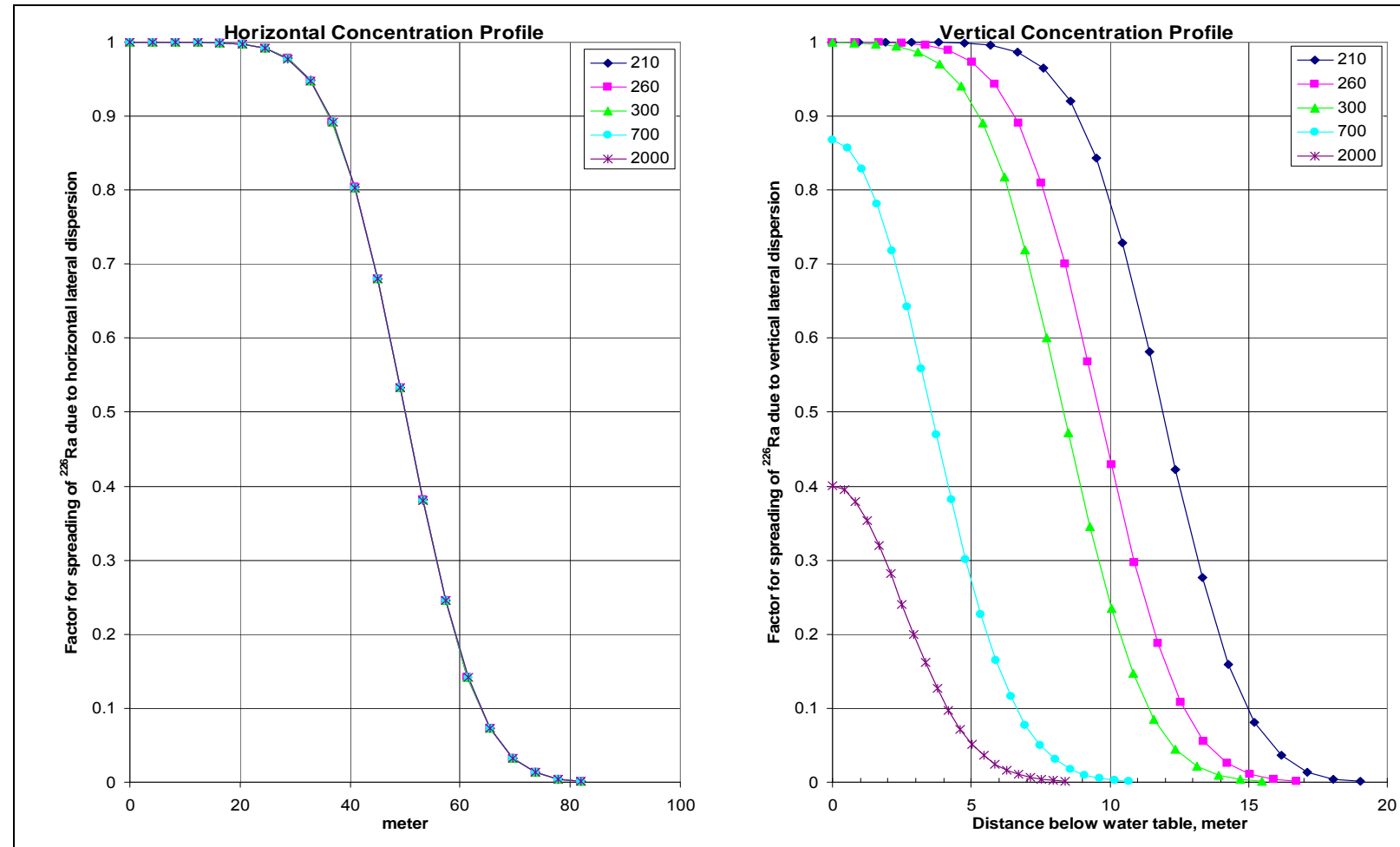

\section{FIGURES C.42 (left) and C.43 (right) Distributions of Radionuclides in the Transverse Plane at the Well}




\section{C.3.4 Relationships between Inputs}

Many of the inputs in the RESRAD-OFFSITE code are not independent. There may be analytical relationships between some variables. In the single-input sensitivity analysis example considered before, the quantity of water intercepted by the surface water body over a year is the product of the hydraulic gradient in the saturated zone, the hydraulic conductivity in the saturated zone, and the width and depth of the aquifer intercepted by the surface water body. The mean residence time of water in the surface water body is the volume of water in the surface water body divided by the quantity of water flowing in and out of the surface water body in a year. If the other inflows into the surface water body are fixed, these two combine to give the relationship between the mean residence time of water in the surface water body and the hydraulic conductivity in the saturated zone. This relationship must be included in the sensitivity analysis in order to accurately assess the sensitivity of peak predicted dose to the hydraulic conductivity. The distributed single-input sensitivity analysis example of Section C.2.2 can be expanded to include this relationship. Any input in the code can be expressed as a function of other (single-valued, distributed, or related) inputs in the "Related inputs" tab of the Uncertainty and Probabilistic Analysis form (Figure C.44). Currently, only the four operations-addition, subtraction, multiplication, and division-can be used to define the relationship. All the inputs to the RESRAD-OFFSITE code are included in the Input Variable dropdown box. This dropdown box and the operation keys are used to key in the relationships between the inputs.

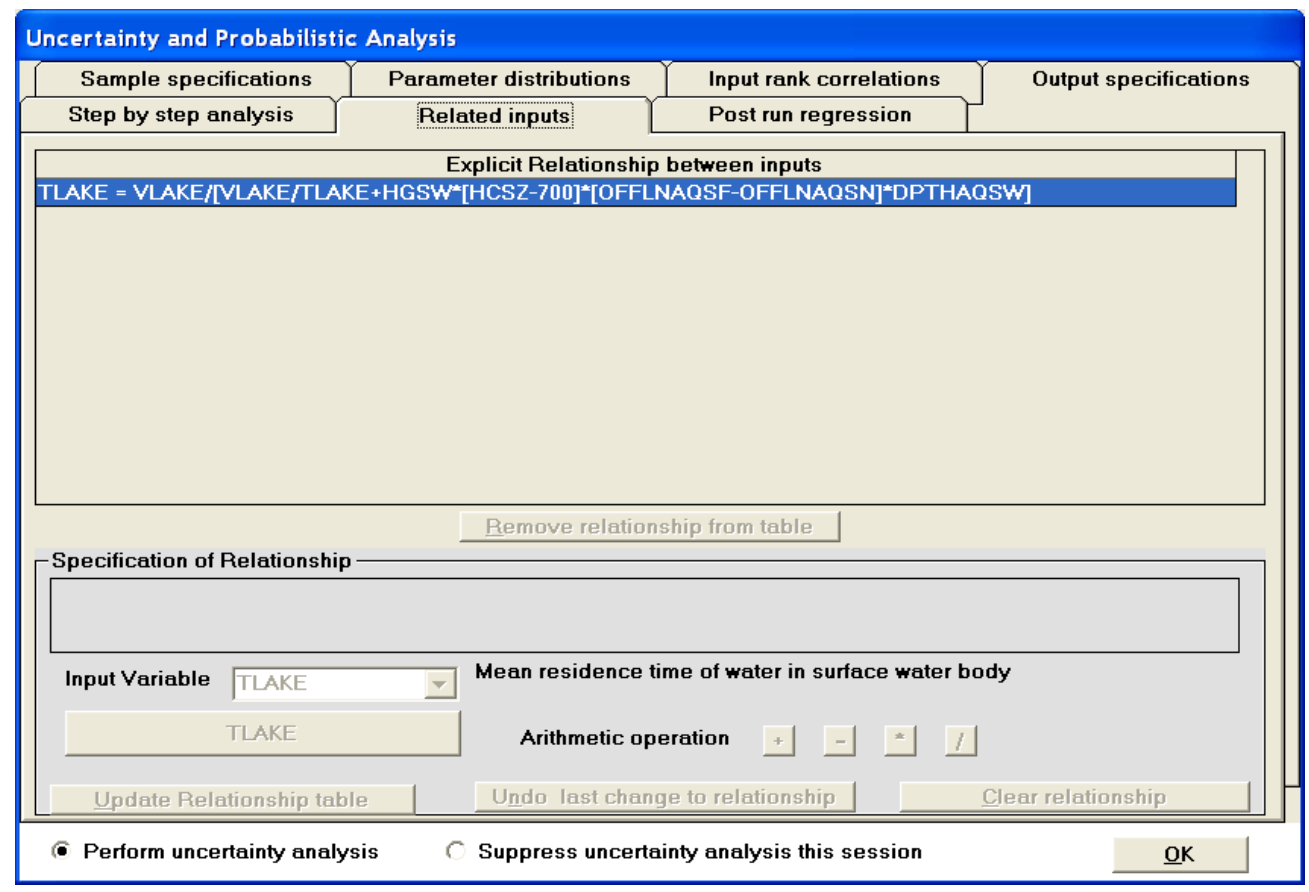

FIGURE C.44 Specifying Relationships between Inputs 


\section{C.3.4.1 Specifying Relationships between Variables}

Relationships can only be specified in "step by step" probabilistic analysis. Distributions must first be specified for all the probabilistic inputs. The input samples for the probabilistic distributions must then be generated in the "Step by step analysis" tab of the Uncertainty and Probabilistic Analysis form. A relationship is specified by first selecting the dependent input from the input variables dropdown box. This places the name of the input variable in the command button below the dropdown box. Clicking that command button transfers the name of the input into box above and adds an equal sign. These steps are illustrated in Figure C.45.

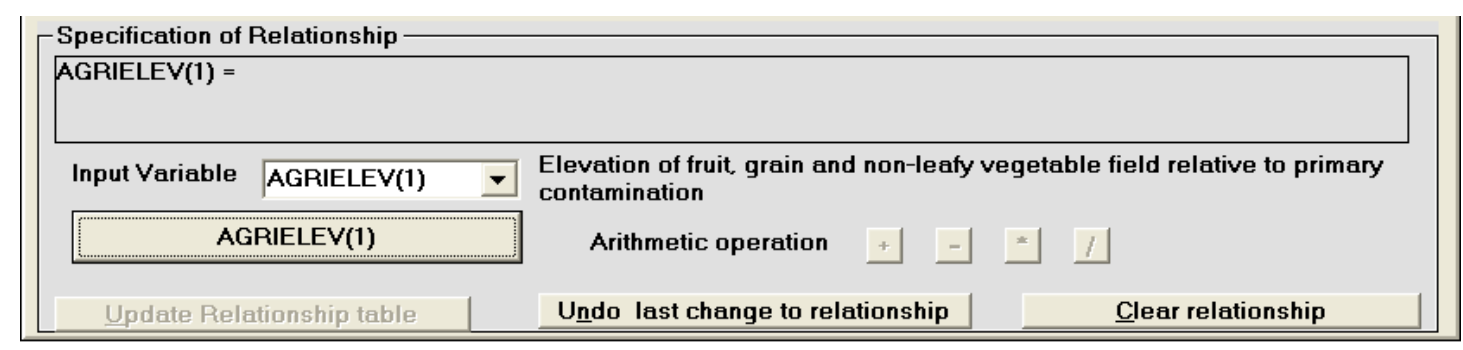

\section{FIGURE C.45 Specifying the Dependent Input of a Relationship}

The right-hand side of the relationship must begin with the name of an input or with a constant. Thus, only the input dropdown box is active and available for selection at this point. The input OFFLNAQF-which is the distance, in the direction perpendicular to the flow, from the center of the contamination to the left edge of the surface water body-is selected in the dropdown box and is transferred into the relationship specification box by clicking the command button as before. The next item that can be added to the specification of the relationship is an arithmetic operator. The buttons,,$+-{ }^{*}$ and /, representing the arithmetic operations of addition, subtraction, multiplication, and division, become active while the input dropdown box becomes inactive at this point (Figure C.46). The expression in the relationship box equating one input to another is the simplest relationship. Therefore, the "Update relationship table" button also becomes active at this point.

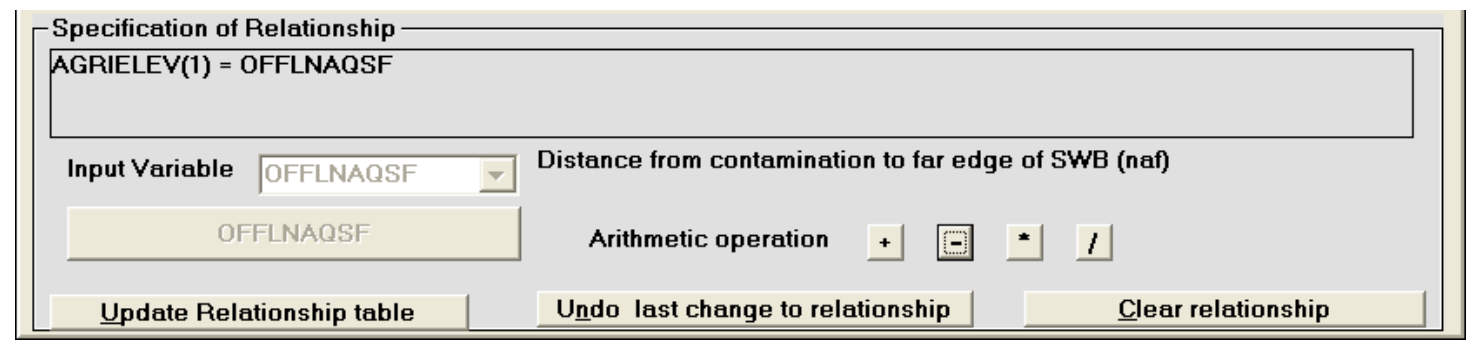

FIGURE C.46 Adding an Independent Input to the Relationship 
The width of the aquifer intercepted by the surface water body is the difference between the distances, in the direction perpendicular to the flow, from the center of the contamination to the left and right edges of the surface water body. The subtraction operator is selected next by clicking the "-" button. This deactivates the arithmetic operations buttons and the "Update relationship table" button, and activates the input dropdown box (Figure C.47).

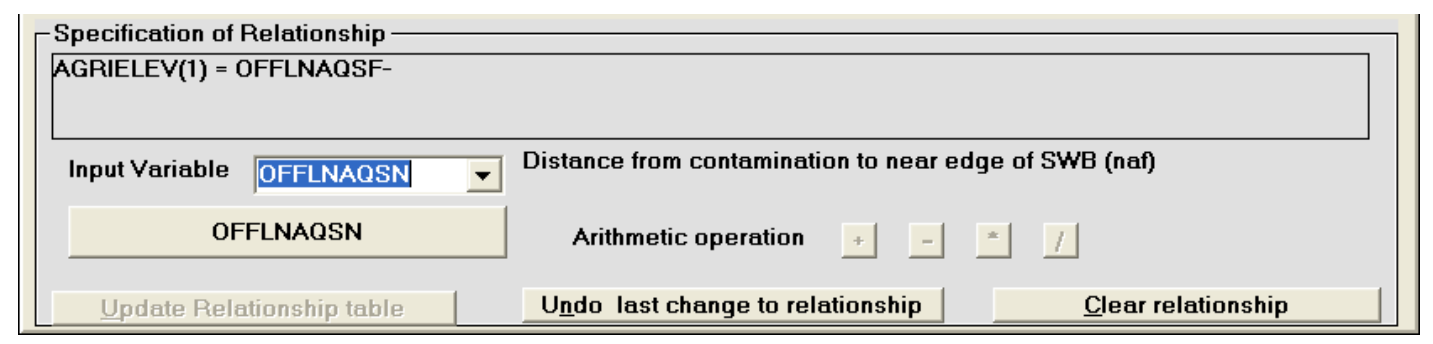

\section{FIGURE C.47 Adding an Arithmetic Operator and Selecting the Second Independent Input to the Relationship}

The input OFFLNAQN-which is the distance, in the direction perpendicular to the flow, from the center of the contamination to the right edge of the surface water body-is selected next and transferred to the expression to complete the desired relationship for the width of the aquifer intercepted by the surface water body (Figure C.48). The width of the aquifer intercepted by the surface water body is not an input in the RESRAD-OFFSITE code. It is therefore not available in the dropdown box. That is why AGRIELEV(1) was chosen as a placeholder variable. This expression is accepted and transferred to the "Relationship table" (Figure C.49) by clicking the "Update relationship table" button (Figure C.48). As seen in Figure C.44, this relationship will be removed from the relationship table after it is used to define the relationship for mean residence time of water in the surface water body.

The additional quantity of water that is intercepted by the surface water body when the hydraulic conductivity is changed from its single deterministic value of $700 \mathrm{~m} / \mathrm{yr}$ to a value sampled from the specified distribution is proportional to the difference between the sampled value and 700 . Any numerical constant, in this case 700 , can be typed into the input dropdown box, as illustrated in Figure C.49. The difference between the sampled value of the hydraulic conductivity in the saturated zone and the deterministic value of 700 is temporarily stored in AGRIELEV(2). This placeholder variable will also be removed from the relationship table. The rate at which water enters the surface water body in the deterministic case is equal to the deterministic volume divided by the deterministic mean residence time.

The rate at which water enters the surface water body when the hydraulic conductivity is different from the deterministic value is the sum of the value in the deterministic case and the product of the hydraulic gradient, the difference in hydraulic conductivity (temporarily stored in 


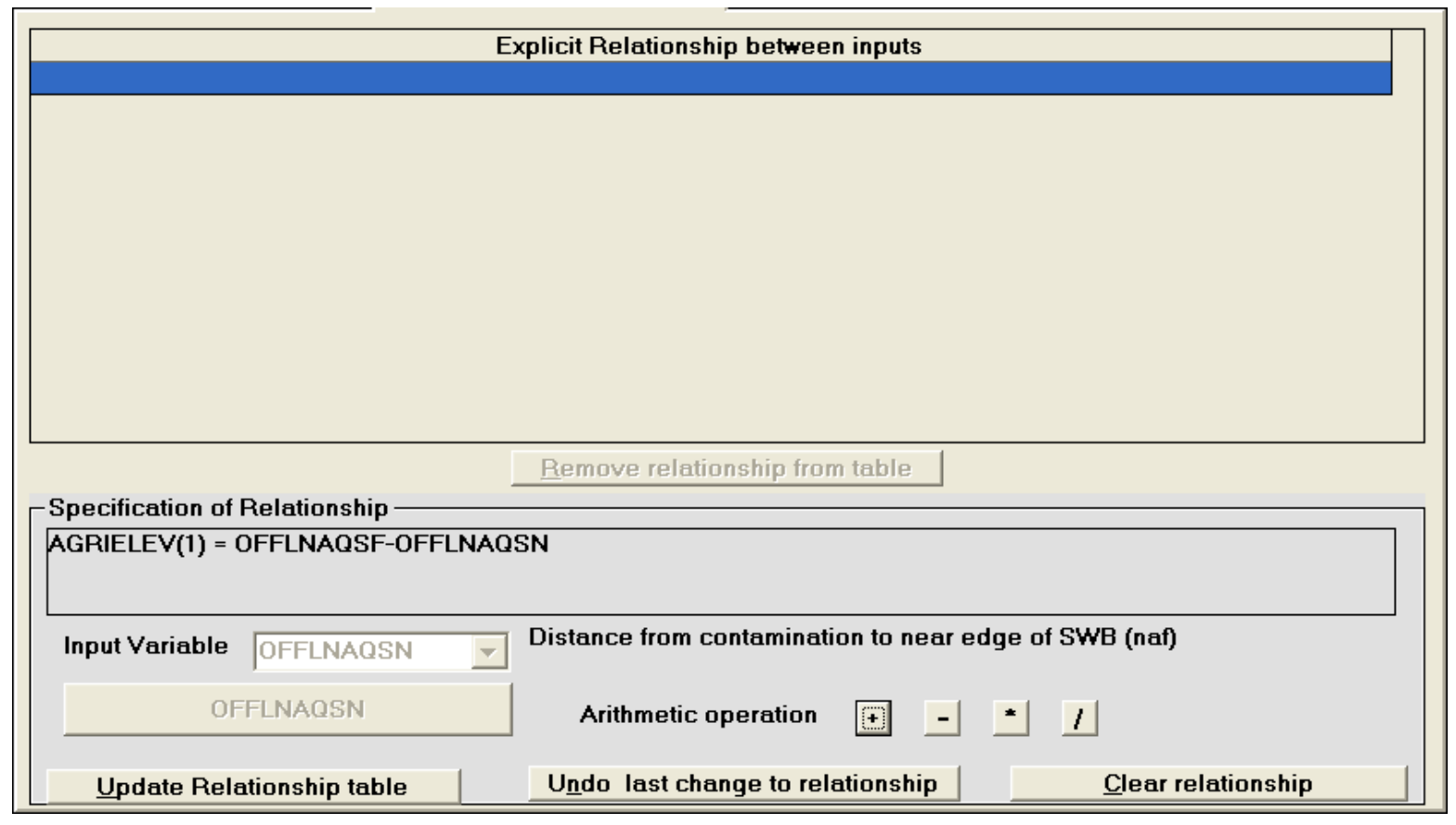

FIGURE C.48 Completing the Specification of a Relationship and Transferring It to the Relationship Table

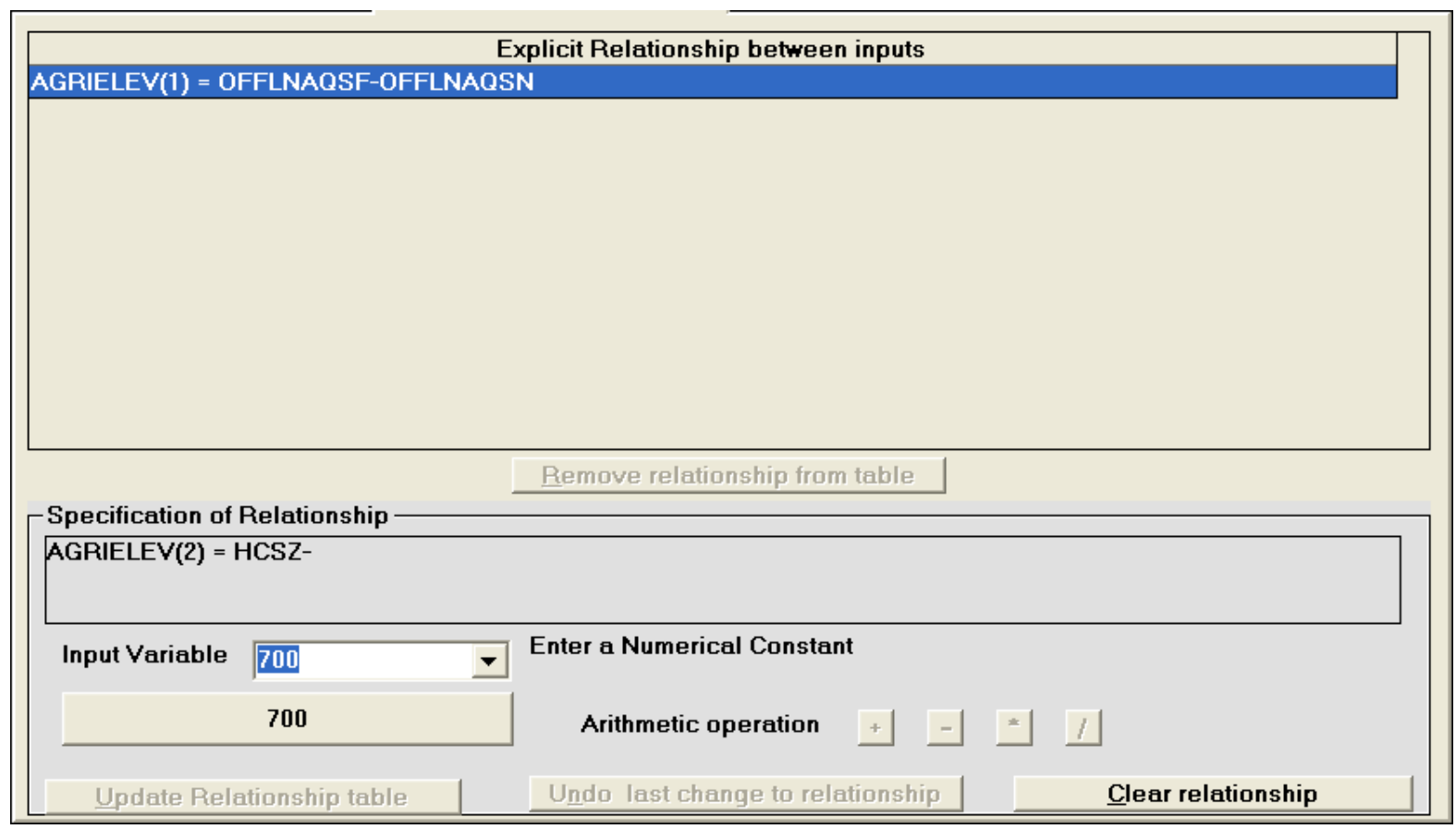

FIGURE C.49 Inputting Numerical Constants in a Relationship 
AGRIELEV(2)), the width of aquifer intercepted by the surface water body (temporarily stored in AGRIELEV(1)), and the depth of aquifer intercepted by the surface water body. This is temporarily stored in AGRIELEV(3).

When an input is selected in the dropdown box, the code first checks whether a relationship has been specified for that input in the Relationship table. If it finds a relationship in the table, it will copy in that relationship into the relationship that is currently being created (Figure C.50). When the placeholder AGRIELEV(2) is selected in the dropdown box and transferred to the current relationship, HCSZ -700, which is the relationship defined for AGRIELEV(2) in the relationship table, is written in the current relationship that is being created for AGRIELEV(3). Likewise, when AGRIELEV(1) is selected in the dropdown box and transferred to the relationship, OFFLNAQF-OFFLNAQN, which is the relationship defined for AGRIELEV(1) in the relationship table, is written in the current relationship being created for AGRIELEV(3).

The relationship for the mean residence time of water can now be specified using the placeholder input AGRIELEV(3). Notice in Figure C.51 that when a previously defined related input is used in the specification of a related input, the expression for the previously defined input is enclosed in square braces in the specification of the current related input. Such use of placeholder inputs facilitates the creation of more complex relationships than would otherwise be possible with just the four arithmetic operators. A more direct way to create these complex relationships will be available in future versions of the code. The placeholder inputs, AGRIELEV(1), AGRIELEV(2), and AGRIELEV(3), are removed from the relationship table once the desired relationship for the mean residence time of water in the lake is transferred to the relationship table (Figure C.44).

The code computes the values for the related inputs as they are being specified. Consider, for example, the placeholder input AGRIELEV(2) (Figures C.49 and C.50). A number of memory spaces, equal to the number of simulations (i.e., observations $\times$ repetitions) are set aside for AGRIELEV(2) when it is defined as a dependent input. When the specification of this placeholder reaches the stage shown in Figure C.49, the value sampled for the hydraulic conductivity in the saturated zone for each simulation is placed in the memory set aside for this placeholder input. When the relationship AGRIELEV(2) $=\mathrm{HCSZ}-700$ is updated into the relationship table, 700 is subtracted from the quantities in those memory spaces. When the situation shown in Figure C.44 is reached there will be 100 values of mean residence time of water in the surface water body in the memory space set aside for TLAKE. These will be related to the probabilistic values sampled for the hydraulic conductivity in that simulation and to the deterministic values of the volume of the lake, the hydraulic gradient in the saturated zone from the contamination to the surface water body, and the width and depth of the aquifer intercepted by the surface water body. Figure C.52 plots against hydraulic conductivity the values computed for the mean residence time of water in the surface water body using the relationship. 


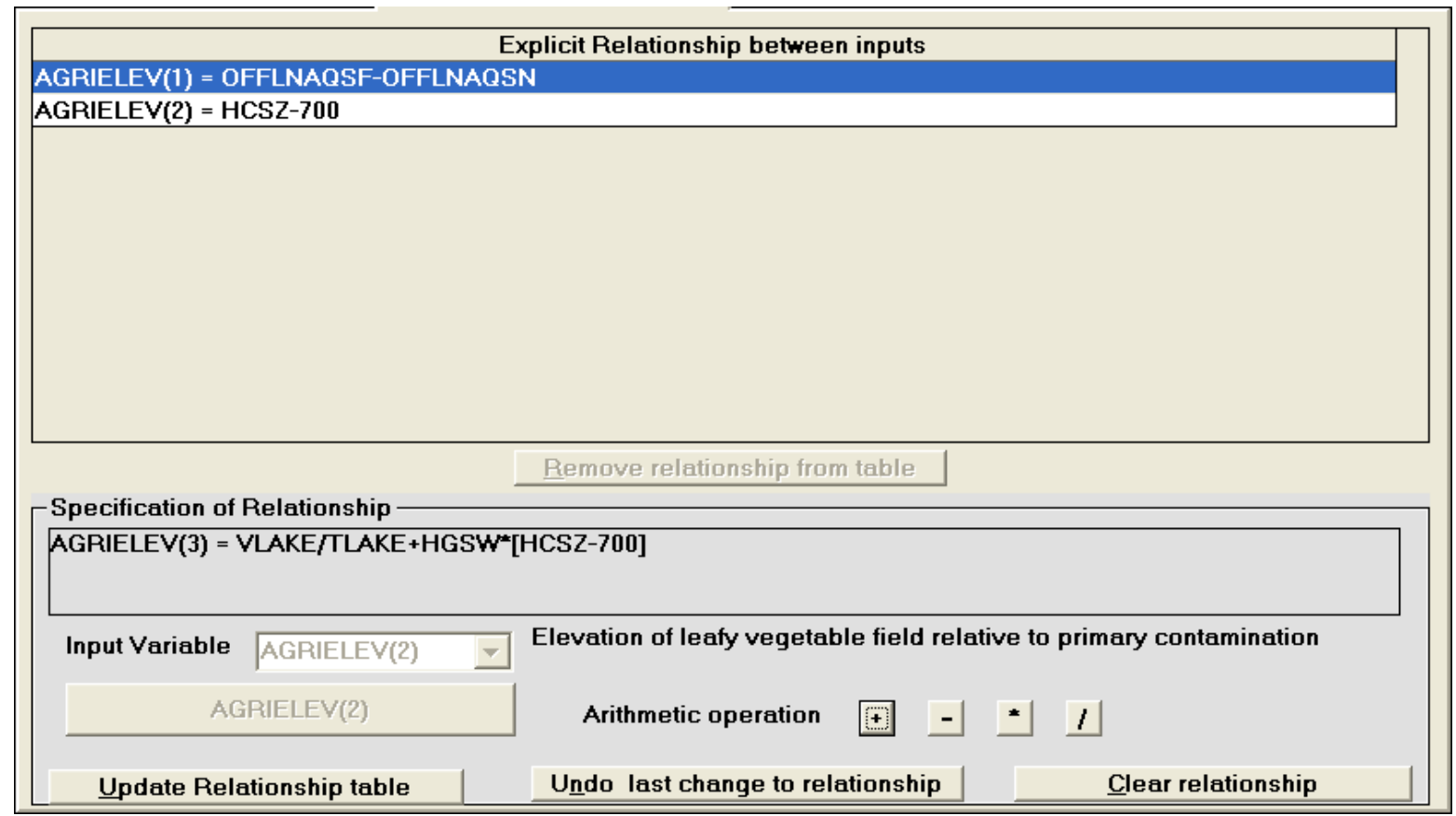

FIGURE C.50 Using a Related Input in the Relationship of Another Input

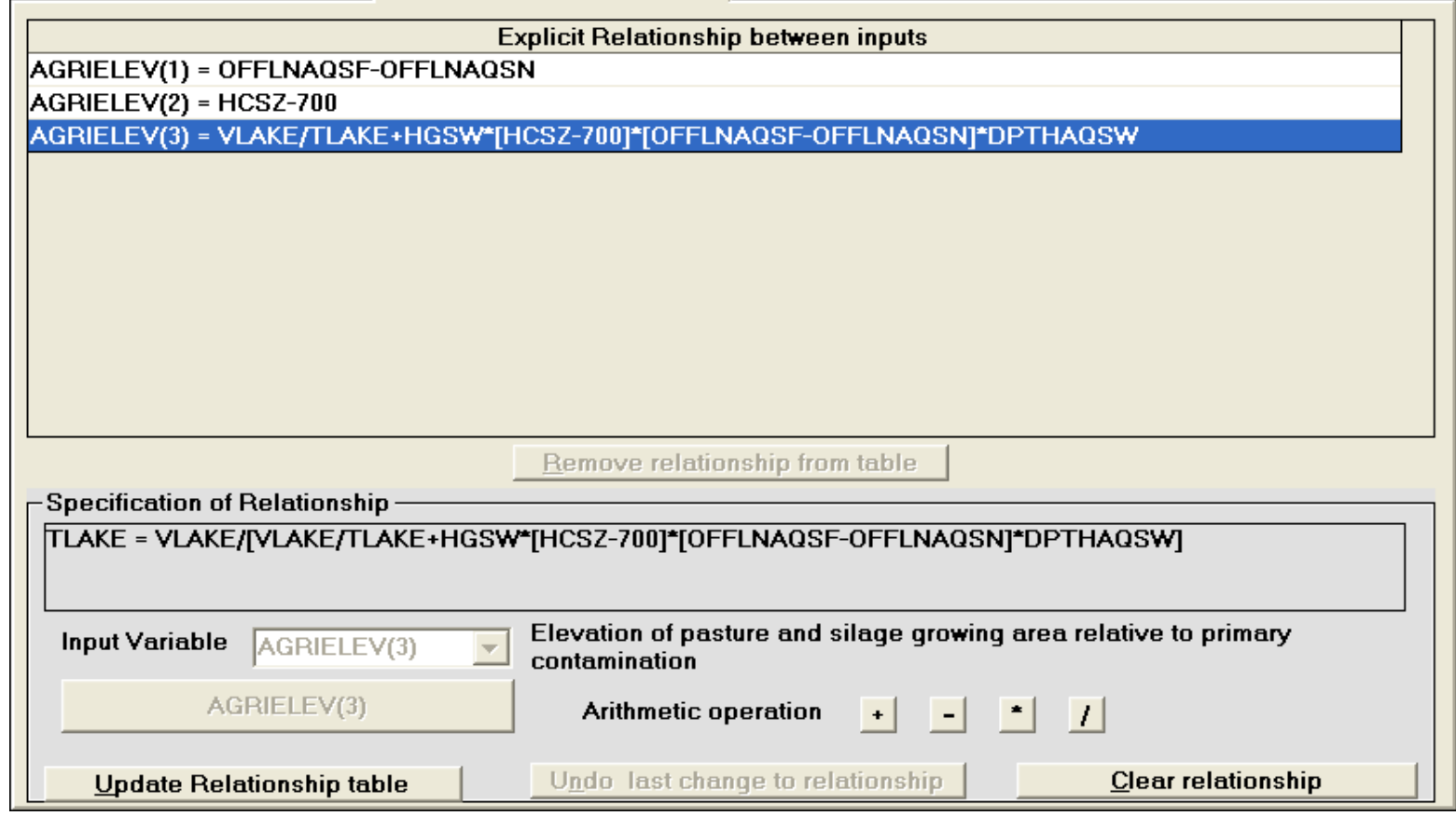

FIGURE C.51 Indirect Introduction of [ ] Using Placeholder Inputs, Enabling Creation of Complex Relationships 


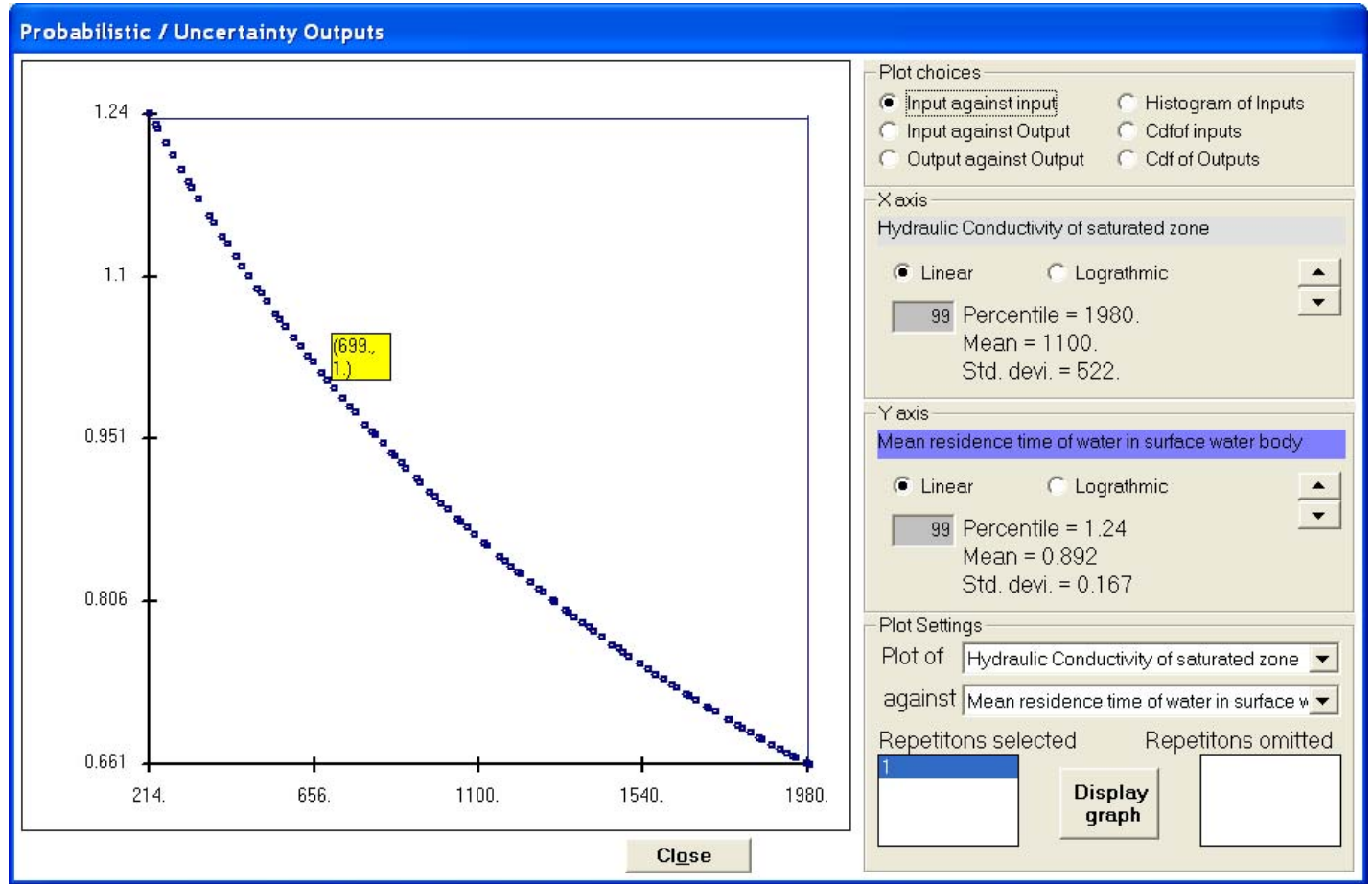

FIGURE C.52 Relationship between Mean Residence Time of Water in the Surface Water Body and the Hydraulic Conductivity in the Saturated Zone

\section{C.3.4.2 Influence of the Relationship on the Peak Predicted Dose}

The computational code is launched from the "Step by step analysis" tab after all the relationships have been specified. The computational code then uses the values computed for the related inputs, the values sampled for the probabilistic inputs, and the deterministic values for the remaining inputs to run the simulations and generates a set of peak predicted doses and peak predicted risks. Figure C.53 shows the variation of peak predicted dose from the ingestion of fish with hydraulic conductivity in the saturated zone, when the relationship between the mean residence time of water in the surface water body and the hydraulic conductivity is specified: RESRAD-OFFSITE models the effects of the larger volume of water that passes through the surface water body as the hydraulic conductivity increases.

There are three major considerations that affect the peak predicted dose from the ingestion of fish as the hydraulic conductivity increases.

1. The faster travel rate reduces the transport time, decreasing the loss from radiological transformations and causing the peak predicted dose from the ingestion of fish to increase with increasing hydraulic conductivity. 


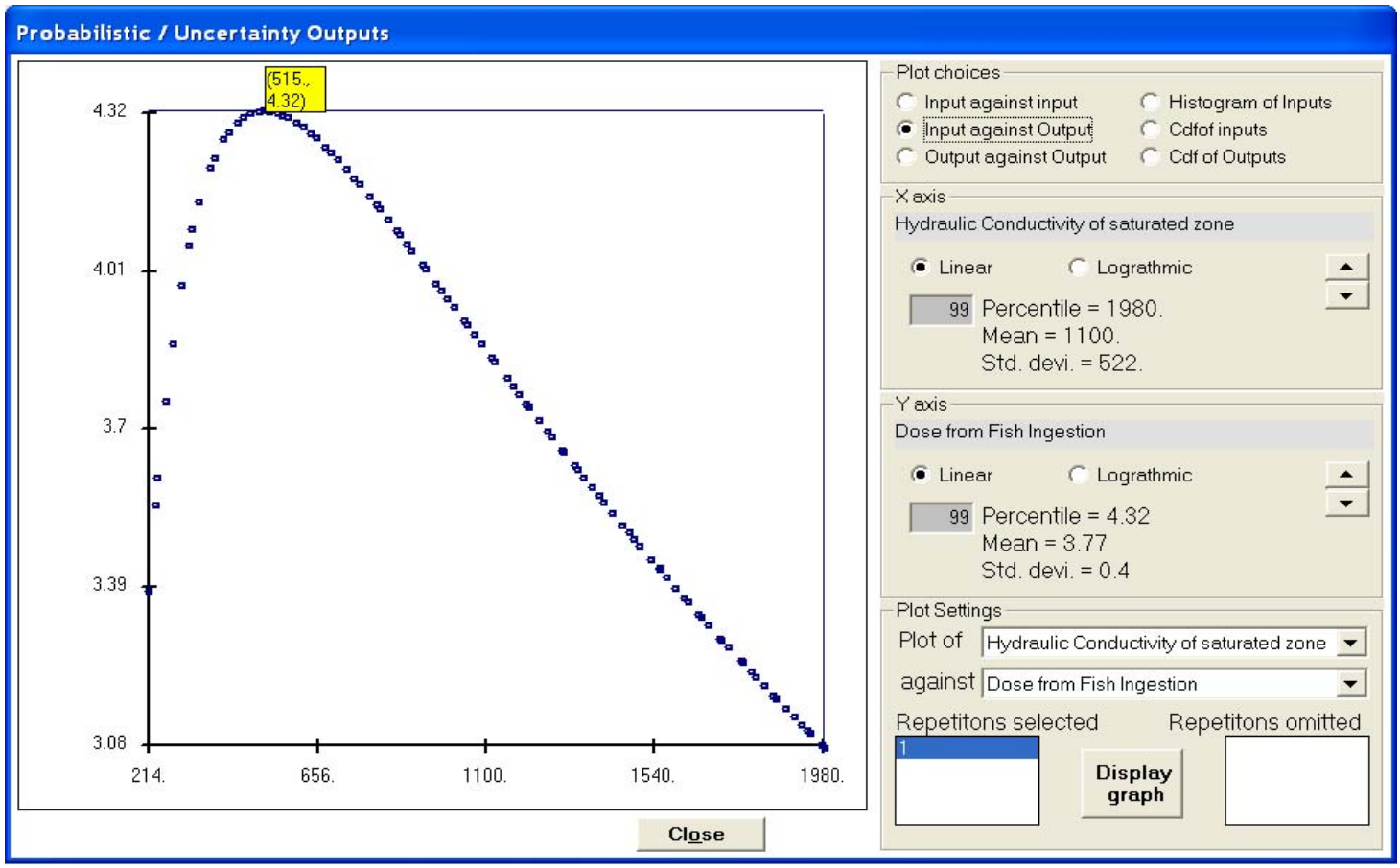

\section{FIGURE C.53 Variation with Hydraulic Conductivity of Peak Predicted Dose from Ingestion of Fish When a Relationship Is Specified between Hydraulic Conductivity and Mean Residence Time of Water in the Surface Water Body}

2. More of the plume passes through the depth of aquifer that is intercepted by the surface water body as the hydraulic conductivity increases, causing the peak predicted dose from the ingestion of fish to increase.

3. The increase in hydraulic conductivity also increases the amount of water that passes through the surface water body in a year, which tends to dilute the radionuclides in the surface water body and to flush them out more quickly, reducing accumulation. As a result, the peak predicted dose from ingestion of fish tends to decrease with increasing hydraulic conductivity.

Initially, the first two processes dominate and the peak predicted dose from the ingestion of fish increases with hydraulic conductivity. As the hydraulic conductivity continues to rise, the third process becomes more important and at some point negates the increase from the first two processes. The peak predicted dose from the ingestion of fish then falls with increasing hydraulic conductivity. Figure C.18 shows the corresponding plot when this relationship was not specified. The third process was not modeled, leading to an increase in peak predicted dose with increasing hydraulic conductivity when the relationship was not specified (Sections C.3.1 and C.3.2). 
Comparing the two figures shows that when the relationship is considered, the peak predicted dose from the ingestion of fish is higher when the hydraulic conductivity is less than the deterministic value, and lower when the hydraulic conductivity is higher than the deterministic value.

Figure C.54 shows the variation of peak predicted dose from all exposure pathways with hydraulic conductivity in the saturated zone when the relationship between hydraulic conductivity and mean residence time of water in the surface water body is specified.

(Figure C.12 shows the corresponding plot when this relationship is not specified.) The fish ingestion pathway makes a noticeable contribution to the peak predicted dose at hydraulic conductivities greater than about $750 \mathrm{~m} / \mathrm{yr}$ in this example for the reasons discussed in Section C.2.2.2. As seen in the preceding paragraphs, this is also the range of hydraulic conductivity in which the doses in Figure C.53 are lower than the doses in Figure C.18. Recall that Figures $\mathrm{C} .53$ and $\mathrm{C} .18$ show the variation of peak predicted dose from the ingestion of fish with hydraulic conductivity with and without consideration of the relationship between the mean residence time of water in the surface water body and hydraulic conductivity, respectively. Thus, Figures C.54 and C.12 differ only at the higher values of hydraulic conductivity, and the curve in Figure C.54 is lower than that in Figure C.12 in that region.

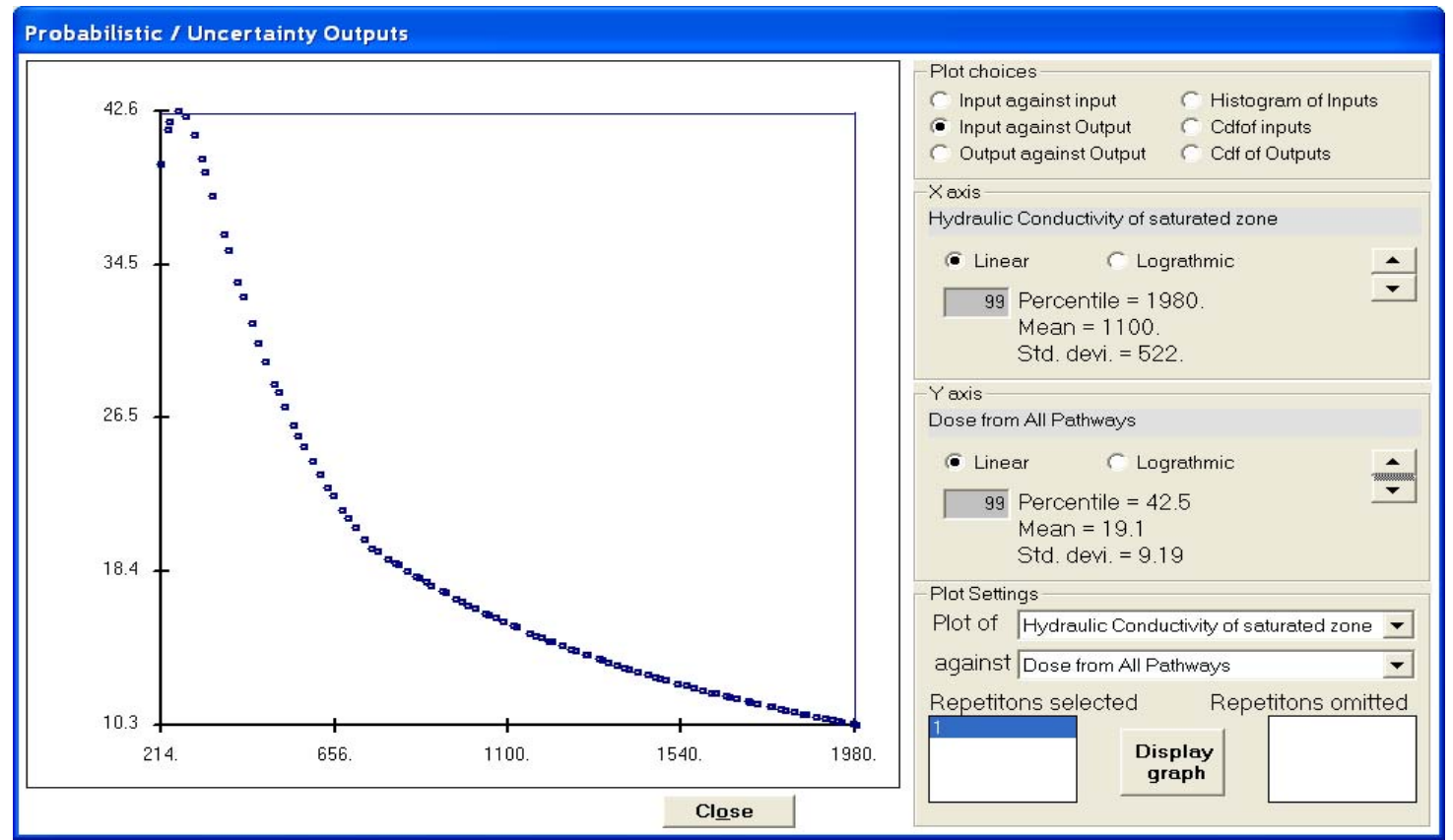

FIGURE C.54 Variation with Hydraulic Conductivity of Peak Predicted Dose from All Pathways When a Relationship Is Specified between Hydraulic Conductivity and Mean Residence Time of Water in the Surface Water Body 


\section{C.3.5 Simultaneous Sensitivity Analysis on Two or More Inputs}

Sections C.3.2 and C.3.3 conclude pointing out the need for performing multi-input sensitivity analysis. This section expands the distributed single-input sensitivity analysis example to illustrate how those can be performed and what outputs are available in RESRAD-OFFITE to determine the sensitivities of peak predicted dose to the different inputs of interest. It progresses gradually to more complex cases to show the difficulties with graphical output, and the need to rely on multivariable regression analysis.

\section{C.3.5.1 Sensitivity Analysis on Two Inputs Using Continuous and Discrete Uniform Distributions}

The discussion in Section C.3.3 suggests that the sensitivity of peak predicted dose to hydraulic conductivity in the saturated zone will depend on the values used for the inputs describing the well: the well depth and the well pumping rate. This section considers simultaneous sensitivity analyses on two inputs; simultaneous sensitivity analyses on three inputs will dealt with in a later section. The example considered in Section C.3.4 is expanded by including a discrete distribution for the depth of the aquifer contributing to the well.

Begin with the input file used for the distributed single-input sensitivity analysis. Save the file under a different name (e.g., "Two Inputs One Discrete.ROF"). Go to the Saturated Zone Hydrology form and place the cursor on the input box for depth of aquifer contributing to the well.

Press the Shift-F8 function key. The "Parameter distributions" tab of the Uncertainty and Probabilistic Analysis form pops up, as shown in Figure C.55.

Click on the Distribution dropdown box and select "Discrete Cumulative." Use six entries to sample depths of $5,6,7,8,9$, and $10 \mathrm{~m}$ with equal probability, as in Figure C.55; click the "Update parameter stats and distribution" button to save this distribution. The reason for using a discrete distribution instead of a continuous distribution will become apparent in Section C.3.5.4, where a continuous distribution is used for the depth of the aquifer contributing to the well. The number of observations needs to be increased in order to see the variation of peak predicted dose with hydraulic conductivity, now that sensitivity analysis is being performed on two inputs. 


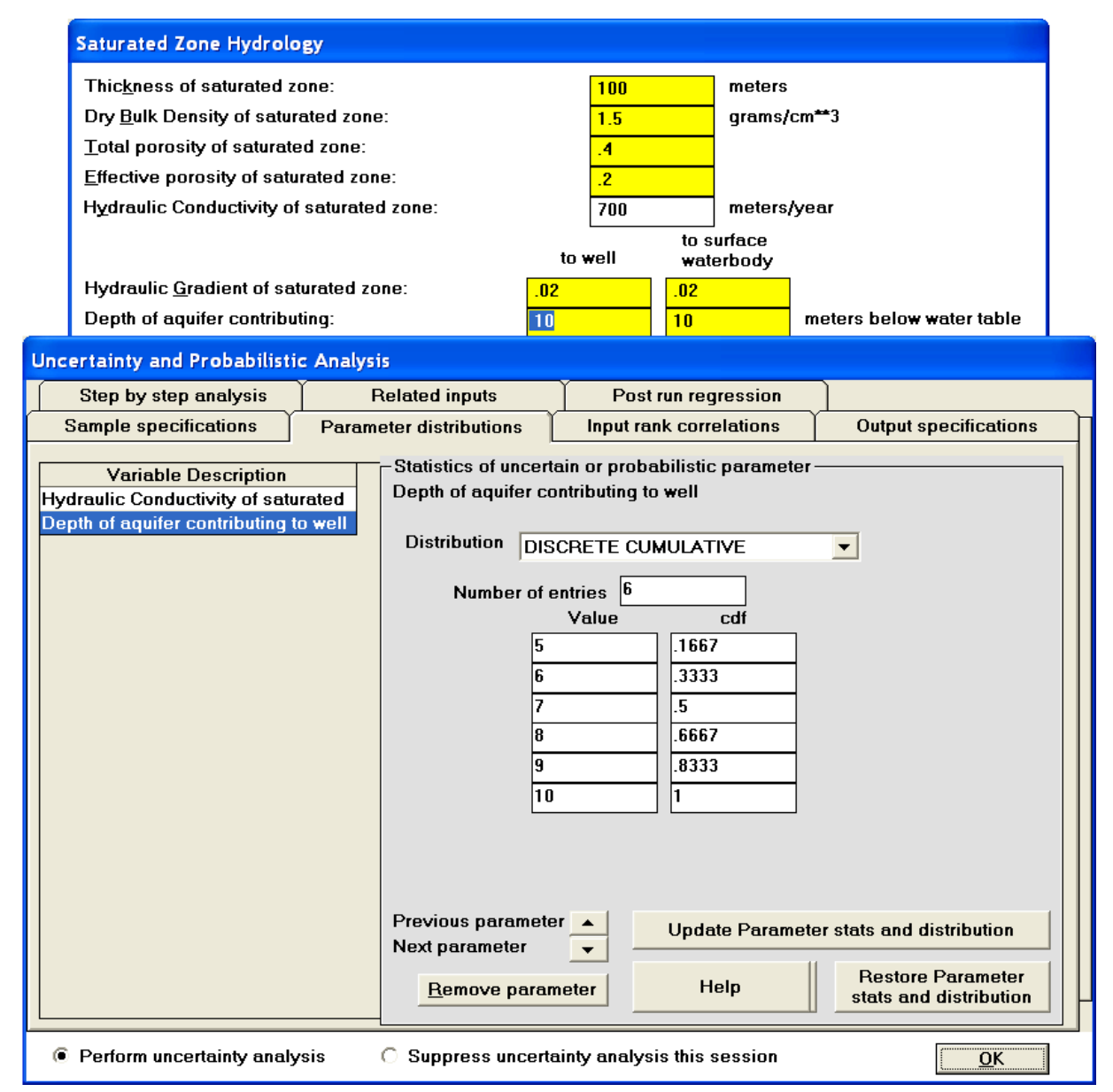

FIGURE C.55 Specifying a Discrete Distribution on an Input

Go to the "Sample specification" tab of the Uncertainty and Probabilistic Analysis form. Increase the number of observations to 600 . Click on the "Generate input samples" button on the "Step by step analysis" tab. The code samples 600 values uniformly in the interval 200 to 2000 for hydraulic conductivity in the saturated zone. It also samples 600 values from the equal probability discrete distribution of $5,6,7,8,9$, and 10 for the depth of aquifer contributing to the well-100 samples at each of the six values. It then combines the samples for the two inputs to make 600 pairs of values for hydraulic conductivity and depth of aquifer contributing to the well. The 600 pairs of values can be viewed by clicking the "View scatter plot of input vs. input" command button in the "Step by step analysis" tab. The resulting screen is shown in Figure C.56. Specify the relationship between the mean residence time of water in the surface water body and the hydraulic conductivity in the saturated zone as in Section C.3.4.1. Click on the "Generate output samples (RESRAD-O)" command button to launch the computational code. 


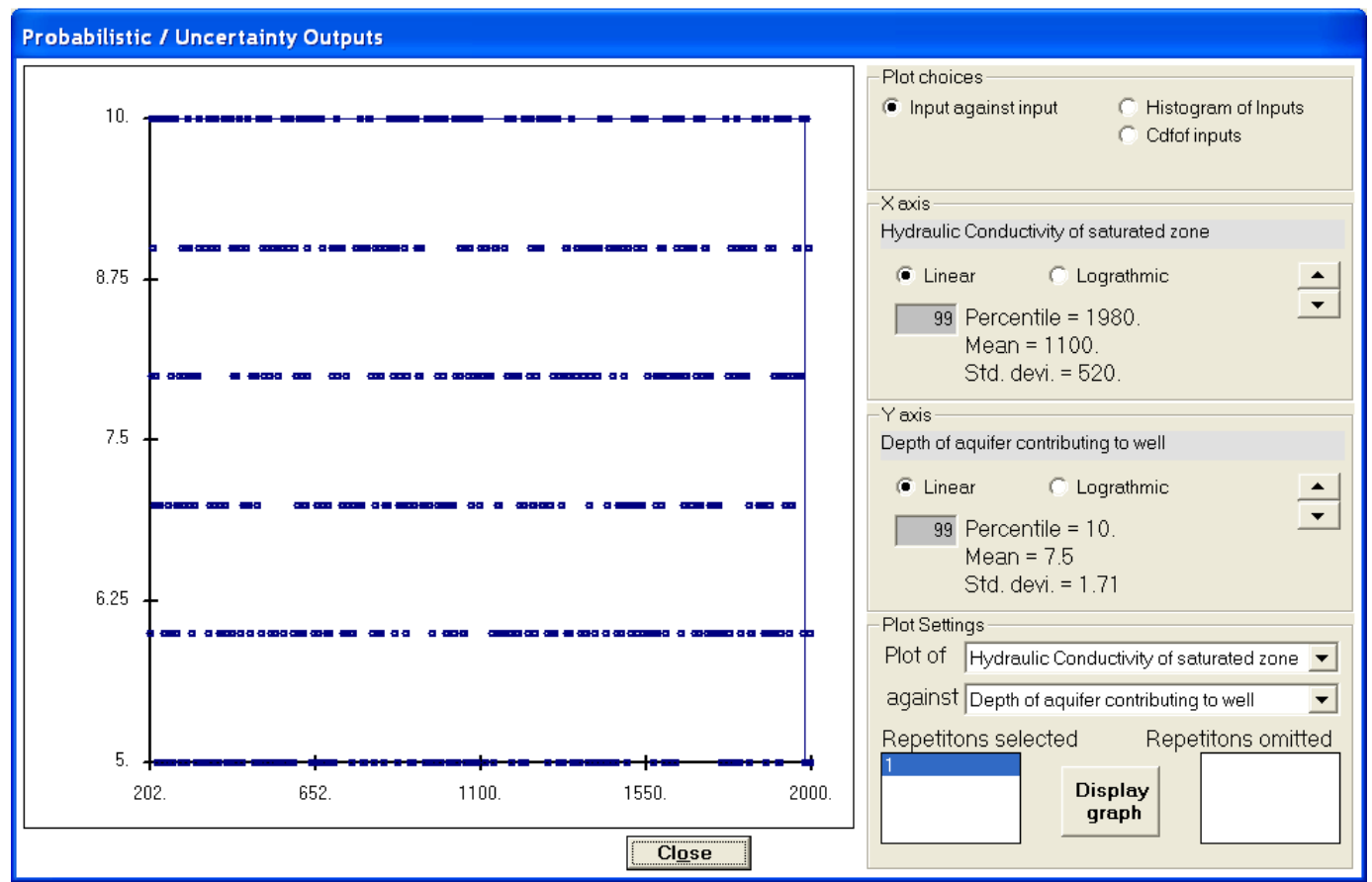

\section{FIGURE C.56 Pairing of Samples in a Two-input Probabilistic Analysis Where One Input Has a Continuous Uniform Distribution and the Other a Discrete Uniform Distribution}

The variation of peak predicted dose from all exposure pathways with hydraulic conductivity can be visualized in the scatter plot of the two. Click the "View scatter plots of output vs. input" button. This displays the Probabilistic/Uncertainty Outputs form. Use the up/down arrows in the "X-axis" frame to change the variable to "Hydraulic conductivity of saturated zone." This displays the plot shown in Figure C.57. The six "curves" correspond to the six discrete depths that were sampled to determine the extent of the aquifer contributing to the well. It is difficult to distinguish the curves in the low range of hydraulic conductivity. This is the region where the peak predicted dose is most sensitive to hydraulic conductivity-some of the curves are rising and others are falling, making it difficult to trace the trend in this region. One way to clarify the plot is to make the code sample more points in this region. 


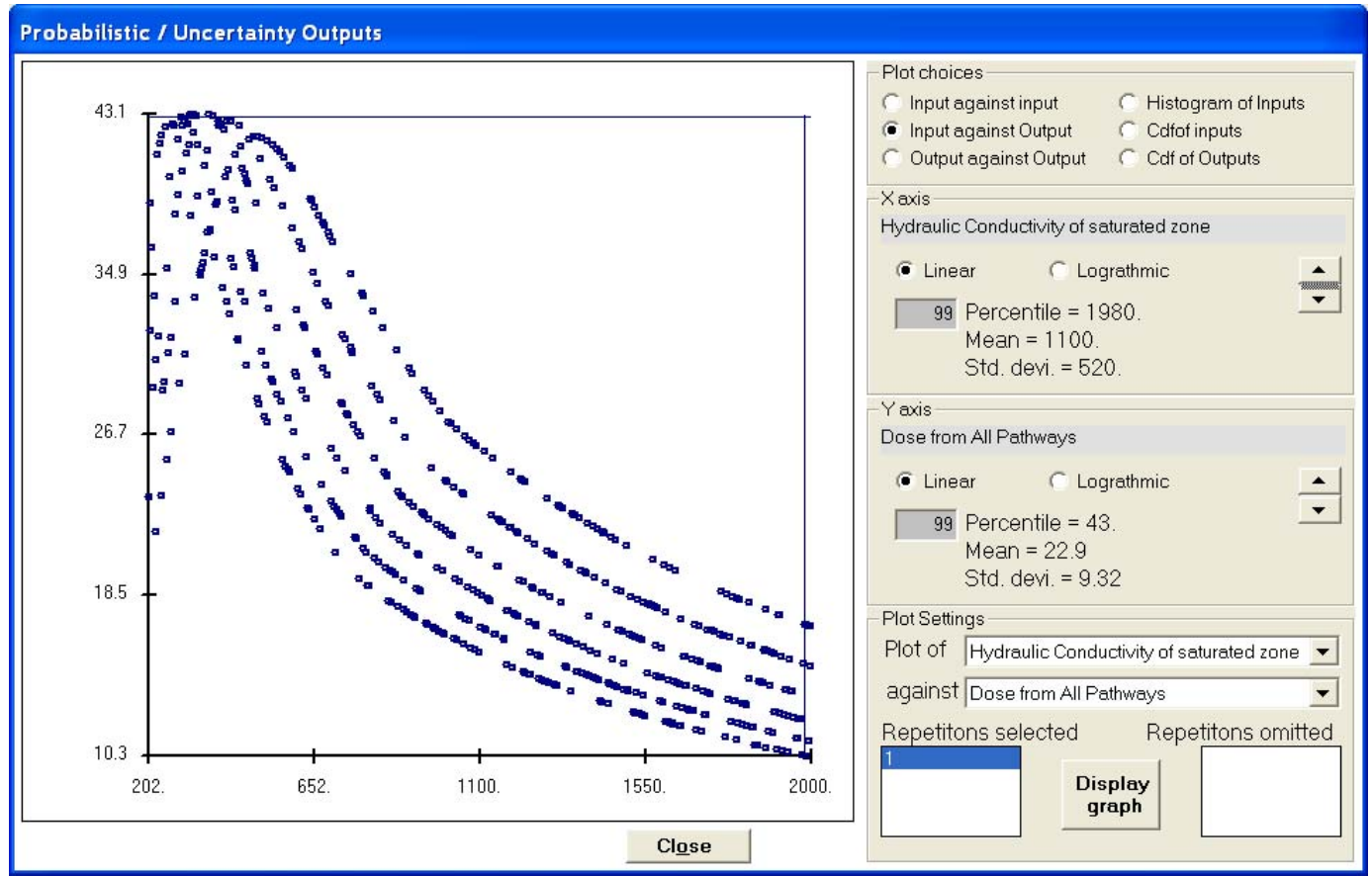

FIGURE C.57 Variation of Peak Predicted Dose with Hydraulic Conductivity at Different Discrete Values of Depth of Aquifer Contributing to the Well

\section{C.3.5.2 Sensitivity Analysis on Two Inputs Using a Continuous Linear Distribution and a Discrete Uniform Distribution}

The values sampled by the code are spread evenly over the range when a uniform distribution is specified for hydraulic conductivity (Figure C.56). The peak predicted dose changes rapidly in the range of hydraulic conductivity 200 to 700 in Figure C.57. More values need to be sampled from this range to show the response of peak predicted dose in this region. This can be achieved by specifying a continuous linear distribution, as shown in Figure C.58. The code will obtain $60 \%$ of the samples for hydraulic conductivity from the range 200 to $700 \mathrm{~m} / \mathrm{yr}$ and the remaining $40 \%$ of the samples from the range 700 to $2000 \mathrm{~m} / \mathrm{yr}$. Save the file under a different name (e.g., "Two Inputs One Discrete Refined.ROF") and generate input samples. The resulting pairs of probabilistic inputs-hydraulic conductivity in the saturated zone and the depth of aquifer contributing to the well-are shown in Figure C.59. The points are very closely spaced in the 200 to $700 \mathrm{~m} / \mathrm{yr}$ range of hydraulic conductivity and are sparsely spaced in the range 700 to $2000 \mathrm{~m} / \mathrm{yr}$. Specify the relationship between the mean residence time of water in the surface water body and the hydraulic conductivity in the saturated zone as in Section C.3.4.1. Click on the "Generate output samples (RESRAD-O)" button to launch the computational code. 


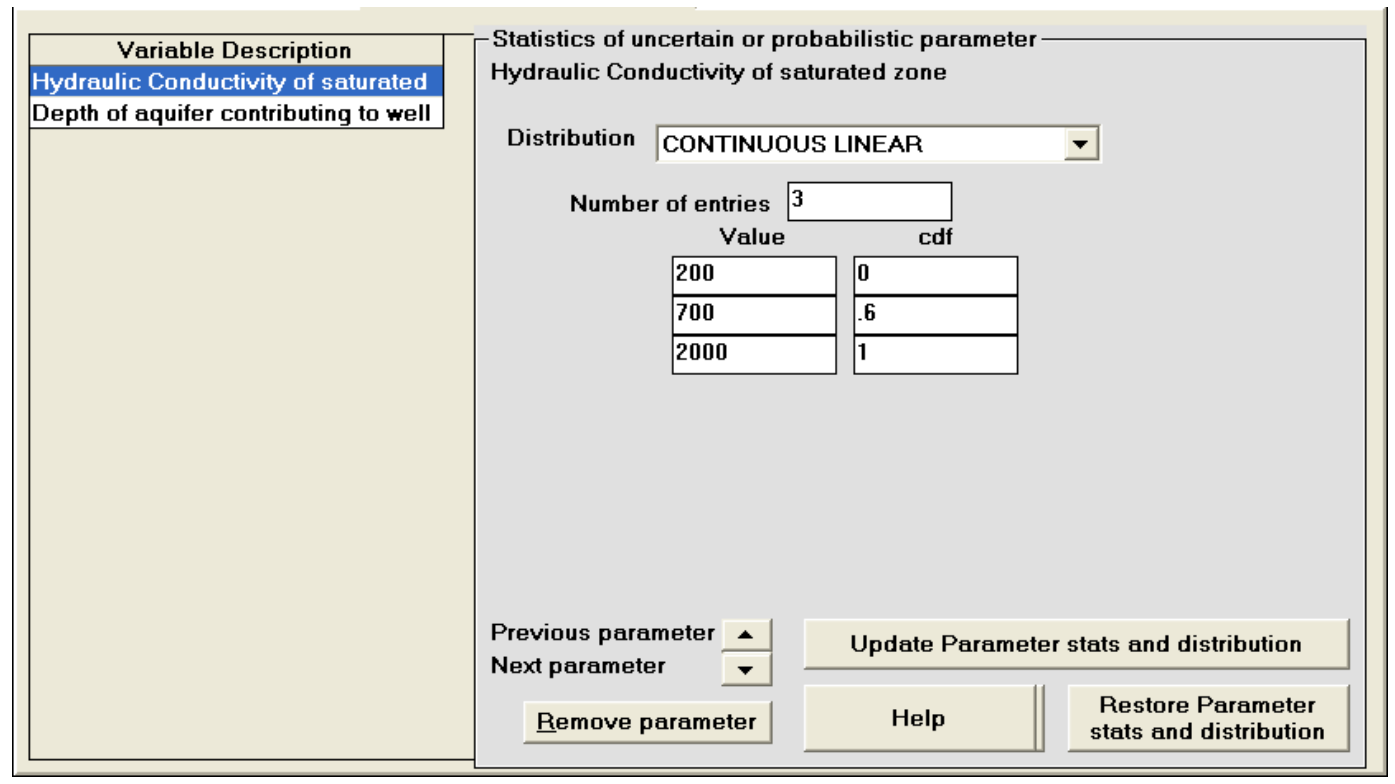

\section{FIGURE C.58 Specifying a Continuous Linear Distribution}

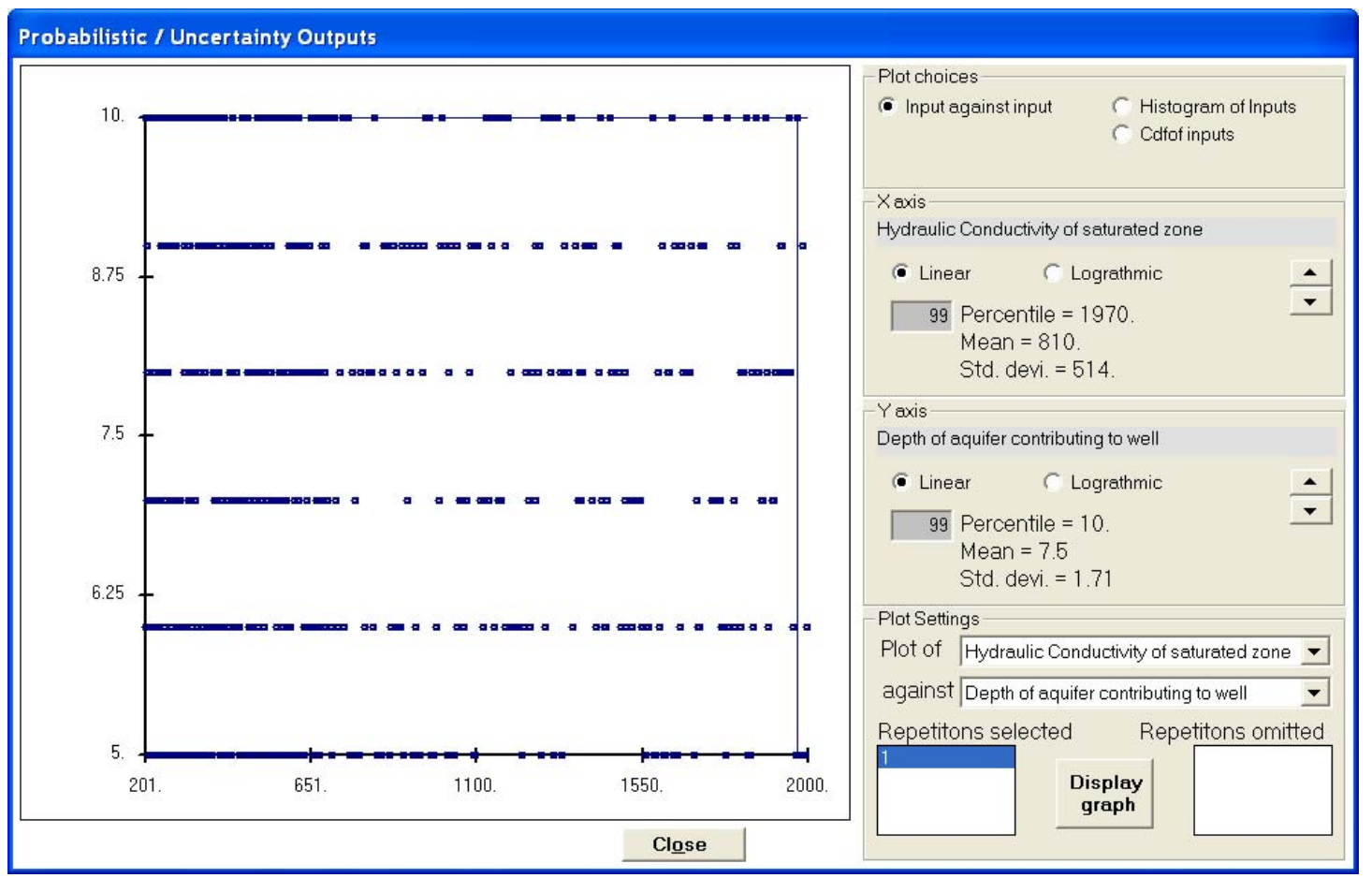

FIGURE C.59 Samples from a Continuous Linear Distribution Showing a Greater Concentration of Samples from One Part of the Range and Sparse Sampling over the Rest of the Range 
The variation of peak predicted dose from all exposure pathways with hydraulic conductivity can now be better visualized in the scatter plot (Figure C.60). Six "curves" correspond to the six discrete depths that were sampled to determine the extent of the aquifer contributing to the well. They have the same tendency: the peak predicted dose increases with hydraulic conductivity, reaches a maximum, and then decreases first rapidly and then more gradually with increasing hydraulic conductivity. The data for these plots are in the peak predicted dose data file (with extension .pds) and the probabilistic input sample data file (with extension .pin). These can be opened in a spreadsheet or transferred into a plotting program.

Figure C.61 focuses on the region where the peak predicted dose changes rapidly. The sensitivity of peak predicted dose to hydraulic conductivity is highly dependent on the depth of aquifer contributing to the well in this range of hydraulic conductivity. This clearly illustrates the need for multi-input sensitivity analysis. A three-point single-input sensitivity analysis in the hydraulic conductivity range of 200 to $600 \mathrm{~m} / \mathrm{yr}$ would have shown an increasing trend if performed with a 5-m depth of aquifer contributing to the well. It would have shown a decreasing trend when performed with a 10-m depth of aquifer contributing to the well. If a three-point single-input sensitivity analysis had been performed at an intermediate value of depth of aquifer contributing to the well, it would have shown a trend where the peak predicted dose falls on either side of the central value of hydraulic conductivity.

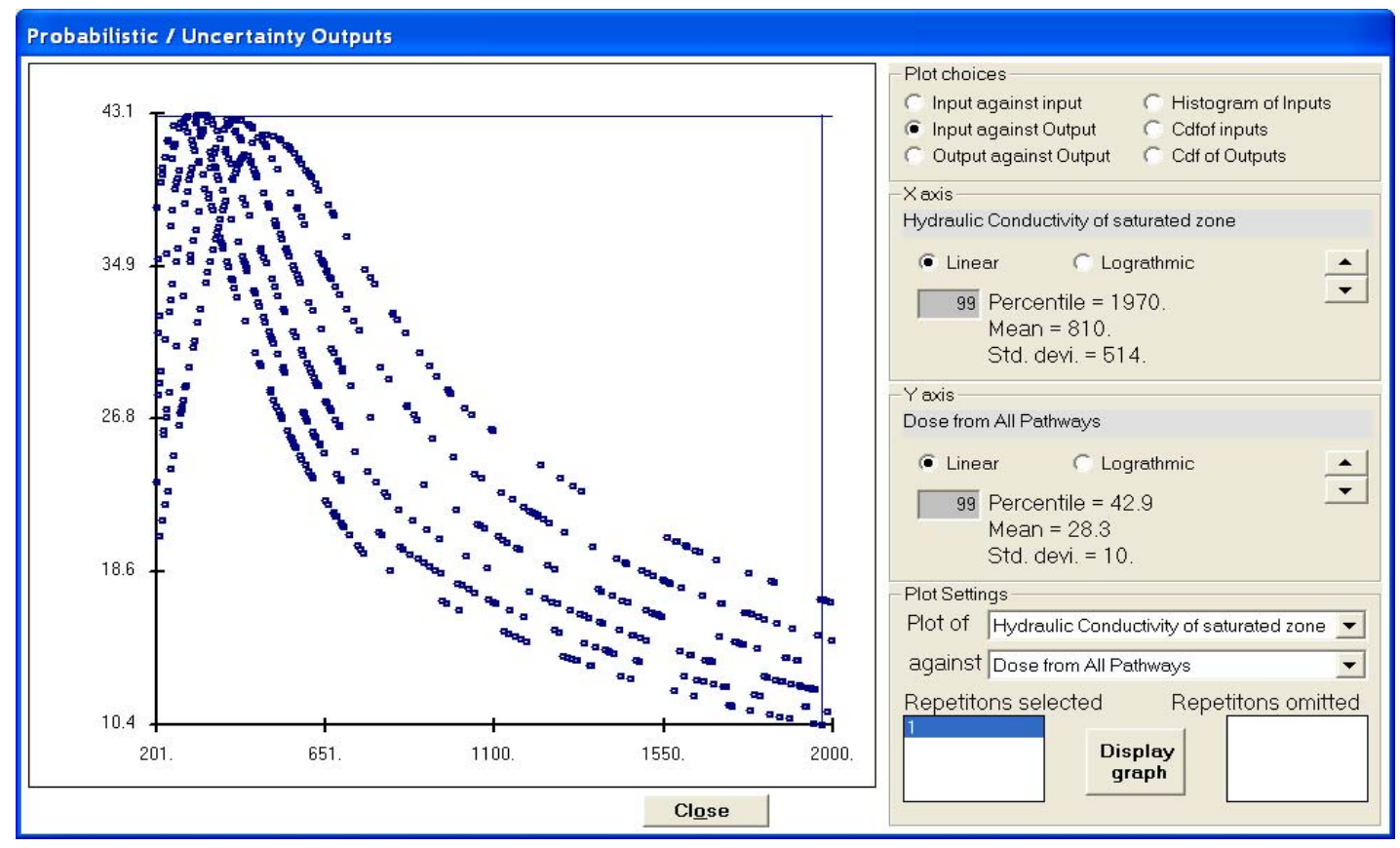

FIGURE C.60 Variation of Peak Predicted Dose with Hydraulic Conductivity at Discrete Values for Depth of Aquifer Contributing to the Well 


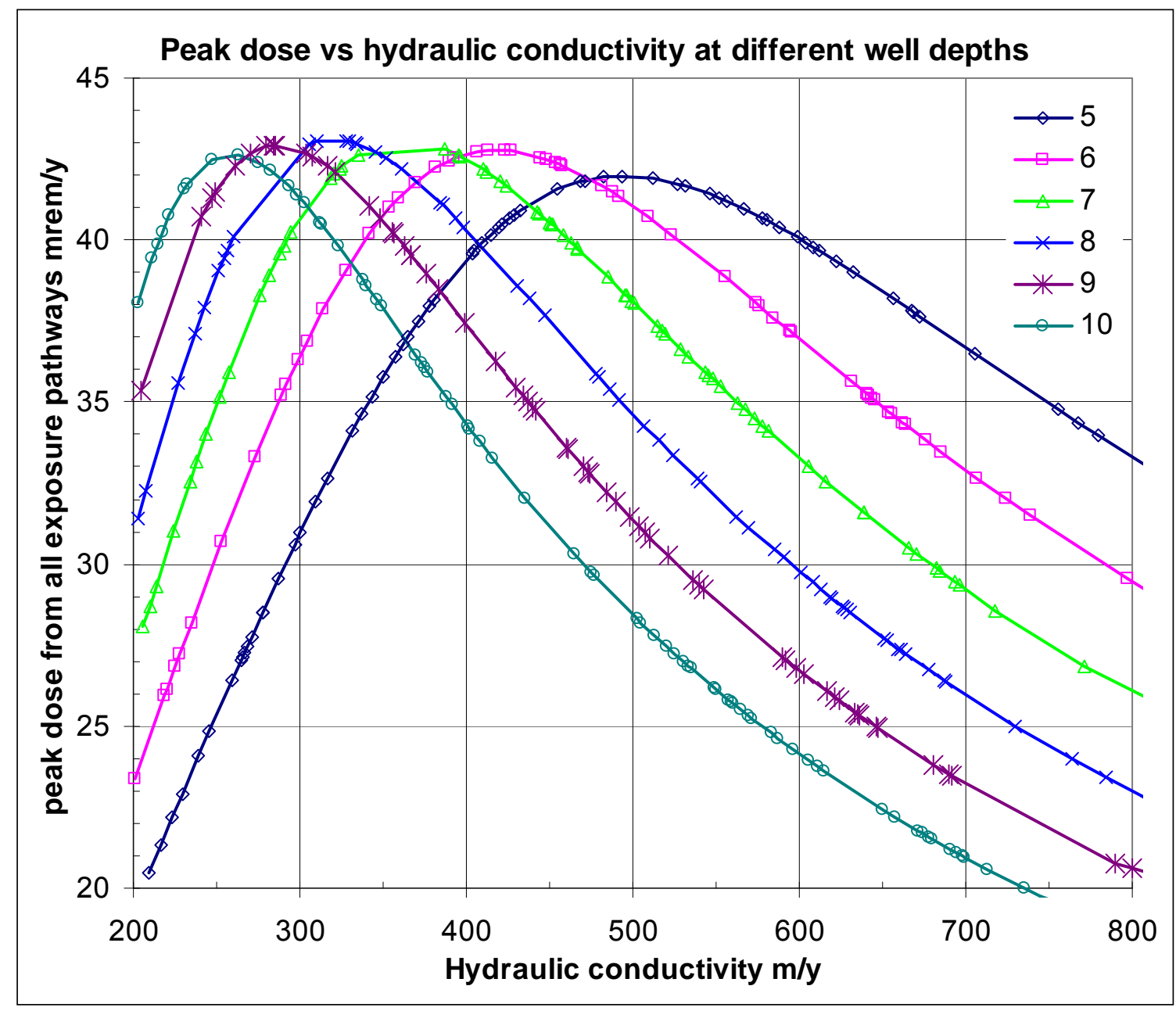

Figure C.61 Variation of Peak Predicted Dose with Hydraulic Conductivity in the Range 200 to $800 \mathrm{~m} / \mathrm{yr}$ at Discrete Values for Depth of Aquifer Contributing to the Well

The scatter plot of peak predicted dose against hydraulic conductivity in the saturated zone shown in Figure C.60 has six curves. A six-valued discrete distribution was specified for the depth of aquifer contributing to the well. It can therefore be inferred that each curve corresponds to a different depth. It is not usual to find distinct curves on this plot because (1) it is a scatter plot; (2) typically, the distributions for the other inputs are not discrete; and (3) distributions are specified for many inputs in a multi-input sensitivity analysis. There is, therefore, no labeling of the curves when they appear in this two-input (one continuous, one discrete) sensitivity analysis, which is a special case of multi-input sensitivity analysis. It is, however, possible to determine which curve corresponds to which depth by viewing the scatter plot of peak predicted dose against the depth of aquifer contributing to the well (Figure C.62). 
The highest value of peak predicted dose at the different depths of aquifer contributing to the well is approximately the same. The peak predicted dose has the greatest range when the depth of aquifer contributing to the well is $10 \mathrm{~m}$. The range of the peak predicted dose decrease as the well depth decreases. This information helps identify the corresponding curves on Figure C.60.

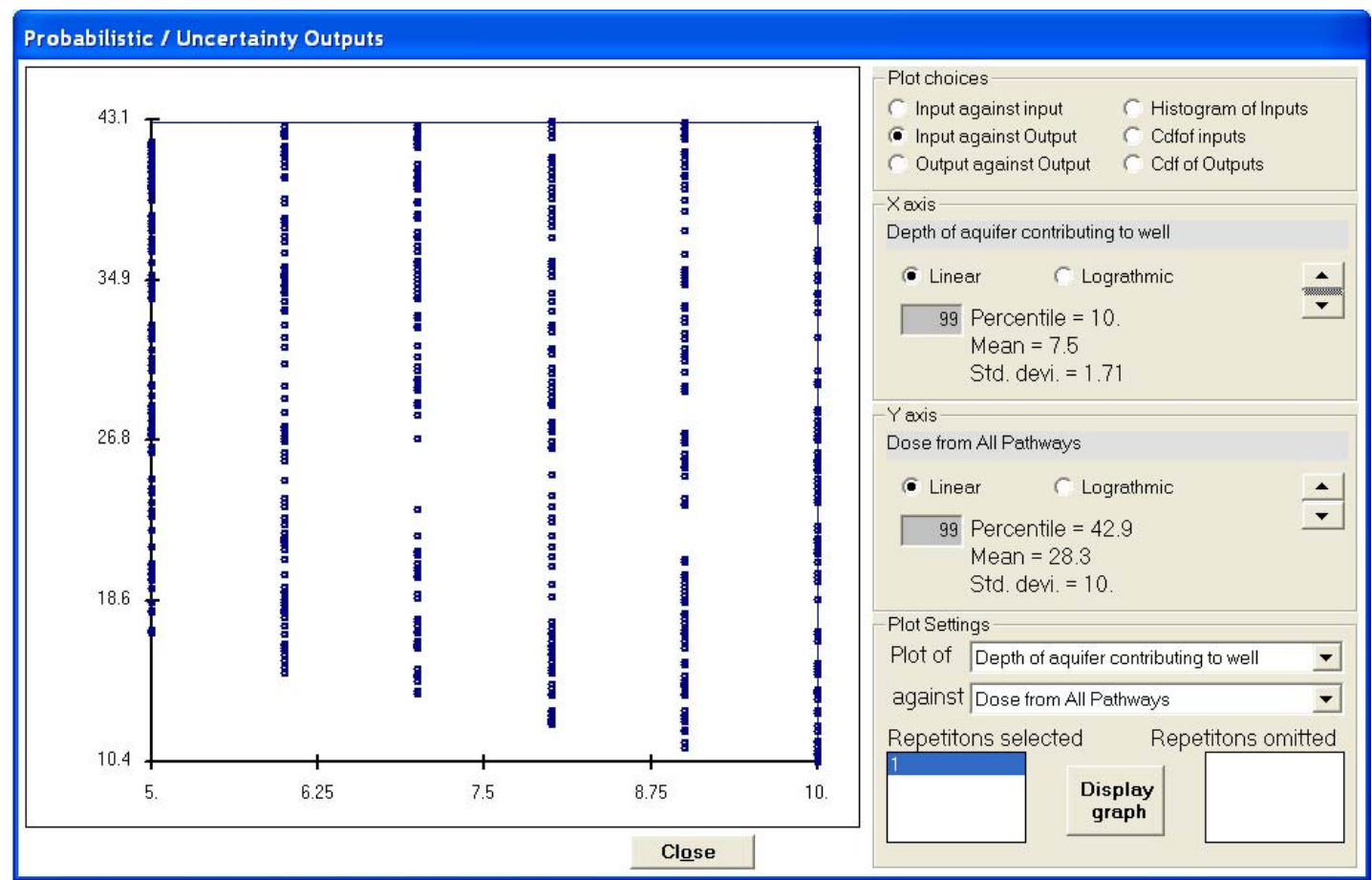

Figure C.62 Range of Peak Predicted Dose at Different Discrete Values for Depth of Aquifer Contributing to the Well Caused by Variation of Hydraulic Conductivity

The scatter plot of peak predicted dose against the depth of aquifer contributing to the well (Figure C.62) does not show how the peak predicted dose varies with the depth of aquifer contributing to the well. There are points representing 100 different values of hydraulic conductivity at each well depth. Because the 100 hydraulic conductivity values used at each depth are different, it is not possible to see how the peak predicted dose varies with depth of the aquifer contributing to the well at a constant value of hydraulic conductivity in the saturated zone. However, the scatter plots of peak predicted dose against hydraulic conductivity (Figures C.60 and C.61) indicate how the peak predicted dose varies with the depth of the aquifer. 
The trend can be seen by looking at the peak predicted doses for the six curves at a specific value of hydraulic conductivity. When the hydraulic conductivity is in the range 500 to $2000 \mathrm{~m} / \mathrm{yr}$, the peak predicted dose decreases as the depth of aquifer contributing to the well increases from 5 to $10 \mathrm{~m}$. When the hydraulic conductivity is in the range 200 to $250 \mathrm{~m} / \mathrm{yr}$, the peak predicted dose increases as the depth of aquifer contributing to the well increases from 5 to $10 \mathrm{~m}$. When the hydraulic conductivity is in the range 250 to $500 \mathrm{~m} / \mathrm{yr}$, the peak predicted dose increases, reaches a maximum, and then decreases as the depth of aquifer contributing to the well increases from 5 to $10 \mathrm{~m}$.

Although we are able see the trend, we cannot visualize the shape of the relationship. That would require a sensitivity analysis with a continuous distribution for the depth of aquifer contributing to the well and a discrete distribution for the hydraulic conductivity. This is left for the reader to do as an exercise.

\section{C.3.5.3 Sensitivity Analysis on Three Inputs Using a Continuous Linear Distribution for One and Discrete Uniform Distributions for the Other Two}

The discussion in Section C.3.3 suggested that the sensitivity of peak predicted dose to hydraulic conductivity in the saturated zone depends not only on the values used for the inputs describing the well, but also on the values used for the inputs that determine the infiltration through the primary contamination. This section expands the previous example further to consider simultaneous sensitivity analyses on three inputs.

The example considered in Section C.3.5.2 is expanded by including a discrete distribution for the evapotranspiration coefficient. Begin with the input file used for the distributed single-input sensitivity analysis. Save the file under a different name (e.g., "Three Inputs Two Discrete.ROF"). Go to the Primary Contamination form containing the physical and hydrological inputs and place the cursor on the input box for evapotranspiration. Press the Shift-F8 function key. The "Parameter distributions" tab of the Uncertainty and Probabilistic Analysis form will appear. Click on the Distribution dropdown box and select "Discrete Cumulative." Use three entries to sample evapotranspiration coefficients of $0.4,0.5$, and 0.6 with equal probability; click the "Update parameter stats and distribution" button to save this distribution.

If the six discrete valued distribution specified in Section C.3.5.2 for the depth of aquifer contributing to the well were to be used, we would expect to see $6 \times 3$ curves. A larger number of simulations would be required to produce the 18 curves. It would also be difficult to make out the 18 curves in the same scatter plot, especially in the regions where the curves intersect or are close together. 
Go to the Saturated Zone Hydrology form and place the cursor on the input box for depth of aquifer contributing to the well. Press the Shift-F8 function key. The "Parameter distributions" tab of the Uncertainty and Probabilistic Analysis form pops up-change it to a discrete distribution with three equally probable values of $5,7.5$, and $10 \mathrm{~m}$. This reduces the anticipated number of distinct curves in the scatter plot of peak predicted dose against hydraulic conductivity to nine.

Click on the "Generate input samples" button on the "Step by step analysis" tab. This launches the sampling code and produces the specified number of probabilistic input sets. Each probabilistic input set contains a sample from each of the three distributions specified for the three inputs. Specify the relationship between the mean residence time of water in the surface water body and the hydraulic conductivity in the saturated zone, as in Section C.3.4.1. Click on the "Generate output samples (RESRAD-O)" button to launch the computational code.

The scatter plot of peak predicted dose against each of the three probabilistic inputs and the related input can be viewed after the code finishes all the simulations. Click the "View scatter plots of output vs. input" button to display the Probabilistic/Uncertainty Outputs form. Use the up/down arrows in the X-axis frame to cycle thorough the four variables. The scatter plot of peak predicted dose against hydraulic conductivity in the saturated zone is shown in Figure C.63. There are three curves in the upper range of hydraulic conductivity-as shown in Section C.3.5.2, these correspond to the three discrete depths that were sampled to determine the extent of the aquifer contributing to the well. Each of these three curves splits into three curves in the lower range of hydraulic conductivity. These correspond to the three levels of evapotranspiration coefficient. The scatter plot of peak predicted dose against evapotranspiration coefficient, Figure C.64, indicates that the upper branch of the three curves corresponds to the higher evapotranspiration coefficient.

The variation of peak predicted dose with hydraulic conductivity in the saturated zone is highly dependent on the values used for the depth of aquifer contributing to the well and for the evapotranspiration coefficient (Figure C.63). This clearly illustrates the need for multi-input sensitivity analysis and highlights the importance of using site-specific or at least siteappropriate values and distributions for all the inputs when performing sensitivity analysis.

The discussion in Section C.3.3 suggested some of the inputs that influence the sensitivity of peak predicted dose to hydraulic conductivity in the saturated zone. These include the depth of aquifer contributing to the well, the well pumping rate, the dispersivities in the saturated zone, the precipitation rate, the runoff and evapotranspiration coefficients, and irrigation rates. If discrete distributions with just two values were specified for each of these eight inputs, there could be $2^{8}(=256)$ curves on the scatter plot of peak predicted dose against hydraulic conductivity in the saturated zone. One obviously cannot differentiate among that many distinct 
curves on one plot. While the idea of performing a multi-input sensitivity analysis with a continuous distribution for one input and discrete distributions for the other inputs that affect the way the first input influences the peak predicted dose is a good way to understand that influence, it is only practical when the number of inputs is small. Even when the number of distinct curves is manageable, the number of simulations needed to produce the curves can be very large. There is also the need to know which inputs to include in the analysis.

Such an analysis, where a continuous distribution is specified for one input and discrete distributions are specified for the other inputs, is useful only in understanding the variation of peak predicted dose with the input for which a continuous distribution was specified. A number of multi-input sensitivity analyses would have to be performed-each one with the continuous distribution on a different input-to gain insight into the manner in which an input of interest affects peak predicted dose.

While this method of multi-input sensitivity analysis might give insight into the influence of an input on the peak predicted dose, it does not provide an easy way to compare the relative influences of a number of inputs. The range of the peak predicted dose over the siteappropriate or site-specific range of the input is a possible measure of the importance of that input (Section C.2.3.3). But the range of the peak predicted dose varies from curve to curve in this method of sensitivity analysis. It is not possible to visually separate out the contributions of the different inputs to the range of the peak predicted dose in the scatter plot. 


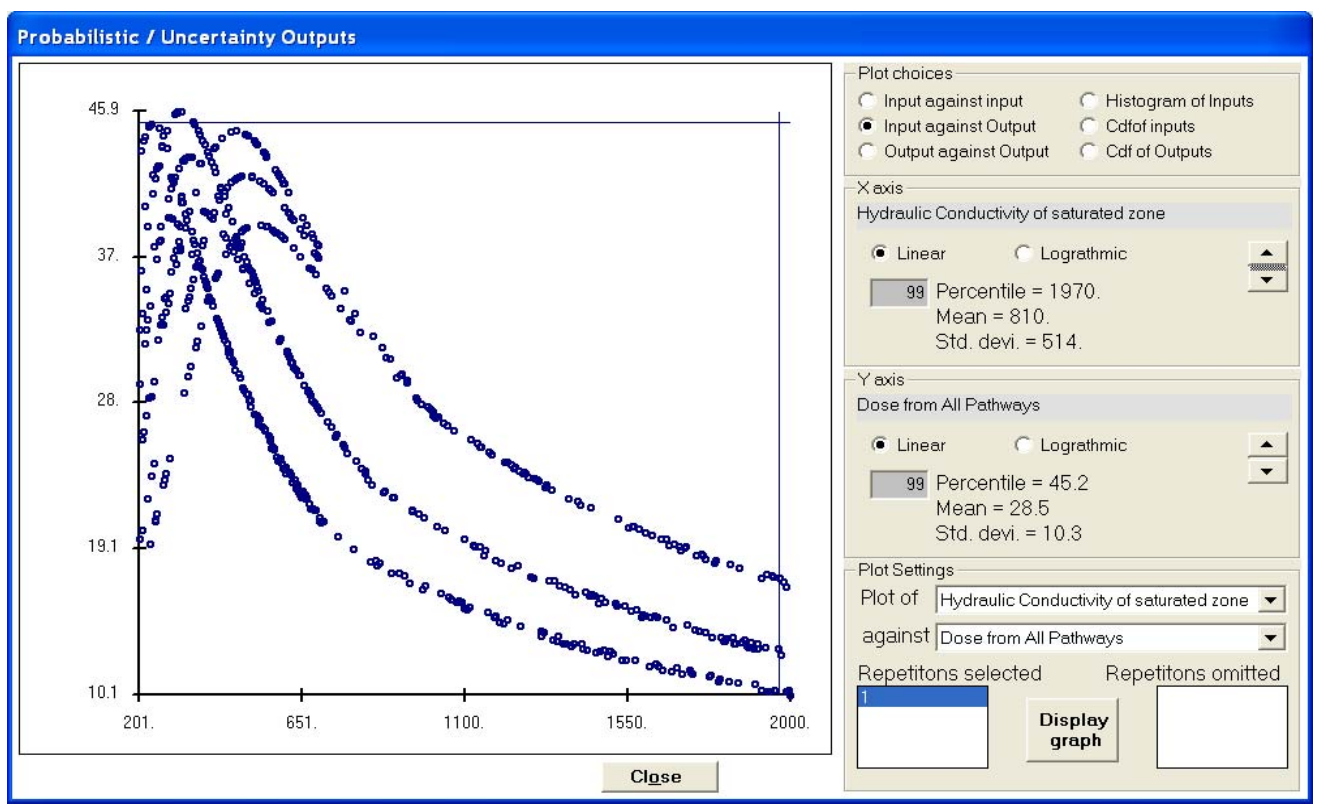

FIGURE C.63 Variation of Peak Predicted Dose with Hydraulic Conductivitythe Scatter Shows the Effect of Sampling the Depth of Aquifer Contributing to the Well and the Evapotranspiration Coefficient

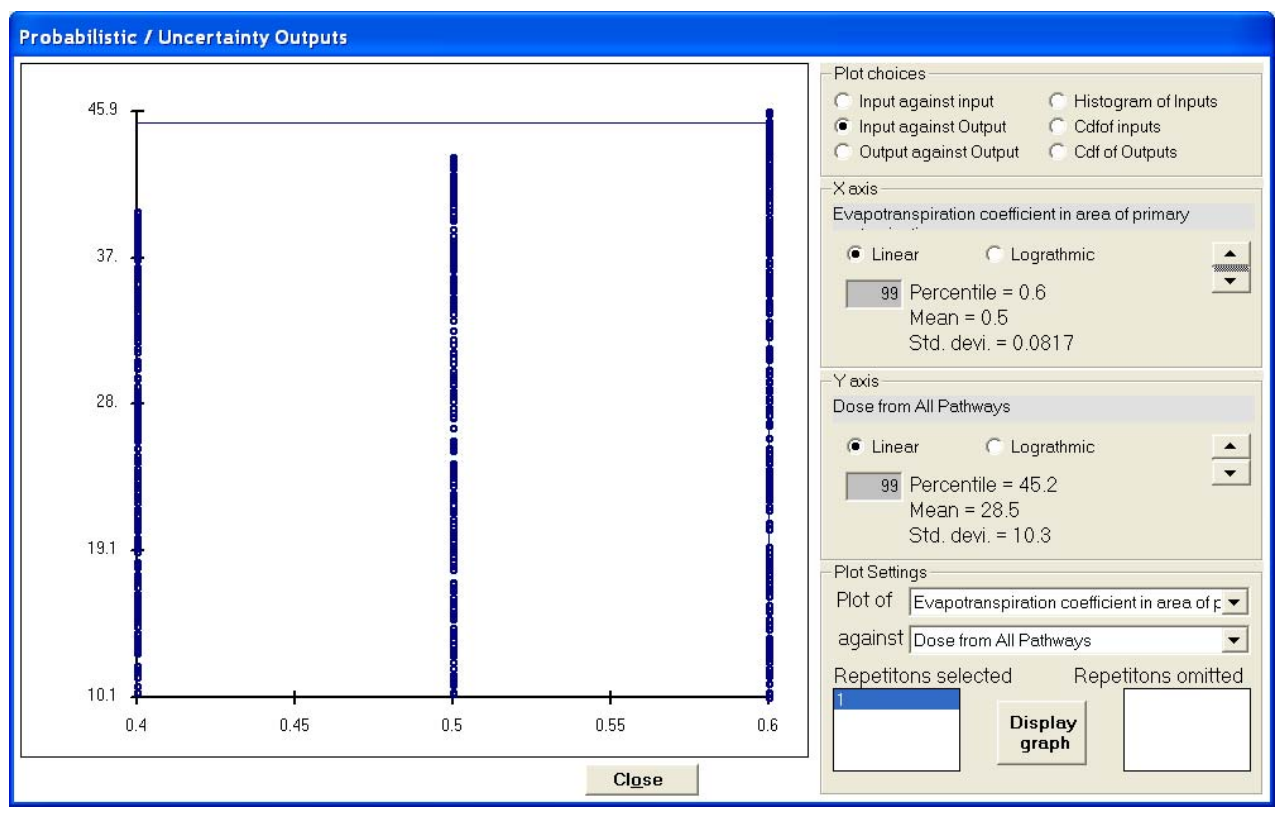

FIGURE C.64 Peak Predicted Dose at Three Values of Evapotranspiration Coefficient-the Range of Peak Predicted Dose Is Due to Sampling the Depth of Aquifer Contributing to the Well and the Hydraulic Conductivity 
The relative influences of different inputs can be gauged from a single sensitivity analysis. If a single analysis is to be used, it is necessary to specify either site-appropriate or site-specific distributions for all the inputs. The site-appropriate or specific distributions are likely to be continuous. A commonly used technique of gauging the relative influences of different inputs involves the following steps:

- Step 1. Perform multi-input sensitivity analysis by specifying site-appropriate or sitespecific distributions for all the inputs.

- Step 2. Perform linear regression analysis between the output, the peak predicted dose, and the distributed inputs. Use the standardized regression coefficient or the standardized rank regression coefficient to identify the inputs that have a significant influence on the variability in the peak predicted dose. (Linear regression is discussed in Section C.3.6.)

- Step 3. Perform a second multi-input sensitivity analysis by specifying the same distributions as in Step 1 for only the inputs that were identified in Step 2.

- Step 4. Compare the distributions of peak predicted dose that resulted from the two multi-input sensitivity analyses preformed in Steps 1 and 3. Repeat Steps 2 and 3 until the distribution of peak predicted dose resulting from Step 3 is close to the distribution of peak predicted dose resulting from Step 1.

This method is incorporated in the RESRAD-OFFSITE code.

\section{C.3.5.4 Sensitivity Analysis on Two Inputs Using Continuous Distributions}

Two-input sensitivity analysis with a view to visually understanding how one of those inputs affects peak predicted dose was illustrated and discussed in Sections C.3.5.1 and C.3.5.2. This section deals with two-input sensitivity focused on evaluating the relative influence of the inputs. A key requirement is to use distributions that are appropriate for the site. The sensitivity analysis is usually performed before significant resources have been expended to develop sitespecific distributions; in fact, the sensitivity analysis identifies the significant inputs so that the limited resources can be focused on identifying site-specific distributions. Thus, distributions that are appropriate for the site, based on factors such as its location, climate, terrain/topography, soil type, and vegetation need to be used-it will be seen in this and subsequent sections that the influence of inputs depends on their distribution. Assume for this example that a uniform distribution from 200 to $2000 \mathrm{~m} / \mathrm{yr}$ is appropriate for hydraulic conductivity and a uniform distribution from 5 to 10 is appropriate for the depth of aquifer contributing to the well. 
Open the file used in Section C.3.5.1, "Two Inputs One Discrete.ROF", and save it as "Two uniform Inputs.ROF." This has the desired distribution for hydraulic conductivity in the saturated zone, but not for the depth of aquifer contributing to the well. View the "Uncertainty/ Probabilistic Interface." Go to the "Parameter distributions" tab of the Uncertainty and Probabilistic Analysis form. Click on "depth of aquifer contributing to the well" entry in the table on the left. Change the distribution to a uniform distribution from 5 to $10 \mathrm{~m}$; click the "Update parameter stats and distribution" button to save these changes.

Click on the "Generate input samples" button on the "Step by step analysis" tab. The code samples 600 values uniformly in the interval 200 to 2000 for hydraulic conductivity in the saturated zone. It also samples 600 values uniformly in the interval of 5 to $10 \mathrm{~m}$ for the depth of aquifer contributing to the well. It then makes 600 pairs of values of hydraulic conductivity and depth of aquifer contributing to the well. The 600 pairs of values can be viewed by clicking the "View scatter plot of input vs. input" button in the "Step by step analysis" tab. The resulting screen is shown in Figure C.65. The pairs of values are distributed evenly over the entire space of the plot, a result of specifying uniform distributions for the two probabilistic inputs and requiring pairing with zero correlation.

Specify the relationship between the mean residence time of water in the surface water body and the hydraulic conductivity in the saturated zone as in Section C.3.4.1. Click on the "Generate output samples (RESRAD-O)" button to launch the computational code. 


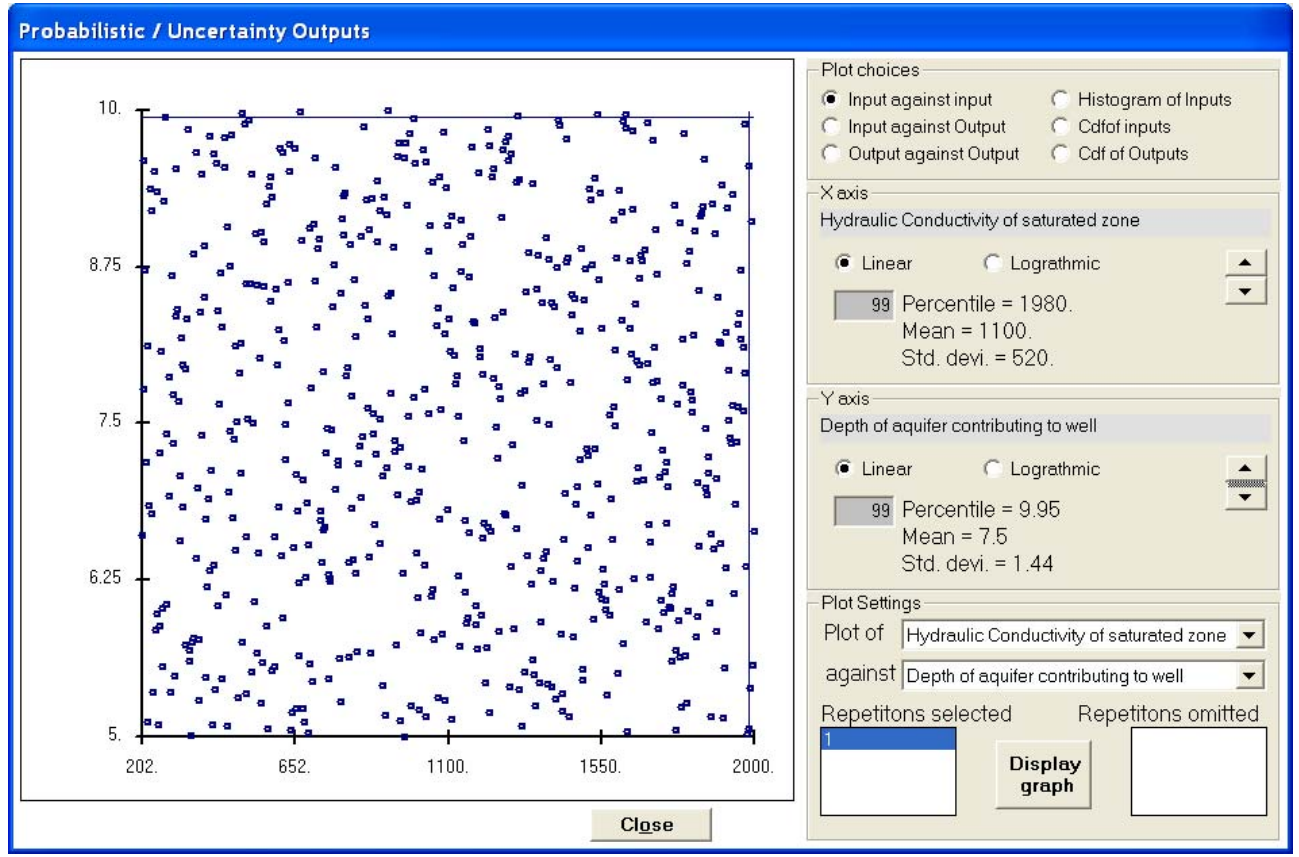

\section{FIGURE C.65 Pairing of Samples in a Two-input Probabilistic Analysis Where Both Inputs Have Continuous Distributions}

The scatter plot of peak predicted dose against each of the two probabilistic inputs and the related input can be viewed after the code finishes all the simulations. Click the "View scatter plots of output vs. input" button to display the Probabilistic/Uncertainty Outputs form. Use the up/down arrows in the X-axis frame to cycle thorough the three variables. The scatter plot of peak predicted dose against hydraulic conductivity in the saturated zone is shown in Figure C.66. This scatter plot shows a strong relationship between the peak predicted dose and the hydraulic conductivity. The 600 points are clustered within a band that first rises and then falls with increasing hydraulic conductivity. The width of the band in the direction parallel to the $y$-axis ranges from 6.5 to $16 \mathrm{mrem} /$ year, compared with a $32.6 \mathrm{mrem} /$ year range of the peak predicted dose (Figure C.68). The peak predicted dose at any saturated hydraulic conductivity varies over a range due to the effects of all the other distributed inputs. In this case, it quantifies the effect of the depth of the aquifer contributing to the well, which is the only other distributed variable. 


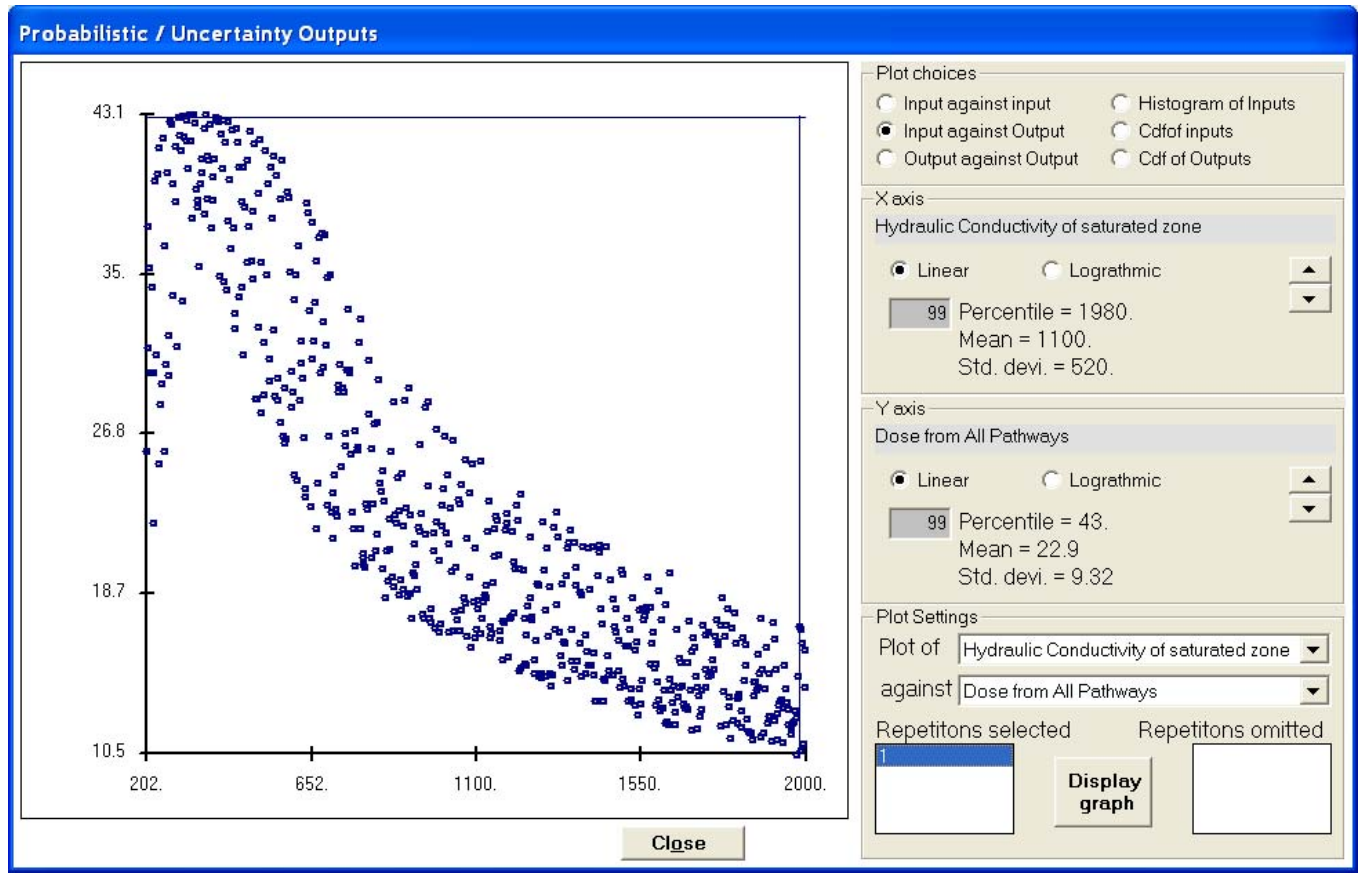

\section{FIGURE C.66 Variation of Peak Predicted Dose with Hydraulic Conductivity-the Scatter Shows the Effect of Sampling the Depth of Aquifer Contributing to the Well}

The scatter plot of peak predicted dose against the depth of the aquifer contributing to the well is shown in Figure C.67. The 600 points are spread out over a wide band. The lower boundary of the band falls with increasing depth of aquifer contributing to the well; the upper boundary is a mild curve that first rises and then falls with increasing depth of aquifer contributing to the well. The width of the band in the direction parallel to the y-axis ranges from 25 to $32 \mathrm{mrem} /$ year. The peak predicted dose at any depth of the aquifer contributing to the well varies over a range due to the effects of all the other distributed inputs. In this case, it quantifies the effect of the hydraulic conductivity in the saturated zone, the only other distributed variable in this analysis.

The range of the dose that is a consequence of the site-specific or site-appropriate distribution of an input is a direct indication of the influence of that input (Section C.2.3.3). In a twodistributed-input sensitivity analysis, the range of peak predicted dose that results from one of the distributed inputs can be determined from the scatter plot of peak predicted dose against the other distributed input. The range can be estimated from the scatter plot by clicking the mouse on the two ends of the band of points at the same x-value, as shown in Figure C.68. Thus, the peak predicted dose ranges from 21.8 to $38 \mathrm{mrem} / \mathrm{yr}$ as the depth of aquifer contributing to the well ranges from 5 to $10 \mathrm{~m}$ at a hydraulic conductivity of $653 \mathrm{~m} / \mathrm{yr}$-a range of $16.2 \mathrm{mrem} / \mathrm{year}$. The range of the peak predicted dose at a few representative hydraulic conductivities can be obtained by applying this procedure on the plot shown in Figure C.66: 17.3 at 200, 7.7 at 360, 
16.2 at $650,9.8$ at $1100,7.9$ at 1550 , and 6.6 at 2000 . This gives an average range of $11 \mathrm{mrem} / \mathrm{yr}$ attributable to the 5 to $10 \mathrm{~m}$ range of depth of aquifer contributing to the well.

Applying the same procedure on the scatter plot of peak predicted dose against depth of aquifer contributing to the well (Figure C.67) gives a $30 \mathrm{mrem} / \mathrm{yr}$ range of peak predicted dose attributable to the 200 to $2000 \mathrm{~m} / \mathrm{yr}$ range of hydraulic conductivity in the saturated zone. In this example, the variations in hydraulic conductivity cause almost three times as much variability in the peak predicted dose as the variations in the depth of aquifer contributing to the well:

$30 \mathrm{mrem} / \mathrm{yr}$ to $11 \mathrm{mrem} / \mathrm{yr}$.

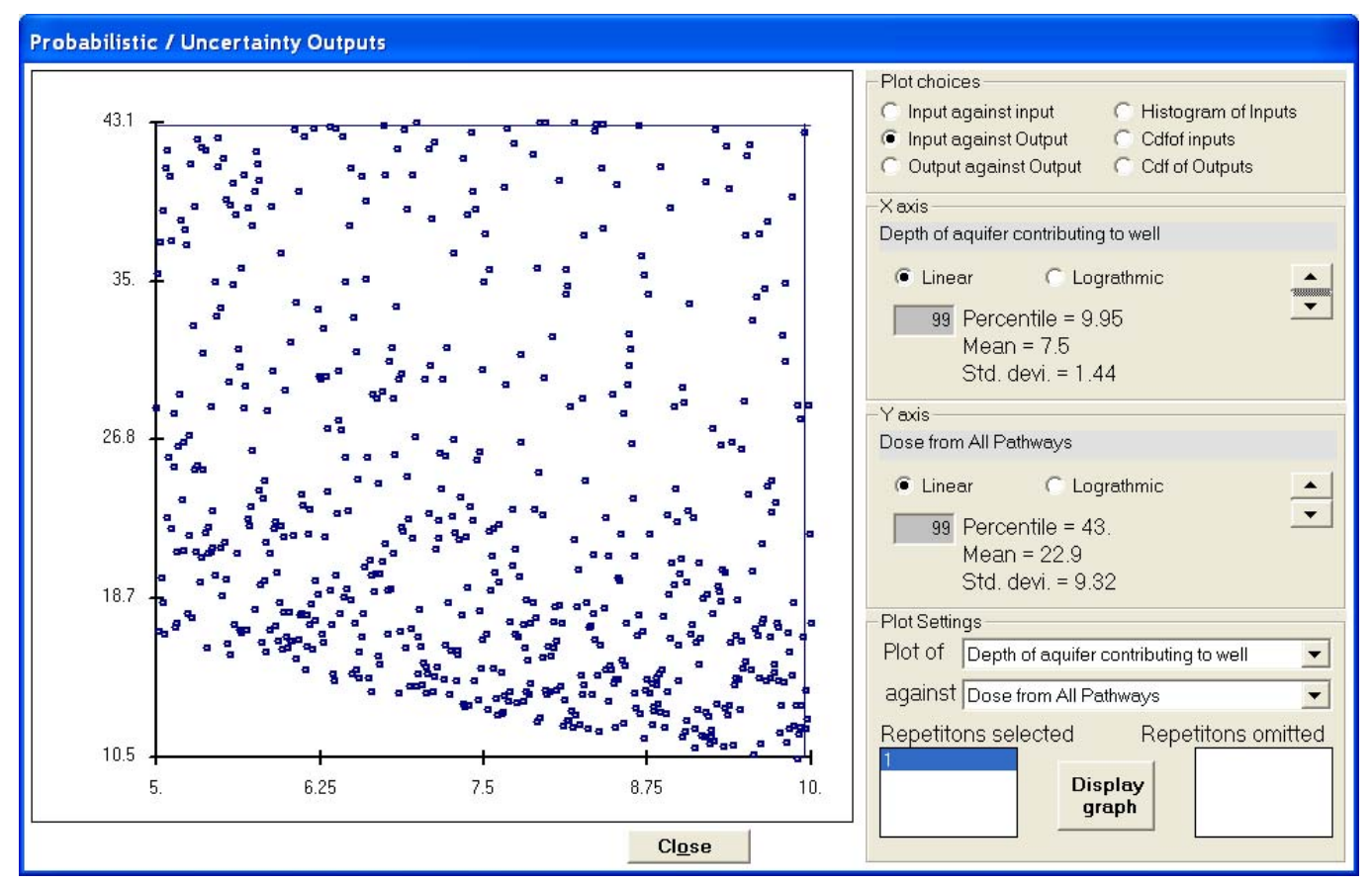

FIGURE C.67 Variation of Peak Predicted Dose with Depth of Aquifer Contributing to the Well-the Scatter Shows the Effect of Sampling the Hydraulic Conductivity 


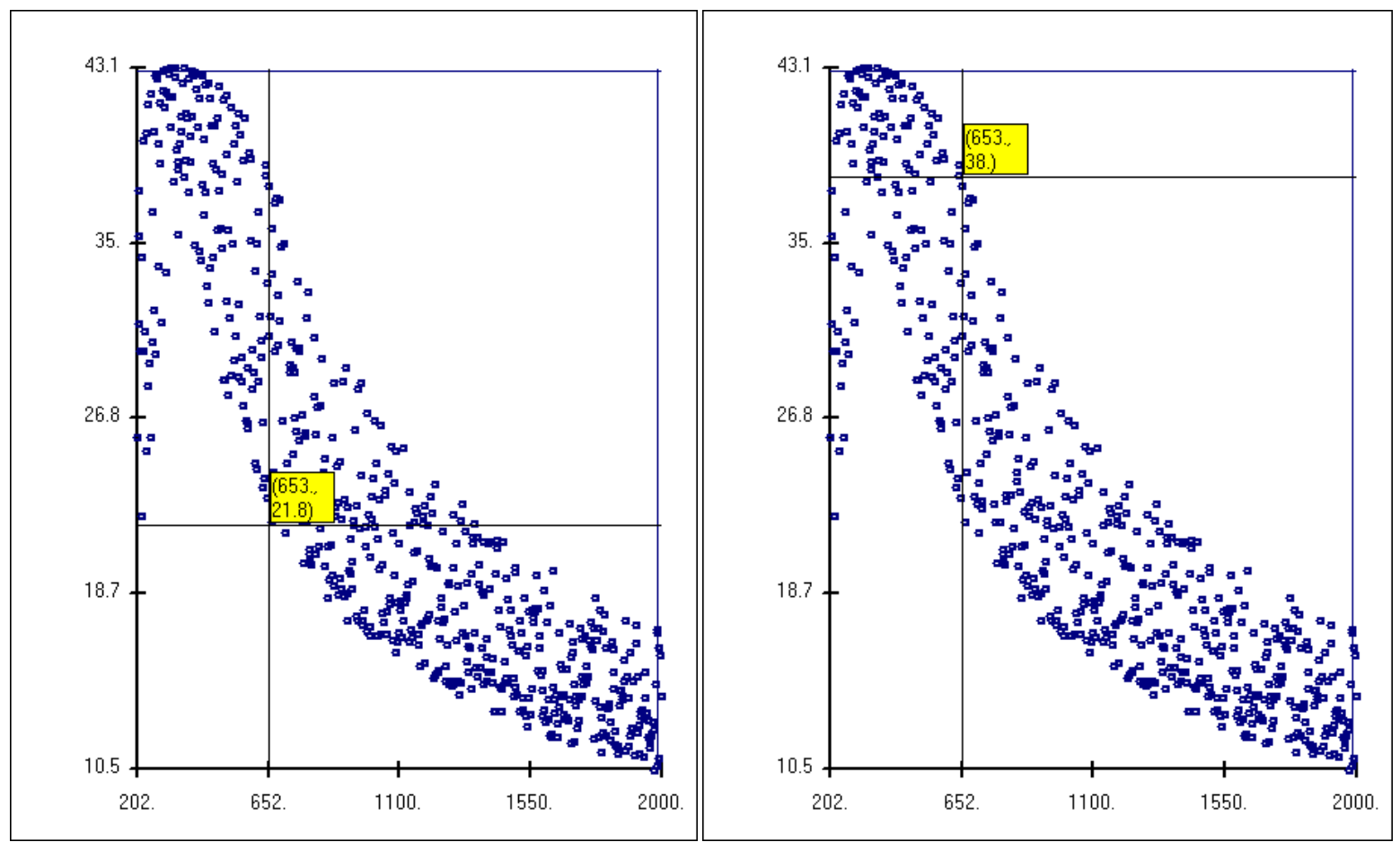

\section{FIGURE C.68 Estimating the Range of Peak Predicted Doses at Any Given Value of X-coordinate}

Another way of estimating the contribution of each distributed input to the variability of the peak predicted dose is by performing linear regression analysis. The regression analyses available in RESRAD-OFFSITE are described in Section C.3.6. That section also discusses the different coefficients that result from those regression analyses. The regression output for this example is shown in Figure C.69. The output-the coefficient of determination-shows that the linear regression on the ranks of the peak predicted dose and the two inputs is able to explain $94 \%$ of the variation in the peak predicted dose in this example. The output-the standardized rank regression coefficients or SRRC — shows that the hydraulic conductivity in the saturated zone causes almost thrice the variability in peak predicted dose as the depth of aquifer contributing to the well: 0.91 compared with 0.34 . 


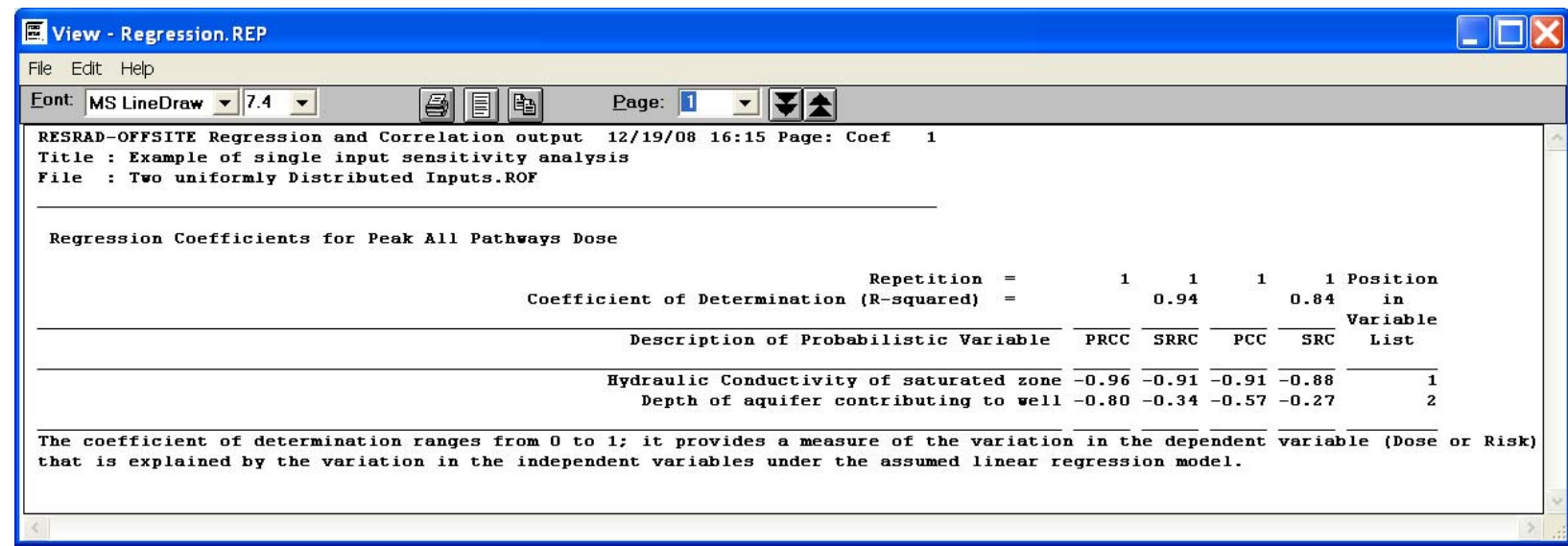

\section{FIGURE C.69 Linear Regression on Standardized Raw and Ranked Data for the Two-input Continuous Distribution Example}

The variability in peak predicted dose that results from a distributed input depends on the distribution used for the input. This is illustrated by repeating the above analysis with a narrower distribution-uniform from 500 to $1000 \mathrm{~m} /$ day - for the hydraulic conductivity in the saturated zone. The regression output and the scatter plots, Figures C.70 through C.72, show that the variabilities in peak predicted dose from the two distributed inputs are about the same. Comparison of the results of the two analyses in this subsection again highlights the need to use site-specific or site-appropriate distributions when performing sensitivity analysis.

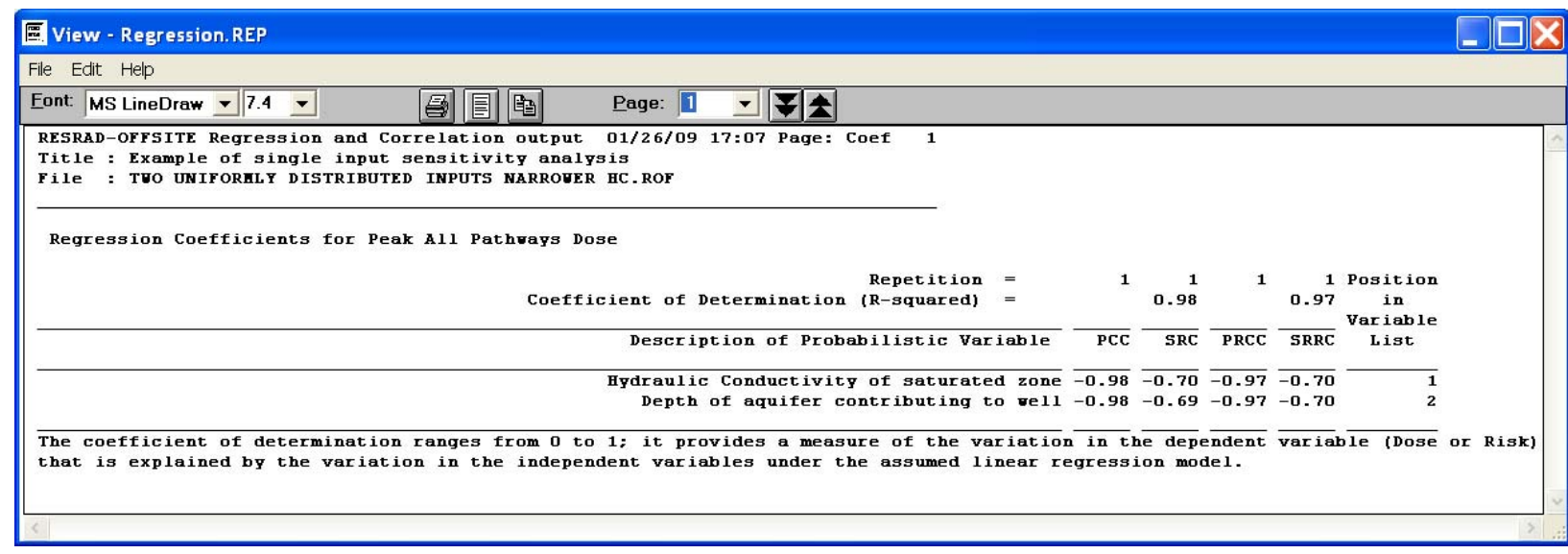

FIGURE C.70 Linear Regression on Standardized Raw and Ranked Data for the Second Two-input Continuous Distribution Example 


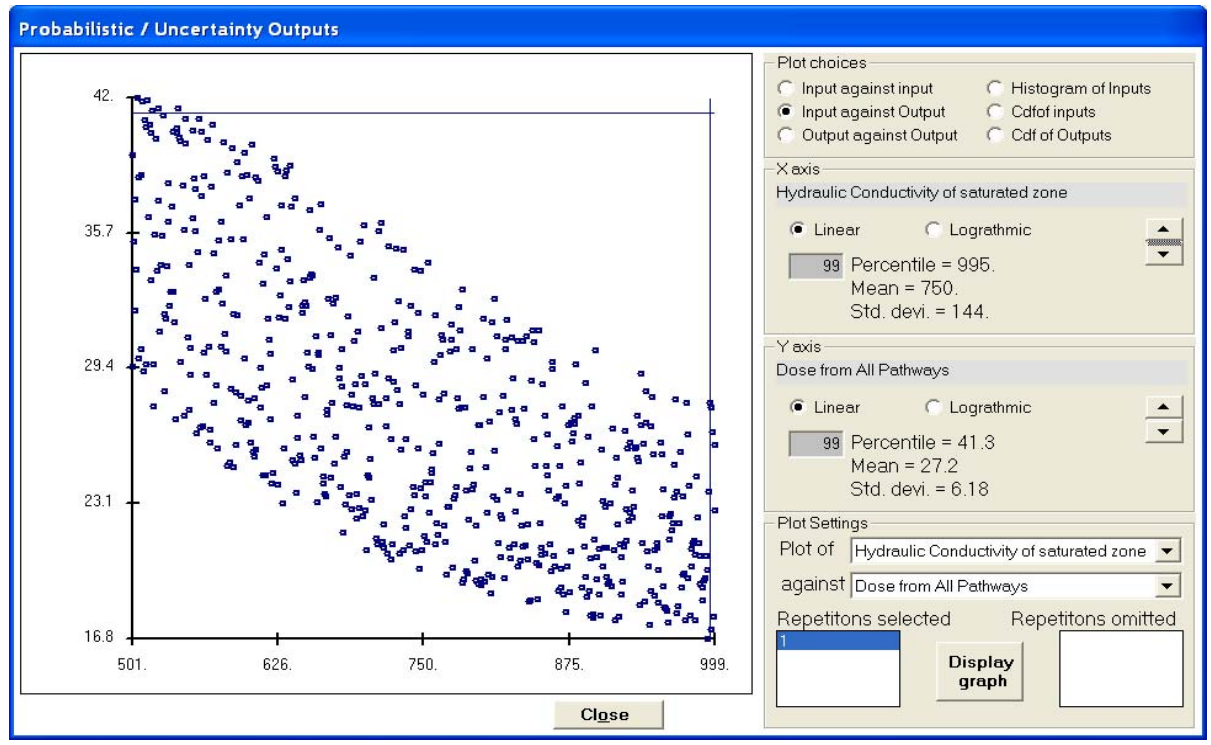

FIGURE C.71 Variation of Peak Predicted Dose with Hydraulic Conductivity-the Scatter Shows the Effect of Sampling the Depth of Aquifer Contributing to the Well for the Second Example Case

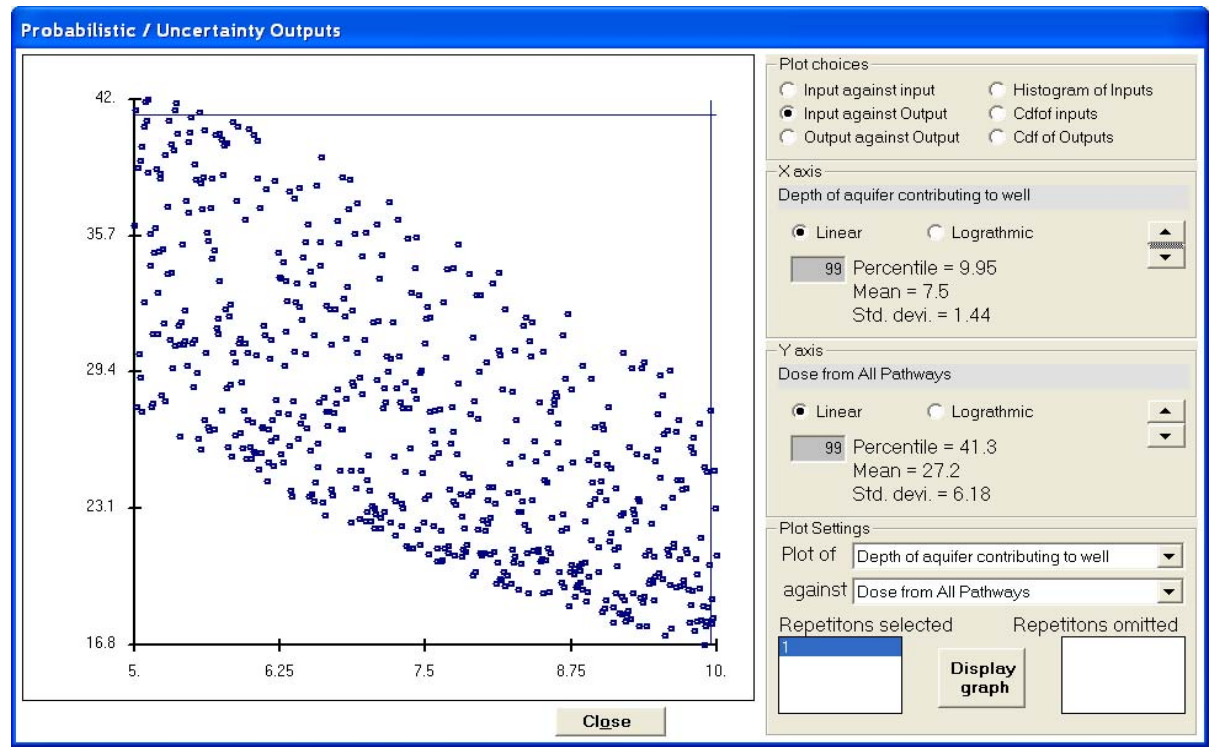

FIGURE C.72 Variation of Peak Predicted Dose with Depth of Aquifer Contributing to the Well-the Scatter Shows the Effect of Sampling the Hydraulic Conductivity for the Second Case 


\section{C.3.5.5 Sensitivity Analysis on Three Inputs Using Continuous Distributions}

A three-input sensitivity analysis using continuous distributions was performed by adding a uniform distribution of 0.4-0.6 for evapotranspiration. The relationship between the residence time in the lake and the saturated hydraulic conductivity was specified as in the previous example after generating the input samples. The regression output and scatter plots of peak predicted dose against the three inputs are shown in Figures C.73 through C.76. These will be discussed in Section C.3.6 to illustrate regression analyses.

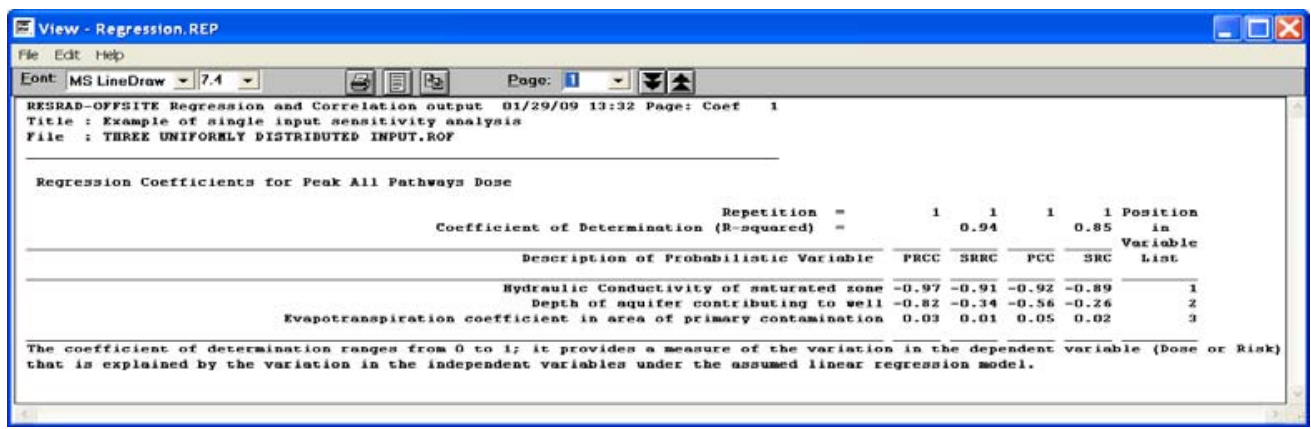

FIGURE C.73 Linear Regression on Standardized Raw and Ranked Data for the Three-input Continuous Distribution Example

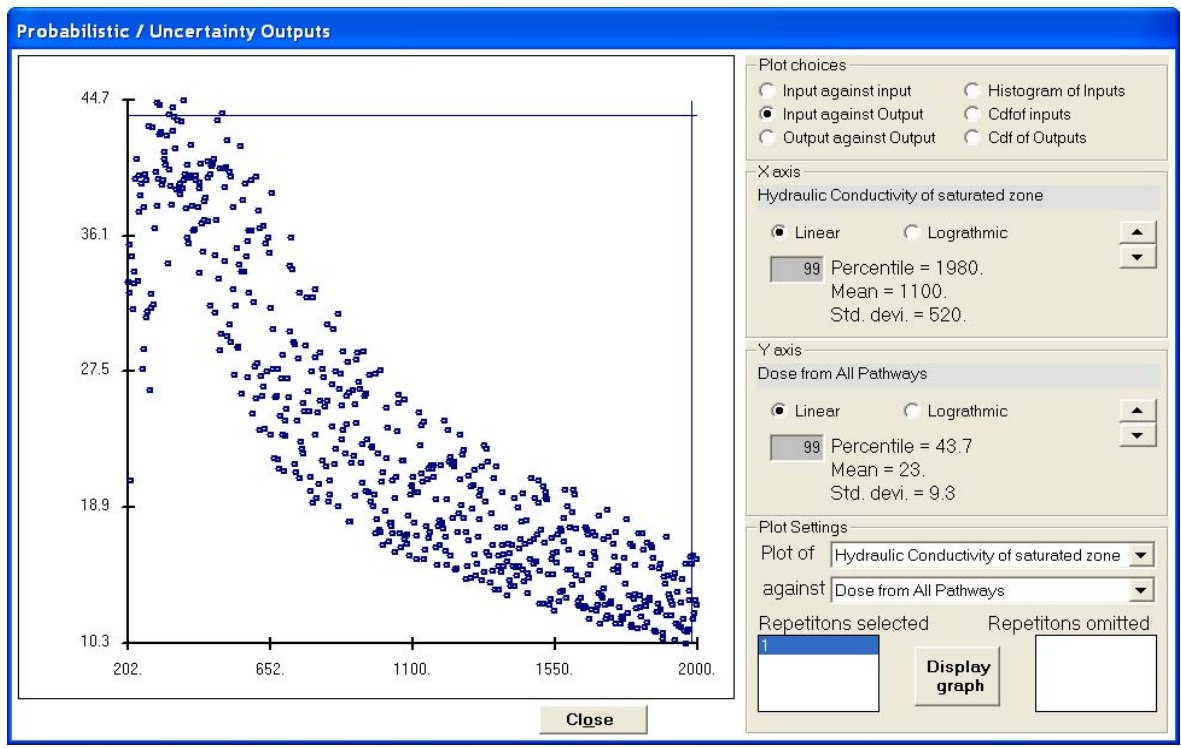

FIGURE C.74 Variation of Peak Predicted Dose with Hydraulic Conductivity-the Scatter Shows the Effect of Sampling the Depth of Aquifer Contributing to the Well and the Evapotranspiration Coefficient 


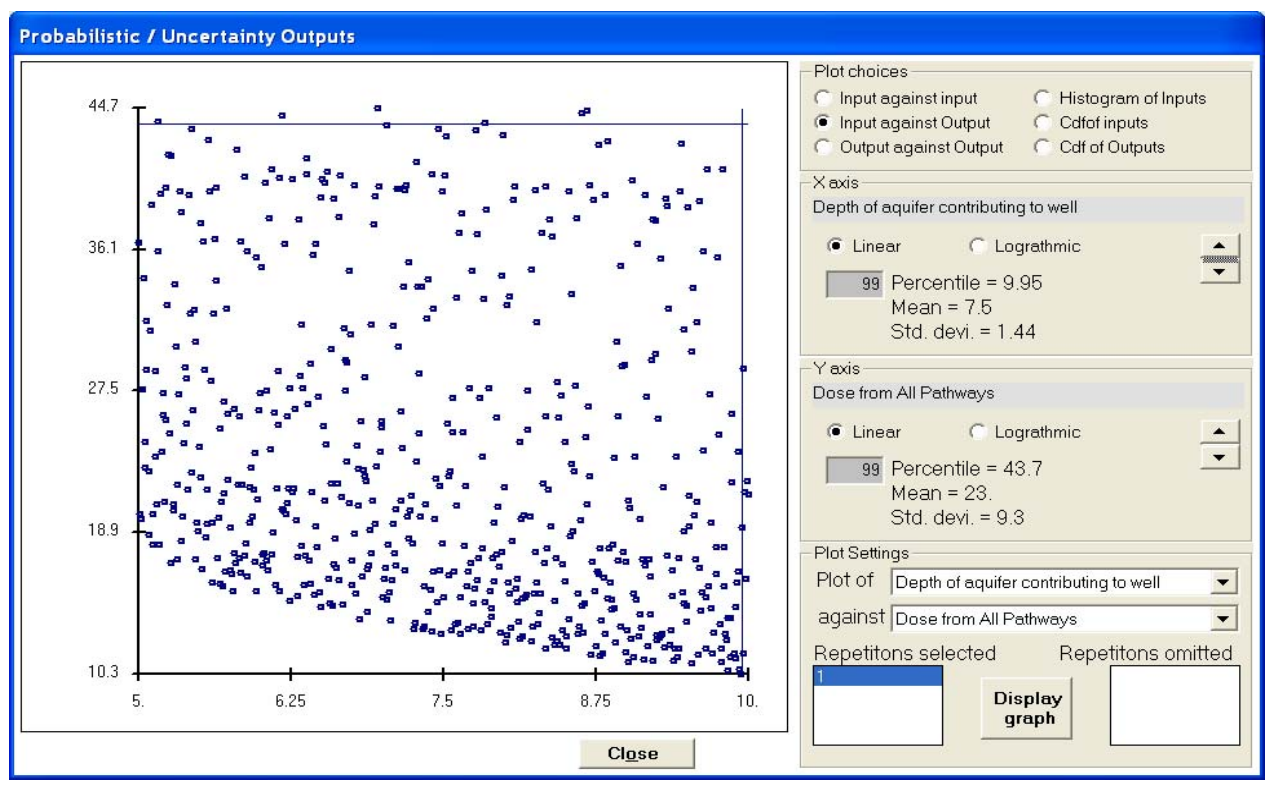

FIGURE C.75 Variation of Peak Predicted Dose with Depth of Aquifer Contributing to the Well-the Scatter Shows the Effect of Sampling the Hydraulic Conductivity and the Evapotranspiration Coefficient

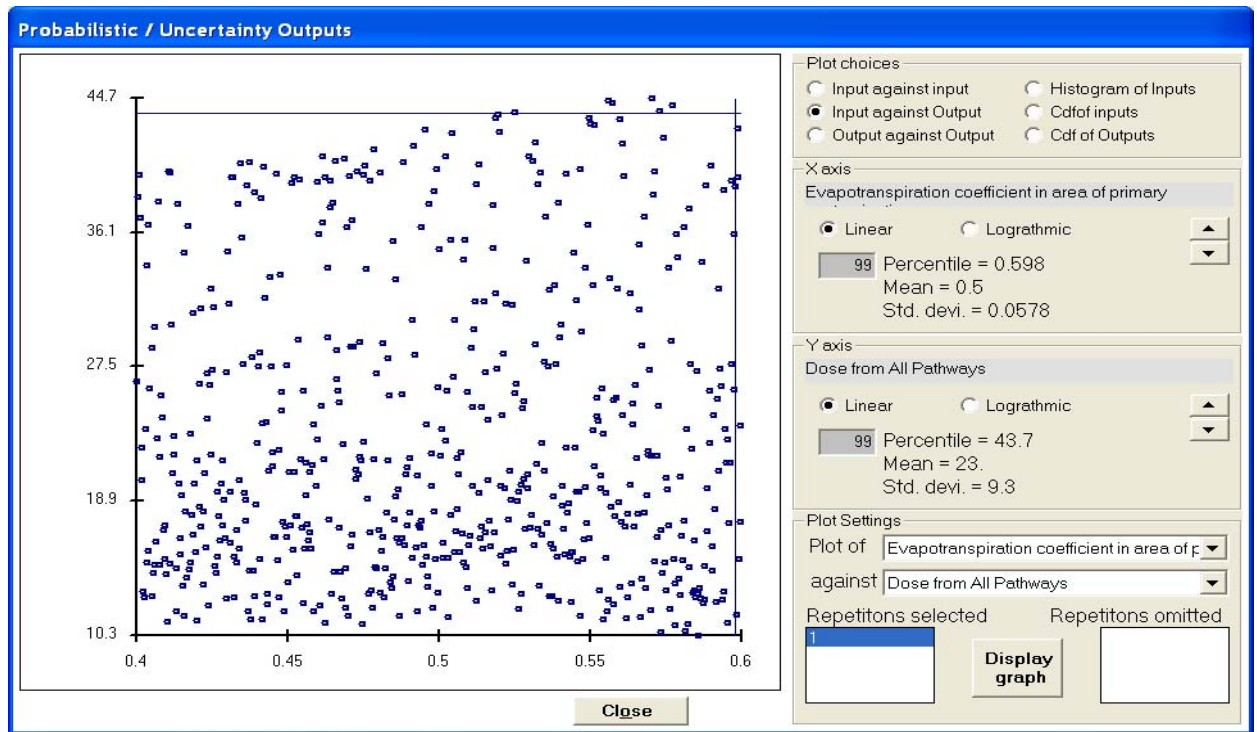

FIGURE C.76 Variation of Peak Predicted Dose with Evapotranspiration Coefficient-the Scatter Shows the Effect of Sampling the Hydraulic Conductivity and the Depth of Aquifer Contributing to the Well 


\section{C.3.6 Linear Regression}

The peak dose predicted by the code depends on the values of the inputs: the peak predicted dose is the dependent variable, the inputs are the independent variables. If all but one of the inputs were fixed and only one input were allowed to vary, it would be easy see the relationship between the dependent and independent variables from a plot of the two (Figure C.77). If the relationship is strictly linear, then quantifying the relationship is straightforward. The relationship can be quantified using any two points on the plot.

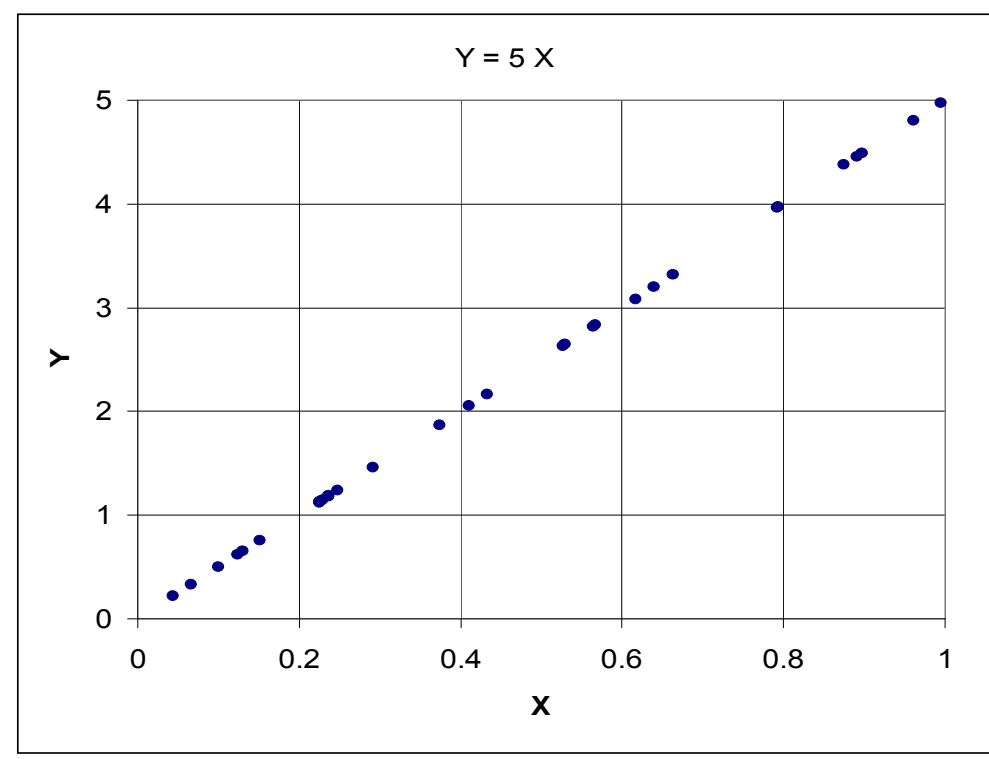

\section{FIGURE C.77 Scatter Plot of a Linear Relationship for a Case Involving Only a Single Variable}

Most of the situations in practice involve more than one independent variable. In this case it is difficult to see the relationship between the dependent and independent variables and quantifying the relationship is not trivial. Linear regression analysis is one of the methods that can be used to quantify the relationship in a multivariable case. The different types of linear regression options available in RESRAD-OFFSITE and the different coefficients in the regression output of the code are described in the following subsections.

\section{C.3.6.1 Linear Regression on Raw Values}

Figures C.78 and C.79 show scatter plots of a dependent variable against each of two independent variables. The first independent variable has a range of 0.2 to 0.4 , while the second has a range of 0.2 to 0.8 . In this example, the dependent variable is one less than five times the first independent variable plus three times the second independent variable. One 
hundred samples of the independent variables and the corresponding values of the dependent variable are shown in Table C.4. Although the dependent variable is a linear function of each of the independent variables, the plots of dependent variable against each independent variable are not straight lines because of the contribution of the other independent variable. Both plots show a tendency of the dependent variable increasing with increasing values of the independent variables. The relationship between the dependent variable and the independent variables in this example can be quantified using a linear regression analysis.
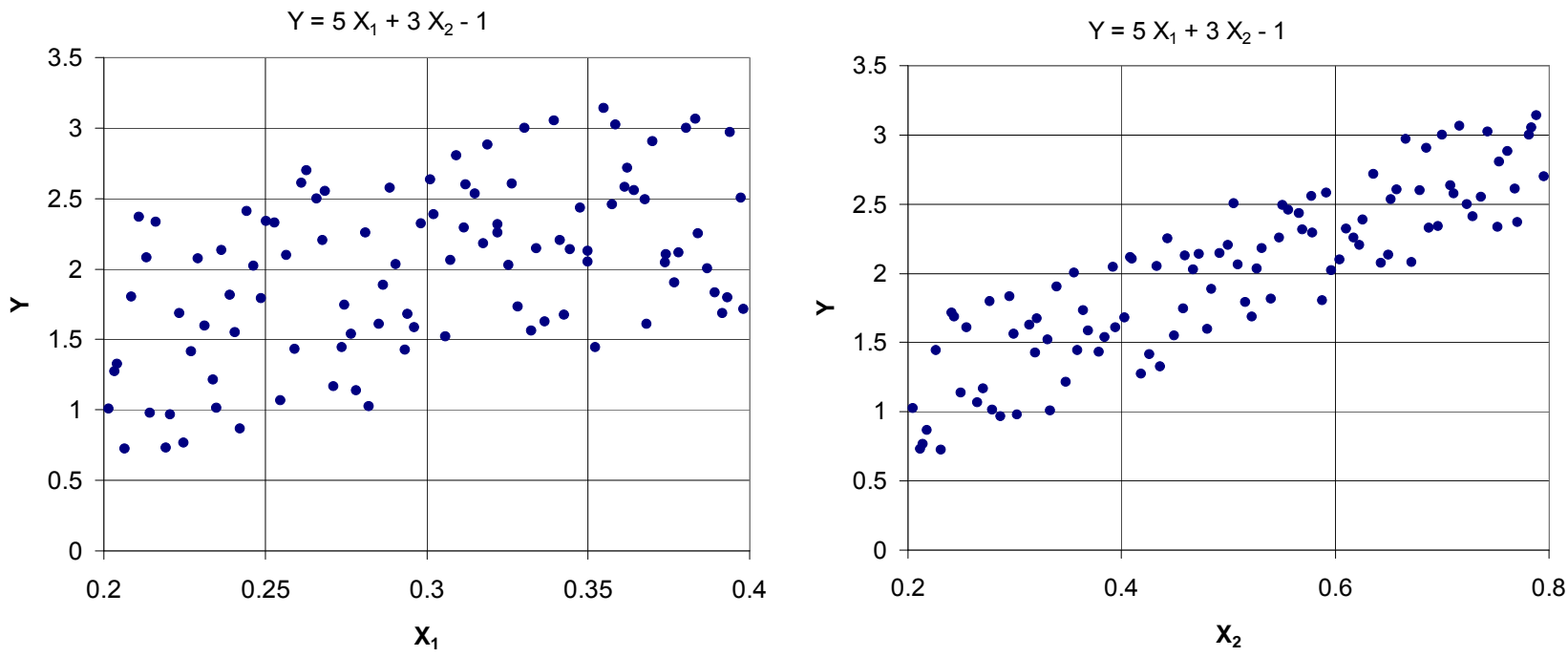

FIGURE C.78 (Left) Scatter Plot of the Dependent Variable and the First Independent Variable for a Case Involving a Linear Relationship with Two Independent Variables

\section{FIGURE C.79 (Right) Scatter Plot of the Dependent Variable and the Second Independent Variable for a Case Involving a Linear Relationship with Two Independent Variables}

The coefficient of determination of the linear regression on the data in this example (Table C.4) was 1.0; this coefficient is an indication of how much of the variation in the dose is explained by the linear regression. In this example the dependent variable is strictly a linear function of the two independent variables. Therefore, the regression between the dependent variable and the two independent variables was able to explain all the variation in the dependent variable in terms of the variation in the two independent variables, so the coefficient of determination was 1. 


\section{TABLE C.4 One Hundred Samples of Data Used in Section 3.6.1 to Illustrate Linear Regression on Raw Values}

\begin{tabular}{|c|c|c|c|c|c|c|c|c|}
\hline$X_{1}$ & $X_{2}$ & $Y=5 X_{1}+3 X_{2}-1$ & $X_{1}$ & $X_{2}$ & $Y=5 X_{1}+3 X_{2}-1$ & $X_{1}$ & $X_{2}$ & $Y=5 X_{1}+3 X_{2}-1$ \\
\hline 0.30930 & 0.75332 & 2.80643 & 0.31508 & 0.65245 & 2.53277 & 0.32822 & 0.36408 & 1.73334 \\
\hline 0.35477 & 0.78836 & 3.13894 & 0.27370 & 0.35838 & 1.44363 & 0.37387 & 0.39191 & 2.04511 \\
\hline 0.34270 & 0.32052 & 1.67508 & 0.23660 & 0.64959 & 2.13178 & 0.36828 & 0.25533 & 1.60738 \\
\hline 0.34448 & 0.47251 & 2.13992 & 0.26774 & 0.62248 & 2.20614 & 0.33953 & 0.78409 & 3.04991 \\
\hline 0.32648 & 0.65787 & 2.60598 & 0.25471 & 0.26501 & 1.06857 & 0.31203 & 0.67937 & 2.59824 \\
\hline 0.37681 & 0.33943 & 1.90235 & 0.38704 & 0.35577 & 2.00252 & 0.20348 & 0.41810 & 1.27171 \\
\hline 0.23911 & 0.53951 & 1.81405 & 0.36208 & 0.63572 & 2.71759 & 0.26590 & 0.72329 & 2.49937 \\
\hline 0.23124 & 0.48010 & 1.59653 & 0.32215 & 0.54773 & 2.25392 & 0.27460 & 0.45755 & 1.74568 \\
\hline 0.22346 & 0.52169 & 1.68235 & 0.29332 & 0.31950 & 1.42511 & 0.32540 & 0.46672 & 2.02715 \\
\hline 0.21625 & 0.75163 & 2.33613 & 0.29615 & 0.36849 & 1.58620 & 0.29044 & 0.52696 & 2.03308 \\
\hline 0.34769 & 0.56596 & 2.43632 & 0.28203 & 0.20445 & 1.02350 & 0.39406 & 0.66622 & 2.96895 \\
\hline 0.33050 & 0.78140 & 2.99670 & 0.34998 & 0.43280 & 2.04829 & 0.39321 & 0.27649 & 1.79550 \\
\hline 0.35235 & 0.22642 & 1.44101 & 0.20153 & 0.33296 & 1.00651 & 0.35743 & 0.55594 & 2.45497 \\
\hline 0.25664 & 0.60419 & 2.09580 & 0.36148 & 0.59171 & 2.58254 & 0.30215 & 0.62616 & 2.38924 \\
\hline 0.39820 & 0.24120 & 1.71460 & 0.20645 & 0.23083 & 0.72472 & 0.27813 & 0.24969 & 1.13971 \\
\hline 0.34127 & 0.49956 & 2.20504 & 0.22475 & 0.21393 & 0.76555 & 0.33244 & 0.29942 & 1.56047 \\
\hline 0.22724 & 0.42613 & 1.41456 & 0.38047 & 0.69975 & 3.00163 & 0.26857 & 0.73646 & 2.55224 \\
\hline 0.36436 & 0.57751 & 2.55435 & 0.33655 & 0.31362 & 1.62362 & 0.28523 & 0.39450 & 1.60968 \\
\hline 0.35001 & 0.45956 & 2.12874 & 0.25022 & 0.69621 & 2.33974 & 0.37425 & 0.41001 & 2.10127 \\
\hline 0.23482 & 0.27932 & 1.01207 & 0.30754 & 0.50867 & 2.06370 & 0.24059 & 0.44920 & 1.55054 \\
\hline 0.20417 & 0.43580 & 1.32827 & 0.24441 & 0.72911 & 2.40940 & 0.37007 & 0.68544 & 2.90667 \\
\hline 0.25912 & 0.37872 & 1.43177 & 0.22920 & 0.64310 & 2.07530 & 0.31765 & 0.53120 & 2.18187 \\
\hline 0.38404 & 0.44306 & 2.24939 & 0.24216 & 0.21814 & 0.86521 & 0.24876 & 0.51573 & 1.79101 \\
\hline 0.24648 & 0.59628 & 2.02124 & 0.28668 & 0.48430 & 1.88630 & 0.37811 & 0.40853 & 2.11616 \\
\hline 0.32193 & 0.56945 & 2.31802 & 0.21322 & 0.67157 & 2.08083 & 0.30588 & 0.33091 & 1.52216 \\
\hline 0.39734 & 0.50508 & 2.50192 & 0.38928 & 0.29530 & 1.83229 & 0.21103 & 0.77040 & 2.36633 \\
\hline 0.33402 & 0.49210 & 2.14638 & 0.35847 & 0.74299 & 3.02131 & 0.23386 & 0.34800 & 1.21328 \\
\hline 0.36776 & 0.55055 & 2.49044 & 0.31154 & 0.57829 & 2.29254 & 0.21427 & 0.30252 & 0.97892 \\
\hline 0.28113 & 0.61754 & 2.25825 & 0.38336 & 0.71664 & 3.06671 & 0.29835 & 0.61064 & 2.32367 \\
\hline 0.27657 & 0.38426 & 1.53563 & 0.30124 & 0.70825 & 2.63097 & 0.20849 & 0.58751 & 1.80499 \\
\hline 0.31883 & 0.76158 & 2.87887 & 0.28857 & 0.71073 & 2.57505 & 0.29426 & 0.40256 & 1.67899 \\
\hline 0.39157 & 0.24306 & 1.68701 & 0.27110 & 0.27058 & 1.16723 & 0.26282 & 0.79544 & 2.70045 \\
\hline \multirow[t]{2}{*}{0.22067} & 0.28673 & 0.96355 & 0.21941 & 0.21158 & 0.73180 & 0.25299 & 0.68777 & 2.32826 \\
\hline & & & 0.26134 & 0.76788 & 2.61035 & & & \\
\hline
\end{tabular}


The partial correlation coefficient between the dependent variable and the first independent variable was 1.0, as was the partial correlation coefficient between the dependent variable and the second independent variable. The partial correlation coefficient is a measure of how linear the relationship is between the dependent variable and one of the independent variables after accounting for the effects of all the other independent variables. In this case it is unity for both cases because the dependent variable is strictly linearly related to each of the independent variables. The correlation coefficients between the dependent variable and each of the two independent variables, on the other hand, would not be equal to unity because of the effects of the other dependent variable. The correlation coefficients were 0.49 (dependent variable and the first independent variable) and 0.88 (dependent variable and the second independent variable) in this example.

The regression coefficients for the first and second independent variables were 5 and 3 , respectively. The regression analysis was able to determine the exact multipliers that were used to compute the dependent variable in this example because of the strict linear relationship that was used in the initial computations of the dependent variable. They would have been close to these values if the computation of the dependent variable had included some random error as well.

The coefficient of determination can be used to gauge how well a particular regression explains the variations in the dependent variable. This can help decide whether to use those particular regression results to select a set of significant inputs. The partial correlation coefficients show how close the relationships between the dependent variable and each of the independent variables are to being linear, but they do not indicate how much influence each independent variable has on the variability of the predicted dose. The regression coefficients show how the dependent variable is related to the independent variables under a linear model.

There are two related considerations that limit their use as indicators of the influence of the corresponding independent variable on the dependent variable: (1) the units of the correlation coefficient and (2) the spread or range of the independent variables. The regression coefficients are represented by $c_{i} s$ in the expression $Y=\sum c_{i} X_{i}$. The units of each regression coefficient $c_{i}$ are the units of the dependent variable, $Y$, divided by the units of the corresponding independent variable, $X_{i}$.

If the independent variables are of different units, so will be the regression coefficients. It is not meaningful to use the numerical values of regression coefficients with different units to decide on the factors that have a significant influence on the predicted dose, because the numerical value can be changed simply by changing the units of the independent variable. For example, a regression coefficient can be changed by a factor of 1000 simply by changing the units of a length-based independent variable from $\mathrm{m}$ to $\mathrm{km}$. 
The contribution of an independent variable to the variation of the dependent variable depends not only on the regression coefficient but also on the spread of the independent variable. If two independent variables are spread out in approximately the same manner, the one with the higher regression coefficient will have a larger influence on the variation of the dependent variable. If the regression coefficients of two independent variables are approximately the same, the one that is more spread out will have a larger influence on the variation of the dependent variable.

The product of the regression coefficient and the measure of the spread of the variable gives a good indication of the effect of the independent variable on the variation of the dependent variable. One way to quantify the spread is by the range - this only describes how far apart the two extreme values are, and is not affected by how the other samples are distributed within that range. The standard deviation of the samples of an independent variable captures the spread of all the samples and is therefore a better measure of the spread.

The numerical values of the regression coefficients for the two independent variables in this example are 5 and 3 . They would be in the units of the dependent variable divided by the units of the corresponding independent variable. The standard deviations of the independent variables are 0.058 and 0.174 , each in the units of the corresponding variable. The products of the correlation coefficients and the corresponding standard deviations are of the same unitsthe units of the dependent variable-and can be compared to gauge the influence of the independent variables on the variability of the dependent variable. It is customary to divide each of these products by the standard deviation of the dependent variable. The resulting nondimensional coefficients, or "standardized regression coefficients," for the two independent variables in this example are 0.48 and 0.87 , respectively. When the first independent variable is changed by a standard deviation of its distribution, the dependent variable is expected to change by 0.48 of its distribution according to the linear regression.

\section{C.3.6.2 Linear Regression on the Ranks of the Values}

Many of the inputs of RESRAD-OFFSITE influence the peak predicted dose in a nonlinear fashion. A linear approximation is applicable when the range of those inputs is small. The deviation from linearity increase as the range of the inputs grows larger. Figures C.80-C.82 show scatter plots of a dependent variable against each of three independent variables. The dependent variable in this example is the sum of the first independent variable, the square of the second independent variable and the inverse of the third independent variable. A linear regression may not be able model the nonlinear relationship especially when the deviation from linearity is large. Each of the three plots shows the relationship between the dependent variable and the corresponding independent variable and also the scatter of the dependent variable due to the other two independent variables. 
The relationship between the dependent variable and the first independent variable is linear (Figure C.80). The contribution of the second independent variable to the dependent variable increases as that variable increases throughout its range. The contribution of the third independent variable to the dependent variable decreases through its range. These relationships are monotonic; there is a one to one relationship between the dependent and independent variables. Monotonic relationships can be linearized by using the ranks of the values instead of the raw values. The rank is the place of a value in a sorted list. Figures C.83C.85 show scatter plots of the ranks of the data of the dependent variable against the ranks of the data of each of the three independent variables.

Linear regression can be performed on the ranks of the data. The resulting coefficient of determination indicates how much of the variation in the rank of the dependent variable is explained by the linear regression model on the ranks. If this is greater than the coefficient of determination of the regression on the raw data, the results of the regression on the ranks of the data can be used to select the inputs that have a significant influence on the dependent variable. The standardized rank regression coefficients are used to identify the inputs that have a significant influence in this case.

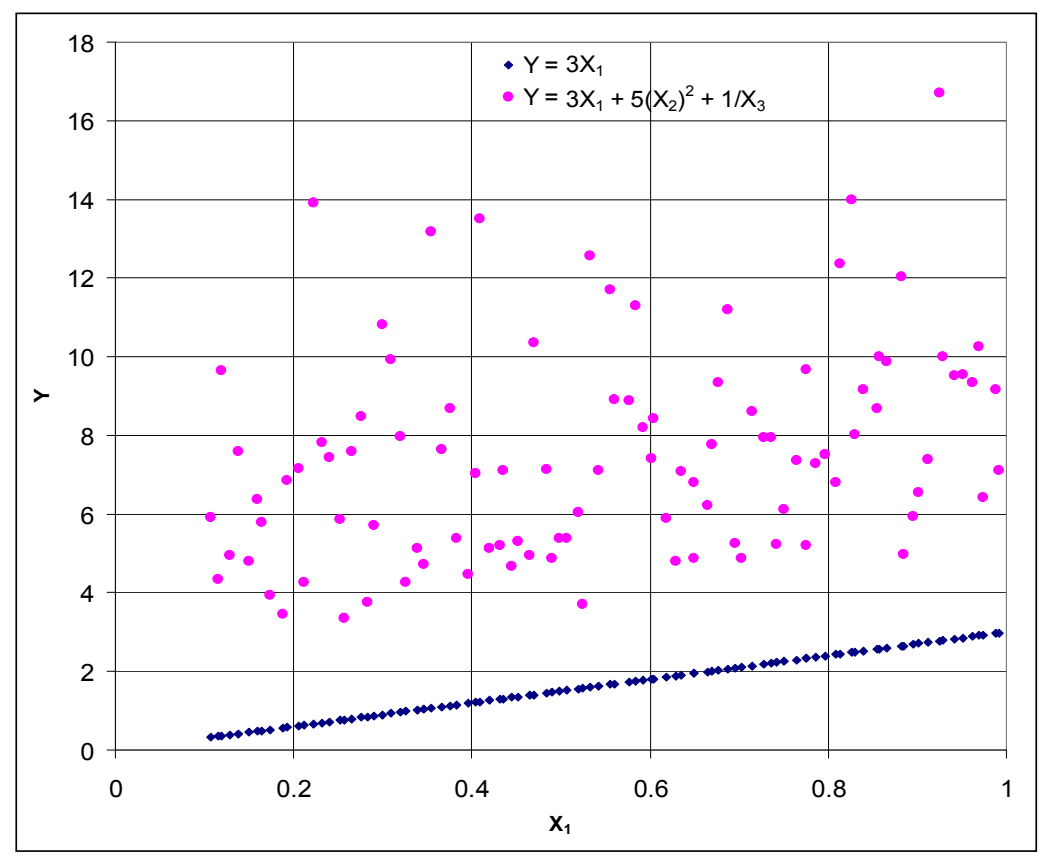

FIGURE C.80 Scatter Plot of the Dependent Variable and the First Independent Variable for a Case Involving Linear and Nonlinear Relationships with Three Independent Variables 


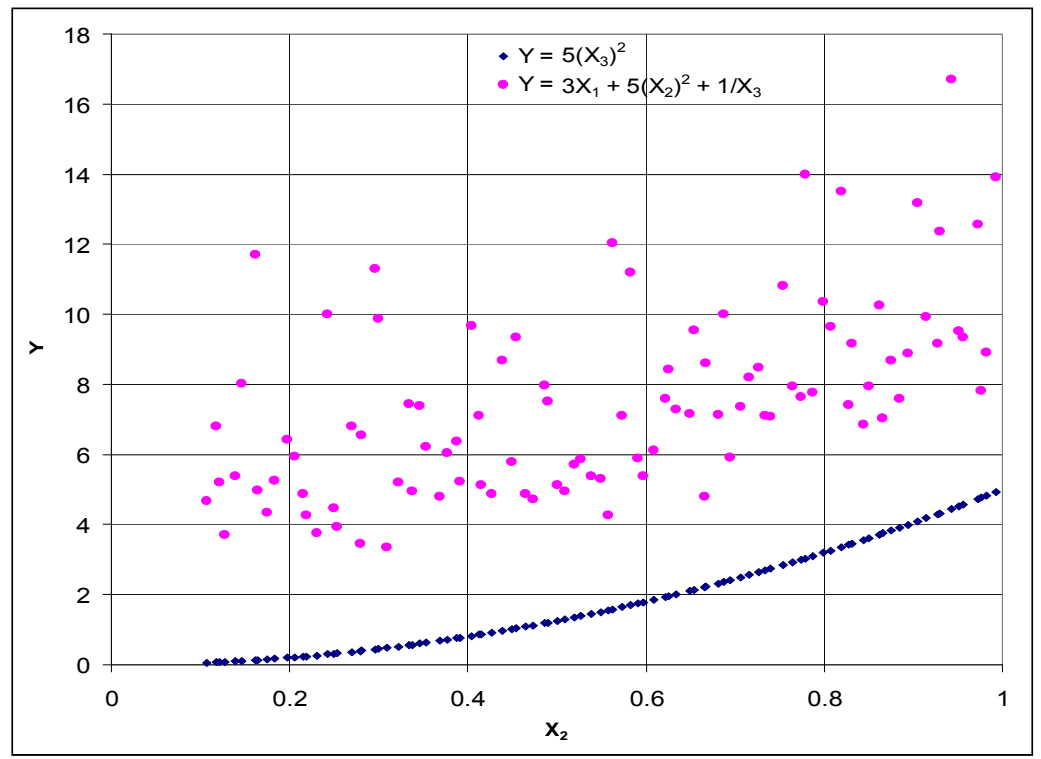

FIGURE C.81 Scatter Plot of the Dependent Variable and the Second Independent Variable for a Case Involving Linear and Nonlinear Relationships with Three Independent Variables

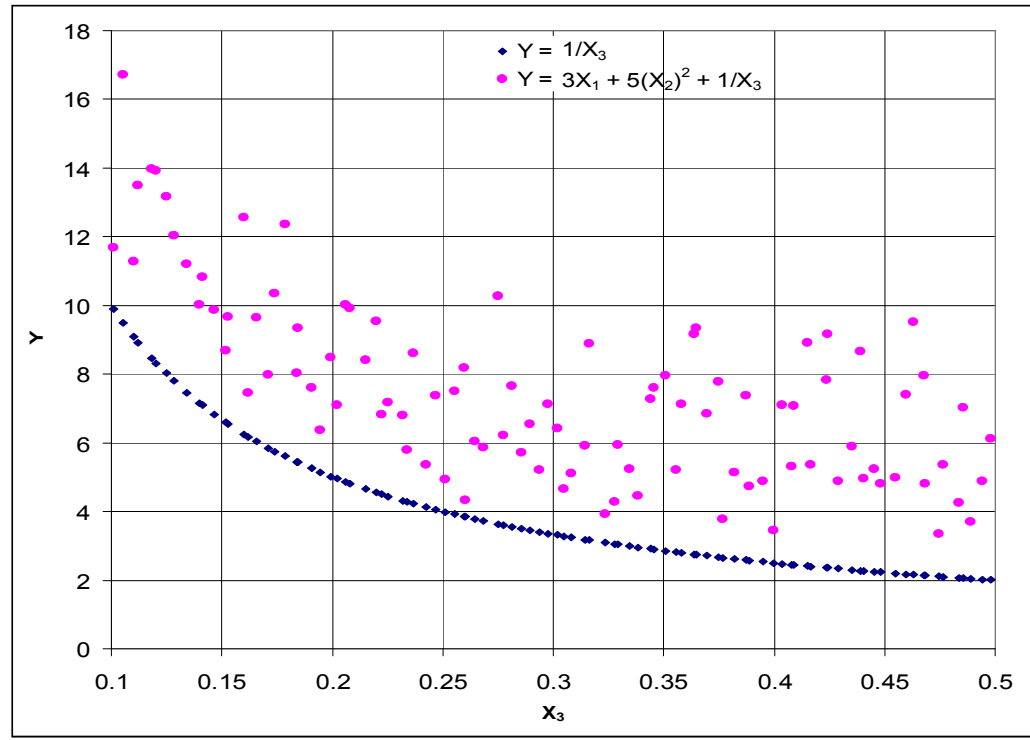

FIGURE C.82 Scatter Plot of the Dependent Variable and the Third Independent Variable for a Case Involving Linear and Nonlinear Relationships with Three Independent Variables 


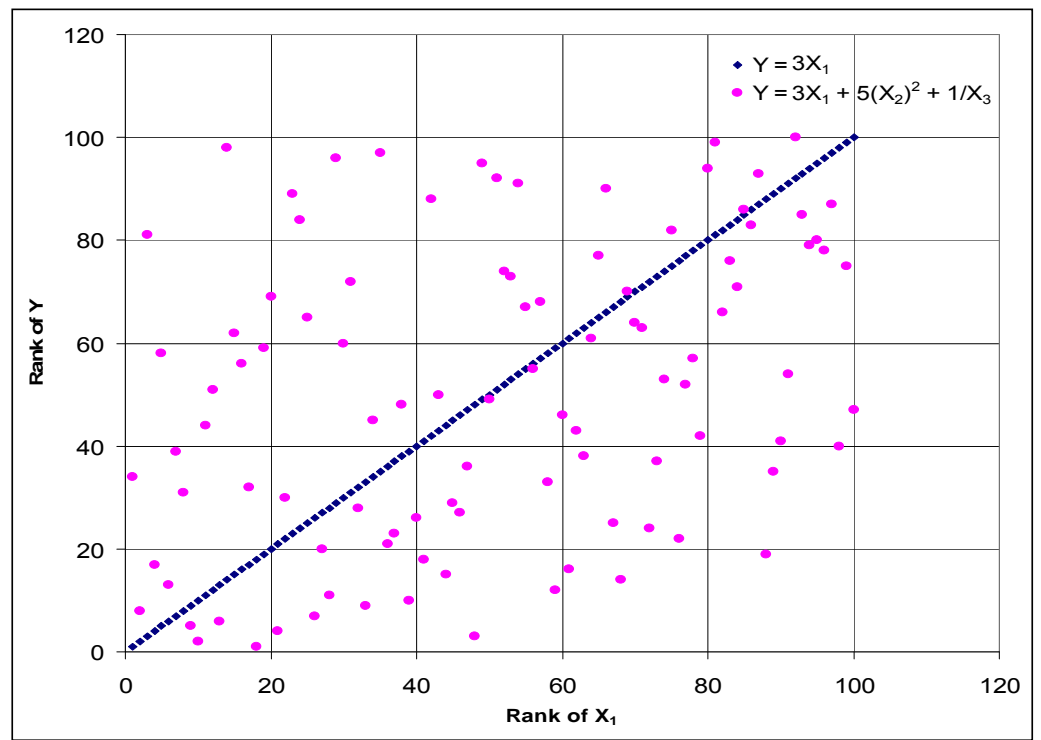

FIGURE C.83 Scatter Plot of the Rank of the Dependent Variable and the Rank of the First Independent Variable for a Case Involving Linear and Nonlinear Relationships with Three Independent Variables

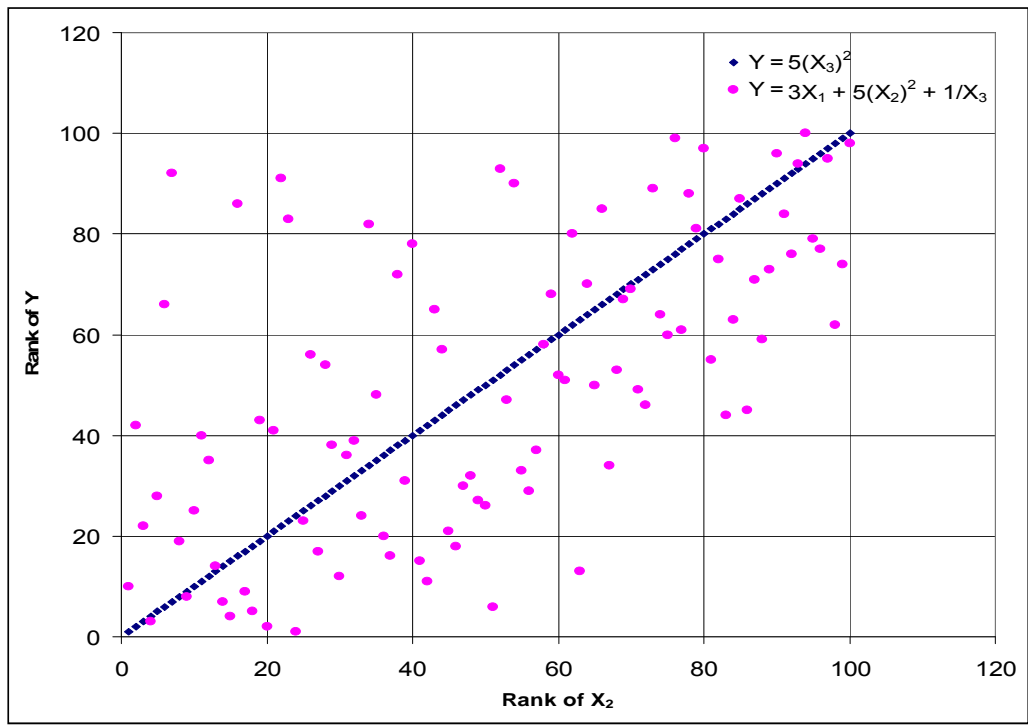

FIGURE C.84 Scatter Plot of the Rank of the Dependent Variable and the Rank of the Second Independent Variable for a Case Involving Linear and Nonlinear Relationships with Three Independent Variables 


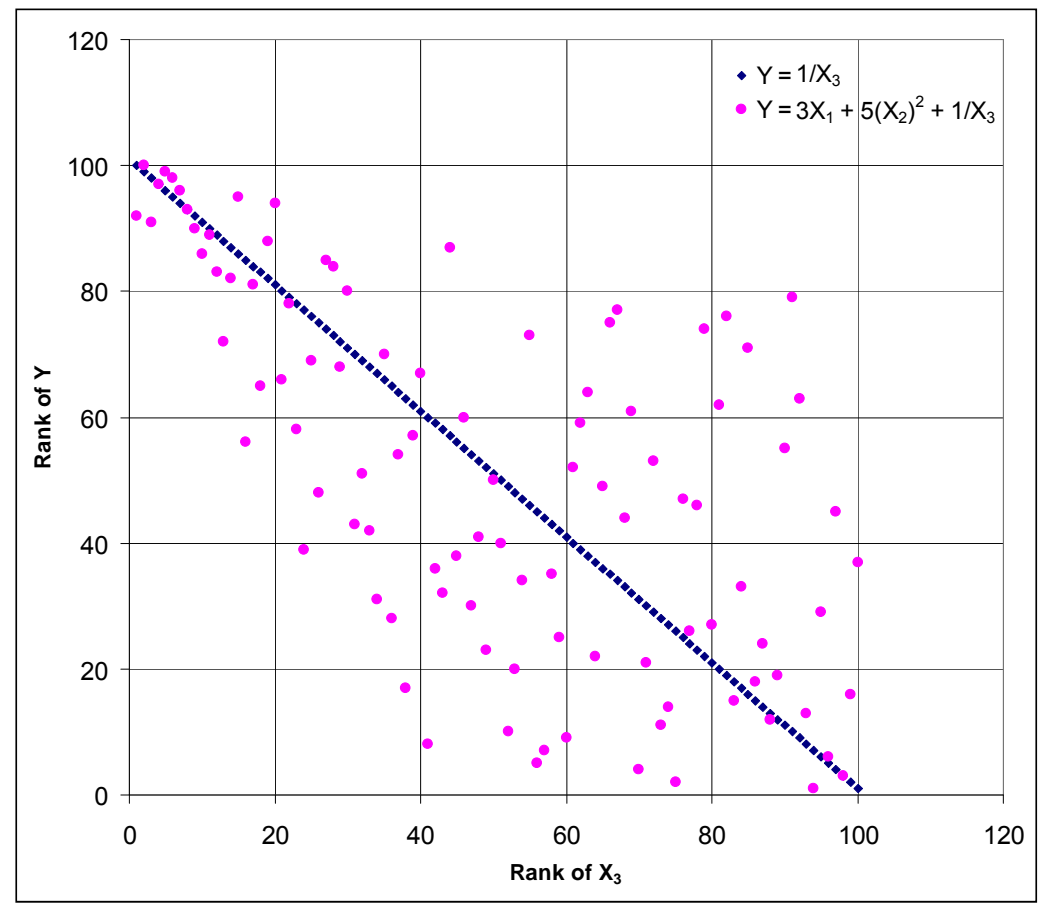

FIGURE C.85 Scatter Plot of the Rank of the Dependent Variable and the Rank of the Third Independent Variable for a Case Involving Linear and Nonlinear Relationships with Three Independent Variables

\section{C.4 Multiple-input Sensitivity Analysis}

The application of the sensitivity analysis tools that were described in the preceding two chapters to identify the sensitive inputs is illustrated by means of a hypothetical scenario in this chapter. The details of the hypothetical scenario are set out in Appendix C.I. The example scenario involves 203 distributed inputs. It is impractical to perform single-input sensitivity on each of these inputs even if one were not aware of the drawbacks associated with the use of single-input sensitivity analysis for the purpose of identifying the set of significant inputs (Sections C.2 and C.3). A multi-input sensitivity analysis involving all 203 distributed inputs can be performed to identify the distributed inputs that make a significant contribution to the variability in the output, the peak predicted dose.

The number of times a distribution is sampled is termed the number of observations in RESRAD-OFFSITE; it is also referred to as the number of realizations. This must be large enough not only to ensure that the samples closely represent the distribution specified for each input, but also to ensure that they represent the multidimensional sample space of the 203 inputs. Each distribution is sampled 500 times in this example. The analysis can be 
repeated with a different set of 500 samples. If the RESRAD-OFFSITE output for the different sets of samples agree (if they are statistically not different), then the number of samples chosen is sufficient for that analysis. The number of samples must be increased if the outputs from the repetitions are statistically different.

The multi-input sensitivity analysis can be performed in stages using the command buttons in the "Step by step analysis" tab of the Uncertainty/Probabilistic Analysis form or it can be preformed all at once using the "run" command. In either case, the cumulative distribution function plots of the inputs can be used to judge whether the number of observations is sufficient to closely represent the distributions specified for the inputs. Figure C.86 is typical of the cumulative distribution function plots of the samples of the inputs used in this example. The plots for the three repetitions are indistinguishable, indicating that the number of observations is sufficient to closely represent the distributions used in this example. The cumulative distribution function plots of the samples of all the inputs must be checked to ensure that the plots of the repetitions are close. It is not as easy to verify that the number of observations chosen is sufficient to accurately represent the grouping in multidimensional space. One way to do this is to check the correlations between the samples of the inputs. Unfortunately, there are 20,503 $(=203 \times 202 \div 2)$ correlations between the 203 inputs—far too many numbers to look at for each repetition! A good check of whether the number of observations used is large enough is the cumulative distribution function of the output, the peak predicted dose. As seen in Figure C.87, the cumulative distribution functions of the peak predicted doses from three repetitions of this example are in good agreement.

\section{C.4.1 Identification of Significant Inputs}

RESRAD-OFFSITE has two aids to help identify the significant inputs: a regression report with the inputs sorted in descending order of significance and scatter plots of output against inputs. The scatter plots are available for all multi-input sensitivity analyses. The user chooses whether or not to perform regression analysis between the output and inputs, as well as whether to perform it on raw data, ranked data, or on both. If regression analysis is performed on both raw and ranked data, the code compares the coefficients of determination for the regression on the raw data and the regression the ranked data. The coefficient of determination measures how well a regression modeled the variation in the output (peak predicted dose). The absolute values of the standardized regression coefficients of the regression, raw or ranked, that has the higher coefficient of determination are used to sort the inputs in descending order of influence. 


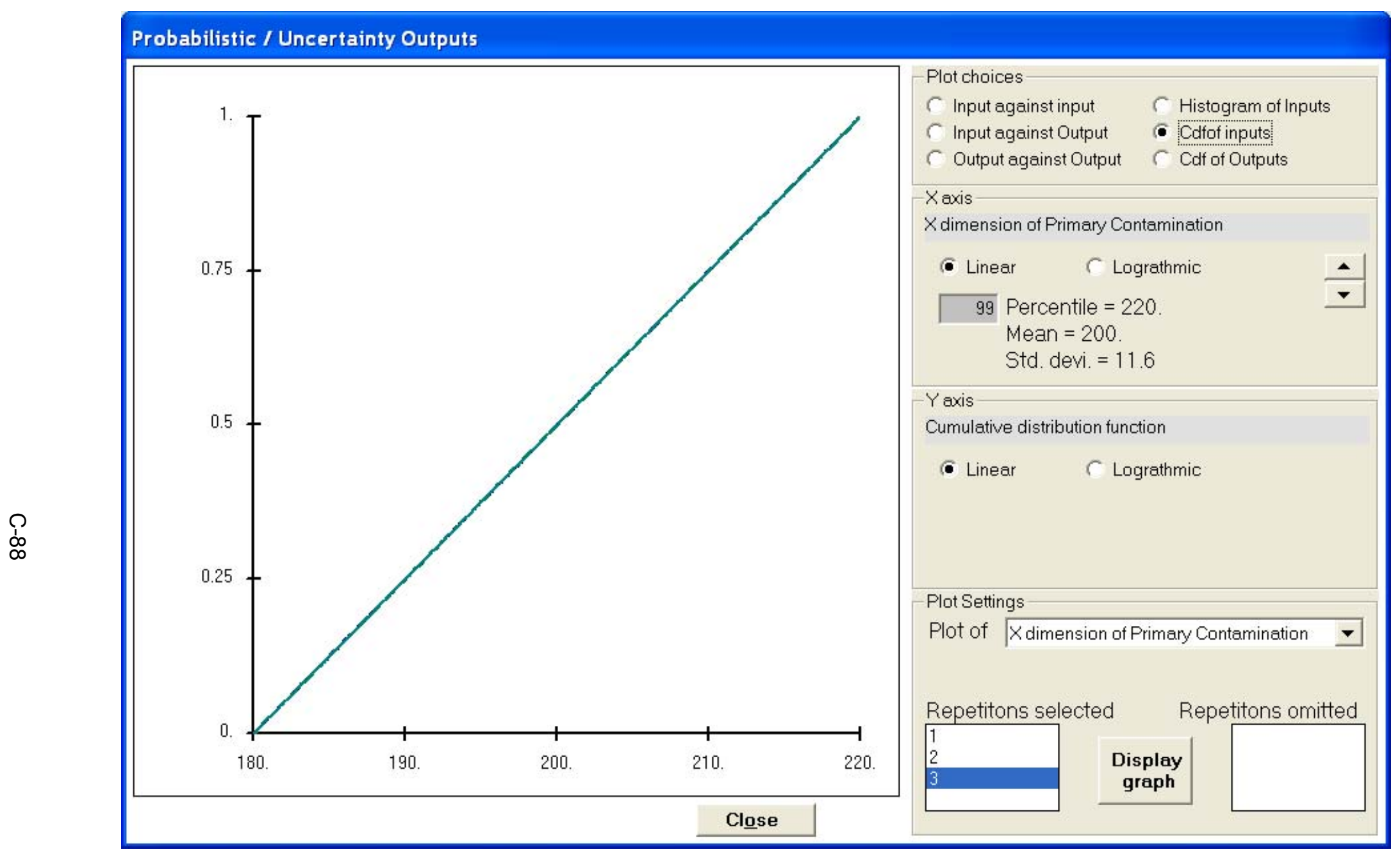

FIGURE C.86 Cumulative Distribution Function of One of the Inputs 


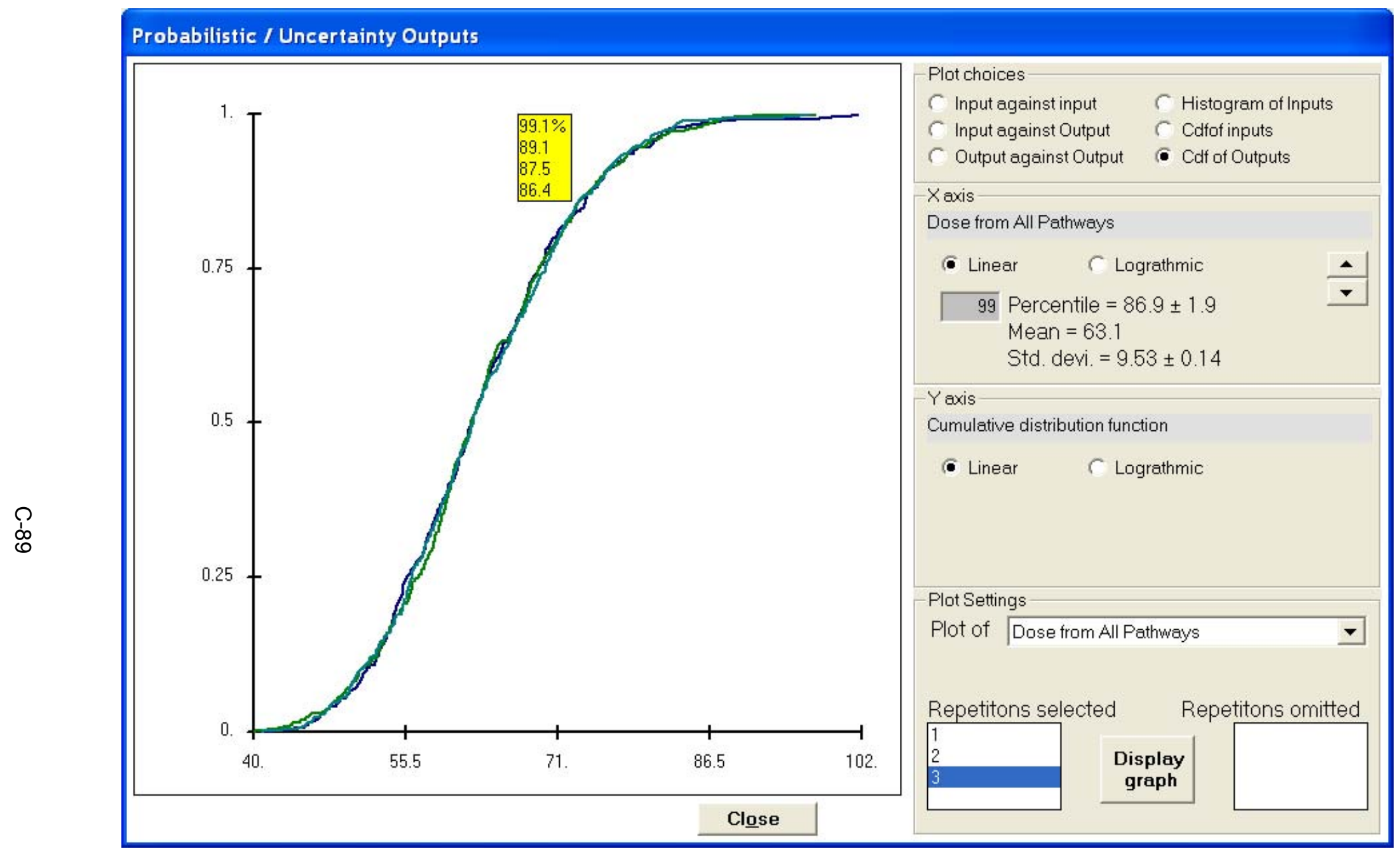

FIGURE C.87 Cumulative Distribution Function of the Output, the Peak Predicted Dose 


\section{C.4.2 Identifying Significant Inputs Using the Regression Report}

Part of the sorted regression report is shown in Figure C.88. The coefficient of determination of the regression on the raw data is just slightly greater than the coefficient of determination of the regression on the ranked data. The inputs are therefore sorted by the absolute values of the standardized regression coefficients. The standardized regression coefficient indicates by how many standard deviations the dose changes, according to the linear regression model of the raw data, when the input changes by a standard deviation. The top two inputs in this example, both relating to the source of water, have standardized regression coefficients of close to 0.4 . The next five inputs are around 0.3 , followed by four inputs with coefficients of 0.2 to 0.1 , and 17 inputs with standardized coefficients of 0.08 to 0.05 . It is easier to see this when the absolute values of the sorted standardized correlation coefficients are plotted in a bar chart, as in Figure C.89. The first 11 inputs are seen to have a significant influence on the variability in the dose; the next 19 have a moderate influence; and the following 10 have a mild influence. The remaining 160 inputs have very little influence on the variability in the dose at this stage. The standardized regression coefficients from the three repetitions are in good agreement, suggesting that the number of samples is sufficient.

\section{C.4.3 Identifying Significant Inputs Using Scatter Plots}

The scatter plots of peak predicted dose against each of the distributed inputs can also be used to identify which inputs have a significant influence on the peak predicted dose. Viewing the scatter plot of peak predicted dose against each distributed input in turn can be tedious when there are a large number of distributed inputs (203 in this case). The up/down scroll arrows in the X-axis frame at the upper right of the scatter plot (Figure C.90) help cycle through the scatter plot. If the distribution specified for the input warrants it, a logarithmic scale must be used for that input. This can slow down the viewing of the scatter plots. Scatter plots for inputs identified as having a significant, moderate, or mild influence on the peak predicted dose are shown in Figures C.90 through C.99. The 11 inputs that have a significant influence can be identified easily from the scatter plots (Figures C.90-C.92). It is difficult to identify the inputs that have a moderate influence on the peak predicted dose because of the scatter caused by variations in the other inputs. 


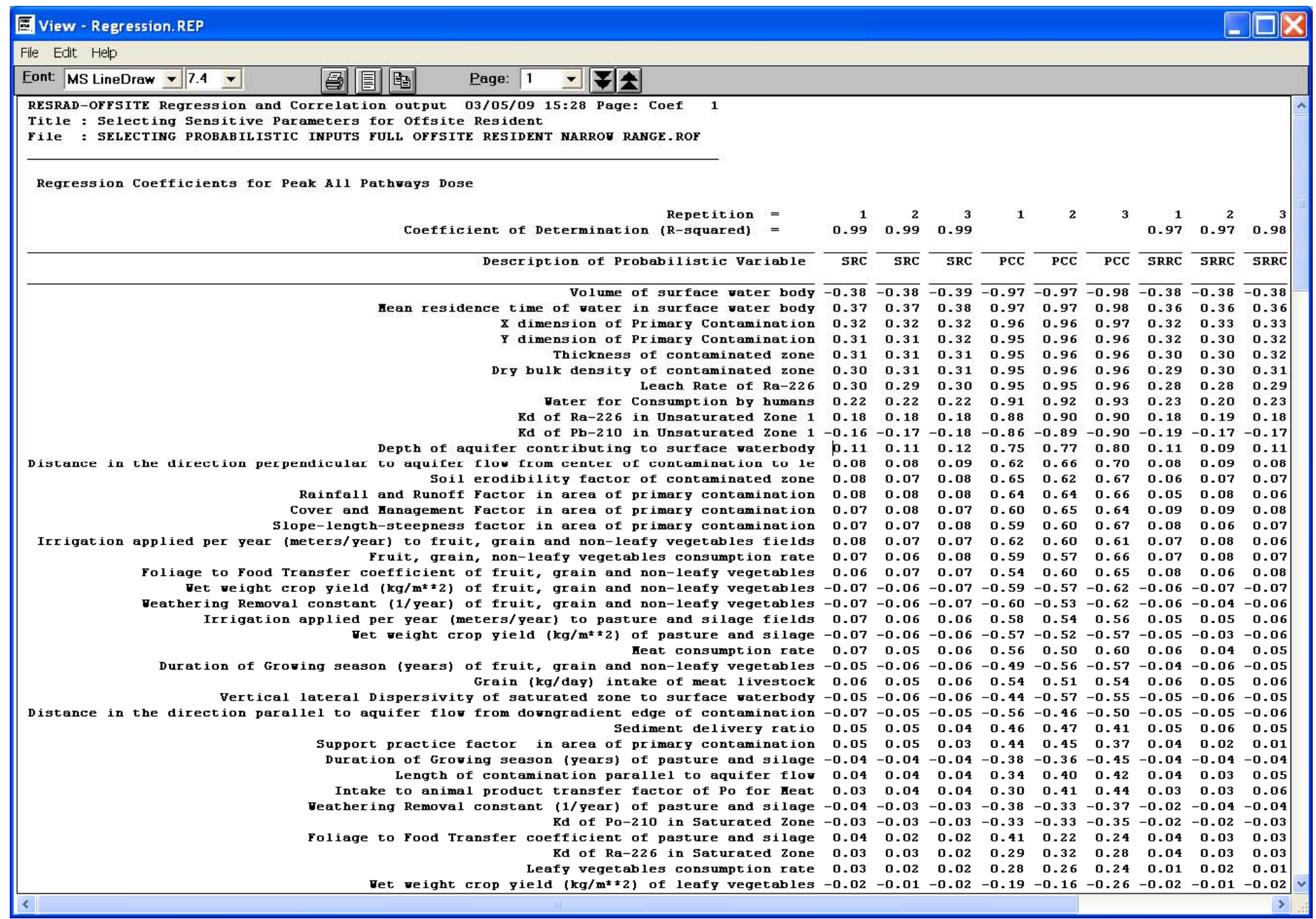

FIGURE C.88 Regression Report 


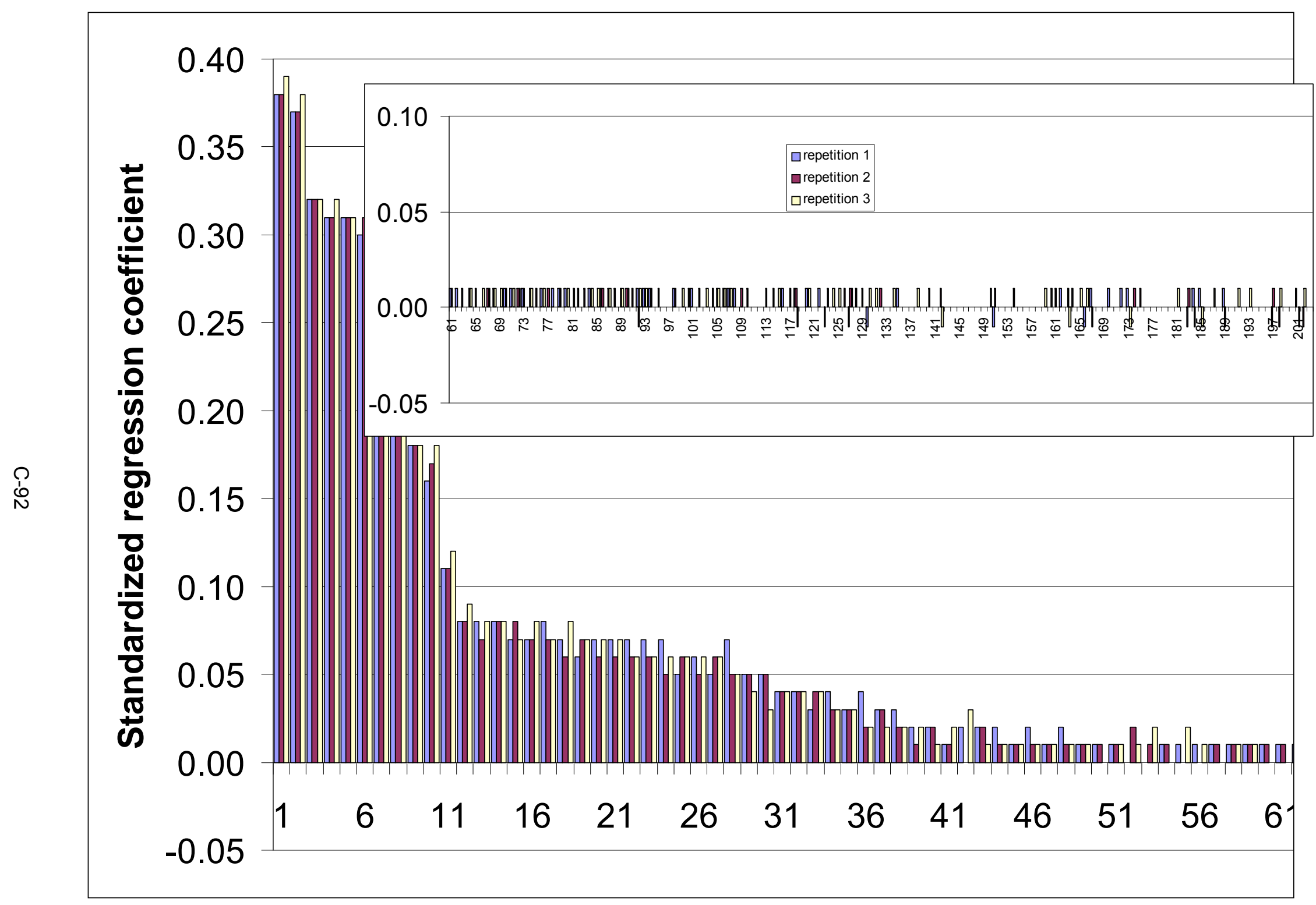

FIGURE C.89 Plot of the Standardized Regression Coefficients from the Regression Report 

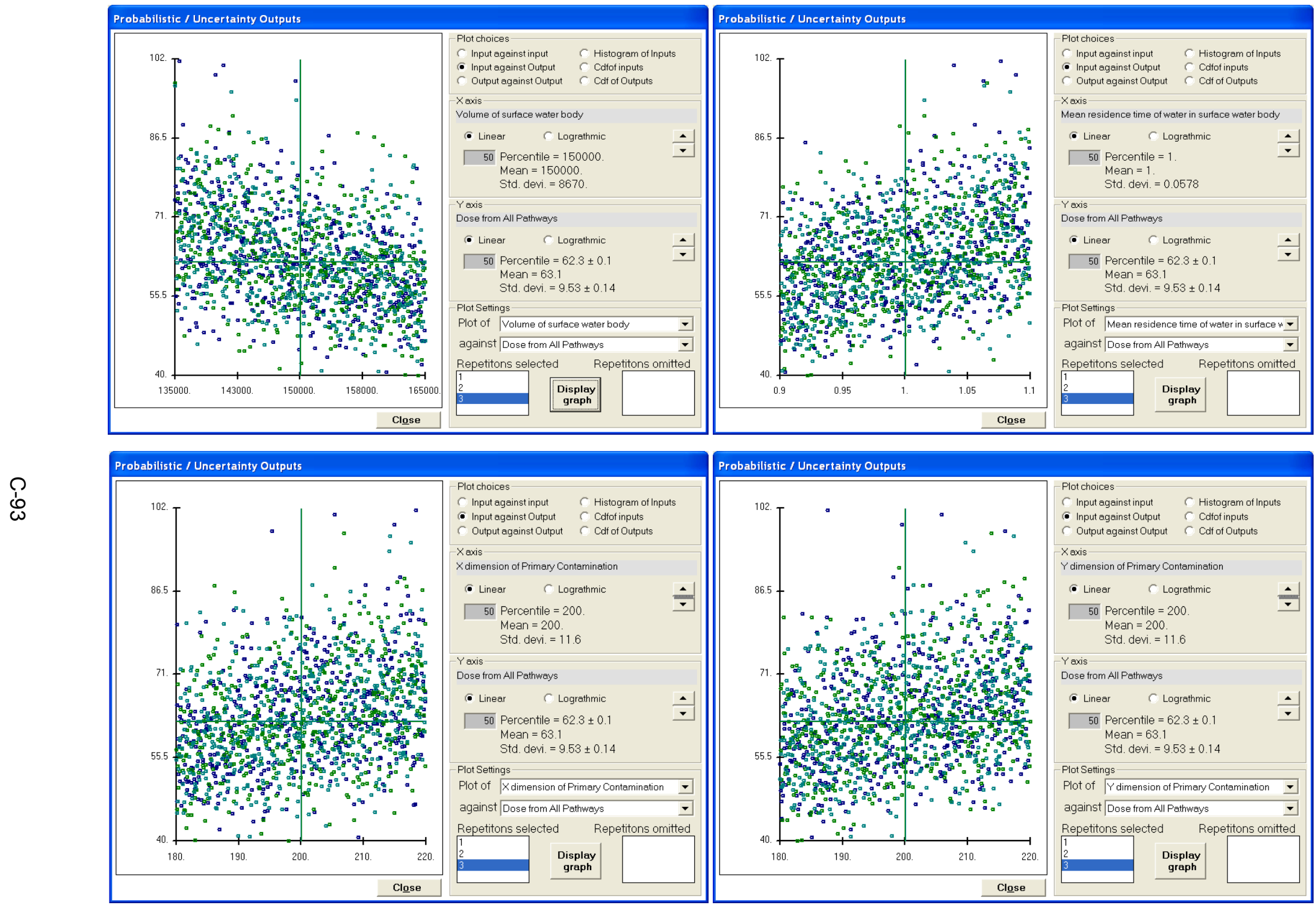

FIGURE C.90 Scatter Plots of Peak Predicted Dose against Inputs Identified as the Four Most Significant by the Regression Analysis 

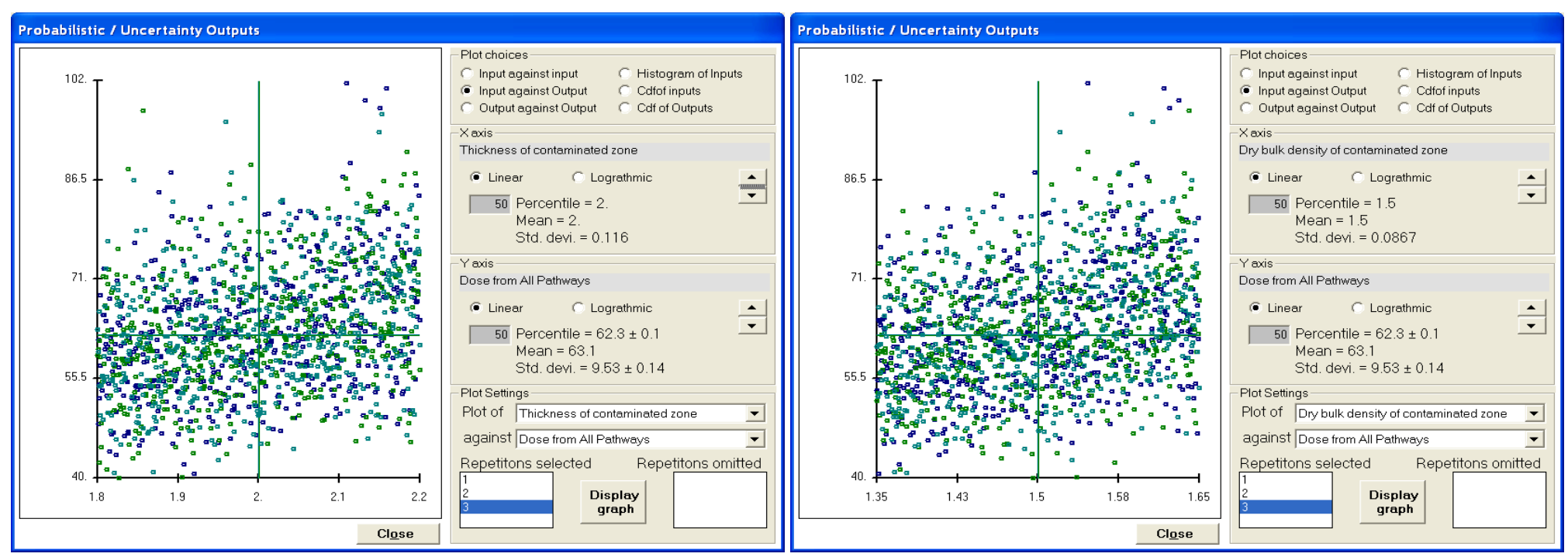

0
1
1
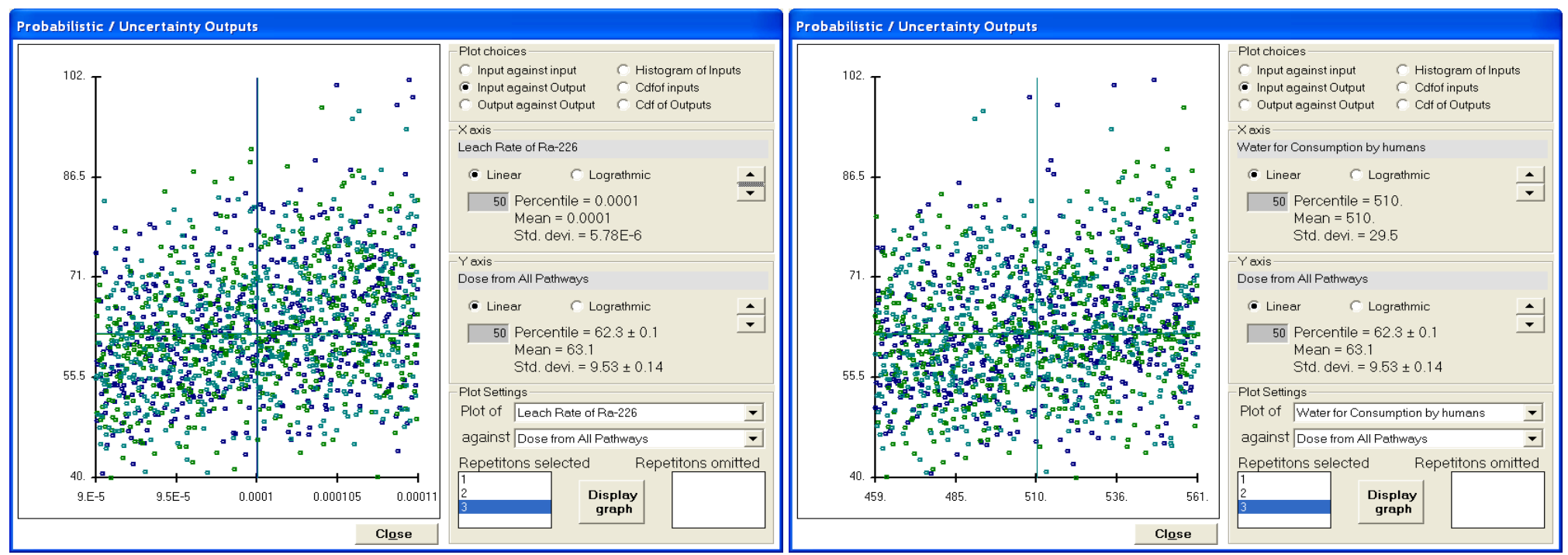

FIGURE C.91 Scatter Plots of Peak Predicted Dose against Inputs Identified as the Second Set of Four Most Significant by the Regression Analysis 

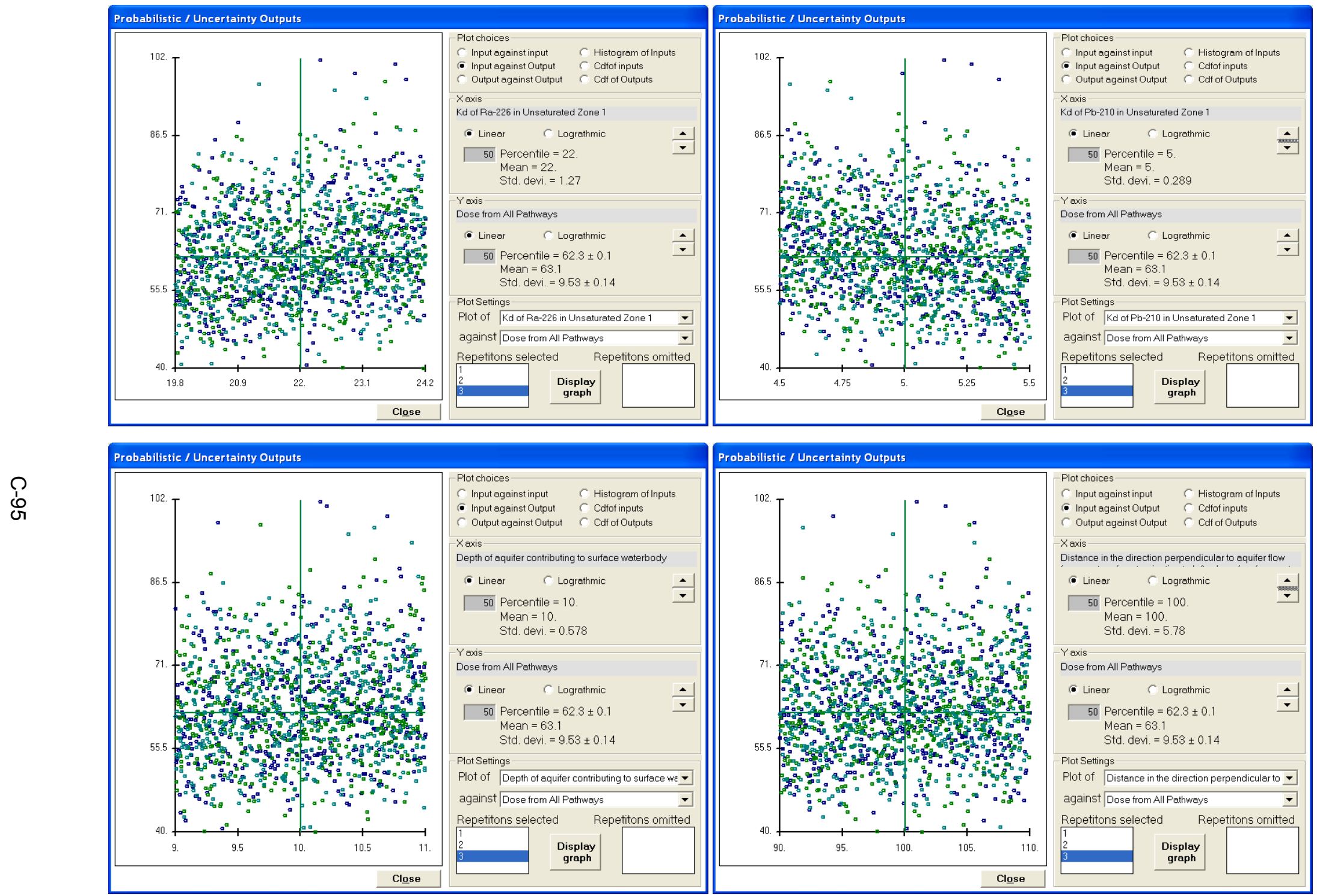

FIGURE C.92 Scatter Plots of Peak Predicted Dose against Inputs Identified as the Third Set of Four Most Significant by the Regression Analysis 

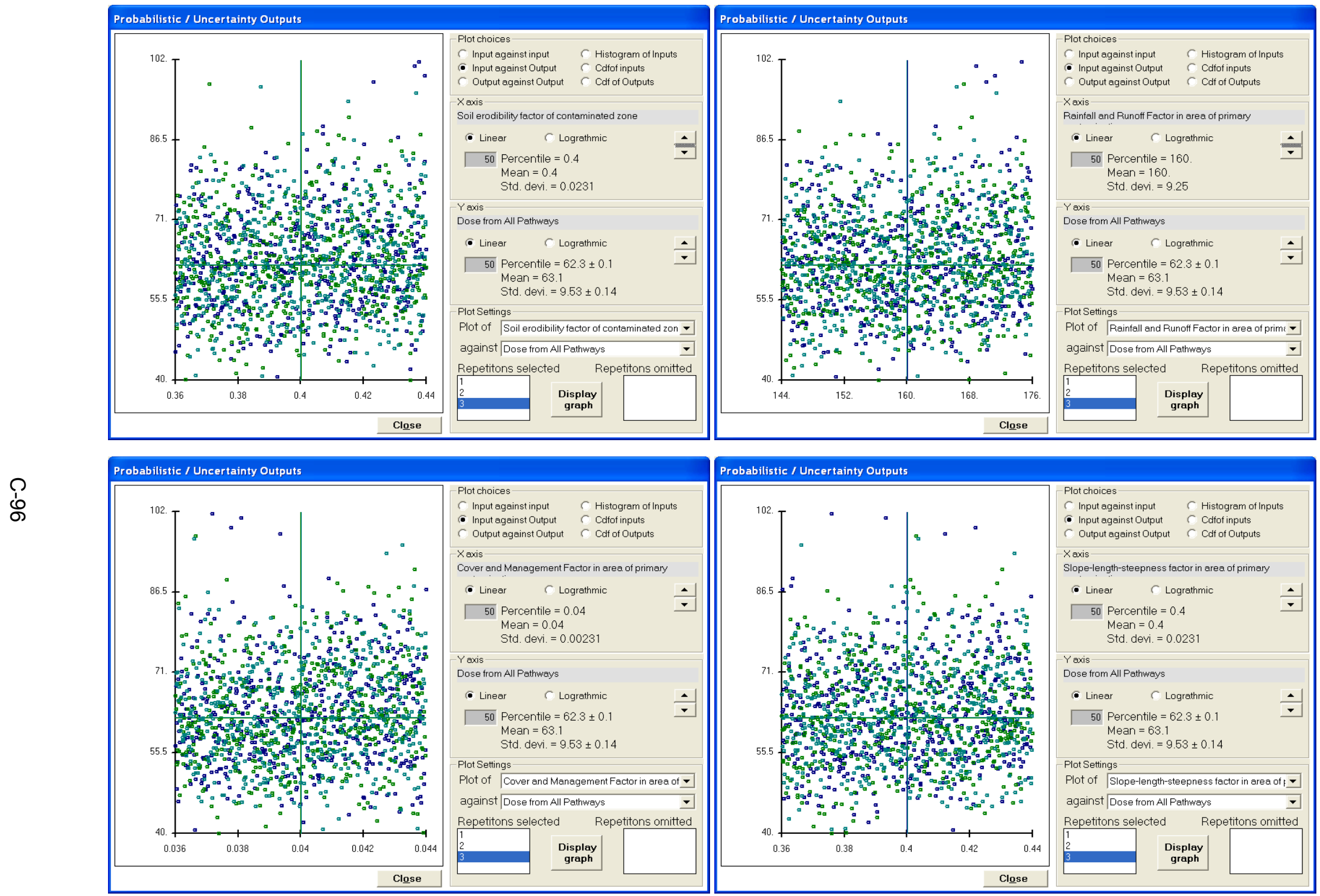

FIGURE C.93 Scatter Plots of Peak Predicted Dose against Inputs Identified as the Fourth Set of Four Most Significant by the Regression Analysis 

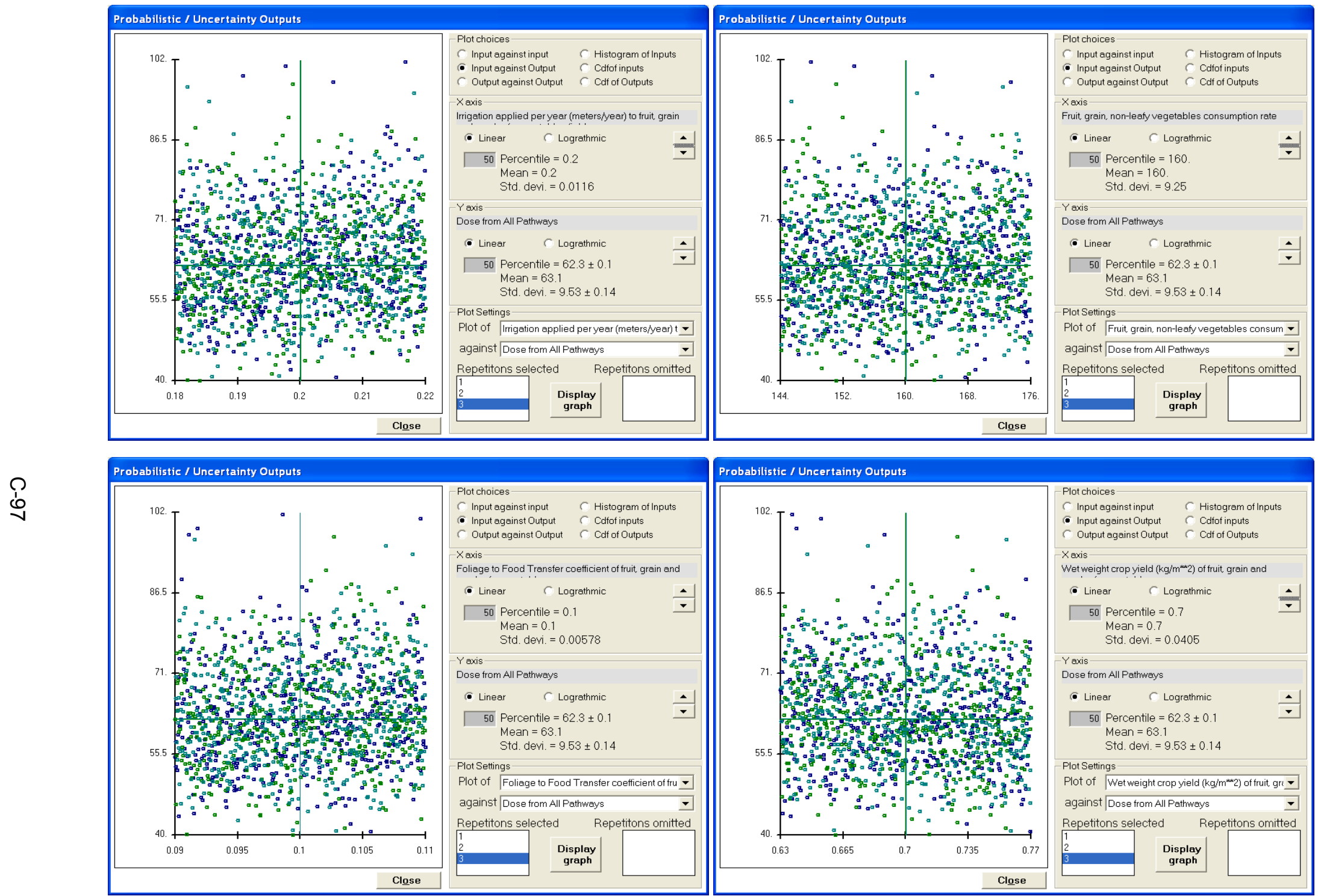

FIGURE C.94 Scatter Plots of Peak Predicted Dose against Inputs Identified as the Fifth Set of Four Most Significant by the Regression Analysis 

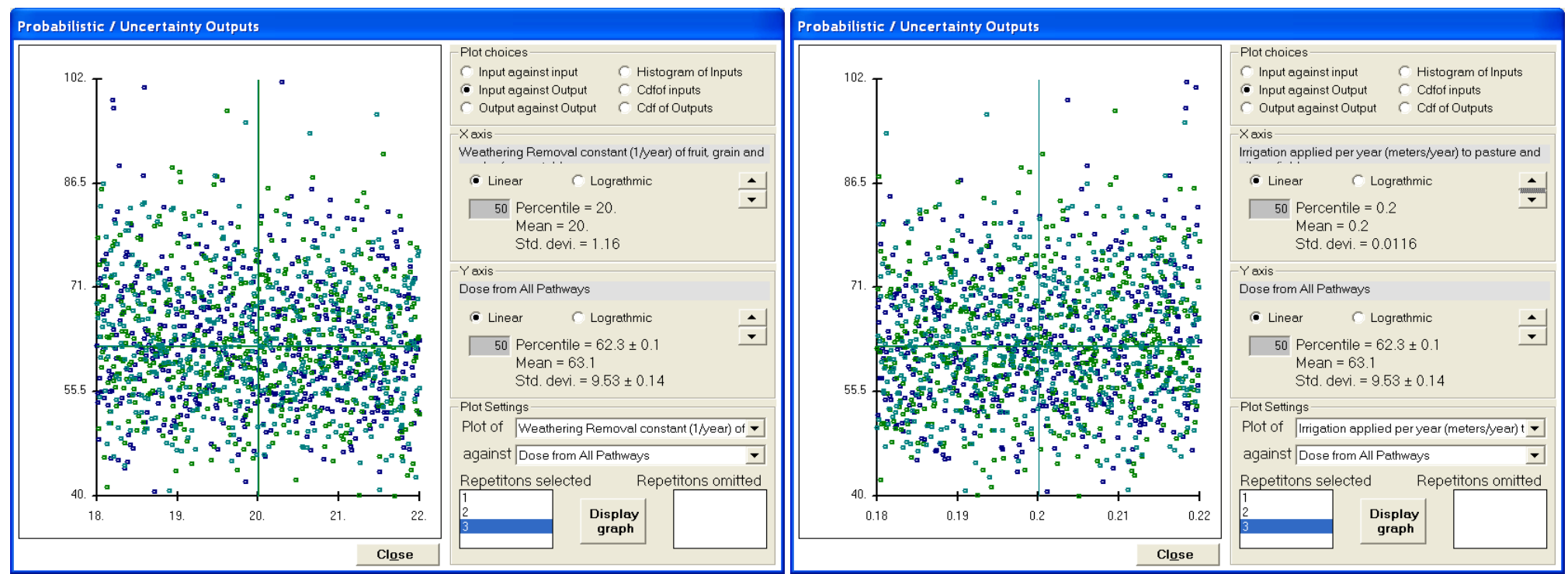

0
0
$\infty$
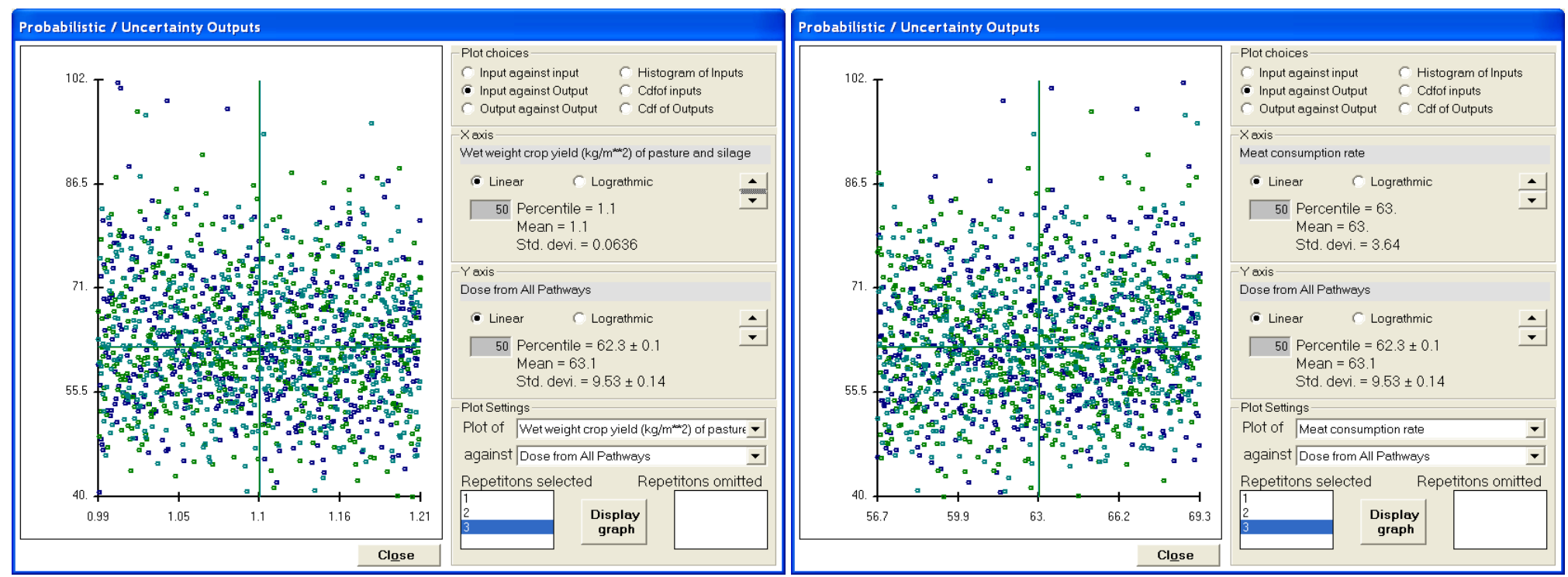

FIGURE C.95 Scatter Plots of Peak Predicted Dose against Inputs Identified as the Sixth Set of Four Most Significant by the Regression Analysis 

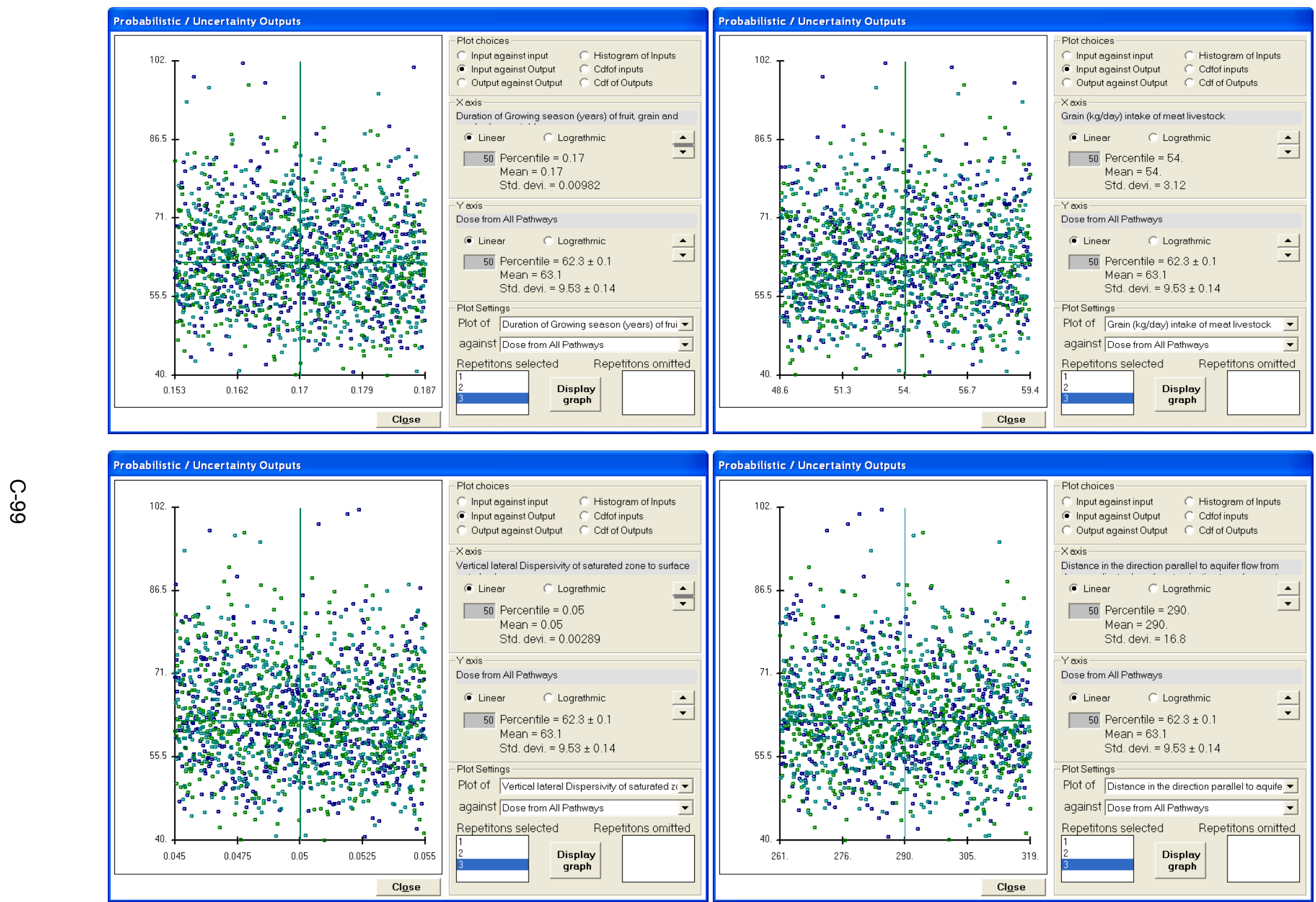

FIGURE C.96 Scatter Plots of Peak Predicted Dose against Inputs Identified as the Seventh Set of Four Most Significant by the Regression Analysis 

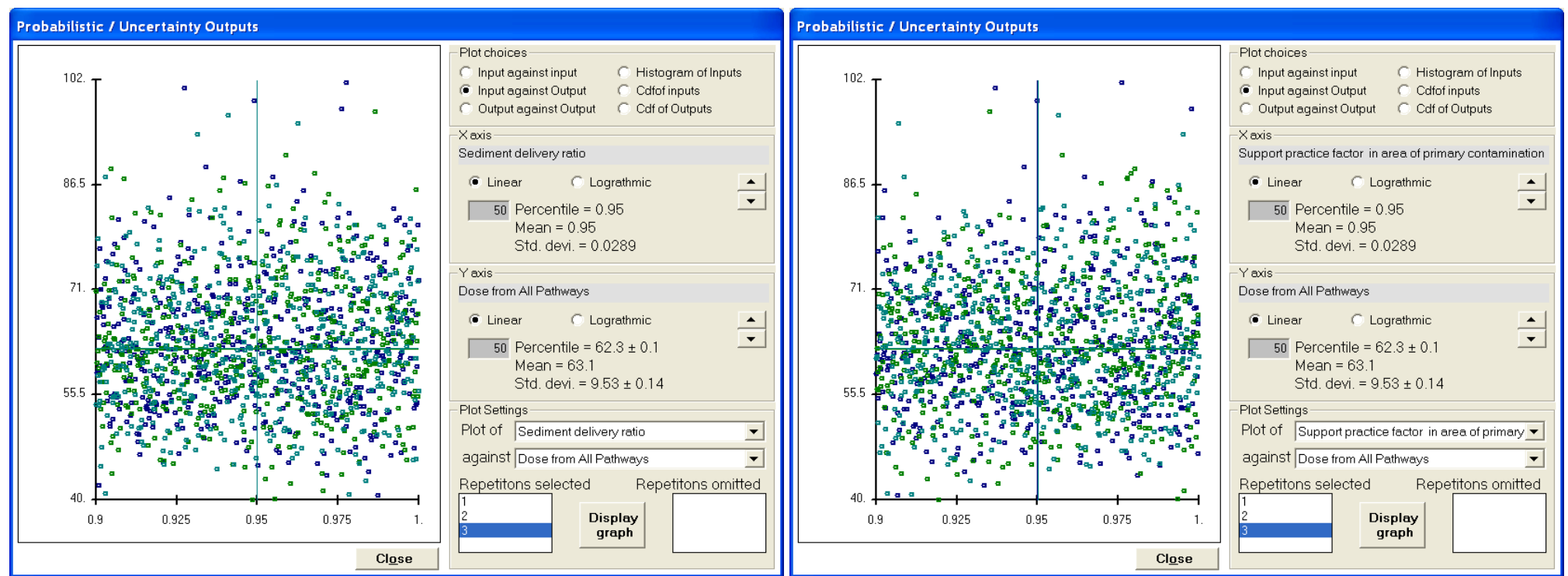

$\stackrel{\stackrel{1}{8}}{8}$
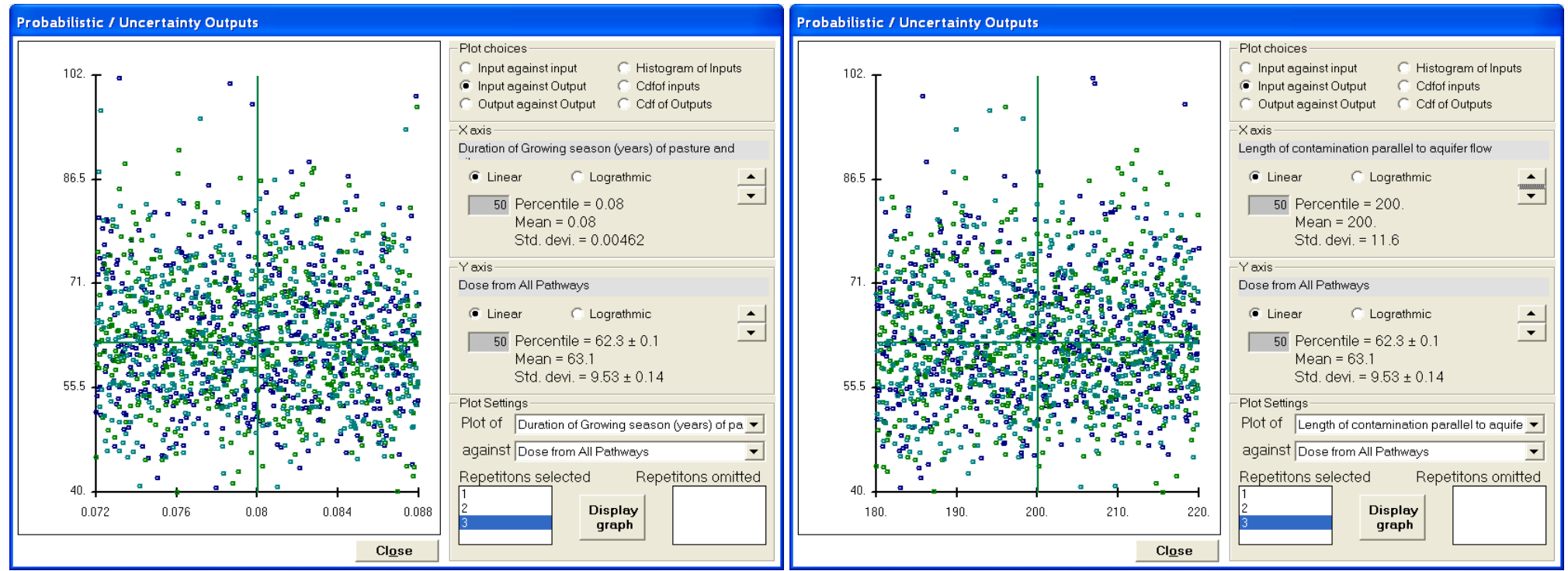

FIGURE C.97 Scatter Plots of Peak Predicted Dose against Inputs Identified as the Eighth Set of Four Most Significant by the Regression Analysis 

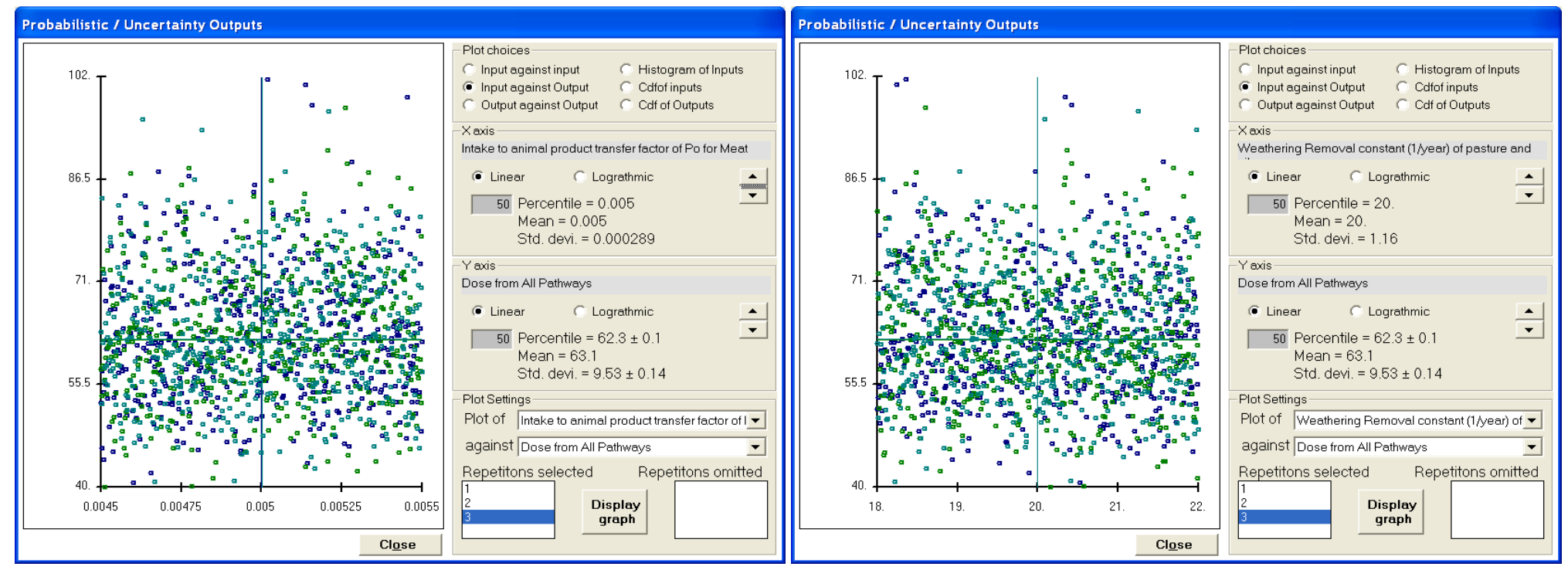

$\stackrel{?}{\stackrel{0}{\rightarrow}}$
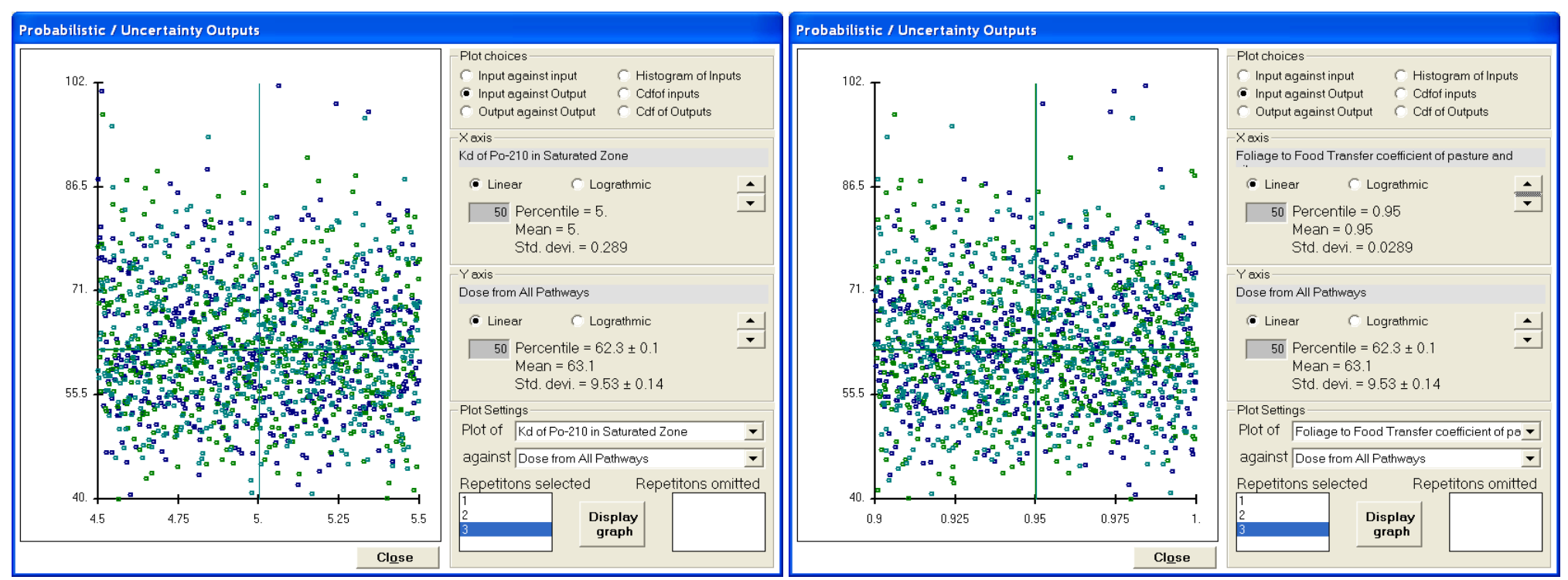

FIGURE C.98 Scatter Plots of Peak Predicted Dose against Inputs Identified as the Ninth Set of Four Most Significant by the Regression Analysis 

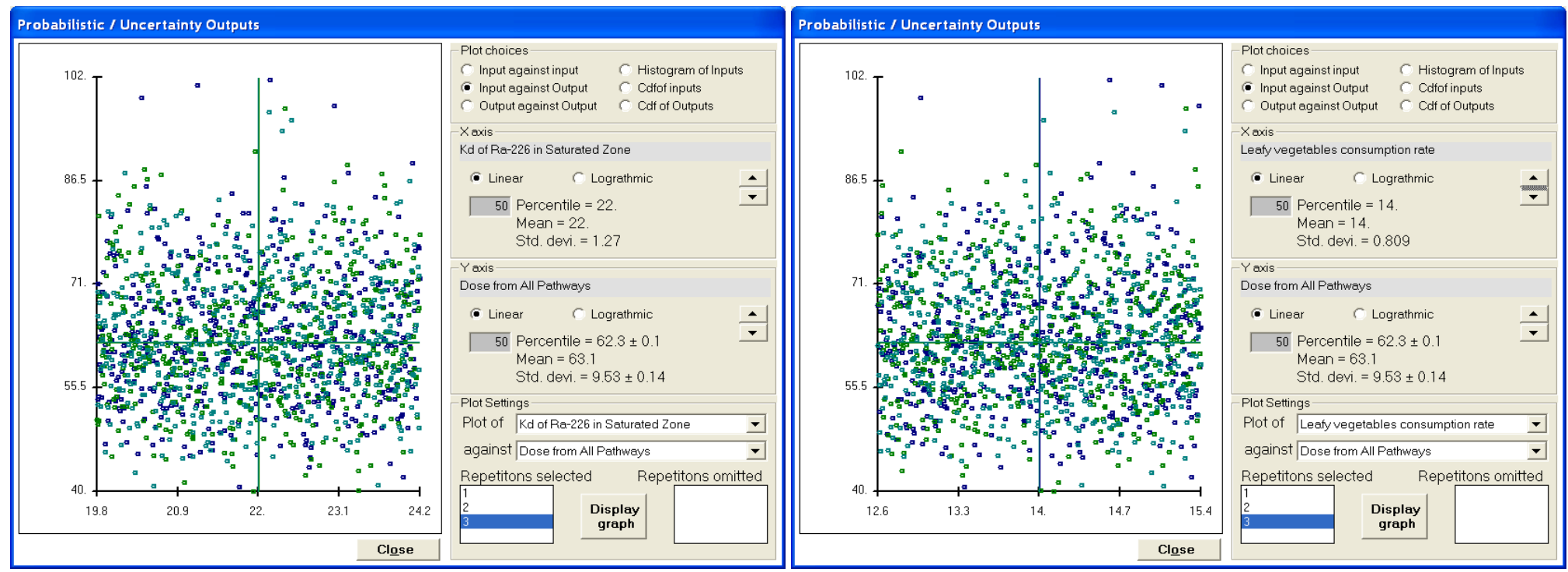

$\stackrel{?}{\stackrel{D}{N}}$
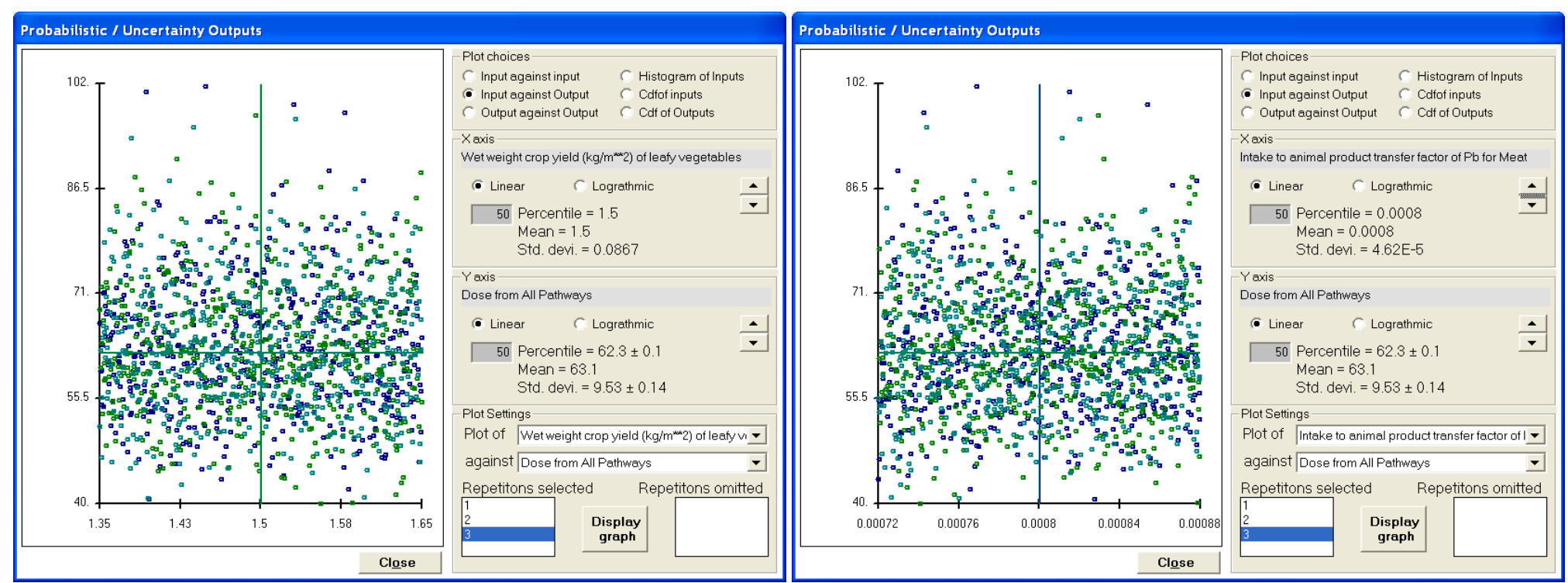

FIGURE C.99 Scatter Plots of Peak Predicted Dose against Inputs Identified as the 10th Set of Four Most Significant by the Regression Analysis 


\section{C.4.4 Verification of Selection of Significant Inputs}

There are two ways of verifying that the set of input identified as having a significant influence on the variability in the dose is in fact responsible for the major part of the variability in the dose. The easier method is to perform a second multi-input sensitivity analysis after deleting the distributions on all but those inputs that were identified as having a significant influence. The more cumbersome method involves performing the second multi-input sensitivity analysis after drastically narrowing the ranges of the distributions for all but those inputs that were identified as having a significant influence.

The advantage of the second method is that the sample values and, more importantly, the pairings of the samples of the initial multi-input sensitivity analysis are retained for the significant inputs. The closeness of the cumulative distribution function plots from the two multi-input sensitivity analyses confirms that none of the inputs that have a significant influence on the variability of the predicted dose has been left out. Figure C.100 compares the cumulative distribution function plots from (1) the multi-input sensitivity analyses on all inputs, (2) the multiinput sensitivity analyses on only the 11 inputs identified as significant, and (3) the multi-input sensitivity analysis with the original distributions for the 11 inputs identified as significant and narrower (by a factor of 100) distributions for those inputs that were not so identified.

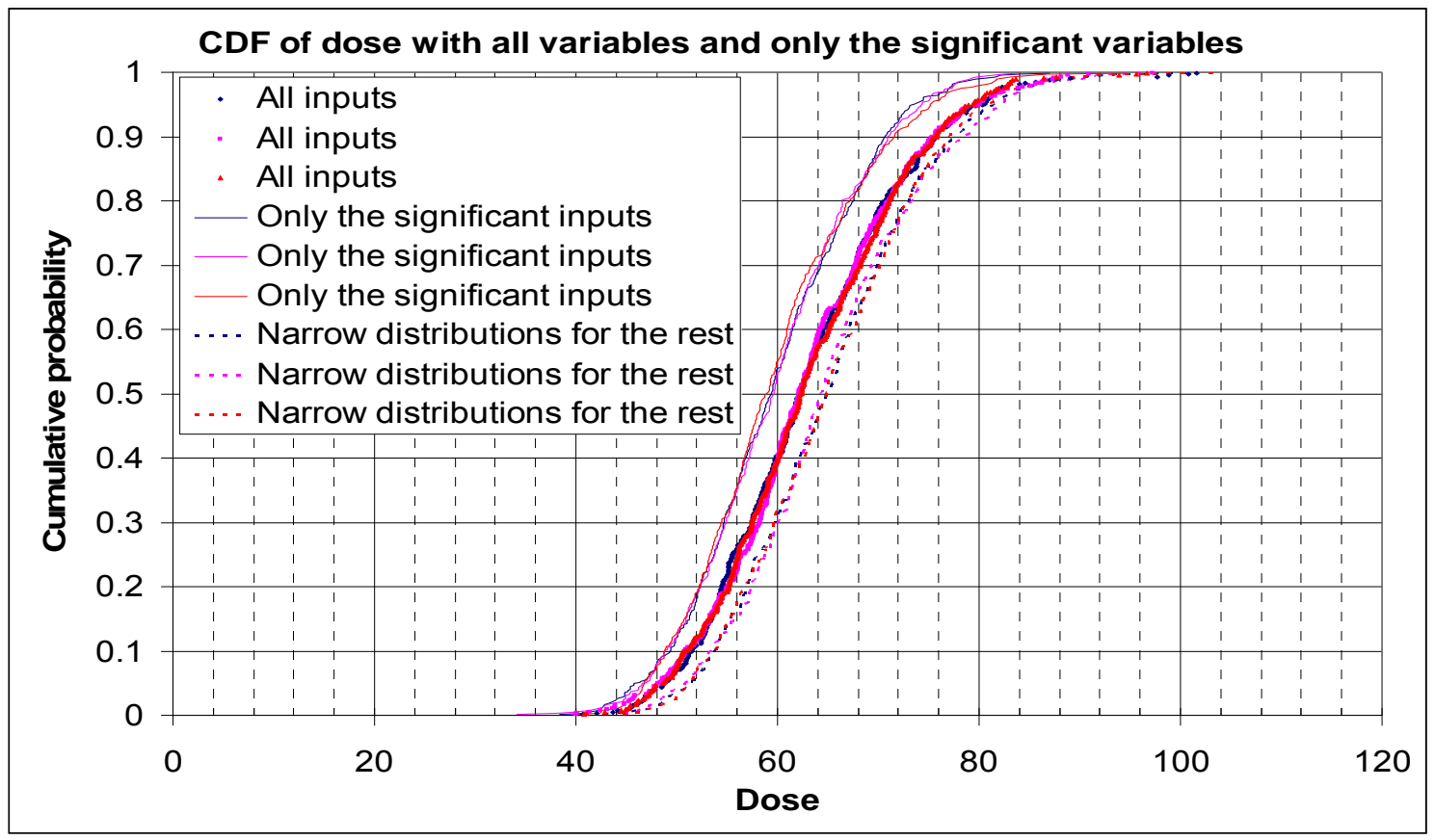

FIGURE C.100 Cumulative Distribution Function Plots from the Three Multi-input Sensitivity Analyses 


\section{C.4.5 Implications of This Set of Influential Inputs}

Three of the 11 inputs identified as having a significant influence on the peak predicted dose in this example relate to the surface water body: volume of surface water body, mean residence time of water in surface water body, and depth of aquifer contributing to surface water body. Five pertain to the primary contamination: $x$-dimension of primary contamination, $y$-dimension of primary contamination, thickness of contaminated zone, dry bulk density of contaminated zone, and leach rate of Ra-226. Two are inputs that affect the rate of transport through the unsaturated zone: Distribution coefficient of Ra-226 in Unsaturated Zone 1 and Distribution coefficient of $\mathrm{Pb}-210$ in Unsaturated Zone 1.

The remaining significant input is quantity of water for consumption by humans. Taken together, this indicates that the variability in the peak predicted dose in this example is mainly from the exposure and transport pathway that involves leaching from the contamination, transport through the unsaturated zone and groundwater to the surface water body, accumulation in the surface water body, and, finally, consumption of water.

The 19 inputs that were identified as having a moderate influence on the peak predicted dose in this example also fall into some broad groups. Three inputs relate to transport in the saturated zone: distance in the direction parallel to aquifer flow from downgradient edge of contamination to the surface water body, distance in the direction perpendicular to aquifer flow from center of contamination to left edge of the surface water body, and vertical lateral dispersivity of saturated zone to surface water body.

Six inputs are used to compute the erosion release from the primary contamination to the surface water body: soil erodibility factor of contaminated zone, rainfall and runoff factor in area of primary contamination, cover and management factor in area of primary contamination, slope-length-steepness factor in area of primary contamination, support practice factor in area of primary contamination, and sediment delivery ratio.

Six inputs are used in the modeling of the contamination and consumption of fruit, grain, and nonleafy vegetables: irrigation applied per year $(\mathrm{m} / \mathrm{yr})$ to fruit, grain, and nonleafy vegetable fields; foliage-to-food transfer coefficient of fruit, grain, and nonleafy vegetables; wet weight crop yield $\left(\mathrm{kg} / \mathrm{m}^{2}\right)$ of fruit, grain, and nonleafy vegetables; weathering removal constant (1/year) of fruit, grain, and nonleafy vegetables; duration of growing season (years) of fruit, grain, and nonleafy vegetables; and fruit, grain, and nonleafy vegetables consumption rate.

Four inputs are used in modeling exposure by the ingestion of meat: irrigation applied per year $(\mathrm{m} / \mathrm{yr})$ to pasture and silage fields, wet weight crop yield $\left(\mathrm{kg} / \mathrm{m}^{2}\right)$ of pasture and silage, grain (kg/day) intake of meat livestock, and meat consumption rate. These show that the erosion 
release, transport through the saturated zone, ingestion of fruit, grain, and nonleafy vegetables, and ingestion of meat also contribute to the variability in the peak predicted dose.

\section{C.4.6 Place of Multi-input Sensitivity Analysis in Probabilistic Analysis}

Site-specific probabilistic analysis is usually an iterative or cyclic process. The first iteration is a multi-input sensitivity analysis using site-appropriate distributions for many of the inputs. Siteappropriate distributions are distributions that are expected to be appropriate for the site based on some general characteristic of the site. The general site characteristics include the location of the site, the climate at the site, the type of soil or soil classification at the site, the topography of the site, and the vegetation at the site. National distributions may be used for some inputs in this first iteration (e.g., for inhalation rate). The results of this first multi-input sensitivity analysis are sets of inputs deemed have a "significant," "moderate," and "mild" influence on the variability in the peak predicted dose.

The next step involves finding site-specific distributions for the inputs identified as having a significant influence on the variability in the peak predicted dose. It might be possible to determine the site-specific distributions for some of the other inputs at a smaller incremental cost at the same time. It is therefore important to bear in mind the inputs that were identified as having a moderate or even mild influence on the variability in the peak predicted dose. Any relationships or correlations between these inputs will also be identified at this time.

The next iteration is another multi-input sensitivity analysis. The site-specific distributions found for the significant inputs and any that were found for the moderate inputs replace the distributions used in the preceding iteration. The relationships and correlations between the inputs are also specified for this iteration of probabilistic analysis. New sets of inputs that have a significant, moderate, and mild influence on the variability in the peak predicted dose will be identified from the output of this iteration.

This process of identifying significant inputs then finding and using site-specific distributions for these significant inputs in the next iteration is repeated until the set of significant inputs identified in an iteration all have site-specific distributions specified for them. At this stage of probabilistic analysis site-specific distributions would have been identified and specified for all the important inputs, relationships between the inputs would have been specified, and correlations between inputs would have been identified and specified. 


\section{C.4.7 Use of National Distributions for the First Iteration}

It is tempting to use national distributions for the first iteration of the probabilistic analysis. This is tried out on the hypothetical scenario discussed in Appendix C.I. The cumulative distribution function plots of the peak predicted dose for the three repetitions with 500 observations each are shown in Figure C.101. A logarithmic scale is used for the $x$-axis, because the distribution for the peak predicted dose spans many orders of magnitude. Although the plots appear to be close, this is misleading because of the logarithmic axis. The three repetitions differ by up to $56 \%$ in the upper half of the plot, compared with less than $5 \%$ difference between the repetitions in Figure C.87. The absolute values of the sorted standardized rank regression coefficients from the regression report are shown in Figure C.102.

There is good agreement between the standardized rank regression coefficients from the repetitions for the three most significant inputs. The differences between the coefficients from the repetitions become large in proportion to the average of the coefficients across the repetitions for the next seven significant inputs. The standardized rank regression coefficients for the 11th most significant input are $-0.06,-0.04$, and 0.01 from the three repetitions; the range is more than twice the mean and even changes sign. Standardized rank regression coefficients of -0.06 and -0.04 would have been significant, especially when the standard deviation of peak predicted dose is as high. The standard deviation of peak predicted dose in this example is in the 1000 to $2000 \mathrm{mrem} / \mathrm{yr}$ range (Figure C.101). The large differences between the standardized rank regression coefficients from the three repetitions call into question the reliability of these numbers.

The differences between the cumulative distribution function plots for the repetitions and the differences between the standardized rank regression coefficients from the repetitions indicates the need to use a larger number of samples (observations) for each repetition. Thus, the analysis was repeated with 5000 observations; this reduced the differences between plots of the cumulative distribution function from the repetitions to $30 \%$. The resulting cumulative distribution plots are in Figure C.103. Plots of the absolute values of the standardized rank regression coefficients from the regression report of inputs are shown in Figure C.104. Part of the regression report is shown in Figure C.105.

There is much more consistency between the standardized rank regression coefficient in this case, which allows the 21 most significant inputs to be identified with confidence. The national distributions generally have a wide range, and the response of the dose is likely to be nonlinearly related to the inputs when the range of the inputs is large. This is evident in the small coefficients of determination, 0.10 to 0.13 , for the regression analysis on the raw data from the repetitions. Using the ranks of the data will yield a linear relationship if the relationship between the raw data is monotonic. The relatively high coefficient of determination of 0.85 for 


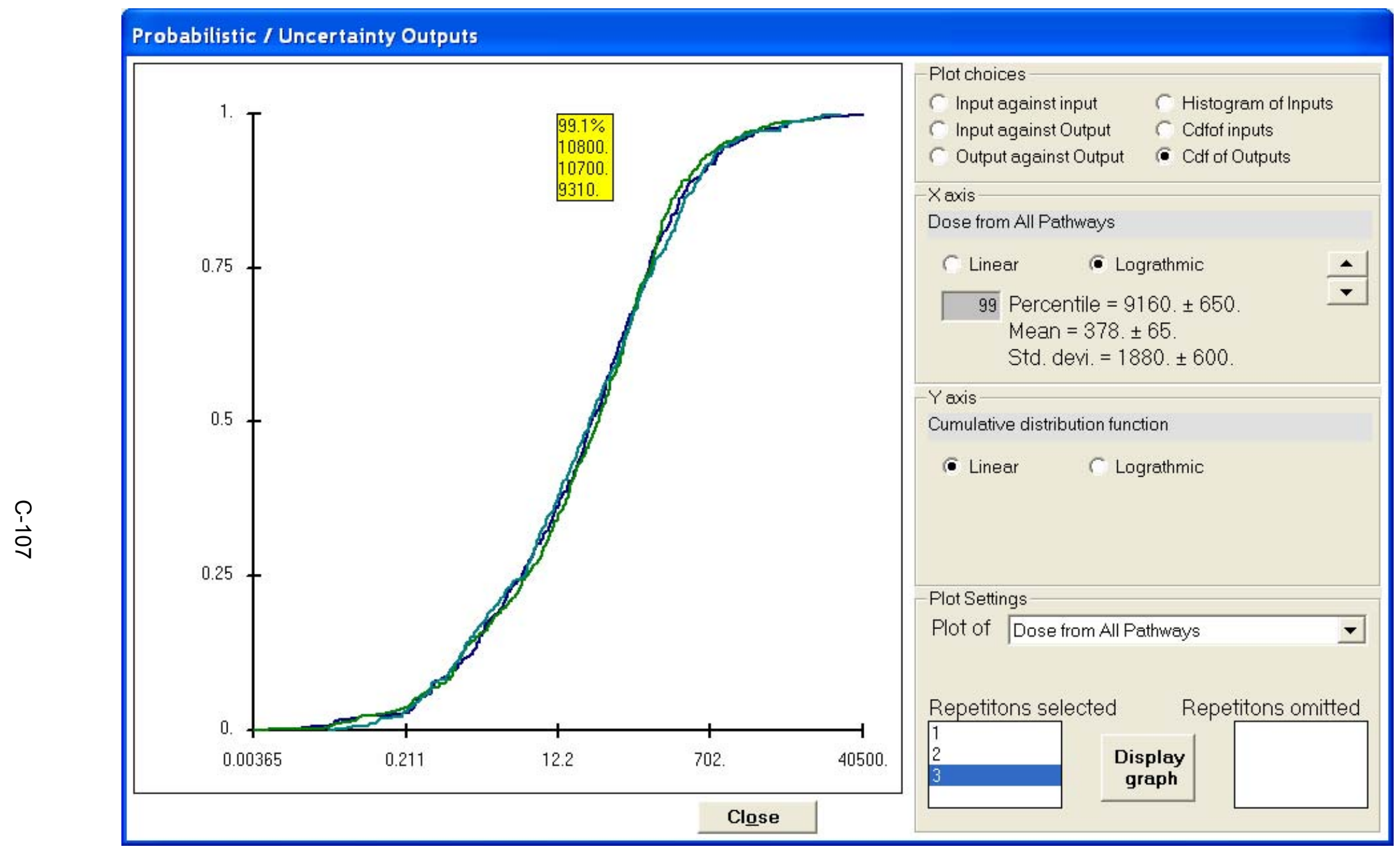

FIGURE C.101 Cumulative Distribution Function of the Output, the Peak Predicted Dose, When National Distributions Are Used to Obtain 500 Samples of Each of the 203 Inputs 


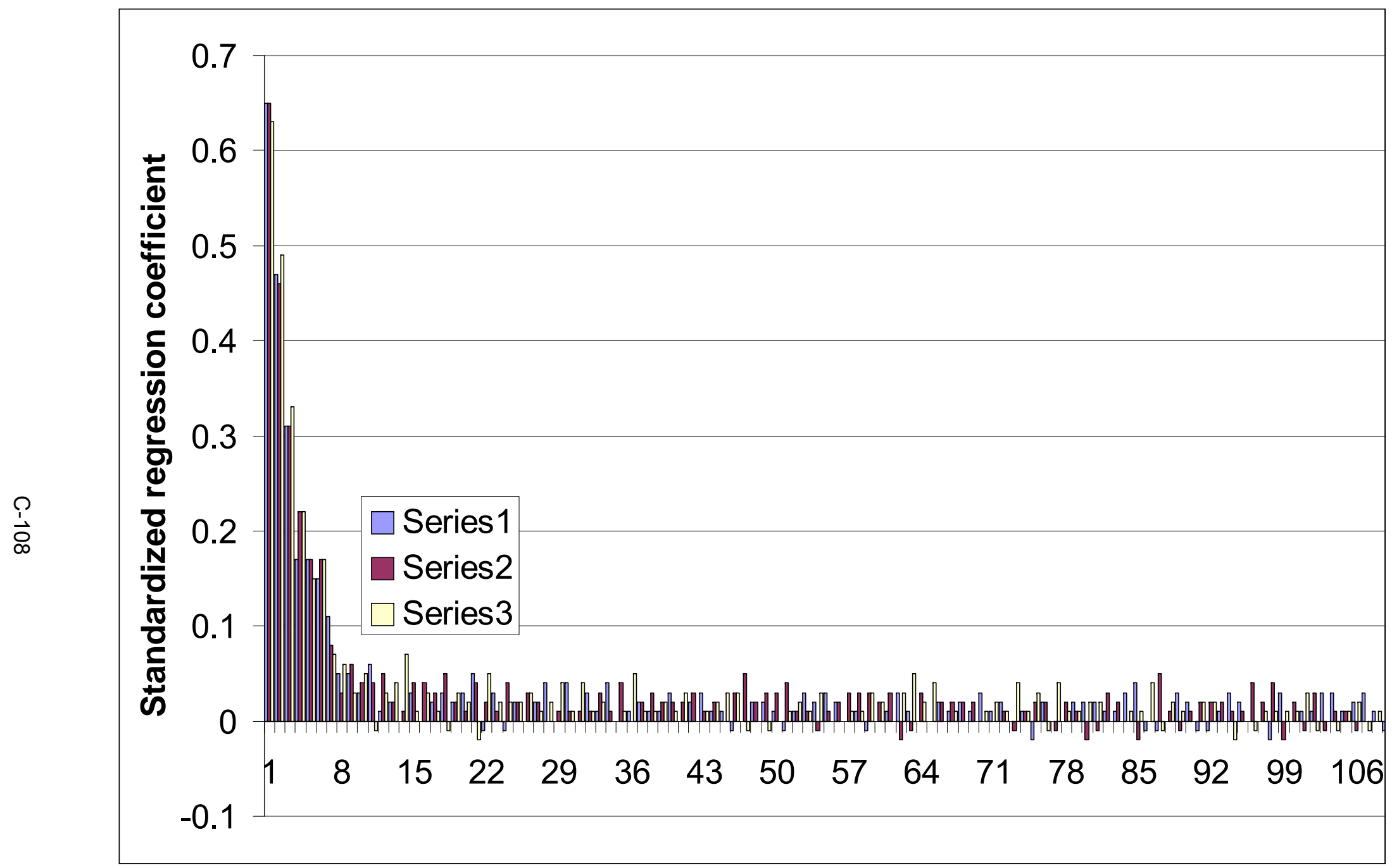

FIGURE C.102 Plot of the Standardized Regression Coefficients from the Regression Report of Sensitivity Analysis Using $\mathbf{5 0 0}$ Observations on National Distribution 


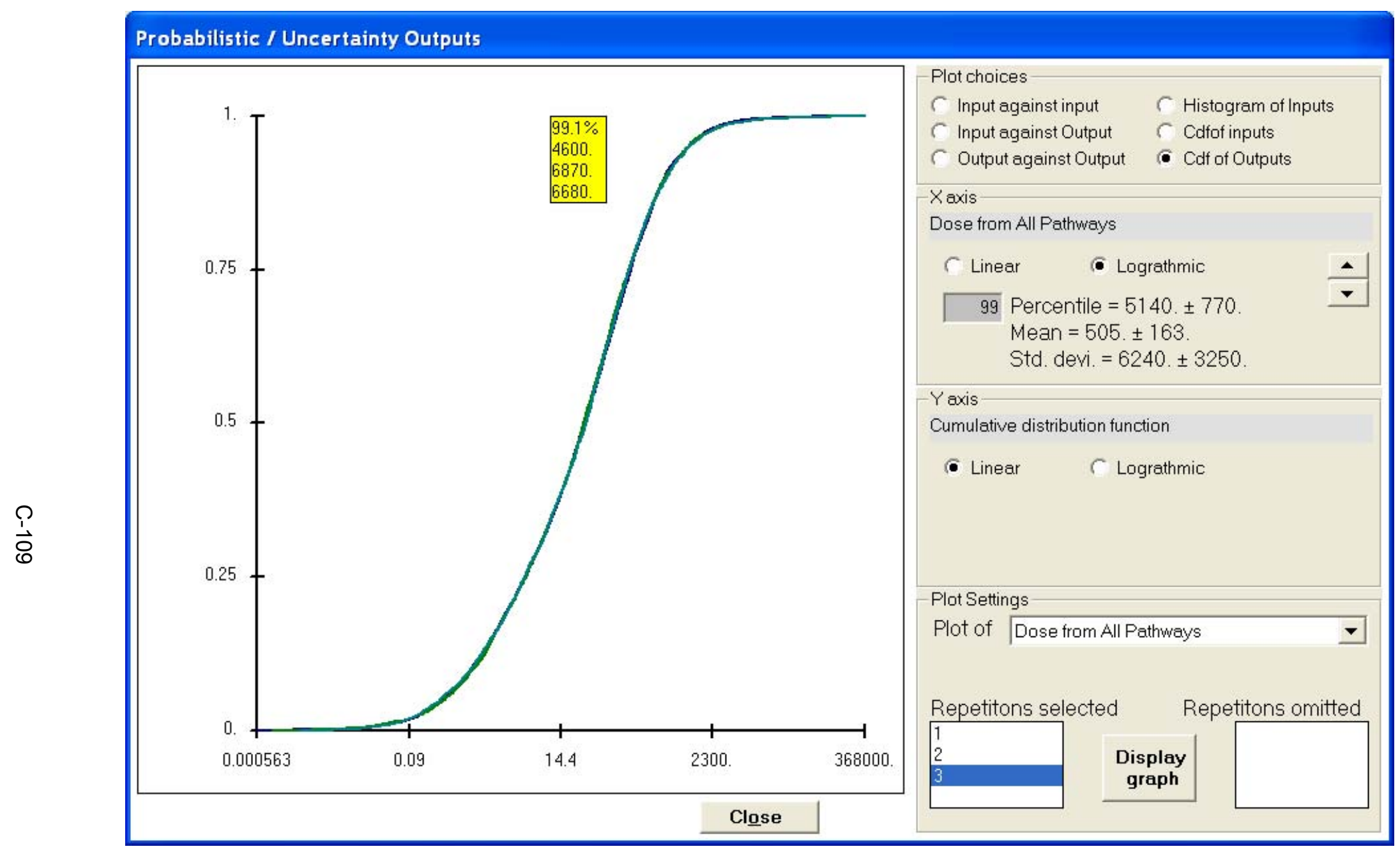

FIGURE C.103 Cumulative Distribution Function of Peak Predicted Dose with 5000 Samples, When National Distributions Are Used for the Inputs 


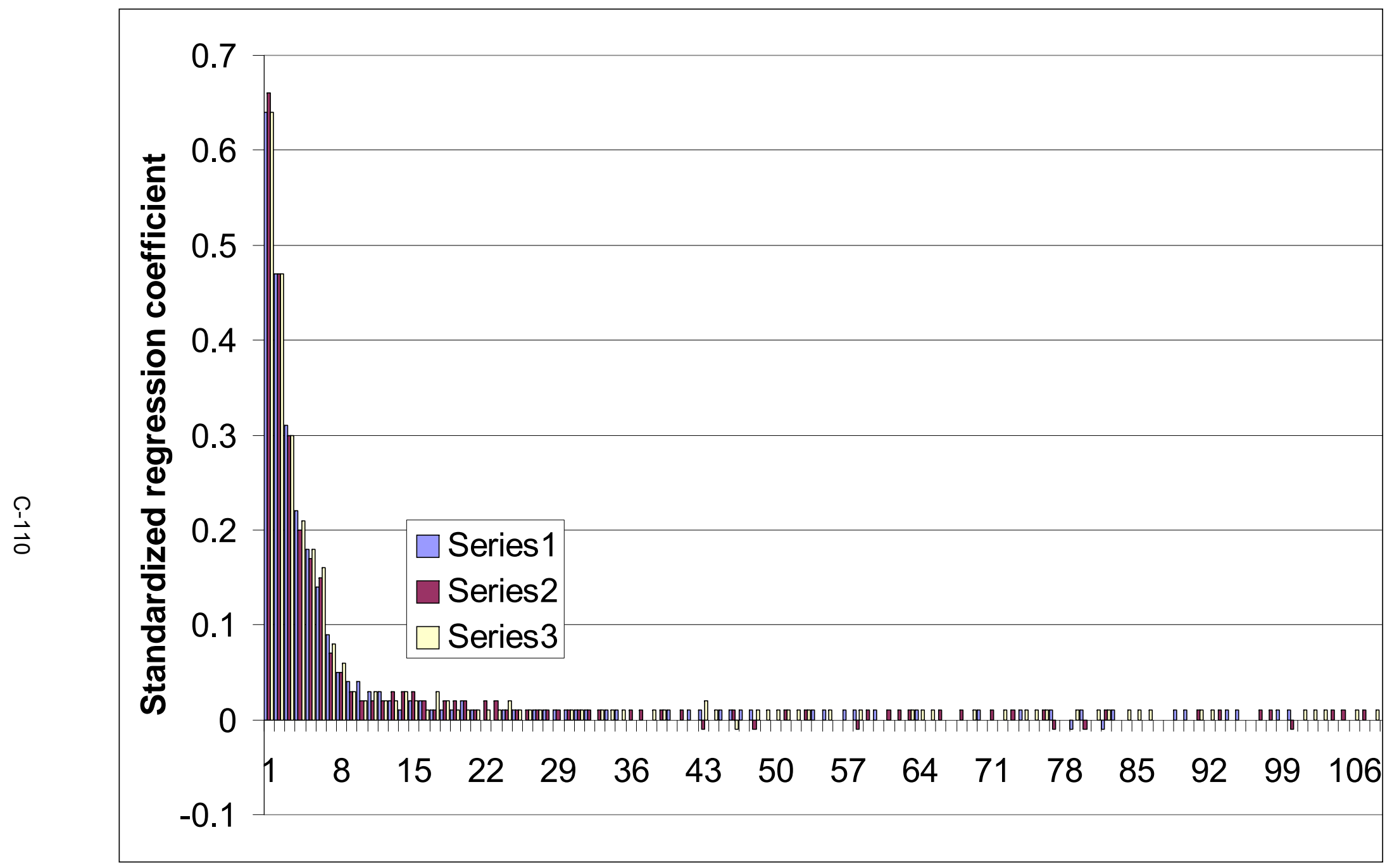

FIGURE C.104 Plot of the Standardized Regression Coefficients from the Regression Report of Sensitivity Analysis Using $\mathbf{5 0 0 0}$ Observations on National Distributions 


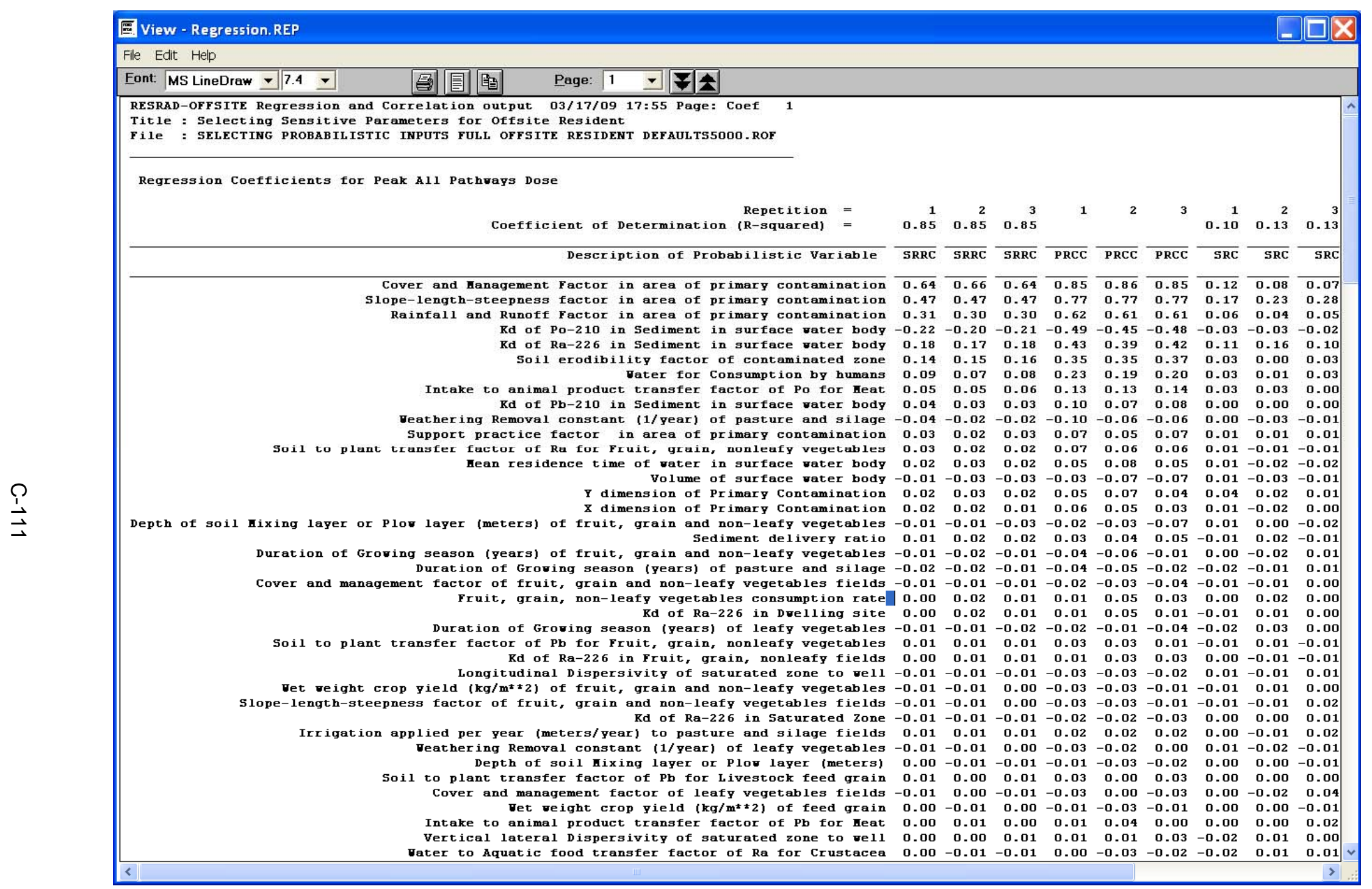

FIGURE C.105 Regression Report of Sensitivity Analysis Using 5000 Observations on National Distributions 
the regression on the ranks of the data indicates that the relationship was close to monotonic. The standardized ranked regression coefficients from the regression are able to explain $85 \%$ of the variation in the ranks of the dose.

Site-specific distributions can then be found for the inputs that were identified as significant in this first iteration. These sites specific distributions would then be used in the next iteration of multi-input sensitivity analysis. A new set of significant inputs is identified from this iteration of the multi-input sensitivity analysis and site-specific distributions are found for any for which national distributions were used in this iteration. If this iterative process were repeated until all the significant inputs identified in an iteration already had site-specific distributions specified from them, would the results be the same as the results from a probabilistic analysis that used site-appropriate distributions for the first iterations? Not necessarily, as can be seen in the following sections.

\section{C.4.8 Implications of This Set of Influential Inputs from Using National Distributions}

The 21 inputs identified fall into five categories. Eight of these inputs are used to model the erosion release from the primary contamination to the surface water body: $y$-dimension of primary contamination, $x$-dimension of primary contamination, cover and management factor in area of primary contamination, slope-length-steepness factor in area of primary contamination, rainfall and runoff factor in area of primary contamination, soil erodibility factor of contaminated zone, support practice factor in area of primary contamination, and sediment delivery ratio.

Five inputs are used to compute the concentration in the surface water body: mean residence time of water in surface water body, volume of surface water body, Distribution coefficient of Po-210 in sediment in surface water body, Distribution coefficient of Ra-226 in sediment in surface water body, and Distribution coefficient of $\mathrm{Pb}-210$ in sediment in surface water body. One input, water for consumption by humans, deals with the ingestion of water. Three inputs are part of the meat ingestion pathway: weathering removal constant (1/year) of pasture and silage, duration of growing season (years) of pasture and silage, and intake to animal product transfer factor of Po for meat.

Four inputs are used to compute the exposure from the ingestion of fruit, grain, and nonleafy vegetables: soil-to-plant transfer factor of Ra for fruit, grain, and nonleafy vegetables; depth of soil mixing layer or plow layer (meters) of fruit, grain and nonleafy vegetables; duration of growing season (years) of fruit, grain, and nonleafy vegetables; and cover and management factor of fruit, grain, and nonleafy vegetable fields. 
This set of influential inputs has much in common with the one identified using the narrower "site-appropriate" distributions. The main exception is that this list does not have any inputs that relate to the leaching release and groundwater transport pathway. This brings up the question in the preceding subsection. The sensitivity analysis was repeated after reducing the influence of the erosion release to the surface water body and the release to the atmosphere. This was achieved by specifying very narrow distributions with very small values for the sediment delivery ratio and for the deposition velocity of dust in the area of primary contamination.

The cumulative distribution function plots and the sorted regression report are shown in Figures C.106 and C.107. The contributions of the erosion release clearly decreased as evidenced by the eight orders of magnitude reduction in the peak predicted dose comparing Figures C.103 and C.106. This also shows that the contribution of the leaching and groundwater transport pathway is predicted to be even lower. The standardized rank regression coefficients for the 27th sorted input in the list are $0.00,0.00$, and 0.02 for the three repetitions; and $0.00,-0.01$, and -0.02 for the 26th sorted input. Inputs 26 and 27 , therefore, are not significant. There is good agreement between the standardized rank regression coefficients in the repetitions for the first 25 sorted inputs; therefore, these can reliably be included in the list of significant inputs.

None of the inputs used to compute the leaching release or the transport by the groundwater pathways appears in this list. Some of the inputs that are used in the groundwater transport calculations have distributions with very wide ranges, as illustrated by the cumulative distribution function plots for the input samples for thickness of the unsaturated zone, the distribution coefficient of radium in the unsaturated zone, the hydraulic gradient, and the hydraulic conductivity in the saturated zone from the contamination to the surface water body (Figures C.108 through C.111). The distribution coefficient of radium in the saturated zone is similar to that shown in Figure C.109. Even with 5000 samples (observations), there apparently were no combinations of input samples that allowed the radionuclides to be transported to the surface water body in the 1000-year time frame of the analysis. Thus, when the national distributions were used, the groundwater-transport-related inputs would not be identified as being significant, even if they were in this example. 


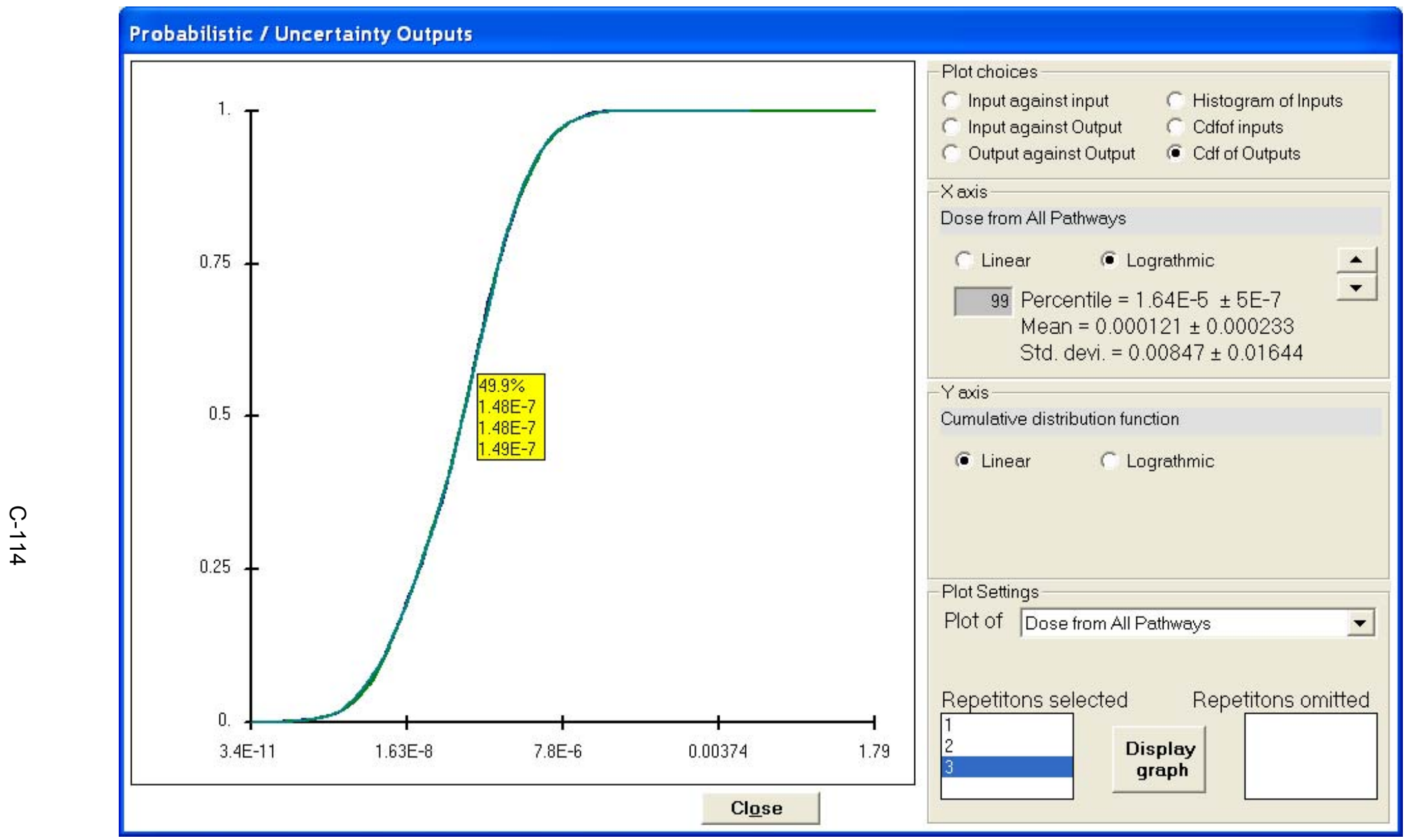

FIGURE C.106 Cumulative Distribution Function of Peak Predicted Dose with 5000 Samples, When National Distributions Are Used for the Inputs after Depressing Erosion Release to Surface Water Body and Release to Atmosphere 


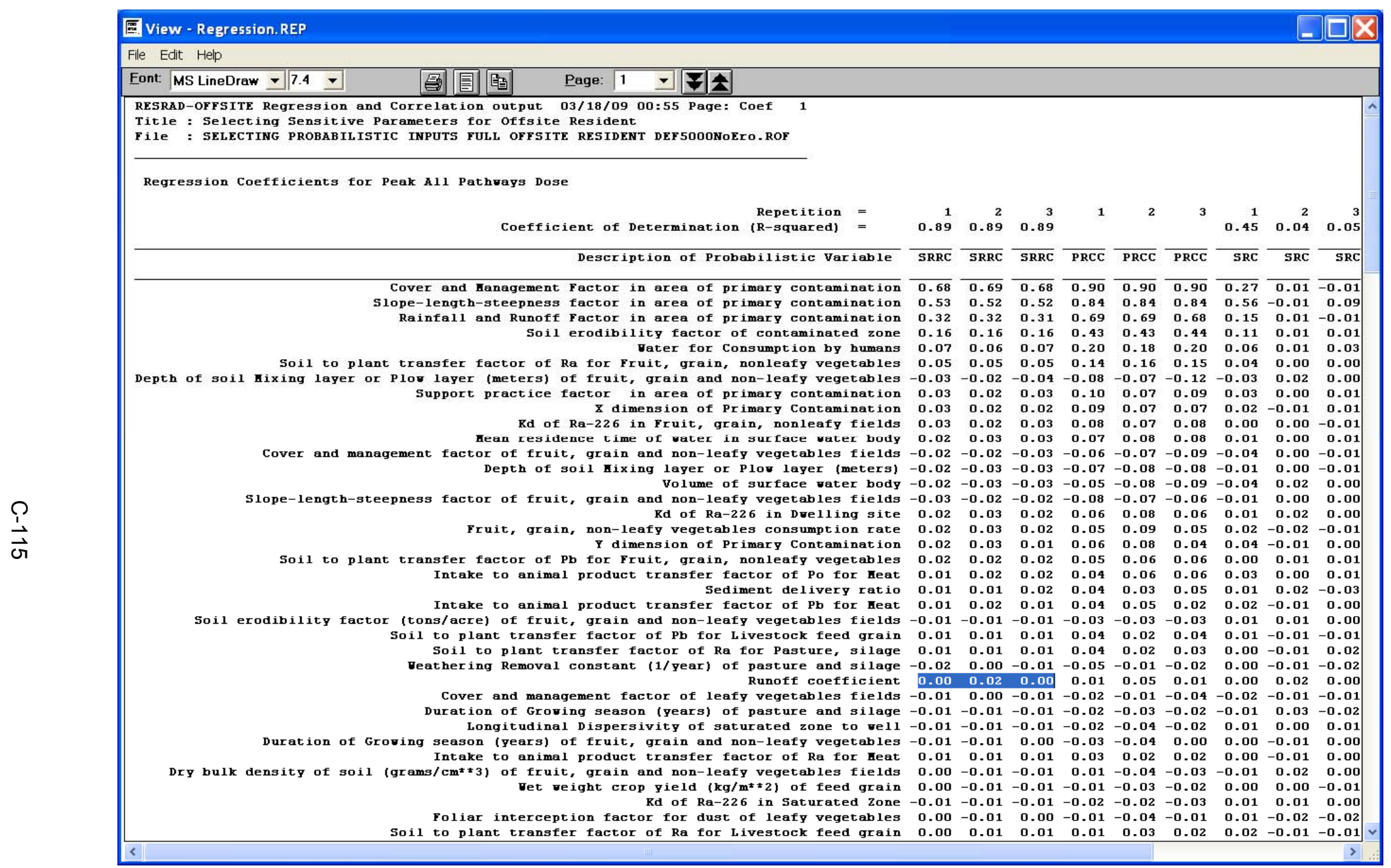

FIGURE C.107 Regression Report of Sensitivity Analysis Using 5000 Observations on National Distributions Inputs after Depressing Erosion Release to Surface Water Body and Release to Atmosphere 


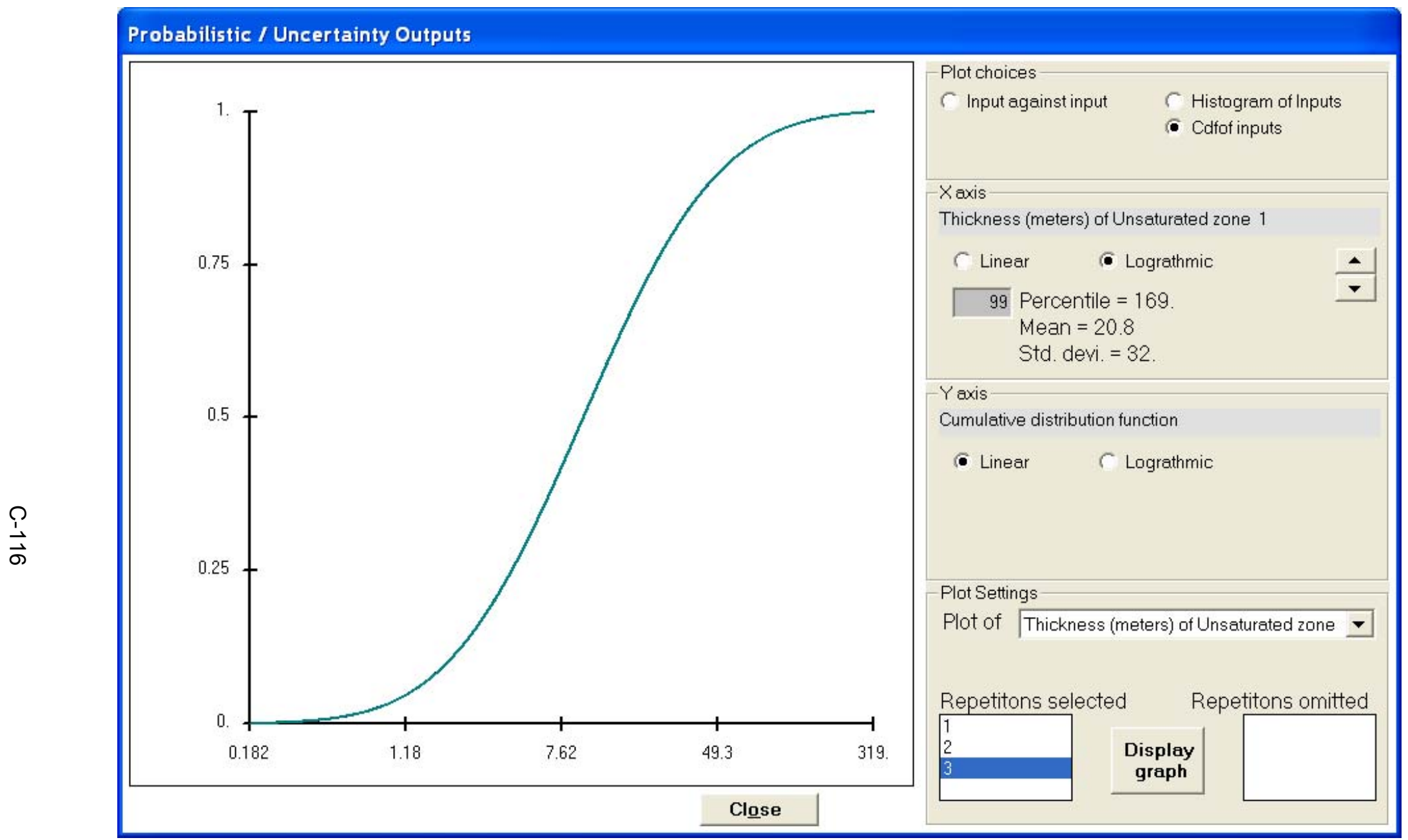

FIGURE C.108 Cumulative Distribution Function Plot of the Input Samples for the Thickness of the Unsaturated Zone 


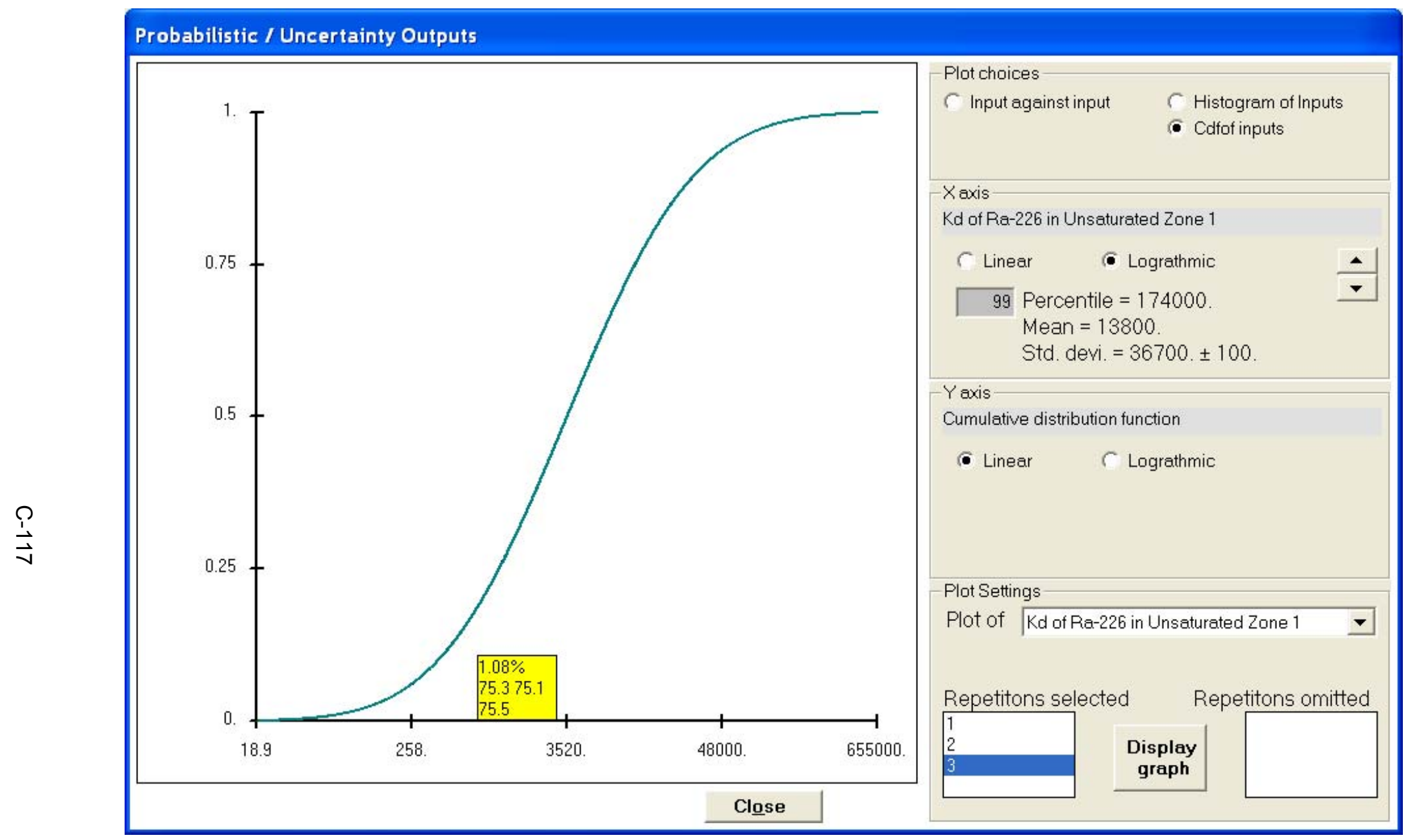

FIGURE C.109 Cumulative Distribution Function Plot of the Input Samples for the Distribution Coefficient for Radium in the Unsaturated Zone 


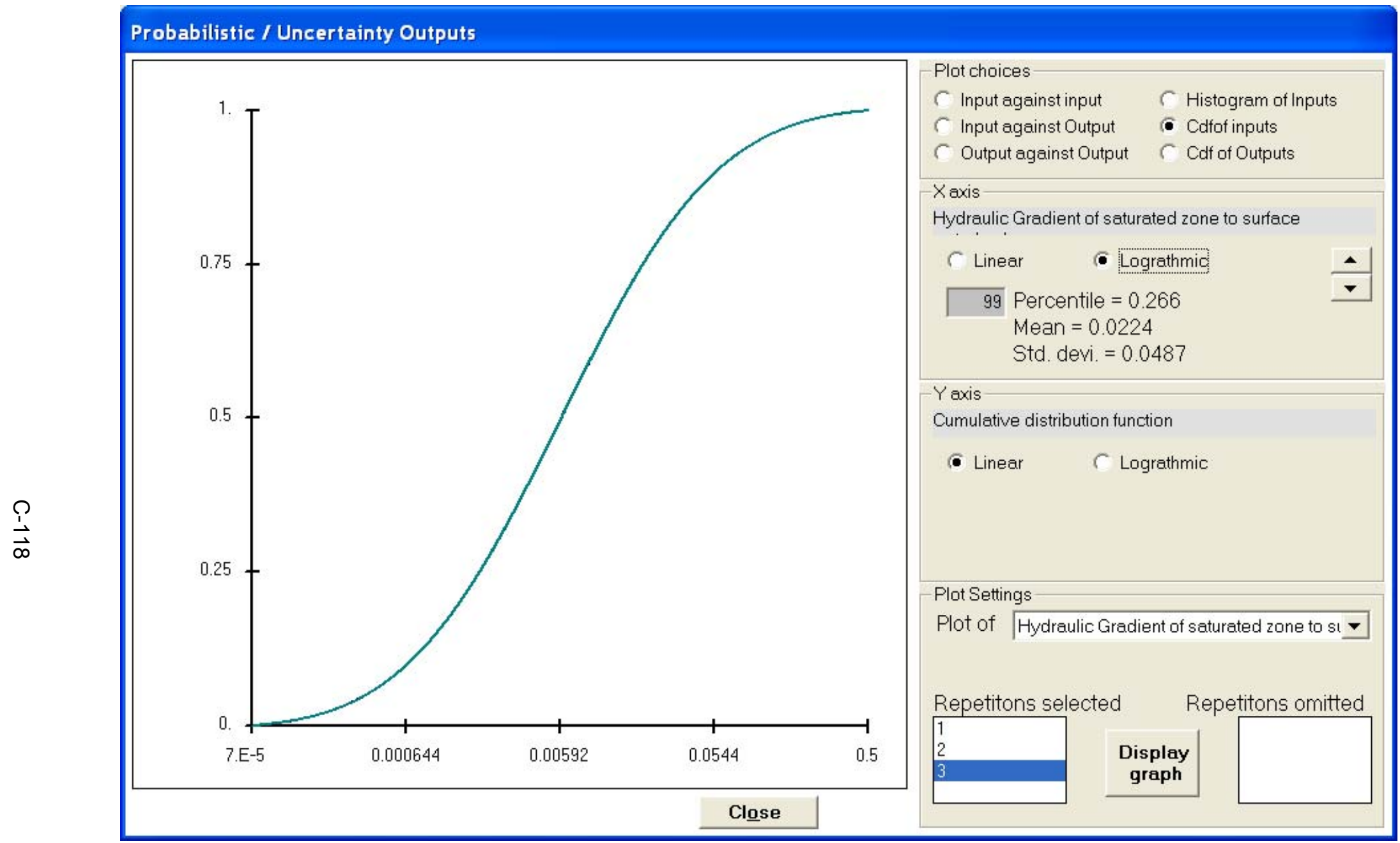

FIGURE C.110 Cumulative Distribution Function Plot of the Input Samples for the Hydraulic Gradient of the Saturated Zone to the Surface Water Body 


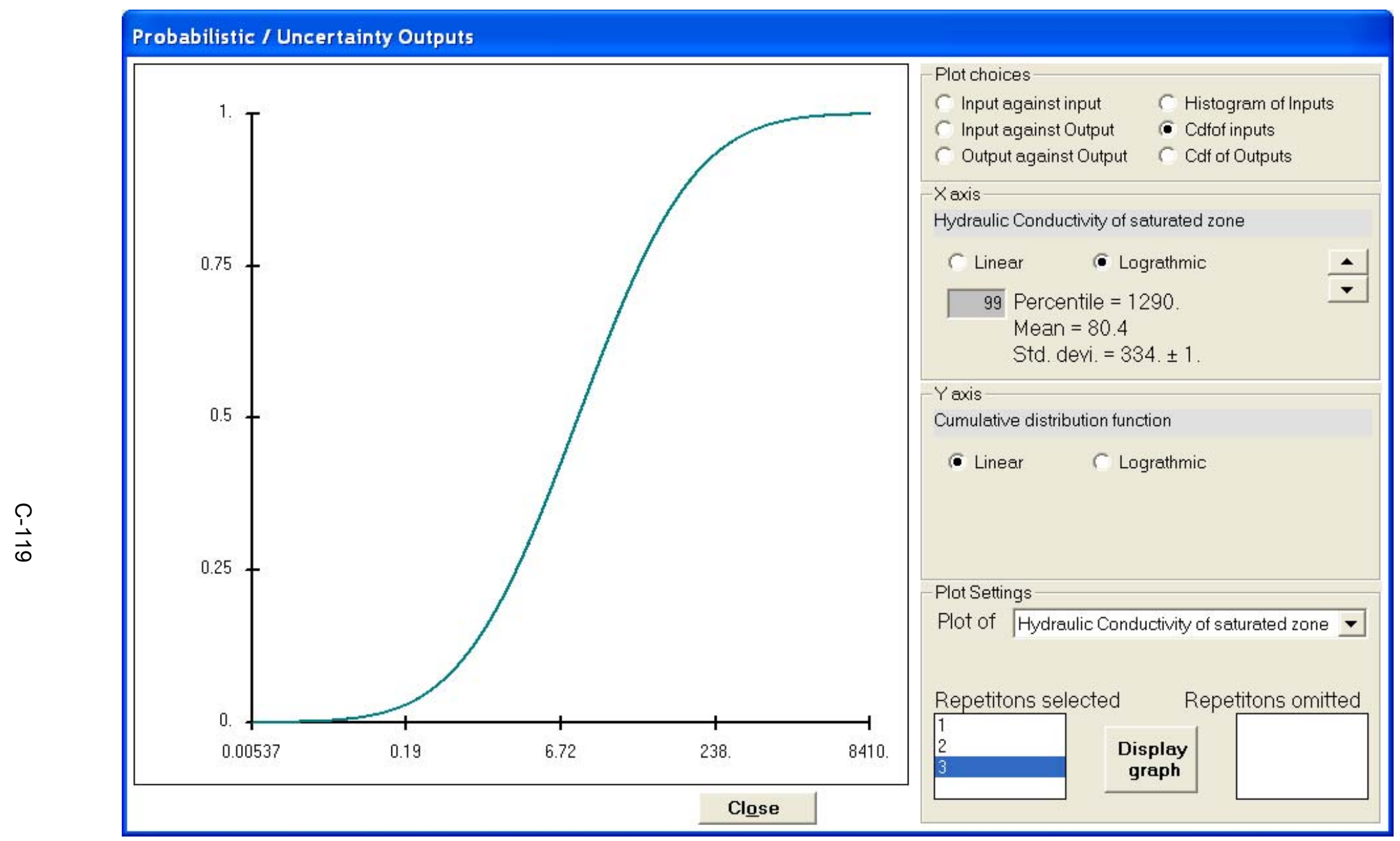

FIGURE C.111 Cumulative Distribution Function Plot of the Input Samples for the Hydraulic Conductivity of the Saturated Zone to the Surface Water Body 


\section{C.5 Summary and Conclusions}

Three methods to perform sensitivity analysis on input parameters using the RESRADOFFSITE code have been demonstrated. The case studies presented are for illustration purposes only-parameter sensitivity may change from site to site. A summary of the advantages and drawbacks of each method follows:

- Three-point single-input sensitivity analysis can be performed easily and quickly. The temporal variations of predicted dose for the three simulations can be viewed on the same plot. Three-point single-input sensitivity analysis cannot take into account interactions among inputs, because just one input is analyzed at a time. It does not give a good indication of how the predicted dose varies over a range of input values because it uses only three simulations. It is also very tedious to compare the results when many inputs need to be analyzed.

- Distributed single-input sensitivity analysis can also be performed easily, but it takes more time than three-point single-input sensitivity analysis to perform because it uses more simulations. The large number of simulations can give a good indication of how the predicted dose varies over a range of the input. As with three-point single-input sensitivity analysis, distributed single-input sensitivity analysis does not take into account interactions among inputs. It is also very tedious to compare results when many inputs need to be analyzed.

- Multi-input sensitivity analysis can be performed using the probabilistic module in the code. Relationships and correlations among inputs can be specified. Interactions among inputs make it difficult to use output vs. input scatter plots to identify all the inputs that have a significant influence on the output. The standardized regression coefficients from a regression analysis between the raw or ranked values of the output and the input can be used to identify the inputs that have a significant influence on the predicted dose. A second multiple-input sensitivity analysis must then be performed to verify the identification of the significant inputs. Identification of significant inputs is an iterative process.

Although both methods of single-input sensitivity analysis (three-point and distributed) are useful in understanding how the RESRAD-OFFSITE code uses an input to model dose, they are usually not very useful for identifying the inputs that have a significant influence on predicted dose or risk. It should be emphasized that, whenever possible, site-appropriate distributions or ranges of parameter values must be used in the sensitivity analysis to identify the inputs that have a significant influence on the predicted dose. Some inputs that actually have a significant influence on the dose may not be identified as such if national distributions with wide ranges are used in the sensitivity analysis. 


\section{Appendix C.I \\ Hypothetical Scenario for Multi-Input Sensitivity Analysis}

\section{Site Layout and Dimensions}

The layout of the hypothetical site and the section through the contaminated layer are depicted in Figures C.I-1 and C.I-2.

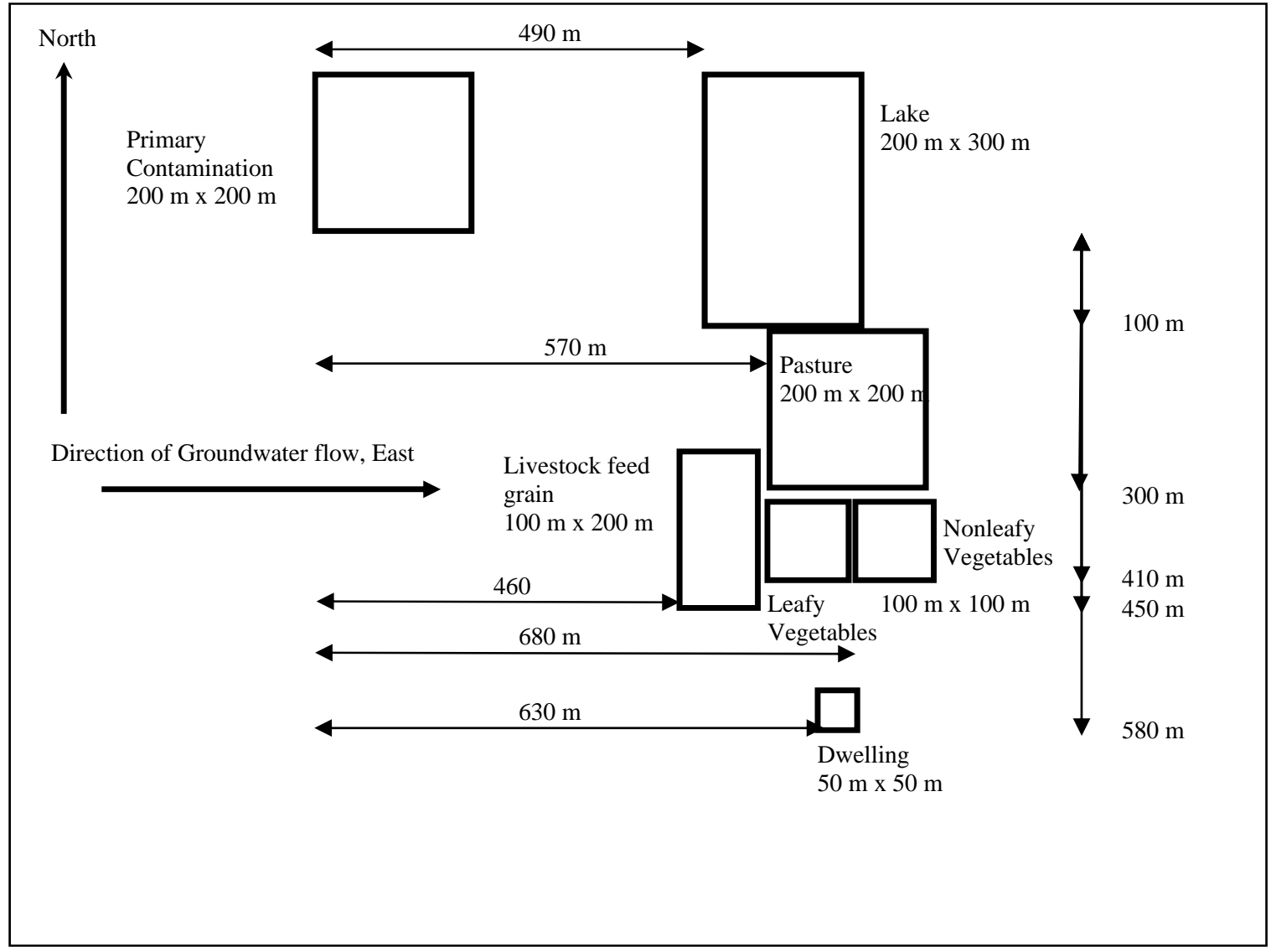

FIGURE C.I-1 Layout of the Hypothetical Site 


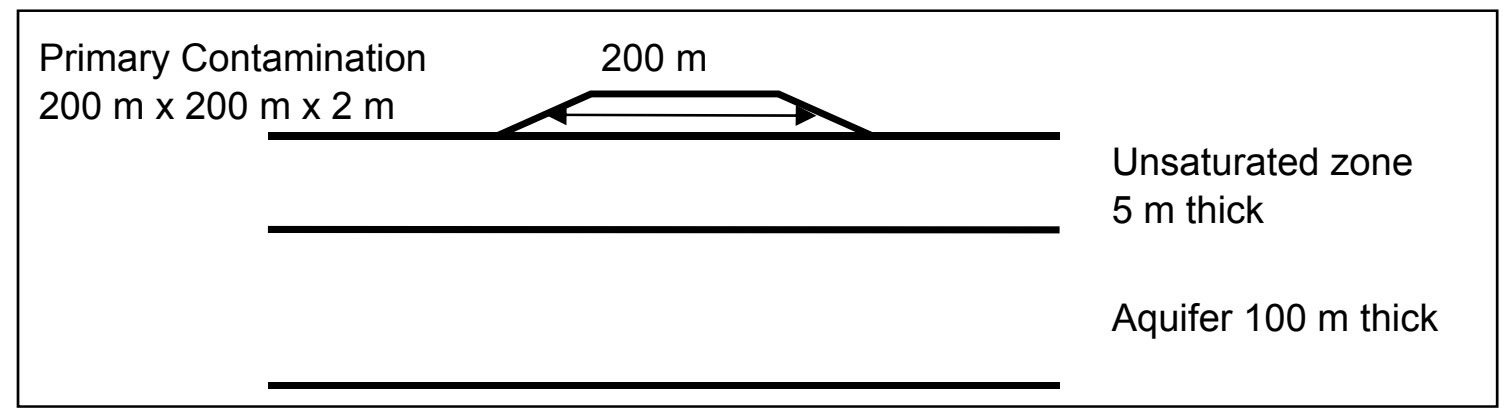

FIGURE C.I-2 The Cross Section of the Primary Contamination and the Soil beneath It

\section{Radionuclides in the Primary Contamination}

The radionuclides in the primary contamination and their concentrations are tabulated below.

${ }^{226} \mathrm{Ra}: 35 \mathrm{pCi} / \mathrm{g}$
${ }^{210} \mathrm{~Pb}: 35 \mathrm{pCi} / \mathrm{g}$
${ }^{210} \mathrm{Po}: 35 \mathrm{pCi} / \mathrm{g}$

\section{Preliminary Data for the Hypothetical Site}

Preliminary data gathered for this site are as given below. All other inputs are assumed to be at the preloaded RESRAD-OFFSITE value for this preliminary analysis.

It is assumed that there is no cover on the pile. The preliminary data indicate that the release of the radionuclides from the waste pile is controlled by the rate of dissolution of the solids in which the radionuclides are embedded; the leach rates for all radionuclides are 0.0001 per year. The distribution coefficients for the radionuclides for both the unsaturated and saturated zones, and for all the offsite locations are as follows:

\footnotetext{
${ }^{226} \mathrm{Ra}: 22 \mathrm{~cm}^{3} / \mathrm{g}$

${ }^{210} \mathrm{~Pb}: 5 \mathrm{~cm}^{3} / \mathrm{g}$

${ }^{210} \mathrm{Po}: 5 \mathrm{~cm}^{3} / \mathrm{g}$
}

The average annual precipitation at this location is $1.25 \mathrm{~m} / \mathrm{yr}$. The primary contamination is not cultivated and is not irrigated. The cover management factor for the primary contamination is 0.04 and the runoff coefficient is 0.5 . This corresponds to an area that is $60 \%$ covered with weeds, short brush, and grass. 
The leafy vegetables are grown in a field that has $20 \%$ slope and is farmed along the contour; so a support practice factor of 0.9 , a cover and management factor of 0.08 and a slope length steepness factor of 4 are appropriate.

The fruit, grain, nonleafy vegetables and livestock feed grain are grown in relatively flat land. The cover management factor is 0.04 for these two areas.

Assume for atmospheric transport purposes that this hypothetical site is located in Peoria, Illinois. The topographical map indicates that the ground level of the livestock feed grain growing area is $15 \mathrm{~m}$ above the ground level at the location of primary contamination. The corresponding differences in elevation for the leafy vegetable growing area and the dwelling site are 20 and 40 meters. All the other offsite locations are at approximately the same elevation as the ground in the vicinity of the primary contamination. A preliminary sensitivity analysis on the grid spacing for this site shows that a grid spacing of $25 \mathrm{~m}$ is adequate for the desired accuracy.

The hydraulic gradient of the groundwater is 0.004 across the entire site and the hydraulic conductivity is $10^{5} \mathrm{~m} / \mathrm{yr}$. The longitudinal, horizontal lateral and vertical lateral dispersivities to the surface water body are estimated to be $8,0.8$ and $0.05 \mathrm{~m}$ respectively.

The surface water body is the source of all water used in this scenario. For groundwater transport purposes, the surface water body is $290 \mathrm{~m}$ along the groundwater flow line, from the down gradient edge of the primary contamination. The right and left edges of the surface water body are at 200 and $100 \mathrm{~m}$ from the groundwater flow line through the center of the primary contamination. This surface water body does not have any edible crustacea or fish.

The individual spends 0.5 of the time inside the dwelling, 0.2 of the time outdoors in the vicinity of the dwelling, 0.08 of the time in each of the vegetable plots and in the livestock grain field, and 0.01 of the time in the pasture. The remainder of time is spent away from the area.

A preliminary (deterministic) analysis of this scenario confirms that the long term dose from this site results from the ${ }^{226} \mathrm{Ra}$ that was at the site because of the shorter half-life of the two other radionuclides that are initially present at the site. The ${ }^{210} \mathrm{~Pb}$ and ${ }^{210} \mathrm{Po}$ that were initially present would have transformed away at the time of the peak in the dose. Thus, these two radionuclides are left out of the probabilistic sensitivity analysis in order to avoid spending computation time on computations that do not affect the final results.

Sections C.2 and C.3 emphasized the need to use site-appropriate if not site-specific distributions when performing sensitivity analysis. This is a hypothetical scenario. Rather than make up hypothetical "site-appropriate" distributions for this hypothetical sites, uniform distributions with a range of 20 percent of the point values, centered about the point values will be used for this example scenario. 
The exercise for this scenario is to perform sensitivity analysis to find the distributed inputs that have a significant impact on the dose. 


\section{Appendix D}

Source Release with Time-delay Options in RESRAD-OFFSITE Version 3.1 



\section{Appendix D}

\section{Source Release with Time-delay Options in RESRAD-OFFSITE Version 3.1}

Version 3.1 of the RESRADOFFSITE code contains a range of options for the release of radionuclides to groundwater in addition to the original release option found in Version 2. These options involve the following:

- One of two release mechanisms, first-order ratecontrolled or instantaneous (equilibrium desorptioncontrolled) release;

- A delay time during which there is no release to groundwater and possibly no release of particulates from the surface soil to either the atmosphere or to surface runoff; and

- A transition time during which different proportions of the radionuclide-bearing material become exposed to the infiltrating moisture.

\section{D.1 Version 2 Release Methodology}

The original source release model of the RESRADOFFSITE code Version 2 is the default option for Version 3 (Figure D.1) and is active when the "Version 2 Release Methodology" box is

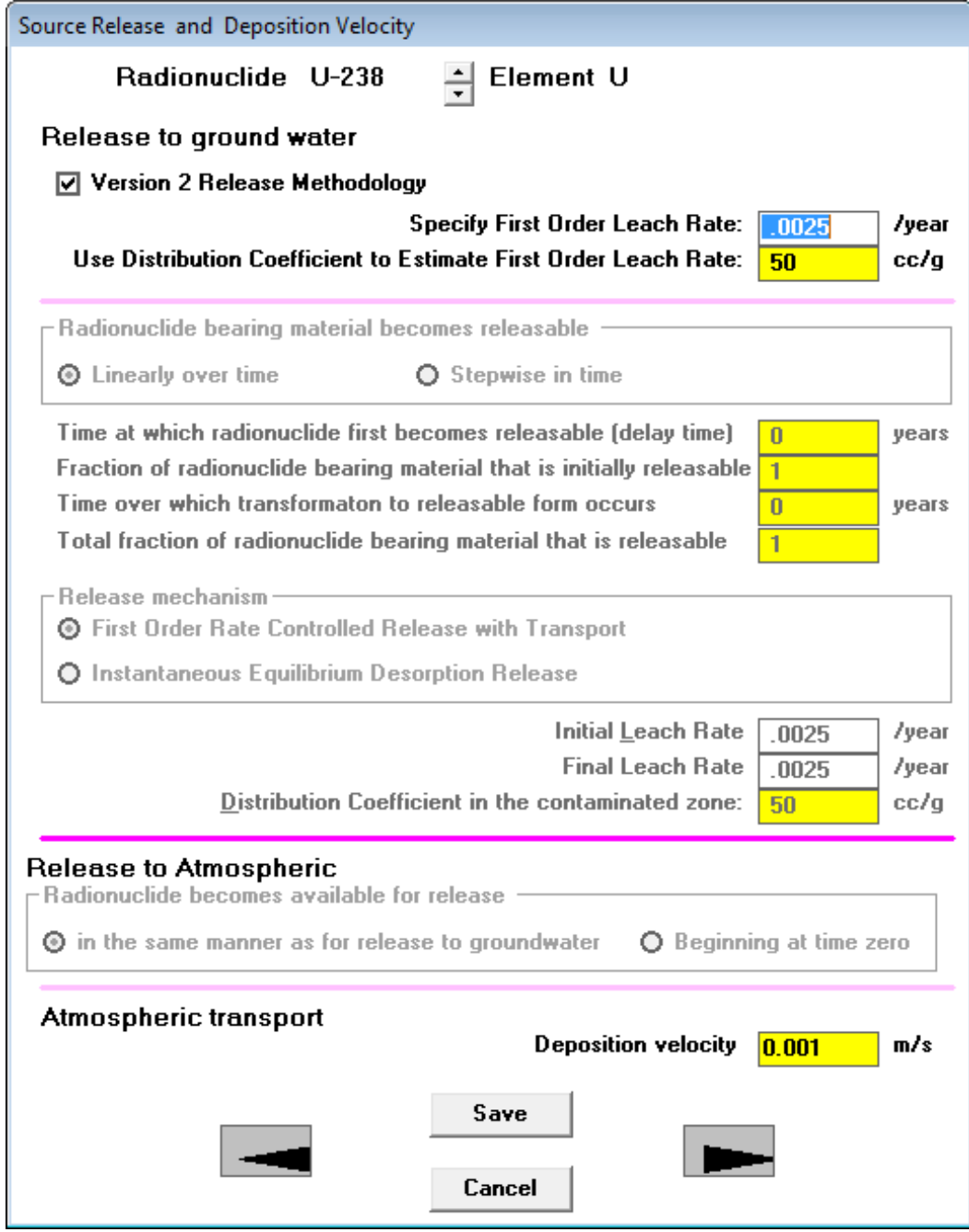

FIGURE D.1 Specifying Inputs for the Version 2 Release Methodology 
checked. In this case, the first-order leach rate specified in the first input box will be used provided that it is not zero and provided that it is less than the upper limit defined by the RESRAD methodology. Otherwise, the first-order leach rate is computed using the userspecified distribution coefficient. The value used by the code is shown in the input echo in the parent dose report (summary.rep).

\section{D.2 Conceptualization of New Release with Time-delay Options}

The new release options conceptualize the material that contains the radionuclide as being in two states or forms: a form that is susceptible to releasing radionuclides to water that infiltrates the primary contamination and a form that is immune (not susceptible) to release. The form that is immune from releasing to infiltrating moisture can also be stipulated to be immune from releasing to the atmosphere. At any time period, a part of the radionuclide-bearing material can be in one form, and the remainder can be in the other form. The concentrations of the radionuclides contained in both the susceptible and immune forms change with time due to radiological transformations (both decay and ingrowth), whereas the concentration of the radionuclides in the susceptible form are also affected by the release to groundwater.

The concentration of the radionuclides (both parent and progeny) contained in the form that is immune to release is computed by the Bateman equation modified for the change in (releasable) form/state, as follows:

$$
s_{k}^{\text {immune }}(t)=\left(1-f_{1}^{r e l}(t)\right) \sum_{i=1}^{k} a_{k, i} \exp \left(-\lambda_{i} t\right) \text {, }
$$

where

$s_{k}^{\text {immune }}(t)$ is the concentration of the $\mathrm{k}^{\text {th }}$ radionuclide of the transformation chain, in the material that is in the form that is immune to release $(\mathrm{pCi} / \mathrm{g})$,

$f_{1}^{r e l}(t)$ is the fraction of the radionuclide-bearing material that is in the releasable form (dimensionless),

$a_{k, i}$ is a set of coefficients defined by the following:

$a_{1,1}=s_{1}(0)$, which is the initial concentration of radionuclide 1 in both forms $(\mathrm{pCi} / \mathrm{g})$,

$a_{k, i}=\frac{\lambda_{k} a_{k-1, i}}{\lambda_{k}-\lambda_{i}}$ for all $1 \leq i<k$, and 


$$
a_{k, k}=-\sum_{i=1}^{k-1} a_{k, i}
$$

$\lambda_{i}$ is the radiological transformation rate of the $\mathrm{i}^{\text {th }}$ radionuclide of the transformation chain $\left(\mathrm{yr}^{-1}\right)$, and

$t$ is the time since the determination of the inventory $(\mathrm{yr})$.

The conceptual model envisions the material to be in the form immune to release for a userspecified period of time. This period of time, sometimes referred to as delay time, can be specified to be zero to model scenarios where some or all of the material is initially in the releasable form. The change of material from the release-immune form to the releasesusceptible form can be instantaneous (fraction at a specified time), or it can be continuous at a constant rate over a user-specified time (fraction/year), or it can even be a combination of the two. In this third scenario, a specified fraction of the material changes instantaneously at the beginning of the period of change followed by additional material changing at a constant rate over the period of change. For scenarios involving instantaneous changes, the user specifies the fractions of material that change to the releasable form at the different times and the time interval between the instantaneous changes. The change at a constant rate is defined by specifying the period of change and the fractions of the material that has changed at the beginning and end of that time period.

The instantaneous increase in the activity of a radionuclide in the releasable form, which results from an instantaneous change in the form of the radionuclide-bearing material from the releaseimmune form to the releasable form, is given by:

$$
C_{k}^{u n r e l e a s a b l e \rightarrow \text { releasable }}(t)=\Delta f_{1}^{r e l}(t) \sum_{i=1}^{k} a_{k, i} \exp \left(-\lambda_{i} t\right) \rho_{p c} A\left(f_{v m} T_{m i x}^{c}(t)+T_{p c}^{u m}(t)\right) 10^{6}
$$

where

$C_{k}^{\text {unreleasabe } \rightarrow \text { releasable }}(t)$ is the instantaneous change in activity of the $\mathrm{k}^{\text {th }}$ radionuclide of the transformation chain at time $t$, from the release-immune form to the releasable form, in units of activity $(\mathrm{pCi})$,

$\Delta f_{1}^{r e l}(t)$ is the instantaneous increase in the fraction of the parent radionuclide-bearing material that is in the releasable form,

$a_{k, i}$ is a set of coefficients defined by the following:

$a_{1,1}=s_{1}(0)$, the initial concentration of the first radionuclide in the transformation chain, in both forms $(\mathrm{pCi} / \mathrm{g})$, 


$$
\begin{aligned}
& a_{k, i}=\frac{\lambda_{k} a_{k-1, i}}{\lambda_{k}-\lambda_{i}} \text { for all } 1 \leq i<k, \text { and } \\
& a_{k, k}=-\sum_{i=1}^{k-1} a_{k, i} .
\end{aligned}
$$

$\lambda_{i}$ is the radiological transformation rate of the $\mathrm{i}^{\text {th }}$ radionuclide of the transformation chain $\left(\mathrm{yr}^{-1}\right)$, $t$ is the time since the determination of the inventory (yr),

$\rho_{p c}$ is the dry bulk density of the primary contamination $\left(\mathrm{g} / \mathrm{cm}^{3}\right)$,

$A$ is the area of the primary contamination $\left(\mathrm{m}^{2}\right)$,

$f_{v m}$ is the volume fraction of the material from the primary contamination in the mixing layer,

$T_{m i x}^{c}(t)$ is the thickness of the contaminated mixing layer $(\mathrm{m})$,

$T_{p c}^{u m}(t)$ is the thickness of the undisturbed primary contamination $(\mathrm{m})$, and

$10^{6}$ is a conversion factor $\left(\mathrm{cm}^{3} / \mathrm{m}^{3}\right)$.

The rate at which the activity of a radionuclide in the releasable form increases as a result of a continuous change of the radionuclide-bearing material from the release-immune form to the releasable form is given by the following:

$$
\dot{C}_{k}^{\text {unreleasable } \rightarrow \text { releasable }}(t)=\frac{f_{1}^{r e l}\left(t_{c}\right)-f_{1}^{r e l}\left(t_{d}\right)}{t_{c}-t_{d}} \sum_{i=1}^{k} a_{k, i} \exp \left(-\lambda_{i} t\right) \rho_{p c} A\left(f_{v m} T_{m i x}^{c}(t)+T_{p c}^{u m}(t)\right) 10^{6}
$$

where

$\dot{C}_{k}^{\text {unreleasable } \rightarrow \text { releasable }}(t)$ is the rate of change of the $\mathrm{k}^{\text {th }}$ radionuclide, from the release-immune form to the releasable form ( $\mathrm{pCi} / \mathrm{yr}$ ),

$f_{1}^{r e l}(t)$ is the fraction of the parent radionuclide-bearing material that is in the releasable form, $t_{d}$ is the time at which the radionuclide-bearing material first changes from the release-immune form to the releasable form (yr),

$t_{c}$ is the time as the radionuclide bearing material finishes changing from the release immune form to the releasable form (yr),

$a_{k, i}$ is a set of coefficients defined by the following:

$a_{1,1}=s_{1}(0)$ is the initial concentration of the first radionuclide in the transformation chain, in both forms $(\mathrm{pCi} / \mathrm{g})$, 


$$
\begin{aligned}
& a_{k, i}=\frac{\lambda_{k} a_{k-1, i}}{\lambda_{k}-\lambda_{i}} \text { for all } 1 \leq i<k, \text { and } \\
& a_{k, k}=-\sum_{i=1}^{k-1} a_{k, i} .
\end{aligned}
$$

$\lambda_{i}$ is the radiological transformation rate $\left(\mathrm{yr}^{-1}\right)$,

$t$ is the time since the determination of the inventory (yr),

$\rho_{p c}$ is the dry bulk density of the primary contamination $\left(\mathrm{g} / \mathrm{cm}^{3}\right)$,

$A$ is the area of the primary contamination $\left(\mathrm{m}^{2}\right)$,

$f_{v m}$ is the volume fraction of the material from the primary contamination in the mixing layer,

$T_{m i x}^{c}(t)$ is the thickness of the contaminated mixing layer $(\mathrm{m})$,

$T_{p c}^{u m}(t)$ is the thickness of the undisturbed primary contamination $(\mathrm{m})$, and

$10^{6}$ is a conversion factor $\left(\mathrm{cm}^{3} / \mathrm{m}^{3}\right)$.

The concentration of the releasable form of the material varies with time and is influenced by the rate of release (leaching), the radiological transformation rates, and the rate of change from the release-immune form to the releasable form. The processes that are considered in modeling the concentration of the releasable form of a radionuclide are as follows. (The mathematical representations of the processes are written considering the activity of the releasable form of the radionuclide in the primary contamination over a time period of $\delta$.)

\section{D.2.1 Change of Radionuclide-Bearing Material from Release-Immune Form to Releasable Form}

The change from the release-immune form to releasable form within the primary contamination is given by the following:

$$
\dot{C}_{i}^{\text {unreleasabe } \rightarrow \text { releasable }}(t) \delta t
$$

where

$\dot{C}_{i}^{u n r e l e a s a b e \rightarrow r e l e a s a b l e}$ is the time-dependent rate of change of the radionuclide from the releaseimmune form to the releasable form ( $\mathrm{pCi} / \mathrm{yr})$. 


\section{D.2.2 Radiological Transformations}

The ingrowth of the releasable form within the primary contamination is given by the following:

$$
\lambda_{i} s_{i-1}^{r}(t) \rho_{b} V \delta t
$$

where

$\lambda_{i}$ is the radiological transformation rate of the radionuclide $\left(\mathrm{yr}^{-1}\right)$,

$s_{i-1}^{r}(t)$ is the releasable concentration of the parent radionuclide in primary contamination $(\mathrm{pCi} / \mathrm{g})$,

$\rho_{b}$ is the dry bulk density of primary contamination $\left(\mathrm{g} / \mathrm{cm}^{3}\right)$, and

$V$ is the volume of primary contamination $\left(\mathrm{cm}^{3}\right)$.

The decay of the releasable form within the primary contamination is given by

$$
\lambda_{i} s_{i}^{r}(t) \rho_{b} V \delta t
$$

where

$\lambda_{i}$ is the radiological transformation rate of the radionuclide $\left(\mathrm{yr}^{-1}\right)$,

$s_{i}^{r}(t)$ is the releasable concentration of the $\mathrm{i}^{\text {th }}$ radionuclide in primary contamination $(\mathrm{pCi} / \mathrm{g})$,

$\rho_{b}$ is the dry bulk density of primary contamination $\left(\mathrm{g} / \mathrm{cm}^{3}\right)$, and

$V$ is the volume of primary contamination $\left(\mathrm{cm}^{3}\right)$.

\section{D.2.3 First-order, Rate-controlled Leaching}

The loss due to leaching is given by

$$
\mu_{i} s_{i}^{r}(t) \rho_{b} V \delta t
$$

where

$\mu_{i}$ is the first-order rate at which the radionuclide is leached out of the mixing layer $\left(\mathrm{yr}^{-1}\right)$, 
$s_{i}^{r}(t)$ is the releasable concentration of the radionuclide in primary contamination $(\mathrm{pCi} / \mathrm{g})$, $\rho_{b}$ is the dry bulk density of primary contamination $\left(\mathrm{g} / \mathrm{cm}^{3}\right)$, and $V$ is the volume of primary contamination $\left(\mathrm{cm}^{3}\right)$.

The net effect of these processes is the change in the releasable activity in the primary contamination given by the following:

$$
\rho_{b} V \frac{d s_{i}^{r}(t)}{d t} \delta t
$$

where

$s_{i}^{r}(t)$ is the releasable concentration of the radionuclide in primary contamination $(\mathrm{pCi} / \mathrm{g})$,

$\rho_{b}$ is the dry bulk density of primary contamination $\left(\mathrm{g} / \mathrm{cm}^{3}\right)$, and

$V$ is the volume of primary contamination $\left(\mathrm{cm}^{3}\right)$.

By equating the combined effects of the processes to the net change, we get the radioactivity balance equation:

$$
\rho_{b} V \frac{d s_{i}^{r}(t)}{d t}=\dot{C}_{i}^{\text {unreleasable } \rightarrow \text { releasable }}(t)+\lambda_{i} s_{i-1}^{r}(t) \rho_{b} V-\lambda_{i} s_{i}^{r}(t) \rho_{b} V-\mu_{i} s_{i}^{r}(t) \rho_{b} V
$$

This formulation simplifies to

$$
\frac{d s_{i}^{r}(t)}{d t}+\left(\lambda_{i}+\mu_{i}\right) s_{i}^{r}(t)=\frac{\dot{C}_{i}^{\text {unreleasable } \rightarrow \text { releasable }}(t)}{\rho_{b} V}+\lambda_{i} s_{i-1}^{r}(t)
$$

This expression can be integrated from 0 to time $t$ to obtain

$s_{i}^{r}(t)=s_{i}^{r}(0) e^{-\left(\lambda_{i}+\mu_{i}\right) t}+\frac{e^{-\left(\lambda_{i}+\mu_{i}\right)(t)}}{\rho_{b} V} \int_{0}^{t} \dot{C}_{i}^{\text {unreleasable } \rightarrow \text { releasable }}(t) e^{\left(\lambda_{i}+\mu_{i}\right) t} d t+\lambda_{i} e^{-\left(\lambda_{i}+\mu_{i}\right) t} \int_{0}^{t} s_{i-1}^{r}(t) e^{\left(\lambda_{i}+\mu_{i}\right) t} d t$ 
The form of $\dot{C}_{i}^{\text {unreleasabe } \rightarrow \text { releasable }}(t)$ needs to be known to solve this equation. It is assumed in the code that $\dot{C}_{i}^{u n r e l e a s a b e \rightarrow \text { releasable }}(t)$ has a linear variation between the known values at time points, as follows:

$$
\dot{C}_{i}^{\text {unreleasabe } \rightarrow \text { releasable }}(t)=\left(\alpha_{i}+\beta_{i} t\right) \rho_{b} V,
$$

where $\alpha_{i}$ and $\beta_{i}$ are constants between each pair of the time points.

\section{D.2.4 Releasable Concentration of the First Member of a Transformation Chain}

Representing the time-dependent deposition rate by a linear function of time and integrating ${ }^{7}$ yields:

$$
s_{1}^{r}(t)=s_{1}^{r}(0) e^{-\left(\lambda_{1}+\mu_{1}\right) t}+\frac{\left(\alpha_{1}-\frac{\beta_{1}}{\lambda_{1}+\mu_{1}}\right)\left(1-e^{-\left(\lambda_{1}+\mu_{1}\right) t}\right)+\beta_{1} t}{\lambda_{1}+\mu_{1}} .
$$

Next, collecting the terms yields:

$$
s_{1}^{r}(t)=A_{1}^{0}+A_{1}^{1} t+C_{1}^{1} e^{-\left(\lambda_{1}+\mu_{1}\right) t},
$$

where

$$
A_{1}^{1}=\frac{\beta_{1}}{\lambda_{1}+\mu_{1}}
$$

7

$$
\begin{aligned}
s_{1}^{r}(t)= & s_{1}^{r}(0) e^{-\left(\lambda_{1}+\mu_{1}\right) t}+\frac{e^{-\left(\lambda_{1}+\mu_{1}\right) t}}{\rho_{b} V} \int_{0}^{t}\left(\alpha_{1}+\beta_{1} t\right) \rho_{b} V e^{\left(\lambda_{1}+\mu_{1}\right) t} d t \\
& =s_{1}^{r}\left(t_{1}\right) e^{-\left(\lambda_{1}+\mu_{1}\right) t}+e^{-\left(\lambda_{1}+\mu_{1}\right) t} \alpha_{i} \frac{e^{\left(\lambda_{1}+\mu_{1}\right) t}-1}{\lambda_{1}+\mu_{1}} \\
& +e^{-\left(\lambda_{1}+\mu_{1}\right) t} \beta_{i}\left(\frac{t e^{\left(\lambda_{1}+\mu_{1}\right) t}-0}{\lambda_{1}+\mu_{1}}-\frac{e^{\left(\lambda_{1}+\mu_{1}\right) t}-1}{\left(\lambda_{1}+\mu_{1}\right)^{2}}\right)
\end{aligned}
$$




$$
\begin{gathered}
A_{1}^{0}=\frac{\alpha_{1}-\frac{\beta_{1}}{\lambda_{1}+\mu_{1}}}{\lambda_{1}+\mu_{1}}=\frac{\alpha_{1}}{\lambda_{1}+\mu_{1}}-\frac{A_{1}^{1}}{\lambda_{1}+\mu_{1}}, \text { and } \\
C_{1}^{1}=s_{1}^{r}(0)-\frac{\alpha_{1}-\frac{\beta_{1}}{\lambda_{1}+\mu_{1}}}{\lambda_{1}+\mu_{1}}=s_{1}^{r}(0)-A_{1}^{0} .
\end{gathered}
$$

\section{D.2.5 Releasable Concentration of the Second Member of a Transformation Chain}

The expression for the second member of the transformation chain is derived by building on the derivation for the first member of the chain, as follows:

$$
\begin{aligned}
s_{2}^{r}(t)=s_{2}^{r}(0) e^{-\left(\lambda_{2}+\mu_{2}\right) t}+ & \frac{\left(\alpha_{2}-\frac{\beta_{2}}{\lambda_{2}+\mu_{2}}\right)\left(1-e^{-\left(\lambda_{2}+\mu_{2}\right) t}\right)+\beta_{2} t}{\lambda_{2}+\mu_{2}} . \\
& +\lambda_{2} e^{-\left(\lambda_{2}+\mu_{2}\right) t} \int_{0}^{t} s_{1}^{r}(t) e^{\left(\lambda_{2}+\mu_{2}\right) t} d t
\end{aligned}
$$

Substituting for the concentration of the first member and integrating ${ }^{8}$ gives the following:

8

$$
\begin{aligned}
& \int_{0}^{t}\left(A_{1}^{0}+A_{1}^{1} t+C_{1}^{1} e^{-\left(\lambda_{1}+\mu_{1}\right) t}\right) e^{\left(\lambda_{2}+\mu_{2}\right) t} d t=A_{1}^{0} \frac{e^{\left(\lambda_{2}+\mu_{2}\right) t}-1}{\lambda_{2}+\mu_{2}} \\
& +A_{1}^{1} \frac{t e^{\left(\lambda_{2}+\mu_{2}\right) t}}{\lambda_{2}+\mu_{2}}-A_{1}^{1} \frac{e^{\left(\lambda_{2}+\mu_{2}\right) t}-1}{\left(\lambda_{2}+\mu_{2}\right)^{2}}+C_{1}^{1} \frac{e^{\left(\lambda_{2}+\mu_{2}-\lambda_{1}-\mu_{1}\right) t}-1}{\lambda_{2}+L_{2}^{o}-\lambda_{1}-\mu_{1}}
\end{aligned}
$$




$$
\begin{gathered}
s_{2}^{r}(t)=s_{2}^{r}(0) e^{-\left(\lambda_{2}+\mu_{2}\right) t}+\frac{\left(\alpha_{2}-\frac{\beta_{2}}{\lambda_{2}+\mu_{2}}\right)\left(1-e^{-\left(\lambda_{2}+\mu_{2}\right) t}\right)+\beta_{2} t}{\lambda_{2}+\mu_{2}} \\
\lambda_{2} \frac{\left(A_{1}^{0}-\frac{A_{1}^{1}}{\lambda_{2}+\mu_{2}}\right)\left(1-e^{-\left(\lambda_{2}+\mu_{2}\right) t}\right)+A_{1}^{1} t}{\lambda_{2}+\mu_{2}}+\lambda_{2} C_{1}^{1} \frac{e^{-\left(\lambda_{1}+\mu_{1}\right) t}-e^{-\left(\lambda_{2}+\mu_{2}\right) t}}{\lambda_{2}+\mu_{2}-\lambda_{1}-\mu_{1}}
\end{gathered}
$$

Collecting the terms yields:

$$
s_{2}^{r}(t)=A_{2}^{0}+A_{2}^{1} t+C_{2}^{1} e^{-\left(\lambda_{1}+\mu_{1}\right) t}+C_{2}^{2} e^{-\left(\lambda_{2}+\mu_{2}\right) t},
$$

where

$$
\begin{aligned}
A_{2}^{1} & =\frac{\beta_{2}}{\lambda_{2}+\mu_{2}}+\frac{\lambda_{2} A_{1}^{1}}{\lambda_{2}+\mu_{2}}, \\
A_{2}^{0} & =\frac{\alpha_{2}-\frac{\beta_{2}}{\lambda_{2}+\mu_{2}}}{\lambda_{2}+\mu_{2}}+\lambda_{2} \frac{A_{1}^{0}-\frac{A_{1}^{1}}{\lambda_{2}+\mu_{2}}}{\lambda_{2}+\mu_{2}} \\
& =\frac{\alpha_{2}}{\lambda_{2}+\mu_{2}}+\frac{\lambda_{2} A_{1}^{0}}{\lambda_{2}+\mu_{2}}-\frac{A_{2}^{1}}{\lambda_{2}+\mu_{2}} \\
C_{2}^{1} & =\frac{\lambda_{2} C_{1}^{1}}{\lambda_{2}+\mu_{2}-\lambda_{1}-\mu_{1}}, \text { and } \\
C_{2}^{2} & =s_{2}^{r}(0)-A_{2}^{0}-C_{2}^{1} .
\end{aligned}
$$

\section{D.2 6 Releasable Concentration of the $\mathrm{j}^{\text {th }}$ Member of a Transformation Chain}

Using the form of the expressions for the first and second members of the chains, the expression for the $j^{\text {th }}$ member of the chain is obtained by induction. The concentration of the $i^{\text {th }}$ member of the transformation chain in offsite soil is as follows: 


$$
s_{i}^{r}(t)=A_{i}^{0}+A_{i}^{1} t+\sum_{k=1}^{i} C_{i}^{k} e^{-\left(\lambda_{k}+\mu_{k}\right) t}
$$

Then, the concentration of the next member, $j$, of the transformation chain is given by:

$$
\begin{aligned}
& s_{j}^{r}(t)=s_{j}^{r}(0) e^{-\left(\lambda_{j}+\mu_{j}\right) t}+\frac{\left(\alpha_{j}-\frac{\beta_{j}}{\lambda_{j}+\mu_{j}}\right)\left(1-e^{-\left(\lambda_{j}+\mu_{j}\right) t}\right)+\beta_{j} t}{\lambda_{j}+\mu_{j}} \\
& +\lambda_{j} e^{-\left(\lambda_{j}+\mu_{j}\right) t} \int_{0}^{t}\left(A_{i}^{0}+A_{i}^{1} t+\sum_{k=1}^{i} C_{i}^{k} e^{-\left(\lambda_{k}+\mu_{k}\right) t}\right) e^{\lambda_{j}+\mu_{j} t} d t
\end{aligned} .
$$

Integration gives the following:

$$
\begin{aligned}
s_{j}^{r}(t)= & s_{j}^{r}(0) e^{-\left(\lambda_{j}+\mu_{j}\right) t}+\lambda_{j} \sum_{k=1}^{i} C_{i}^{k} \frac{e^{-\left(\lambda_{k}+\mu_{k}\right) t}-e^{-\left(\lambda_{j}+\mu_{j}\right) t}}{\lambda_{j}+\mu_{j}-\lambda_{k}-\mu_{k}} \\
& +\frac{\left(\alpha_{j}+\lambda_{j} A_{i}^{0}-\frac{\beta_{j}+\lambda_{j} A_{i}^{1}}{\lambda_{j}+\mu_{j}}\right)\left(1-e^{-\left(\lambda_{j}+\mu_{j}\right) t}\right)+\left(\beta_{j}+\lambda_{j} A_{i}^{1}\right) t}{\left(\lambda_{j}+\mu_{j}\right)} \\
s_{j}^{r}(t)= & s_{j}^{r}(0) e^{-\left(\lambda_{j}+\mu_{j}\right) t}+\frac{\left(\alpha_{j}-\frac{\beta_{j}}{\lambda_{j}+\mu_{j}}\right)\left(1-e^{-\left(\lambda_{j}+\mu_{j}\right) t}\right)+\beta_{j} t}{\lambda_{j}+\mu_{j}} \\
& +\lambda_{j} \frac{\left(A_{i}^{0}-\frac{A_{i}^{1}}{\lambda_{j}+\mu_{j}}\right)\left(1-e^{-\left(\lambda_{j}+\mu_{j}\right) t}\right)+A_{i}^{1} t}{\lambda_{j}+\mu_{j}}+\lambda_{j} \sum_{k=1}^{i} C_{i}^{k} \frac{e^{-\left(\lambda_{k}+\mu_{k}\right) t}-e^{-\left(\lambda_{j}+\mu_{j}\right) t}}{\lambda_{j}+\mu_{j}-\lambda_{k}-\mu_{k}}
\end{aligned}
$$

Collecting the terms yields:

$$
s_{j}^{r}(t)=A_{j}^{0}+A_{j}^{1} t+\sum_{k=1}^{j} C_{j}^{k} e^{-\left(\lambda_{k}+\mu_{k}\right) t}
$$


where

$$
\begin{aligned}
A_{j}^{1} & =\frac{\beta_{j}}{\lambda_{j}+\mu_{j}}+\frac{\lambda_{j} A_{j-1}^{1}}{\lambda_{j}+\mu_{j}}=\frac{\beta_{j}+\lambda_{j} A_{j-1}^{1}}{\lambda_{j}+\mu_{j}}, A_{1}^{1}=\frac{\beta_{1}}{\lambda_{1}+\mu_{1}}, \\
A_{j}^{0} & =\frac{\alpha_{j}-\frac{\beta_{j}}{\lambda_{j}+\mu_{j}}}{\lambda_{j}+\mu_{j}}+\lambda_{j} \frac{A_{j-1}^{0}-\frac{A_{j-1}^{1}}{\lambda_{j}+\mu_{j}}}{\lambda_{j}+\mu_{j}} \\
& =\frac{\alpha_{j}}{\lambda_{j}+\mu_{j}}+\frac{\lambda_{j} A_{j-1}^{0}}{\lambda_{j}+\mu_{j}}-\frac{A_{j}^{1}}{\lambda_{j}+\mu_{j}} \\
& =\frac{\alpha_{j}+\lambda_{j} A_{j-1}^{0}-A_{j}^{1}}{\lambda_{j}+\mu_{j}}, \\
A_{1}^{0} & =\frac{\alpha_{1}-\frac{\beta_{1}}{\lambda_{1}+\mu_{1}}}{\lambda_{1}+\mu_{1}}=\frac{\alpha_{1}}{\lambda_{1}+\mu_{1}}-\frac{A_{1}^{1}}{\lambda_{1}+\mu_{1}}, \\
C_{j}^{k}= & \frac{\lambda_{j} C_{j-1}^{k}}{\lambda_{j}+\mu_{j}-\lambda_{k}-\mu_{k}} \text { for all } k \neq j, \text { and } \\
C_{j}^{j}= & s_{j}^{r}(0)-A_{j}^{0}-\sum_{k=1}^{j-1} C_{j}^{k}, C_{1}^{1}=s_{1}^{r}(0)-\frac{\alpha_{1}-\frac{\beta_{1}}{\lambda_{1}+\mu_{1}}}{\lambda_{1}+\mu_{1}}=s_{1}^{r}(0)-A_{1}^{0} .
\end{aligned}
$$

The release from the contaminated material into the infiltration water is computed by applying the first-order leach rate to the inventory of releasable radionuclides, as follows:

$$
R_{k}(t)=\mu_{k} s_{k}^{r}(t) \rho_{p c} A\left(f_{v m} T_{m i x}^{c}(t)+T_{p c}^{u m}(t)\right) 10^{6}
$$

where

$R_{k}(t)$ is the rate of release of the $\mathrm{k}^{\text {th }}$ radionuclide, from the releasable form to the infiltrating water ( $\mathrm{pCi} / \mathrm{yr})$.

$\mu_{k}$ is the first order release rate of the $\mathrm{k}^{\text {th }}$ radionuclide from the releasable form to the infiltrating water $\left(\mathrm{yr}^{-1}\right)$,

$t$ is the time since the determination of the inventory (yr), 
$\rho_{p c}$ is the dry bulk density of the primary contamination $\left(\mathrm{g} / \mathrm{cm}^{3}\right)$,

$A$ is the area of the primary contamination $\left(\mathrm{m}^{2}\right)$,

$f_{v m}$ is the volume fraction of the material from the primary contamination in the mixing layer

(dimensionless)

$T_{m i x}^{c}(t)$ is the thickness of the contaminated mixing layer $(\mathrm{m})$,

$T_{p c}^{u m}(t)$ is the thickness of the undisturbed primary contamination $(\mathrm{m})$,

$10^{6}$ is a conversion factor $\left(\mathrm{cm}^{3} / \mathrm{m}^{3}\right)$.

The release rate for the equilibrium desorption release option can be obtained by using a very high value for the first-order leach rate. They can also be computed more directly as being equal to the rate of change from the release-immune form to the releasable form. The direct method is preferable because using a high leach rate to model the equilibrium desorption requires a much larger number of time points.

\section{D.3 Three Release Options Described in Chapter 2}

The three release options of Version 3.0 beta discussed in Chapter 2 of this report-namely, First-Order Release with Transport, Equilibrium Release, and Uniform Release-are limiting cases of the more versatile releases in Version 3.1.

\section{D.3.1 Comparison of First-Order Release with Transport}

The first-order release with transport discussed in Chapter 2 can be modeled in Version 3.1 using the following procedures (and as illustrated in Figure D.2). First, uncheck the "Version 2 Release Methodology" check box to activate the new release options. Verify that the four input boxes-Time at which radionuclide first becomes releasable (delay time), Fraction of radionuclide bearing material that is initially releasable, Time over which transformation to releasable form occurs, and Total fraction of radionuclide bearing material that is releasable are at their default values of $0,1,0,1$, respectively. Verify that the "First Order Rate Controlled Release with Transport" radio button is selected in the "Release mechanism" frame. Next, specify the desired leach rate in the "Initial Leach Rate" and "Final Leach Rate" input boxes. Finally, specify the distribution coefficient in the contaminated zone. 


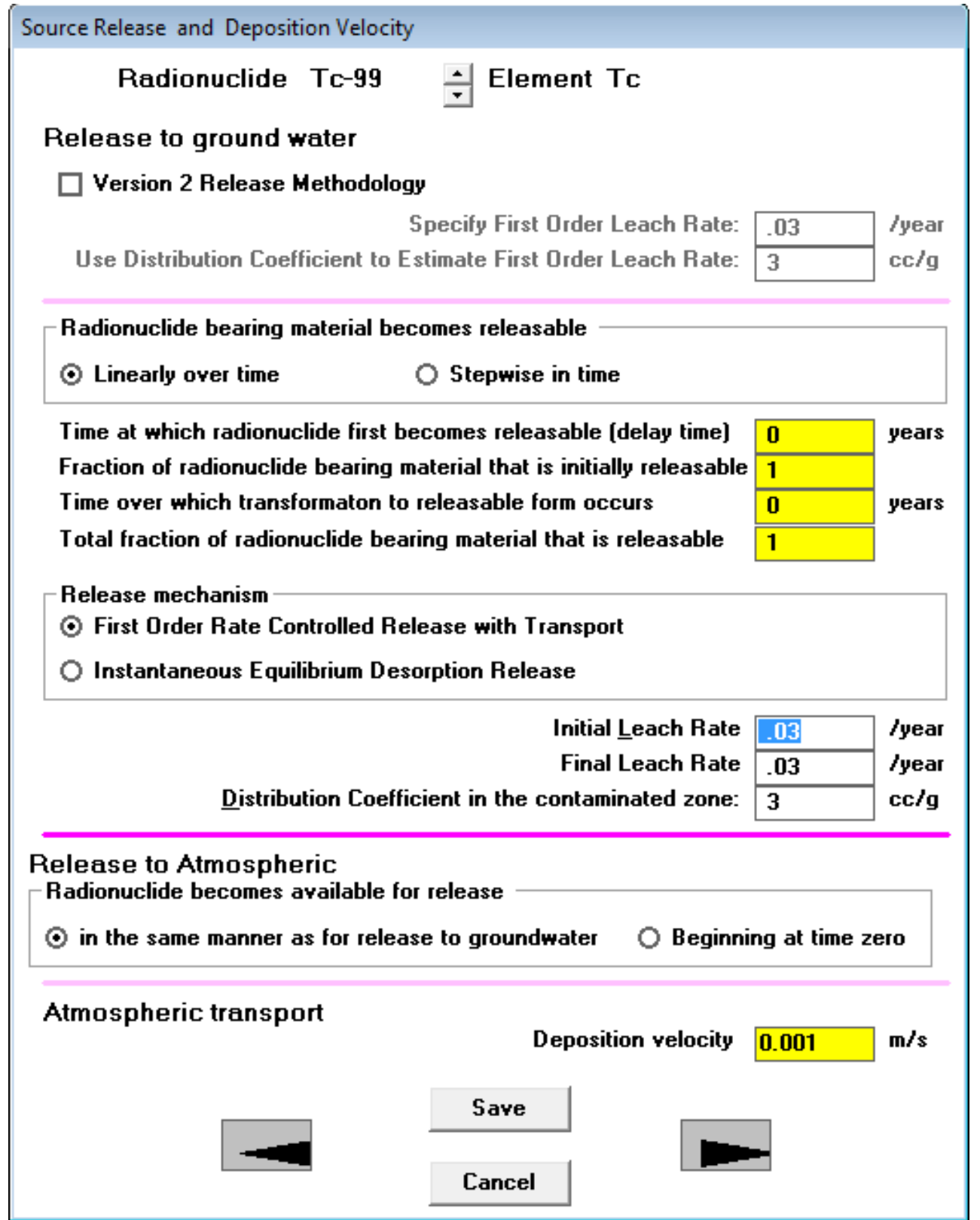

FIGURE D.2 Specifying Inputs for First-Order, Rate-Controlled Release
of Material That Initially Is Entirely in the Releasable Form

The temporal plots of component (pathway) doses obtained from Version 3.1 for this case are compared with the temporal plots of component doses obtained for the first-order release with transport of Version 3.0 beta in Figure D.3. There are no differences between the two plots in Figure D.3. 


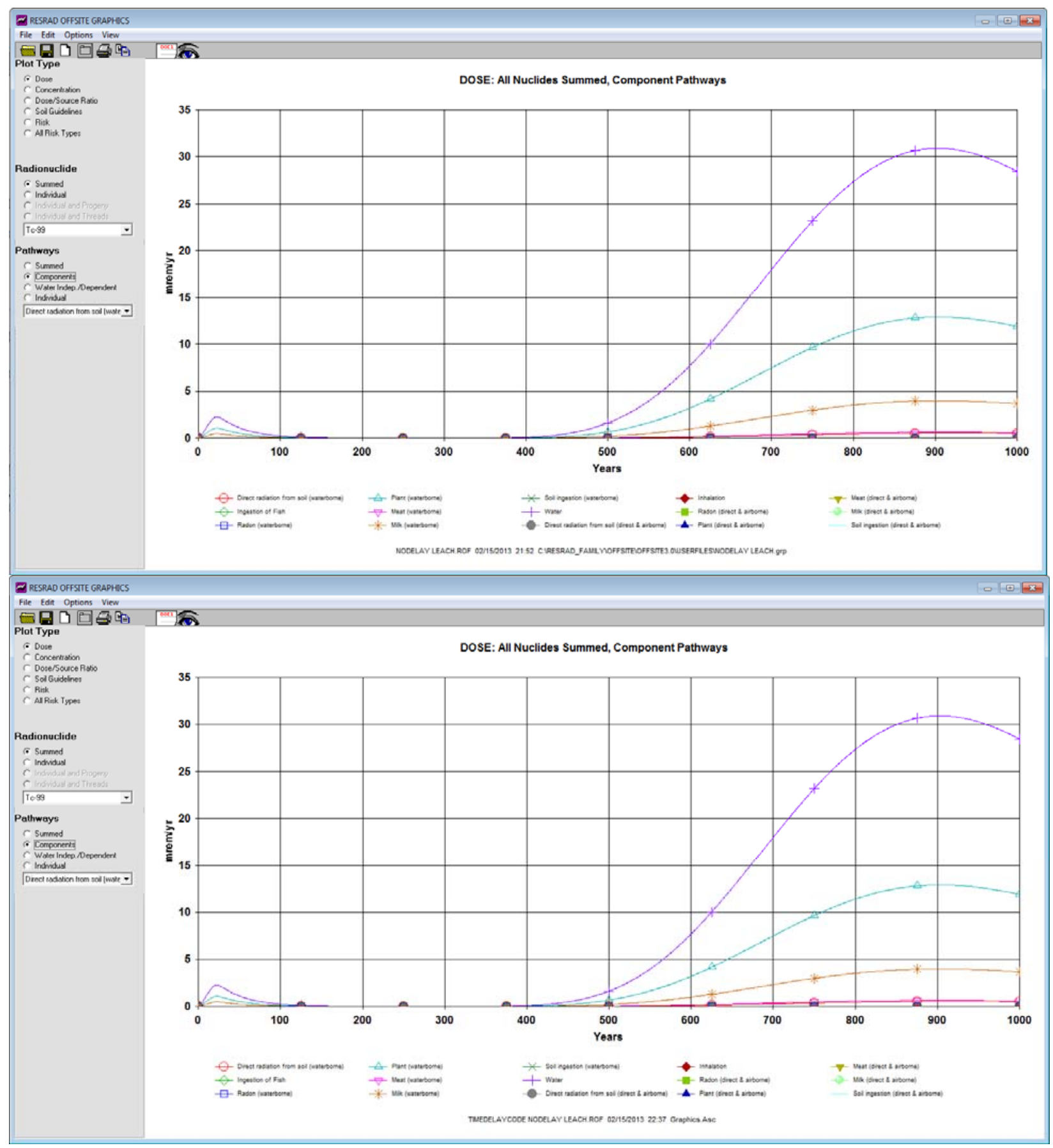

FIGURE D.3 Comparison of Pathway Doses Predicted by Version 3.1 with the Doses Predicted by the Previously Benchmarked 3.0 Beta Version of RESRAD-OFFSITE for a First-Order, Rate-Controlled Release 


\section{D.3.2 Comparison of Equilibrium Release}

The equilibrium release discussed in Chapter 2 can be modeled in Version 3.1 using the following procedures (and as illustrated in Figure D.4). First, uncheck the "Version 2 Release Methodology" check box to activate the new release options. Verify that the four input boxesTime at which radionuclide first becomes releasable (delay time), Fraction of radionuclide bearing material that is initially releasable, Time over which transformation to releasable form occurs, and Total fraction of radionuclide bearing material that is releasable are at their default values of $0,1,0,1$, respectively. Click the "Instantaneous Equilibrium Desorption Release" radio button in the "Release mechanism" frame. Next, specify the desired distribution coefficient in the contaminated zone.

The temporal plots of component (pathway) doses obtained from Version 3.1 for the equilibrium desorption release discussed in Chapter 2 are compared with the temporal plots of component doses obtained from Version 3.0 beta in Figure D.5. There are no differences between the two plots in Figure D.5. 
Source Release and Deposition Velocity

\section{Radionuclide Tc-99 $\div$ Element Tc}

Release to ground water

Version 2 Release Methodology

Specify First Order Leach Rate:

Use Distribution Coefficient to Estimate First Order Leach Rate:

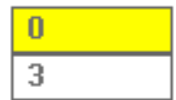

/year

$\mathrm{cc} / \mathrm{g}$

Radionuclide bearing material becomes releasable

C Linearly over time

Stepwise in time

Time at which radionuclide first becomes releasable [delay time]

Fraction of radionuclide bearing material that is initially releasable

Time over which transformaton to releasable form occurs

Total fraction of radionuclide bearing material that is releasable

\begin{tabular}{|l|}
\hline 0 \\
\hline 1 \\
\hline 0 \\
\hline \hline 1 \\
\hline
\end{tabular}

years

years

Release mechanism

First Order Rate Controlled Release with Transport

$\odot$ Instantaneous Equilibrium Desorption Release

Initial Leach Rate

Final Leach Rate

Distribution Coefficient in the contaminated zone:

\begin{tabular}{|l|l|}
\hline 0 & $\begin{array}{l}\text { /year } \\
\text { /year } \\
\text { cc/g }\end{array}$ \\
\hline 3 & \\
\hline
\end{tabular}

Release to Atmospheric

- Radionuclide becomes available for release

$\odot$ in the same manner as for release to groundwater

Beginning at time zero

Atmospheric transport

Deposition velocity 0.001 $\mathrm{m} / \mathrm{s}$

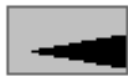

Save

Cancel

FIGURE D.4 Specifying Inputs for Equilibrium Desorption Release of Material That Initially Is Entirely in the Releasable Form 


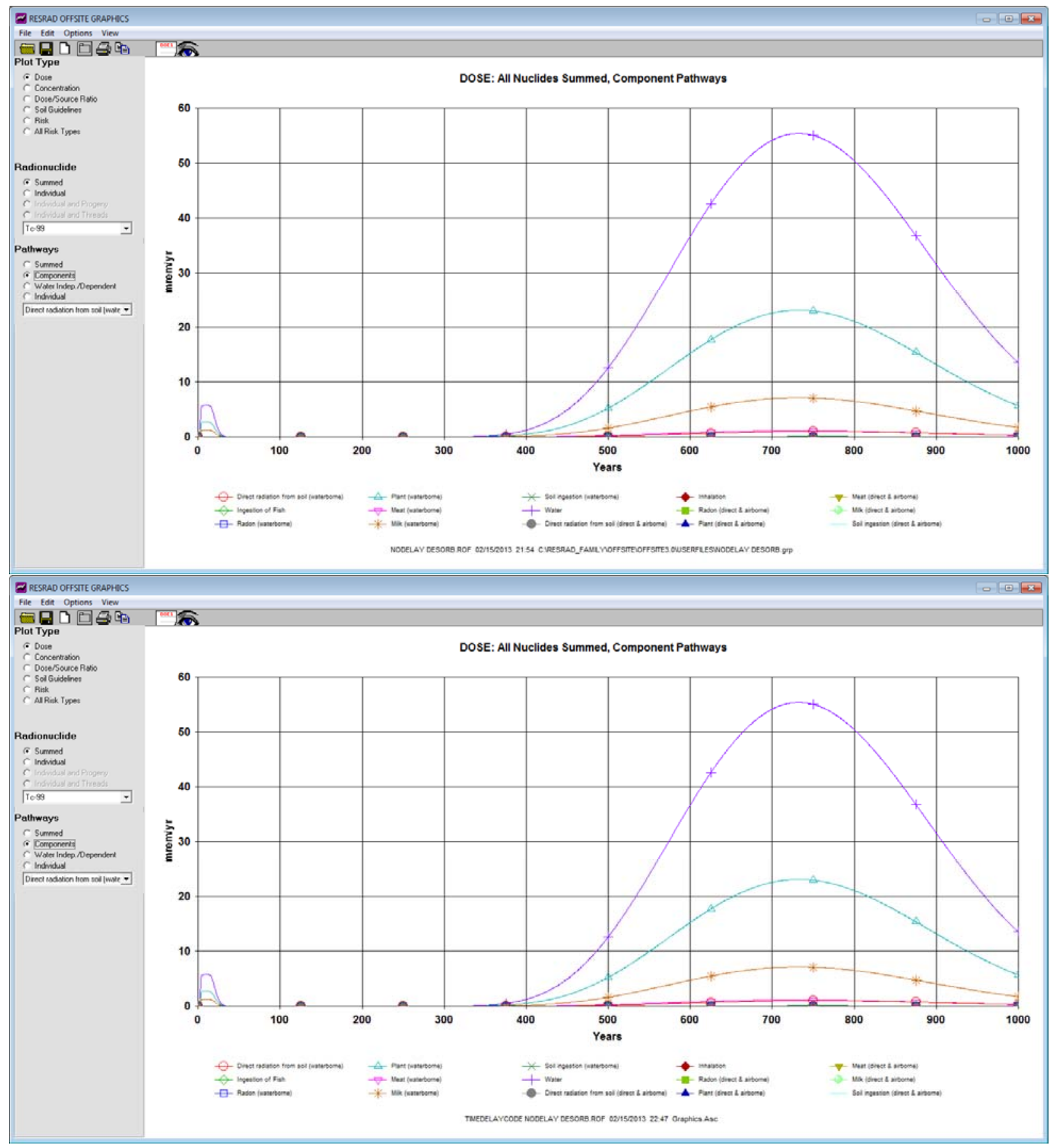

FIGURE D.5 Comparison of Pathway Doses Predicted by Version 3.1 with the Doses Predicted by the Previously Benchmarked 3.0 Beta Version of RESRADOFFSITE for an Equilibrium Desorption Release 


\section{D.3.3 Comparison of Uniform Release}

The Uniform Release discussed in Chapter 2 can be modeled in Version 3.1 using the following procedures (and as illustrated in Figure D.6). First, uncheck the "Version 2 Release Methodology" check box to activate the new release options. Verify that the two input boxes Time at which radionuclide first becomes releasable (delay time) and Fraction of radionuclide bearing material that is releasable are at their default values of 0 and 1, respectively. Specify a value of 0 for the Fraction of radionuclide bearing that is initially releasable. Click the "Instantaneous Equilibrium Desorption Release" radio button in the "Release mechanism"

frame. Next, specify the desired distribution coefficient in the contaminated zone and the Time over which transformation to releasable form occurs.

The temporal plots of component (pathway) doses obtained from Version 3.1 for this case are compared with the temporal plots of component doses obtained from Version 3.0 beta of RESRAD-OFFSITE in Figure D.7. There are no differences between the two plots in Figure D.7. 


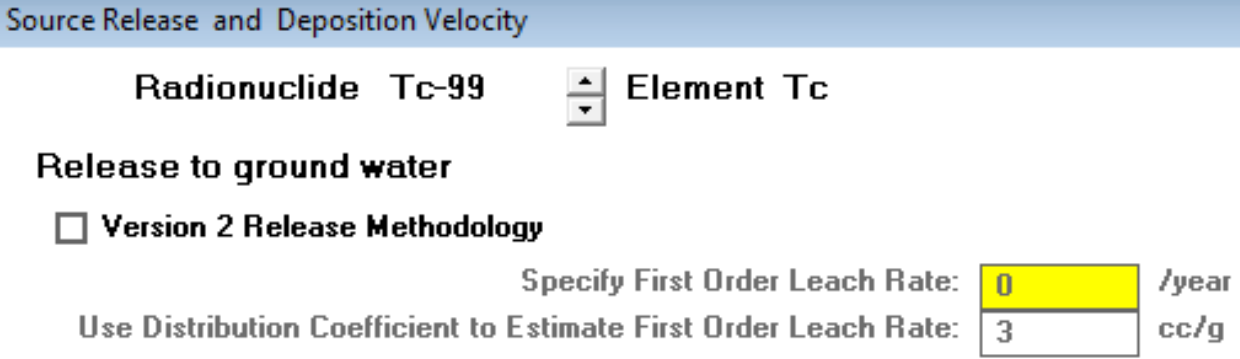

-Radionuclide bearing material becomes releasable

$\odot$ Linearly over time

Stepwise in time

Time at which radionuclide first becomes releasable [delay time] Fraction of radionuclide bearing material that is initially releasable Time over which transformaton to releasable form occurs

Total fraction of radionuclide bearing material that is releasable

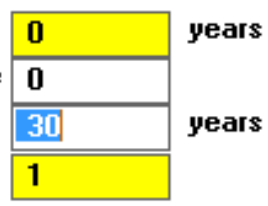

-Release mechanism

First Order Rate Controlled Release with Transport

C Instantaneous Equilibrium Desorption Release

Initial Leach Rate

Final Leach Rate

Distribution Coefficient in the contaminated zone:

\begin{tabular}{|l|l|}
\hline 0 & /year \\
\cline { 1 - 1 } & /year \\
\cline { 1 - 1 } & $\mathrm{cc} / \mathrm{g}$ \\
\hline
\end{tabular}

Release to Atmospheric

- Radionuclide becomes ayailable for release

$\odot$ in the same manner as for release to groundwater

\section{Beginning at time zero}

Atmospheric transport

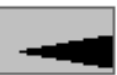

Save

Cancel

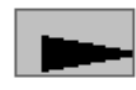

FIGURE D.6 Specifying Inputs for Uniform Release of Material That Initially Is Entirely in the Releasable Form 


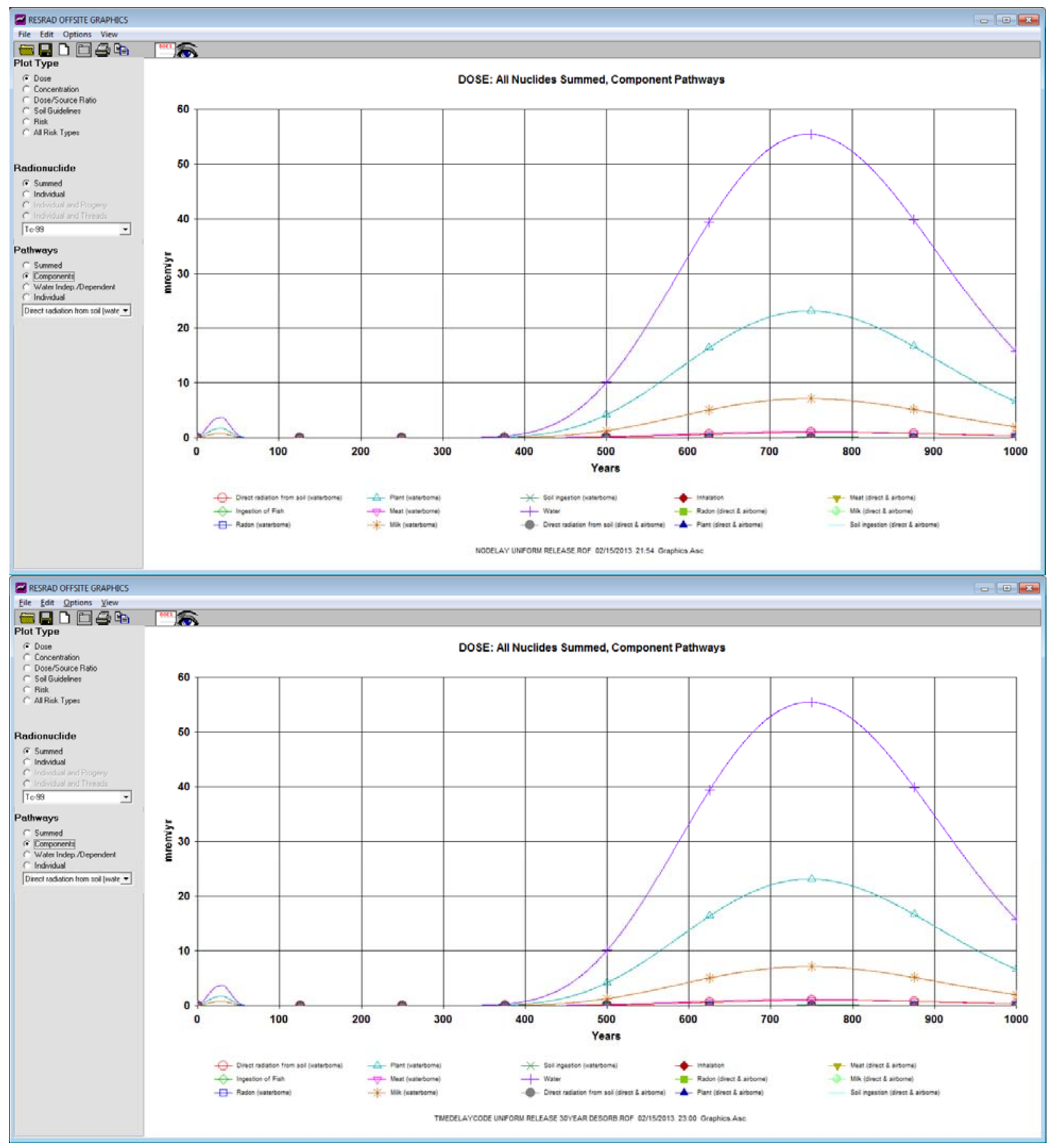

FIGURE D.7 Comparison of Pathway Doses Predicted by Version 3.1 with the Doses Predicted by the Previously Benchmarked 3.0 Beta Version of RESRADOFFSITE for a Uniform Release 


\section{D.4 General Release Options in Version 3.1}

There are four main general release options in Version 3.1, which are obtained by combining one of the two release mechanisms and one of the two temporal profiles of how the radionuclide-bearing material becomes susceptible to release. A first-order release in which radionuclide-bearing material becomes available for release linearly over time is specified, as shown in Figure D.8. No release occurs over a specified period of time-for the first 250 years, in this example. The radionuclides in a specified fraction of the material-one tenth in this example-become available for release at that time. Then, the radionuclides in another fraction of the material become available for release over a specified period of time $-75 \%$ over the next 100 years, in this example. Two first-order leach rates can be specified: the first applies at the beginning of the release, and the second applies after the end of the second period of time. The leach rate varies uniformly over time in the intervening period of time.

Next, a first-order release in which radionuclide-bearing material becomes susceptible for release stepwise over time is specified, as shown in Figure D.9. There are two differences between this case and the preceding case. First, the radionuclides in two specified fractions of the material become available for release at two specified times; for the inputs shown in this example, the radionuclides in $10 \%$ of the material become releasable at 250 years, whereas the radionuclides in another $75 \%$ of the material become susceptible to release in 350 years.

Second, the radionuclides in the first fraction of material are released at the initial leach rate, whereas the radionuclides in the second fraction of material are released at the final leach rate. The leach rates for the radionuclides in the two fractions of material do not change over time. 


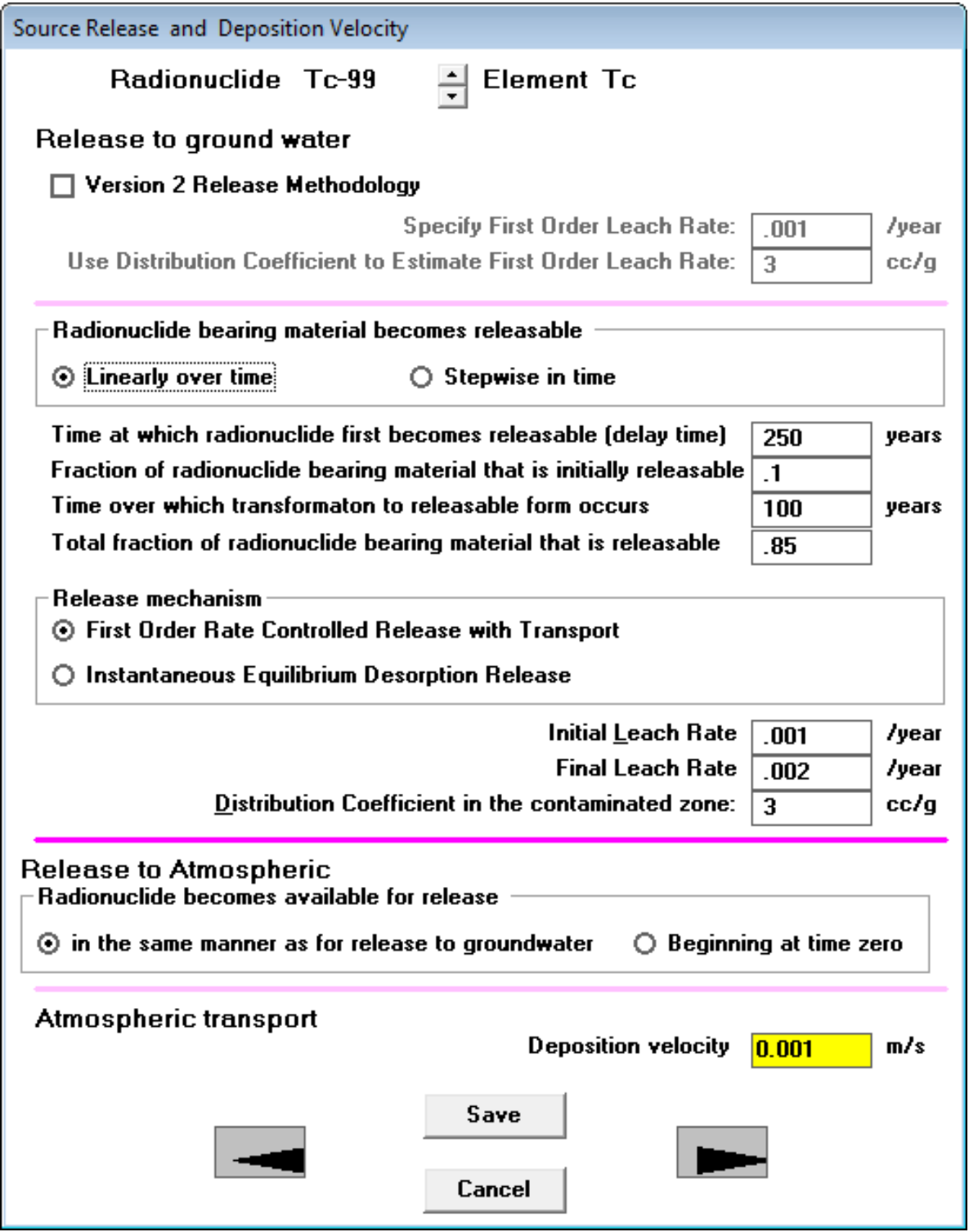

FIGURE D.8 A First-Order Release with Radionuclide-Bearing Material Becoming Available Linearly Over Time 


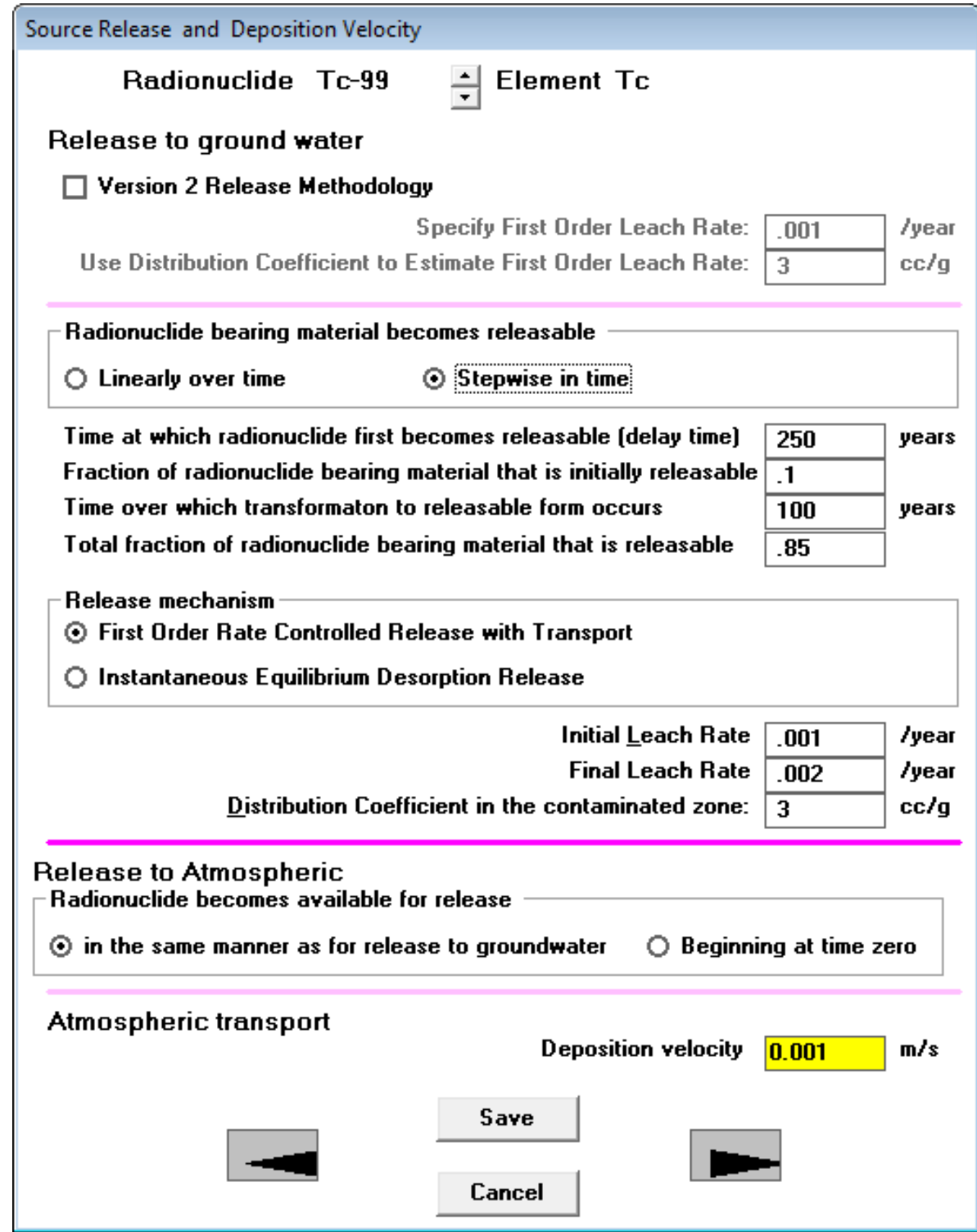

\section{FIGURE D.9 A First-Order Release with Radionuclide-Bearing Material Becoming Available Stepwise in Time}

Examples of instantaneous equilibrium desorption controlled releases in which radionuclidebearing material becomes available for release linearly over time or at two discrete times are shown in Figures D.10 and D.11. The radionuclides that become available for release at any time are distributed between the solids in the soil and the soil moisture according to the specified distribution coefficient at that time. If the primary contamination is conceptualized as a mixture of soil and an ion-exchange medium on which the radionuclides are adsorbed, then 
weighted values need to be input for the distribution coefficients and the physical characteristics of the primary contamination.

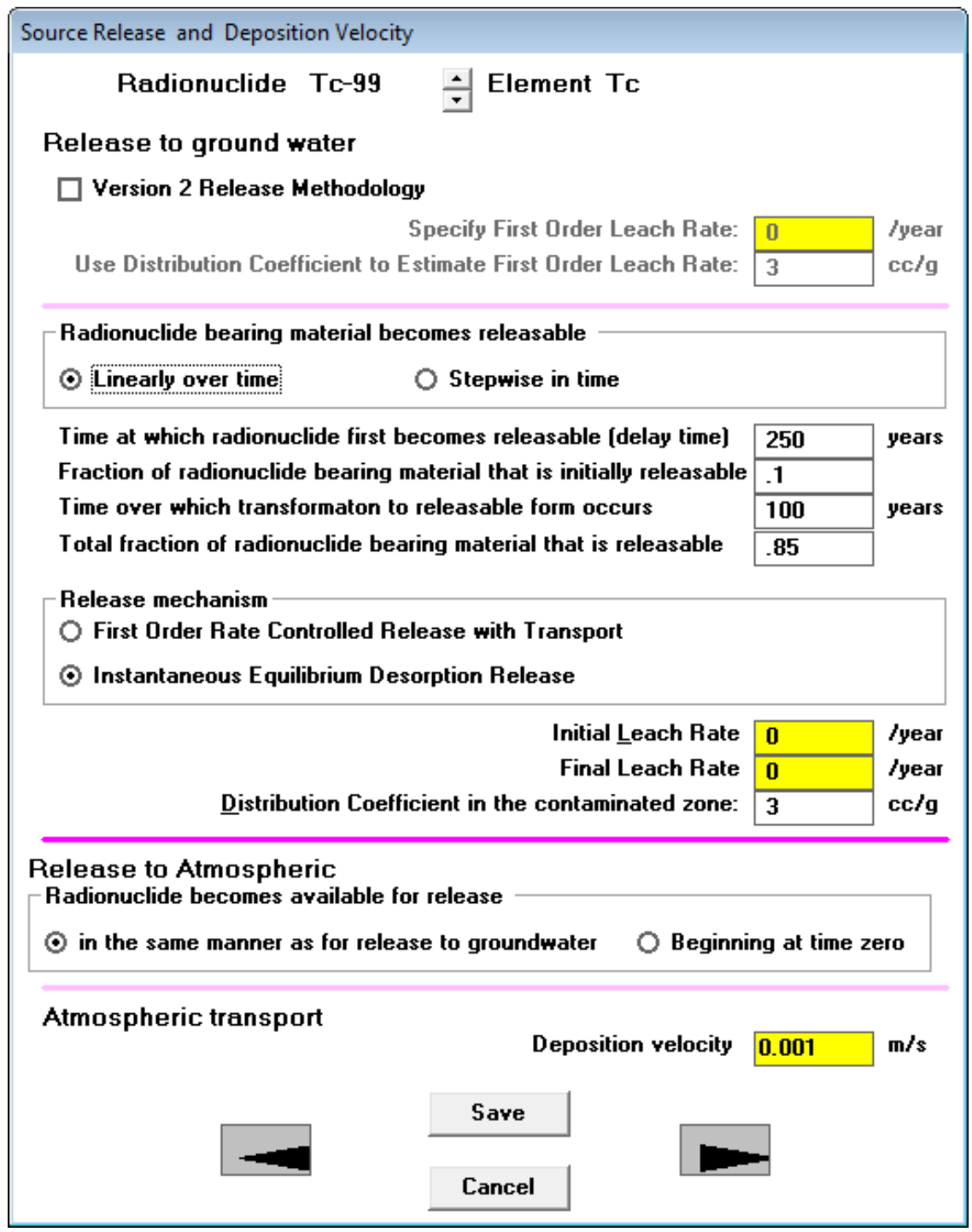
$\begin{aligned} & \text { FIGURE D.10 } \text { An Instantaneous Equilibrium Desorption } \\ & \text { Release in Which Radionuclide-Bearing Material } \\ & \text { Becomes Available for Release Linearly in Time }\end{aligned}$ 


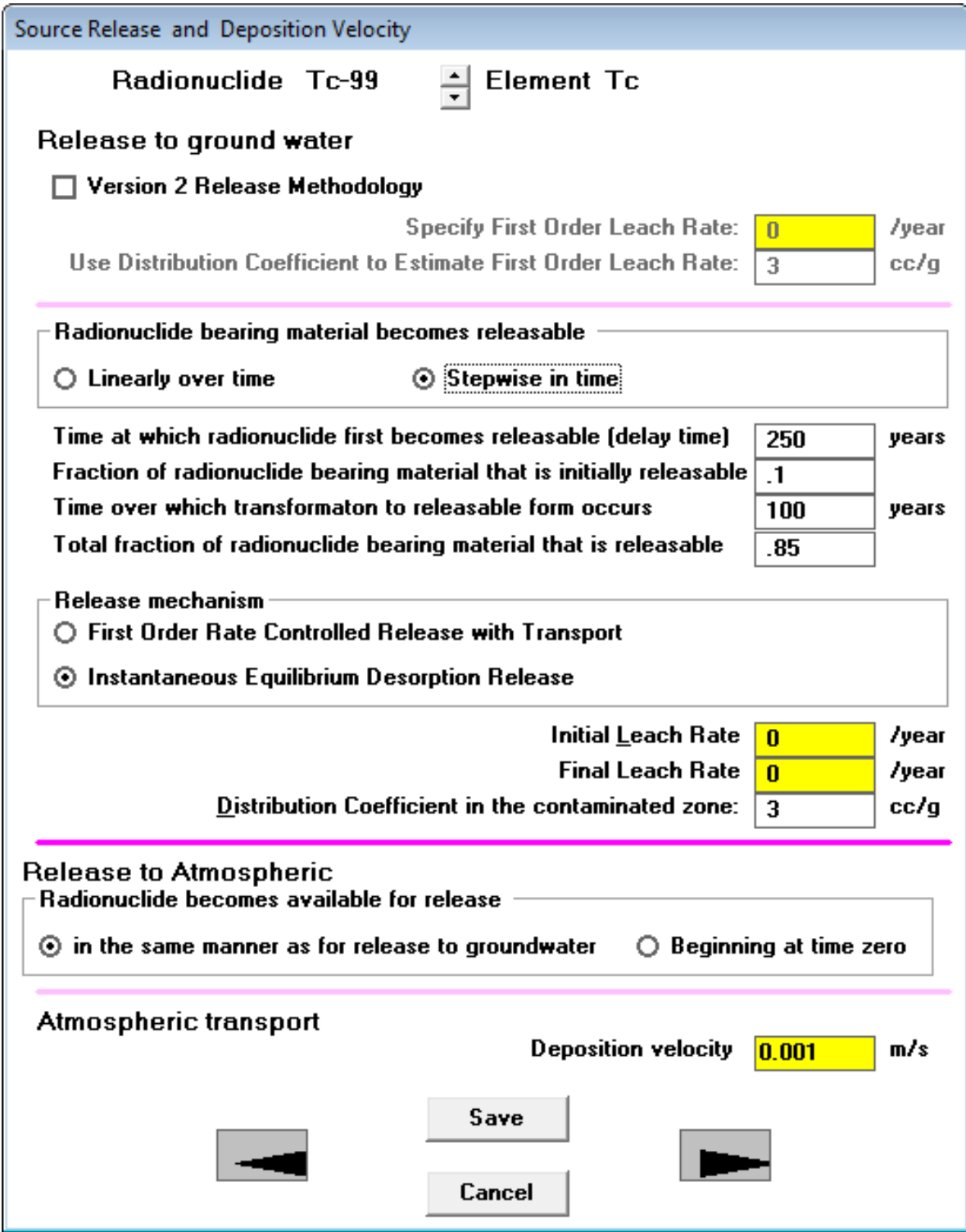

FIGURE D.11 An Instantaneous Equilibrium Desorption Release in Which Radionuclide-Bearing Material Becomes Available for Release Stepwise in Time

\section{D.5 Release of Progeny Radionuclides}

The same release mechanism specified for an initially present radionuclide is also applied to its progeny but at the leach rates and distribution coefficients specified for the progeny. If users desire to model different mechanisms of release for the parent and progeny, then the first-order, 
rate-controlled release mechanism must be chosen and a very high leach rate must be specified for those radionuclides for which an instantaneous desorption release is desired. The time interval between intermediate time points will need to be made small enough to model the rapid release accurately.

\section{D.6 Releases from the Surface Soil}

The RESRAD-OFFSITE code computes the release of particulates to the atmosphere and to runoff using the radionuclide concentration in the surface layer. By default, only the material in the release-susceptible form is released from the surface layer. The user can choose to include both the release-susceptible form and the release-immune form in the atmospheric release calculations. The radionuclides that are released into the soil moisture in the primary contamination can resorb onto the solids in soil; these radionuclides are not included in the calculations of the releases from the surface soil even though some of those radionuclides may still be within the mixing layer.

\section{D.7 Connecting the Conceptual Options to Practical Situations}

Section 3.2.1 of this report discusses which of the three options of Version 3 might best represent the release from different waste materials. This section expands on that topic to include the features added in RESRAD-OFFSITE Version 3.1.

Engineered barriers, such as impermeable covers, waste containers, and grouting of the voids around and within the waste are designed to prevent water from infiltrating into the wastebearing material for certain periods of time. With the passage of time, however, some of these barriers, containers, and waste forms might deteriorate and moisture could penetrate them. The effect of engineered barriers in preventing release for a period of time can be modeled in RESRAD-OFFSITE by specifying a delay time. The period of time during which the engineered barrier is expected to be fully effective in preventing release should be entered in the delay time input captioned "Time at which radionuclides first become releasable."

When the engineered barrier starts to fail, it is likely to lose its integrity gradually. Most of a given barrier would still be effective when it first starts to deteriorate to the extent that moisture is able to start penetrating it. Only a small fraction of the waste material is likely to be exposed to this moisture at that time. This situation can be modeled by specifying the fraction of waste material that is exposed at the time when the barrier first fails in the input captioned "Fraction of radionuclide bearing material that is initially releasable." 
The engineered barrier could continue to deteriorate, and the amount of waste material that is subject to release would increase gradually with time. This somewhat compromised state can be modeled in RESRAD-OFFSITE by specifying the time period over which the barrier is expected to fail gradually in the input box captioned "Time over which transition to releasable form occurs" and by selecting the "linearly over time" option in the "Radionuclide bearing material becomes releasable" frame. If there are multiple engineered barriers, some of the waste would continue to be isolated. The input captioned, "Total fraction of radionuclide bearing material available for release" can be used to model this scenario.

Version 3.1 of RESRAD-OFFSITE also has the option to model the change in the releasable fraction of waste material as occurring stepwise in time. This option can be used to model situations where there are different types of waste material containing the same radionuclide in the disposal area.

Section 3.2.1 of the report discusses the choice of the release mechanisms-first-order, ratecontrolled release or instantaneous desorption-controlled release-that might be appropriate for modeling the release from different waste materials. Recall that the uniform release option discussed in that section is modeled in Version 3.1 by selecting the instantaneous desorption equilibrium release option in which radionuclide-bearing material becomes available for release linearly with time. For some wastes, either of these release mechanisms could apply depending on the chemical conditions of the waste and infiltrating moisture. Ion exchange resins could be used to illustrate this choice. If the partitioning between the resin and the infiltrating moisture occurs rapidly relative to the movement of moisture, then the instantaneous desorption equilibrium release option would be appropriate. If, on the other hand, the partitioning occurs slowly relative to the movement of moisture, the first-order, rate-controlled release option would be appropriate.

There are studies on source term releases for radionuclides on ion-exchange resins (Akers 2002, Robertson et al., 2000) and solubility and leaching of radionuclide releases from decommissioned sites (Felmy et al., 2002, 2003). These reports may be useful when using RESRAD-OFFSITE Version 3.1 source release models to simulate radionuclides' release mechanisms from waste disposal sites.

\section{D.8 References for Appendix D}

Akers, D.W., 2002, "Release of Radionuclides and Chelating Agents from Full-System Decontamination lon-Exchange Resins," NUREG/CR-6766, March.

Felmy, A.R., D. Rai, S.A. Hartley, and V.L. LeGore, 2002, "Solubility and Leaching of Radionuclides in Site Decommissioning Management Plan (SDMP) Slags," NUREG/CR-6632, February. 
Felmy, A.R., V.L. LeGore, and S.A. Hartley, 2003, "Solubility and Leaching of Radionuclides in Site Decommissioning Management Plan (SDMP) Soil and Ponded Wastes," NUREG/CR-6821, June.

Robertson, D.E., S.L. Pratt, and C.W. Thomas, 2000, "Adsorption and Desorption Behavior of Selected 10 CFR Part 61 Radionuclides from Ion Exchange Resins by Waters of Different Chemical Composition," NUREG/CR-6647, U.S. Nuclear Regulatory Commission, Washington, D.C., 20555-0001, July. 
This page is intentionally left blank. 


\section{Appendix E}

\section{Users' Guide to RESRAD-OFFSITE Code Area Factors}





\section{Appendix E}

\section{Users' Guide to RESRAD-OFFSITE Code Area Factors}

The area factor is the ratio of the dose from the whole (large or wide) area of primary contamination divided by the dose from a small (hot spot or elevated activity) area within the large (wide) area. The small (elevated activity) area is referred to as the elevated measurement comparison (EMC) area in the Multi-Agency Radiation Survey and Site Investigation Manual (MARSSIM) (MARSSIM Manual 2000). The area factor can be used in conjunction with MARSSIM applications to determine the Derived Concentration Guideline Level (DCGL) for a

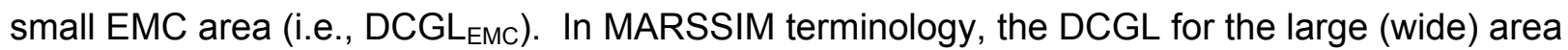
is called DCGLw. The DCGL is inversely proportional to the corresponding dose; therefore the area factor is equivalent to the quotient of $D C G L_{E M C}$ divided by $D C G L_{W}$, as shown in Equation E.1. The area factors are especially useful in field applications for quick determination of $D C G L_{E M C}$, which is simply the product of area factor and the DCGL .

$$
\begin{aligned}
\text { Area Factor } & =\frac{\text { Dose from wide Area of primary contamination }}{\text { Dose from small area of elevated activity }} \\
& =\frac{D C G L_{E M C}}{D C G L_{W}}
\end{aligned}
$$

RESRAD-OFFSITE code Version 3 provides users with an easy and flexible way to obtain the area factors for an offsite receptor scenario using the probabilistic analysis feature of the code. The first step is to prepare an input file just as in the case of a regular RESRAD-OFFSITE run. The layout of the site is specified in the site layout form or in the map interface, and siteappropriate parameter values are included for all of the variables in the RESRAD-OFFSITE interface. The "Generate Area Factors" command is then chosen from the File menu to display the Area Factors form (Figure E.1). The user can then specify parameters related to area factor calculations. The $X$ dimension of the small area of elevated contamination is always sampled. The $Y$ dimension of the small area of elevated contamination can either be sampled or it can be made proportional to the $X$ dimension. If the $Y$ dimension is to be sampled, the correlation between the samples of the $X$ and $Y$ dimensions can be specified. The location of the small area of elevated contamination can be fixed at the center of the primary contamination, or it can be allowed to move within the confines of the original primary contamination. 


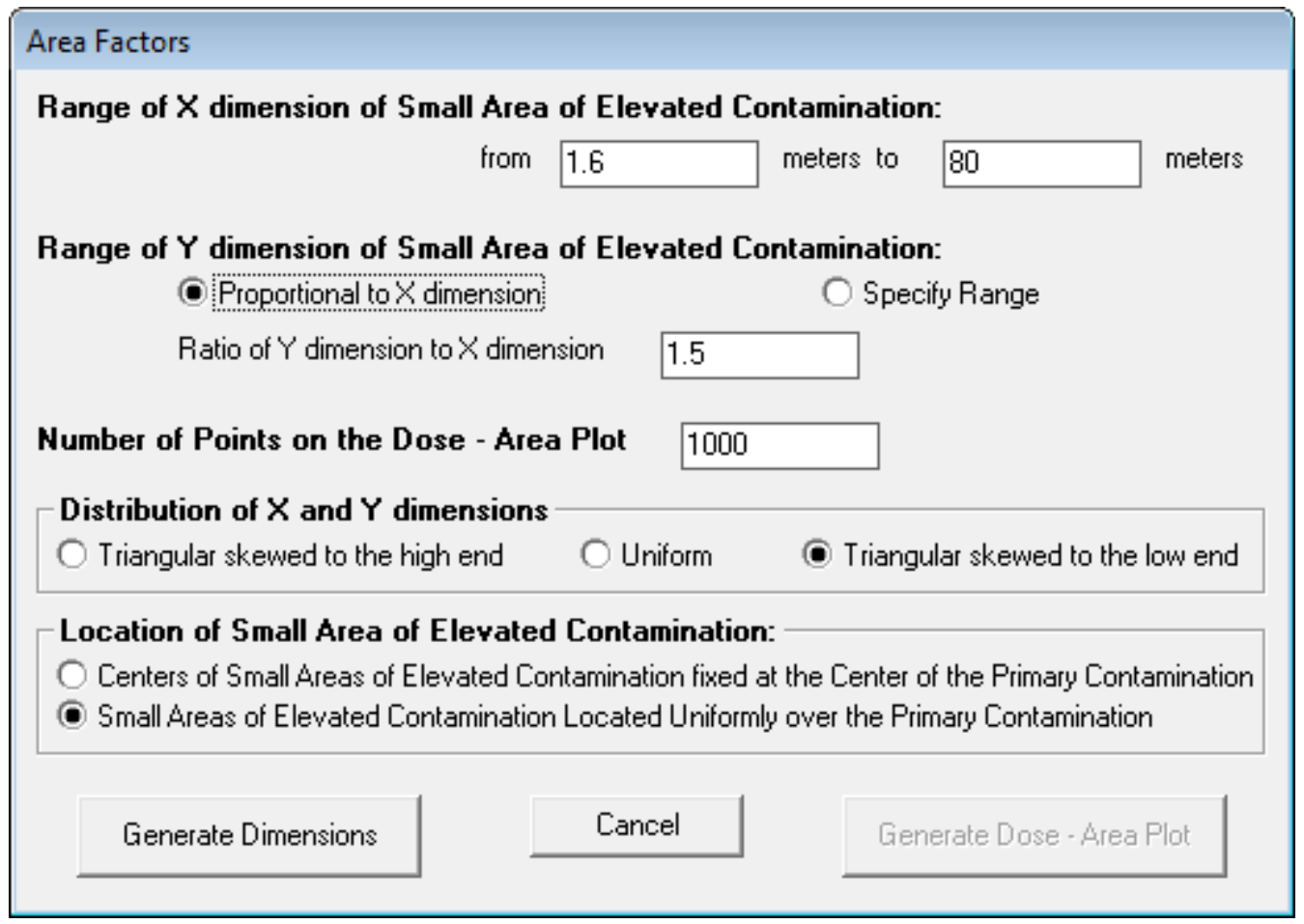

FIGURE E.1 Area Factors Form - Default Options

\section{E.1 The Range of the X Dimension of the Small Area of Elevated Contamination}

By default, the $X$ dimension of the small area of elevated contamination will be obtained by sampling a triangular distribution skewed to the low end ranging from 1/50 to 1 times the $X$ dimension of the primary contamination. The user can change the lower and upper limits; however, the upper limit may not exceed the $X$ dimension of the primary contamination, that is, the $X$ dimension of the small area of elevated contamination cannot be larger than the $X$ dimension of the primary contamination (wide area).

\section{E.2 The Range of the Y Dimension of the Small Area of Elevated Contamination}

By default, the $Y$ dimension for the small area of elevated contamination is set to be proportional to the $X$ dimensions of the small area of elevated contamination. The default proportionality constant is the ratio of the $Y$ dimension of the primary contamination to the $X$ dimension of the primary contamination (Figure E.1). The user may change the proportionality constant; 
however, the code will not then check to ensure that the resulting $Y$ dimension of the small area of elevated contamination is less than the $Y$ dimension of the primary contamination.

Alternatively, the $Y$ dimension can be sampled in the same manner as the $X$ dimension. If this option is chosen (Figure E.2), by default the $Y$ dimension will be sampled from a uniform distribution ranging from $1 / 50$ to 1 times the $Y$ dimension of the primary contamination. The user can change the lower and upper limits; however, as mentioned previously, the upper limit may not exceed the $Y$ dimension of the primary contamination. In this case, the user can specify the rank correlation coefficient between the $X$ and $Y$ dimensions of the small area of elevated contamination; the default value of 0.99 will produce results that are similar to the default proportionality option. A negative rank correlation coefficient would simulate small areas of elevated contamination that are elongated in either the $\mathrm{X}$ or $\mathrm{Y}$ directions.

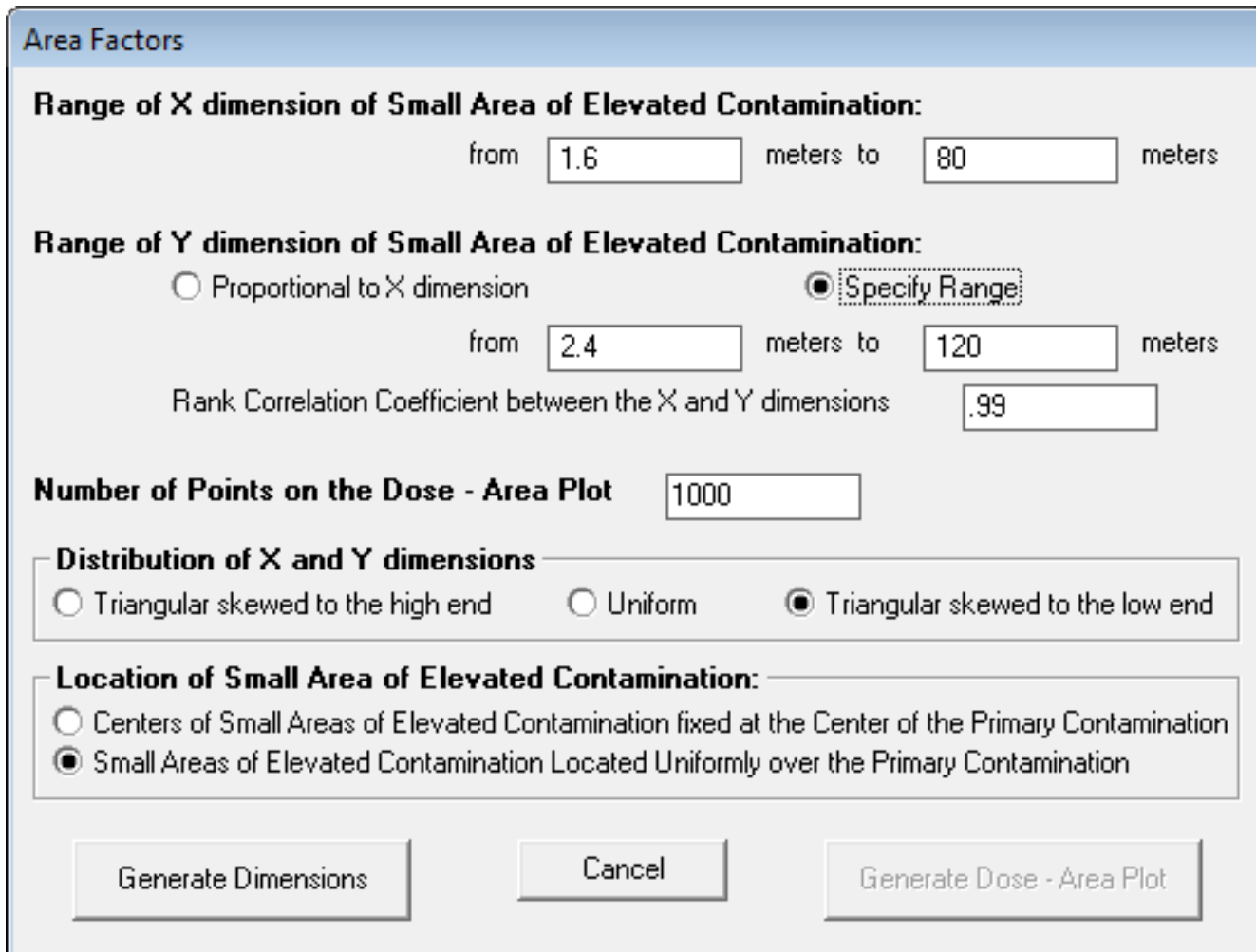

FIGURE E.2 Area Factors Form - Option to Specify the Range of the Y Dimension of the Small Area of Elevated Contamination 


\section{E.3 Distribution of the $X$ and $Y$ Dimensions of the Small Area of Elevated Contamination}

Three options are available for the distribution of the dimensions of the small area of elevated contamination: (1) triangular distribution skewed to the high end, (2) uniform distribution, and (3) triangular distribution skewed to the low end. Option 1 (i.e., triangular distribution skewed to the high end) will result in uniform spacing of the sampled contamination area. This result can be seen from the cumulative distribution function of the area that was produced in the top graph of Figure E.3. 


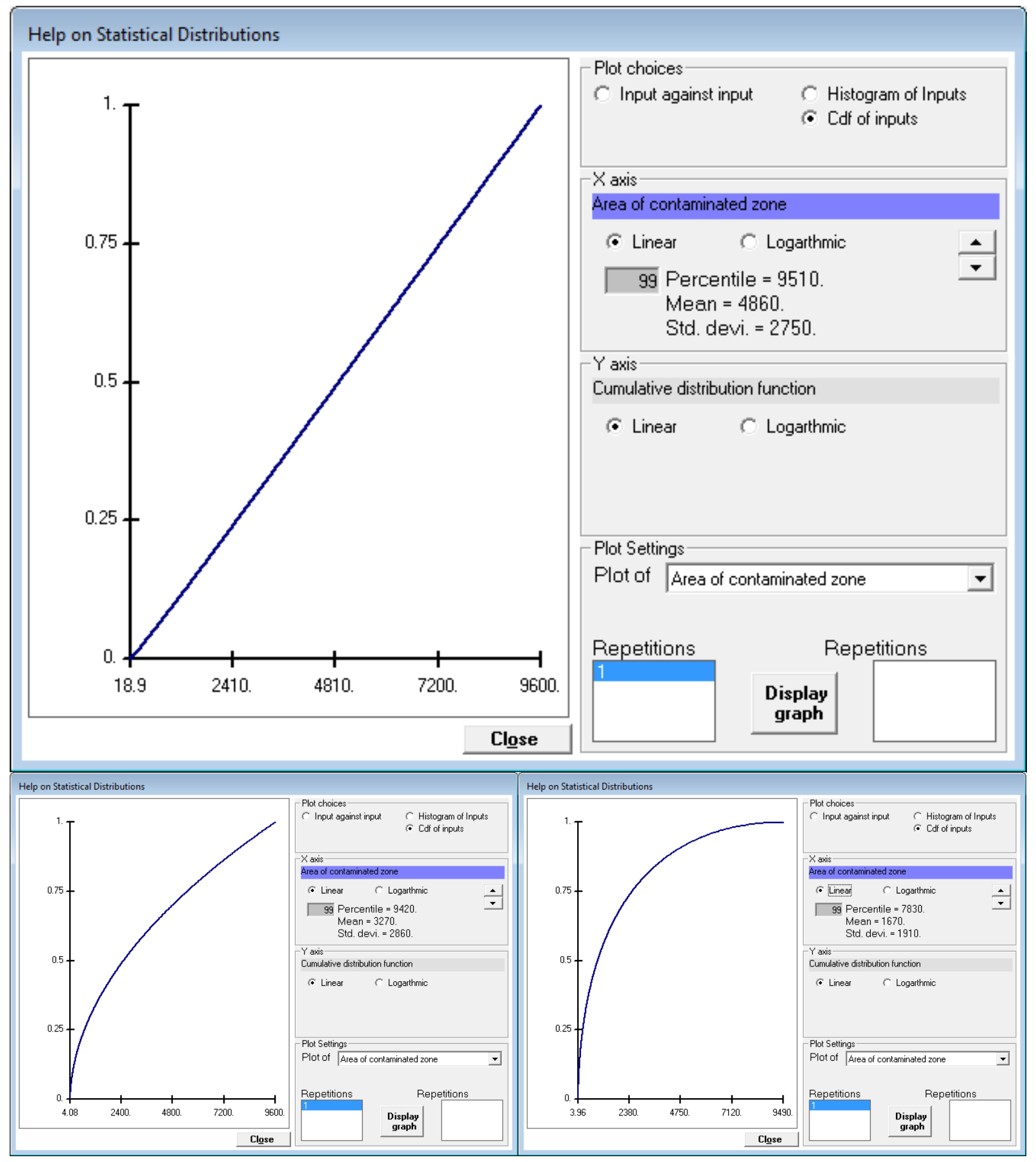

FIGURE E.3 Distribution of Area for the Small Area of Elevated Contamination Using the Three Distributions Options for Sampling the Dimensions of the Small Area of Elevated Contamination: triangular skewed to the high end (top), uniform (bottom left), and triangular skewed to the low end (bottom right) 
Option 2 (i.e., sampling the dimensions uniformly) will result in a distribution of the contamination area that is skewed to the lower end. The distribution of the sampled area will be skewed even more toward the low end if Option 3 (i.e., triangular distribution that is skewed to the low end) is selected. Either Option 2 or Option 3 would be appropriate if the areas of elevated contamination are likely to be small and a user desires to sample a large number of small areas.

\section{E.4 Location of the Center of the Small Area of Elevated Contamination}

There are two choices for the location of the center (or centroid) of the small area of elevated contamination: it can be fixed at the center of the primary contamination, or it can be allowed to vary. The fixed option will produce a tighter curve between the dose and the small area of elevated contamination. Under the variable option, the small area of elevated contamination is constrained to be within the primary contamination. Thus, the center has to lie within a rectangle with dimensions that are the difference between the corresponding dimensions of the primary contamination and the small area of elevated contamination. The code samples two unit uniform distributions and uses those samples to locate the center of the small area of elevated contamination. A sample value of zero for the $X$ location would place the left edge of the small area of elevated contamination on the left boundary of the primary contamination; a unit value would place the right edge of the small area of elevated contamination on the right boundary of the primary contamination. Likewise, a sample value of zero for the $Y$ location would place the lower edge of the small area of elevated contamination on the lower boundary of the primary contamination; a unit value would place the top edge of the small area of elevated contamination on the upper boundary of the primary contamination. Because the calculated dose will vary with the location of the small area of elevated contamination, the first option (centers fixed) could produce area factors that are higher than reasonable, whereas the second option (moving centers) will produce conservative area factors.

\section{E.5 Number of Points on the Dose - Area Plot}

This parameter specifies the number of times the distribution of the dimensions of the area of elevated contamination is to be sampled. The default value is 1,000. A smaller value can be used if the dose-area relationship is expected to be in a tight band or a single curve; this result will be the case if the $Y$ dimension of the small area of elevated contamination is set to be proportional to the $X$ dimension and the center is fixed. A larger value might be required if the dose-area relationship is expected to be in the shape of a wide band. This result could be the case when both dimensions of the small area of elevated contamination are sampled and/or the location of these small areas of elevated contamination was allowed to vary. 


\section{E.6 Generate Dimensions}

The Generate Dimensions command button is used to generate the layout-dimensions and location-of each sample of area of elevated contamination and the offsite transport distances from each sample of area of elevated contamination to the offsite receptor locations. The Generate Dimension command must be clicked after the user does the following: specifies the limits and type of distribution for the $X$ dimension of the small area of elevated contamination, chooses the method of determining the $Y$ dimension and specifying the required values for that method, selects the desired location option for the centers of the small areas of elevated contamination, and sets the number of samples. This command button first launches the probabilistic sampling code to sample all of the specified distributions. That information is then read by the interface, which uses the sampling results to develop the relationships for the offsite transport distances. Three types of plots of the dimension that were generated can be viewed before proceeding to generate the dose from each area of elevated contamination: scatter plots, histograms, and cumulative distribution functions. These plots are used to verify that the desired layout and transport distances have been generated. The Generate Dose-Area Plot command button becomes active after the layout and transport distances have been generated (Figure E.4).

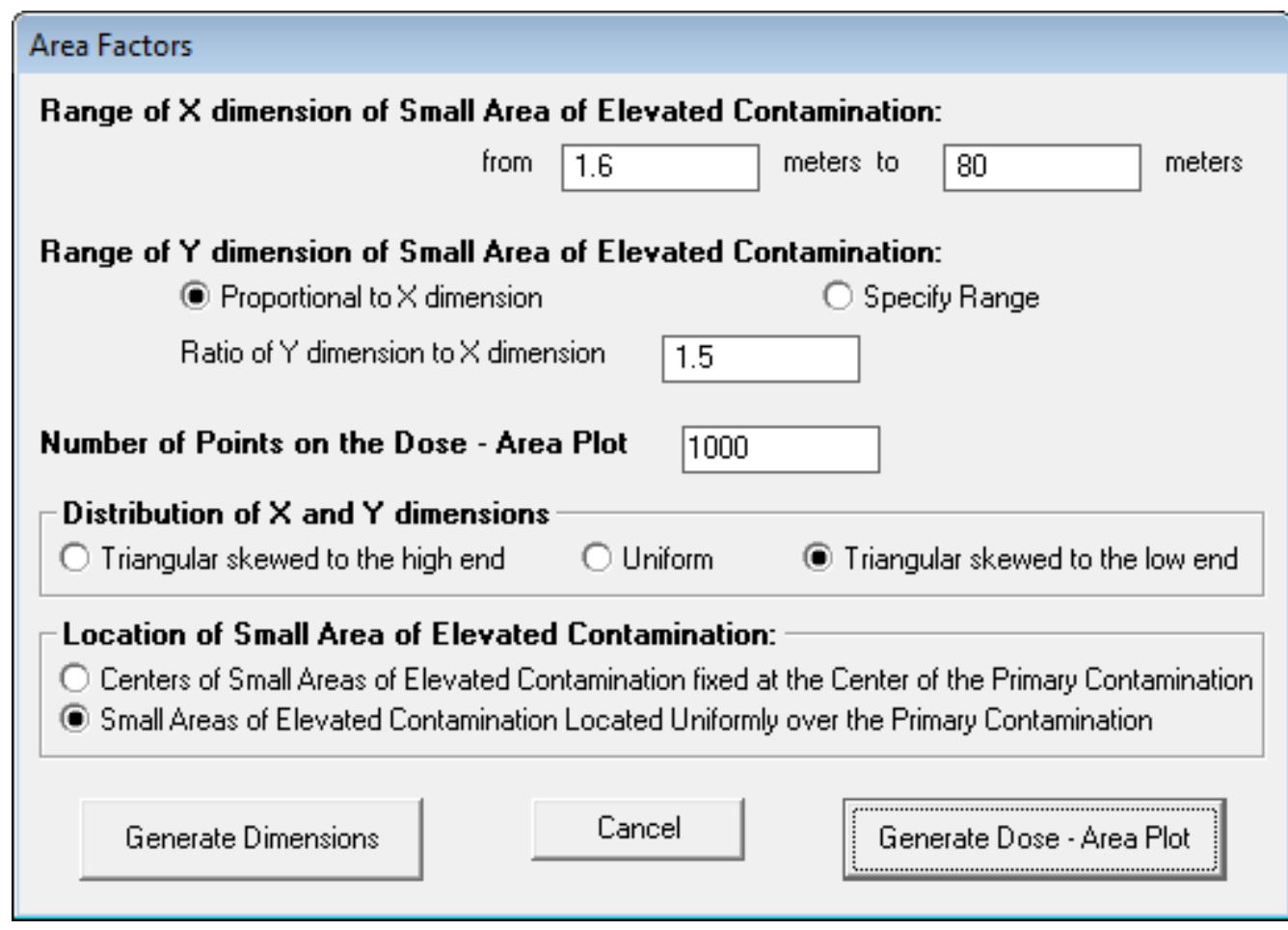

FIGURE E.4 Area Factors Form - Generate Dose-Area Plot Command Button Activated 


\section{E.7 Generate Dose - Area Plot}

This command launches the computational code of RESRAD-OFFSITE to process the inputs for each of the sampled areas of elevated contamination, generating the dose from each of the areas of elevated contamination. A scatter plot of dose against the area of elevated contamination is displayed at the end of the run. Four sample plots, obtained by using the four options, for three different radionuclides (i.e., Cs-137, Sr-90, and Tc-99) are shown in Figures E.5 through E.7. The four options are: (a) the $Y$ dimensions of the small area of elevated contamination are proportional to the $X$ dimension of the small area of elevated contamination with the center of the area of elevated contamination coinciding with the center of the whole area of primary contamination; (b) the $Y$ dimensions of the small area of elevated contamination are proportional to the $X$ dimension, and the area of elevated contamination is located within the whole area of primary contamination but is not constrained to be concentric; (c) the $Y$ dimensions of the small area of elevated contamination are sampled and paired with the samples of the $X$ dimension at a specified correlation and the center of the area of elevated contamination coincides with the center of the whole area of primary contamination; and (d) the $Y$ dimensions of the small area of elevated contamination are sampled and paired with the samples of the $X$ dimension at a specified correlation and the area of elevated contamination is located within the whole area of primary contamination but is not constrained to be concentric. The area factor is the ratio of the dose from the large (wide) area of primary contamination divided by the dose from the smaller area of elevated contamination within the large (wide) area. The calculated area factors with the corresponding areas are saved in a file named "AreaFactorText.REP." This file contains a list of up to 21 pairs of areas and area factors for each radionuclide analyzed. When more than 21 samples are used (the default is 1,000 ), the code groups the samples into 21 intervals based on area. Then the code determines the area within each interval for which the dose/area ratio is the greatest. These 21 areas and doses are used to generate the area factor text report, AreaFactorText.REP. 


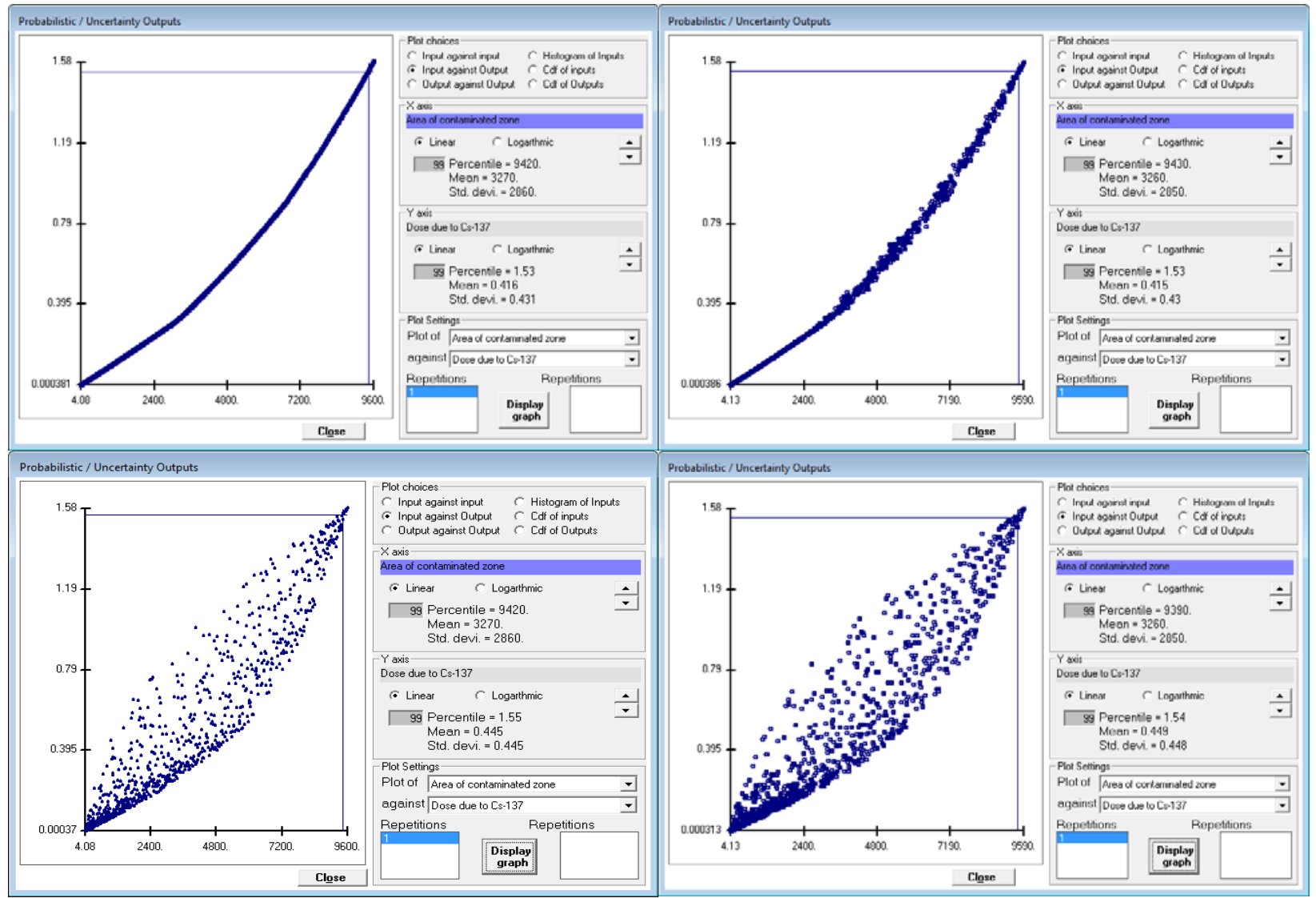

FIGURE E.5 Scatter Plot of Dose against Area of Contamination for Cs-137 Where the Direct External Exposure Is the Dominant Pathway. (a) Top left, $Y$ dimension is proportional to the $X$ dimension; the center of the area of elevated contamination is located at the center of the area of primary contamination. (b) Bottom left, $Y$ dimension is proportional to the $X$ dimension; area of elevated contamination is located anywhere within the area of primary contamination. (c) Top right, $X$ and $Y$ dimensions are highly correlated (rank regression coefficient 0.99 ); the center of area of elevated contamination is located at the center of the area of primary contamination. (d) Bottom right, $X$ and $Y$ dimensions are highly correlated (rank regression coefficient 0.99 ); the area of elevated contamination is located anywhere within the area of primary contamination. 


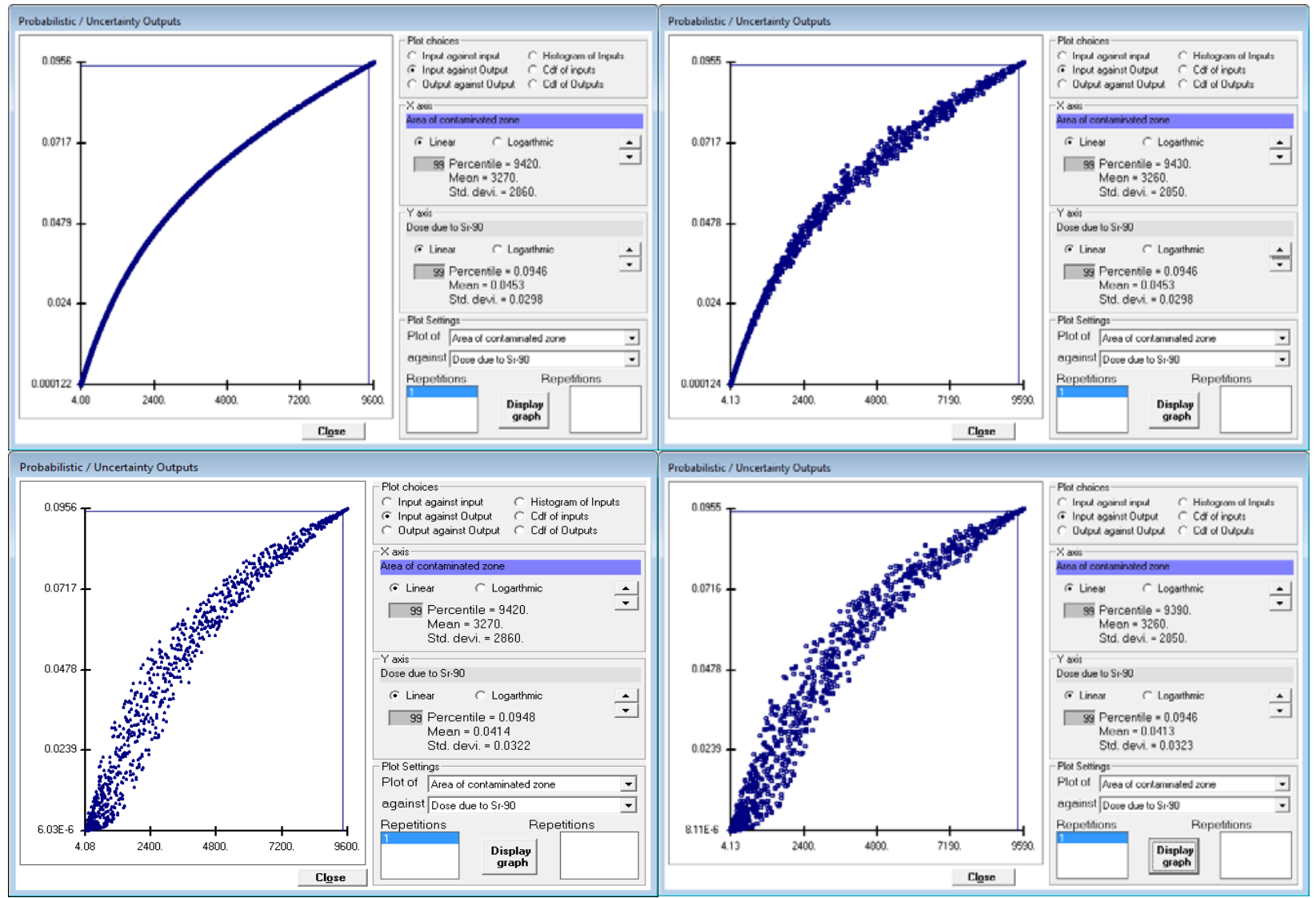

FIGURE E.6 Scatter Plot of Dose against Area of Contamination for Sr-90 for Which Groundwater Transport Is the Dominant Route of Exposure. (a) Top left, $Y$ dimension is proportional to the $X$ dimension; the center of the area of elevated contamination is located at the center of the area of primary contamination. (b) Bottom left, $Y$ dimension is proportional to the $X$ dimension; the area of elevated contamination is located anywhere within the area of primary contamination. (c) Top right, $X$ and $Y$ dimensions are highly correlated (rank regression coefficient 0.99 ); the center of the area of elevated contamination is located at the center of the area of primary contamination. (d) Bottom right, $X$ and $Y$ dimensions are highly correlated (rank regression coefficient 0.99 ); the area of elevated contamination is located anywhere within the area of primary contamination. 


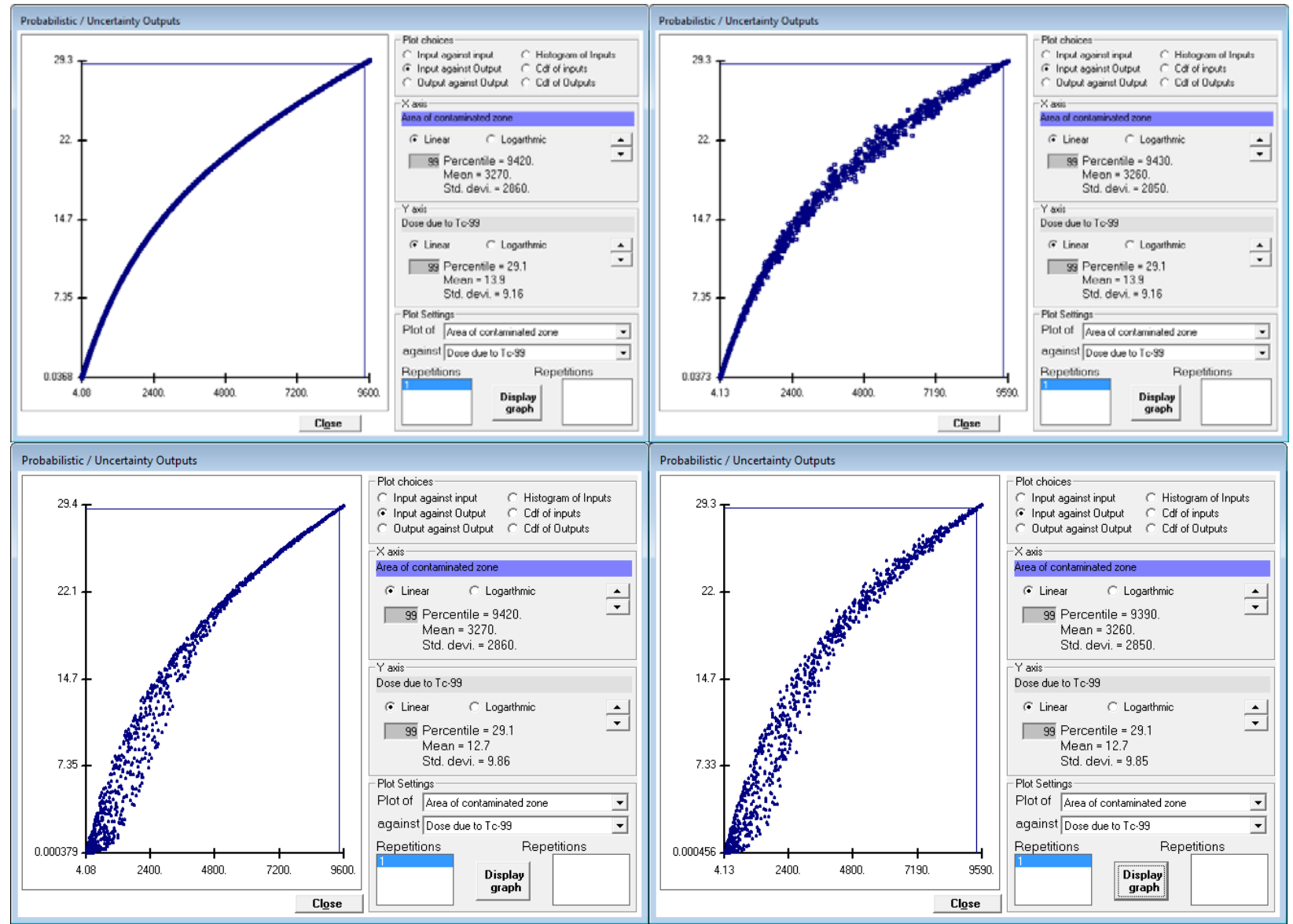

Figure E.7 Scatter Plot of Dose against Area of Contamination for Tc-99 for Which Groundwater Transport Is the Dominant Route of Exposure. (a) Top left, $Y$ dimension is proportional to the $X$ dimension; the center of the area of elevated contamination is located at the center of the area of primary contamination. (b) Bottom left, $Y$ dimension is proportional to the $X$ dimension; the area of elevated contamination is located anywhere within the area of primary contamination. (c) Top right, $X$ and $Y$ dimensions are highly correlated (rank regression coefficient 0.99 ); the center of the area of elevated contamination is located at the center of the area of primary contamination. (d) Bottom right, $X$ and $Y$ dimensions are highly correlated (rank regression coefficient 0.99 ); the area of elevated contamination is located anywhere within the area of primary contamination. 


\section{E.8 Discussion}

The area factors generated by the RESRAD-OFFSITE code Version 3, as listed in the "AreaFactorText.REP" file, are for the specific scenario analyzed. They are derived on the basis of pathways selected and parameter values used for that particular case. Therefore, for different scenarios, the calculated area factors will most likely be different. Area factors, which are radionuclide-specific, describe the extent to which a radionuclide concentration can exceed the DCGL $w$ and still comply with the release criteria. In the "AreaFactorText.REP" file, the area factors for different radionuclides are listed separately in tabular format. The radionuclidespecific area factor $\left(\mathrm{AF}_{\mathrm{i}}\right)$ for radionuclide $i$ can be used with the wide area, radionuclide-specific $D C G L_{W_{i}}$ to calculate the small area $D C G L_{E M C_{i}}$ as in Equation E.2.

$$
\text { Area Factor }_{i}=\frac{D C G L_{E M C_{i}}}{D C G L_{W_{i}}}
$$

If there are multiple radionuclides in the contaminated area, the sum of fractions rule can be used to determine whether the dose criterion is exceeded or not.

\section{E.9 Reference for Appendix E}

MARSSIM Revision 1, 2000, Multi-Agency Radiation Survey and Site Investigation Manual (MARSSIM), NUREG-1575, Rev. 1, EPA 402-R-97-016, Rev. 1, DOE/EH-0624, Rev. 1, August. 